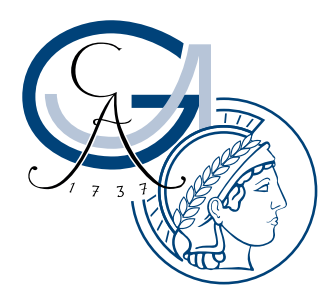

\title{
Reconstitution of Doa10-mediated ER-associated protein degradation with purified components
}

\author{
Dissertation \\ for the award of the degree \\ "Doctor rerum naturalium" \\ of the Georg-August-Universität Göttingen \\ within the doctoral program Molecular Biology \\ of the Georg-August University School of Science (GAUSS)
}

submitted by

Claudia C. Schmidt

from Landshut, Germany

Göttingen, 2019 


\section{Thesis Advisory Committee}

Dr. Alexander Stein (Supervisor, Reviewer)

Research Group Membrane Protein Biochemistry

Max Planck Institute for Biophysical Chemistry, Göttingen, Germany

Prof. Dr. Blanche Schwappach-Pignataro (Reviewer)

Department of Molecular Biology

University Medical Center, Göttingen, Germany

\section{Prof. Dr. Holger Stark}

Research Group Structural Dynamics

Max Planck Institute for Biophysical Chemistry, Göttingen, Germany

\section{Extended Thesis Advisory Committee}

\section{Dr. Alex Faesen}

Research Group Biochemistry of Signal Dynamics

Max Planck Institute for Biophysical Chemistry, Göttingen, Germany

\section{Prof. Dr. Peter Rehling}

Department of Cellular Biochemistry

University Medical Center Göttingen, Germany

\section{Prof. Dr. Henning Urlaub}

Research Group Bioanalytical Mass Spectrometry

Max Planck Institute for Biophysical Chemistry, Göttingen, Germany

Date of oral examination: 25.11 .2019 
Herewith I declare that I prepared the doctoral thesis entitled Reconstitution of Doa10-mediated ER-associated protein degradation with purified components on my own and with no other sources and aids than quoted.

Claudia Schmidt

Göttingen, October 2019 



\section{Abstract}

ER-associated protein degradation (ERAD) is a protein quality control pathway at the ER membrane mediated by membrane-embedded ubiquitin ligase complexes. During ERAD, membrane and luminal proteins are ubiquitinated and retrotranslocated back into the cytosol where they are degraded by the proteasome. The conserved ubiquitin ligase Doa10 mediates degradation of ER membrane proteins as well as cytosolic and nuclear proteins. It acts with the E2 enzymes Ubc6 and Ubc7. How Doa10 substrates are recognized, ubiquitinated and retrotranslocated is not well understood.

In this thesis, I have established a system to investigate the mechanism of Doa10mediated ERAD using purified components from S. cerevisiae. Membrane proteins are hereby reconstituted into separate sets of phospholipid vesicles and co-reconstituted upon SNARE-mediated fusion. Using this approach, I was able to recapitulate ubiquitination and extraction of the tail-anchored membrane protein Ubc6 which is itself unstable and degraded in a Doa10-dependent manner. In the presence of Doa10, I observe spontaneous retrotranslocation of the transmembrane (TM) anchor of Ubc6. A folded luminal domain attached to Ubc6 inhibits spontaneous retrotranslocation. Extraction can be restored upon ubiquitination and extraction by the Cdc48/UN complex. My results show that Doa10 is a retrotranslocase and indicate that Doa10 and Cdc48 cooperate in extraction of membrane proteins.

In order to characterize structural elements in Doa10, I tested Doa10 truncations as well as previously described Doa10 mutants in the reconstituted system. My results show that the Doa10 TM domain is required for ubiquitination and spontaneous retrotranslocation of Ubc6. The tested Doa10 mutants did not show any differences. Thus, to identify interaction sites between the TM domains of Ubc6 and Doa10, a site-specific photocrosslinking approach was optimized.

To extend conclusions obtained from Ubc6 to another Doa10 substrate, ERAD of the tail-anchored membrane protein Sbh2 was investigated. To characterize the machinery required for ERAD of Sbh2, we first performed a screen, as the machinery for cytosolic, but not membrane-bound Doa10 substrates has been characterized in screens until now. The screen identified described ERAD components as the most conclusive hits. Thus, I next characterized Sbh2 in a reconstituted system and identified a minimal machinery for Doa10-mediated ubiquitination of Sbh2.

In conclusion, this thesis provides mechanistic insights into Doa10-mediated ERAD of membrane proteins. It also provides a tool for co-reconstituting membrane proteins for studying ERAD as well as other membrane-associated processes in the future. 


\section{Contents}

$\begin{array}{lll}\text { Abstract } & \text { V }\end{array}$

1 Introduction 1

1.1 Protein degradation by the ubiquitin proteasome system . . . . . . . 1

1.1.1 The E1/E2/E3 enzymatic cascade for ubiquitination . . . . . 1

1.1.2 Degradation by the proteasome . . . . . . . . . . . . 2

1.1.3 The UPS operates with multiple pathways for protein degrada-

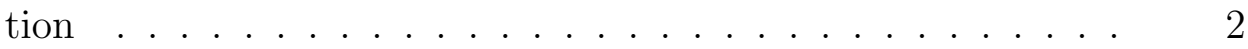

1.2 ER-associated protein degradation (ERAD) . . . . . . . . . 3

1.2.1 ERAD substrates . . . . . . . . . . . . . . . . . 4

1.2.2 The Hrd1 complex . . . . . . . . . . . . . . . . . . . . . . . 11

1.2.3 The Doa10/TEB4 complex . . . . . . . . . . . . . . . . 14

1.2.4 Substrate recognition . . . . . . . . . . . . . . 16

1.2.5 Retrotranslocation of substrates . . . . . . . . . . . 19

1.2.6 Questions regarding the mechanism of Doa10-mediated ERAD 22

1.3 Reconstitution approaches to study the function of membrane proteins 23

1.4 Aims . . . . . . . . . . . . . . . . . . 26

2 Materials and Methods $\quad 27$

2.1 Materials . . . . . . . . . . . . . . . . . . 27

2.2 General methods for DNA cloning and protein expression . . . . . . . 29

2.3 Reconstitution of Doa10-mediated ERAD of Ubc6 . . . . . . . . . . . 30

2.3.1 Strains used for protein expression . . . . . . . . . . . . . 30

2.3 .2 Constructs . . . . . . . . . . . . . . . . 30

2.3.3 Expression and purification of proteins . . . . . . . . . . 32

2.3.4 Sortase-mediated labeling . . . . . . . . . . . . . 35

2.3.5 Reconstitution into proteoliposomes . . . . . . . . . . . 36

2.3.6 Ubiquitination assays . . . . . . . . . . . . . . . . . . . . 39

2.3.7 Measuring extraction by the Cdc48-complex . . . . . . . . . 40 
2.3.8 Assays for release by Doa10 . . . . . . . . . . . . . . . . . 41

2.3.9 Ubc6-SBP . . . . . . . . . . . . . . . . . . . . . . 42

2.3.10 Analysis . . . . . . . . . . . . . . . . . . 4 44

2.4 Optimization of SNARE-mediated fusion assay . . . . . . . . . . 44

2.4.1 Constructs . . . . . . . . . . . . . . . . . . . . . 44

2.4.2 Expression and purification of $\mathrm{TF}_{\mathrm{o}} \mathrm{F}_{1}$ ATP synthase . . . . . 45

2.4.3 Reconstitution of $\mathrm{TF}_{\mathrm{o}} \mathrm{F}_{1}$ ATP synthase . . . . . . . . . . 45

2.4.4 Purification of Doa10 by size-exclusion chromatography . . . . 46

2.5 Doa10 TD mutants . . . . . . . . . . . . . . . . . 46

2.5.1 Constructs . . . . . . . . . . . . . . . . 46

2.5.2 Purification and reconstitution of Doa10 variants containing a mutation in the TD domain . . . . . . . . . . . . 46

2.5.3 Ubiquitination assay . . . . . . . . . . . . . . . . . . 47

2.5.4 Release assay using an anti-A488 antibody . . . . . . . . . 47

2.6 Site-specific photocrosslinking . . . . . . . . . . . . 47

2.6.1 Constructs and strains . . . . . . . . . . . . . . . . 47

2.6 .2 Expression of $\mathrm{Ubc} 6_{\mathrm{BpA}}$ variants . . . . . . . . . . . 48

2.6.3 Reconstitution of Ubc6 $6_{\mathrm{BpA}}$ variants and Doa10 . . . . . . . . 49

2.6.4 Photocrosslinking . . . . . . . . . . . . . . . . . . 49

2.6.5 Mass spectrometry . . . . . . . . . . . . . 50

2.7 Tandem fluorescent timer screen . . . . . . . . . . . . . 51

2.7.1 Constructs and strains . . . . . . . . . . . . . . 51

2.7.2 Microscopy . . . . . . . . . . . . . . . . . 52

2.7.3 Analysis ....................... 53

2.7.4 Cycloheximide chase ............... 54

2.7.5 Analysis of stability of tFT-Sbh2 and tFT-Sbh1 using a microplate reader . . . . . . . . . . . . . . 55

2.8 Purification and reconstitution of Sbh2 . . . . . . . . . . 55

2.8.1 Constructs and strains . . . . . . . . . . . . . . . 55

2.8.2 Expression and purification of Sbh2 . . . . . . . . . 55

2.8.3 Fluorescent labeling of Sbh2 . . . . . . . . . . . 56

2.8.4 Reconstitution of Sbh2 . . . . . . . . . . . 56

2.8.5 Ubiquitination assay . . . . . . . . . . . . 56

3 Retrotranslocation of a tail-anchored membrane protein by the ubi$\begin{array}{ll}\text { quitin ligase Doa10 } & 58\end{array}$

3.1 Introduction . . . . . . . . . . . . . . . . 59 
3.2 Results . . . . . . . . . . . . . . . . . . . 61

3.2.1 Membrane-reconstitution of Doa10 and Ubc6 . . . . . . . . . 61

3.2.2 Mono-Ubiquitination of Ubc6 by Doa10 . . . . . . . . . . . . 62

3.2.3 Ubc7-dependent polyubiquitination of Ubc6 . . . . . . . . 64

3.2.4 Sequential autoubiquitination of Doa10 . . . . . . . . 66

3.2.5 Membrane extraction by the Cdc48 ATPase complex . . . . . 67

3.2.6 Doa10 facilitates membrane release of Ubc6 . . . . . . . . . 69

3.2.7 Structural elements in Doa10 required for retrotranslocation and ubiquitination . . . . . . . . . . . . . . . 73

3.2.8 Structural determinants in Ubc6 . . . . . . . . . . . . . . 75

3.2.9 A folded luminal domain prevents spontaneous release . . . . 78

3.2.10 Cdc48 action breaks luminal interactions . . . . . . . . . . . . 79

3.3 Discussion . . . . . . . . . . . . . . . . . . . . . . . 81

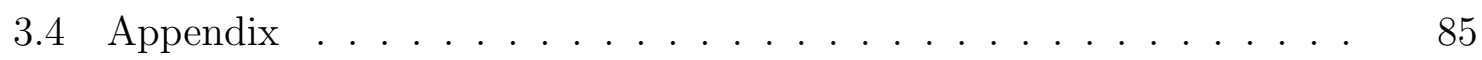

4 Optimization of SNARE-mediated fusion assay $\quad 97$

4.1 Stability of liposomes after SNARE-mediated fusion . . . . . . . . . . 98

4.1.1 Optimization of lipid to protein ratio . . . . . . . . . . . 98

4.1.2 Optimization of purification method for Doa10 . . . . . . . . 102

4.2 Identification of a suitable fluorescent label for Ubc6 . . . . . . . . . 104

5 Identification of structural elements important for Doa10 function 106

5.1 Characterization of Doa10 variants containing mutations in the TD domain . . . . . . . . . . . . . . . . . 106

5.2 Identification of interaction sites of Ubc6 with Doa10 by photocrosslinking . . . . . . . . . . . . . . . . . . . 109

6 Analysis of Doa10-mediated ERAD of Sbh2 114

6.1 Identification of components necessary for degradation of Sbh2 . . . . 114

6.2 Reconstitution of Doa10-mediated ERAD of Sbh2 . . . . . . . . . . 121

6.2.1 Identification of a suitable Sbh2 construct for reconstitution studies ............................... 121

6.2.2 Purification and reconstitution of Sbh2 . . . . . . . . . 123

6.2.3 Characteristics of Doa10-mediated ubiquitination of Sbh2 . . . 124

7 Discussion $\quad 126$

7.1 Co-reconstitution of membrane proteins by SNARE-mediated membrane fusion . . . . . . . . . . . . . . . . . 
7.1.1 SNARE-mediated fusion enables high co-reconstitution efficiencies . . . . . . . . . . . . . . . . .

7.1.2 SNARE-mediated fusion allows the use of a lipid composition mimicking the ER membrane . . . . . . . . . . . . 127

7.1.3 Liposome instability is a result of low lipid to protein ratios . 128

7.2 A minimal system for Ubc6 polyubiquitination . . . . . . . . . . . . . 129

7.2.1 Mono-ubiquitination of Ubc6 depends on its autoubiquitination activity and is required for subsequent Ubc7/Cue1-mediated polyubiquitination . . . . . . . . . . . . 129

7.2.2 Proteasomal degradation of Ubc6 might have a regulatory role $\quad 130$

7.3 Mechanistic insights into retrotranslocation of membrane proteins . . 131

7.3.1 Cdc48/UN extracts polyubiquitinated Ubc6 . . . . . . . . 131

7.3.2 Translocation systems contain a driving force and often a protein conduit . . . . . . . . . . . . . . . . . . 131

7.3.3 Doa10 facilitates retrotranslocation of Ubc6 . . . . . . . . 132

7.3.4 Luminal unfolding occurs concomitant with protein extraction 134

7.3.5 Role of the retrotranslocase activity of Doa10 . . . . . . . . . 134

7.3.6 The role of protein conduits for dislocation and insertion of membrane proteins into the lipid bilayer . . . . . . . . . . . . . 135

7.3.7 Role of Ubx2 and Dfm1 . . . . . . . . . . . . . . . . 136

7.4 Towards structural insights of Doa10 . . . . . . . . . . . . 137

7.4.1 Function of the TD-domain . . . . . . . . . . . . 138

7.4.2 Identification of interaction sites of the TM anchor of Ubc6 with

Doa10 by site-specific crosslinking . . . . . . . . . . . . . 139

7.5 The machinery for ERAD of Sbh2 . . . . . . . . . . . . . . . . 140

7.5.1 Tandem fluorescent timer screens are a useful screening tool . 141

7.5.2 Screening for components required for degradation of Sbh2 . 141

7.5.3 Potential role of the deubiquitinase Ubp3 . . . . . . . . . . . . 142

7.5.4 Minimal machinery for ubiquitination of Sbh2 . . . . . . . 143

7.5.5 Ubc6 and Ubc7 have different functions . . . . . . . . . . . . . 144

7.6 Outlook . . . . . . . . . . . . . . . . . . . . 144

8 Supplement $\quad 146$

$\begin{array}{ll}\text { Acknowledgements } & 187\end{array}$

$\begin{array}{lr}\text { Published work } & 189\end{array}$ 


\section{List of Figures}

1.1 Localization of the ERAD ubiquitin ligases . . . . . . . . . . . . . 4

1.2 Substrates of Doa10-mediated ERAD . . . . . . . . . . . . . . . 8

1.3 The Asi, Hrd1 and Doa10 complex and its components . . . . . . . . 13

3.1 Doa10 catalyzes intramolecular mono-ubiquitination of Ubc6 at multiple

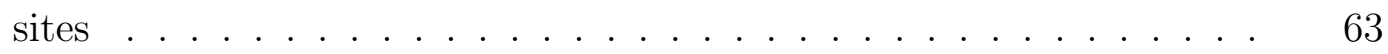

3.2 Polyubiquitination of Ubc6 by Doa10, Cue1 and Ubc7 . . . . . . . . 65

3.3 The Cdc48 complex catalyzes membrane extraction of polyubiquitinated Ubc6 . . . . . . . . . . . . . . . . . . . . . 68

3.4 Release of Ubc6 from Doa10 containing liposomes . . . . . . . . . . . 71

3.5 Structural determinants of retrotranslocation in Doa10 . . . . . . . . 74

3.6 Structural determinants in Ubc6 . . . . . . . . . . . . . . . . . 77

3.7 Influence of a luminal folded domain bound to Ubc6 . . . . . . . . . 80

A1 Related to Figure $3.1 \ldots \ldots \ldots$. . . . . . . . . . . 85

A2 Related to Figure $3.2 \ldots \ldots \ldots$. . . . . . . . . . . . . 87

A3 Related to Figure $3.3 \ldots \ldots \ldots$. . . . . . . . . . . . . . . . . 89

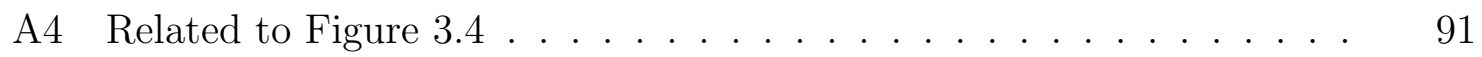

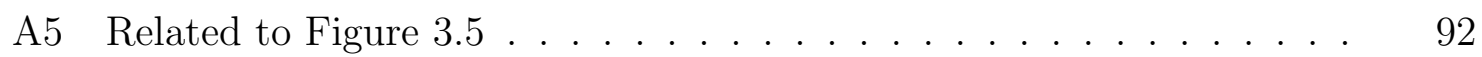

A6 Related to Figure $3.6 \ldots \ldots$. . . . . . . . . . . . . . . . . . . 93

A7 Related to Figure $3.7 \ldots \ldots \ldots \ldots$. . . . . . . . . . . . . 95

4.1 Titration of SNARE-concentration for SNARE-mediated fusion assay 100

4.2 Co-reconstitution of $\mathrm{TF}_{\mathrm{o}} \mathrm{F}_{1}$ ATP synthase with Syb into liposomes . . 101

4.3 Titration of ATP synthase and Doa10 for SNARE-mediated fusion assay 102

4.4 Testing SEC and sucrose density gradient centrifugation as purification method for Doa10 . . . . . . . . . . . . . . . . . . . . 103

4.5 Influence of the C-terminal fluorescent label of Ubc6 on substrate behaviour . . . . . . . . . . . . . . . . . 105

5.1 Ubiquitination of Ubc6 in the presence of Doa10 $\mathrm{E} 633 \mathrm{D}$, Doa10 $\mathrm{E} 633 \mathrm{Q}$ or Doa10 WT . . . . . . . . . . . . . . . . . . . . . . 108 
5.2 Release of Ubc6 by Doa10 TD mutants . . . . . . . . . . . . . . . . . 109

5.3 Crosslinking of Ubc6 $6_{\mathrm{BpA}}$ variants with Doa10 . . . . . . . . . . . 111

5.4 Photocrosslinking of liposomes containing Ubc6 6 M249BpA-A488 and Doa10 after incubation with anti-A488 antibody . . . . . . . . . . . 113

6.1 Characterization of tFT screen analysis . . . . . . . . . . . . . 117

6.2 Comparison of results for the 348 selected strains of both screens . . . 118

6.3 Identified hits in the tFT screen . . . . . . . . . . . . . . . . . . 119

6.4 Effect of UBP3 deletion on Sbh2 stability . . . . . . . . . . . . . 120

6.5 Degradation of Sbh2 variants in wildtype and $\Delta$ doa10 cells . . . . . . 122

6.6 Degradation of Sbh2 $2_{89 \mathrm{C}}$ in wildtype, $\Delta$ doa10 and $\Delta$ ubc6 cells . . . . . 123

6.7 Fluorescent labeling and reconstitution of Sbh2 . . . . . . . . . . . . 124

6.8 Ubiquitination of Sbh2 in a reconstituted system . . . . . . . . . . 125

7.1 Hydropathy profile of Ubc6 and Syb . . . . . . . . . . . . . . 133

S1 Purification of ATP synthase . . . . . . . . . . . . . . . 146

S2 Purification of Ubc6 $\mathrm{B}_{\mathrm{BpA}}$ variants . . . . . . . . . . . . . . . . 147

S3 Direct co-reconstitution of Ubc6 and Doa10 . . . . . . . . . . . . . 148

S4 Distribution of cells per well . . . . . . . . . . . . . . . . . . . . . 149

S5 Localization of tFT-Sbh2 in $\Delta \mathrm{ssm} 4, \Delta \mathrm{ubp} 3$ and $\Delta \mathrm{ygl} 214 \mathrm{w}$ cells $\ldots . .150$ 


\section{List of Tables}

2.1 Lipids used in this thesis . . . . . . . . . . . . . . . . . . . . 27

2.2 Detergents used in this thesis . . . . . . . . . . . . . . 27

2.3 Miscellanous materials used in this thesis . . . . . . . . . . . . 28

2.4 Materials used for culturing E. coli and S. cerevisiae in this thesis . . 29

2.5 Commercial kits used in this thesis . . . . . . . . . . . . . . . . 29

4.1 Molecular weight of A488, DL680 and DL800 maleimide . . . . . . 104

S1 Sequence coverage of Doa10 and Ubc6 after sequential digest with trypsin and chymotrypsin . . . . . . . . . . . . . . . . . 151

S2 Plasmids used in this thesis . . . . . . . . . . . . . . . . 152

S3 S. cerevisiae strains used in this thesis . . . . . . . . . . . . . . 153

S4 Oligonucleotides used for DOA10 cloning . . . . . . . . . . . . . 154

S5 Oligonucleotides used for UBC6 cloning . . . . . . . . . . . . . . . . . 155

S6 Oligonucleotides used for generating Ubc6 $6_{\mathrm{BpA}}$ variants . . . . . . . . 156

S7 Oligonucleotides used for cloning CUE1 and GET3 . . . . . . . . . 157

S8 Oligonucleotides used for SBH2 cloning . . . . . . . . . . . . 158

S9 Oligonucleotides used for deleting chromosomal UBP3 . . . . . . . . . 159 


\section{Abbreviations}

aa

A488

DL680

DL800

ER

ERAD

E. coli

f.c.

FPP

HA

INM

LD

S. cerevisiae

SBP

TM

UPR

UPS
Amino acid

AlexaFluor488

DyLight680

DyLight800

Endoplasmic reticulum

ER-associated protein degradation

Escherichia coli

Final concentration

Farnesylpyrophosphate

Hemagglutinin

Inner nuclear membrane

Lipid droplet

Saccharomyces cerevisiae

Streptavidin-binding peptide

Transmembrane

Unfolded protein response

Ubiquitin proteasome system 


\section{Introduction}

\subsection{Protein degradation by the ubiquitin proteasome system}

Proteins carry out a vast amount of different functions in the cell. The cell controls their biogenesis and abundance transcriptionally and post-transcriptionally. Protein degradation is thereby an important post-transcriptional mechanism that controls protein biogenesis as well as protein half-life and that occurs via different pathways. Proteins are delivered to the lysosome for degradation by autophagy and other pathways. In addition, the ubiquitin proteasome system operates by selectively degrading proteins.

\subsubsection{The E1/E2/E3 enzymatic cascade for ubiquitination}

Covalent modification of proteins with ubiquitin serves as a signal for proteasomal degradation. The $8.5 \mathrm{kDa}$ protein ubiquitin is attached to substrates by an enzymatic cascade that is also employed to attach ubiquitin-like proteins. The ubiquitin-activating enzyme (E1) activates ubiquitin. It first catalyzes the adenylation of the carboxy terminus of ubiquitin using ATP and subsequently forms a thioester bond between the catalytic cysteine of the E1 enzyme and the C-terminus of ubiquitin. The activated ubiquitin is then transferred to the active-site cysteine of a ubiquitin-conjugating enzyme (E2). Ubiquitin ligases (E3) bind to this ubiquitin-bound E2 enzyme as well as to substrates. Ubiquitin transfer to the substrate occurs by formation of an isopeptide bond between the carboxy-terminus of ubiquitin and the $\varepsilon$-amino group of a lysine residue of the substrate. E3 ubiquitin ligases usually do not only act as a scaffold for the E2 enzyme and the substrate, but also stimulate the ubiquitin transfer activity of the E2 enzyme. Different types of ubiquitin ligases exist. RING ligases contain a catalytic RING domain that stimulates ubiquitin transfer. In contrast, other ubiquitin ligase classes such as HECT and RBR ligases bind ubiquitin via a thioester bond, before transfer to the substrate occurs (Kleiger and Mayor, 2014).

Apart from lysine modification, also non-canonical ubiquitination on serine, threonine and cysteine residues as well as the amino terminus has been observed (Finley et al., 2012). Ubiquitin chains are formed by repeated action of E1, E2 and E3 enzymes and can be formed via one of the 7 lysine residues of ubiquitin as well as its amino-terminus. Different linkage types serve as different signals within the cell and 
thus create a ubiquitin code. K48-linked ubiquitin chains are the main signal for proteasomal degradation (Chau et al., 1989; Xu et al., 2009; Grice and Nathan, 2016). The ubiquitin code has proteolytic and non-proteolytic functions. Besides proteasomal degradation, ubiquitination also serves as a signal for lysosomal degradation via the ESCRT-pathway. Moreover, ubiquitination can affect the localization of a protein or recruit binding partners. The ubiquitin code is read by ubiquitin binding domains that bind specific ubiquitin signals, and can be erased by deubiquitinating enzymes (Komander and Rape, 2012).

\subsubsection{Degradation by the proteasome}

Polyubiquitinated substrates are degraded by the proteasome. The $26 \mathrm{~S}$ proteasome is a 2.5 MDa complex that consists of a 20S catalytic subunit and two 19S regulatory subunits. The $20 \mathrm{~S}$ catalytic core forms a cylindric structure that contains proteolytically active subunits that face the inside of the cylinder. The 19S regulatory subunit binds polyubiquitinated substrates, deubiquitinates them and unfolds them before translocating them into the $20 \mathrm{~S}$ catalytic subunit for proteolysis. It contains ubiquitin receptors that bind polyubiquitinated substrates (Rpn10, Rpn13 and probably Rpn1), a deubiquitinating enzyme (Rpn11) as well as a hexameric ring consisting of AAA ATPases (Rpt1-6) that unfold the substrate using ATP-hydrolysis (Grice and Nathan, 2016; Kleiger and Mayor, 2014).

Moreover, additional non-proteasomal factors as Rad23 and Dsk2 function in recruiting substrates to the proteasome by binding to K48-linked ubiquitin chains via their UBA domain and to the proteasome via their UBL domain (Grice and Nathan, 2016).

\subsubsection{The UPS operates with multiple pathways for protein degradation}

Whereas S. cerevisiae has only one E1 enzyme (Uba1), it has 11 E2 enzymes and 60 to 100 putative E3 ubiquitin ligases (Finley et al., 2012). In humans, more than 600 genes are predicted to encode for ubiquitin ligases (Li et al., 2008). Ubiquitination systems are localized throughout the cell to ensure protein degradation. Examples from S. cerevisiae are used in the following section to illustrate the cellular distribution of ubiquitin ligases.

In the nucleus, the ubiquitin ligases San1, the Asi-complex as well as Doa10 ubiquitinate substrates (Enam et al., 2018). In the endoplasmic reticulum (ER), the ubiquitin ligases Doa10 and Hrd1 function in ER-associated protein degradation (ERAD). Recently, a pathway similar to ERAD has been identified in the Golgi, where the 
Dsc ubiquitin ligase has been shown to ubiquitinate Orm2 and target it for degradation (Schmidt et al., 2019). A protein degradation pathway using the UPS has been also identified in the outer membrane of chloroplasts (Ling et al., 2019). Moreover, a cooperative pathway eliminates mistargeted tail-anchored proteins from mitochondria which requires the ER-localized ubiquitin ligase Doa10 (Dederer et al., 2019; Matsumoto et al., 2019). Cytosolic protein quality control involves multiple ubiquitin ligases, such as Ubr1 and Doa10 (Amm et al., 2014; Ravid et al., 2006; Furth et al., 2011). These examples show that E1/E2/E3 systems are distributed throughout the cell, ensuring protein degradation.

\subsection{ER-associated protein degradation (ERAD)}

ERAD is a protein quality control pathway at the ER. In ERAD, substrates are recognized, ubiquitinated, retrotranslocated into the cytosol and degraded by the proteasome (Zattas and Hochstrasser, 2014). Conserved, membrane-embedded ubiquitin ligase complexes recognize and ubiquitinate substrates. In S. cerevisiae, three ubiquitin ligase complexes have been identified that operate in ERAD - the Hrd1, Doa10 and Asi complex. Whereas Hrd1 exclusively localizes to the ER membrane, Doa10 localizes to the ER membrane as well as the inner nuclear membrane (INM) (Deng and Hochstrasser, 2006). The Asi complex is localized at the INM (Khmelinskii et al., 2014; Foresti et al., 2014). Figure 1.1 illustrates the localization of those ubiquitin ligases complexes. ERAD substrates are very diverse and it has been shown that different ligase complexes recognize different substrates. After ubiquitination of substrates by different ligase complexes, all ERAD pathways converge on the step of retrotranslocation into the cytosol by the AAA ATPase Cdc48 (p97 in metazoans) in complex with its cofactors Ufd1 and Npl4 (Rabinovich et al., 2002; Jarosch et al., 2002; Ye et al., 2001; Bays et al., 2001; Huyer et al., 2004).

This thesis investigates the mechanism of ERAD mediated by the ubiquitin ligase Doa10. Therefore, the focus of this introduction will be on Doa10-mediated ERAD. However, many features of ERAD are common for all ERAD pathways and thus the Hrd1 as well as the Asi complex will be introduced and used for comparison. This thesis investigates ERAD in the model organism S. cerevisiae. Many ERAD components are conserved and have homologs in the mammalian system (Zattas and Hochstrasser, 2014). Mammalian ERAD is quite more complex. More than 10 ubiquitin ligases have already been implicated in ERAD in mammalian cells (Olzmann et al., 2012). As $S$. cerevisiae was the model organism studied in this thesis, this introduction will focus 


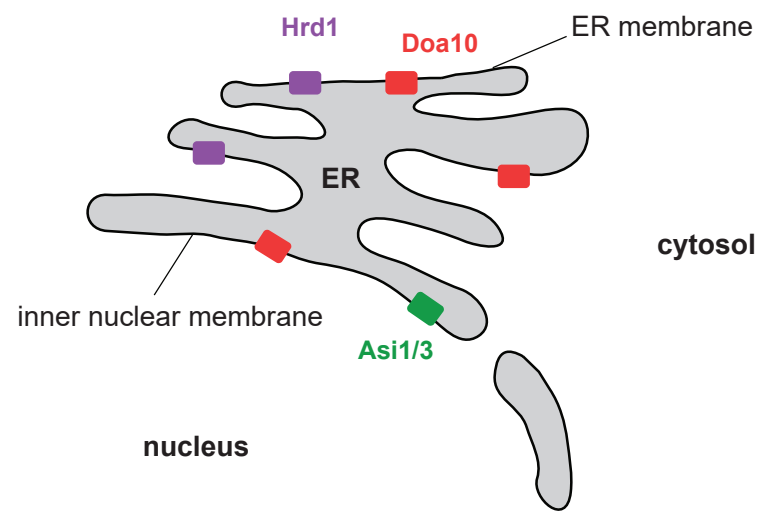

Figure 1.1: Localization of the ERAD ubiquitin ligases. Scheme illustrating the localization of the ubiquitin ligases Doa10 (red), Hrd1 (purple) and Asi1/Asi3 (green). Hrd1 localizes to the ER membrane, Asi1/3 to the inner nuclear membrane, and Doa10 to both, the ER and inner nuclear membrane.

on ERAD in S. cerevisiae, but draw parallels to ERAD in other eukaryotes.

Over the last years, the list of substrates that are degraded by ERAD has been growing. These discoveries have provided many insights into the different functions of ERAD. In this introduction, I will first give an overview of the identified substrates and thereby illustrate the different functions of ERAD (section 1.2.1). Next, the machinery involved in ERAD will be introduced and the characteristics of the conserved ubiquitin ligase complexes Hrd1 as well as Doa10 explained in more detail (section 1.2.2 and 1.2.3). Following this overview over ERAD substrates and the ERAD machinery, I will summarize the available literature on two steps of ERAD whose mechanism is not well understood and has been investigated during this thesis: recognition and retrotranslocation of substrates.

\subsubsection{ERAD substrates}

The ERAD machinery has a wide substrate range. Studies on different substrates have elucidated many functions of protein degradation by ERAD. ERAD has been extensively studied using model substrates which contain point mutations that render the protein constitutively misfolded. Those, in contrast to their wildtype version, are degraded by ERAD. It has been further shown that also subunits that are not assembled into complexes are recognized by ERAD. These observations led to the identifcation of ERAD as a quality control pathway for the biogenesis of proteins destined for the secretory pathway (Lippincott-Schwartz, 1988; Bonifacio and Lippincott-Schwartz, 1991; 
Meusser et al., 2005). Moreover, ERAD plays an important role in regulating the abundance of enzymes of the sterol synthesis pathway that reside in the ER membrane. Upon accumulation of certain metabolites, they are recognized by the ERAD machinery. Moreover, also proteins that are mislocalized or destined to other organelles such as lipid droplets are degraded by ERAD (Ruggiano et al., 2014; Mehrtash and Hochstrasser, 2018). These studies indicate that ERAD has a role in maintaining the identity of organelles. Examples of such substrates are described in more detail in the next sections.

\subsubsection{ERAD is a quality control pathway for the biogenesis of proteins destined for the secretory pathway}

ERAD has been identified by studying the degradation of proteins destined for the secretory pathway that contain a mutation and thus are retained in the ER and degraded by ERAD.

Proteins destined for the secretory pathway are synthesized at the ER. Soluble and membrane proteins are translocated into the ER or integrated into the ER membrane via different pathways (for review, see Barlowe and Miller (2013)). Translocation across the ER membrane occurs through the Sec61-translocon (Rapoport et al., 2017). Tail-anchored (TA) proteins are targeted to the ER posttranslationally in a Sec61independent manner, by the GET-pathway (Stefanovic and Hegde, 2007; Schuldiner et al., 2008; Mateja and Keenan, 2018). Recent studies have shown that many targeting pathways act in parallel and have overlapping functions. One example is the recently identified EMC-complex, an ER-resident protein complex that can also target TA-proteins to the ER (Jonikas et al., 2009; Guna et al., 2017). It has been proposed that it can also function during SRP-dependent translocation to ensure the correct topology of multipass transmembrane proteins, e.g. GPCRs (Chitwood et al., 2018).

In the ER, polypeptides undergo folding into tertiary structures and assemble into protein complexes. The oligosaccharyltransferase (OST) complex attaches an oligosaccharide onto consensus sequences (N-linked glycosylation). Chaperone systems assist in protein folding. The Hsp70 (heat shock protein) Kar2 (BiP in mammals) interacts with hydrophobic regions of substrates. Protein disulfide isomerases (PDIs) and prolyl peptidyl cis-trans isomerases (PPIs) ensure that the polypeptide assembles into its correct tertiary structure (Braakman and Hebert, 2013; Barlowe and Miller, 2013).

Proteins that do not fold into their correct tertiary structure and are thus misfolded are degraded by ERAD. A mutant version of the vacuolar protease Carboxypeptidase $\mathrm{Y}$ that contains a single point mutation is a substrate of Hrd1 (Hiller et al., 1996; 
Finger et al., 1993; Bordallo et al., 1998). Also misfolded membrane proteins have been described as Hrd1 substrates, like the ATP-binding cassette transporter Pdr5 containing a point mutation (C1427Y) (Plemper et al., 1998). Doa10 also recognizes mutant versions of proteins such as the a-factor ATP-binding cassette transporter Ste6 containing a premature STOP codon (Ste6*) (Loayza et al., 1998; Vashist and Ng, 2004) and a mutant version of the plasma membrane ATPase Pma1 (D378S) (Wang and Chang, 2003, 1999; Han et al., 2007).

These initial studies have identified some key rules of substrate selection by different ubiquitin ligase complexes. Whereas ER-luminal proteins are exclusively recognized by the Hrd1 complex, both Doa10 and Hrd1 recognize ER membrane proteins. Vashist and $\mathrm{Ng}$ identified ERAD pathways based on model substrates that harbor a mutation (Vashist and Ng, 2004). The authors show that whereas substrates with a mutated and thus misfolded domain in the ER-lumen (ERAD-L) or ER membrane (ERAD-M) are degraded by Hrd1, substrates with a misfolded cytosolic domain (ERAD-C) are degraded by Doa10.

Further evidence for a role of ERAD in degradation of terminally misfolded proteins comes from studies that show that ERAD is linked to the unfolded protein response (UPR). When misfolded proteins accumulate in the ER, UPR is triggered. ER-stress sensors activate transcription factors that lead to downregulation of general protein biosynthesis as well as upregulation of factors involved in protein folding (Hetz and Papa, 2018). Two observations link ERAD to the UPR. It has been shown that components of the Hrd1 complex are upregulated during the UPR (Friedlander et al., 2000; Travers et al., 2000). Moreover, double deletion of DOA10 and HRD1 and thus defective ERAD leads to a pronounced induction of the UPR (Swanson et al., 2001; Jonikas et al., 2009). These studies show the importance of ERAD in degradation of misfolded proteins.

Besides ERAD, also autophagy takes place at the ER which leads to degradation of ER membrane material as well as ER proteins in the lysosome (Grumati et al., 2018). This process, termed ER-phagy, has been also shown to remove aggregated proteins which cannot be cleared by ERAD (Ishida et al., 2009; Kruse et al., 2006). Moreover, it has been shown that ERAD and ER-phagy can function in parallel for the degradation of certain proteins (Schultz et al., 2018).

After translocation into the ER, polypeptides assemble into multi-subunit complexes. It has been shown that certain proteins are degraded when they do not assemble into a complex. Sbh2 is part of the Ssh1 translocon in S. cerevisiae (Finke et al., 1996). When Sbh2 is overexpressed or when its interaction partner Sss1 is deleted, 
it becomes unstable and is degraded in a Doa10-dependent manner (Habeck et al., 2015). Interestingly, its homolog Sbh1 which is part of the Sec61 translocon becomes a Doa10 substrate when OPI3, an enzyme involved in synthesis of phosphatidylcholine, is deleted. OPI3 deletion leads to changes in lipid composition and a reduced interaction of Sbh1 with Sec61 (Shyu et al., 2019).

Concluding, ERAD is a protein quality control pathway that controls the biogenesis of proteins destined for the secretory pathway. Doa10 mediates degradation of model substrates harboring a misfolded domain as well as unassembled subunits (Figure 1.2A).

\subsubsection{ERAD regulates the abundance of enzymes for sterol synthesis}

The ER is a major compartment for synthesis of sterols. Cholesterol synthesis in animals as well as ergosterol synthesis in fungi is regulated by multiple mechanisms. Besides transcriptional control, it has been shown that ERAD degrades certain lipid synthesis enzymes in a feedback regulation (Espenshade and Hughes, 2007).

Sterols are synthesized at the ER from Acetyl-CoA by the mevalonate pathway. Condensation of three Acetyl-CoA molecules leads to 3-methylglutaryl-Coenzyme A (HMG-CoA) which is then reduced to mevalonate by HMG-CoA reductase. Through subsequent steps, farnesylpyrophosphate (FPP) is formed that is the precursor for sterols as well as other molecules such as dolichol and ubiquinone. Squalene, formed from two molecules of FPP, is converted into squalene epoxide by squalene monooxygenase under consumption of oxygen. The downstream product lanosterol is then in multiple steps converted to ergosterol in fungi or cholesterol in animals (Klug and Daum, 2014; Espenshade and Hughes, 2007).

HMG-CoA reductase as well as squalene monooxygenase are ERAD substrates in response to accumulation of downstream products. S. cerevisiae has two isozymes of HMG-CoA reductase, Hmg1 and Hmg2. It has been shown that Hmg2 is a substrate of Hrd1 (Hampton, 1994; Hampton et al., 1996; Bays et al., 2001) and degraded in a regulated manner by two signals. Hmg2 interacts with the INSIG Nsg1 in the presence of lanosterol. This interaction protects it from ERAD (Theesfeld and Hampton, 2013). When lanosterol levels are low, Nsg1 is degraded and a second control mechanism becomes important: In the absence of its interaction partner Nsg1, Hmg2 is degraded by ERAD when the downstream product FPP accumulates, but rendered stable when FPP levels are low (Garza et al., 2009b; Theesfeld and Hampton, 2013). The FPP-derivative geranylgeranyl pyrophosphate is most likely the endogenous signal for ERAD-mediated degradation of Hmg2 (Garza et al., 2009b; Theesfeld and Hampton, 2013; Wangeline and Hampton, 2018). 


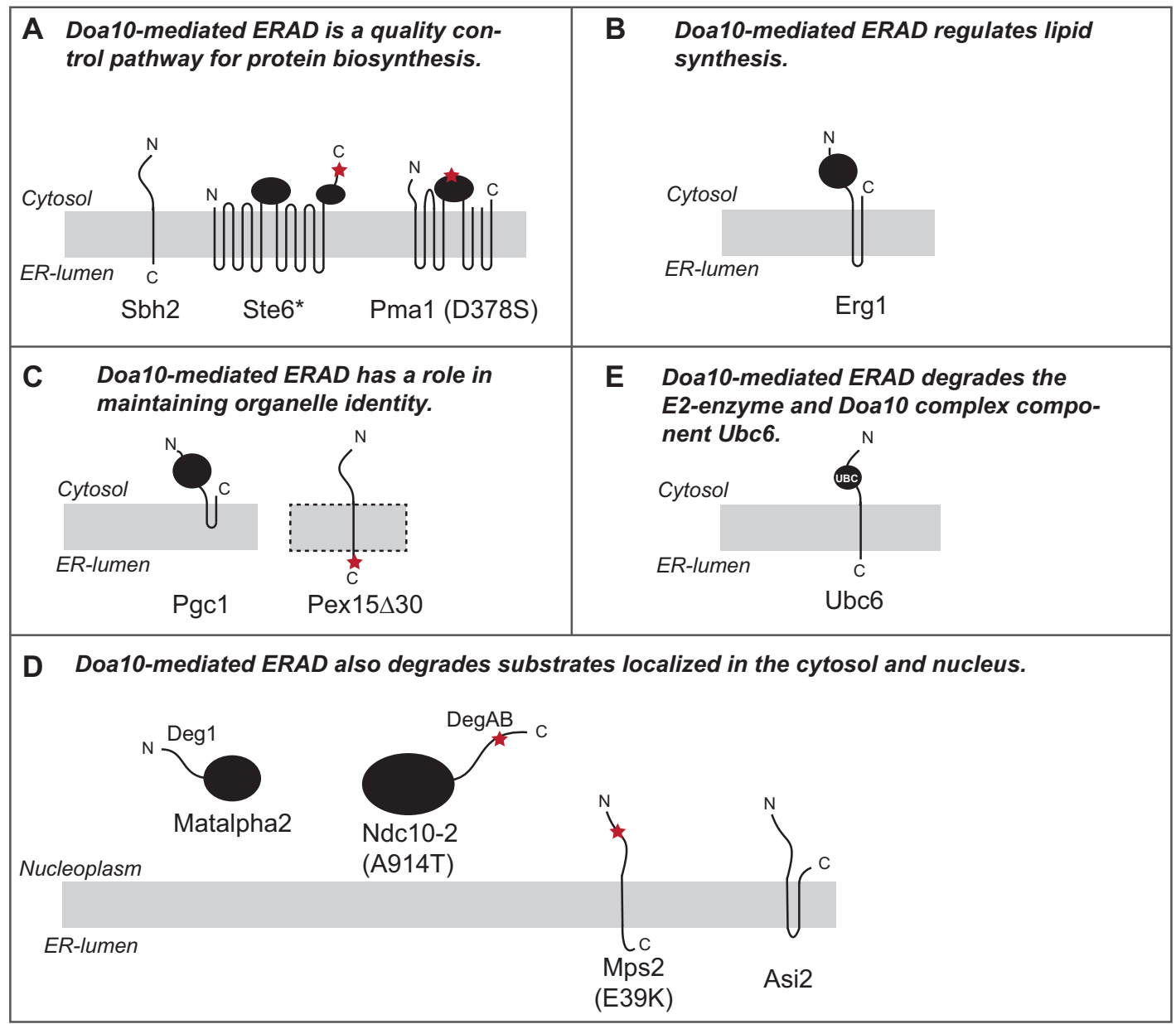

Figure 1.2: Substrates of Doa10-mediated ERAD. Doa10 recognizes a plethora of different substrates as discussed in this section (1.2.1). Here, substrates are schematically illustrated and presented in groups showing different functions of Doa10-mediated ERAD, as (A) quality control of protein biosynthesis, (B) regulation of lipid synthesis, (C) maintenance of organelle identity, (D) degradation of the E2 enzyme Ubc6 and (E) cytosolic and nuclear quality control. Asterisks indicate a mutation (point mutation or deletion of a fragment). The lipid bilayer is indicated by a grey bar. Dots around lipid bilayer indicate that it is unclear if Doa10 recognizes this substrate in a membrane-inserted state. This is the case for Pex15 $\Delta 30$ that due to its mislocalization to mitochondria is extracted by Msp1 and then most likely ubiquitinated by Doa10. See text for more details.

Moreover, the squalene monooxygenase Erg1 is degraded in a Doa10-dependent manner when the downstream product lanosterol accumulates (Foresti et al., 2013). Interestingly, homologs of Erg1 in mammals and plants are also degraded in a Doa10dependent manner, indicating that the function of Doa10 in lipid regulation is con- 
served. When cholesterol-deprived human cells are treated with cholesterol, otherwise stable squalene monooxygenase $\mathrm{SM}$ is rapidly degraded in a process dependent on the Doa10 homolog TEB4/MARCH6 (Foresti et al., 2013; Zelcer et al., 2014; Gill et al., 2011). In Arabidopsis thaliana, a mutation in squalene monooxygenase causing a growth defect can be rescued by a mutation in the SUD-1 gene. SUD1 shows sequence similarity to DOA10 and TEB4 (Doblas et al., 2013).

In accordance with the involvement of Doa10 in regulating the abundance of a lipid synthesis enzyme, deletion of DOA10 changes the cellular lipid profile. In the absence of Doa10, cells contain less ergosterol but accumulate intermediates. This effect is even more pronounced when the enzymes involved in esterification of sterol intermediates, ARE1 and ARE2, are also deleted (Foresti et al., 2013).

In summary, ERAD has a role in regulating lipid synthesis by degrading lipid synthesis enzymes in a feedback regulation. Degradation of squalene monooxygenase is thereby a conserved process mediated by Doa10 (Figure 1.2B).

\subsubsection{ERAD maintains organelle identity}

Lipid droplets (LDs) are lipid reservoirs that consist of a lipid monolayer and a hydrophobic core containing neutral lipids like triacylglycerols and sterol esters and are formed at the ER. The lipid monolayer harbors proteins that are associated with the LD either through an amphipathic helix or a hydrophobic hairpin. LD proteins containing such a hydrophobic hairpin are targeted to the LD via insertion into the ER (Olzmann and Carvalho, 2018). The PG (phosphatidylglycerol)-specific phospholipase Pgc1 is primarily localized to lipid droplets (Ruggiano et al., 2016; Kubalová et al., 2019) and is important for degradation of the anionic phospholipid PG. Upon deletion of PGC1, PG accumulates on lipid droplets, but also in the ER and in mitochondria indicating that Pgc1 has a broad role in regulating cellular PG levels (Kubalová et al., 2019). The ER-localized fraction of Pgc1 is subjected to Doa10-mediated ERAD. Recognition by Doa10 seems to be specific for the ER-localized pool of Pgc1, as in the absence of LDs, the degradation of Pgc1 is enhanced whereas when oleate is fed to stimulate LD synthesis, Pgc1 is stable. Moreover, Doa10 plays a role in degradation of other LD proteins such as Dga1 and Yeh1 (Ruggiano et al., 2016).

Proteins localized to mitochondria are also degraded in a Doa10-dependent manner. Recently, it has been shown that TA-proteins that are mislocalized to mitochondria are degraded by the proteasome. This degradation is dependent on the mitochondrial AAA ATPase Msp1 as well as Doa10 (Dederer et al., 2019; Matsumoto et al., 2019). Msp1 seems to act prior to ubiquitination and upon DOA10 deletion, substrates accumulate 
at the ER, suggesting that TA-proteins are transferred to the ER by Msp1 and then ubiquitinated by Doa10 (Matsumoto et al., 2019).

Interestingly, a mitochondrial Pgc1 pool and activity has been reported (Simocková et al., 2008; Kubalová et al., 2019) and it is therefore possible that Doa10 also has a role in clearance of mitochondrially localized Pgc1. Recent evidence suggests that by degrading a pool of Pgc1 localized to the ER and possibly to mitochondria, Doa10 influences the activity of Pgc1 and thus turnover of PG. Pgc1 activity depends on its localization. Wheres it is inactive in lipid droplets, it is active when inserted into a lipid bilayer such as the ER- or mitochondrial membrane (Kubalová et al., 2019). Indeed, upon deletion of Doa10 or Msp1, the phospholipase activity of a mitochondrial fraction is increased (Kubalová et al., 2019). Thus, Pgc1 turnover might be a an important control mechanism to regulate Pgc1 activity.

Overall, these studies indicate that Doa10 plays an important role in maintaining the identity of organelles by degrading mislocalized proteins or selected pools of proteins (Figure 1.2C).

\subsubsection{Role of ERAD ubiquitin ligases in cytosolic and nuclear protein quality control}

The ERAD machinery is not only involved in degradation of proteins in the ER, but also proteins with cytosolic or nuclear localization (Figure 1.2D). The transcription factor Mat $\alpha 2$ is a Doa10 substrate (Swanson et al., 2001). It contains a 76 amino acid stretch termed Deg1 that is sufficient to transform a stable protein into a Doa10 substrate (Chen et al., 1993). Doa10 was identified by screening for components required for degradation of a Deg1-containing reporter construct, Deg1-Ura3 (Swanson et al., 2001). In line with its function in degrading a transcription factor, Doa10 also localizes to the inner nuclear membrane and functions in the nucleus (Deng and Hochstrasser, 2006). Another example is a mutant version of the kinetochore protein Ndc10 (Ndc10-2) that is degraded in a Doa10-dependent manner (Kopski and Huffaker, 1997; Ravid et al., 2006). Besides those soluble proteins, the inner nuclear membrane proteins Asi2 as well as a mutant version of Mps2 have been also identified as Doa10 substrates (Boban et al., 2014, 2015; McBratney and Winey, 2002; Kreft et al., 2006). Many artificial degrons fused to the amino- or carboxy-terminus of reporter proteins have been identified for Doa10 (Gilon et al., 2000; Ravid et al., 2006; Khmelinskii et al., 2012; Kats et al., 2018) and it has been shown that soluble proteins are degraded in a Doa10-dependent manner in the nucleus as well as the cytoplasm (Deng and Hochstrasser, 2006; Ravid et al., 2006; Samant et al., 2018). Also Hrd1 has been 
implicated in cytosolic quality control (Samant et al., 2018).

Besides Doa10, also the Asi-complex functions in quality control at the inner nuclear membrane. The Asi-complex consists of Asi2 and the RING domain containing proteins Asi1 and Asi3 (Foresti et al., 2014). It is involved in degradation of the transcription factor Spt1 (Forsberg et al., 2001; Boban et al., 2006; Zargari et al., 2007; Omnus and Ljungdahl, 2014; Khmelinskii et al., 2014). Moreover, it targets membrane proteins at the inner nuclear membrane for degradation. The E2 enzymes Ubc6 and Ubc7 (with its cofactor Cue1) are involved in Asi-mediated ERAD (Foresti et al., 2014; Khmelinskii et al., 2014). Those E2 enzymes are further described in the following sections (section 1.2.2 and 1.2.3). A scheme of the components of the Asi complex is shown in Figure 1.3A. As ER membrane proteins as well as vacuolar proteins have been identified as substrates, it was hypothesized that the Asi complex degrades mislocalized substrates at the INM (Foresti et al., 2014; Khmelinskii et al., 2014). Smoyer et al. (2019) have recently identified INM proteins as substrates of the Asi complex suggesting a broader role in protein quality conrol at the INM. In contrast to Doa10 and Hrd1, the Asi complex does not have homologs in higher eukaryotes (Foresti et al., 2014).

Concluding, ERAD has multiple roles in protein quality control. Doa10-mediated ERAD contributes to quality control of protein biogenesis as well as regulation of lipid synthesis. Moreover, it plays a role in maintaining the identity of organelles and contributes to cytosolic and nuclear protein quality control.

\subsubsection{The Hrd1 complex}

Doa10, Hrd1 as well as Asi1 and Asi3 are RING ubiquitin ligases. RING domains bind to E2 ubiquitin conjugating enzymes and catalyze the direct transfer of ubiquitin from the E2 enzyme to the substrate (Deshaies and Joazeiro, 2009). The catalytic RING domain contains a consensus sequence of cysteine and histidine residues that coordinate two Zinc atoms (Freemont et al., 1991; Deshaies and Joazeiro, 2009).

The ubiquitin ligase Hrd1 has been identified in a screen for components necessary for degradation of Hmg2 (HMG-CoA reductase degradation) (Hampton et al., 1996). Another screen using a CPY* reporter construct also identified Der3 (Knop et al., 1996) which was later shown to be identical to Hrd1 (Bordallo et al., 1998). Hrd1 acts with the E2 enzyme Ubc7 (Bays et al., 2001). Ubc7 is anchored to the ER membrane via the membrane protein Cue1 (Biederer et al., 1997) and activates Ubc7 (Bazirgan and Hampton, 2008). Cue1 activates Ubc7 by binding to Ubc7 via its UB7R domain (Metzger et al., 2013; Bagola et al., 2013) and binds to ubiquitin chains via its CUE domain allowing for processive ubiquitination (Bagola et al., 2013; 
von Delbrück et al., 2016). Ubc7 catalyzes the formation of K48-linked polyubiquitin chains (Bazirgan and Hampton, 2008; Bagola et al., 2013). Besides Ubc7, Ubc1 has been shown to be partially required for some substrates (Bays et al., 2001).

Hrd1 is part of a complex consisting of Hrd1, Hrd3, Usa1 and Der1 (Carvalho et al., 2006). Figure 1.3B depicts the components of the Hrd1 complex. Hrd1 contains 8 transmembrane (TM) segments and a cytosolic, carboxy-terminal RING domain (Schoebel et al., 2017). It interacts with Hrd3 which consists of a large luminal domain and a carboxy-terminal TM segment. This interaction is required for Hrd1 stability. Upon deletion of HRD3, Hrd1 is unstable and degraded (Gardner et al., 2000). Usa1 contains two TM segments and an amino-terminal Ubl-domain. It has been shown that it is required for the interaction of Hrd1 with the multipass TM protein Der1 (Carvalho et al., 2006; Horn et al., 2009). Interestingly, Usa1 and Der1 are required for degradation of ERAD-L, but not for ERAD-M substrates suggesting that different subcomplexes of Hrd1 exist (Carvalho et al., 2006). 


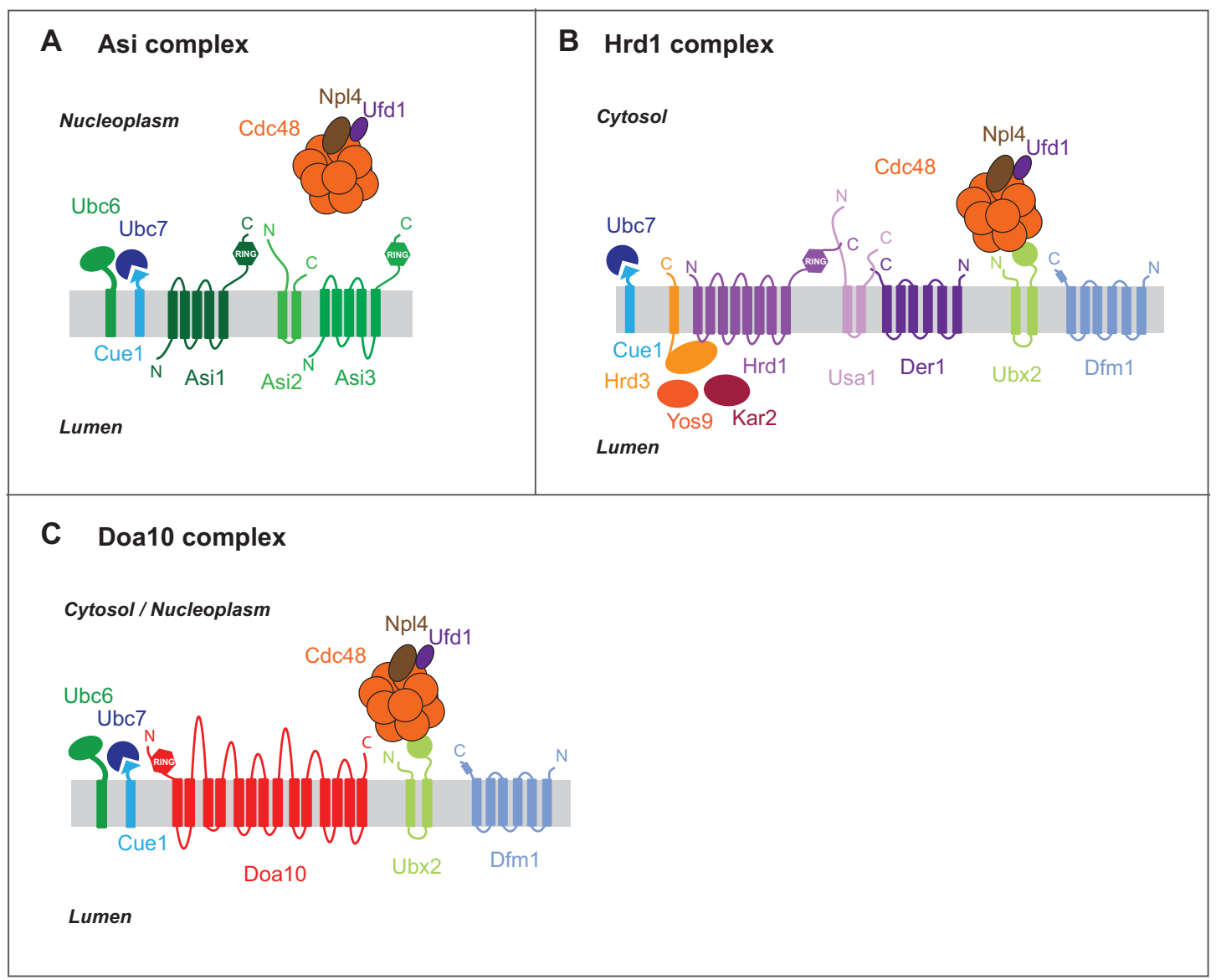

Figure 1.3: The Asi, Hrd1 and Doa10 complex and its components. The complex components of the Doa10, Hrd1 and Asi complex are schematically illustrated. (A) The Asi complex is localized at the inner nuclear membrane. It consists of the two RING domain containing proteins Asi1 and Asi3, and Asi2. The Cdc48-complex is also required for degradation of Asi-substrates. (B) Hrd1 interacts with Hrd3 which is together with Yos9 and Kar2 involved in substrate recognition. Moreover, Usa1 links the membrane protein Der1 to the complex. Polyubiquitinated substrates are extracted by the Cdc48-complex. Ubx2 has been also identified as part of the Hrd1 complex. (C) Doa10 functions with the E2 enzymes Ubc6 and Ubc7 which is anchored to the ER membrane by its co-factor Cue1. Doa10 contains 14 TM segments and both the amino-terminus containing the RING domain as well as the carboxy-terminus face the cytosol. Doa10 is not only localized at the ER membrane but also at the INM. Polyubiquitinated substrates are extracted by the Cdc48-complex containing the co-factors Npl4 and Ufd1. Cdc48 is recruited to the Doa10-complex by Ubx2. Dfm1 contains a carboxy-terminal SHP-box and plays a role in retrotranslocation. Scheme for Doa10 and Hrd1 complex modified from Zattas and Hochstrasser (2014). Figure for Asi complex based on topological analysis (Zargari et al., 2007; Boban et al., 2006) and studies identifiying components involved in Asi-mediated degradation (Foresti et al., 2014; Khmelinskii et al., 2014). See text for further details. 


\subsubsection{The Doa10/TEB4 complex}

Yeast Doa10 has a molecular mass of $151 \mathrm{kDa}$ and contains an amino-terminal RING domain (Swanson et al., 2001). Doa10 contains probably 14 TM segments with both termini facing the cytosol (Kreft et al., 2006). Figure 1.3C shows a scheme of the components of the Doa10 complex which will be introduced in the following sections.

\subsubsection{Doa10 acts with the E2 enzymes Ubc6 and Ubc7}

The E2 enzymes Ubc6 and Ubc7 as well as its cofactor Cue1 have been identified in several screens conducted to identify components necessary for Doa10-mediated degradation. The Deg1-Ura3 reporter construct was used both in a mutagenesis screen which initially identified Doa10 as well as a genetic screen which tested 4753 deletion strains (Swanson et al., 2001; Ravid et al., 2006). A screen for suppressors of the temperature-sensitive ndc10-2 mutation identified as well Ubc6, Ubc7, Cue1 and Doa10 (Kopski and Huffaker, 1997; Ravid et al., 2006).

Doa10 functions with the E2 enzymes Ubc6 and Ubc7 (Swanson et al., 2001). Ubc6 is a tail-anchored membrane protein (Sommer and Jentsch, 1993). Whereas deletion of UBC7 leads to complete stabilization of ERAD substrates, UBC6 deletion often has less strong effects, as observed for Ste6*, Erg1 and Sbh2 (Huyer et al., 2004; Foresti et al., 2013; Habeck et al., 2015). However, Ubc6 is absolutely required for ERAD when substrates lack lysine residues. It has been shown that Ubc6 ubiquitinates those substrates via the hydroxylgroups of serines or threonines (Boban et al., 2015; Weber et al., 2016). In line with this, an Sbh1 variant lacking lysine residues is still a Doa10 substrate (Shyu et al., 2019). Moreover, the function of Ubc6 and Ubc7 has been investigated using autoubiquitination of the RING domain as a readout (Weber et al., 2016). Using cytosolic fragments of Ubc6, Ubc7 and the Doa10 RING domain, the authors observed that Ubc6 and Ubc7 act sequentially. Whereas Ubc6 primes the RING domain with ubiquitin, Ubc7 forms a polyubiquitin chain. Such a sequential ubiquitination mechanism by separate E2 enzymes has been also observed for other ubiquitin ligases (Deshaies and Joazeiro, 2009). One example is the ubiquitination of cyclin B mediated by the anaphase promoting complex (APC/C). Reconstitution studies showed that whereas Ubc4 monoubiquitinates cyclin B at multiple sites, Ubc1 catalyzes polyubiquitination on preattached ubiquitins (Rodrigo-Brenni and Morgan, 2007).

Ubc6 itself is unstable and degraded in a Doa10-dependent manner (Walter et al., 2001; Swanson et al., 2001). Degradation of Ubc6 is dependent on its own activ- 
ity, as catalytically inactive Ubc6 is stable even when active Ubc6 is expressed as well (Walter et al., 2001). Requirement for catalytic activity can be bypassed by mono-ubiquitination, as a Ubc6 construct containing a ubiquitin moieity fused to the amino-terminus of Ubc6 is degraded in a Doa10-dependent manner (Weber et al., 2016). This indicates that Ubc6 monoubiquitination mediated by its autoubiquitination activity generates a signal for Doa10-dependent degradation.

Besides the ubiquitination machinery, the ER membrane protein Ubx2 as well as Cdc48 and its cofactors Ufd1 and Npl4 have been identified as part of the Doa10 complex and Hrd1 complex (Carvalho et al., 2006). Moreover, the multipass transmembrane protein Dfm1 has been shown to interact with Doa10 as well as Hrd1 and is involved in ERAD of Doa10 and Hrd1-substrates (Stolz et al., 2010; Neal et al., 2018). The Cdc48-complex as well as Ubx2 and Dfm1 are introduced in more detail in section 1.2.5 where retrotranslocation is discussed.

\subsubsection{Structural elements of Doa10}

Besides the RING domain, two conserved elements have been described for Doa10. Doa10 contains a conserved 16-residue C-terminal element (CTE). Deletion of this region or mutation of a conserved asparagine (N1314A) results in impaired degradation due to impaired ubiquitination of substrates. This has been shown for soluble and membrane-bound substrates containing the Deg1 degron or a degron derived from Ndc10-2 (DegAB) (Zattas et al., 2016). The role of this CTE for ERAD of membrane proteins is however unclear. Whereas Zattas et al. show that disrupting the CTE does not affect degradation of Ubc6 or Ste6* ${ }^{*}$, Kreft et al. show that Ubc6 degradation is impaired when a Doa10 version lacking the CTE is expressed (Zattas et al., 2016; Kreft et al., 2006).

Moreover, Doa10 contains a conserved domain spanning the TM segments 5-7 termed the TEB4-Doa10 (TD) domain (Swanson et al., 2001). The TD domain seems to be important for Doa10 function, as mutations in this region lead to stabilization of Doa10 substrates (Kreft and Hochstrasser, 2011).

\subsubsection{The mammalian Doa10 ortholog TEB4/MARCH6}

The mammalian Doa10 ortholog TEB4/MARCH6 probably has a similar topology as Doa10, with both termini facing the cytosol and contains a TD-domain (Swanson et al., 2001; Kreft et al., 2006). It localizes to the ER and has E3 ubiquitin ligase activity (Hassink et al., 2005; Zattas et al., 2016). It has been shown that TEB4 itself is unstable and its degradation is dependent on its autoubiquitination activity (Hassink et al., 
2005). It also contains a CTE that seems to be important for its function as mutation of the conserved asparagine of the CTE (N890A) impairs degradation of TEB4 (Zattas et al., 2016). The E2 enzymes Ubc6 and Ubc7 each have two mammalian homologs, Ube2j1, Ube2j2 and Ube2g1, Ube2g2, respectively (Kostova et al., 2007). Recently E2 enzymes involved in TEB4-mediated degradation of squalene monooxygenase have been identified. Ube2j2 and Ube2g2, but not Ube2j1 are involved in degradation of squalene monooxygenase (Tan et al., 2019).

\subsubsection{Substrate recognition}

A major question in ERAD is how substrates are recognized, especially given the diversity of substrates. Several features in substrates are important for proteolytic processing. First, they have to contain a region that is recognized by the ubiquitin ligase as well as sites where ubiquitination can occur. Moreover, the substrate has to contain an unstructured region that is, in addition to ubiquitination, required for proteasomal processing (Ravid and Hochstrasser, 2008). A sequence that can be transferred onto a stable protein to intiate its degradation is termed degron (Varshavsky, 1991; Ravid and Hochstrasser, 2008). Degrons thus are defined as elements that are directly or indirectly recognized by a ubiquitin ligase and lead to ubiquitination and subsequent degradation of the substrate. In this definition, the ubiquitination site as well as the unstructured region required for proteasomal degradation, do not have to reside within the degron but can be also within the rest of the protein whose stability is determined by the degron (Ravid and Hochstrasser, 2008). It becomes clear that different ligase complexes are specialized to recognize different features. Examples of degrons have provided insight into how substrates are recognized.

\subsubsection{Recognition of a luminal degron}

For ERAD-L substrates, a bipartite signal consisting of a misfolded region as well as an N-glycan structure is recognized by the ERAD machinery. In the ER lumen, Hrd3 interacts with misfolded substrates and also binds to Kar2 and the luminal protein Yos9 (Carvalho et al., 2006; Denic et al., 2006; Gauss et al., 2006). Yos9 is a lectin that recognizes glycosylated proteins and it has been also shown to recognize misfolded proteins (Bhamidipati et al., 2005; Szathmary et al., 2005; Kim et al., 2005). During N-linked glycosylation, an oligosaccharide chain composed of three glucose (Glc), nine mannose (Man) and two N-acetylglucosamine (GlcNAc) moieities $\left(\mathrm{Glc}_{3} \mathrm{Man}_{9} \mathrm{GlcNAc}_{2}\right)$ is transferred onto the protein. The glycan is further processed by glucosidases. This process is linked to chaperones. Substrates containing at least 
one glucose are bound by sugar-binding chaperones called lectins. In a second step, mannosidases trim the glycan structure to $\mathrm{Man}_{8} \mathrm{GlcNac}_{2}$. Folded proteins are usually exported with a $\mathrm{Man}_{8} \mathrm{GlcNac}_{2}$ structure. Misfolded proteins however are further processed by the mannosidase Htm1/Mnl1 in complex with the disulfide isomerase Pdi, resulting in a $\mathrm{Man}_{7} \mathrm{GlcNac}_{2}$ glycan that is recognized by Yos9 (Nakatsukasa et al., 2001; Jakob et al., 2001; Gauss et al., 2011; Liu et al., 2016; Pfeiffer et al., 2016).

\subsubsection{Recognition of an intramembrane degron}

For ERAD-M substrates, it has been shown that hydrophilic residues in the Hrd1 TM region are important for substrate degradation indicating that Hrd1 recognizes an intramembrane degron (Sato et al., 2009).

Doa10 also recognizes intramembrane degrons. The carboxy-terminal region comprising the TM anchor of Sbh2 and the hydrophobic hairpin of Pgc1 are necessary and sufficient for their Doa10-dependent degradation (Habeck et al., 2015; Ruggiano et al., 2016). The TM anchor of Ubc6 is also probably important for its degradation. When the Ubc6 TM anchor is attached to the stable cytosolic E2 enzyme Ubc4, this fusion protein is degraded in a Doa10-dependent manner (Walter et al., 2001). However, the Ubc6 TM anchor seems to be not sufficient for Doa10-dependent degradation, as a construct containing the soluble Ura3 fused to the Ubc6 TM anchor is stable (Kreft and Hochstrasser, 2011). This is in agreement with experiments showing that catalytically inactive Ubc6 is stable. Concluding, the TM anchor as well as catalytic activity are required for degradation of Ubc6. These studies show that Doa10 can recognize intramembrane degrons. It has been shown that mutations in the TD-domain affect Ubc6 degradation, but not the degradation of soluble or membrane proteins that contain the soluble Deg1 degron suggesting that the TD-domain of Doa10 is involved in recognition of intramembrane degrons (Kreft and Hochstrasser, 2011).

\subsubsection{Recognition of a cytosolic degron}

Several degrons have been identified for soluble substrates of Doa10. It has been shown that an amphipathic helix in Deg1 is important for Doa10-dependent degradation (Johnson et al., 1998). Since then, more soluble degrons have been identified like the synthetic CL1 degron as well as a 55 aa carboxy-terminal part of the mutant protein Ndc10-2 (DegAB). Disrupting the hydrophobic surface of predicted amphipathic helices interferes with Doa10-mediated degradation of those substrates (Gilon, 1998; Gilon et al., 2000; Furth et al., 2011). 


\subsubsection{Exposure of degrons}

It is still unclear how substrates are recognized in response to certain metabolites. This is best understood for the Hrd1 substrate HMG-CoA reductase. Studies indicate that the presence of the isoprenoid GGPP results in conformational changes in Hmg2 (Wangeline and Hampton, 2018). These structural changes probably expose a degron that is recognized by the Hrd1 complex. For degradation of squalene monooxygenase, an amphipathic helix localized at the N-terminal region seems to be important for its TEB4-dependent degradation (Zelcer et al., 2014).

A common feature of substrate recognition is masking of a degron by interaction partners. The degron of Sbh2 and Sbh1 only gets exposed when it does not interact with the translocon complex (Habeck et al., 2015; Shyu et al., 2019). Moreover, Mat $\alpha 2$ is stable in diploid cells when it interacts with Mata1 (Johnson et al., 1998). By interacting with the INSIG Nsg1, Hmg2 is protected from Hrd1-mediated ERAD in $S$. cerevisiae (Flury et al., 2005).

\subsubsection{Role of chaperones}

ER-luminal and cytosolic chaperones are involved in ERAD. The ER-localized chaperone Hsp70 Kar2 is involved in ERAD-L. It keeps substrates soluble and forms a complex with Hrd3/Yos9 (Nishikawa et al., 2001; Denic et al., 2006).

It is unclear which role chaperones play in substrate recognition by Doa10. Chaperones might play a role in substrate recognition and delivery to Doa10. Moreover, it is possible that chaperones are involved in maintaining substrate solubility after ubiquitination as well as extraction from the ER membrane. It has been hypothesized that the C-terminal element of Doa10 serves as a platform for chaperone binding (Kreft and Hochstrasser, 2011).

The Hsp70 chaperone Ssa1 as well as its Hsp40 co-chaperones Ydj1, Hlj1 and Sis1 have been shown to play a role in Doa10-mediated ERAD. Ssa1 is required for degradation of substrates containing an Ndc10-derived degron or the synthetic CL1 degron (Metzger et al., 2008; Furth et al., 2011; Shiber et al., 2013). Moreover, the co-chaperone Sis1 is required for normal degradation of substrates containing Ndc10derived degrons or Deg1. Interestingly, Ubc6 degradation is independent of Sis1 (Shiber et al., 2013). Ssa1 and Ydj1/Hlj1 are also required for normal degradation of Ste6* and Pma1 D378S (Huyer et al., 2004; Nakatsukasa et al., 2008; Han et al., 2007).

Several studies indicate that chaperones play a role in the interaction of substrates with Doa10. When a temperature-sensitive mutant of Sis1 (sis1-85) is expressed, a 
membrane protein containing an Ndc10-derived degron (Vma12-DegAB) does not interact with Doa10 in contrast to wildtype cells (Shiber et al., 2013). Moreover, substrates containing a CL1 or Ndc10-derived degron as well as mutant versions of Ste6 and Pma1 are not ubiquitinated when chaperones are deleted or mutant versions expressed (Metzger et al., 2008; Furth et al., 2011; Shiber et al., 2013; Nakatsukasa et al., 2008; Han et al., 2007).

\subsubsection{Summary}

In summary, ERAD recognizes exposed degrons which can be localized in the ER lumen, ER membrane or INM as well as the cytosol. Exposure of hydrophobic residues as well as posttranslational modification by glycosylation can serve as a signal for degradation. Moreover, chaperones at the luminal and cytosolic side are involved in substrate recognition at least for some ERAD substrates. Additionally, competition of ubiquitination and DUB-mediated deubiquitination might contribute to substrate discrimination (Zhang et al., 2013).

For Doa10-mediated ERAD, examples for intramembrane degrons as well as soluble degrons exist. It is unclear how those degrons are recognized. Doa10 might recognize those degrons directly. Alternatively, additional factors might be involved in substrate recognition. Chaperones might play a role, especially in the recognition of soluble degrons. As no screen has been carried out for components required for degradation of Doa10 substrates that are membrane proteins, we might be still missing components of the degradation machinery. It is possible that for intramembrane degrons, additional factors might be required for recognition of those substrates and delivery to Doa10.

\subsubsection{Retrotranslocation of substrates}

ERAD of ER luminal and membrane proteins requires a retrotranslocation step in which ubiquitinated substrates are retrotranslocated from the ER into the cytosol by the Cdc48 ATPase in complex with its cofactors Ufd1 and Npl4 (Cdc48/UN) (Rabinovich et al., 2002; Jarosch et al., 2002; Ye et al., 2001; Bays et al., 2001; Huyer et al., 2004). Whereas the role of Cdc48/UN was initially discovered by studying Hrd1 substrates, it has been shown that the Cdc48 complex is also required for ERAD of membrane proteins mediated by the Asi complex (Foresti et al., 2014) and Doa10 complex (Huyer et al., 2004; Ravid et al., 2006; Wang and Chang, 2003; Ruggiano et al., 2016; Foresti et al., 2013). Mechanistically, this process of retrotranslocation is poorly understood. The following sections summarize the current understanding of this process. 


\subsubsection{The Cdc48/Ufd1 Npl4 complex}

Cdc48 is a hexameric ATPase that belongs to the AAA family (ATPases associated with diverse cellular activities). Each Cdc48 subunit consists of an N-terminal domain and two ATPase domains (D1 and D2) that form two rings (Bodnar and Rapoport, 2017a). The cofactors Ufd1 and Npl4 associate with Cdc48 at the top side of the D1 ring. Npl4 associates with Cdc48 by contacting the D1 ring as well as the N-terminal domain (Bodnar et al., 2018; Twomey et al., 2019). Ufd1 contains a ubiquitin-binding domain (UT3), an unstructured UT6 domain that can bind to Npl4 and an SHP motif interacting with Cdc48 (Hetzer et al., 2001). Npl4 has a ubiquitin binding domain that binds to K48, but not K63-linked ubiquitin chains that contain at least 6 linked ubiquitin moieities (Tsuchiya et al., 2017).

Reconstitution and structural studies have recently expanded our understanding of the mechanism of the Cdc48/UN complex. Cdc48 gets recruited to polyubiquitinated substrates via its cofactors Ufd1/Npl4 (Stein et al., 2014; Bodnar and Rapoport, 2017b). Subsequently, the substrate is unfolded by ATP-hydrolysis in the D2 domain and translocation of the substrate through the central pore of Cdc48 (Bodnar and Rapoport, 2017b; Twomey et al., 2019). A structure of the Cdc48/UN complex bound to a polyubiquitinated substrate shows that an unfolded ubiquitin moieity is present in the pore, suggesting that unfolding of ubiquitin initiates unfolding of the substrate (Twomey et al., 2019). Substrate release from Cdc48 requires the deubiquitinating enzyme Otu1 and ATP-hydrolysis in the D1 domain. Reconstitution experiments have shown that Otu1 deubiquitination is not complete, leaving up to 10 ubiquitin moieties attached to the substrate (Stein et al., 2014; Bodnar and Rapoport, 2017b) which are probably important for recognition by proteasome receptors.

\subsubsection{Recruitment of the Cdc48-complex by Ubx2}

Cdc48, Ufd1, Npl4 as well as the membrane protein Ubx2 are part of the Doa10 and Hrd1 complex (Carvalho et al., 2006). Multiple studies have shown that Ubx2 is important for ERAD and that it recruits Cdc48 to the ER membrane. Ubx2 contains a UBA-domain and a UBX-domain and interacts with polyubiquitinated proteins as well as with Cdc48 (Schuberth et al., 2004). Whereas UBA-domains have been shown to interact with ubiquitin, UBX-domains can interact with the N-terminal domain of Cdc48 (Scott et al., 2014; Schuberth and Buchberger, 2008). In vivo, Ubx2 is required for the interaction of Cdc48 with Hrd1 as well as Doa10, indicating that it recruits Cdc48 to the ubiquitin ligase (Schuberth and Buchberger, 2005; Neuber et al., 2005). 
Interestingly, interaction between Doa10 and Ubx2 only occurs when Doa10 is active, indicating that Ubx2 is recruited to polyubiquitinated substrates or Doa10, potentially via its UBA-domain (Neuber et al., 2005). Deletion of UBX2 affects the degradation of multiple ERAD substrates (Schuberth and Buchberger, 2005; Neuber et al., 2005). For Doa10 substrates, the requirement for Ubx2 has been shown for Ubc6 and Ste6* (Neuber et al., 2005; Nakatsukasa and Kamura, 2016). In agreement with Ubx2 being a recruitment factor, it has been shown in a reconstituted system that Ubx2 binds to mono- and poly-ubiquitinated Hrd1 and that it can recruit the Cdc48/UN complex (Stein et al., 2014).

Experiments using microsomes have provided useful insights into retrotranslocation. In these in vitro assays, microsomes are incubated with cytosol. Soluble and retrotranslocated proteins are separated from membranes by ultracentrifugation. Subsequently, substrate is detected in both fractions by immunoprecipitation followed by blotting against ubiquitin (Neal et al., 2019). Such assays have been performed for the Hrd1 substrate Hmg2 as well as the Doa10 substrate Ste6*. The retrotranslocation of Hmg2 and Ste6* is dependent on Cdc48, as no retrotranslocation occurs when microsomes are prepared from cdc48-3 cells (Garza et al., 2009a; Nakatsukasa et al., 2008). Also Ubx2 has been shown to be required for retrotranslocation of those substrates (Garza et al., 2009a; Nakatsukasa and Kamura, 2016).

\subsubsection{A protein conduit for retrotranslocation}

Whereas it is established that Cdc48 provides the driving force for retrotranslocation, the identity and role of protein conduits in this process is not clear yet.

Retrotranslocation of ERAD-L substrates requires the substrate to fully cross the ER membrane. Recent studies indicate that Hrd1 forms a channel for retrotranslocation of substrates. Upon Hrd1 overexpression, Hrd3, Der1 as well as Usa1 are not required for substrate degradation indicating that Hrd1 alone can form such a channel (Carvalho et al., 2010). Moreover, recent reconstitution studies as well as a cryo-electron microscopy structure of a Hrd1/Hrd3 complex suggest that Hrd1 forms a protein conduit for luminal substrates (Baldridge and Rapoport, 2016; Schoebel et al., 2017).

It is unclear if a protein conduit is involved in retrotranslocation of membrane proteins. Candidates for such a protein conduit are the membrane-embedded ubiquitin ligases Hrd1 and Doa10 themselves as well as the multipass membrane protein Dfm1.

Dfm1 as well as the Hrd1-complex component Der1 are rhomboid pseudoproteases. They are homologous to rhomboid proteases but lack an active site (Avci and Lemberg, 
2018). Dfm1 localizes to the ER and contains an SHP-box motif that is probably involved in binding the N-terminal domain of Cdc48 (Hitt and Wolf, 2004). Dfm1 interacts with Hrd1 as well as Doa10 (Stolz et al., 2010). Moreover, Hrd1 complex components, but not Doa10 were identified by immunoprecipitation of Dfm1 (carboxyterminally tagged with calmodulin peptide and protein A module) followed by mass spectrometric identification (Goder et al., 2008). However, previous Doa10 and Hrd1 immunoprecipitation experiments have not identified Dfm1 (Carvalho et al., 2006). Two observations make it difficult to interpret some studies about Dfm1. First, early studies (Sato and Hampton, 2006; Goder et al., 2008) have used a carboxy-terminally hemagglutinin (HA)-tagged Dfm1 construct that has later been shown to be unstable in contrast to wildtype Dfm1 (Stolz et al., 2010). Moreover, a DFM1 deletion is suppressed when substrates are overexpressed. It has been shown that this suppression is due to duplication of chromosome XV leading to elevation of Hrd1 levels (Neal et al., 2018). This might explain why other studies did not identify a role of Dfm1 in ERAD (Sato and Hampton, 2006; Goder et al., 2008).

Using a microsome-based assay as described above, it has been shown that a selfubiquitinating ERAD substrate that does not require ubiquitination by Hrd1, is retrotranslocated independent of the presence of Hrd1. In contrast, it is only retrotranslocated in the presence of Dfm1 suggesting that Dfm1 forms a protein conduit for ERADM substrates (Garza et al., 2009a; Neal et al., 2018). Neal et al. have also shown that Dfm1 is required for retrotranslocation of Ste6* ${ }^{*}$. These results are in agreement with stabilization of Ste6* in the absence of Dfm1 (Stolz et al., 2010). Further studies are necessary to understand the function of Dfm1 in retrotranslocation of Ste ${ }^{*}$ and to test if Dfm1 is also involved in degradation of other Doa10 substrates.

Besides Dfm1, it has been hypothesized that Doa10 forms a protein conduit for retrotranslocation of membrane proteins, due to its large TM region comprising 14 TM segments (Swanson et al., 2001). The role of Doa10 in retrotranslocation has not been investigated yet and thus it remains unclear if Doa10 has besides its role as a ubiquitin ligase such a function in retrotranslocation of substrates.

\subsubsection{Questions regarding the mechanism of Doa10-mediated ERAD}

We lack a mechanistic understanding of processes such as substrate recognition, ubiquitination and retrotranslocation during Doa10-mediated ERAD. Moreover, it is unclear if the components identified are sufficient for Doa10-mediated ERAD. The following questions remain to be answered:

- How are substrates recognized? In one model, substrates are directly recog- 
nized by Doa10. Alternatively, chaperones or adaptors recognize substrates and deliver them to Doa10. Moreover, it is unclear how substrates such as Erg1 are recognized in a regulatory manner upon accumulation of certain metabolites.

- What is the role of Ubc6 and Ubc7 in substrate ubiquitination? Ubc6 and Ubc7 have been shown to have different functions. However, Ubc6/Ubc7mediated ubiquitination of Doa10 substrates has not been investigated yet in a reconstituted system. Moreover, it is unclear how Doa10 coordinates these two E2 enzymes.

- What is the function of Ubc6 turnover? Ubc6 has a short half life and is degraded in a Doa10-dependent manner, but the physiological relevance of this process is unclear.

- How does Cdc48/UN extract substrates? Cdc48/UN action at the cytosolic side leads to extraction of transmembrane and ER-luminal domains of substrates. How this is achieved is unclear. Moreover, whereas it is known that Ubx2 can recruit Cdc48 to the ERAD-machinery, its precise function is unclear.

- How is retrotranslocation coordinated? It is unclear if a protein conduit exists for retrotranslocation of membrane proteins. Doa10 as well as Dfm1 might have such a role in retrotranslocation.

To address these questions and get mechanistic insight, reconstitution studies with purified components were used in this thesis. Different approaches exist for the reconstitution of reactions consisting of multiple membrane proteins.

\subsection{Reconstitution approaches to study the function of mem- brane proteins}

Reconstitution of reactions with membrane proteins requires the presence of amphiphilic molecules that shield the TM segments from the aqueous solution and therefore prevent aggregation of the protein. Different model membrane systems exist that can be used to incorporate membrane proteins into lipid bilayers such as unilamellar phospholipid vesicles (liposomes) and nanodiscs which are self-assembled structures of a lipid bilayer surrounded by a protein scaffold (Civjan et al., 2003). Reconstitution protocols usually are based on the solubilization of lipids with detergent, addition of membrane proteins and subsequent detergent removal to allow for incorporation of the membrane proteins 
into the spontaneously forming lipid bilayer. The conditions for this protocol (e.g. detergent used, concentration of proteins and lipids) mostly depend on the membrane protein used and have to be determined experimentally (reviewed by Shen et al., 2013).

For the reconstitution of pathways, often multiple membrane proteins have to be co-reconstituted. To overcome the problem of limited conditions for co-reconstitution as well as to avoid interaction of membrane proteins before reconstitution into the lipid bilayer, approaches based on fusion of liposomes have been developed. In those assays, proteins are reconstituted into separate liposomes and co-reconstituted upon fusion of liposomes.

In cells, many fusion events are mediated by SNARE (soluble N-ethylmaleimidesensitive factor attachment protein receptors) proteins. SNAREs are anchored to the membrane via a TM segment or a lipid anchor and contain a conserved SNARE motif that can be classified into Qa, Qb, Qc and R-motifs. SNAREs form heterotypic complexes between two membranes (QabcR complexes). During neuronal exocytosis, synaptic vesicles containing the SNARE synaptobrevin 2 (Syb) fuse with the plasma membrane where the SNAREs SNAP25-A (SNAP25) and syntaxin-1A (syntaxin) are localized (reviewed by Jahn and Scheller, 2006). In a reconstituted system using liposomes, they are sufficient to catalyze fusion (Weber et al., 1998). SNARE-based systems have been used to develop assays for co-reconstitution of membrane proteins.

Nordlund et al. co-reconstituted parts of the respiratory chain from E. coli into liposomes (Nordlund et al., 2014). $\mathrm{F}_{1} \mathrm{~F}_{0}$ ATP synthase was co-reconstituted into liposomes with SNAP25/syntaxin and bo3-oxidase with synaptobrevin. As a readout for fusion, the authors measured the ATP-production upon addition of the electron donors DTT and ubiquinol Q1. ATP-production was specific for the presence of SNAREs indicating co-reconstitution. Later, Biner et al. (2016) showed that membrane proteins of the respiratory chain can be also co-reconstituted in the absence of SNAREs, by fusing liposomes with co-reconstituted lipids of opposite charge. They have also used this approach for co-reconstitution of different membrane proteins into giant unilamellar vesicles (GUVs). To do so, liposome sets containing different membrane proteins were fused with GUVs. For reconstition of the membrane protein bacteriorhodopsin into GUVs, another approach has been reported. Kahya et al. (2001) reconstituted bacteriorhodopsin into liposomes and used a small peptide that when anchored to the membrane serves as a fusogen to fuse liposomes with GUVs. Moreover, SNAREmediated fusion was employed to deliver the $\mathrm{F}_{0} \mathrm{~F}_{1}$ ATP synthase (from the thermophilic Bacillus PS3) co-reconstituted with SNAREs to synaptic vesicles that contain Syb (Preobraschenski et al., 2014). In this study, an acceptor SNARE complex was used that 
has been shown to catalyze efficient membrane fusion in vitro (Pobbati, 2006; Hernandez et al., 2012). This complex termed $\Delta \mathrm{N}$ complex consists of syntaxin, SNAP25 and a C-terminal Syb fragment that gets displaced by full-length Syb upon formation of the trans-SNARE complex. The $\Delta \mathrm{N}$ complex is more efficient in fusion than the individual SNARE proteins, as syntaxin and SNAP25 alone form 2:1 complexes which blocks the binding of synaptobrevin (Pobbati, 2006).

Although all of these studies report co-reconstitution of membrane proteins, the co-reconstitution efficiency that they achieved is not known. This can be only determined by biochemical assays such as pulldowns of individual proteins after fusion or other functional readouts that show a fusion-specific activity relative to the total protein content. To reconstitute ERAD and study reactions such as ubiquitination, a high co-reconstitution efficiency is required. We therefore decided to use the well studied SNARE-fusion system, in particular the highly fusogenic $\Delta \mathrm{N}$ complex with synaptobrevin, to co-reconstitute the membrane proteins Doa10, Ubc6 and Cue1. 


\subsection{Aims}

During this thesis, I have established a reconstituted system using purified components and liposomes. Using this system, the following topics have been addressed:

\section{Reconstitution of Doa10-mediated ERAD of Ubc6 (Chapter 3 and 4)}

I have investigated the behaviour of Ubc6 as E2 enzyme and substrate and recapitulated Doa10-mediated polyubiquitination of Ubc6. Moreover I have recapitulated retrotranslocation of Ubc6 by the Cdc48/UN-complex. I observe that Doa10 acts as a retrotranslocase that facilitates removal of Ubc6 from the membrane.

\section{Identification of structural elements important for Doa10 function} (Chapter 5)

I have tested the behaviour of previously described Doa10 mutants in ubiquitination of Ubc6 as well as their retrotranslocase activity. Moreover, I have optimized a protocol for site-specific photocrosslinking to identify interaction sites between the TM anchor of Ubc6 and Doa10 in collaboration with Iwan Parfentev (Laboratory of Prof. Urlaub, MPI for Biophysical Chemistry, Göttingen).

\section{Analysis of Doa10-mediated ERAD of Sbh2 (Chapter 6)}

We have performed a screen to identify components necessary for degradation of Sbh2 in collaboration with Ákos Farkas (Laboratory of Prof. Schwappach-Pignataro, University Medical Center, Göttingen). In combination with reconstitution studies, we have identified the minimal machinery for ubiquitination of Sbh2. 


\section{Materials and Methods}

\subsection{Materials}

The following section lists materials used in this thesis.

Table 2.1: Lipids used in this thesis.

\begin{tabular}{|c|c|c|}
\hline Name & Company & $\begin{array}{l}\text { Catalog } \\
\text { number }\end{array}$ \\
\hline $\begin{array}{l}\text { 1-palmitoyl-2-oleoyl-glycero3-phosphocholine } \\
\text { (POPC) }\end{array}$ & $\begin{array}{l}\text { Avanti Polar } \\
\text { Lipids }\end{array}$ & $850457 \mathrm{P}$ \\
\hline $\begin{array}{l}\text { 1,2-dioleoyl-sn-glycero-3-phosphoethanolamine } \\
\text { (DOPE) }\end{array}$ & $\begin{array}{l}\text { Avanti Polar } \\
\text { Lipids }\end{array}$ & $850725 \mathrm{P}$ \\
\hline $\begin{array}{l}\text { 1,2,-dioleoyl-sn-glycero-3-phospho-L-serine } \\
\text { (DOPS) }\end{array}$ & $\begin{array}{l}\text { Avanti Polar } \\
\text { Lipids }\end{array}$ & $840035 \mathrm{P}$ \\
\hline $\begin{array}{l}\text { 1,2-dioleoyl-sn-glycero-3-phosphoethanolamine-N- } \\
\text { (biotinyl) }\end{array}$ & $\begin{array}{l}\text { Avanti Polar } \\
\text { Lipids }\end{array}$ & $870282 \mathrm{P}$ \\
\hline $\begin{array}{l}\text { 1,2-dioleoyl-sn-glycero-3-phosphoethanolamine-N- } \\
\text { (lissamine rhodamine B sulfonyl) (Rhd-PE) }\end{array}$ & $\begin{array}{l}\text { Avanti Polar } \\
\text { Lipids }\end{array}$ & $810150 \mathrm{P}$ \\
\hline Ergosterol ( $\geq 95 \%$, HPLC) & Sigma-Aldrich & 45480 \\
\hline
\end{tabular}

Table 2.2: Detergents used in this thesis.

\begin{tabular}{l|l} 
Name & Company \\
\hline \hline Anapoe-X-100 (Triton X-100) & Anatrace \\
Decyl Maltose Neopentyl Glycol (DMNG) & Anatrace \\
n-Decyl $\beta$-maltoside Glycon Biochemicals (DM) & Glycon Biochemicals \\
GDN101 (GDN) & Anatrace \\
n-Octyl $\beta$-D-glucopyranoside (OG) & Glycon Biochemicals \\
Sodium cholate hydrate, $\geq 99 \%$ & Sigma-Aldrich
\end{tabular}


Table 2.3: Miscellanous materials used in this thesis.

\begin{tabular}{|c|c|c|}
\hline Name & Company & Catalog number \\
\hline ATP & PanReac AppliChem & A1348 \\
\hline AlexaFluor488 maleimide & $\begin{array}{l}\text { Thermo Fisher } \\
\text { Scientific }\end{array}$ & A10254 \\
\hline BSA: Bovine serum albumin & PanReac AppliChem & A1391 \\
\hline Coomassie Brilliant blue G-250 & PanReac AppliChem & A3480 \\
\hline DyLight 680 maleimide & $\begin{array}{l}\text { Thermo Fisher } \\
\text { Scientific }\end{array}$ & 46618 \\
\hline DyLight 800 maleimide & $\begin{array}{l}\text { Thermo Fisher } \\
\text { Scientific }\end{array}$ & 46621 \\
\hline Dithiothreitol (DTT) & Formedium & DTT025 \\
\hline HisPur NiNTA resin & $\begin{array}{l}\text { Thermo Fisher } \\
\text { Scientific }\end{array}$ & 88223 \\
\hline Nycodenz & Alere Technologies & 1002424 \\
\hline Pepstatin A & Peptide Institute & 4397 \\
\hline Phenylmethyl sulphonyl fluoride (PMSF) & Carl Roth & 6367 \\
\hline $\begin{array}{l}\text { Pierce Complete Protease Inhibitor } \\
\text { Tablets }\end{array}$ & $\begin{array}{l}\text { Thermo Fisher } \\
\text { Scientific }\end{array}$ & A32963 \\
\hline Pierce Detergent removal spin columns & $\begin{array}{l}\text { Thermo Fisher } \\
\text { Scientific }\end{array}$ & 87777 \\
\hline $\begin{array}{l}\text { Pierce High Capacity Streptavidin } \\
\text { Agarose }\end{array}$ & $\begin{array}{l}\text { Thermo Fisher } \\
\text { Scientific }\end{array}$ & 20361 \\
\hline Pierce Streptavidin Magnetic Beads & $\begin{array}{l}\text { Thermo Fisher } \\
\text { Scientific }\end{array}$ & 88817 \\
\hline $\begin{array}{l}\text { Tris(2-carboxyethyl)-phosphin- } \\
\text { hydrochlorid (TCEP) }\end{array}$ & Sigma-Aldrich & $\mathrm{C} 4706$ \\
\hline Trypsin & Roche & 11047841001 \\
\hline Ubiquitin (WT) & Boston Biochem & $\mathrm{U}-100 \mathrm{Sc}$ \\
\hline Ubiquitin (K0) & LifeSensors & SI209 \\
\hline Ubiquitin (K48R) & Enzo Lifesciences & $\begin{array}{l}\text { BML-UW8615- } \\
0001\end{array}$ \\
\hline Ubiquitin (K48) & Enzo Lifesciences & $\begin{array}{l}\text { BML-UW0235- } \\
0001\end{array}$ \\
\hline
\end{tabular}


Table 2.4: Materials used for culturing E. coli and S. cerevisiae in this thesis.

\begin{tabular}{l|l|l} 
Name & Company & $\begin{array}{l}\text { Catalog } \\
\text { number }\end{array}$ \\
\hline \hline $\begin{array}{l}\text { 4-Benzoyl-L-phenylalanine (BpA) } \\
\text { D-(+)-Galactose }\end{array}$ & $\begin{array}{l}\text { Iris Biotech } \\
\text { PanReac AppliChem }\end{array}$ & A1131 \\
CSM quadruple dropout (-HTLU) & Formedium & DCS1389 \\
Histidine & Formedium & DOC0142 \\
Leucine & Formedium & DOC0154 \\
L-(+)-Arabinose & Sigma-Aldrich & A3256 \\
IPTG, dioxane free & Formedium & IPTG025 \\
D-Mannitol & Serva & 28410.02 \\
Uracil & Formedium & DOC0214 \\
Tryptophan & Formedium & DOC0186 \\
YEP broth & Formedium & CCM0410 \\
Yeast Nitrogen Base (YNB) & US Biological Life Sciences & C19032801
\end{tabular}

Table 2.5: Commercial kits used in this thesis.

\begin{tabular}{l|l} 
Name & Company \\
\hline \hline Gibson Assembly Master Mix & New England Biolabs \\
Q5 Site-Directed Mutagenesis Kit & New England Biolabs \\
B-PER Bacterial Protein Extraction Reagent & Thermo Fisher Scientific \\
MasterPure Yeast DNA Purification Kit & Epicentre (Lucigen)
\end{tabular}

\subsection{General methods for DNA cloning and protein expression}

For cloning, the Gibson assembly kit and the Site-directed mutagenesis kit (both from New England Biolabs) were used according to the manufacturer's protocol. Oligonucleotides used for cloning are listed in the Supplement (Tables S4, S5, S6, S7, S8, S9). Plasmids used in this study are listed in Table $\mathrm{S} 2$, and $S$. cerevisiae strains are listed in Table S3. NEB 5-alpha Competent E. coli (New England Biolabs) were used for cloning purposes. All bacterial transformations were carried out according to the manufacturer's protocol. Yeast transformations were carried out using an adapted protocol from Gietz et al. (1995). Briefly, cells were diluted to an $\mathrm{OD}_{600}$ of 0.2 and grown until an $\mathrm{OD}_{600}$ of approximately 1 . After harvesting (3000 x g, 5 min, RT) cells were washed 
in $\mathrm{H}_{2} \mathrm{O}$. The cell pellet was then resuspended in $1 \mathrm{~mL} 100 \mathrm{mM}$ lithium acetate. Cells were pelleted (table centrifuge, max. speed, $5 \mathrm{~s}$ ) and the cell pellet resuspended again in $100 \mathrm{mM}$ lithium acetate $(500 \mu \mathrm{L}$ for $50 \mathrm{~mL}$ yeast culture). The cell suspension was aliquoted into $50 \mu \mathrm{L}$ aliquots, again pelleted and the supernatant removed. Subsequently, a transformation mix was added, consisting of $240 \mu \mathrm{L}$ polyethylene glycol (50\% (w/v)), $36 \mu \mathrm{L}$ lithium acetate (1 M stock), $25 \mu \mathrm{L}$ single-stranded carrier DNA $(2 \mathrm{mg} / \mathrm{mL}$ stock) and $50 \mathrm{\mu L}$ of DNA (approximately $200 \mathrm{ng}$ ). The suspension was vortexed $(1 \mathrm{~min})$ and subsequently incubated at $30^{\circ} \mathrm{C}(30 \mathrm{~min})$. After heat shock at $42^{\circ} \mathrm{C}$ (20 min), the transformation mix was removed (max. speed, 5s, table top centrifuge). For plasmid transformations, the cells were resuspended directly in $\mathrm{H}_{2} \mathrm{O}$ and plated onto selective plates. For transformation of DNA-fragments for in vivo homologous recombination, the cells were resuspended in rich medium (YPD), incubated at $30^{\circ} \mathrm{C}$ for $2 \mathrm{~h}$, and then after removing the YPD and resuspending in $\mathrm{H}_{2} \mathrm{O}$ plated on selective plates.

Bacteria were grown in LB medium (Miller, 1992) for cloning purposes and in Terrific Broth (TB) medium (Hobbs and Tartoff, 1987) for expression, unless otherwise indicated. When needed, antibiotics were added with the following final concentrations: ampicillin $(100 \mu \mathrm{g} / \mathrm{mL})$, kanamycin $(40 \mu \mathrm{g} / \mathrm{mL})$, chloramphenicol $(34 \mu \mathrm{g} / \mathrm{mL})$, unless otherwise stated.

\subsection{Reconstitution of Doa10-mediated ERAD of Ubc6}

This section is part of the manuscript that is a part of this thesis (Chapter 3) and has been written by Claudia Schmidt.

\subsubsection{Strains used for protein expression}

For protein expression in E. coli, BL21-CodonPlus (DE3)-RIPL competent cells (Agilent) were used. Where indicated, BL21 (DE3) competent cells (NEB) were used instead. To express Doa10 in S. cerevisiae, a DOA10 deletion strain derived from BY4741 was used.

\subsubsection{Constructs}

\subsubsection{Expression of Doa10 in $S$. cerevisiae}

DOA10 constructs were cloned into the $2 \mu$ plasmid pRS426 containing a Gal1 promotor (Mumberg et al., 1994), and appended with a C-terminal tobacco etch virus (TEV) 
protease cleavage site followed by a streptavidin-binding peptide (SBP) tag (Keefe et al., 2001), in which the single lysine was mutated to arginine, and a short sequence for Sortase-mediated labeling with fluorescent dyes (Popp et al., 2009). The full Cterminal tag for Doa10 had the sequence GSGENLYFQSGGGMDERTTGWRGGHVVEGLAGELEQ LRARLEHHPQGQREPLPETGG. As full-length DOA10 is toxic for E. coli (Mandart et al., 1994), the DOA10 sequence was split in two parts and cloned into two separate plasmids, similarly to as described before (Swanson et al., 2001). Sequences coding for Doa10 $1-468^{-S B P}$ and Doa10 $225-1319^{-S B P}$, were cloned into a pRS426-pGal1 plasmid using XhoI/SpeI restriction sites (plasmids \#376 and \#375, respectively). A plasmid containing full-length (fl) Doa10 was subsequently generated in S. cerevisiae by homologous recombination. To do so, plasmid \#375 was linearized (starting from Doa10 residue 225) and an N-terminal fragment was generated from plasmid \#376 (Doa10 residues 1-257) by PCR. The N-terminal fragment contained 80 to 100 nt overlaps with the linearized plasmid. Both PCR-products were co-transformed into S. cerevisiae. Correct homologous recombination was confirmed by sequencing of the PCR-amplified insert after preparation of total DNA of the generated strain (yAST112). The construct for expression of Doa10 $434-1319$ contained an N-terminal SBP-SUMO* tag (Liu et al., 2008) and a C-terminal Sortase (LPETGG) tag.

\subsubsection{Expression of proteins in $E$. coli}

UBC6 from $S$. cerevisiae and its variants were cloned into the K27SUMO vector using the SfoI restriction site (Stein et al., 2014). This vector encodes an N-terminal His H- $^{-}$ SUMO-tag. Ubc6 and its variants were appended with a C-terminal LPETGG tag for Sortase-mediated labeling. To clone a construct for expression of Ubc6 $6_{\mathrm{SybTM}}$ and Sybubc6TM, the sequence for the cytosolic Ubc6 domain (aa 232-250) was fused to the sequence for the Syb TM domain (aa 96-116). Vice versa, the cytosolic Syb domain (aa 1-95) was fused to the Ubc6 TM domain (aa 232-250). Constructs containing a Syb TM domain contained a linker between the TM domain and the LPETGG tag for efficient Sortase labeling with the sequence GSGSATGSGGS. To clone a construct for expression of Ub-Ubc6 $6_{\mathrm{C} 87 \mathrm{~A}}$ and $\mathrm{Ub}-\mathrm{Ubc} 6_{\mathrm{C} 87 \mathrm{~A} / \mathrm{SybTM}}$, the sequence encoding ubiquitin ${ }_{\mathrm{V} 76}$ (aa 1-76) was inserted between the sequence encoding the His ${ }_{14}$-SUMO tag and Ubc6. For efficient Ulp1-cleavage, a linker sequence (coding for GSG) was inserted between the His $_{14}$-SUMO tag and ubiquitin (Vasic et al., 2020).

To generate a construct for expression of SUMO-Ubc6, a C-terminal TEV-cleavage site flanked by linker sequences was introduced between the Ubc6 TM domain and the LPETGG tag resulting in GSGS-ENLYFQS-SGLPETGG. To generate a construct for 
expression of Ubc6-SBP, Ubc6 was engineered with a C-terminal TEV-cleavage site separated from Ubc6 by a linker (GSGENLYFQSGGG) followed by an SBP-tag and a tag (LPETGG) for Sortase-mediated labeling. The coding sequence for His 14 -SUMOUbc6-SBP was inserted into a pET39b(+) vector (Novagen) right after the DsbA signal sequence.

The expression construct for Cue1 has been described previously (Vasic et al., 2020).

The coding sequence for Get3 (and Get3 ${ }_{\mathrm{D} 57 \mathrm{~N}}$ ) was inserted into the K27SUMO vector using the SfoI restriction site. The expression construct contained an N-terminal His $_{14}$-SUMO tag. An expression construct encoding for Get3 $3_{\text {I193D }}$ was expressed from a pET28 vector (kind gift from Blanche Schwappach-Pignataro). All constructs for expression of SNAREs from rattus norvegicus have been previously described (Hernandez et al., 2012; Stein et al., 2007).

\subsubsection{Expression and purification of proteins}

\subsubsection{In $S$. cerevisiae}

Expression of Doa10 was essentially performed as described previously for Hrd1 (Stein et al., 2014). Yeast cells were grown in minimal synthetic medium containing $2 \%$ (w/v) Glucose and amino acid drop-out supplements at $30^{\circ} \mathrm{C}$. An overnight-culture (grown for 24h) was diluted 1:50 into fresh medium. After 24h of growth, expression was induced by addition of yeast extract, peptone and galactose to final concentrations of $1 \%, 2 \%$, and $2 \%(\mathrm{w} / \mathrm{v})$, respectively. After 17-19 h of induction, the cells were harvested at $3000 \mathrm{x} \mathrm{g}$, washed once with $\mathrm{ddH}_{2} \mathrm{O}$, resuspended in a minimal amount of $\mathrm{H} 2 \mathrm{O}$ and stored at $-80^{\circ} \mathrm{C}$.

A membrane fraction was prepared as described previously (Stein et al., 2014), with some modifications. Briefly, $150 \mathrm{~g}$ of cells were resuspended in $900 \mathrm{~mL}$ of cold $\mathrm{H}_{2} \mathrm{O}$ and incubated with $2 \mathrm{mM}$ DTT for $15 \mathrm{~min}$ on ice. All subsequent steps were done at $4^{\circ} \mathrm{C}$. The cells were then pelleted at $3000 \mathrm{x}$ g and resuspended in lysis buffer $(20 \mathrm{mM}$ HEPES/KOH pH 7.5, $5 \mathrm{mM}$ potassium acetate, $600 \mathrm{mM}$ mannitol, $0.5 \mathrm{mM}$ EDTA). PMSF (1 mM) and Pepstatin A $(2 \mu \mathrm{M})$ were added freshly. The cell suspension was then added to a bead beating chamber (total volume $=300 \mathrm{~mL}$ ) filled up to $1 / 3$ with zirkonia beads. Cells were lysed in a Bead Beater (Biospec Products) with cycles of $20 \mathrm{~s}$ on and $2 \mathrm{~min}$ breaks in between for $50 \mathrm{~min}$. Beads were filtered off, and the lysate was centrifuged at $1,500 \mathrm{x}$ g for $10 \mathrm{~min}$. The supernatant was subsequently pelleted at $40,000 \mathrm{x}$ g for $45 \mathrm{~min}$ (Ti45 rotor). The pelleted crude membrane fraction was resuspended in $200 \mathrm{~mL}$ lysis buffer by douncing and again pelleted at 180,000 x $\mathrm{g}$ for 
$30 \mathrm{~min}$ (Ti45 rotor). The pellet was resuspended in $40 \mathrm{~mL}$ and snap-frozen in liquid nitrogen for storage at $-80^{\circ} \mathrm{C}$. The total protein concentration of the membrane fraction was determined using the Pierce ${ }^{\mathrm{TM}} 660 \mathrm{~nm}$ Protein Assay (Thermo Scientific).

To purify Doa10-SBP, the membrane fraction was solubilized with $1.3 \%(\mathrm{w} / \mathrm{v})$ GDN (Anatrace) at a protein concentration of $3-4 \mathrm{mg} / \mathrm{mL}$ in $20 \mathrm{mM}$ Hepes/KOH pH 7.4, $300 \mathrm{mM} \mathrm{KCl}, 0.5 \mathrm{mM}$ TCEP, $5 \mathrm{mM}$ magnesium acetate, supplemented with $1 \mathrm{mM}$ PMSF and 1 Pierce Complete EDTA-free protease inhibitor cocktail (Roche) per $100 \mathrm{~mL}$ solubilization volume. After $1 \mathrm{~h}$ solubilization, insoluble material was pelleted at 40,000 rpm for $30 \mathrm{~min}$ (Ti45 rotor). The supernatant was added to $4 \mathrm{~mL}$ Pierce High Capacity Streptavidin Agarose slurry (Thermo Scientific) and incubated for $3 \mathrm{~h}$. The beads were then filtered off and washed with 4 x $25 \mathrm{~mL}$ of wash buffer (20 mM HEPES/KOH pH 7.4, $150 \mathrm{mM} \mathrm{KCl,} 5 \mathrm{mM}$ magnesium acetate, $0.5 \mathrm{mM}$ TCEP, $150 \mu \mathrm{M}$ GDN). Doa10-SBP was eluted with wash buffer supplemented with $2 \mathrm{mM}$ biotin. Doa10-SBP was further purified by sucrose density gradient ultracentrifugation. Gradients were prepared with two solutions where the less dense solution contained GDN (solution A: 20 mM Hepes/KOH pH 7.4, $150 \mathrm{mM} \mathrm{KCl,} 2 \mathrm{mM}$ magnesium acetate, $10 \%$ (w/v) sucrose, 0.5 mM TCEP, $100 \mu \mathrm{M}$ GDN, solution B: $20 \mathrm{mM}$ Hepes/KOH pH 7.4, $150 \mathrm{mM} \mathrm{KCl,} 2 \mathrm{mM}$ magnesium acetate, 25\% (w/v) sucrose, $0.5 \mathrm{mM}$ TCEP). Gradients were prepared using a gradient mixer (Gradient Master, Biocomp Instruments) at RT and kept at $4^{\circ} \mathrm{C}$ before. $500 \mu \mathrm{L}$ were removed from the top of gradient, and an equal volume of sample was loaded on top of the gradient. After centrifugation at 40,000 rpm (19 h, slow break, SW41Ti rotor), the gradient was harvested in $500 \mu \mathrm{L}$ fractions. Doa10-containing fractions were concentrated with Amicon Ultra Centrifugal Filters (Merck) using a $100 \mathrm{kDa}$ cut-off. The same protocol was used for purification of Doa10 ${ }_{1-468-S B P}$ and SBP-SUMO*-Doa10 $434-1319$.

\subsubsection{In $E$. coli}

For bacterial expression, an overnight culture was diluted 1:50 into Terrific Broth and grown at $37^{\circ} \mathrm{C}$. At an $\mathrm{OD}_{600}$ of 0.5 , the cells were shifted to $18^{\circ} \mathrm{C}$ and expression induced with $0.5 \mathrm{mM}$ IPTG. After approximately $20 \mathrm{~h}$ of induction, cells were harvested at $4000 \mathrm{rpm}$, resuspended in buffer I30 (50 mM Tris/ $\mathrm{HCl} \mathrm{pH} 8.0$ (at $\left.4^{\circ} \mathrm{C}\right), 500 \mathrm{mM} \mathrm{NaCl}$, $30 \mathrm{mM}$ Imidazole) and stored at $-20^{\circ} \mathrm{C}$.

A bacterial membrane fraction was prepared as described previously (Vasic et al., 2020).

Ubc6 and its variants were purified via an N-terminal His ${ }_{14}-\mathrm{SUMO}$ tag. Ubc6 was purified as described previously (Vasic et al., 2020). 
To purify Ubc6-SBP, an additional purification step was included to ensure that only full-length Ubc6-SBP was purified. After size-exclusion chromatography, the protein was diluted to $0.5 \mathrm{mg} / \mathrm{mL}$ and bound to Pierce High Capacity Streptavidin Agarose (Thermo Scientific). After washing the beads with buffer used during the size exclusion chromatography, Ubc6-SBP was eluted with buffer supplemented with $2 \mathrm{mM}$ biotin. Eluted protein was then used for Sortase-mediated labeling.

To purify SUMO-Ubc6 (containing a C-terminal TEV cleavage site), protein was eluted from the Ni-NTA resin with wash buffer containing $500 \mathrm{mM}$ imidazole, and then as described above further purified by size-exclusion chromatography (Superdex 200).

To purify Get3, bacterial lysate was cleared by ultracentrifugation (40,000 rpm, $45 \mathrm{~min}, 4^{\circ} \mathrm{C}$, Ti45 rotor) and the supernatant incubated with Ni-NTA slurry $(6 \mathrm{ml}$ slurry for $6 \mathrm{~L}$ of culture) for $2 \mathrm{~h}$. Beads were filtered off and washed with 4 x $50 \mathrm{~mL}$ buffer I30 and $50 \mathrm{~mL}$ of buffer I10 $\left(20 \mathrm{mM}\right.$ Tris/ $\mathrm{HCl} \mathrm{pH} 8.0$ (at $\left.4^{\circ} \mathrm{C}\right), 200 \mathrm{mM} \mathrm{NaCl}$, $10 \mathrm{mM}$ Imidazole). Get3 was eluted from beads by cleavage with Ulp1as described above. The elution was supplemented with $1 \mathrm{mM}$ DTT and further purified by sizeexclusion chromatography using a Superdex 200 HiLoad 16/60 column (GE Healthcare) equilibrated with $20 \mathrm{mM}$ HEPES/KOH pH 7.4, $200 \mathrm{mM} \mathrm{NaCl}, 1 \mathrm{mM}$ DTT.

Cue1 was purified as described previously (Vasic et al., 2020). Uba1, Ubc7, Cdc48 and Ufd1/Npl4 were purified as described (Stein et al., 2014).

To express the t-SNARE complex, plasmids encoding syntaxin-1a (aa 183-288), synaptobrevin-2 (aa 49-96) (pETDuet-1 vector) and SNAP-25A (pET28a vector) were co-transformed into BL21 (DE3) E. coli cells (NEB) and expressed as described previously (Stein et al., 2007). Briefly, at an $\mathrm{OD}_{600}$ of 0.5 , the cells were shifted to $18^{\circ} \mathrm{C}$ and induced with $0.5 \mathrm{mM}$ IPTG. After approximately $20 \mathrm{~h}$ of induction, the cells were harvested at $4000 \mathrm{rpm}$, resuspended in Buffer $\mathrm{I} 8\left(50 \mathrm{mM}\right.$ Tris $/ \mathrm{HCl} \mathrm{pH} 8.0$ at $4^{\circ} \mathrm{C}$, $500 \mathrm{mM} \mathrm{NaCl}, 8 \mathrm{mM}$ Imidazole) and stored at $-20^{\circ} \mathrm{C}$. After cell lysis using a microfluidizer (in the presence of $1 \mathrm{mM}$ PMSF and Complete protease inhibitor), the lysate was cleared by ultracentrifugation (40,000 rpm, $30 \mathrm{~min}$, Ti45 rotor). The pellet was resuspended in Buffer I8 supplemented with 5\% (w/v) sodium cholate, $2 \mathrm{M}$ urea, $200 \mathrm{mM}$ sucrose and $1 \mathrm{mM}$ PMSF. After solubilization for $30 \mathrm{~min}$ at RT, insoluble material was pelleted by ultracentrifugation $\left(40,000 \mathrm{rpm}, 30 \mathrm{~min}, 4^{\circ} \mathrm{C}\right.$, Ti45 rotor). Ni-NTA slurry ( $6 \mathrm{~mL}$ for $6 \mathrm{~L}$ of culture) was added to the supernatant and incubated for $3 \mathrm{~h}$ at $4^{\circ} \mathrm{C}$ while rotating. Beads were filtered off and washed with $4 \times 50 \mathrm{~mL}$ wash buffer (20 mM Tris/ $\mathrm{HCl} \mathrm{pH} 8.0$ (at $4^{\circ} \mathrm{C}$ ), $500 \mathrm{mM} \mathrm{NaCl}, 8 \mathrm{mM}$ imidazole, $200 \mathrm{mM}$ sucrose, $2 \%$ (w/v) octyl glucoside (OG, Glycon)). Protein was eluted with wash buffer supplemented with $400 \mathrm{mM}$ Imidazole. $1 \mathrm{mM}$ DTT and $0.05 \mathrm{mg} / \mathrm{mL}$ of thrombin (100x stock 
prepared in $50 \%(\mathrm{w} / \mathrm{v})$ glycerol) were added to the elution fractions and incubated at $4^{\circ} \mathrm{C}$ overnight. After thrombin-cleavage, the solution was diluted until the conductivity reached $15 \mathrm{mS} / \mathrm{cm}$ with buffer A (20 mM Tris/HCl pH 7.4 (RT), $1 \mathrm{mM}$ DTT, $200 \mathrm{mM}$ sucrose, $2 \%(\mathrm{w} / \mathrm{v}) \mathrm{OG})$. The protein was further purified by ion exchange chromatography on a MonoQ column (GE healthcare) equilibrated with $20 \mathrm{mM}$ Tris/HCl pH 7.4 (RT), $150 \mathrm{mM} \mathrm{NaCl}, 1 \mathrm{mM}$ DTT, $200 \mathrm{mM}$ sucrose, 2\% (w/v) OG and eluted in a gradient until $450 \mathrm{mM} \mathrm{NaCl}$ (elution at approximately $25 \mathrm{mS} / \mathrm{cm}$ ).

To express Syb, a plasmid encoding His $_{6}$-thrombin-Syb was transformed into BL21 (DE3) E. coli cells (NEB). Expression and preparation of a membrane fraction were done as described above, in buffer I15 (50 mM Tris/HCl pH 8.0, $500 \mathrm{mM} \mathrm{NaCl}, 15 \mathrm{mM}$ imidazole). The membrane fraction was solubilized in buffer I15 supplemented with $2.5 \%(\mathrm{w} / \mathrm{v})$ sodium cholate for $30 \mathrm{~min}$. After clearing the lysate by ultracentriguation, the supernatant was incubated with Ni-NTA slurry $(6 \mathrm{~mL}$ for $6 \mathrm{~L}$ culture) for $3 \mathrm{~h}$. Beads were filtered off and washed with 2 x $50 \mathrm{~mL}$ wash buffer $\mathrm{I} 15$ supplemented with $1.5 \%(\mathrm{w} / \mathrm{v})$ sodium cholate and subsequently with $4 \times 50 \mathrm{~mL}$ wash buffer $\mathrm{I} 15$ supplemented with $5 \mathrm{mM}$ decylmaltoside (DM, Anatrace). Protein was eluted with wash buffer supplemented with $400 \mathrm{mM}$ imidazole and $5 \mathrm{mM}$ DM. $0.05 \mathrm{mg} / \mathrm{mL}$ of thrombin was added to the elution fractions. The solution was dialyzed overnight against $10 \mathrm{mM}$ MOPS, $50 \mathrm{mM} \mathrm{NaCl}, 1 \mathrm{mM}$ DTT, $1 \mathrm{mM}$ EDTA pH 7.0 (10 kDa MWCO). The protein was further purified by ion exchange chromatography on a MonoS column (GE healthcare) equilibrated with $10 \mathrm{mM}$ MOPS, $50 \mathrm{mM} \mathrm{NaCl}, 1 \mathrm{mM}$ EDTA and $1 \mathrm{mM}$ DTT pH 7.0 and eluted in a salt gradient to $500 \mathrm{mM} \mathrm{NaCl}$.

\subsubsection{Sortase-mediated labeling}

Proteins were labeled at their C-terminal LPETGG tag with the previously described technique sortase-mediated transpeptidation (Popp et al., 2009). A peptide with the sequence GGGC was labeled at its cysteine residue with a maleimide dye. Peptide dissolved in $100 \mathrm{mM}$ Hepes/KOH pH 7.4 was added to dye (DyLight680 maleimide or DyLight800 maleimide, Thermo Scientific) in 1.5-fold molar excess. After labeling at RT for $2 \mathrm{~h}$, the reaction was stopped with $10 \mathrm{mM}$ DTT. To label peptide with AlexaFluor 488 C5 Maleimide (Thermo Scientific), peptide and dye were both dissolved in $100 \mathrm{mM}$ Hepes/KOH $\mathrm{pH} 7.4$ and then mixed in a 1:1 molar ratio.

A pentamutant P94R/D160N/D165A/K190E/K196T of SrtA (sortase) from S. aureus was purified from $E$. coli (Chen et al., 2011).

To label proteins, 3-fold molar excess of labeled peptide, $10 \mathrm{mM} \mathrm{CaCl} 2$ and SortA were added to the protein. SortA was added to $1 / 7$ of the total concentration of re- 
actants (peptide and protein). After labeling for $16-20 \mathrm{~h}$ at $4^{\circ} \mathrm{C}$, the reaction was separated by size-exclusion chromatography. To label Doa10 purified in GDN, biotin elution fractions were labeled for $2 \mathrm{~h}$ at $4^{\circ} \mathrm{C}$ prior to density gradient ultracentrifugation.

\subsubsection{Reconstitution into proteoliposomes}

\subsubsection{Preparation of protein-free liposomes}

The following lipids were purchased from Avanti Polar Lipids: 16:0-18:1 PC (POPC, 1palmitoyl-2-oleoyl-glycero-3-phosphocholine), 18:1 ( $\Delta$ 9-Cis) PE (DOPE, 1,2-dioleoylsn-glycero-3-phosphoethanolamine), 18:1 PS (DOPS, 1,2,-dioleoyl-sn-glycero-3-phospho-L-serine), 18:1 Biotinyl PE (Biotinyl-PE, 1,2-dioleoyl-sn-glycero-3-phosphoethanolamine-N-(biotinyl)), 18:1 Liss Rhod PE (Rhd-PE, 1,2-dioleoyl-sn-glycero-3-phosphoethanolamine-N-(lissamine rhodamine B sulfonyl)). Ergosterol ( $\geq 95 \%$, HPLC) was purchased from Sigma-Aldrich.

Large unilamellar liposomes were prepared by reverse-phase evaporation as described (Hernandez et al., 2012). Briefly, lipids were dissolved in chloroform and mixed at a molar ratio of 60:20:10:10 (POPC : DOPE : DOPS : Ergosterol). Chloroform was subsequently removed using a rotary evaporator by lowering the pressure step-wise to 20 mbar. The lipid film was then dissolved in $1 \mathrm{~mL}$ diethyl ether (when preparing $1 \mathrm{~mL}$ of liposomes with a final concentration of $20 \mathrm{mM}$ lipid) and $300 \mu \mathrm{L}$ of buffer $\mathrm{L}(20 \mathrm{mM}$ HEPES/KOH pH 7.4, $150 \mathrm{mM} \mathrm{KCl,} 5 \mathrm{mM}$ magnesium acetate) was added. The sample was sonicated for 1 min on ice (Branson Sonifier 450, 100\% duty cycle, microtip limit 1). Afterwards the ether was removed at 500 mbar. After $10 \mathrm{~min}, 700 \mu \mathrm{L}$ of buffer $\mathrm{L}$ was added and the pressure was gradually decreased to 100 mbar until diethyl ether was completely removed. The volume was adjusted to $1 \mathrm{~mL}$ with $\mathrm{H}_{2} \mathrm{O}$. The resulting lipid suspension was extruded through a polycarbonate filter (11x through a $0.4 \mu \mathrm{M}$ filter, 21x through a $0.1 \mu \mathrm{M}$ filter) using the Mini extruder kit (Avanti Polar Lipids). Protein-free liposomes were used for up to 2 weeks after preparation.

For experiments with a pulldown via co-reconstituted biotinylated lipids, lipids were used in a molar ratio of $57.5: 20: 10: 10: 0.5: 2$ (POPC : DOPE : DOPS : Ergosterol : Rhd-PE : Biotinyl-PE).

\subsubsection{Reconstitution of proteins into liposomes}

To reconstitute proteins into liposomes, protein-free liposomes were mixed with detergent and proteins and subsequently incubated for $1 \mathrm{~h}$ at RT prior to detergent 
removal. The detergent concentration used for solubilization can be described by the R-value (Rigaud and Lévy, 2003). The R-value is defined as the ratio of the total detergent concentration $\left(\mathrm{D}_{\text {total }}\right)$ above the critical micellar concentration $\left(\mathrm{D}_{\mathrm{CMC}}\right)$ and the total lipid concentration ([lipid]):

$$
R=\frac{D_{\text {total }}-D_{C M C}}{[\text { lipid }]}
$$

To co-reconstitute Ubc6 and t-SNARE, protein-free liposomes (4 mM final lipid concentration) were mixed with OG (R-value of 2), proteins and buffer D (buffer L supplemented with $1 \mathrm{mM}$ DTT). t-SNARE and Ubc6 were reconstituted at a molar lipid:protein ratio of 1000 and 2000, respectively. After incubation for $1 \mathrm{~h}$ at RT, the detergent was removed by dialysis. The mix was dialysed against a 1000x volume of buffer D at RT in 2 steps using dialysis cassettes (16 h with $2000 \mathrm{kDa}$ cut-off, $2 \mathrm{~h}$ with 10,000 kDa cut-off). Biobeads (SM-2 resin, Bio-Rad) were added to the buffer to bind OG $(2 \mathrm{~g} / \mathrm{L})$. When Ubc6 ${ }_{\text {SybTM }}$ and Ubc6 WT were co-reconstituted with t-SNARE, both Ubc6 variants were reconstituted at a molar lipid : protein ratio of 2000.

To reconstitute Doa10 (purified in GDN), Cue1 and Syb, protein-free liposomes (4 mM final lipid concentration) were mixed with DM (R-value of 0.55 ), proteins and buffer T (buffer L supplemented with $0.1 \mathrm{mM}$ TCEP). Doa10, Cue1 and Syb were reconstituted at a molar lipid : protein ratio of 5000, 20000 and 2000, respectively. After incubation for $1 \mathrm{~h}$ at RT, the detergent was removed by incubation with resin from Pierce detergent removal spin columns (Thermo Scientific) in 3 subsequent steps (45 mg washed resin to $130 \mu \mathrm{L}$ reconstitution mix in each step). Resin incubation was performed while rotating the sample, at RT for 20 min each. The protocol was the same when Cue1 was omitted. Doa10 truncations were reconstituted at the same lipid : protein ratio, also when both Doa10 truncations were co-reconstituted for the rescue experiment.

After reconstitution into separate liposomes, Ubc6 and Doa10 were subsequently co-reconstituted by SNARE-mediated fusion. Both sets of liposomes were diluted 1:10 into buffer $\mathrm{T}$ and incubated for $1 \mathrm{~h}$ at $30^{\circ} \mathrm{C}$. To inhibit fusion, liposomes containing t-SNARE were preincubated with 7-fold excess of a soluble Syb fragment $\left(\mathrm{Syb}_{1-95}\right)$ for 5 min at RT prior to addition of liposomes containing Syb.

For the experiment in Figure 3.6A, Doa10 (purified in DMNG) was co-reconstituted

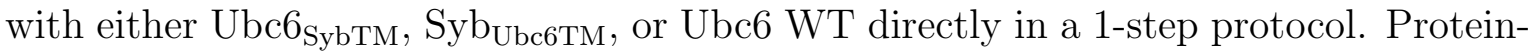
free liposomes (10 mM final lipid concentration) were mixed with DMNG (R-value of 1.5), proteins and buffer T. Ubc6 and Doa10 were both reconstituted at a molar 
lipid:protein ratio of 10,000. After incubation for $1 \mathrm{~h}$ at $\mathrm{RT}$, the detergent was removed by incubation with prewashed Pierce detergent removal spin columns in 3 subsequent steps (1 spin column for $100 \mu \mathrm{L}$ reconstitution mix in each step). Incubation was performed at RT for 10, 20 and $30 \mathrm{~min}$ and the sample eluted by centrifugation at 3,500 rpm for $2 \mathrm{~min}$ in a table top centrifuge. To reconstitute Ubc6 or its variants alone, protein-free liposomes (10 $\mathrm{mM}$ final lipid concentration) were mixed with $\mathrm{OG}$ (R-value of 2.0), proteins and buffer T. Ubc6 was reconstituted at a molar lipid:protein ratio of 10,000. After incubation for $1 \mathrm{~h}$ at $\mathrm{RT}$, the detergent was removed by resin from Pierce detergent removal spin columns in 3 subsequent steps (40, 60, $60 \mathrm{mg}$ resin to $160 \mu \mathrm{L}$ reconstitution mix in step 1, 2 and 3, respectively). Resin incubation was performed while rotating the sample, at RT for 20 min each and the sample eluted by centrifugation at $3,500 \mathrm{rpm}$ for $2 \mathrm{~min}$ in a table top centrifuge.

\subsubsection{Flotation of liposomes}

To test for reconstitution of proteins, liposomes were floated in a Nycodenz step gradient. Nycodenz stocks were prepared in buffer L. $50 \mu \mathrm{L}$ of liposomes were mixed with $50 \mu \mathrm{L}$ of $80 \%$ (w/v) Nycodenz and overlaid with $40 \mu \mathrm{L}$ of 30 and $15 \%$ (w/v) Nycodenz and $40 \mu \mathrm{L}$ of buffer L. The gradients were ultracentrifuged at 50,000 rpm for $1 \mathrm{~h}$ at $4^{\circ} \mathrm{C}$ (S55-S rotor). The gradient was disassembled in 6 fractions, starting from the top of the gradient. Fractions were analyzed by SDS-PAGE.

\subsubsection{Protease protection}

To check the orientation of Ubc6 reconstituted into liposomes, trypsin protease was used. Liposomes were diluted (1:10 in buffer D) and incubated with $6.6 \mathrm{\mu g} / \mathrm{mL}$ trypsin (Roche) at RT. The detergent control contained in addition 1\% Triton-X100 (TX100, Anatrace, Anapoe-X-100). The reaction was stopped with $4 \mathrm{mM}$ PMSF and samples were analyzed by SDS-PAGE.

The orientation of Doa10-SBP or Doa10 $1-468^{-S B P}$ in liposomes was determined by assessing the accessibility of the C-terminal TEV-cleavage site to TEV-protease. Liposomes were diluted 1:10 into buffer D and incubated with $10 \mu \mathrm{M}$ TEV-protease at RT. The detergent control contained in addition 1\% Triton-X100. The reaction was stopped by addition of SDS-sample buffer and samples were analyzed by SDS-PAGE and subsequent western-blotting against the SBP-tag. To determine the orientation of SBP-SUMO*-Doa10 ${ }_{434-1319}$, the accessibility of the N-terminal SBP-SUMO* to Ulp1* protease was assessed (protocol as described above for TEV-protease). 
The reconstitution quality of Ubc6 was assessed with a Ubc6 construct containing an N-terminal His $_{14}$-SUMO tag and a C-terminal TEV-cleavage site followed by a fluorescent dye (SUMO-Ubc6 DL680 $)$. Liposomes containing SUMO-Ubc6 DL680 $_{\text {and }}$ tSNARE were diluted into buffer T 1:20 (final concentration (f.c.) of $0.1 \mu \mathrm{M}$ Ubc6). Ulp1 and/or TEV-protease were added to a f.c. of $10 \mu \mathrm{M}$ each.

\subsubsection{Pulldowns}

For pulldown experiments via the SBP-tag of Doa10, $20 \mu \mathrm{L}$ of the fusion reaction (supplemented with $0.25 \mathrm{mg} / \mathrm{mL}$ bovine serum albumin (BSA)) were incubated with $20 \mu \mathrm{L}$ of Pierce Streptavidin Magnetic Beads (Thermo Scientific) prewashed with buffer B (buffer T supplemented with $0.25 \mathrm{mg} / \mathrm{mL} \mathrm{BSA}$ ). After binding for $1 \mathrm{~h}$ (rotating, RT), the supernatant of the binding reaction was taken off, the beads washed three times with $100 \mu \mathrm{L}$ of buffer B and bound proteins eluted with $20 \mu \mathrm{L}$ of buffer B supplemented with $2 \mathrm{mM}$ biotin. Samples from input, supernatant and elution fractions were analyzed by SDS-PAGE.

\subsubsection{Ubiquitination assays}

All ubiquitinations reactions were performed at $30^{\circ} \mathrm{C}$ in a thermocycler. The fusion reaction was diluted 1:2 (f.c. of $0.1 \mu \mathrm{M}$ Ubc6, $0.02 \mu \mathrm{M}$ Cue1 and $0.04 \mu \mathrm{M}$ Doa10). The following components were used at the indicated concentrations unless stated otherwise: $0.1 \mu \mathrm{M}$ Uba1 (E1), $1 \mu \mathrm{M}$ Ubc7, $120 \mu \mathrm{M}$ ubiquitin (from S. cerevisiae, R\&D Systems) and $2.5 \mathrm{mM}$ ATP. All reactions contained $0.1 \mathrm{mg} / \mathrm{mL}$ BSA. Ubiquitination reactions were performed at $30^{\circ} \mathrm{C}$. The ubiquitin mutants K0 (Lifesensors), K48 and K48R (both from Enzo Life Sciences) are derived from human ubiquitin. To test the effect of the presence of the Cdc48/UN-complex on ubiquitination, $0.1 \mu \mathrm{M}$ Cdc48 (hexamer) and/or $0.1 \mu \mathrm{M}$ Ufd1/Npl4 were included in the reaction. Reactions were stopped by adding reducing SDS-sample buffer and samples were analyzed by SDS-PAGE. For analysis, the non-ubiquitinated band was quantified and normalized to the 0 min timepoint.

\subsubsection{Analysis of ubiquitination reactions}

To analyze the chain profile, the fluorescence intensity was quantified along a vertical axis starting from the top of the gel using the line scan function in Image J (Figure 3.2B and $3.3 \mathrm{C}$ ). When different Ubc6-variants were compared (Ubc6 WT vs Ubc6 SybTM $_{\text {), }}$ ) the line scan values were normalized to the integral of the whole scan (Figure 3.6I), to account for different Sortase labeling efficiencies. To quantify the ubiquitination 
kinetics for mono-, di-, tri- and tetraubiquitination, each band corresponding to one, two, three and four ubiquitins was quantified for every time point and normalized to the non-ubiquitinated band at timepoint 0 min (Figure A5A). To calculate the total number of ubiquitins transferred, the values for one to four ubiquitins obtained above were summed up for each timepoint (Figure 3.5F and 3.6F).

\subsubsection{Measuring extraction by the Cdc48-complex}

Liposomes prepared with protein-free liposomes containing $2 \mathrm{~mol} \%$ biotinyl-PE and $0.5 \mathrm{~mol} \%$ Rhd-PE. Liposomes were fused and subsequently ubiquitinated as described above. To immobilize liposomes after ubiquitination, the ubiquitination reaction was diluted $1: 2$ to a final lipid concentration of $0.4 \mathrm{mM}$ total lipid (f.c. of $0.05 \mu \mathrm{M}$ Ubc6) and BSA was added to a f.c. of $0.25 \mathrm{mg} / \mathrm{ml}$. The diluted mix was then added to an equal volume of Pierce Streptavidin Magnetic Beads (Thermo Scientific, prewashed with buffer B). After incubation for $1 \mathrm{~h}$ at RT (rotating), the unbound fraction was removed and the beads were subsequently washed $3 \mathrm{x}$ with buffer $\mathrm{B}$. The beads were then resuspended in the same volume of buffer B and $30 \mu \mathrm{L}$ of the suspension aliquoted in a PCR-strip. The buffer was removed and the beads resuspended in $1 \mathrm{x}$ extraction mixes or $1 \mathrm{x}$ SDS sample buffer. 1x extraction mixes contained $0.1 \mu \mathrm{M}$ Cdc48 (hexamer), $0.1 \mu \mathrm{M} \mathrm{Ufd} 1 / \mathrm{Npl} 4$ and $0.25 \mathrm{mg} / \mathrm{mL}$ BSA. Beads were incubated for $30 \mathrm{~min}$ at $30^{\circ} \mathrm{C}$. The supernatant was removed (containing extracted and soluble proteins). After washing the beads $3 \mathrm{x}$ with buffer $\mathrm{B}$, the bound proteins were eluted by adding $30 \mu \mathrm{L}$ of $1 \mathrm{x}$ SDS sample buffer.

Samples of the supernatant and the elution fractions were analyzed by SDS-PAGE. To quantify the liposome immobilization efficiency, the Rhodamine fluorescence was measured in a Tecan Genios Pro microplate reader using 550/10 nm and 590/20 nm for excitation and emission, respectively. To quantify the protein immobilization efficiency, the DyLight680 (Ubc6/ Ub-Ubc6 ${ }_{\mathrm{C} 87 \mathrm{~A}}$ ) and Dylight800 (Doa10) fluorescence was measured using the Odyssey scanner (384-well plate, transparent bottom, Corning REF3655).

\subsubsection{Analysis of extraction reactions}

To quantify the extraction efficiency of $\mathrm{Ub}-\mathrm{Ubc} 6_{\mathrm{C} 87 \mathrm{~A}}$ relative to its ubiquitination status (Figure A3G), Ubc6 bands corresponding to Ub-Ubc6 ${ }_{\mathrm{C} 87 \mathrm{~A}}$ modified with 1 to 10 ubiquitins were quantified separately and normalized to the corresponding band of the input sample (beads treated with sample buffer). 
To quantify the fraction of Ubc6 in the supernatant (Figure $3.3 \mathrm{G}$ and $3.3 \mathrm{H}$ ), the fluorescence intensity for Ubc6 modified with 0 - 5 ubiquitins and for Ubc6 modified with more than 5 ubiquitins was quantified by drawing a single rectangular box around the respective area using Image Studio and subsequently normalized to the Input (beads treated with sample buffer). For Ub-Ubc6 $6_{\mathrm{C} 87 \mathrm{~A}}$, the unmodified band was counted as monoubiquitinated.

\subsubsection{Assays for release by Doa10}

\subsubsection{Get3}

Liposomes were prepared with protein-free liposomes containing 2 mol\% biotinyl-PE and $0.5 \mathrm{~mol} \%$ Rhd-PE and fused as described above. The fusion reaction was diluted 1:2 into buffer $\mathrm{T}$ (f.c. Ubc6 $=0.1 \mu \mathrm{M}$ ) and incubated with an excess of Get3 (f.c. $10 \mu \mathrm{M}$ ). After incubation at RT for $16 \mathrm{~h}$, the reaction was diluted to a f.c. of lipid to $0.4 \mathrm{mM}$ (1:2 dilution) and $0.25 \mathrm{mg} / \mathrm{mL}$ BSA added. The diluted mix was then added to an equal volume of Pierce Streptavidin Magnetic beads (Thermo Scientific, prewashed with buffer B). After binding for $1 \mathrm{~h}$, the supernatant was removed. Input and supernatant samples were analyzed by SDS-PAGE, and the Rhodamine fluorescence measured as described above.

For the turbidity assay, Ubc6 (in 0.03\% DDM) was diluted 1:25 into buffer L (f.c. of $1.8 \mu \mathrm{M}$ Ubc6) in the presence or absence of Get3 (f.c. $1.8 \mu \mathrm{M}$ or $3.6 \mu \mathrm{M}$ ). The optical density at $360 \mathrm{~nm}$ was measured using a UV-2401PC spectrophotometer (Shimadzu Corporation).

\subsubsection{Protease protection}

For this experiment, both sets of fusion liposomes were diluted 1:5 for the fusion reaction (instead of 1:10). The fusion reaction was diluted 1:2 into buffer $\mathrm{T}$ and incubated with $2 \mu \mathrm{M}$ Ulp1. For the subsequent TEV-cleavage, the Ulp1-cleaved sample was diluted 1:2 again (f.c. of $0.1 \mu \mathrm{M}$ Ubc6) and incubated with $10 \mu \mathrm{M}$ TEV-protease. During TEV-cleavage, $0.5 \mathrm{mM}$ DTT was present. Detergent controls contained $1 \%$ TX100. Reactions were stopped by adding SDS sample buffer.

\subsubsection{Antibody}

AlexaFluor 488 fluorescence was measured in a Tecan Genios Pro microplate reader using 495/10 nm and 535/25 $\mathrm{nm}$ for excitation and emission, respectively. $30 \mu \mathrm{L}$ of the fusion reaction was measured in a 96-well plate (Corning, REF 3686) with a f.c. 
of Ubc6 $6_{\mathrm{A} 488}$ of $0.2 \mu \mathrm{M}$. After the signal was stable, the measurement was stopped and anti-Alexa Fluor 488 polyclonal antibody (Invitrogen, \#A-11094) was added (diluted 1:15) and the measurement started again. After approx. $40 \mathrm{~min}, 1 \mu \mathrm{L}$ TX100 (f.c. 1\%) was added to solubilize the liposomes. To analyze the fluorescence traces, the three measurements (equilibration, antibody and detergent addition) were merged. The background (stabilized A488 signal after detergent addition) was subtracted from all measurements. The fluorescence traces were subsequently normalized to the average signal of the last 10 timepoints before antibody addition. To quantify the fraction of released Ubc6, the difference between the normalized values of the samples with and without Doa10 30 min after antibody addition was calculated.

To test for the release of the Ubc6-swap mutants, liposomes containing Doa10 co-reconstituted with Ubc6, Ubc6 ${ }_{\text {SybTM }}$ or Syb Ubc6TM were first subjected to a pulldown via the SBP-tag of Doa10. Liposomes were diluted 1:8 (f.c. Ubc6 $0.125 \mu \mathrm{M}$, $0.25 \mathrm{mg} / \mathrm{mL} \mathrm{BSA}$ ) and $50 \mu \mathrm{L}$ of diluted liposomes were added to $50 \mu \mathrm{L}$ of Pierce Streptavidin Magnetic Beads (Thermo Scientific) prewashed with buffer B. For the controls, Ubc6-only liposomes were also diluted 1:8 (in the presence or absence of 1:8 diluted Doa10-liposomes) and incubated with beads. After binding for $1 \mathrm{~h}$ (rotating, RT), the supernatant of the binding reaction was taken off, the beads washed three times with $200 \mu \mathrm{L}$ of buffer B and bound Doa10-liposomes eluted with $40 \mu \mathrm{L}$ of buffer B supplemented with $2 \mathrm{mM}$ biotin. Samples from input, supernatant and elution fractions were analyzed by SDS-PAGE. $30 \mu \mathrm{L}$ of eluted fractions (Doa10-containing liposomes) were then added into a 96-well plate. Liposomes lacking Doa10 (containing

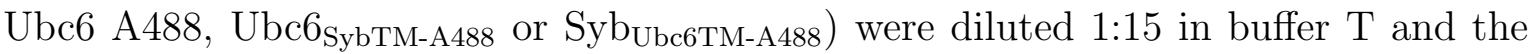
antibody quenching assay was carried out as described above.

\subsubsection{Ubc6-SBP}

To reconstitute Ubc6-SBP into proteoliposomes, Ubc6-SBP was preincubated with a 1.25-fold molar excess of tetrameric streptavidin (NEB) for $15 \mathrm{~min}$ at RT in the presence of $0.03 \%$ DDM to allow for complex formation. The reconstitution conditions were otherwise the same as for the co-reconstitution of Ubc6 and t-SNARE $(2 \mu \mathrm{M}$ Ubc6-SBP and $2.5 \mu \mathrm{M}$ Streptavidin).

To asses the orientation of Ubc6-SBP in liposomes (Figure A7B), a TEV-protection assay was carried out as described above, except that liposomes were diluted 1:5.

To test if the biotinylated nanobody (anti-GFP, construct for expression kindly provided by Dirk Görlich; purified and biotinylated essentially as described in (Pleiner et al., 2018)) is completely biotinylated (Figure A7C), $20 \mu \mathrm{L}$ of biotinylated nanobody 
(f.c. $10 \mu \mathrm{M}$, supplemented with $0.25 \mathrm{mg} / \mathrm{mL}$ BSA) were incubated with $20 \mu \mathrm{L}$ magnetic Streptavidin beads that were prewashed with buffer B or prewashed with buffer B supplemented with $10 \mathrm{mM}$ biotin. After binding for $45 \mathrm{~min}$ at RT, the supernatant was taken off. Samples of input and both supernatants were analyzed by SDS-PAGE and stain-free scanning using a GelDoc EZ Imager.

For the antibody quenching assay, the fusion reaction was first incubated with $1.5 \mathrm{\mu M}$ biotin or biotinylated nanobody for $10 \mathrm{~min}$ at RT, before the A488-measurements were started as described above. A 6-fold excess of of biotin over streptavidin was used. Fluorescence traces were processed and analyzed as described above.

To check if biotinylated nanobody is capable of releasing Streptavidin from Ubc6SBP, a flotation assay was used. Liposomes containing Ubc6-SBP and t-SNARE were incubated with Streptavidin for 5 min at RT (f.c. of $0.9 \mu \mathrm{M}$ Streptavidin to $1.4 \mu \mathrm{M}$ Ubc6-SBP). A 10-fold excess of biotin or biotinylated nanobody was then added and after incubation for another $5 \mathrm{~min}$, a sucrose density gradient $(40 \%(\mathrm{w} / \mathrm{v}), 30 \%(\mathrm{w} / \mathrm{v})$, $15 \%(\mathrm{w} / \mathrm{v})$ and buffer T layer) was assembled. Flotation was carried out as described above. Samples were analyzed by SDS-PAGE and stain-free scanning using a GelDoc EZ Imager. Streptavidin and Ubc6-SBP levels were quantified using ImageJ. Intensity values for Streptavidin were normalized to Ubc6-SBP levels and subsequently to the buffer control.

To measure extraction of Ubc6-SBP by the Cdc48-complex, fusion and ubiquitination reactions were carried out as described above, except that fusion liposomes were diluted 1:5 instead of 1:10 for the fusion reaction (f.c. of $0.2 \mu \mathrm{M}$ Ubc6 during ubiquitination). After $30 \mathrm{~min}$ of ubiquitination, the reactions were aliquoted into a 96-well plate, $2.5 \mathrm{mM}$ ATP were added again and the measurement of the fluorescence of A488 was started (plate reader preheated to $30^{\circ} \mathrm{C}$ ). After $10 \mathrm{~min}$, anti-A488 antibody (1:15 diluted) and $3 \mu \mathrm{L}$ of 10x Cdc48-complex were added. 10x Cdc48-complex mix contained $10 \mu \mathrm{M}$ Cdc48 (hexamer), $10 \mu \mathrm{M}$ Ufd1/Npl4 as well as $1 \mathrm{mM}$ ATP. To quantify the fraction of extracted Ubc6-SBP, fluorescence traces were processed as described above and the difference between the normalized values of samples with and without Cdc48/UN 30 min after antibody addition was calculated.

To test, if Streptavidin stays in the liposome lumen during extraction (Figure 3.7G), samples were floated after the extraction assay in a Nycodenz step gradient as described above. The samples were prepared with the following modifications: To ensure sufficient detection levels, the liposomes were diluted 1:3.3 for the fusion reaction. The ubiquitination reaction contained the fusion reaction (diluted 1:2, f.c. Ubc6 $=0.3 \mu \mathrm{M}$, f.c. Doa10 $=0.12 \mu \mathrm{M}$ ) and the ubiquitination machinery components (concentrations 
as described above). The extraction assay was done in the plate reader as described above, in the presence of $0.3 \mu \mathrm{M} \mathrm{Cdc48/UN} \mathrm{complex} \mathrm{(f.c.} \mathrm{Ubc6}=0.25 \mu \mathrm{M}$ ). After $30 \mathrm{~min}, 2 \mathrm{mM}$ biotin was added to $50 \mu \mathrm{L}$ sample, a Nycodenz step gradient was assembled and the flotation carried out as described above. Samples were analyzed by SDS-PAGE and stain-free scanning using a GelDoc EZ Imager. Streptavidin levels were quantified using ImageJ. Intensity values were normalized to the sample without Cdc48/UN.

\subsubsection{Analysis}

Samples were mixed with SDS sample buffer (stock used as $3 \mathrm{x}$ contained $12 \%(\mathrm{w} / \mathrm{v}$ ) SDS, 30\% (w/v) glycerol, 0.05\% Coomassie blue G-250, $150 \mathrm{mM}$ Tris/HCl pH 7.0 and $6 \%(\mathrm{v} / \mathrm{v}) \beta$-mercaptoethanol for reducing sample buffer (Schägger and von Jagow, 1987; Schägger, 2006). Samples were heated at $70^{\circ} \mathrm{C}$ (Streptavidin-containing samples were boiled, samples were heated at $50^{\circ} \mathrm{C}$ when Doa10 was visualized) and analyzed by SDS-PAGE using CRITERION TGX stain-free precast gels (Bio-Rad).

Fluorescent proteins were detected using an Odyssey scanner (Li-Cor) for DyLight680 and DyLight800-labeled proteins, and an FLA-700 fluorescence scanner (Fujifilm) for A488-labeled proteins. To detect streptavidin, samples were run on CRITERION TGX stain-free precast gels (Bio-Rad) and scanned with a GelDoc EZ Imager (Bio-Rad). Colloidal Coomassie staining was used (Dyballa and Metzger, 2009). SBPtagged proteins were analyzed by western blotting were indicated. After transfer on a nitrocellulose membrane using the Trans-Blot Turbo Transfer System (Bio-Rad), the membrane was blocked with 5\% skim milk powder (dissolved in TBS-T) for $1 \mathrm{~h}$ at RT. A 1:2,500 dilution of anti-SBP antibody (clone 20, mouse monoclonal, MAB10764, Millipore), and a 1:15,000 dilution of secondary antibody (goat anti-mouse, IRDye 800 CW or IRDye 680RD) were used for detection. Gels were quantified using ImageStudio Lite (Li-Cor). Fiji (ImageJ) was used for quantification of ubiquitin chain profiles (plot profile function) as well as streptavidin (gel analyzer function) (Schindelin et al., 2012).

\subsection{Optimization of SNARE-mediated fusion assay}

\subsubsection{Constructs}

A construct for expression of ATP-synthase $\left(\mathrm{TF}_{\mathrm{o}} \mathrm{F}_{1}\right)$ from Bacillus sp. PS3 (pTR19ASDS) was a kind gift from Reinhard Jahn and has been described (Suzuki et al., 2002). Other constructs used in this section are described in section 2.3.2.1 and 2.3.2.2. 


\subsubsection{Expression and purification of $\mathrm{TF}_{\mathrm{o}} \mathrm{F}_{1}$ ATP synthase}

ATP-synthase $\left(\mathrm{TF}_{\mathrm{o}} \mathrm{F}_{1}\right)$ from Bacillus sp. PS3 was expressed and purified as described previously (Suzuki et al., 2002; Schenck et al., 2009). Briefly, ATP-synthase was constitutively expressed in E. coli DK8 cells. Cells cultured in TB-medium were harvested when they reached an $\mathrm{OD}_{600}$ of 2 . Cells were lysed in buffer containing $50 \mathrm{mM}$ Tris/ $\mathrm{HCl}$ $\left(\mathrm{pH} 8.0\right.$ at $\left.4^{\circ} \mathrm{C}\right), 0.5 \mathrm{mM}$ EDTA. After incubation with lysoyzme $(60 \mathrm{mg}$ for $150 \mathrm{~mL}$ lysate) at $37^{\circ} \mathrm{C}$ for $1 \mathrm{~h}$, the cells were lysed by sonication ( 4 x $30 \mathrm{~s}$, microtip limit $2,50 \%$ duty cycle). Subsequently, DNase $(1 \mathrm{\mu g} / \mathrm{mL}) 250 \mathrm{mM} \mathrm{Na}_{2} \mathrm{SO}_{4}$ and $0.7 \%$ (w/v) sodium cholate were added and stirred for $20 \mathrm{~min}$ at RT. The lysate was cleared by centrifugation $\left(20,000 \mathrm{x} \mathrm{g}, 25 \mathrm{~min}, 4^{\circ} \mathrm{C}\right)$. The pellet was resuspended in buffer containing $1 \%$ (w/v) DDM, $40 \mathrm{mM}$ Tris/HCl (pH 7.8 at $4^{\circ} \mathrm{C}$ ), $100 \mathrm{mM} \mathrm{KCl,} 20 \mathrm{mM}$ Imidazole, $5 \mathrm{mM}$ $\mathrm{MgCl}_{2}$ and 1 Complete protease inhibitor tablet (EDTA-free). After solubilization for $1 \mathrm{~h}$ at $4^{\circ} \mathrm{C}$, unsolubilized material was pelleted by centrifugation $(20,000 \mathrm{x} \mathrm{g}, 25 \mathrm{~min}$, $4^{\circ} \mathrm{C}$ ). The supernatant was incubated with Ni-NTA beads (6 mL slurry). After incubation for $2.5 \mathrm{~h}\left(4^{\circ} \mathrm{C}\right)$, the beads were filtered off and washed with $4 \times 50 \mathrm{~mL}$ wash buffer (40 mM Tris/ $\mathrm{HCl} \mathrm{pH} 7.8$ (at $4^{\circ} \mathrm{C}$ ), $100 \mathrm{mM} \mathrm{KCl,} 20 \mathrm{mM}$ imidazole, $5 \mathrm{mM} \mathrm{MgCl}_{2}$ and $0.1 \%(\mathrm{w} / \mathrm{v}) \mathrm{DDM}$ ). Elution was carried out using a buffer containing $40 \mathrm{mM}$ Tris $/ \mathrm{HCl}$ $\left(\mathrm{pH} 7.8\right.$ at $\left.4^{\circ} \mathrm{C}\right), 50 \mathrm{mM} \mathrm{KCl}, 250 \mathrm{mM}$ Imidazole, $5 \mathrm{mM} \mathrm{MgCl}_{2}$ and $0.05 \%(\mathrm{w} / \mathrm{v})$ DDM. The elution fractions were dialyzed against buffer containing $20 \mathrm{mM}$ Hepes, $20 \mathrm{mM} \mathrm{NaCl}, 5 \mathrm{mM} \mathrm{MgCl} 2$ and $0.05 \%$ (w/v) DDM (10 kDa cut-off). The sample was loaded onto a MonoQ column equilibrated with dialysis buffer and further purified by size-exclusion chromatography using a Superdex200 10/300 column (GE Healthcare) equilibrated with $20 \mathrm{mM}$ HEPES/KOH (pH 7.4), $100 \mathrm{mM} \mathrm{KCl,} 2 \mathrm{mM} \mathrm{MgCl} 2$ and $0.05 \%(\mathrm{w} / \mathrm{v})$ DDM. The $\mathrm{TF}_{\mathrm{o}} \mathrm{F}_{1}$ complex eluted in one peak and was stored in the fridge for a week, or frozen after supplementing the sample with $50 \%$ (w/v) glycerol.

\subsubsection{Reconstitution of $\mathrm{TF}_{\mathrm{o}} \mathrm{F}_{1}$ ATP synthase}

ATP synthase was co-reconstituted with Syb into liposomes. The procedure was the same as for Doa10, Syb liposomes (described in section 2.3.5.1 and 2.3.5.2). To test the effect of concentration of reconstituted membrane proteins on liposome stability, ATP-synthase was reconstituted with different lipid:protein ratios $(2000,4000,8000)$. As described for Doa10 Syb liposomes, the lipid:Syb ratio was 2000 (f.c. of lipid $4 \mathrm{mM}$ ). ATP synthase, Syb liposomes or Doa10, Syb liposomes were subsequently fused with Ubc6, dN liposomes (reconstituted as described in section 2.3.5.2) following the fusion protocol described in section 2.3.5.2. Reconstitution quality was assessed by flotation 
in a Nycodenz step gradient (described in section 2.3.5.3).

\subsubsection{Purification of Doa10 by size-exclusion chromatography}

For Figure 4.4, two purification approaches for Doa10 (sucrose gradient centrifugation and size exclusion chromatography) were compared. Doa10 was purified in GDN as described in section 2.3.3.1. Instead of loading the biotin elution fractions onto a sucrose gradient, the sample was loaded on a Superose6 10/300 column (GE Healthcare) equilibrated with $20 \mathrm{mM}$ Hepes/KOH (pH 7.4), $150 \mathrm{mM} \mathrm{KCl,} 0.5 \mathrm{mM}$ TCEP and $0.15 \mathrm{mM}$ GDN. Peak fractions were pooled and concentrated using Amicon Ultra Centrifugal Filters (Merck) with a 100 kDa cut-off.

\subsection{Doa10 TD mutants}

\subsubsection{Constructs}

To generate S. cerevisiae strains which express Doa10 TD mutants (E633D, E633Q, G636R and P638A G642A), the pRS426-pGal1 plasmid harboring the sequence encoding for

Doa10 $225-1319$-SBP (\#375) was modified using the Q5 site-directed mutagenesis kit (NEB). The plasmids were subsequently linearized and transformed with a Doa10 Nterminal fragment as described in section 2.3.2.1. Other constructs used in this section are described in section 2.3.2.1 and 2.3.2.2.

\subsubsection{Purification and reconstitution of Doa10 variants containing a mu- tation in the TD domain}

Doa10 variants containing a mutation in the TD domain were purified in GDN, as described for wildtype Doa10 (section 2.3.3.1). For the ubiquitination assay (Figure 5.1), Doa10 variants were purified via size exclusion chromatography (described in section 2.4.4). For the release assay (Figure 5.2), Doa10 variants purified via sucrose gradient centrifugation were used.

Doa10 variants were co-reconstituted with Syb with and without Cue1 (for ubiquitination and release assay, respectively) and subsequently fused with liposomes containing Ubc6 and t-SNARE. The reconstitution protocol was the same as for WT Doa10 (described in section 2.3.5.2), except that the lipid:protein ratio for Cue1 was lower (2000). 


\subsubsection{Ubiquitination assay}

Cue1, Syb liposomes or liposomes containing Syb, Cue1 co-reconstituted with either Doa10 WT, Doa10 E633D $_{\text {or Doa10 }}$ E33Q were fused to liposomes containing Ubc6 DL800 $_{2}$ and t-SNARE (both liposome sets diluted 1:5 in buffer $\mathrm{T}$ ). The fusion reaction was diluted 1:2 (f.c. of $0.2 \mu \mathrm{M}$ Ubc6, $80 \mathrm{nM}$ Doa10, $0.2 \mu \mathrm{M}$ Cue1) and mixed with ubiquitination machinery (f.c. $0.1 \mu \mathrm{M}$ Uba1, $0.5 \mu \mathrm{M}$ Ubc7, $60 \mu \mathrm{M}$ ubiquitin, $2.5 \mathrm{mM}$ ATP). Ubiquitination reactions were performed at $30^{\circ} \mathrm{C}$ in a thermocycler. Reactions were stopped by adding reducing sample buffer to an aliquot of the reaction. Analysis of ubiquitination reactions was performed as described in section 2.3.6.

\subsubsection{Release assay using an anti-A488 antibody}

Syb-only liposomes or liposomes containing Syb co-reconstituted with either Doa10 WT, Doa10 $\mathrm{E} 633 \mathrm{D}_{\mathrm{D}}$ or Doa10 $\mathrm{E} 633 \mathrm{Q}_{\mathrm{Q}}$ were fused to liposomes containing $\mathrm{Ubc6}_{\mathrm{A} 488}$ and tSNARE (both liposome sets diluted 1:10 in buffer $\mathrm{T}$ ). The fluorescence quenching assay based on quenching of the A488 epitope by an anti-A488 antibody was then carried out as described in section 2.3.8.3.

\subsection{Site-specific photocrosslinking}

\subsubsection{Constructs and strains}

To generate a construct for expression of Ubc6 variants used for site-specific photocrosslinking, the sequence encoding for UBC6 from $S$. cerevisiae was inserted into the $\mathrm{pBAD} / \mathrm{HisC}$ vector (Invitrogen). An N-terminal His ${ }_{14}-\mathrm{SUMO}$ tag as well as a C-terminal Sortase tag (LPETGG) were further introduced. Subsequently, different residues of UBC6 were mutated to the amber STOP codon (TAG). All cloning steps were carried out using the Gibson Assembly and Site-directed Mutagenesis kit (NEB).

The generated plasmids encoding for Ubc6 variants containing an amber codon at specific sites were co-transformed with a plasmid encoding a tRNA synthetase/tRNA pair for the incorporation of the photocrosslinker p-benzoyl-l-phenylalanine into proteins in E coli at the position of the amber codon. This plasmid (pEVOL-pBpF) was a gift from Peter Schultz (Addgene plasmid \# 31190; http://n2t. net/addgene: 31190; RRID:Addgene_31190) (Chin et al., 2002). The E. coli strain C321. $\Delta$ A.exp used for expression. Its release factor 1 is deleted and thus this strain does not lead to termination of the translation at amber STOP codons. C321. $\triangle$ A.exp was a gift from George Church (Addgene plasmid \# 49018) (Lajoie et al., 2013). 
Other constructs used in this section are described in section 2.3.2.1 and 2.3.2.2.

\subsubsection{Expression of Ubc6 $6_{\mathrm{BpA}}$ variants}

\subsubsection{Expression}

An ON-culture was diluted 1:50 into LB-medium supplemented with Ampicillin $(100 \mu \mathrm{g} / \mathrm{L})$ and chloramphenicol $(37 \mu \mathrm{g} / \mathrm{L})$ and grown at $37^{\circ} \mathrm{C}$. At an $\mathrm{OD}_{600}$ of 0.5 , expression was induced by addition of $0.2 \%(\mathrm{w} / \mathrm{v})$ L-arabinose (Sigma-Aldrich). At the same time, BpA (Iris Biotech) was added to the medium (f.c. $0.2 \mathrm{mM}, 1000 \mathrm{x}$ stock was freshly prepared in $0.5 \mathrm{M} \mathrm{NaOH}$ ). Cells were induced at $18^{\circ} \mathrm{C}$, overnight. In the morning, cells were harvested (4000 rpm, $15 \mathrm{~min}, 4^{\circ} \mathrm{C}$ ).

\subsubsection{Test expression}

A test expression was carried out to test if expression of Ubc6 occurs specifically when the unnatural amino acid p-benzoyl-l-phenylalanine (BpA) is present (Figure S2). To do so, expression was carried out in $7 \mathrm{~mL}$ of medium, as described above. For cell lysis and protein extraction, the B-PER reagent (Thermo) was used. A working solution was made by supplementing the B-PER stock with $1 \%$ (w/v) DDM, 1 mM PMSF and $1 \mathrm{mM}$ EDTA (pH 8.0). Each cell pellet was resuspended in $500 \mu \mathrm{L}$ working solution and incubated for $1 \mathrm{~h}$ (RT, rotating). Unsolubilized material was subsequently pelleted by ultracentrifugation ( $\mathrm{S} 45 \mathrm{~A}$ rotor, $15 \mathrm{~min}, 45,000 \mathrm{rpm}, 4^{\circ} \mathrm{C}$ ). A sample from the supernatant was subsequently incubated with Ulp1 (f.c. of $1 \mu \mathrm{M}$ ) for $30 \mathrm{~min}$ (RT). Samples of solubilization reaction, supernatant after centrifugation and the Ulp1treated sample were analyzed by SDS-PAGE and Coomassie blue staining.

\subsubsection{Purification of Ubc6 $6_{\mathrm{BpA}}$ variants}

Expression was carried out in $500 \mathrm{~mL}$ LB medium (description see above). To lyse the cells, the pellet was resuspended in buffer $\mathrm{N} 500\left(50 \mathrm{mM}\right.$ Tris $/ \mathrm{HCl}\left(\mathrm{pH} 8.0\left(4^{\circ} \mathrm{C}\right)\right)$, $500 \mathrm{mM} \mathrm{NaCl}, 30 \mathrm{mM}$ imidazole) supplemented with $20 \mathrm{mM}$ magnesium acetate, $1 \mathrm{mM}$ PMSF, $1 \mathrm{mg} / \mathrm{mL}$ lysozyme and $0.1 \mathrm{mg} / \mathrm{mL}$ DNaseI (total volume of $30 \mathrm{~mL}$ ). After incubation for $30 \mathrm{~min}\left(4^{\circ} \mathrm{C}\right)$, the suspension was sonicated ( $50 \%$ duty cycle, 4 x 30 strokes). Cell debris and unbroken cells were removed by centrifugation (2000 x g, $20 \mathrm{~min}$, $4^{\circ} \mathrm{C}$ ). The supernatant was subsequently ultracentrifuged (Ti70 rotor, $42000 \mathrm{rpm}, 4^{\circ} \mathrm{C}$, $30 \mathrm{~min}$ ) to pellet a membrane fraction. The pellet was solubilized in buffer N500 supplemented with $1 \%(\mathrm{w} / \mathrm{v}$ ) DDM and $1 \mathrm{mM}$ PMSF (total volume of $23 \mathrm{~mL}$ ). After solubilization for $1 \mathrm{~h}\left(4^{\circ} \mathrm{C}\right)$, unsolubilized material was pelleted (Ti70 rotor, 41,000 rpm, 
$4^{\circ} \mathrm{C}, 30 \mathrm{~min}$ ). The supernatant was incubated with Ni-NTA resin (1 mL of slurry, $3 \mathrm{~h}$, $4^{\circ} \mathrm{C}$ ). Next, the beads were filtered off and washed with 3 x $25 \mathrm{~mL}$ buffer N500 supplemented with $0.03 \%$ (w/v) DDM and with 3 x $25 \mathrm{~mL}$ of buffer N250 (50 mM Tris/HCl, $250 \mathrm{mM} \mathrm{NaCl}, 30 \mathrm{mM}$ Imidazole) supplemented with $0.03 \%$ DDM. Ubc6 was eluted from beads by cleavage with the SUMO protease Ulp1. To do so, $1 \mu \mathrm{M}$ Ulp1 was added to beads resuspended in buffer N250 and incubated overnight at $4^{\circ} \mathrm{C}$. Afterwards the flowthrough was collected and $2 \mathrm{mM}$ DTT was added to the elution fractions. Ubc6 $6_{\mathrm{BpA}}$ variants were not further purified but directly used for reconstitution.

\subsubsection{Reconstitution of Ubc $6_{\mathrm{BpA}}$ variants and Doa10}

Doa10 (purified in DMNG) was co-reconstituted with Ubc6 $_{\mathrm{BpA}}$ variants directly in a 1-step protocol. Protein-free liposomes (4 mM final lipid concentration) were mixed with DMNG (R-value of 1.5), proteins and buffer T. Ubc6 variants and Doa10 were both reconstituted at a molar lipid:protein ratio of 2000. After incubation for $1 \mathrm{~h}$ at RT, the detergent was removed by incubation with prewashed Pierce detergent removal spin columns in 2 subsequent steps ( 1 spin column for $180 \mu \mathrm{L}$ reconstitution mix in each step). Incubation was performed at RT for 15 min per step and the sample eluted by centrifugation at 3,500 rpm for $2 \mathrm{~min}$ in a table top centrifuge.

\subsubsection{Photocrosslinking}

For photocrosslinking, Doa10 liposomes co-reconstituted with Ubc6 $6_{\mathrm{BpA}}$ variants were transferred to a 96-well microplate (PS, transparent,\#655101, Greiner). Each sample was split into two wells (à $70 \mu \mathrm{L}$ ). The microplate was placed on ice and then exposed to UV (365 nm, 4 Joule, Bio-Link BLX 365 (peqlab)). The sample was subsequently enriched for Doa10 for easier detection on SDS-PAGE by pulldown via the SBP-tag of Doa10. To do so, $130 \mu \mathrm{L}$ of the crosslinking reaction were supplemented with $10 \mathrm{mM}$ DMNG to solubilize the liposomes. This was necessary, as the binding capacity of magnetic streptavidin beads is higher in detergent than when Doa10 is reconstituted into liposomes. After solubilization for $10 \mathrm{~min}$ at RT, the sample was added to $130 \mathrm{\mu L}$ of beads (pre-washed with buffer containing $20 \mathrm{mM}$ Hepes/KOH (pH 7.4), $150 \mathrm{mM}$ $\mathrm{KCl}, 5 \mathrm{mM}$ magnesium acetate, $0.1 \mathrm{mM}$ TCEP, $10 \mathrm{mM}$ DMNG). The binding reaction was incubated for $45 \mathrm{~min}$ (RT, rotating). Subsequently, the supernatant was removed, and the beads resuspended in $30 \mu \mathrm{L}$ of $2 \mathrm{x}$ sample buffer to elute bound proteins. The elution fractions were subsequently analyzed by SDS-PAGE and Coomassie blue staining. 


\subsubsection{Mass spectrometry}

Protocol for mass-spectrometric analysis was provided by Iwan Parfentev (Laboratory of Prof. Urlaub, MPI for Biophysical Chemistry, Göttingen).

\subsubsection{Sequential tryptic and chymotryptic in-gel digest}

Shifted bands in the SDS-PAGE gel corresponding to cross-linked Doa10 and Ubc6 were cut out and digested as described before (Shevchenko et al., 1996) with the following modifications. After overnight tryptic digestion, $20 \mu \mathrm{l}$ of digestion buffer $(50 \mathrm{mM}$ ammonium bicarbonate, $5 \mathrm{mM} \mathrm{CaCl} 2$ ) was added and gel pieces were crushed with a spatula to increase the surface to volume ratio. Tryptic peptides were released from the gel pieces by $3 \times 5$ min sonication in a water bath. As a second digestion step, $0.5 \mu \mathrm{g}$ chymotrypsin was added per sample and digestion was continued for $4 \mathrm{~h}$ at room temperature, followed by peptide extraction and drying in a Speedvac concentrator (Eppendorf, Hamburg, Germany).

\subsubsection{LC-MS analysis}

LC-MS acquisition was performed on a Q Exactive HF-X hybrid quadrupole-Orbitrap mass Spectrometer with a front-end Dionex UltiMate 3000 RSLCnano system (both Thermo Fisher Scientific, Waltham, USA). Dried peptides were resuspended in 5\% ACN, $0.1 \%$ TFA (v/v) and analysed by LC-MS in technical triplicates. Chromatographic separation was achieved on a C18 PepMap100 $\mu$-Precolumn $(0.3$ x 5 mm, $5 \mu \mathrm{m}$, Thermo Fisher Scientific, Waltham, USA), and an in-house packed main column $(75 \mu \mathrm{m} \times 30 \mathrm{~cm})$ at $300 \mathrm{nl} / \mathrm{min}$ flow rate. The chromatographic gradient was conducted for 37 min from $10 \%$ to $36 \%$ buffer B ( $80 \%$ ACN, $0.08 \%$ formic acid, v/v) followed by an increase to $60 \%$ buffer B for 6 min. The overall method duration was $58 \mathrm{~min}$. MS1 scans were acquired with 60,000 resolution, 1 x $10^{6}$ automatic gain control (AGC) target, and $50 \mathrm{~ms}$ maximum injection time from 350 to $2,000 \mathrm{~m} / \mathrm{z}$ scan range. The 30 most abundant precursor ions were selected individually with a $1.4 \mathrm{~m} / \mathrm{z}$ isolation window and were fragmented with a normalized collision energy of 30. MS2 scans were acquired with 30,000 resolution, $5 \times 10^{3}$ minimum and $2 \times 10^{5}$ target AGC, $200 \mathrm{~ms}$ maximum injection time and a fixed first mass of $110 \mathrm{~m} / \mathrm{z}$. Precursor charges of 1 and larger than 8 were excluded from isolation and fragmentation. No dynamic exclusion was set. 


\subsubsection{Database search}

Acquisition files were converted to mgf format with Proteome Discoverer 2.1 and were subjected for database searching with pLink1 (Yang et al., 2012) with the following parameters: Carbamidomethylation on cysteine as fixed and oxidation on methionine as variable modification, enzymatic C-terminal cleavage after FYWL (Chymotrypsin) and KR (Trypsin), up to 11 missed cleavages, definition of BPA as amino acid 'B' (monoisotopic mass: 251.0946), cross-linker reactivity of B to every other amino acid without mass addition, and no FDR cut-off. Protein sequences of Doa10 and Ubc6 were provided for database searching. Obtained spectral identifications were evaluated manually.

\subsection{Tandem fluorescent timer screen}

\subsubsection{Constructs and strains}

To create an Sbh2 construct that is N-terminally tagged with an sfGFP-mCherry tag for ectopic expression, the sequence encoding for SBH2 from $S$. cerevisiae was inserted

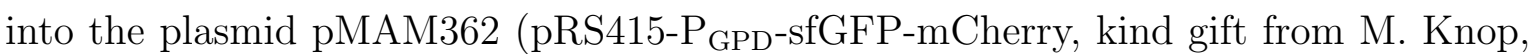
(Khmelinskii et al., 2016)) (plasmid \# 511). The same strategy was used to create an Sbh1 construct that is N-terminally tagged with an sfGFP-mCherry tag for ectopic expression (plasmid \#515).

To tag chromosomal SBH2, a plasmid containing the NatMX6 cassette upstream of a GPD promotor (pGPD) and an sfGFP(superfolder GFP)-mCherry was generated. Therefore, the N-terminal tagging cassette containing plasmid pFA6a-NatMX6p3nmt1-3xFLAG (Source: D. Moazed, Harvard Medical School, Boston, USA) was linearized (without p3nmt1-3xFLAG) and the sequence for p $_{\mathrm{GPD}}$-sfGFP-mCherry was inserted by Gibson Assembly (\# 590). From this plasmid, the whole tagging cassette (NatMX6-p(GPD)-sfGFP-mCherry-HA) was amplified. Moreover, 500 bp long PCR-products were generated of the upstream and downstream region of SBH2 (see primer table). Subsequently, an SBH2 tagging cassette was generated from these PCRproducts using the Gibson Assembly kit. A PCR-amplified product from this Gibson

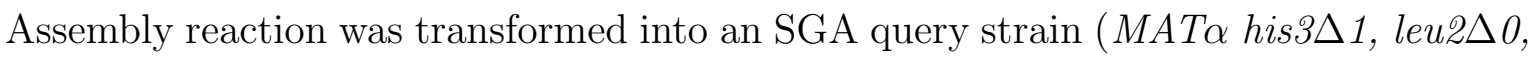

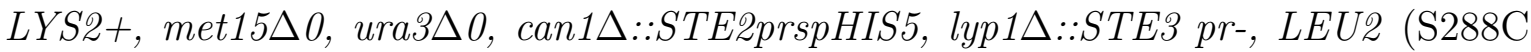
background), kind gift from Maya Schuldiner). Correct tagging was confirmed by sequencing after PCR-amplification of the region of interest after genomic DNA preparation (MasterPure Yeast DNA Purification Kit, Epicentre). 
Two libraries were used for the screen. The first library contained 4,815 haploid deletion mutants (Giaever et al., 2002). Moreover, the DAmP (decreased abundance by mRNA perturbation) library (for essential genes) was used (Schuldiner et al., 2005). The generated SGA query strain was mated with strains by SGA-based crossing that was carried out as described previously (Tong and Boone, 2006, 2007). In short, library strains were mated with the generated query strain on rich medium plates. The resulting diploid strains were selected for all applicable markers, then induced to sporulate on nitrogenstarvation plates. After sporulation, haploid strains were first selected for auxotrophic, then for antibiotic markers to yield the final modified haploid strain. As a counterselection against any remaining diploid cells, all plates after sporulation contained the toxic amino acid analogs Canavanine and Thyalisine. The SGA-based crossing was carried out by Ákos Farkas who also provided this protocol (Laboratory of Prof. Schwappach-Pignataro, University Medical Center, Göttingen).

To delete chromosomal UBP3, the KanMX6 cassette was amplified from pFA6aKanMX6 (Source: D. Moazed, Harvard Medical School, Boston, USA). Moreover, 300 bp PCR-products were generated of the upstream and downstream region of UBP3. Subsequently, a UBP3 tagging cassette was generated from these PCR-products using the Gibson Assembly kit. A PCR-amplified product from this Gibson Assembly reaction was transformed into BY4741. Correct tagging was confirmed by analysis of PCR-products generated by using primers two once amplify the whole UBP3 region as well as by using a primer which anneals to the KanMX6-cassette to amplify a product that is only present when the tagging worked.

\subsubsection{Microscopy}

Stationary-phase cells were diluted into 384-well glass-bottom microtiter plates (Matriplate, Brooks Life Science Systems) containing low fluorescence minimal media (Reference number: CYN6502; FORMEDIUM Ltd.) supplemented with methionine, histidine, uracil and leucine, and allowed to grow for four hours at $30^{\circ} \mathrm{C}$. The plates were automatically imaged at $30^{\circ} \mathrm{C}$ on an Imaging Machine 03-dual (Acquifer) widefield high-content screening microscope, equipped with a white LED array for bright field imaging, an LED fluorescence excitation light source, an sCMOS camera (2048 x 2048 pixels) and a stationary plate holder in combination with movable optics. Images were acquired with $470 \mathrm{~nm}$ (Ex 469/35, Em 525/39, dichroic 497) and $590 \mathrm{~nm}$ (Ex 590/20, Em 628/32, dichroic 607) filter cubes or without filter cube for brightfield images using 3 z-slices $(\mathrm{dz}=1 \mathrm{\mu m})$ and a 40x CFI Super Plan Fluor ELWD N.A. 0.60 (Nikon) objective. The focal plane was automatically detected in the brightfield channel us- 
ing a yeast autofocus algorithm. Experiment was performed by Ákos Farkas (UM, Göttingen) who provided this protocol.

\subsubsection{Analysis}

\subsubsection{Image analysis}

For image analysis, cells were automatically identified using a script written in KNIME (https://www.knime.com) and the fluorescence per cell quantified, similarly to a method described previously (Jonikas et al., 2009). In short, using an ImageJ (Schneider et al., 2012) macro in KNIME, the difference of two brightfield images $(+/-1 \mu \mathrm{M}$ from focal plane) was used to identify segments (cells). Background areas containing no irregularities were identified based on areas of image with low variance. Previously identified cells were removed if they overlapped with the calculated background area. Cells were also filtered for circularity, variance in the brightfield signal within the cell, the range of brightfield signal values within cells in in order to remove cells from analysis that contained irregularities or were stacked on each other. Outliers in size, i.e. too big or small cells were also excluded. Fluorescent channel background was estimated based on the fluorescent signal observed in the background areas identified with the brightfield images, then subtracted from the fluorescent channel image. Cells identified in the brightfield channels, as described above, were used to calculate the pixel distribution for each cell in the fluorescent channel. Outliers with too little variance in the fluorescent signal or too high signal intensity were removed in order to exclude dead cells. Using the different statistical parameters obtained for the distribution of pixel intensities within each cell, the average value for each statistic was calculated for the whole image. This analysis was performed by Ákos Farkas (UM, Göttingen) who provided this protocol.

\subsubsection{Selection of hits for repeated screen}

In a first screen, 5614 strains were initially analyzed. From those strains, 348 strains were selected to repeat the screen. The following criteria were applied to select strains for the repeated analysis. Strains which had either a GFP signal higher than 1750, an mCherry signal higher than 75 or a ratio higher than 0.07 were selected ( 1245 strains). Subsequently a GO-term analysis was performed using the PANTHER tool for gene list analysis (Mi et al., 2018). This classification was used to remove genes which have a function probably unrelated to ERAD (based on localization or function). 


\subsubsection{Identification of hits}

To compare the results of both screens and also identify strains in which Sbh2 is stabilized, both the mCherry intensities as well as the GFP intensities of both repeated were compared using scatter plots. Hits were identified as such if both the mCherry as well as the GFP intensity were higher than the respective 3rd quartile value from the first screen (GFP: 1653, mCherry: 63). Analysis was done using the software Origin, (Version 2018b, OriginLab Corporation, Northampton, MA, USA).

\subsubsection{Cycloheximide chase}

Cycloheximide chase assays (Hampton, 1994) were carried out as described (Gardner et al., 1998; Buchanan et al., 2016) with the following modifications. Briefly, cells were diluted into drop-out medium to an $\mathrm{OD}_{600}$ of 0.2 and grown to an $\mathrm{OD}_{600}$ of 0.5 at $30^{\circ} \mathrm{C}$. Subsequently, the cells were pelleted $(1500 \mathrm{x} \mathrm{g}, 5 \mathrm{~min}, \mathrm{RT})$ and resuspended to an $\mathrm{OD}_{600}$ of 5 . After growing for further $30 \mathrm{~min}$, cycloheximide was added (f.c. of $0.25 \mathrm{mg} / \mathrm{mL})$ and incubated in a waterbath $\left(30^{\circ} \mathrm{C}\right.$, with occasional shaking of the tubes). After indicated timepoints, $1 \mathrm{~mL}$ samples were taken, immediately placed on ice and the cells pelleted ( $1 \mathrm{~min}$, max speed, $4{ }^{\circ} \mathrm{C}$, table top centrifuge). The cell pellet was washed with $1 \mathrm{~mL}$ of ice cold water and subsequently flash frozen in liquid nitrogen (storage at $-80^{\circ} \mathrm{C}$ ).

For analysis of the cycloheximide chase, a total protein extract was prepared as described (Printen and Sprague, 1994). Briefly, the cells were resuspended in $100 \mu \mathrm{L}$ of cracking buffer ( $8 \mathrm{M}$ urea, $50 \mathrm{~g} / \mathrm{L}$ SDS, $40 \mathrm{mM}$ Tris/HCl pH 6.8, $0.1 \mathrm{mM}$ EDTA, $0.4 \mathrm{mg} / \mathrm{mL}$ Bromophenol Blue) supplemented with $1 \%$ (v/v) $\beta$-Mercaptoethanol, $10 \mathrm{mM}$ PMSF, $1.25 \mathrm{x}$ of Complete protease inhibitor (Thermo) stock solution (prepared as 25x stock, according to manufacturer's instructions). Resuspended cells were transferred to $2 \mathrm{~mL}$ screw-cap tubes (Sigma-Aldrich, REF 27000) filled with zirkonia beads (Roth, $0.5 \mathrm{~mm}$ ). After incubation for $10 \mathrm{~min}$ at $70^{\circ} \mathrm{C}$, the cells were broken using the BeadRupter (Omni Beadruptor 24, Biolab Products) (1 round, $30 \mathrm{~s}$, maximum output $(\mathrm{S}=8)$ ). Afterwards, the samples were centrifuged (5 min, RT, max. speed in table top centrifuge) and a sample of the supernatant $(5 \mu \mathrm{L})$ was analyzed by SDS-PAGE and subsequent immunoblotting. Immunoblotting was performed as described in section 2.3.10. Antibodies were dissolved in TBS-T containing $5 \%(\mathrm{w} / \mathrm{v})$ skim milk powder. The following primary antibodies were used: anti-HA (mouse, polyclonal, kind gift from R. Jahn (23.06.00), dilution used: 1:5000); anti-tubulin (clone 3A2, mouse, monoclonal, Synaptic Systems, REF 302211, 1:2500 dilution used); anti- 
Pgk1 (22C5D8, mouse, REF 459250, Invitrogen, dilution used: 1:2500). Secondary antibodies were used as described in section 2.3.10.

\subsubsection{Analysis of stability of tFT-Sbh2 and tFT-Sbh1 using a microplate reader}

To analyze the GFP and mCherry intensity of strains harboring a plasmid for ectopic expression of tFT-Sbh2 and tFT-Sbh1 using a microplate reader (as in Figure 6.4), $500 \mu \mathrm{L}$ of an ON-culture were harvested (table top centrifuge, max. speed, $1 \mathrm{~min}$, RT) and the pellet washed twice with $\mathrm{H}_{2} \mathrm{O}$. Subsequently, $30 \mu \mathrm{L}$ of each sample was added to a 96-well plate (Corning, REF 3686) and the fluorescence was measured with a Tecan Genios Pro microplate reader using 550/10 nm and 612/20 nm for excitation and emission to measure the mCherry intensity, and 495/10 $\mathrm{nm}$ and 535/25 $\mathrm{nm}$ for excitation and emission to measure the GFP intensity.

\subsection{Purification and reconstitution of Sbh2}

\subsubsection{Constructs and strains}

To generate a construct for expression of Sbh2, it was engineered with an N-terminal His $_{14}$-SUMO-tag. The coding sequence for this construct was inserted into the pET39b $(+)$ vector (Novagen) right after the DsbA signal sequence using Gibson Assembly (resulting in plasmid \#695). For protein expression, E. coli BL21-CodonPlus (DE3)-RIPL competent cells (Agilent) were used.

\subsubsection{Expression and purification of Sbh2}

$\mathrm{Sbh} 2_{\mathrm{S} 4 \mathrm{C}}$ was expressed in RIPL cells using an auto-induction protocol as described previously (Studier, 2005). Shortly, an ON-culture was diluted into complex auto-inducing medium (ZYM-5052, metals included) supplemented with $100 \mathrm{\mu g} / \mathrm{L}$ kanamycin and grown for $48 \mathrm{~h}$ at $25^{\circ} \mathrm{C}$ (expression in $6 \mathrm{~L}$ medium resulted in approximately $130 \mathrm{~g}$ of cells). Cells were harvested and a membrane fraction was prepared as described in section 2.3.3.2. The purification was carried out at $4^{\circ} \mathrm{C}$. The membrane fraction was solubilized in buffer $\operatorname{Im} 20\left(50 \mathrm{mM}\right.$ Tris $/ \mathrm{HCl}\left(\mathrm{pH} 8.0\right.$ at $\left.4^{\circ} \mathrm{C}\right), 500 \mathrm{mM} \mathrm{NaCl}, 20 \mathrm{mM}$ Imidazole) supplemented with $1 \mathrm{mM}$ PMSF, $200 \mathrm{mM}$ Sucrose and 1\% (w/v) Sucrose (270 ml total volume). After incubation for $1 \mathrm{~h}$, unsolubilized material was pelleted by ultracentrifugation (Ti45 rotor, $30 \mathrm{~min}, 40,000 \mathrm{rpm}$ ). Ni-NTA resin ( $8 \mathrm{~mL}$ of slurry for $6 \mathrm{~L}$ expression) was added to the supernatant and incubated for $3 \mathrm{~h}$. Beads were 
subsequently filtered off and washed with 4 x $50 \mathrm{~mL}$ of buffer Im20 supplemented with $200 \mathrm{mM}$ Sucrose and $0.03 \%$ (w/v) DDM. To elute Sbh2 $2_{\mathrm{S} 4 \mathrm{C}}, 1 \mu \mathrm{M}$ Ulp1 was added to beads resuspended in wash buffer and incubated for 30 min. Afterwards the flowthrough was collected and $10 \mathrm{mM}$ DTT added. Sbh2 $2_{\mathrm{S} 4 \mathrm{C}}$ was further purified by size-exclusion chromatography using a Superdex 200 10/300 column (GE Healthcare) equilibrated with $20 \mathrm{mM}$ HEPES/KOH pH 7.4, $250 \mathrm{mM} \mathrm{NaCl}, 0.03 \%$ (w/v) DDM, $200 \mathrm{mM}$ Sucrose and $0.1 \mathrm{mM}$ TCEP. Peak fractions were concentrated using Amicon Ultra Centrifugal Filters (Merck) with a 10 kDa cut-off. Fractions were directly used for fluorescent labeling.

\subsubsection{Fluorescent labeling of Sbh2}

To fluorescently label $\mathrm{Sbh} 2_{\mathrm{S} 4 \mathrm{C}}$ via maleimide chemistry, Sbh2 $2_{\mathrm{S} 4 \mathrm{C}}, 2.5$ fold molar excess of DyLight800 Maleimide (Thermo Scientific) was added. The dye was removed using Sephadex G-25 (Superfine, Sigma Aldrich) as medium, equilibrated with buffer containing 20 mM HEPES/KOH pH 7.4, $250 \mathrm{mM} \mathrm{NaCl}$ 0.03\% (w/v) DDM, $200 \mathrm{mM}$ Sucrose and $0.1 \mathrm{mM}$ TCEP.

\subsubsection{Reconstitution of Sbh2}

Sbh2 $2_{\text {S4C-DL800 }}$ was co-reconstituted with Doa10 (purified in DMNG) with and without Cue1 directly in a 1-step protocol. Protein-free liposomes $(9.5 \mathrm{mM}$ final lipid concentration) were mixed with DMNG (R-value of 1.5), proteins and buffer T. Sbh2 $2_{\mathrm{S} 4 \mathrm{C}-\mathrm{DL} 800}$, Doa10 and Cue1 were all reconstituted at a molar lipid:protein ratio of 10,000. After incubation for $1 \mathrm{~h}$ at RT, the detergent was removed by incubation with prewashed Pierce detergent removal spin columns in 2 subsequent steps (1 spin column for $180 \mu \mathrm{L}$ reconstitution mix in each step). Incubation was performed at RT for 15 min per step and the sample eluted by centrifugation at 3,500 rpm for $2 \mathrm{~min}$ in a table top centrifuge.

\subsubsection{Ubiquitination assay}

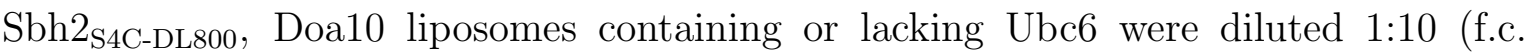
$0.1 \mu \mathrm{M}$ Sbh2, $0.1 \mu \mathrm{M}$ Doa10, $0.1 \mu \mathrm{M}$ Ubc6) and incubated with the rest of the ubiquitination machinery (f.c. $0.1 \mu \mathrm{M}$ Uba1, $1 \mu \mathrm{M}$ Ubc7, $1 \mu \mathrm{M}$ Cue1, $120 \mu \mathrm{M}$ ubiquitin) in the presence of $0.1 \mathrm{mg} / \mathrm{mL}$ BSA. Where indicated, a soluble Ubc6 fragment (aa 1-231) was added (f.c. $1.8 \mu \mathrm{M}$ ). The reaction was started by addition of $2.5 \mathrm{mM}$ ATP. Ubiquitination reactions were performed at $30^{\circ} \mathrm{C}$ in a thermocycler. Reactions were stopped by adding non-reducing sample buffer to an aliquot of the reaction. Analysis 
of ubiquitination reactions was performed as described in section 2.3.6. 


\title{
3. Retrotranslocation of a tail-anchored membrane protein by the ubiquitin ligase Doa10
}

\author{
Claudia C. Schmidt ${ }^{1}$, Vedran Vasic ${ }^{1}$, Alexander Stein ${ }^{1 *}$
}

1 Research Group Membrane Protein Biochemistry

Max Planck Institute for Biophysical Chemistry, Am Fassberg 11,

D-37077 Göttingen, Germany

* Correspondence: alexander.stein@mpibpc.mpg.de (A.S.)

\section{Manuscript}

Detailed author contribution of Claudia C. Schmidt:

- Experimental work and analysis for all figures (Figures 3.1 - 3.7, A1 - A7).

- The manuscript has been written together with Alexander Stein.

Vedran Vasic has performed preliminary experiments to establish the SNARE-mediated fusion assay.

A modified version of this manuscript has been published in:

Schmidt, C. C., Vasic, V., and Stein, A. (2020). Doa10 is a membrane protein retrotranslocase in ER-associated protein degradation. Elife, 9, e56945 (DOI: 10.7554/eLife.56945). 


\begin{abstract}
In ER-associated protein degradation luminal and membrane proteins are poly-ubiquitinated, extracted from the membrane by the Cdc48 ATPase, and degraded by the proteasome. How hydrophobic trans-membrane (TM) segments move from the membrane into the cytosol is unknown. Here, we show using purified proteins reconstituted in phospholipid vesicles that the ubiquitin ligase Doa10 facilitates movement of a tailanchored membrane protein into the cytosol. The substrate's TM segment interacts with the membrane-embedded domain of Doa10 and then passively moves into the aqueous phase. No other membrane component is required. Passive translocation of the substrate is prevented when the luminal C-terminus interacts with a binding partner. However, membrane extraction is restored when the substrate is polyubiquitinated allowing the Cdc48 ATPase to pull on it. Our results indicate that Doa10 forms a conduit for the translocation of hydrophobic TM segments. Cdc48 cooperates with Doa10 by unfolding a luminal polypeptide segment concomitant with membrane extraction.
\end{abstract}

\title{
3.1 Introduction
}

The endoplasmic reticulum (ER) is a major site for protein and lipid synthesis in eukaryotic cells. Proteins destined for the endomembrane system and secretory pathway are translocated into the ER lumen or integrated into the ER membrane where they undergo protein folding. Two conserved membrane embedded ubiquitin ligase complexes have evolved that cope with the problem of protein misfolding and regulate protein abundance in the ER, the Hrd1 and Doa10 complexes. They recognize misfolded proteins, catalyze their polyubiquitination and recruit the Cdc48 complex. The Cdc48 complex, composed of the AAA ATPase Cdc48 (called p97 or VCP in metazoans), its cofactors Ufd1 and Npl4, and the recruitment factor Ubx2, mediates extraction of ubiquitinated proteins from the ER membrane (Bodnar and Rapoport, 2017b; Neuber et al., 2005; Schuberth and Buchberger, 2005; Ye et al., 2001), releasing them into the cytoplasm where proteasomal degradation occurs. Collectively this pathway is called ER associated protein degradation (ERAD) (Mehrtash and Hochstrasser, 2018; Christianson and Ye, 2014; Ruggiano et al., 2014).

ERAD substrates have a vast variety of different topologies; they can be luminal soluble proteins, or membrane proteins with lesions in either a luminal domain, the transmembrane (TM) domain, or a cytosolic domain. Substrates take different routes depending on the localization of the misfolded domain relative to the ER mem- 
brane (Carvalho et al., 2006; Huyer et al., 2004; Taxis et al., 2003; Vashist and Ng, 2004). The Hrd1 complex mediates ubiquitination of substrates with misfolded luminal and TM domains, and acts in concert with other proteins, like Hrd3, Usa1, and members of the Derlin protein family. A growing body of evidence suggests that Hrd1 forms a retrotranslocon required for the passage of substrates across the ER membrane, but the Derlins Der1 and Dfm1 likely also play an important role in this process (Baldridge and Rapoport, 2016; Carvalho et al., 2010; Mehnert et al., 2013; Neal et al., 2018).

Doa10, originally described in S. cerevisiae, appears to be ubiquitously conserved in eukaryotes (Swanson et al., 2001). Homologs have been described in plants (called SUD1 in Arabidopsis thaliana), animals (called TEB4 or MARCH6 in mammals), and have been identified in other unicellular eukaryotes (Doblas et al., 2013; Swanson et al., 2001). In S. cerevisiae, Doa10 is a $150 \mathrm{kDa}$ protein with 14 transmembrane segments (Kreft et al., 2006). Apart from its N-terminal RING-CH domain bearing the E3 ligase catalytic activity, Doa10 contains two highly conserved regions, the TD (for TEB4-Doa10) domain comprising TM segments 5-7 (Swanson et al., 2001), and a C-terminal cytoplasmic element (Zattas et al., 2016). Substrates of Doa10 are very diverse. They include soluble proteins of the cyto- and nucleoplasm, but also ER membrane proteins (Ravid et al., 2006). While for many of these substrates cytoplasmic elements are recognized, as is the case for the Matalpha- and Ndc10-derived degrons Deg1 and DegAB (Furth et al., 2011; Swanson et al., 2001), Doa10 also recognizes TM degrons, e.g. the tail-anchored (TA) membrane protein Sbh2, an accessory component of the Ssh1 translocon (Habeck et al., 2015). Like Hrd1, Doa10 also regulates the abundance of enzymes involved in sterol metabolism. Squalene monooxygenase is degraded in a Doa10 dependent manner in plants, fungi and animals in response to cellular sterol load (Doblas et al., 2013; Foresti et al., 2013). Moreover, in S. cerevisiae, Doa10 restricts the localization of the phosphatidylglycerol phospholipase Pgc1 and probably other lipid droplet proteins by targeting ER localized Pgc1 for proteasomal degradation (Ruggiano et al., 2016), indicating a broader role for Doa10 in maintaining organelle identity.

Doa10 works in concert with two ubiquitin conjugating enzymes, the tail-anchored membrane protein Ubc6 and the soluble cytoplasmic protein Ubc7 (Swanson et al., 2001). Ubc7 is anchored to the ER membrane by Cue1 (Biederer et al., 1997). Experiments with soluble cytoplasmic fragments of Ubc6 and Doa10 showed that these two E2 enzymes have different roles in the build-up of poly-ubiquitin chains (Weber et al., 2016). Ubc6 initiates ubiquitin chains by transferring the first ubiquitin moiety onto Doa10, whereas Ubc7 extends ubiquitin chains with mainly K48 linkage. Pheno- 
types of ubc6 deletion are often more pronounced when substrates lack lysine residues for ubiquitin attachment via isopeptide bonds. In this case, Ubc6 has been shown to ubiquitinate the hydroxyl-group of serine and threonine residues (Boban et al., 2015; Weber et al., 2016).

Importantly, Ubc6 is itself an unstable protein and degraded in a Doa10-dependent manner (Swanson et al., 2001; Walter et al., 2001). Instability of Ubc6 is linked to its catalytic activity. If the active-site Cys87 is mutated to Ser or to Ala, which interferes with E1-dependent ubiquitin loading, Ubc6 is stabilized even if a second, catalytically active form of Ubc6 is present. N-terminal fusion with ubiquitin bypasses the requirement for catalytic activity, suggesting that Ubc6 with a ubiquitin attached to a site other than the active site becomes a substrate to Doa10 (Weber et al., 2016). No completely soluble, luminal substrates of Doa10 have been described, but degradation of membrane bound Doa10 substrates requires retrotranslocation of protein parts into the cytosol. While it is established that this reaction requires the Cdc48 complex (Ravid et al., 2006), it is unknown if Doa10 acts as a retrotranslocase that facilitates substrate extraction or whether it merely serves as a scaffold that recognizes substrates and brings them in close proximity to the ubiquitination machinery. Furthermore, it is not known if Doa10-mediated ERAD of membrane proteins requires additional factors apart from Doa10 itself, the components of the ubiquitination machinery, and the Cdc48 complex. For instance, it has been suggested that the rhomboid-like Derlin Dfm1 plays a role in retrotranslocation of Doa10 substrates (Neal et al., 2018).

Here, we have investigated the mechanism of Doa10-mediated ERAD of Ubc6. We establish a reconstituted system using purified components, and identify the minimal machinery required for polyubiquitination and membrane extraction of Ubc6. Using this system, we show that Doa10 acts as a retrotranslocase by facilitating release of the TM anchor of Ubc6 from the membrane. The Cdc48 complex breaks non-covalent luminal interactions and thus drives extraction while membrane integrity is preserved.

\subsection{Results}

\subsubsection{Membrane-reconstitution of Doa10 and Ubc6}

We first sought to establish a reconstituted system that faithfully recapitulates observations made on ERAD of Ubc6 in intact yeast cells. To this end, we purified S. cerevisiae Ubc6 from E. coli (Figure A1A and A1B), and fluorescently labeled it at the carboxyl terminus by sortase-mediated transpeptidation (Popp and Ploegh, 2011). Yeast Doa10 with a C-terminal streptavidin-binding peptide (SBP) was purified from $S$. cerevisiae 
by streptavidin affinity chromatography followed by sucrose gradient centrifugation or gel filtration (Figure A1C and A1D). We reconstituted Doa10 and Ubc6 into separate liposome sets. To enable subsequent mixing of the two membranes and delivery of Ubc6 to Doa10, both proteins were co-reconstituted with complementary SNARE proteins engineered to efficiently catalyze membrane fusion in the absence of SNARE-regulatory proteins (Figure 3.1A) (Hernandez et al., 2012; Pobbati, 2006). This approach has two advantages over a direct co-reconstitution. First, it allows us to characterize Ubc6 liposome preparations and then fuse them with liposomes containing Doa10 or mutants of it. Second, it avoids non-native protein-protein interactions that might occur due to different conformational flexibilities in lipid-detergent micelles during direct co-reconstitution. Flotation in Nycodenz step-gradients showed that proteins were efficiently reconstituted (Figure A1E and A1F). Mixing of the two liposome sets resulted in SNARE-dependent interaction of Doa10 and Ubc6 liposomes, as shown by precipitation of Doa10-containing liposomes (Figure A1G). Protease-protection experiments showed that Doa10 was reconstituted mostly in the correct orientation (Figure A1H), and that Ubc6 was randomly oriented (Figure A1I-K).

\subsubsection{Mono-Ubiquitination of Ubc6 by Doa10}

We first investigated ubiquitination of Ubc6 in the absence of Ubc7/Cue1. In the presence of the E1 Uba1, ubiquitin, and ATP, about 50\% of Ubc6 is readily loaded on its active site cysteine, as shown in non-reducing SDS-PAGE, corresponding to the fraction of correctly oriented Ubc6 (Figure 3.1B). We also observed generation of Ubc6ubiquitin adducts that were insensitive to reducing agent, indicating E3-independent autoubiquitination of Ubc6, as previously described for a soluble fragment of Ubc6 (Weber et al., 2016). 
A

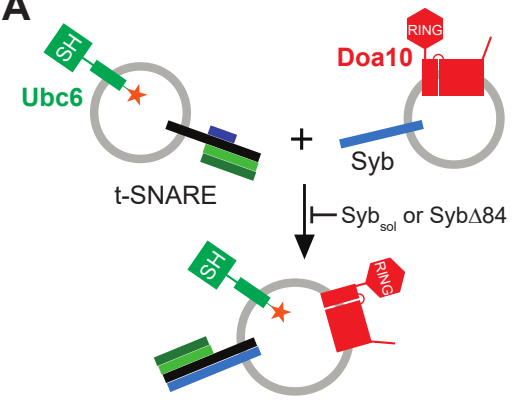

C

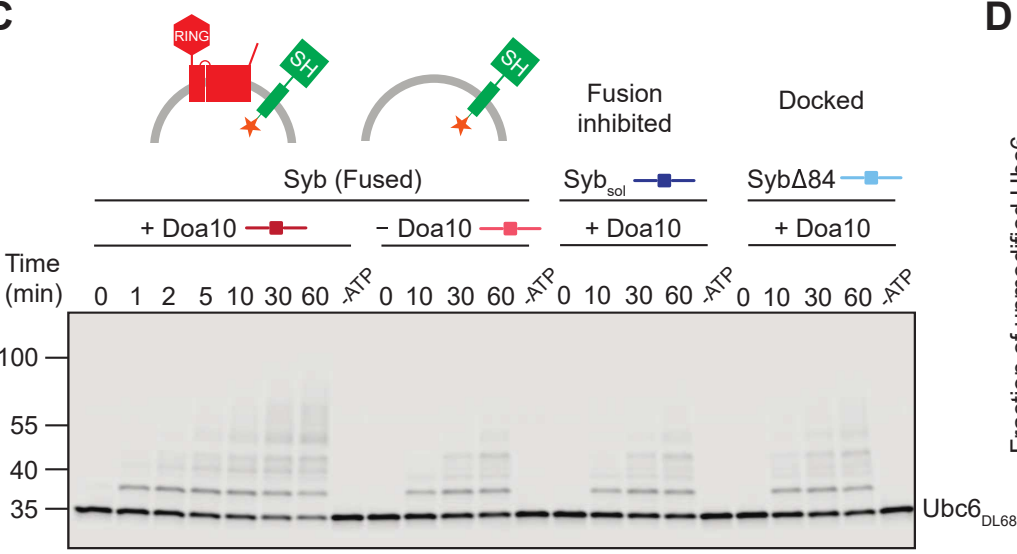

B

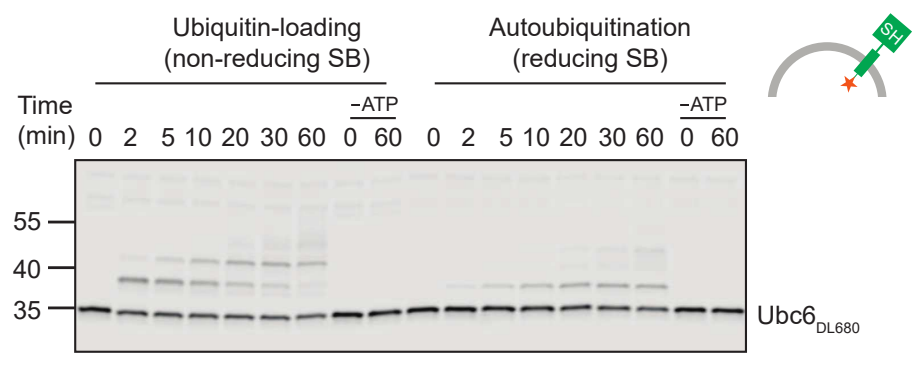

D

Figure 3.1: Doa10 catalyzes intramolecular mono-ubiquitination of Ubc6 at multiple sites. (A) Scheme for SNARE-mediated delivery of Ubc6 (green) to Doa10 (red). t-SNARE denotes a complex of Syntaxin 1A, SNAP25-A, and a stabilizing fragment of Synaptobrevin 2 (Syb) (Pobbati, 2006). Addition of a soluble fragment of $\mathrm{Syb}\left(\mathrm{Syb}_{\text {sol }}\right)$ inhibits SNARE interaction, while replacing Syb with the mutant version Syb $\Delta 84$ arrests fusion at a docked state (Hernandez et al., 2012). SH in Ubc6 indicates the identity of the active site amino acid (Cys87) which is replaced in some experiments by Ala. (B) Time course of E1-mediated ubiquitin-loading of fluorescently labeled Ubc6 $6_{\text {DL680 }}$ analyzed by SDS-PAGE under non-reducing (left) and reducing conditions (right) and fluorescence scanning. Reactions lacking ATP are denoted as -ATP. (C) Time course of ubiquitination of Ubc6 in the presence or absence of Doa10. Liposomes containing Doa10 and Ubc6 ${ }_{\mathrm{DL} 680}$ as depicted in (A) were mixed to allow for fusion. Where indicated, fusion was inhibited using either a soluble Syb fragment $\left(\mathrm{Syb}_{\text {sol }}\right)$ or a Syb mutant (Syb 844$)$. Liposomes were then incubated with $100 \mathrm{nM} \mathrm{E1,} 120 \mu \mathrm{M}$ ubiquitin, and $2.5 \mathrm{mM}$ ATP (Final concentrations (f.c.) of $40 \mathrm{nM}$ Doa10 and $100 \mathrm{nM}$ Ubc6). For each reaction, a 60 min sample in the absence of ATP is shown. Samples were analyzed by SDS-PAGE and fluorescence scanning. (D) Quantification (mean and SD) of unmodified Ubc6 from three experiments as in (C).

We next generated liposomes containing Doa10 and Ubc6 using the fusion system and subsequently added E1, ubiquitin, and ATP. In contrast to the slow E3independent autoubiquitination, ubiquitination in the presence of Doa10 occurred much faster resulting in ubiquitination of $30 \pm 5 \%$ of Ubc6 within 2 min that proceeded with 
slower kinetics afterwards (Figure 3.1C and 3.1D). For efficient and fast ubiquitination of Ubc6 by Doa10, both proteins need to reside in the same membrane, as inhibition of fusion with a soluble v-SNARE fragment $\left(\mathrm{Syb}_{\mathrm{sol}}\right)$ or replacing Syb with a mutant version that only supports liposome docking (Syb $\Delta 84$, (Hernandez et al., 2012)) resulted in auto-ubiquitination as observed in the absence of Doa10.

Higher molecular weight species of Ubc6 largely correspond to multiple monoubiquitinations, as we observed almost no difference in the ubiquitination pattern when we performed the same reaction with a ubiquitin mutant in which all lysines are mutated to arginine $\left(\mathrm{Ub}_{\mathrm{K} 0}\right)$ and that thus cannot form ubiquitin chains (Figure A1L). Together, these results show that Ubc6, loaded at the active site with ubiquitin is a substrate for Doa10-mediated ubiquitin transfer onto a different residue of Ubc6.

\subsubsection{Ubc7-dependent polyubiquitination of Ubc6}

Doa10-dependent degradation of Ubc6 requires the activity of another ubiquitin-conjugating enzyme, Ubc7, and its adapter Cue1 (Walter et al., 2001). We therefore investigated how Ubc7/Cue1 affect ubiquitination of Ubc6. We purified full-length Cue1 from E. coli and co-reconstituted it together with Doa10 (Figure A2A-C). When we fused such liposomes with Ubc6-liposomes and then added E1, Ubc7, ubiquitin, and ATP, we observed formation of high molecular weight ubiquitin adducts (Figure 3.2A-C), representing polyubiquitin chains with predominantly K48 linkage (Figure A2D). Ubc7 and Cue1 act in concert, as in the absence of either protein, ubiquitination patterns were indistinguishable from reactions without both proteins (Figure 3.2A-C). When fusion was inhibited or Doa10 excluded entirely, no ubiquitination beyond E3-independent autoubiquitination was observed.

In intact yeast cells, only catalytically active Ubc6 is a Doa10 substrate, but amino-terminal fusion of ubiquitin to Ubc6 bypasses this requirement, suggesting that monoubiquitinated Ubc6 is a substrate for Ubc7-dependent polyubiquitination (Weber et al., 2016). To test if our reconstituted system recapitulates this behavior, we compared ubiquitination of WT Ubc6, the catalytically inactive Ubc6 $6_{\mathrm{C} 87 \mathrm{~A}}$, and the N-terminal ubiquitin fusion to $\mathrm{Ubc} 6_{\mathrm{C} 87 \mathrm{~A}}\left(\mathrm{Ub}-\mathrm{Ubc} 6_{\mathrm{C} 87 \mathrm{~A}}\right)$ by Doa10 in the presence of Ubc7/Cue1 (Figure A2E for reconstitution controls). We observed no ubiquitination of $\mathrm{Ubc}_{\mathrm{C} 87 \mathrm{~A}}$, but robust polyubiquitination of $\mathrm{Ub}-\mathrm{Ubc} 6_{\mathrm{C} 87 \mathrm{~A}}$ (Figure $3.2 \mathrm{D}$ and $3.2 \mathrm{E}$ ). 
A
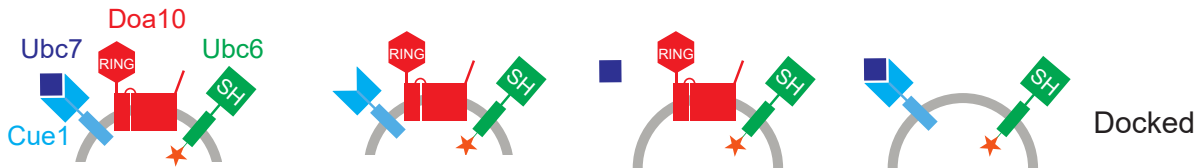

Doa10, Cue1,

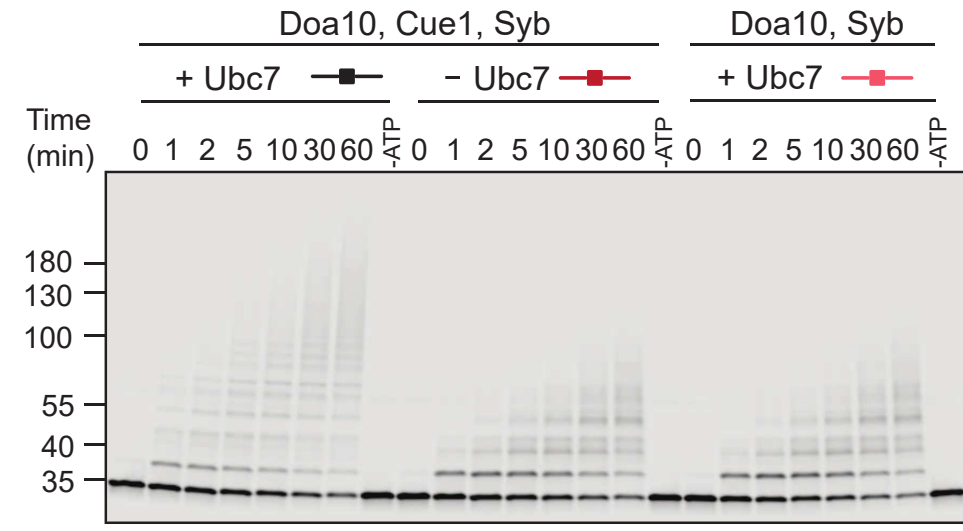

$\underline{\text { Cue1, Syb Syb } \Delta 84}$

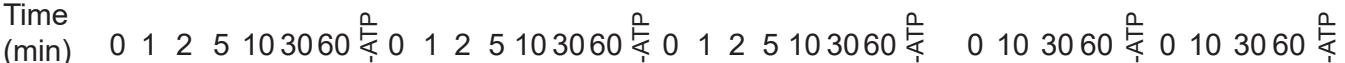

B

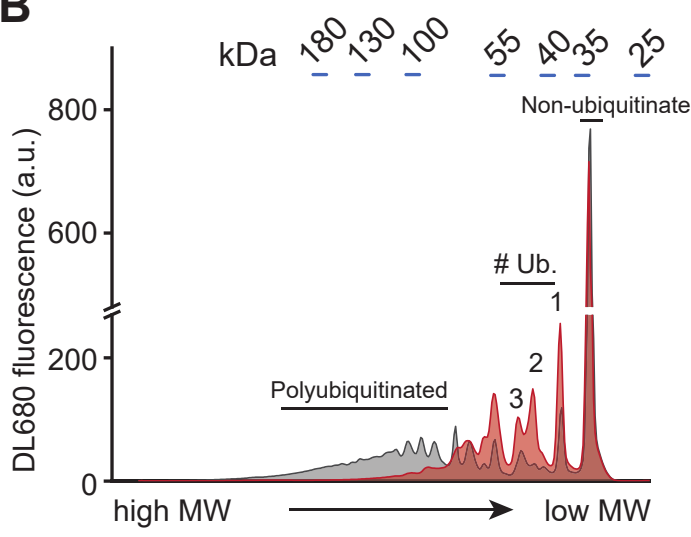

C $\mathrm{Ubc6}_{\mathrm{DL} 680}$

D

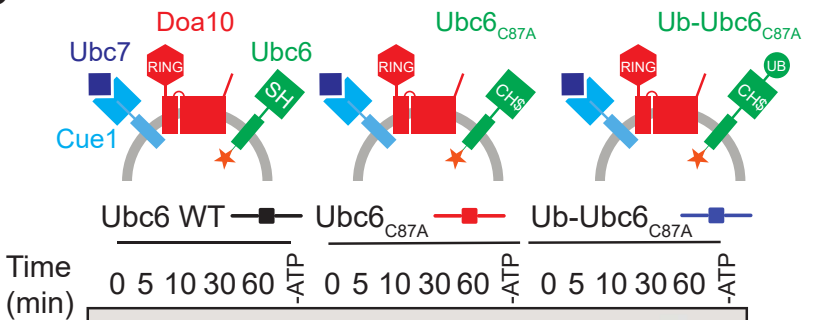

(min) 05103060 妾 05103060 妾 05103060 章

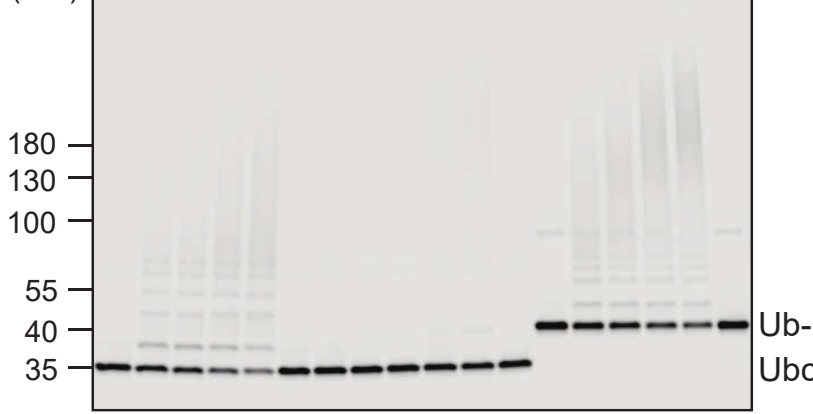

Figure 3.2: Polyubiquitination of Ubc6 by Doa10, Cue1 and Ubc7. (continued)

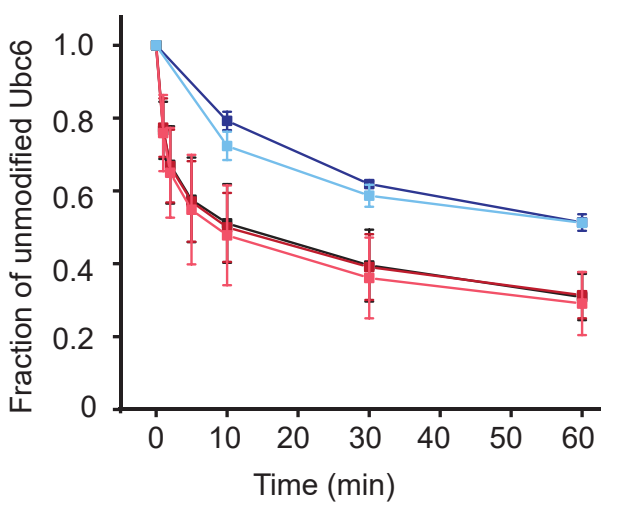

E

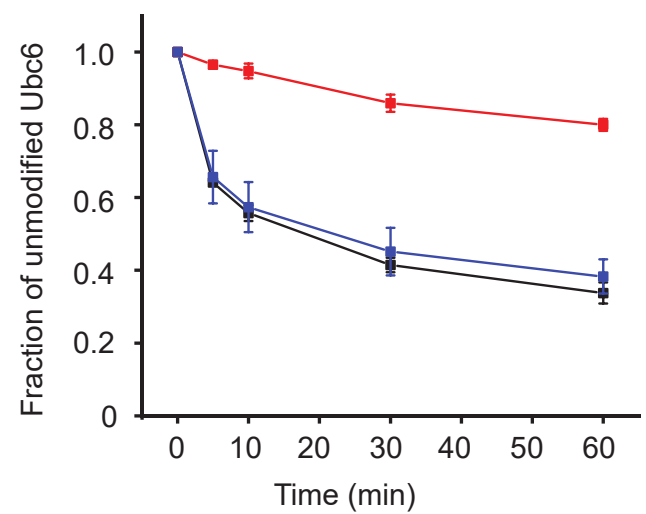

b-Ubc6 $6_{\text {C87A-DL680 }}$

DL680 (WT/C87A) 
Figure 3.2 (continued): (A) Time course of ubiquitination of Ubc6 in the presence or absence of Doa10, Cue1 and Ubc7. Liposomes as indicated were incubated with ubiquitination machinery (f.c. of $40 \mathrm{nM}$ Doa10, $10 \mathrm{nM}$ Cue1, $1 \mu \mathrm{M}$ Ubc7, $100 \mathrm{nM}$ Ubc6, $120 \mu \mathrm{M}$ ubiquitin, and $2.5 \mathrm{mM}$ ATP). For each reaction, a 60 min sample in the absence of ATP is shown. Samples were analyzed by SDSPAGE and fluorescence scanning. (B) Analysis of ubiquitin-chain length on Ubc6 in the presence of Doa10 and Cue1, with (black) and without (red) Ubc7. Linescans were performed on fluorescence images of two representative gel samples as in $(\mathrm{A})$ at $\mathrm{t}=30 \mathrm{~min}$. The approximate position in the gel is indicated by the molecular weight marker on top of the graph. \# ub. denotes number of ubiquitin moieties attached. (C) Quantification (mean and SD) of the fraction un-modified Ubc6 from three experiments as in (A). Coloring as indicated in (A). (D) Time-course of ubiquitination of Ub-Ubc6 $6_{\mathrm{C} 87 \mathrm{~A}}$ compared to Ubc6 WT and Ubc6 $_{\mathrm{C} 87 \mathrm{~A}}$ in the presence of Doa10, Cue1, and Ubc7. Concentrations as in (A) were used. (E) Quantification (mean and SD) of unmodified Ubc6 variants from three experiments as in (D). Coloring as indicated in (D).

Together, these results establish that polyubiquitination of Ubc6 occurs sequentially, first requiring active site loading of Ubc6, followed by a Doa10-catalyzed Ubc6ubiquitination, followed by Ubc7/Cue1-dependent polyubiquitination. In line with the notion that Ubc7 only acts after mono-ubiquitination of Ubc6, kinetics of total Ubc6 turnover were independent of the presence of Ubc7/Cue1 (Figure 3.2C). These results agree with observations made in intact cells and indicate that our reconstituted system faithfully recapitulates the in vivo ubiquitination pathway (Kreft and Hochstrasser, 2011; Walter et al., 2001; Weber et al., 2016).

\subsubsection{Sequential autoubiquitination of Doa10}

Autoubiquitination of E3 RING ligases is a commonly observed reaction in vitro, although its physiological significance is often unclear. We also measured how the presence or absence of Ubc6 or Ubc7/Cue1 affects autoubiquitination of Doa10. To this end, liposomes containing fluorescently labeled Doa10 (Doa10 DL800) and Cue1 were fused with liposomes with or without Ubc6, and subsequently incubated with E1, ubiquitin and ATP, in the absence or presence of Ubc7. High molecular weight ubiquitin adducts of Doa10 were only observed in the presence of both Ubc6 and Ubc7, whereas only smaller ubiquitin adducts were observed with Ubc6 as the only E2 (Figure A2F and $\mathrm{A} 2 \mathrm{G}$ ). When we omitted Ubc6, we observed some Doa10 polyubiquitination, but the reaction was strongly impaired. This result confirms that Ubc6 and Ubc7 act sequentially in the build-up of polyubiquitin chains on Doa10, as previously shown for soluble protein fragments (Weber et al., 2016). 


\subsubsection{Membrane extraction by the Cdc48 ATPase complex}

Our results so far establish a minimal system for efficient polyubiquitination of Ubc6 by Doa10 that requires the E1 Uba1, ubiquitin, Ubc7, Cue1, and ATP. Next, we investigated under which conditions Ubc6 is a substrate for Cdc48 complex-mediated extraction from the membrane. We first tested the effect of the Cdc48 complex on polyubiquitination of Ubc6. In the presence of Cdc48 complex, ubiquitin chains on Ubc6 remain shorter while the kinetics of Ubc6 turnover are unaffected (Figure 3.3AC). Accordingly, no effect on Ubc6 ubiquitination in the absence of Ubc7 was seen (Figure A3A and A3B). This effect was only observed when Cdc48 and Ufd1/Npl4 (UN) were present, but not when either UN or Cdc48 were omitted. Furthermore, ubiquitin chains only remained short when Cdc48 was catalytically active, as seen from a reaction in which a Walker B mutant in the D2 ring of Cdc48 (Cdc48 $8_{\text {E588A }}$ ) was used. We hypothesized that this effect was due to the extraction of Ubc6 from the membrane once ubiquitin chains of sufficient length were formed, thereby removing Ubc6 from the vicinity of Doa10 and interrupting further chain elongation.

To test this directly, we performed experiments in which we staged ubiquitination and the Cdc48/UN-dependent reaction (Figure 3.3D). We first incubated liposomes containing Doa10, Cue1 and Ubc6 with E1, Ubc7, ubiquitin, and ATP for 20 min and then stopped the reaction by addition of EDTA. Liposomes were then immobilized to magnetic beads via co-reconstituted biotinylated lipids (see Figures A3C-F for controls on the efficiency of immobilization). After washing to remove soluble ubiquitination machinery, we incubated the beads with either buffer or Cdc48/UN for $15 \mathrm{~min}$ and analyzed bound and released material by SDS-PAGE (Figure 3.3E). Since under this condition, ubiquitination of Ubc6 is relatively inefficient, we performed the same experiment with Ub-Ubc6 $6_{\mathrm{C} 87 \mathrm{~A}}$ (Figure 3.3F). Extraction efficiency was dependent on the length of ubiquitin chains, with five ubiquitin moieties being minimally required (Figure A3G). In the presence of Cdc48/UN, $45 \pm 17 \%$ of Ubc6 molecules with more than five attached ubiquitin moieties were extracted, compared to $15 \pm 4 \%$ in the absence of the Cdc48 complex (Figure 3.3G). No extraction above this background was observed when either Cdc48 or UN were omitted. Furthermore, ATP hydrolysis by the Cdc48 complex was necessary, as Cdc48 E588A was inactive. Polyubiquitin chains were required because we found no extraction above background when ubiquitination was performed in the absence of Ubc7 (Figure $3.3 \mathrm{E}$ and $3.3 \mathrm{H}$ ). Similar observations were made for Ub-Ubc6 ${ }_{\mathrm{C} 87 \mathrm{~A}}$ (Figure $3.3 \mathrm{~F}$ and $3.3 \mathrm{G}$ ). 

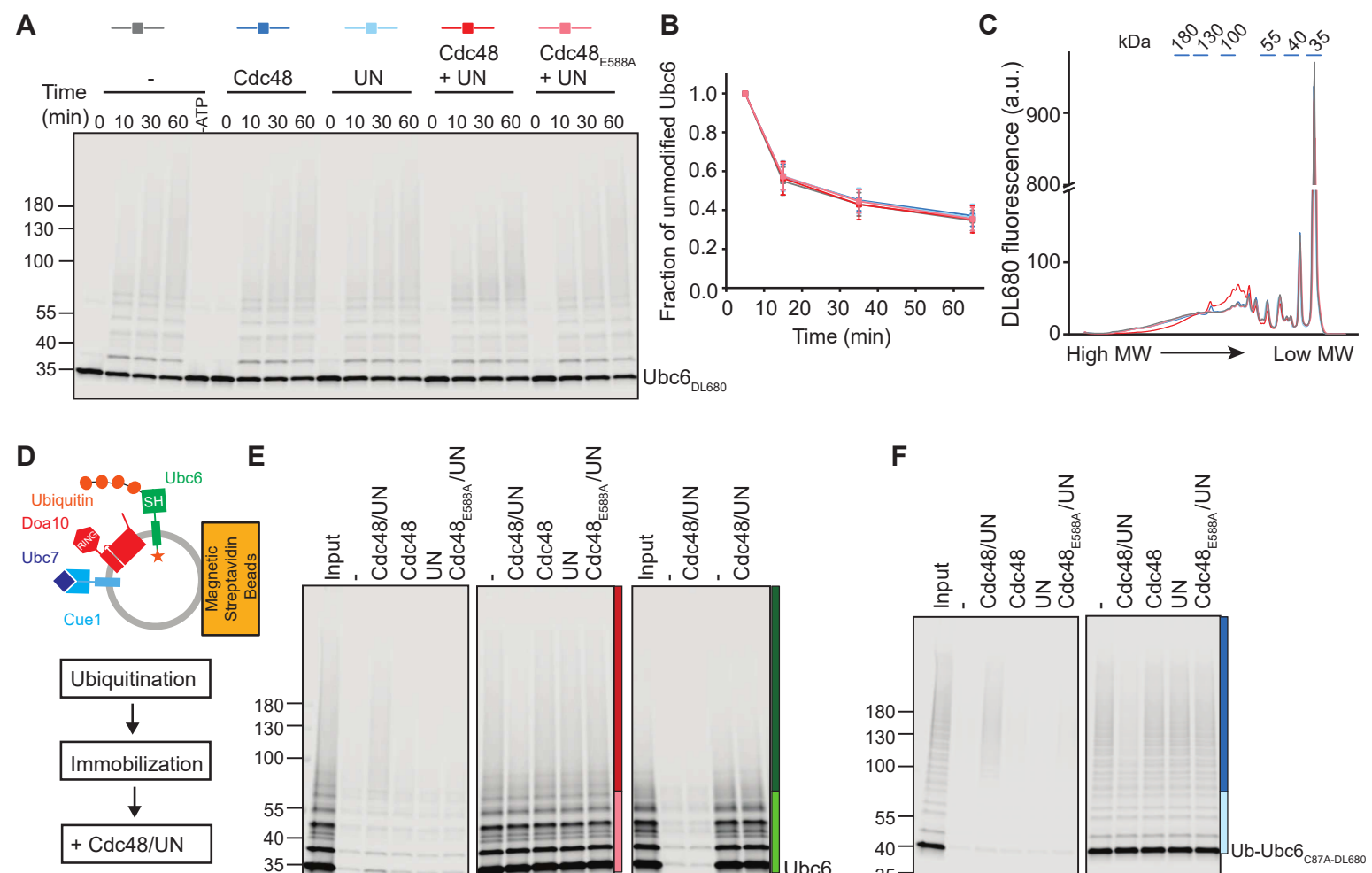

E

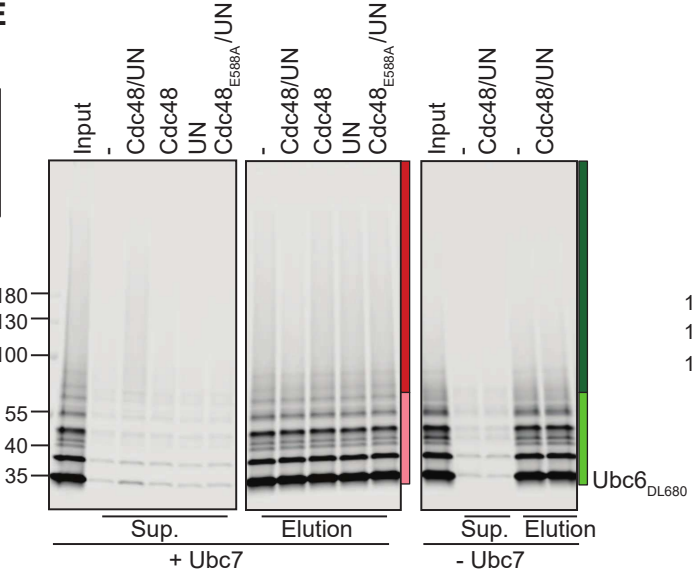

$\mathbf{F}$

G

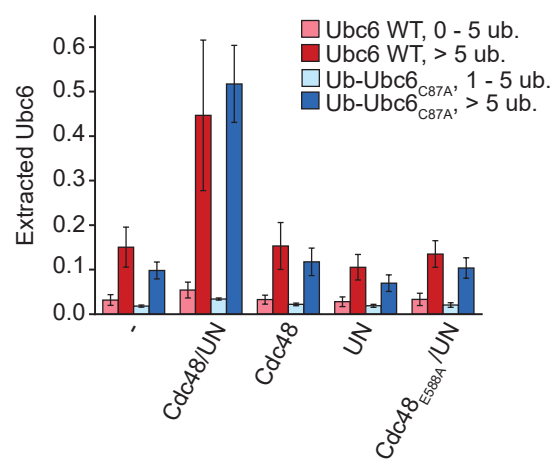

H

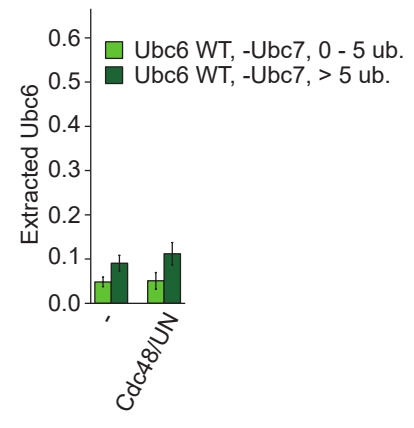

Figure 3.3: The Cdc48 complex catalyzes membrane extraction of polyubiquitinated Ubc6. (A) Time-course of Ubc6 polyubiquitination in the presence of the indicated components. Ubc6 liposomes were fused with liposomes containing Cue1 and Doa10, incubated with $100 \mathrm{nM} \mathrm{E1,} 1 \mu \mathrm{M}$ Ubc7, $120 \mu \mathrm{M}$ ubiquitin, $2.5 \mathrm{mM}$ ATP, and $100 \mathrm{nM}$ of the indicated Cdc48 complex components (f.c. of $100 \mathrm{nM}$ Ubc6, $10 \mathrm{nM}$ Cue1, $40 \mathrm{nM}$ Doa10). UN for Ufd1/Npl4 heterodimer. A 60 min sample in the absence of ATP is shown for the reaction in the absence of Cdc48 or UN. Samples were analyzed by SDS-PAGE and fluorescence scanning. (B) Quantification (mean and SD) of unmodified Ubc6 from three experiments as in (A). Coloring as indicated in (A). (C) Analysis of ubiquitin-chain length on Ubc6 at $30 \mathrm{~min}$ from an experiment as in (A). Coloring as indicated in (A). (continued) 
Figure 3.3 (continued): (D) Explanatory scheme for experiments presented in (E) and (F). After fusion and ubiquitination, liposomes were immobilized to streptavidin magnetic beads via biotinylated lipids. One beads equivalent was removed, and bound protein eluted with SDS sample buffer (Input). Beads were then incubated with $100 \mathrm{nM}$ of the components as indicated in (E) and (F). After $30 \mathrm{~min}$, the solution was removed (Sup.). Material that remained bound to beads was eluted with SDS sample buffer (Elution). All samples were analyzed by SDS-PAGE and fluorescence scanning. (E) Ubc6 liposomes (100 nM) were fused and ubiquitinated as before. Samples were then treated as described in (D). (F) As in (E) but with Ub-Ubc6 ${ }_{\mathrm{C} 87 \mathrm{~A}}$ instead of Ubc6. (G) Quantification (mean and SD) of three experiments as in (E) and (F). Ubiquitinated species in gels as in $(\mathrm{E})$ and $(\mathrm{F})$ were categorized according to ubiquitin chain length (light colors for 0-5 ubiquitin adducts, dark colors for longer chains), and the signal in the supernatant normalized to that in the input. Red for Ubc6, blue for UbUbc6 $6_{\mathrm{C} 87 \mathrm{~A}}$. (H) Quantification (mean and $\mathrm{SD}$ ) of three experiments as in (E), when ubiquitination was performed in the absence of Ubc7.

We also analyzed if autoubiquitinated Doa10 is extracted by the Cdc48 complex (Figure A3H-K). In reactions with WT Ubc6, in which Doa10 is efficiently autoubiquitinated, we found a small fraction of total Doa10 in the unbound fraction (Figure A3J), raising the possibility that at least some Ubc6 is co-extracted with Doa10. However, when Ubc6 was replaced by Ub-Ubc6 $6_{\mathrm{C} 87 \mathrm{~A}}$, which prevents Doa10 autoubiquitination (Figure A3H) and completely abolishes Doa10 extraction (Figure A3K), extraction efficiency for Ub-Ubc6 ${ }_{\mathrm{C} 87 \mathrm{~A}}$ was unchanged (Figure $3.3 \mathrm{~F}$ and $3.3 \mathrm{G}$ ), showing that coextraction only accounts for a minor fraction of extracted protein.

\subsubsection{Doa10 facilitates membrane release of Ubc6}

Our results so far establish a minimal system for polyubiquitination and membrane extraction of Ubc6. However, it remains unclear if Doa10 acts as a retrotranslocase that provides a specific conduit for the movement of a substrate out of the membrane and, thus, reduces the energy required for Cdc48-mediated extraction. We hypothesized that retrotranslocase activity would result in some spontaneous disengagement of Ubc6 from the membrane that would normally remain undetected because of reinsertion. We reasoned that factors that stabilize released Ubc6, but do not provide a direct pulling force like Cdc48, should prevent reinsertion and thus drive the equilibrium towards the released state.

We used the chaperone Get3 to test if Ubc6 is spontaneously released from Doa10 containing liposomes. Get3 is involved in membrane targeting of tail-anchored membrane proteins and wraps around the hydrophobic TM anchor (Mateja et al., 2015). 
In a turbidity assay, we showed that Get3 prevents aggregation of Ubc6 when diluted into detergent-free buffer confirming that Get3 chaperones Ubc6 (Figure A4A). We then incubated liposomes containing Doa10 and Ubc6 with Get3, immobilized liposomes to beads (Figure A4B), and determined the amount of Ubc6 in the unbound fraction. When Ubc6 liposomes were fused to Doa10 liposomes, $43 \pm 5 \%$ of Ubc6 was detected in the unbound fraction (Figure 3.4A, 3.4B and $\mathrm{A} 4 \mathrm{C}$ ), corresponding to an almost quantitative release of correctly oriented Ubc6. When fusion was inhibited, or when we used Syb liposomes lacking Doa10, only 7-9\% were found in the supernatant, probably corresponding to the fraction of improperly reconstituted protein (Figure A1J and $\mathrm{A} 1 \mathrm{~K})$. In the absence of Get3, or when we used a Get3 mutant defective in tailanchored membrane protein binding (Get3 $3_{\mathrm{I} 193 \mathrm{D}}$, (Mateja et al., 2015)), we observed no or drastically reduced release, respectively. We observed no difference between WT Get3 and the ATPase-deficient mutant Get3 ${ }_{\mathrm{D} 57 \mathrm{~N}}$, as previously shown for the holdase activity of Get3 (Voth et al., 2014). Thus, Ubc6 is released in a Doa10-dependent manner. Doa10 mediates spontaneous release of its substrate Ubc6. A chaperone that traps a non-membrane bound state is sufficient to drive spontaneous release. Importantly, this activity of Doa10 does not require the substrate to be ubiquitinated.

To test for spontaneous release in the absence of a trap, we used SUMO-Ubc6 $6_{\text {DL680 }}$, an amino-terminal fusion of SUMO to Ubc6, in which we additionally introduced a TEV protease cleavage site between the luminal carboxyl terminus of Ubc6 and the fluorescent dye (Figure 3.4C). Addition of Ulp1 readily clips off the SUMO-tag resulting in Ubc6 $6_{\text {DL680 }}$, and thus identifies correctly-oriented Ubc6. We fused SUMO-Ubc6 $6_{\text {DL680 }}$ liposomes with Doa10-liposomes and then added Ulp1 to cleave correctly oriented Ubc6. We then added TEV protease and compared cleavage of correctly and wrongly oriented Ubc6 over time. About $70 \%$ of Ubc6 $6_{\mathrm{DL} 680}$ is cleaved within $30 \mathrm{~min}$ (Figure $3.4 \mathrm{D}$ and $3.4 \mathrm{E})$. In contrast, in liposomes lacking Doa10, only $20 \%$ of Ubc6 $6_{\mathrm{DL} 680}$ is accessible to TEV protease, corresponding to the not properly reconstituted Ubc6 (Figure A1J and A1K). Wrong-side out SUMO-Ubc6 ${ }_{\text {DL680, }}$ which exposes the TEV cleavage site on the outside of liposomes, is completely accessible to TEV protease and cleaved with faster kinetics, indicating that protease accessibility over time is not due to slow protease action. This experiment confirms that the carboxyl terminus of Ubc6 is retrotranslocated in a Doa10-dependent manner even in the absence of a trap. 
A

A

क T
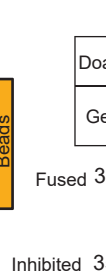

Inhibited 35

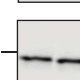

C

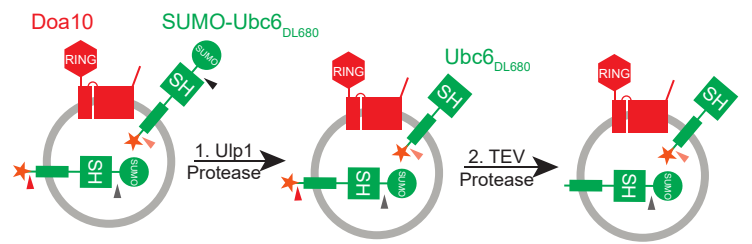

D

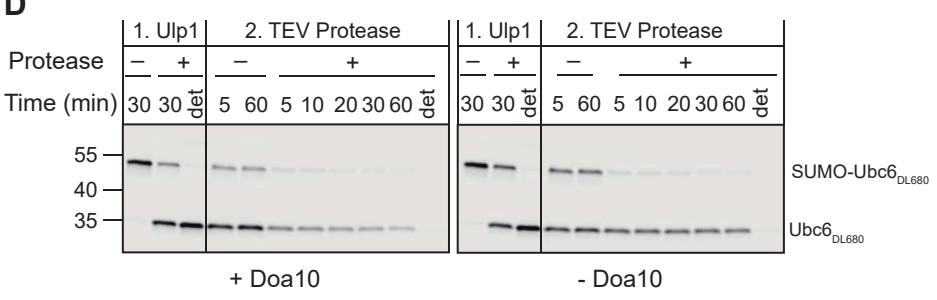

$\mathbf{F}$

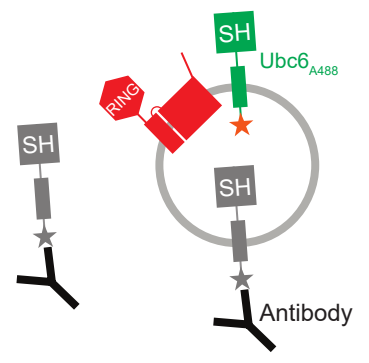

G

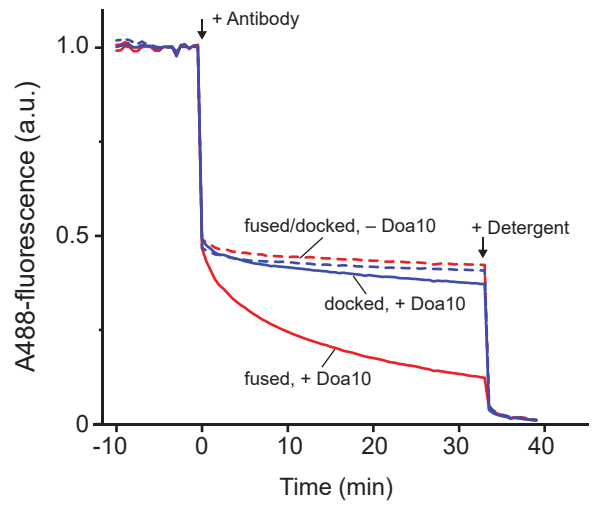

B

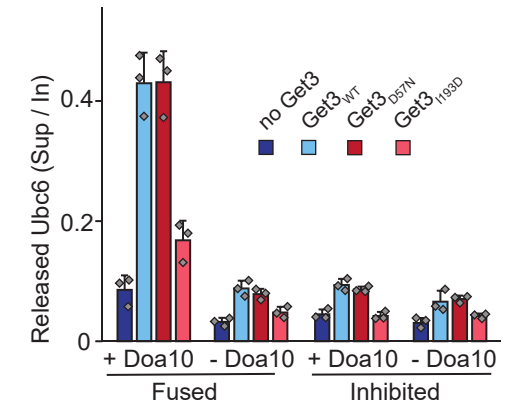

E

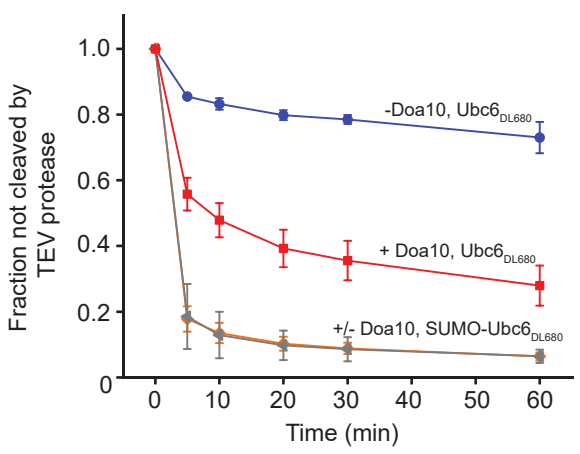

H

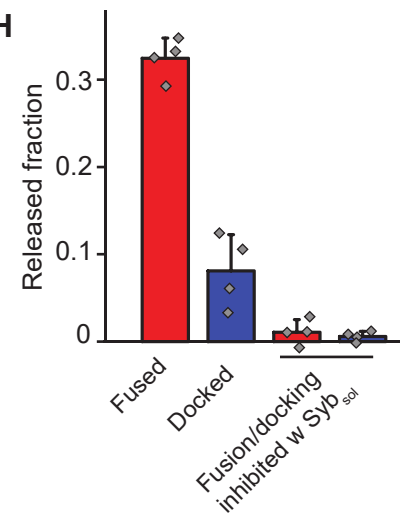

Figure 3.4: Release of Ubc6 from Doa10 containing liposomes. (A) Release of Ubc6 in the presence of different Get3 variants. Ubc6 ${ }_{\mathrm{DL} 680}$ liposomes were incubated with liposomes with or without Doa10, in the absence (Fused) or presence of $\mathrm{Syb}_{\mathrm{sol}}$ (Inhibited), immobilized to streptavidin magnetic beads via biotinylated lipids, and incubated for $16 \mathrm{~h}$ with either buffer or $10 \mu \mathrm{M}$ of either WT Get3 (WT), an ATPase deficient mutant of Get3 (D57N) or a Get3 mutant that cannot efficiently interact with Ubc6 (I193D) (f.c. of $100 \mathrm{nM}$ Ubc6, 40 $\mathrm{nM}$ Doa10). Input and supernatant samples were analyzed by SDS-PAGE and fluorescence scanning. (B) Quantification (mean and SD) of three experiments as in (A). (C) Schematic depiction of the experiment shown in (D) and (E). Ubc6 was fused at the N-terminus to a SUMO tag, and a TEV protease cleavage site inserted between the C-terminus of Ubc6 and the fluorescent dye (DL680, indicated as a star). Ulp1 protease cleaves off the SUMO moiety (black arrow head), TEV protease the fluorescent dye (red arrow head). Liposomes were first incubated with Ulp1 to identify correctly oriented Ubc6, followed by incubation with TEV protease. (continued) 
Figure 3.4 (continued): (D) Accessibility of TEV cleavage site in Ubc6.

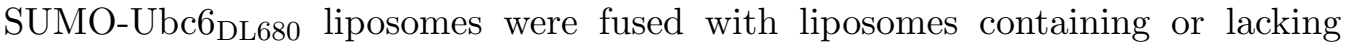
Doa10, followed by incubation with either buffer or Ulp1 in the presence or absence of solubilizing amounts of detergent (det) (lanes 1-3). Ulp1-treated liposomes were then incubated with buffer (lanes 4 and 5), TEV protease (lanes 6-10) or TEV protease and detergent (lane 11), and aliquots were taken at the indicated times. Samples were analyzed by SDS-PAGE and fluorescence scanning. (E) Quantification (mean and SD) of three experiments as in (D). Band intensities from samples treated with TEV protease were normalized to the corresponding band intensities of samples without TEV protease (-). (F) Schematic representation of antibody quenching experiment. A fluorescence quenching anti-AlexaFluor488 antibody quenches fluorescence of dye molecules attached to wrong-side out Ubc6 $\mathrm{A}_{488}$ or Ubc6 $6_{4488}$ that has disengaged from the membrane (grey), whereas dye attached to correctly oriented Ubc6 $\mathrm{A}_{488}$ (green) is shielded from antibody. (G) Time-course of fluorescence quenching experiments as depicted in (F). Liposomes containing Ubc6 ${ }_{A 488}$ were incubated with liposomes without (dashed lines) or with (solid lines) Doa10, co-reconstituted with either Syb (red) or the fusion-deficient $\operatorname{Syb} \Delta 84$ mutant (blue). Addition of the quenching antibody or of solubilizing amount of detergent (Triton X-100) indicated by arrows. (H) Quantification (mean and SD) of four experiments as in $(G)$. Released fraction is defined as $\mathrm{F}_{- \text {Doa10 }}(30 \mathrm{~min})$ $\mathrm{F}_{\text {+Doa10 }}(30 \mathrm{~min})$. Additionally, quantification of experiments as in $(\mathrm{G})$, but in the presence of $\mathrm{Syb}_{\text {sol }}$ is also shown.

We employed a third experimental system to show retrotranslocation, which is based on quenching of an AlexaFluor488 fluorophore (A488) by an antibody. We hypothesized that binding of the antibody to a carboxyl-terminal dye should prevent reinsertion and thus act as a trap similar to Get3 (Figure $3.4 \mathrm{~F}$ ). When we mixed Ubc6 ${ }_{\mathrm{A} 488^{-}}$and Doa10liposomes, but inhibited fusion with either $\mathrm{Syb}_{\text {sol }}$ or $\mathrm{Syb} \Delta 84$, and then added the antiA488 antibody, we observed a sudden decrease in fluorescence by $50 \%$, corresponding to the fraction of wrong-side out protein that exposes its C-terminus to the outside of liposomes. Upon solubilization of liposomes with detergent, the antibody quenches the fluorescence of all A488 epitopes (Figure 3.4G). When liposomes were allowed to fuse, and we then added anti-A488 antibody, again, a sudden decrease in fluorescence was seen, but this time followed by a slower decrease in fluorescence to about $10 \%$ of the original fluorescence signal within $30 \mathrm{~min}$. Thus, in the presence of Doa10, the luminally-encapsulated part of Ubc6 becomes accessible to the antibody. The antibody not only acts as a reporter system for release, but - similar to Get3 - drives release to completion by stabilizing the released state and preventing re-insertion. Together, Get3 capture assay, protease protection assay, and the antibody accessibility assay show that Doa10 facilitates movement of the Ubc6 TM from the membrane to the aqueous phase, and thus reveal its retrotranslocase activity. 


\subsubsection{Structural elements in Doa10 required for retrotranslocation and ubiquitination}

We sought to define structural elements in Doa10 important for retrotranslocation and ubiquitination of Ubc6. To this end, we generated two truncated versions of Doa10 either encompassing only the RING-CH domain and the first two TM segments, or starting with the cytoplasmic loop region before TM3 (Doa10 ${ }_{1-468}$ and Doa10 $434-1319$, respectively). The sites of truncation were chosen based on the finding that in the yeast Kluyveromyces lactis Doa10 is expressed as two separate polypeptides with similar boundaries (Stuerner et al., 2012). We then generated liposomes containing either full-length Doa10, Doa10 $1-468$, or Doa10 $434-1319$ (see Figure A1F-H for characterization of liposomes), fused these liposomes with $\mathrm{Ubc}_{4} \mathrm{~A}_{48}$-containing liposomes, and tested for antibody-accessibility of the fluorescent dye on Ubc6, as before. In this assay, a Doa10 version containing TM segments 3-14 was as efficient as full-length Doa10, whereas a construct containing only the first two segments of Doa10 resulted in only minor quenching above background (Figure 3.5A and 3.5B). Similar observations were made, when we tested for accessibility of TEV protease to a luminal cleavage site (Figure 3.5C). From these experiments we conclude that TM segments 3-14 in Doa10 are sufficient to mediate retrotranslocation.

Next, we asked the question how Ubc6 ubiquitination is affected by the removal of TM segments 3-14. To this end, we prepared liposomes containing either full-length Doa10, only Doa10 $1-468$, or Doa10 $1-468$ and Doa10 $434-1319$. When such liposomes were fused with liposomes containing Ubc6, and incubated with E1, ubiquitin, and ATP, we observed significantly less ubiquitination in the case of Doa10 $1_{1-468}$ (Figure 3.5D and 3.5E). This effect was even more pronounced when the total amount of ubiquitin transferred onto Ubc6 was quantified (Figure 3.5F and A5). Similarly, Ubc7/Cue1dependent polyubiquitination of Ub-Ubc6 $6_{\mathrm{C} 87 \mathrm{~A}}$ was significantly reduced when Doa10 was replaced with Doa10 $1-468$ (Figure 3.5G and 3.5H). Less efficient ubiquitination by Doa10 1-468 was not due to a defect in its E3 activity, because co-reconstitution of Doa10 $434-1319$ restored ubiquitination efficiency (Figure 3.5D-H). We conclude that the Doa10 region that includes TMs 3-14 plays a role in ubiquitination of Ubc6. However, whether this reflects a specific interaction with the TM of Ubc6 is unclear. 


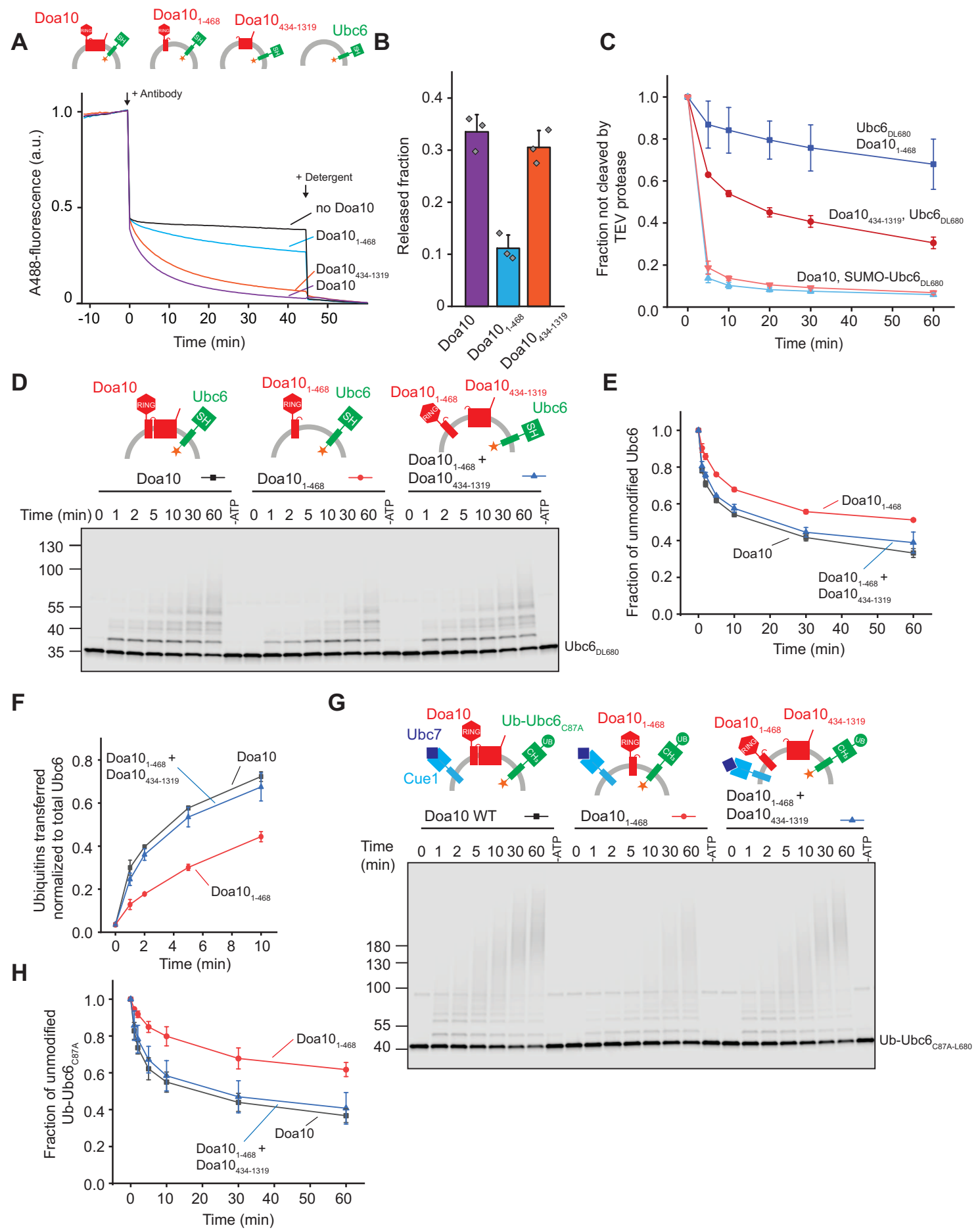

Figure 3.5: Structural determinants of retrotranslocation in Doa10. (A) Using the fusion system, Ubc6 ${ }_{\mathrm{A} 488}$ was reconstituted with the indicated Doa10 versions. Fluorescence traces upon addition of a fluorescence quenching antibody are shown. Antibody or detergent were added at the indicated times. (B) Quantification (mean and SD) of three experiments as in (A). Released fraction is defined as $\mathrm{F}_{- \text {Doa10 }}(30 \mathrm{~min})-\mathrm{F}_{+ \text {Doa10 }}(30 \mathrm{~min})$. (continued) 
Figure 3.5 (continued): (C) Accessibility of TEV protease to an encapsulated TEV cleavage site in the presence of different Doa10 variants as described in

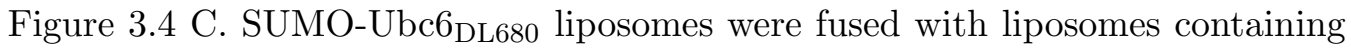
either Doa101-468 (blue) or Doa10434-1319 (red), and then treated with Ulp1 to distinguish right-side out from wrong-side out reconstituted Ubc6 (dark and light colors, respectively). TEV protease was added and samples at different time points analyzed by SDS-PAGE and fluorescence scanning. Band intensities from samples treated with TEV protease were normalized to the corresponding band intensities of samples without TEV protease. (D) Time-course of Ubc6 ubiquitination with different Doa10 truncations in the absence of Ubc7. Liposomes of the indicated compositions were incubated with $100 \mathrm{nM} \mathrm{E} 1,120 \mu \mathrm{M}$ ubiquitin, and $2.5 \mathrm{mM}$ ATP (f.c. of $100 \mathrm{nM} \mathrm{Ubc6,} 10 \mathrm{nM}$ Cue1 and $40 \mathrm{nM}$ of Doa10-variants). A $60 \mathrm{~min}$ sample in the absence of ATP is shown for each reaction. Samples were analyzed by SDS-PAGE and fluorescence scanning. (E) Quantification (mean and SD) of unmodified Ubc6 from three experiments as in (D). (F) Quantification (mean and $\mathrm{SD}$ ) of total ubiquitin-transfer relative to Ubc6 from three experiments as in (D). Intensities of Ubc6 with one to four ubiquitin moieties attached (see Figure A5A) were summed up for each time point and normalized to total Ubc6 in the reaction. (G) Time-course of Ub-Ubc6 $6_{\mathrm{C} 87 \mathrm{~A}}$ ubiquitination with different Doa10 truncations in the presence of Ubc7. Liposomes of the indicated compositions were fused to Ub-Ubc6 ${ }_{\mathrm{C} 87 \mathrm{~A}}$ liposomes. Experiment as in (D), but with $1 \mu \mathrm{M} \mathrm{Ubc7.} \mathrm{A} 60 \mathrm{~min}$ sample in the absence of ATP is shown for each reaction. Samples were analyzed as in (D). (H) Quantification (mean and SD) of unmodified Ub-Ubc6 ${ }_{\mathrm{C} 87 \mathrm{~A}}$ from three experiments as in $(\mathrm{G})$.

\subsubsection{Structural determinants in Ubc6}

To gain further insight into the role of the Ubc6 TM for retrotranslocation and ubiquitination, we compared Ubc6 with mutants in which either the TM anchor or the cytoplasmic parts were replaced with the corresponding segments of synaptobrevin

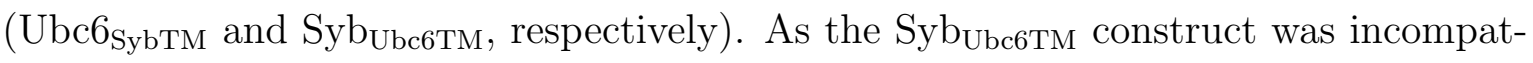
ible with the SNARE-mediated fusion assay, we directly co-reconstituted Doa10 with Ubc6 or its variants (Figure A6A). To remove liposomes that only contained Ubc6 but not Doa10, we affinity-purified Doa10 containing liposomes via the C-terminal SBP-tag (Figure A6B). We then used these liposomes to test for accessibility of A488 attached to the C-terminus of Ubc6 $6_{\text {SybTM }}$ or Sybubc6TM. As shown in Figure 3.6A and 3.6B, the fluorescent dye was only accessible to the antibody in a Doa10-dependent manner when attached to the Ubc6 TM, whereas the dye attached to the Syb TM remained inaccessible.

We also compared retrotranslocation of Ubc6 and Ubc6 $6_{\text {SybTM }}$ in the fusion assay. However, this time we co-reconstituted both Ubc6 and Ubc6 $6_{\text {SybTM }}$ with t-SNARE into the same liposomes, but once labeled Ubc6 (Ubc6 $6_{\mathrm{A} 488}$, Ubc6 $\left.6_{\text {SybTM }}\right)$ and in a separate 
set of liposomes Ubc6 SybTM $_{\text {(Ubc6, Ubc6 }}$ SybTM-A488 $_{\text {) }}$ (Figure A6C). We then fused with liposomes either with or without Doa10. Only the dye attached to the Ubc6 TM was accessible to antibody in a Doa10-dependent manner (Figure 3.6C-D). Importantly, this label-swap excludes that accessibility is due to liposome leakage over time.

Together, these experiments show that the identity of the TM segment is important for retrotranslocation, which might be due to a specific recognition of the TM anchor of Ubc6 by Doa10. Alternatively, failure of spontaneous, Doa10-dependent membrane disengagement of Ubc6 $6_{\text {SybTM }}$ might be explained by the higher hydrophobicity of the Syb TM compared to the Ubc6 TM.

To further understand the role of the TM anchor in Ubc6, we tested its role in

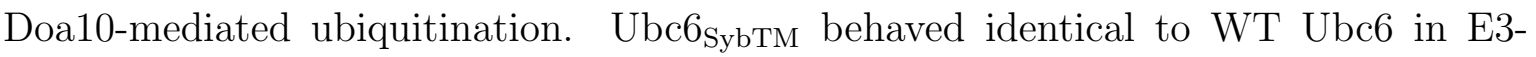
independent autoubiquitination, showing that its E2 activity was not impaired (Figure $\mathrm{A} 6 \mathrm{D}, \mathrm{E}, \mathrm{G})$. In the presence of Doa10, but without Ubc7 or Cue1, we observed minor changes in the turnover of Ubc6 at early time points (Figure 3.6F and A6F), but uncovered a more drastic impediment when we compared the total amount of ubiquitin

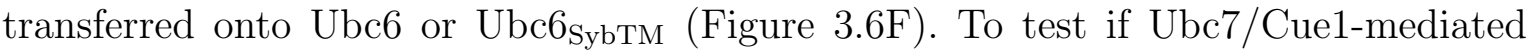
polyubiquitination was affected by the identity of the TM anchor, we again employed N-terminal ubiquitin fusions to either $\mathrm{Ubc}_{\mathrm{C} 87 \mathrm{~A}}$ or $\mathrm{Ubc6}_{\mathrm{C} 87 \mathrm{~A} / \mathrm{SybTM}}\left(\mathrm{Ub}-\mathrm{Ubc} 6_{\mathrm{C} 87 \mathrm{~A}}\right.$ or Ub-Ubc6 $6_{\mathrm{C} 87 \mathrm{~A} / \mathrm{Syb} T \mathrm{M}}$, respectively, Figure A6G) that bypass initial ubiquitination of Ubc6. We found no significant difference in the kinetics of initial ubiquitin transfer to both proteins (Figure 3.6G and 3.6H), but ubiquitin chains remained shorter when the Ubc6 TM was replaced (Figure 3.6G and 3.6I). Thus, the Ubc6 TM anchor contributes to the efficient build-up of polyubiquitin chains, indicating a more efficient recruitment to Doa10. 

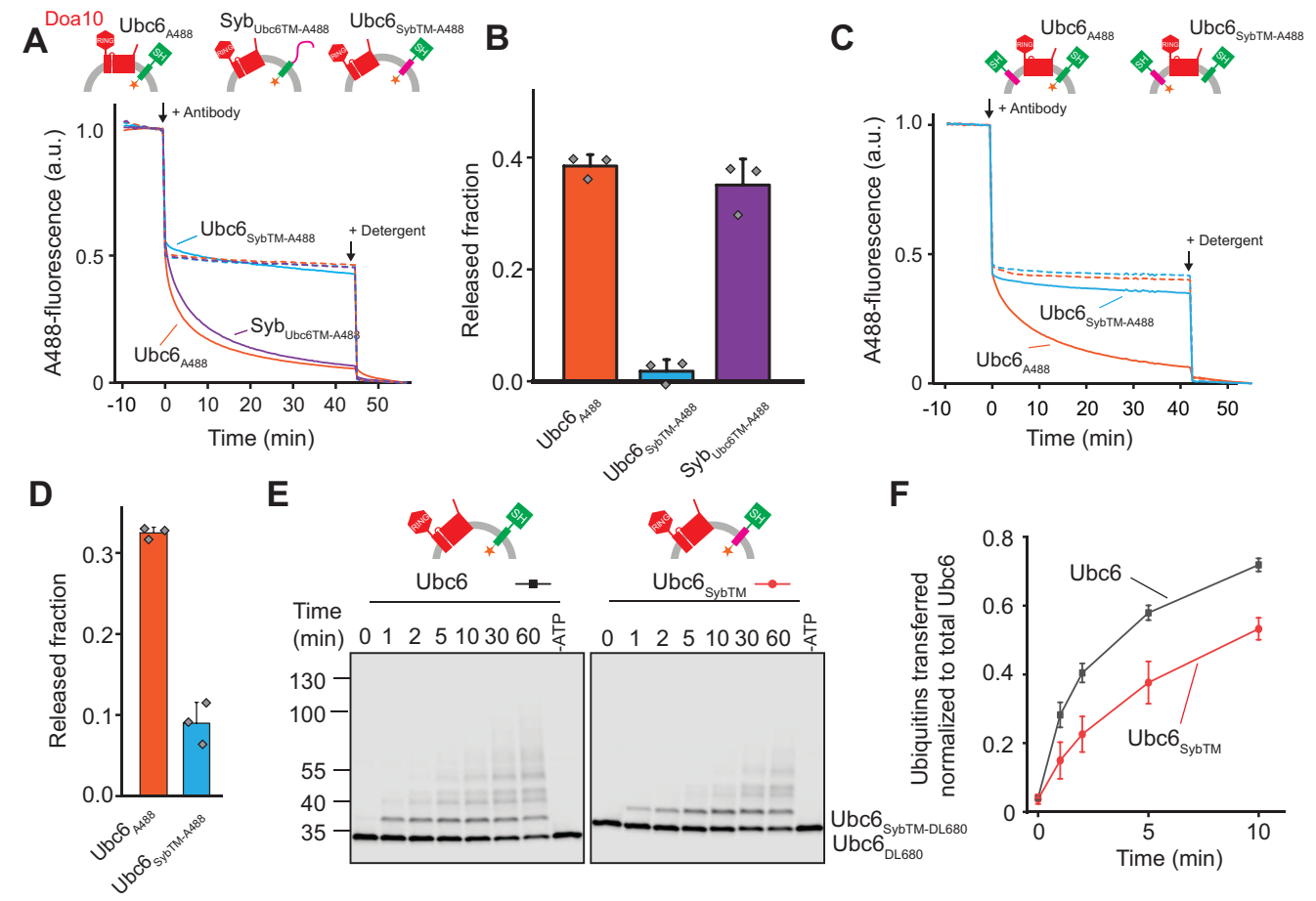

E
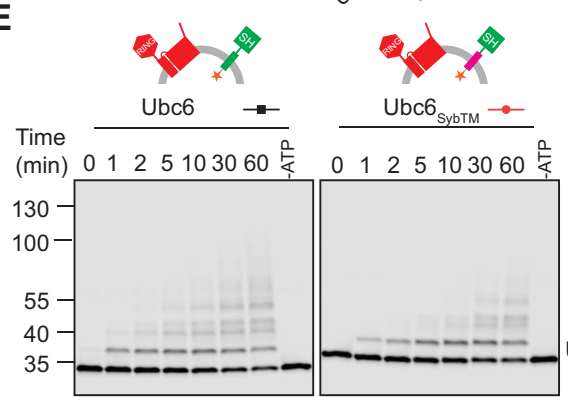

$\mathbf{F}$
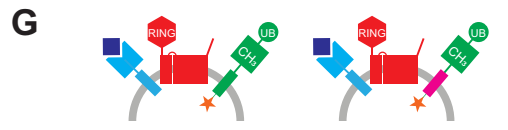

H
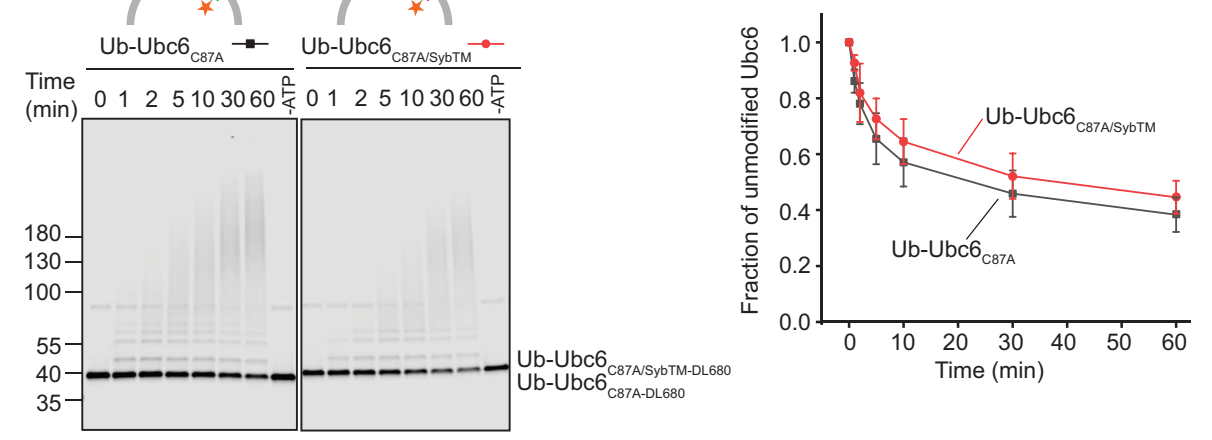

I

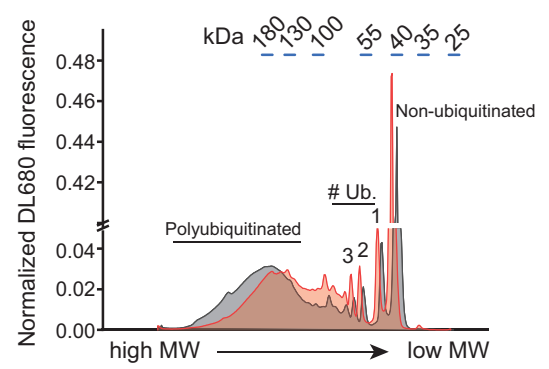

Figure 3.6: Structural determinants in Ubc6. (continued) 
Figure 3.6 (continued): (A) Fluorescence traces of Ubc6 $\mathrm{A}_{488}$ (orange),

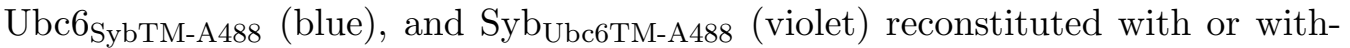
out Doa10 (solid and dashed lines, respectively) upon addition of a fluorescence quenching antibody. Antibody and detergent were added at the indicated times. (B) Quantification (mean and SD) of three experiments as in (A). Released fraction is defined as $\mathrm{F}_{- \text {Doa } 10}(30 \mathrm{~min})-\mathrm{F}_{+ \text {Doa10 }}(30 \mathrm{~min})$. (C) Antibody quenching experiment as in (A) with liposomes containing Ubc6 and Ubc6 $6_{\text {SybTM }}$, and with or without Doa10 (solid and dashed lines, respectively), generated using the fusion system. In separate liposome populations, A488 was either attached to Ubc6 $\left(\mathrm{Ubc6}_{\mathrm{A} 488}\right)$ or $\mathrm{Ubc6}_{\mathrm{SybTM}}\left(\mathrm{Ubc6}_{\text {SybTM-A488 }}\right)$. Arrows indicate the time when antibody or solubilizing detergent were added. (D) Quantification (mean and SD) of three experiments as in $(\mathrm{C})$. Released fraction is defined as $\mathrm{F}_{- \text {Doa } 10}(30 \mathrm{~min})$ - $\mathrm{F}_{+ \text {Doa10 }}(30 \mathrm{~min})$. (E) Time course of Ubc6 WT or Ubc6 $6_{\text {SybTM }}$ ubiquitination in the absence of Ubc7. Doa10 liposomes were fused with liposomes containing the indicated Ubc6 version, and then incubated with E1, ubiquitin and ATP, as before. A 60 min sample in the absence of ATP is shown for each reaction. Samples were analyzed by SDS-PAGE and fluorescence scanning. (F) Quantification (mean and SD) of total ubiquitin-transfer to Ubc6 or Ubc6 $6_{\text {SybTM }}$ from three experiments as in (E). Intensities of Ubc6 variants with one to four ubiquitin moieties attached were summed up for each time point and normalized to total Ubc6 in the reaction. $(\mathbf{G})$ Time-course of Ub-Ubc6 $6_{\mathrm{C} 87 \mathrm{~A}}$ and Ub-Ubc6 $6_{\mathrm{C} 87 \mathrm{~A} / \mathrm{SybTM}}$ ubiquitination in the presence of Ubc7/Cue1. Indicated liposomes generated with the fusion system were incubated with $100 \mathrm{nM} \mathrm{E1,} 1 \mu \mathrm{M} \mathrm{Ubc7,} 120 \mu \mathrm{M}$ ubiquitin, and $2.5 \mathrm{mM}$ ATP (f.c. of $100 \mathrm{nM}$ Ubc6 variants, $10 \mathrm{nM}$ Cue1, $40 \mathrm{nM}$ Doa10), and analyzed as in (E). (H) Quantification (mean and SD) of unmodified UbUbc6 ${ }_{\mathrm{C} 87 \mathrm{~A}}$ or Ub-Ubc6 ${ }_{\mathrm{C} 87 \mathrm{~A} / \mathrm{SybTM}}$ from three experiments as in $(\mathrm{G})$. (I) Analysis of ubiquitin-chain length on Ub-Ubc6 $6_{\mathrm{C} 87 \mathrm{~A}}$ (black) or Ub-Ubc6 $6_{\mathrm{C} 87 \mathrm{~A} / \mathrm{SybTM}}$ (red) at $30 \mathrm{~min}$. Line-scans were performed on fluorescence images of two representative gel samples as in $(\mathrm{G})$. The approximate position in the gel is indicated by the molecular weight marker on top of the graph. \# Ub. denotes the number of ubiquitin moieties attached.

\subsubsection{A folded luminal domain prevents spontaneous release}

Ubc6 is a relatively simple substrate, in the sense that it contains a C-terminal TM segment but no luminal domain. To gain further insight into the retrotranslocase activity of Doa10, we next asked the question how the presence of an additional luminal polypeptide segment or an interaction with another luminal protein affect retrotranslocation.

To directly test how a folded luminal domain affects retrotranslocation, we appended an SBP-tag to the C-terminus of Ubc6, and formed a complex with streptavidin. As before, the protein was labeled with A488 (Ubc6-SBP ${ }_{\mathrm{A} 488}$, Figure 3.7A). This design has the advantage that the high affinity SBP-streptavidin interaction $(\mathrm{Kd}=2.5$ $\mathrm{nM}$, (Keefe et al., 2001)) is broken in the presence of biotin, allowing us to use the same 
liposomes to distinguish between the effect of the SBP-tag and binding of streptavidin to it. As shown in Figure A7A and A7B, liposome incorporation and orientation of Ubc6-SBP ${ }_{\mathrm{A} 488}$ were similar to $\mathrm{Ubc6}_{\mathrm{A} 488}$, with about $50 \%$ correctly oriented. When we fused these liposomes with Doa10-containing liposomes and added anti-A488 antibody, we observed no spontaneous release (Figure 3.7B and 3.7C). In contrast, when we added biotin shortly before addition of the antibody, we observed Doa10-dependent quenching over time as before, indicating that biotin led to the dissociation of streptavidin from SBP and that Ubc6-SBP $\mathrm{A}_{488}$ is spontaneously released despite the presence of the SBP-tag. This was only the case when free, membrane permeable biotin was used, but not when biotin was attached to a protein that cannot pass the membrane. Importantly, addition of biotinylated protein led to the dissociation of streptavidin from wrong-side out Ubc6-SBP ${ }_{\mathrm{A} 488}$ showing that the biotinylated protein indeed dissociates the SBP-tag from streptavidin (Figure A7C-E). Together, these experiments show that interaction with a second protein on the luminal side of the membrane, mimicking the presence of a folded domain, acts as an anchor and prevents spontaneous release of Ubc6.

\subsubsection{Cdc48 action breaks luminal interactions}

Finally, we tested if this anchoring activity of SBP-streptavidin can be overcome by the Cdc48 complex. Liposomes containing Ubc6-SBP A488-streptavidin, Doa10, and Cue1, were incubated with ubiquitination mix with or without Ubc7, followed by the addition of Cdc48 complex and anti-A488 antibody (Figure 3.7D for experimental outline). Only in the presence of Ubc7 and Cdc48 complex, A488-epitopes were accessible to the antibody (Figure 3.7E and 3.7F and A7F). In the absence of ubiquitin, when Ubc7 was omitted, or when we used the catalytically inactive $\mathrm{Cdc} 48_{\mathrm{E} 588 \mathrm{~A}}$, no fluorescence quenching above background was observed. Importantly, streptavidin remained encapsulated in liposomes in reactions where Ubc6-SBP $\mathrm{A} 488_{8}$ was extracted (Figure $3.7 \mathrm{G}$ and 3.7H). Together these results show that while the interaction with streptavidin inhibits spontaneous Doa10-dependent release, Cdc48/UN acting on the cytoplasmic side of the membrane on polyubiquitin chains breaks non-covalent luminal interactions and thus drives extraction while preserving membrane integrity. 

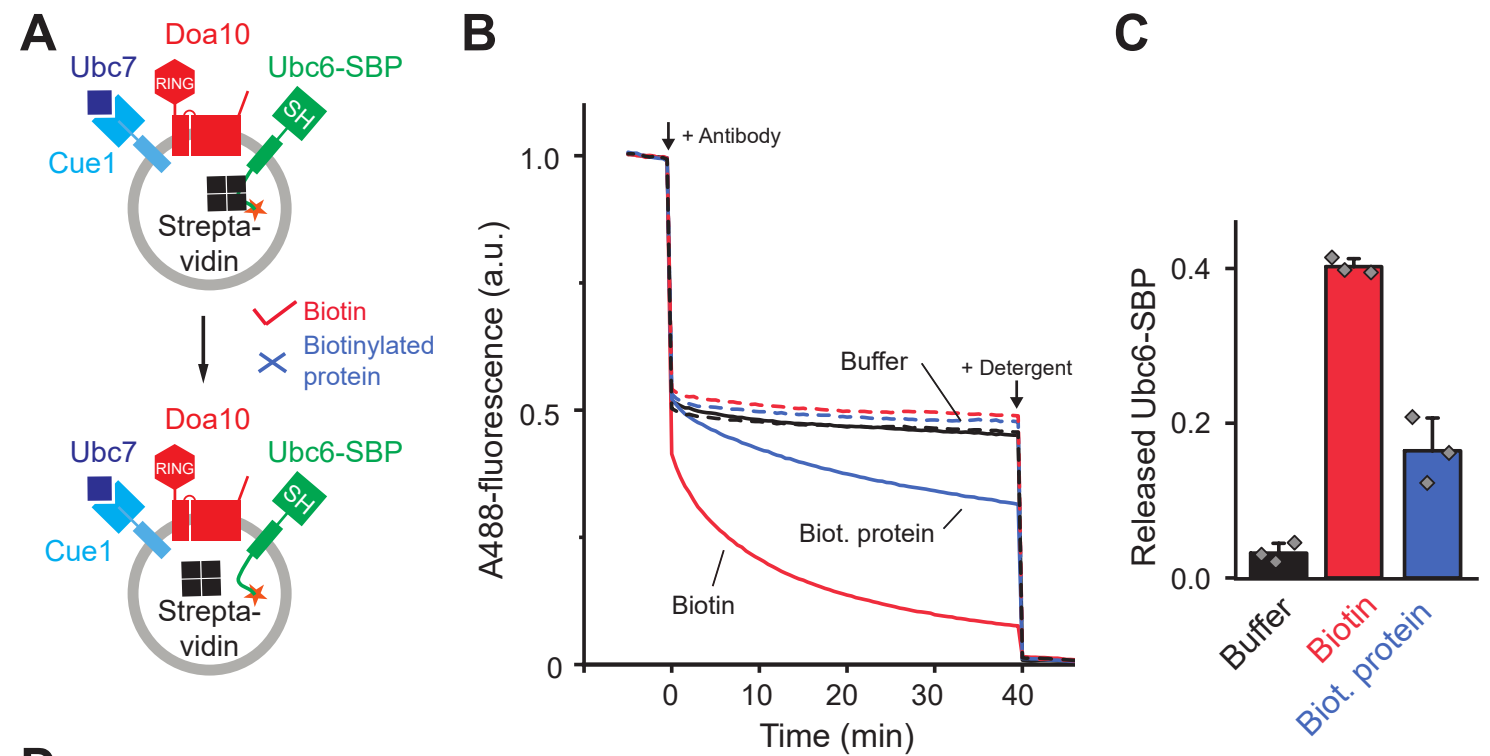

D

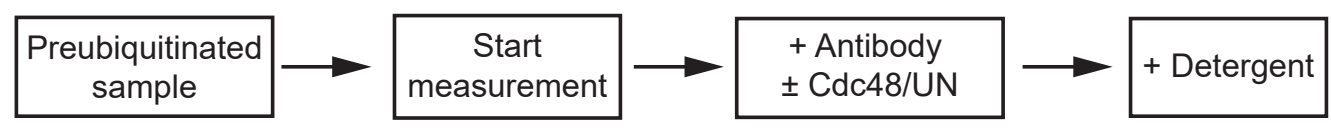

E

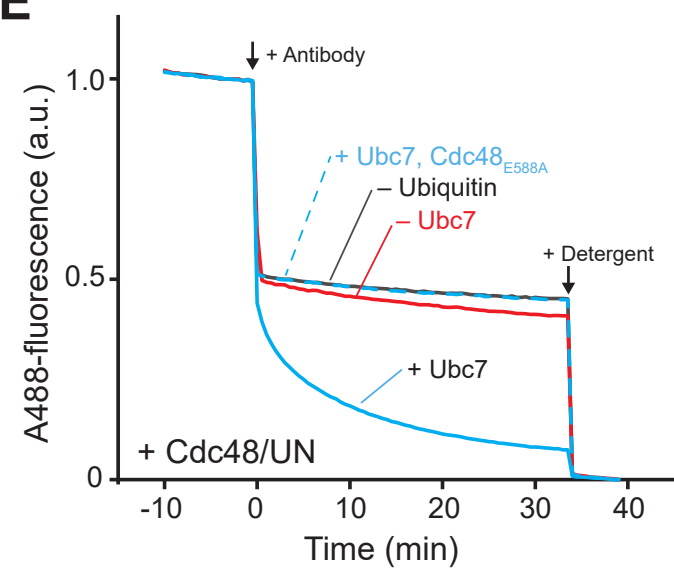

$\mathbf{F}$

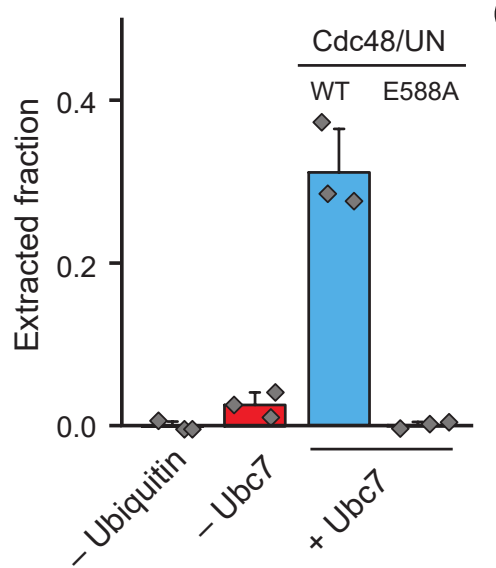

G

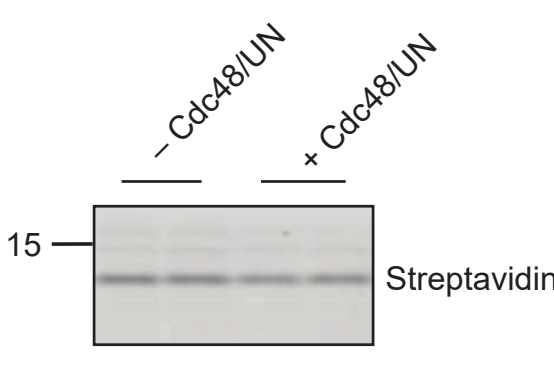

H

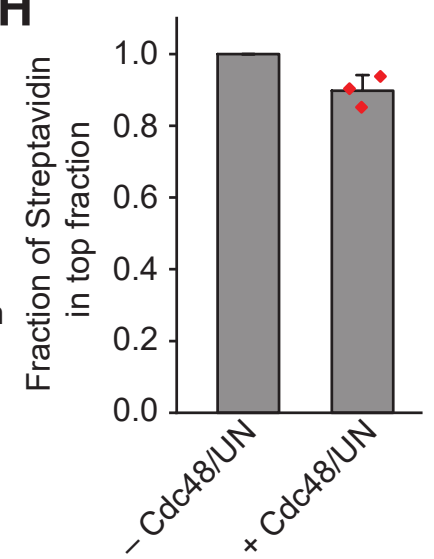

Figure 3.7: Influence of a luminal folded domain bound to Ubc6 (continued) 
Figure 3.7 (continued): (A) Scheme for experiment in (B) and (C). Ubc6 was appended at the C-terminus with streptavidin-binding peptide (SBP) tag and a complex with streptavidin was formed. Membrane permeable biotin releases streptavidin from SBP, whereas a biotinylated protein cannot access the liposome lumen. (B) Liposomes as in (A), with or without Doa10 (solid and dashed lines, respectively) were incubated with either buffer (black), biotin (red) or a biotinylated protein (blue). Antibody or detergent (det) were added at the indicated times. (C) Quantification (mean and SD) of three experiments as in (B). Released fraction is defined as $\mathrm{F}_{- \text {Doa10 }}(30 \mathrm{~min})-\mathrm{F}_{+ \text {Doa10 }}(30 \mathrm{~min})$. (D) Outline for experiments shown in $(\mathrm{E})$ and $(\mathrm{F})$. (E) Liposomes as depicted in (A) were incubated with complete ubiquitination mix (blue), or a mix lacking either Ubc7 (red) or ubiquitin (grey). A488 fluorescence was recorded and quenching antibody was added together with or without Cdc48 complex (left and right panels, respectively). Where indicated by dashed blue lines, Cdc48 was replaced with Cdc48 ${ }_{\text {E588A }}$. UN for Ufd1/Npl4 heterodimer. (F) Quantification (mean and SD) of fluorescence quenching from three experiments as in (E). Released fraction is defined as $\mathrm{F}_{-\mathrm{Cdc} 48}(30 \mathrm{~min})-\mathrm{F}_{+\mathrm{Cdc} 48}(30 \mathrm{~min})$. (G) Liposomes as depicted in (A) were incubated with ubiquitination mix, followed by incubation with or without Cdc48/UN. After addition of biotin, liposomes were then floated in a Nycodenz gradient. Co-floating streptavidin was detected in SDS-PAGE using stain-free technology. Two replicates are shown for each condition. (H) Quantification (mean and SD) of the relative amount of streptavidin co-floating from three experiments as in $(\mathrm{G})$. Each data point represents the mean of two replicates as shown in $(\mathrm{G})$.

\subsection{Discussion}

The ubiquitin ligase Doa10 is required for ERAD of membrane proteins with different topologies, ranging from tail-anchored proteins such as Sbh2, to misfolded mutants of multipass transmembrane proteins like Ste6 and Pma1 (Huyer et al., 2004; Loayza et al., 1998; Wang and Chang, 2003). In addition, the tail-anchored E2 enzyme Ubc6 is a substrate of Doa10 (Swanson et al., 2001). It has been postulated that, in addition to its role in the build-up of polyubiquitin chains, Doa10 is a retrotranslocase that facilitates the transport of luminal and transmembrane polypeptide segments during extraction by the AAA ATPase Cdc48 (Swanson et al., 2001).

Here, we provide direct evidence that Doa10 has a retrotranslocase activity. Using proteoliposomes with reconstituted purified proteins, we identify the minimal machinery that recapitulates the ubiquitination pathway leading to polyubiquitination of Ubc6 by Doa10 and extraction of polyubiquitinated Ubc6 from the membrane. The reaction starts with ubiquitin loading of Ubc6 at its active site cysteine by the E1 Uba1. Doa10 catalyzes transfer of this activated ubiquitin to another residue of Ubc6 (Figure 3.1). 
This transfer occurs spontaneously, but is greatly accelerated by the E3 activity of Doa10. E3-independent autoubiquitination has previously been described for other E2 enzymes in vitro (David et al., 2010), but the functional relevance has only been investigated for a few cases. Ubc7 is normally anchored to the ER membrane via Cue1. In the absence of Cue1, Ubc7 is degraded by the proteasome after assembly of a polyubiquitin chain on its active site cysteine (Ravid and Hochstrasser, 2007). The E2 UbcH10 mediates APC-dependent ubiquitination of cyclin A during G1 phase of the cell cycle. Cyclin A levels are regulated by proteasomal degradation of UbcH10. UbcH10 ubiquitination depends on its catalytic site cysteine and APC/C (Rape and Kirschner, 2004). It is conceivable that degradation of Ubc6 is a mechanism to control overall activity of Doa10. In this view, Ubc6 degradation would be more pronounced when other substrates are less abundant, leading in turn to lower Ubc6 levels and decreased likelihood of off-target ubiquitination. Ubc6 instability might also be a consequence of its apparently highly active ubiquitin-loaded state that is capable of ubiquitinating Ser and Thr, residues that are usually poorer ubiquitination substrates (Weber et al., 2016).

Mono-ubiquitinated Ubc6 is a substrate for Ubc7/Cue1-mediated polyubiquitination with K48 ubiquitin linkages (Figure 3.2). A ubiquitin-fused Ubc6 variant bypasses the requirement for catalytic activity of Ubc6, as shown both in vivo and in our reconstituted system (Weber et al., 2016). The Cdc48 complex, composed of the AAA ATPase Cdc48 and its co-factors Ufd1 and Npl4, is required and sufficient to extract polyubiquitinated but not mono- or multiubiquitinated Ubc6 from the membrane. ATP hydrolysis by Cdc48 provides the driving force for extraction (Figure 3.4). Attachment of five ubiquitin moieties appears to be minimally required for efficient Cdc48 action, in agreement with recent mechanistic studies on Cdc48 mediated unfolding (Bodnar and Rapoport, 2017b). In our system, Ubx2 was not required for Cdc48 mediated membrane extraction of Ubc6, although Ubx2 has been shown to interact with Doa10 and deletion of ubx2 stabilizes Ubc6 (Neuber et al., 2005). We speculate that Ubx2 increases the efficiency of Cdc48 recruitment to sites of retrotranslocation, an activity probably not required in a system lacking any component that would compete for Cdc48. Alternatively, Ubx2 might only be required in the context of several other Cdc48 binding co-factors that have been shown to interact hierarchically with Cdc48 (Haenzelmann et al., 2011), e.g. to prevent deubiquitination by Otu1 at the site of retrotranslocation (Stein et al., 2014).

Protein translocation requires a driving force. In ERAD, ubiquitination coupled to the unfoldase activity in the Cdc48 ATPase provides this driving force. In addition, 
protein translocases often provide a conduit for facilitated movement of a polypeptide chain across membrane. We show that Doa10 facilitates movement of the TM of Ubc6, in the absence of ubiquitination and Cdc48 action, which results in exposure of a luminal fluorescent dye, a TEV-protease cleavage site or capture of the TM by a chaperone (Figure 3.4). In these assays, binding of an antibody or capture by Get3 prevent back-sliding or re-insertion and thereby drive retrotranslocation. A similar replacement of the driving force for translocation has been used to investigate translocation by the Sec61 complex, where binding of antibodies can replace BiP to drive import of prepro-alpha-factor into proteoliposomes (Matlack et al., 1999). Facilitated movement is blocked when the luminal carboxy terminus of Ubc6 is engaged in a protein-protein interaction (Figure 3.7), but this block is overcome by Cdc48 action on ubiquitinated Ubc6, suggesting that unfolding occurs concomitant with retrotranslocation and does not have to occur prior to it.

Using Doa10 truncations and Ubc6/Syb swap mutants, we have analyzed structural determinants of ubiquitination and retrotranslocation. We show that the TM of Ubc6 is sufficient for its retrotranslocation by Doa10 (Figure 3.6), and that replacing the Ubc6 TM with the one from Syb abolishes spontaneous retrotranslocation and impairs ubiquitination of Ubc6. While the total lack of spontaneous retrotranslocation might be explained by higher hydrophobicity of the Syb TM, impairment of ubiquitination by both, Doa10 truncations and Ubc6 TM replacement argue for an interaction of the TM domains. This agrees with a study by Sommer and colleagues, who showed that attaching the TM of Ubc6 to Ubc4, renders this fusion construct unstable, although a direct involvement of Doa10 was not investigated (Walter et al., 2001).

How exactly Doa10 facilitates release of Ubc6 from the membrane is unclear. While our data show that this activity resides within the TM domain of Doa10 comprised of TM segments 3-14 (Figure 3.5), structural information on Doa10 is necessary to further elucidate the mechanism of Doa10's retrotranslocase activity. During extraction of a protein from the membrane, an energetic barrier has to be overcome depending on the hydrophobicity of the TM domain of substrates (Guerriero et al., 2017). Doa10 facilitates the retrotranslocation of Ubc6 and we hypothesize that it has such a role also for other substrates. Hampton and colleagues recently showed that deletion of the Derlin Dfm1, a homolog of the mammalian Derlin-1, impairs degradation of the Doa10 substrate Ste6* $^{*}$ (Neal et al., 2018). Thus, additional factors might be required for retrotranslocation of more complex substrates such as multispanning membrane proteins. Our newly-established reconstituted system provides a useful tool for studying the functions of such factors on a mechanistic level. 
Chapter 3. Retrotranslocation of a tail-anchored membrane protein by the ubiquitin ligase Doa10

\section{Acknowledgements}

We thank Blanche Schwappach-Pignataro (University Medical Center Göttingen, Germany) for providing Get3 constructs, Iris Bickmeyer and Nupur Nupur for technical assistance, and Tom Rapoport and Blanche Schwappach-Pignataro for comments on the manuscript. This work was supported by the European Research Council (ERC) under the Horizon2020 research and innovation program (grant 677770), by the Deutsche Forschungsgemeinschaft SFB1190, P15 (both to AS), and the Boehringer Ingelheim Fonds (to VV). 


\subsection{Appendix}

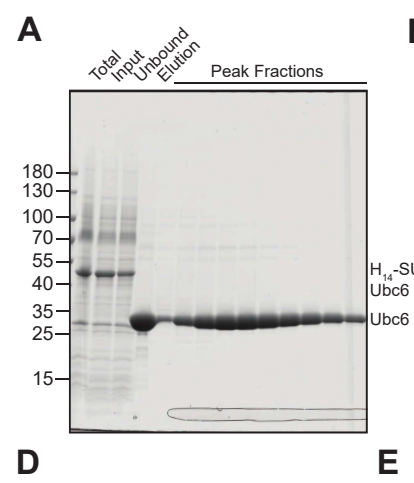

B

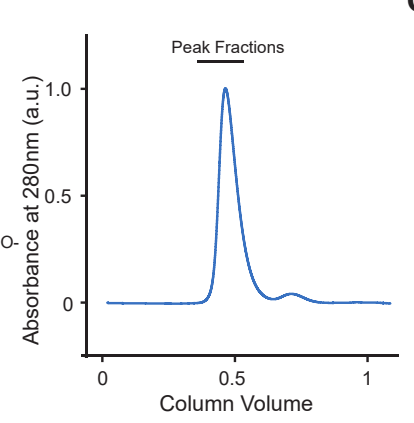

C

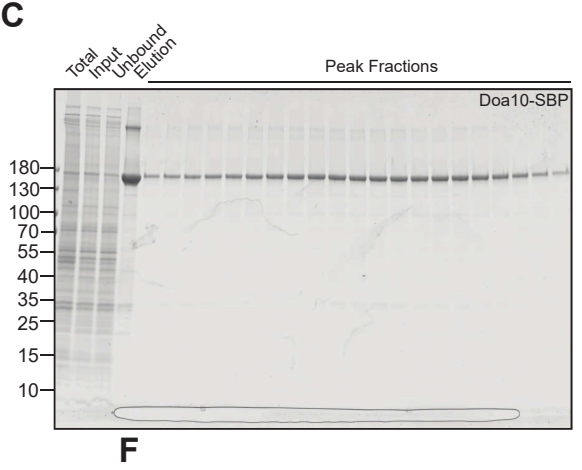

G
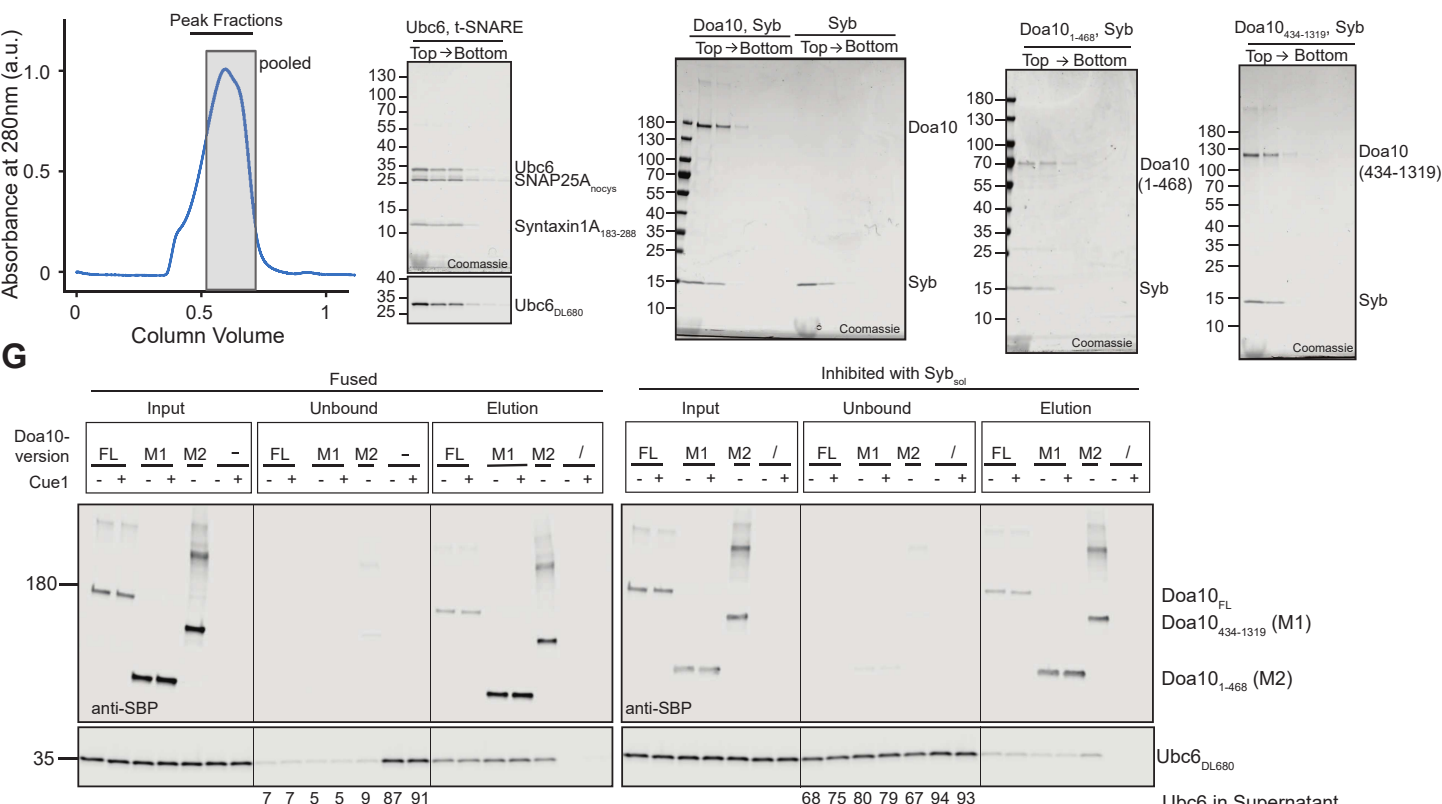

nhibited with Syb

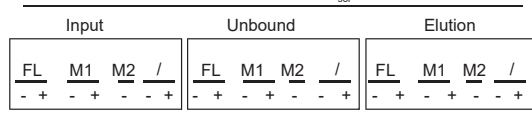

H
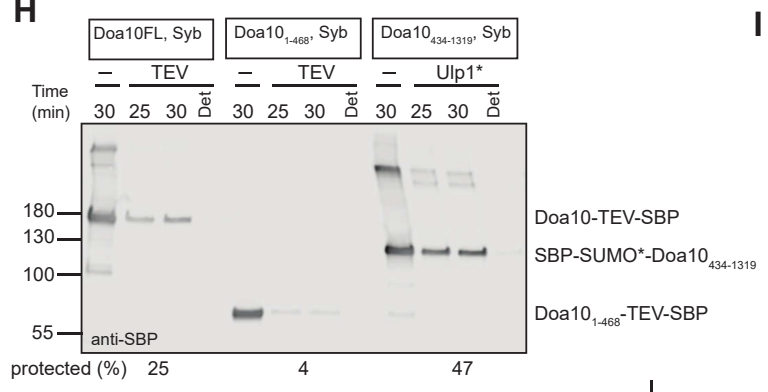

J

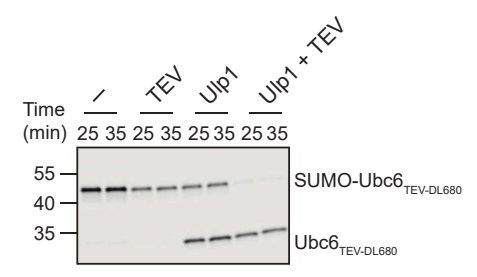

K

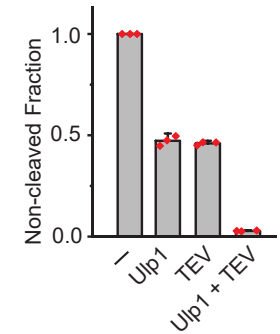

I
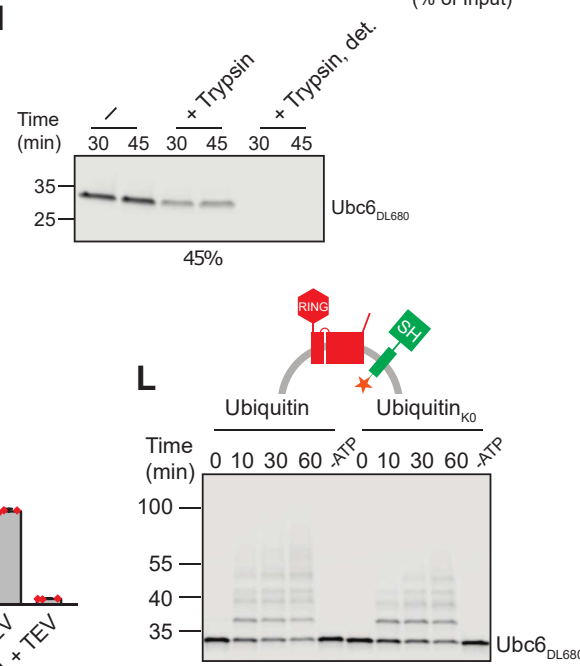

Figure A1: Related to Figure 3.1. (continued) 
Figure A1 (continued): (A) Coomassie Blue stained SDS-PAGE showing purification of Ubc6 from E. coli. Ubc6 was expressed as a His $14-\mathrm{SUMO}$ fusion protein with a C-terminal LPETGG tag for sortase A mediated transpeptidation with a fluorescently labeled GlyGlyGlyCys peptide. First, a membrane fraction was prepared and solubilized with the detergent dodecylmaltoside (Total). After ultracentrifugation, Ubc6 was purified by immobilized nickel ion chromatography (Input, Unbound). Ubc6 was eluted by incubation of Ni-NTA beads with Ulp1 (Elution). The protein was further purified by size-exclusion chromatography (B). (B) Chromatogram of size exclusion chromatography for Ubc6 purification shown in (A) on a Superdex 200 column. (C) Coomassie Blue stained SDS-PAGE showing purification of SBP-tagged Doa10 from S. cerevisiae by streptavidin-affinity chromatography and size exclusion chromatography (Superose 6 column). (D) Chromatogram of size-exclusion chromatography for Doa10 purification shown in (C). Shaded area indicates fractions that were pooled. (E) Liposomes with co-

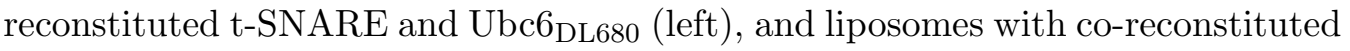
Doa10 and synaptobrevin (Syb, right) were subjected to a Nycodenz step gradient. After ultracentrifugation, the gradient was fractionated and fractions analyzed by SDS-PAGE and Coomassie Blue staining (top) and fluorescence scanning (bottom). (F) Liposomes containing Syb and truncated versions of Doa10 used in Figure 3.5 were analyzed as in (E). (G) Analysis of SNARE-dependent interaction of liposomes. Liposomes containing Syb and different SBP-tagged Doa10 versions were mixed with Ubc6/t-SNARE liposomes in the absence (Fused) or presence (Inhibited) of $\mathrm{Syb}_{\mathrm{sol}}$. Liposomes were then precipitated using streptavidin magnetic beads, washed, and eluted with biotin. Input, unbound, and elution fractions were analyzed by SDS-PAGE followed by Western blotting using an anti-SBP antibody to detect Doa10 variants (top), or fluorescence scanning to detect Ubc6 $6_{\mathrm{DL} 680}$ (bottom). Numbers below unbound fractions indicate the depletion of Ubc6 $6_{\mathrm{DL} 680}$ relative to input fractions. (H) Liposomes containing different Doa10 versions were subjected to either TEV protease (Doa10-TEV-SBP and Doa101-468-TEV-SBP) or Ulp1star (SBP-SUMOstar-Doa10 $434-1319$ ) for the indicated times in the absence or presence of Triton-X100 (det), and analyzed by SDS-PAGE and Western blotting using an anti-SBP antibody. Percentages at the bottom indicate the fraction of protein remaining undigested in the absence of detergent and thus indicate the fraction with wrong-side out orientation.

(I) Liposomes containing Ubc6 $6_{\mathrm{DL} 680}$ and t-SNARE were subjected to tryptic digest for the indicated times in the absence or presence of Triton-X100 (det). Samples were analyzed by SDS-PAGE and fluorescence scanning. (J) To evaluate the reconstitution of Ubc6, we engineered a TEV cleavage site between the TM anchor of Ubc6 and its carboxy-terminal fluorescence label (DyLight680, DL680). In correctly oriented Ubc6, this cleavage site resides in the liposome lumen. To distinguish correctly from wrong-side out reconstituted Ubc6, we also fused a

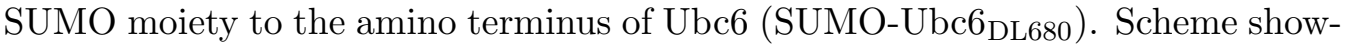

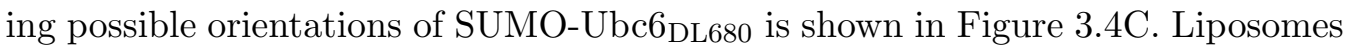
containing SUMO-Ubc6 $6_{\text {DL680 }}$ were incubated with either TEV protease, Ulp1 or both proteases for the indicated times. Samples were analyzed by SDS-PAGE and fluorescence scanning. (K) Quantification (mean and SD) of (J). Three experiments as in $(\mathrm{J})$ were quantified. $53 \pm 2 \%$ of SUMO-Ubc6DL680 was accessible to Ulp1 generating Ubc6DL680. (continued) 
Figure A1 (continued): TEV protease cleaved $54 \pm 8 \%$, showing that about $10 \%$ of Ubc6 was accessible to both proteases indicating that this fraction was not properly reconstituted but probably sticking to the liposome surface. (L) Time course of reactions as in Figure 3.1C, but with either wild-type ubiquitin or a ubiquitin mutant with all Lys mutated to Arg (Ubiquitin ${ }_{\mathrm{K} 0}$ ).

A

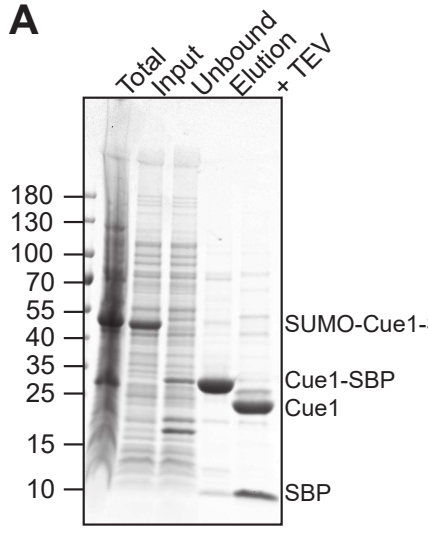

B

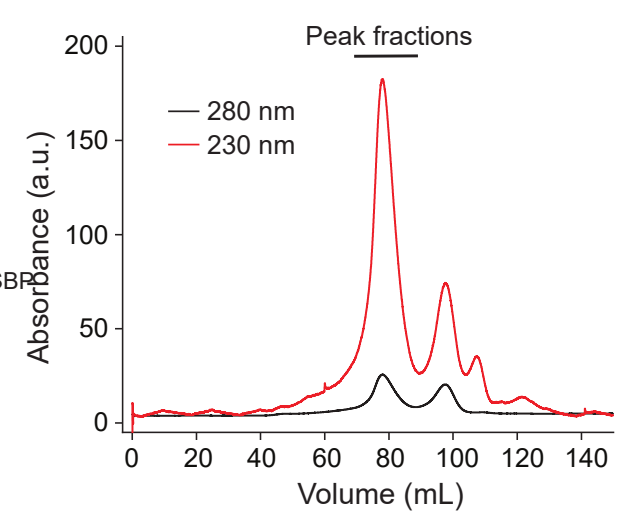

C

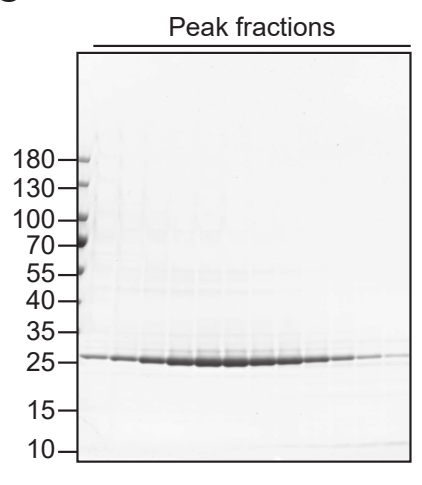

D
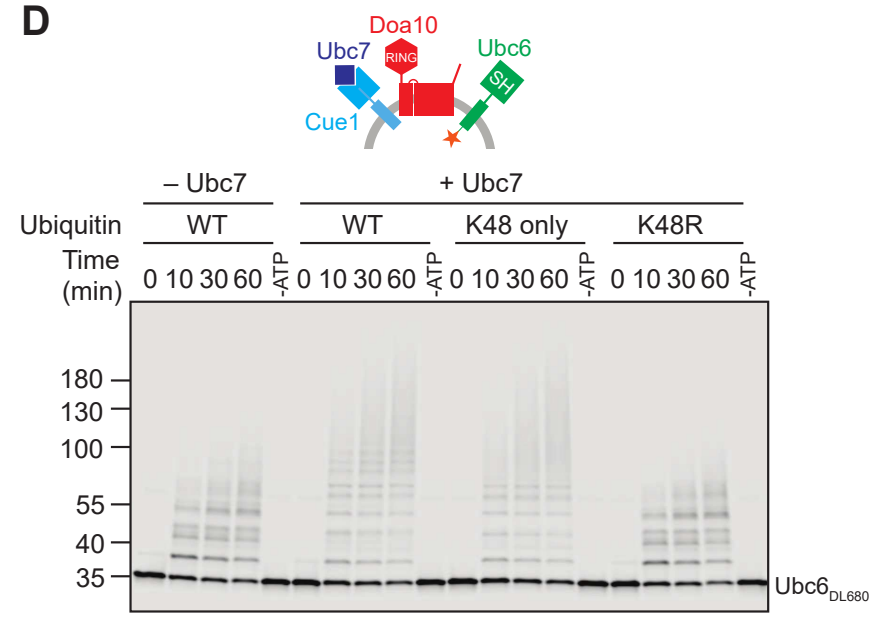

$\mathbf{F}$
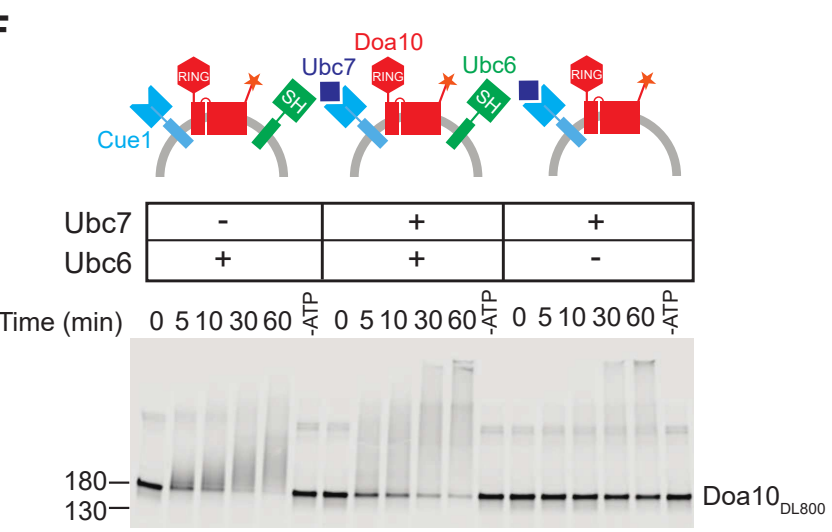

$\mathbf{E}$

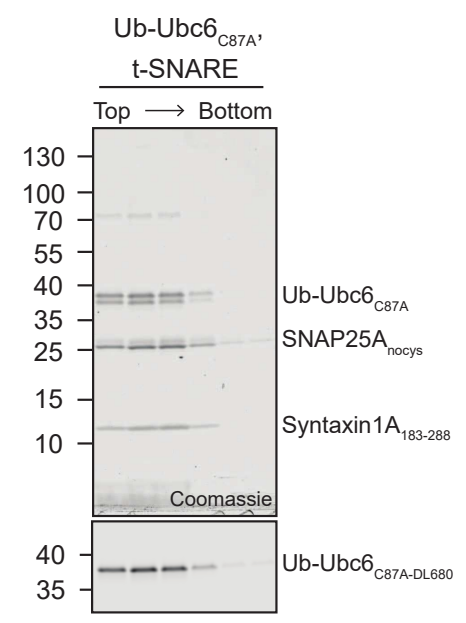

G

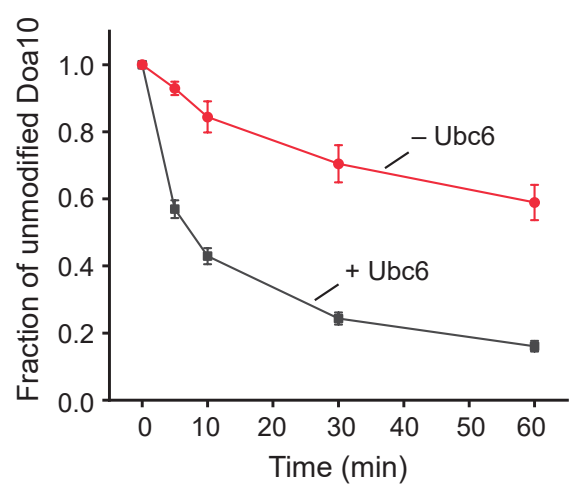

Figure A2: Related to Figure 3.2. (continued) 
Figure A2 (continued): (A-C) Purification of Cue1 from E. coli. (A) Coomassie Blue stained SDS-PAGE showing purification of Cue1. Cue1 was expressed with a signal peptide from DsbA followed by a SUMO tag, Cue1, and a C-terminal SBP-tag. A decylmaltoside-solubilized membrane fraction (Total, Input) was supplemented with Ulp1 and Cue1-SBP purified by streptavidin affinity chromatography (Unbound, Elution). Following removal of the SBP-tag with TEV protease (+ TEV). (B) Chromatogram of size-exclusion chromatography on Superdex S200 column for Cue1 purification. As Cue1 only weakly absorbs at $280 \mathrm{~nm}$ (black), the absorbance at $230 \mathrm{~nm}$ (red) is additionally shown. Peak fractions analyzed by SDS-PAGE are indicated by a black bar at the top of the chromatogram. (C) Coomassie Blue stained SDS-PAGE showing peak fractions of size exclusion chromatography shown in (B). (D) Time course of ubiquitination of Ubc6 $6_{\text {DL680 }}$ analyzed by SDS-PAGE and fluorescence scanning. Doa10/Cue1 li-

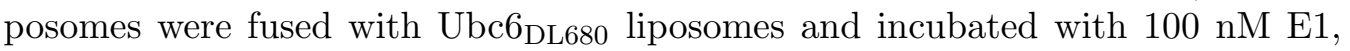
$120 \mu \mathrm{M}$ ubiquitin, and $2.5 \mathrm{mM}$ ATP in the presence or absence of $1 \mu \mathrm{M}$ Ubc7 (f.c. of $100 \mathrm{nM}$ Ubc6, $40 \mathrm{nM}$ Doa10, $10 \mathrm{nM}$ Cue1). Where indicated, ubiquitin was replaced with either K48R ubiquitin or a ubiquitin mutant in which all lysines but K48 were mutated to arginine (K48 only). For each condition a 60 min sample of a reaction lacking ATP was analyzed. (E) Liposomes with co-reconstituted tSNARE and Ub-Ubc6 C87A-DL680 were subjected to a Nycodenz step gradient. After ultracentrifugation, the gradient was fractionated and analyzed by SDS-PAGE and Coomassie Blue staining (top) and fluorescence scanning (bottom). (F) Time course of Doa10 autoubiquitination in the presence of Ubc6 and Ubc7/Cue1. Liposomes containing Cue1 and a C-terminally sortase-labeled Doa10 (Doa10 ${ }_{\text {DL800 }}$ ) were fused with liposomes lacking or containing Ubc6, and incubated with $100 \mathrm{nM}$ E1, $120 \mu \mathrm{M}$ ubiquitin, $2.5 \mathrm{mM}$ ATP, and with our without $1 \mu \mathrm{M}$ Ubc7 (f.c. of $100 \mathrm{nM}$ Ubc6, $40 \mathrm{nM}$ Doa10, $10 \mathrm{nM}$ Cue1). Samples were analyzed by SDS-PAGE and fluorescence scanning. For each condition a $60 \mathrm{~min}$ sample of a reaction lacking ATP was analyzed. (G) Quantification (mean and SD) of unmodified Doa10 ${ }_{\text {DL800 }}$ from three experiments as in $(\mathrm{F})$. 
A

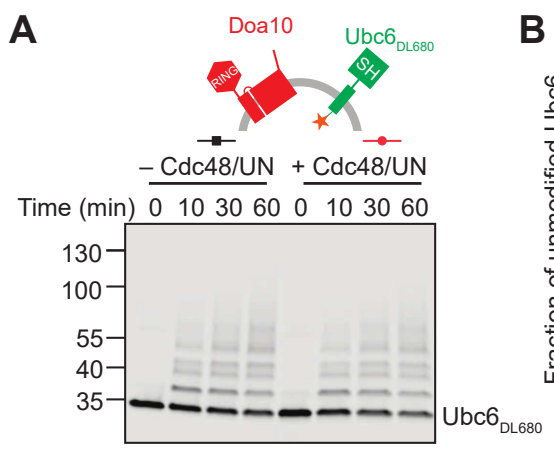

D

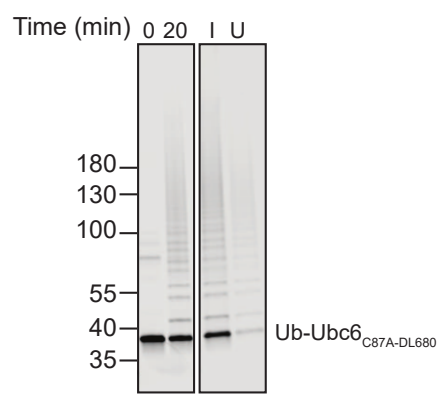

G

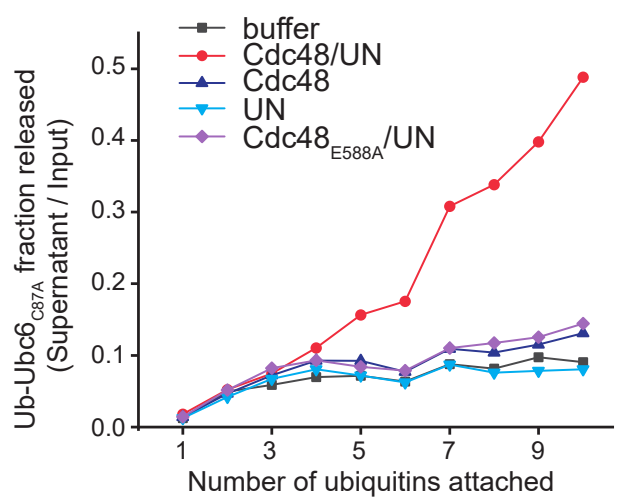

$\mathbf{J}$

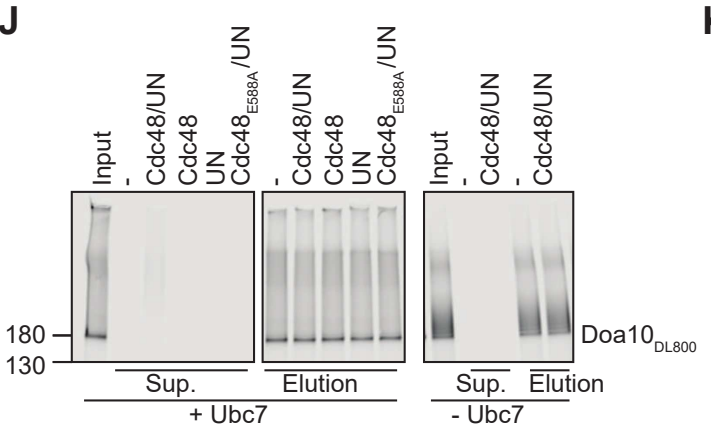

H
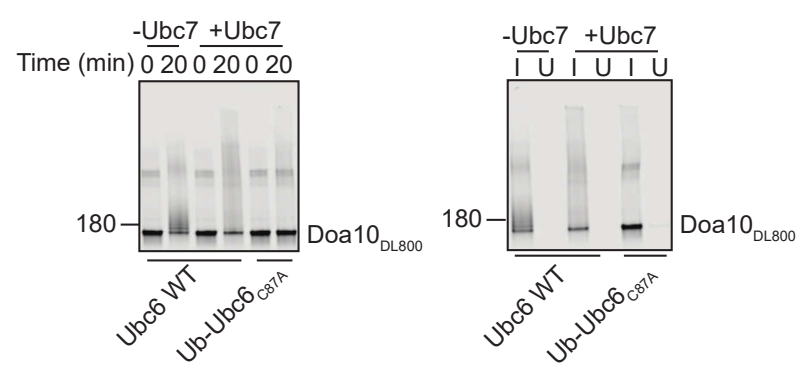

K

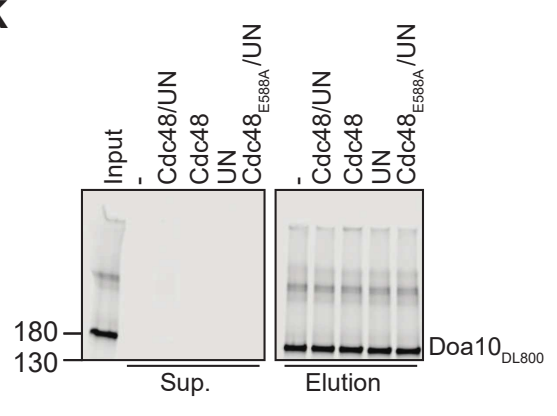

Figure A3: Related to Figure 3.3. (continued) 
Figure A3 (continued): (A) Time course of Ubc6 ubiquitination by Doa10 in the absence of Ubc7, with or without Cdc48 complex. Doa10/Cue1 liposomes were fused with Ubc6 liposomes, and incubated with $100 \mathrm{nM} \mathrm{E} 1,120 \mu \mathrm{M}$ ubiquitin, and $2.5 \mathrm{mM}$ ATP (f.c. of $100 \mathrm{nM}$ Ubc6, $40 \mathrm{nM}$ Doa10, $10 \mathrm{nM}$ Cue1). Samples were analyzed by SDS-PAGE and fluorescence scanning. For each condition a 60 min sample of a reaction lacking ATP was analyzed. (B) Quantification (mean and SD) of unmodified Ubc6 $6_{\mathrm{DL} 680}$ from three experiments as in (A). Coloring as indicated in (A). (C) Samples from ubiquitination and immobilization under conditions described in Figure 3.3D. Doa10/Cue1 liposomes were fused with Ubc6 liposomes, and incubated with E1, ubiquitin, and ATP with or without Ubc7. After 20 min the reaction was stopped by adding EDTA. Liposomes were then immobilized to streptavidin magnetic beads and Input (I) and unbound (U) fractions analyzed SDS-PAGE and fluorescence scanning. (D) As in (C) but with Ub-Ubc6 $6_{\mathrm{C} 87 \mathrm{~A}}$ instead of WT Ubc6. (E) Quantification (mean and SD) of protein immobilization efficiency from three experiments as in (C) and (D). In these experiments, Doa10 was fluorescently labelled with DyLight800. (F) Quantification (mean and SD) of the efficiency of liposome immobilization in experiments described in Figure 3.3 and A3. Liposomes contained 0.5 mol\% Rhodamine-PE. Rhodamine-PE content of immobilizations shown in (C) and (D) was quantified (Input and Unbound). In addition, Rhodamine content in supernatants from extraction reactions shown in Figure 3.3 E,F was determined. (G) Comparison of extraction efficiency of Ub-Ubc6 $6_{\mathrm{C} 87 \mathrm{~A}}$ modified with ubiquitin chains of different length as shown in Figure 3.3F. (H) Ubiquitination of Doa10 ${ }_{\text {DL800 }}$ with or without Ubc7 under conditions described in Figure 3.3 D. Doa10/Cue1 liposomes were fused with Ubc6 or Ub-Ubc6 ${ }_{\mathrm{C} 87 \mathrm{~A}}$ liposomes, and incubated with E1, ubiquitin, and ATP with or without Ubc7. After 20 min the reaction was stopped by adding EDTA. (I) Liposomes from reactions as in $(\mathrm{H})$ were immobilized to streptavidin magnetic beads and input (I) and unbound (U) fractions analyzed SDS-PAGE and fluorescence scanning. (J) Cdc48-dependent extraction of Doa10. Immobilized liposomes from reactions as in (H) and (I) were treated with the indicated components. Input, unbound (Sup.) and SDS sample buffer elutions were analyzed by SDS-PAGE and fluorescence scanning. (K) As in $(\mathrm{J})$, but with Ub-Ubc6 ${ }_{\mathrm{C} 87 \mathrm{~A}}$ instead of WT Ubc6. 
A

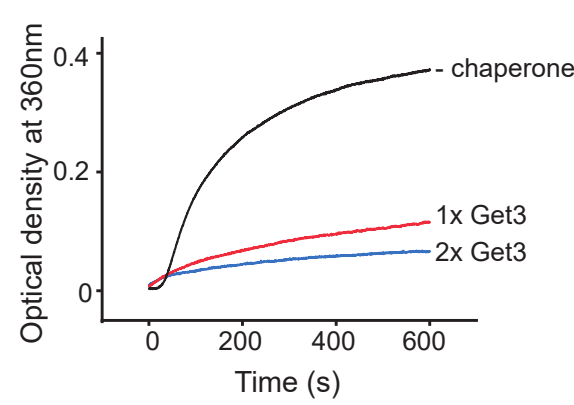

C

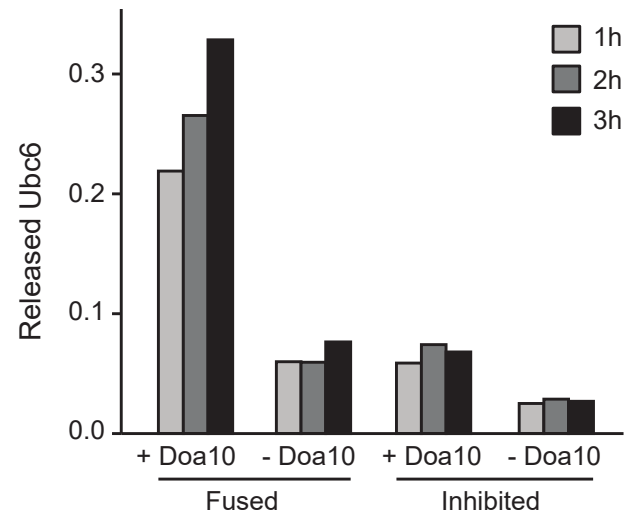

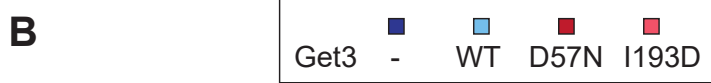

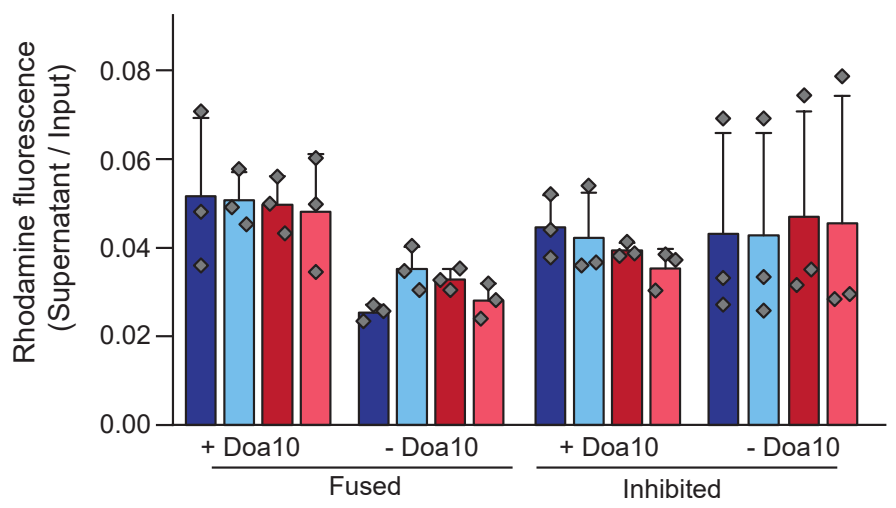

Figure A4: Related to Figure 3.4. (A) Ubc6 in $0.03 \%$ (w/v) dodecyl maltoside was diluted 25 -fold (f.c. $1.8 \mu \mathrm{M}$ ) into either detergent-free buffer (black), or buffer containing 1.8 or $3.6 \mu \mathrm{M}$ Get3 (red and blue, respectively). Optical density was measured at $360 \mathrm{~nm}$. (B) Immobilization efficiency of liposomes used in Figure 3.4 A,B was quantified (mean and SD) via co-reconstituted fluorescent Rhodamine-PE. (C) Release of Ubc6 $6_{\text {DL680 }}$ in the presence of Get3, as in Figure 3.4 A,B, but for shorter incubation times. 


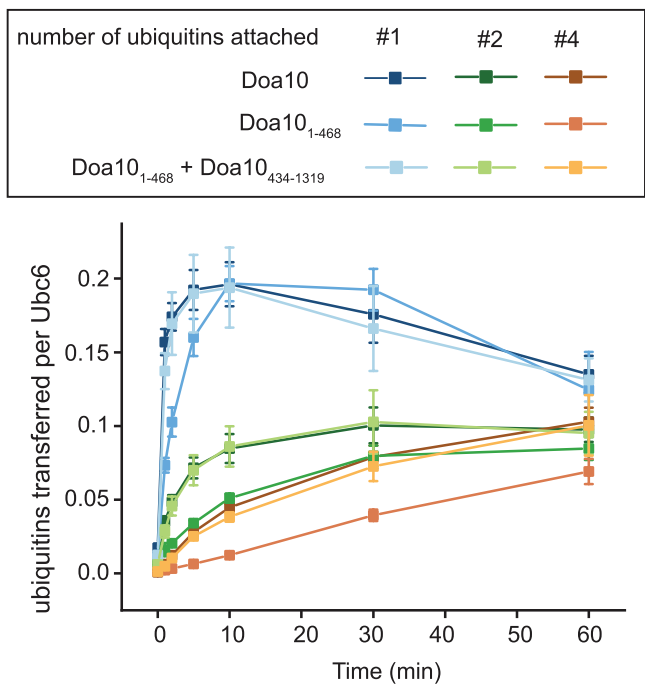

Figure A5: Related to Figure 3.5. Quantification (mean and SD) of mono(blue), di- (green), and tetra (brown)-ubiquitinated Ubc6 in the presence of different Doa10 variants (quantified from three experiments as in Figure 3.5 D). These data points were used to generate graphs in Figure 3.5 F. 


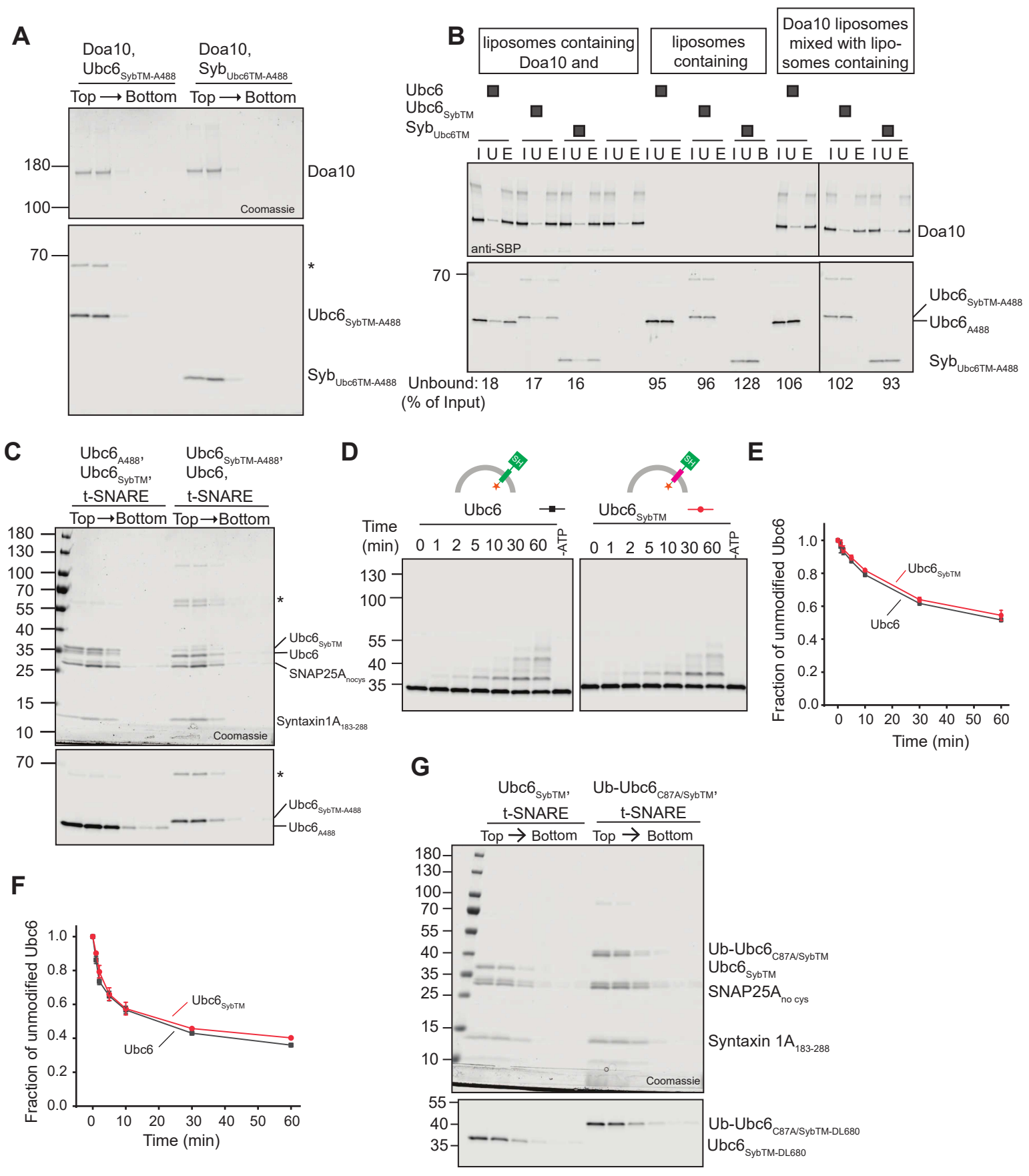

Figure A6: Related to Figure 3.6. (A) Doa10 liposomes co-reconstituted

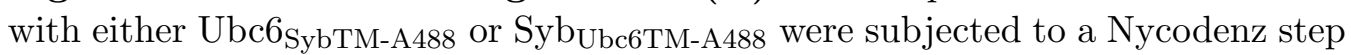
gradient. After ultracentrifugation, the gradient was fractionated and analyzed by SDS-PAGE and Coomassie Blue staining (top) and fluorescence scanning (bot-

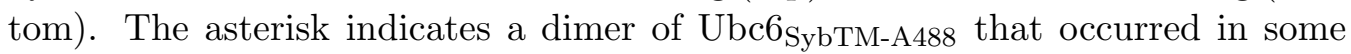
sortase mediated labeling reactions of this construct. (continued) 
Figure A6 (continued): (B) Affinity-purification of Doa10-SBP liposomes. Liposomes containing Doa10 and the indicated Ubc6/Syb chimera were immobilized to streptavidin magnetic beads and eluted with biotin. Input (I), unbound (U) and elution (E) fractions were analyzed by SDS-PAGE and by either Western Blot using an anti-SBP antibody (Doa10-SBP), or fluorescence scanning (Ubc6 chimera). As an indication of the specificity of the pull-down, we also tested for binding of liposomes lacking Doa10, and for co-purification of Ubc6 liposomes, when Doa10SBP and Ubc6 were reconstituted in separate liposome sets. Numbers at the bottom indicate the percentage of Ubc6 in the unbound fraction. (C) Liposomes containing t-SNARE, Ubc6 $6_{\text {SybTM }}$ and Ubc6, either labeled on Ubc6 $6_{\text {SybTM }}$ or Ubc6 were subjected to a Nycodenz step gradient. After ultracentrifugation, the gradient was fractionated and analyzed by SDS-PAGE and Coomassie Blue staining (top) and fluorescence scanning (bottom). The asterisk indicates a dimer of Ubc6 $6_{\text {SybTM-A } 488}$ that occurred in some sortase mediated labeling reactions of this construct. (D) Time course of E3-independent autoubiquitination of Ubc6 and Ubc6 $6_{\text {SybTM. }}$. Liposomes containing the indicated Ubc6 variants $(100 \mathrm{nM})$ were incubated with $100 \mathrm{nM} \mathrm{E1}, 120 \mu \mathrm{M}$ ubiquitin, and $2.5 \mathrm{mM}$ ATP. For each condition a 60 min sample of a reaction lacking ATP was analyzed. Samples were analyzed by SDS-PAGE and fluorescence scanning. (E) Quantification (mean and SD) of unmodified Ubc6 or Ubc6 ${ }_{\text {SybTM }}$ from three experiments as in (D). (F) Quantification (mean and SD) of unmodified Ubc6 or Ubc6 SybTM $_{\text {from three }}$ experiments as in Figure 3.6 E. (G) Liposomes with t-SNARE co-reconstituted with either Ubc6 $6_{\text {SybTM }}$ or Ub-Ubc6 $6_{\mathrm{C} 87 \mathrm{~A} / \text { /SybTM }}$ were subjected to a Nycodenz step gradient. After ultracentrifugation, the gradient was fractionated and analyzed by SDS-PAGE and Coomassie Blue staining (top) and fluorescence scanning (bottom). 
A

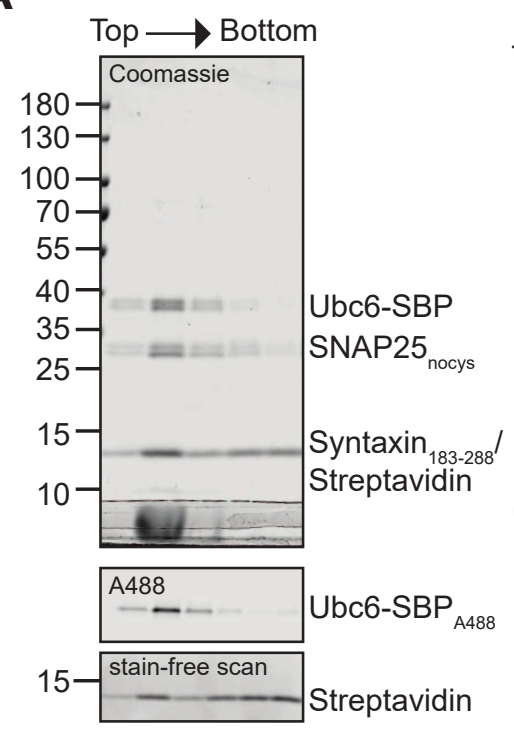

$\mathbf{E}$

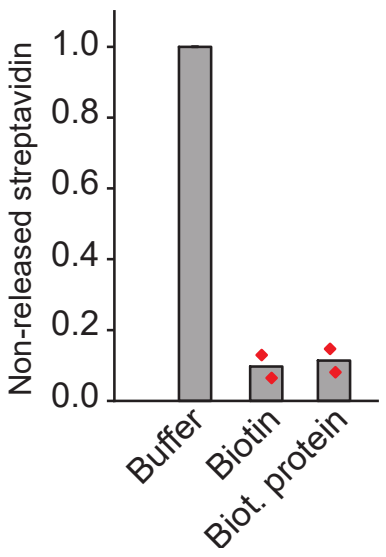

B

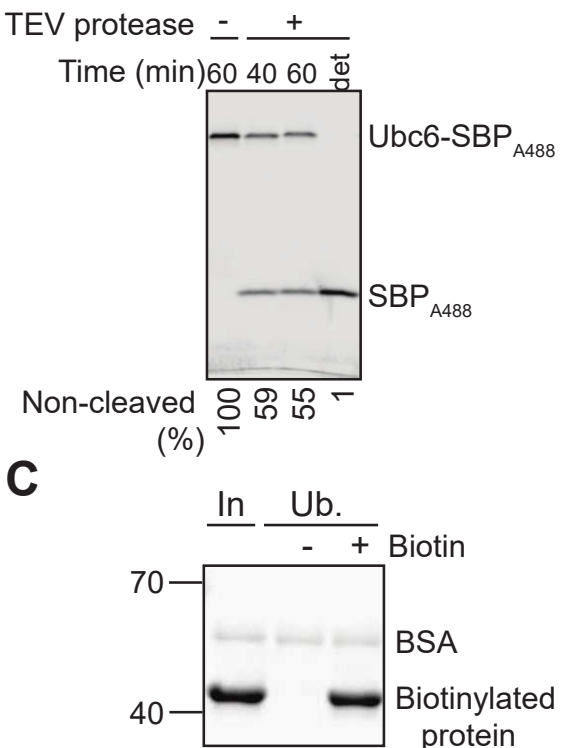

$\mathbf{F}$

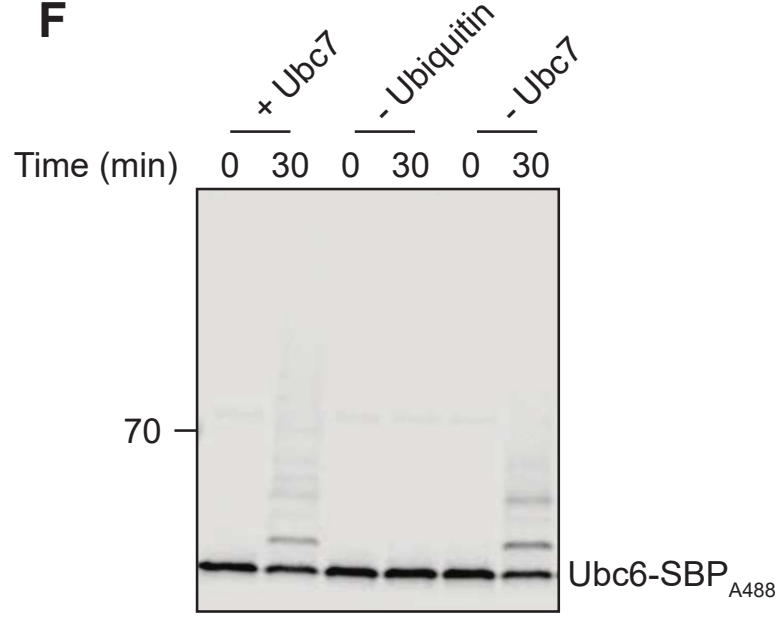

Figure A7: Related to Figure 3.7. (A) Liposomes containing t-SNARE and Ubc6-SBP $\mathrm{A}_{488}$ in complex with streptavidin were subjected to a Nycodenz step gradient. After ultracentrifugation, the gradient was fractionated and analyzed by SDS-PAGE and Coomassie Blue staining (top), fluorescence scanning (middle), and using stain-free dye technology (bottom) to distinguish between streptavidin and Syntaxin, which is not visible with this technique. (B) The orientation of Ubc6-SBP ${ }_{\mathrm{A} 488}$ in liposomes was assessed by testing for accessibility of TEV-protease to the TEV-cleavage site between the Ubc6 TM anchor and the SBP-tag. Liposomes were incubated with buffer or TEV protease with or without TX-100 (det) for the indicated times. Samples were analyzed by SDS-PAGE and fluorescence scanning. Numbers at the bottom indicate the fraction of protein wrong-side out oriented. (continued) 
Figure A7 (continued): (C) Streptavidin affinity pulldown of biotinylated protein in the presence and absence of biotin showing complete biotinylation of the protein. Samples of Input (In) and unbound (Ub) fractions were analyzed by SDSPAGE and scanning of the gel using stain-free dye technology. (D) Liposomes containing Ubc6-SBP 4488 were incubated with streptavidin and buffer / biotin / biotinylated protein, and subjected to a Nycodenz step gradient. After ultracentrifugation, the top fraction was analyzed by SDS-PAGE and the gel scanned using stain-free dye technology to visualize streptavidin. Ubc6 gives a strong signal here, because of the fluorescent dye that is also detected with the scanner used. (E) Quantification of co-floating streptavidin from two experiments as in (D). Streptavidin signal was first normalized to Ubc6-SBP signal and then normalized to the signal in buffer only control. (F) Ubiquitination of Ubc6-SBP $\mathrm{A}_{488}$. Samples from ubiquitination reaction from experiments as in Figure 3.7 E were analyzed by SDS-PAGE and fluorescence scanning. 


\section{Optimization of SNARE-mediated fusion assay}

This thesis provides an approach to co-reconstitute membrane proteins by SNAREmediated fusion which is introduced in Chapter 3. Ubc6 was hereby co-reconstituted with Doa10 and Cue1. Establishing this method required the optimization of multiple parameters which was focused on two major requirements for the fusion system. First, the generated fused liposomes should contain a stable lipid bilayer. The lipid bilayer separates two different environments, the liposome lumen representing the ER-lumen and the outside aqueous phase representing the cytosol. To maintain those different environments, it is important that the lipid bilayer does not allow passage or leakage of molecules such as proteins, and thus is stable. This aspect is also of importance for the fluorescence-quenching assay presented in Chapter 3. An antibody against the AlexaFluor488 label was hereby used to quench the fluorescence of carboxy-terminally labeled Ubc6 $6_{4488}$. Upon spontaneous retrotranslocation of Ubc6, the antibody gets access to the dye and quenches its fluorescence. This assay was used to identify the retrotranslocase activity of Doa10. As the experimental setup relies on the antibody only being present on the outside, and not having access to the liposome lumen, it is important that the lipid bilayer does not allow passage of the antibody into the liposome lumen and thus is stable. Using this fluorescence-quenching assay as a readout, I have tested if liposomes are stable after fusion. This readout is a useful tool to address this question, as in the absence of Doa10, the antibody should have access only to wrongly oriented Ubc6 $6_{\mathrm{A} 488}$.

As a second requirement for the reconstituted fusion system, the fluorescent label attached to the carboxy-terminus of Ubc6 should not interfere with the behaviour of Ubc6. With this in mind, I have compared different fluorescent dyes for carboxyterminal labeling of Ubc6 with a Ubc6-variant that is labeled at its cytosolic side and thus does not contain any C-terminal label. I then tested if Doa10 retrotranslocates these Ubc6 variants. In Chapter 3, three assays are shown to measure spontaneous retrotranslocation by Doa10. Both the fluorescence-quenching assay as well as the protease protection assay are not useful to study retrotranslocation of differently labeled Ubc6-variants as they rely on the presence of a carboxy-terminal label at Ubc6. In contrast, the chaperone-binding assay, which is based on Get3 binding to released Ubc6, is a suitable method and was therefore used to examine the effect of different 
fluorescent labels on release of Ubc6.

In the following sections, the results of these two optimization strategies are presented.

\subsection{Stability of liposomes after SNARE-mediated fusion}

Using the fluorescence quenching as a readout, I have identified and optimized two main parameters for liposome stability. First, the concentration of reconstituted membrane proteins plays a role and I thus optimized the lipid to protein ratio for reconstitution of SNAREs. Moreover, I have tested the effect of reconstituting multipass transmembrane proteins, to be able to reconstitute Doa10 without affecting liposome stability. I have moreover compared different purification strategies for Doa10 and tested for the compatibility with the SNARE-mediated fusion system.

\subsubsection{Optimization of lipid to protein ratio}

To assess the effect of protein concentration in liposomes, I prepared Ubc6 $6_{\mathrm{A} 488}$, tSNARE liposomes and Syb liposomes lacking Doa10 and incubated them to allow for SNARE-mediated fusion. I then measured the A488 fluorescence upon addition of anti-A488 antibody (anti-A488). If liposomes are stable, the antibody should only bind to wrongly oriented Ubc $6_{\mathrm{A} 488}$ and therefore quench about $50 \%$ of the total fluorescence. However, when a lipid:t-SNARE ratio of 400 is used (dashed black lines), the fluorescence decreases quickly to $45 \%$ but subsequently decreases further (Figure 4.1A and $4.1 \mathrm{~B}$ ). This further slow decrease could be due to antibody slowly accessing the liposome lumen and thus indicates liposome instability. Slight instability can be also observed when fusion is inhibited (Figure 4.1C and 4.1D). In contrast, at lower SNARE concentrations, the signal stays stable over $30 \mathrm{~min}$. At a lipid:t-SNARE ratio of 1000 or 2000 (dashed blue and red lines), the signal stays stable at both tested lipid:Syb ratios (1000 and 2000) (Figure 4.1).

To test if these SNARE concentrations still ensure high co-reconstitution efficiency after fusion, I fused $\mathrm{Ubc}_{\mathrm{A} 488}$, t-SNARE liposomes with Doa10, Syb liposomes and again measured the A488 fluorescence upon anti-A488 addition. As Doa10-mediated release is a readout for co-reconstitution of Ubc6 with Doa10, I tested for Doa10-mediated release at different SNARE-concentrations. Whereas a lipid:t-SNARE concentration of 2000 compromises the release efficiency (red solid lines), a lipid:t-SNARE concentration of 1000 leads to complete release efficiency at both tested Syb concentrations (blue solid lines), indicating co-reconstitution (Figure 4.1). SNARE concentrations 
that led to complete release efficiency in the presence of Doa10 and no instability in the absence of Doa10 were chosen for all experiments (lipid:tSNARE 1000, lipid:Syb 2000). Importantly, the accessibility of anti-A488 to Ubc6 $\mathrm{A}_{488}$ in the presence of Doa10 is not due to leakage but due the exposure of the carboxy-terminus of Ubc6 to the outside, as the signal stays stable when a control protein is co-reconstituted with Doa10

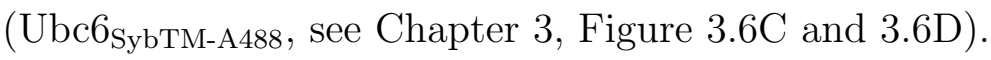




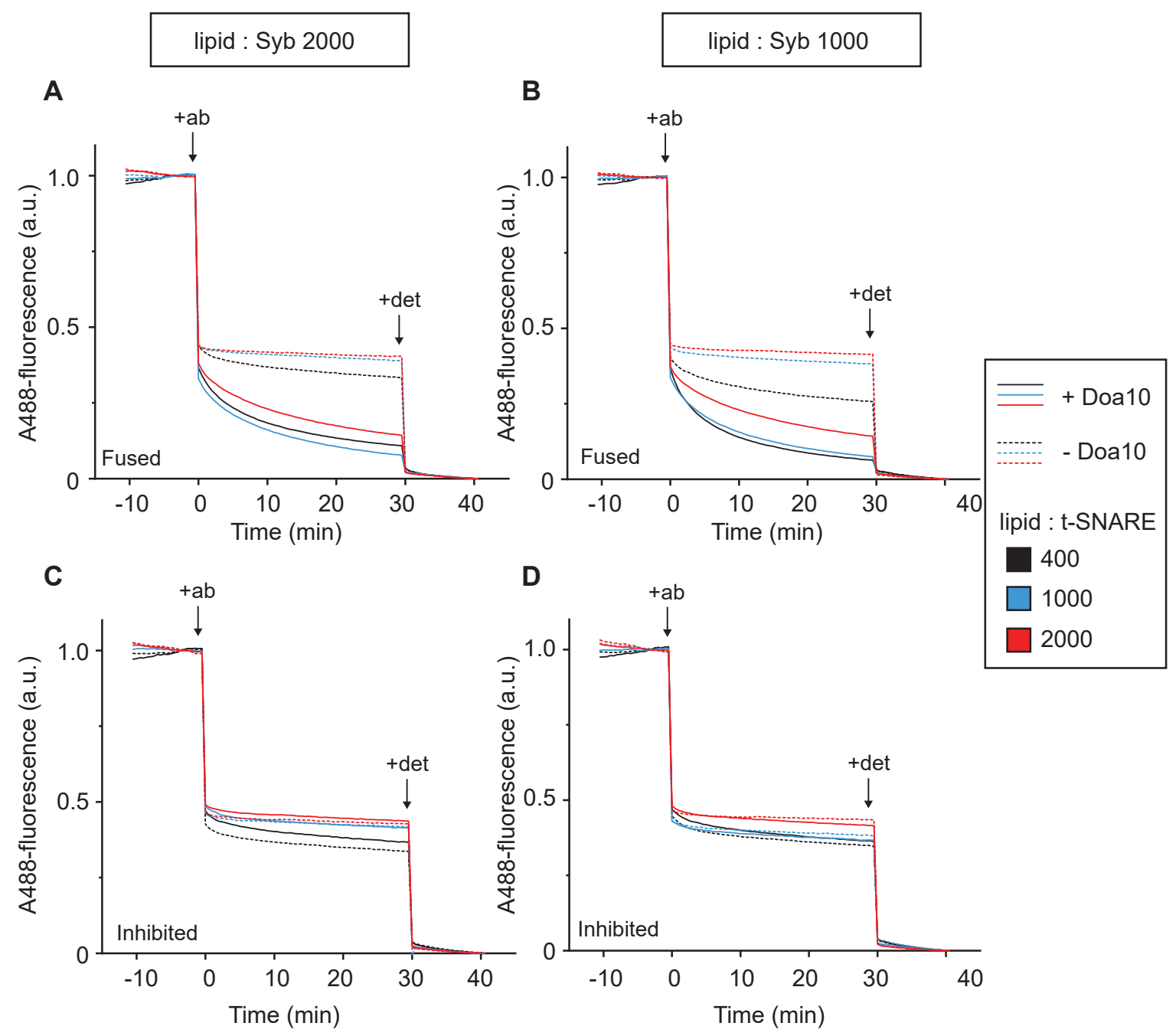

Figure 4.1: Titration of SNARE-concentration for SNARE-mediated fusion assay. Liposomes containing Ubc6 $6_{4} 488$ and t-SNARE were incubated with Syb liposomes containing (solid) or lacking (dashed lines) Doa10 (lipid:Doa10 ratio of 2000) to allow for SNARE-mediated fusion. The A488 fluorescence upon incubation with anti-A488 antibody ( + ab) was measured. Different lipid:protein ratios for reconstitution of SNAREs were tested. Graphs show results for reconstitution of Syb using a lipid:protein ratio of 2000 (A, C) and 1000 (B, D). Lipid:t-SNARE ratios of 400 (black), 1000 (blue) and 2000 (red) were tested. Where indicated, fusion was inhibited with a soluble Syb fragment (C, D). Samples without Doa10 are indicated with a dashed line. Where indicated, detergent was added (+det) to solubilize the liposomes.

These results show that the concentration of reconstituted proteins influences the stability of liposomes. Doa10 contains a large TM domain containing 14 TM segments. The presence of Doa10 could therefore also affect the stability of liposomes due to its transmembrane domain. I tested next the effect of the presence of multiple transmem- 
brane segments on liposome stability using the fluorescence-quenching assay again. To be able to distinguish between liposome instability and release of Ubc6, a multipass protein was used as a control protein which does not interact with Ubc6. $\mathrm{TF}_{\mathrm{o}} \mathrm{F}_{1}$ ATP synthase is a multi-subunit complex that consists of a soluble $\mathrm{F}_{1}$ region (subunits $\alpha_{3} \beta_{3} \gamma \delta \epsilon$ ) and a membrane anchored $\mathrm{F}_{\mathrm{o}}$ region (subunits $\mathrm{ab}_{2} \mathrm{c}_{10}$ ). The c-subunits form a ring consisting of $20 \mathrm{TM}$ segments (Guo et al., 2019). I therefore chose the ATP synthase as a suitable multipass TM control protein to test the effect of the presence of multiple transmembrane segments on liposome stability.

I purified $\mathrm{F}_{\mathrm{o}} \mathrm{F}_{1}$ ATP synthase from thermophilic Bacillus sp. PS3 ( $\mathrm{TF}_{\mathrm{o}} \mathrm{F}_{1}$ ) as described previously (Suzuki et al., 2002; Schenck et al., 2009), (Figure S1). ATP synthase was subsequently co-reconstituted with Syb into liposomes. ATP synthase and Syb co-floated in a Nycodenz step gradient indicating that they were both reconstituted into liposomes (see Figure 4.2).

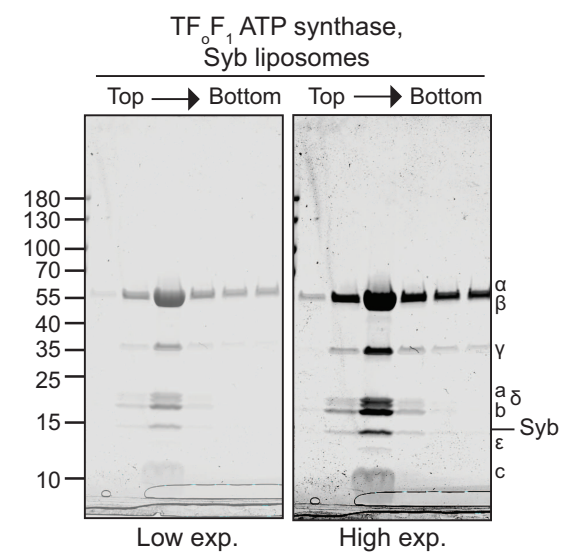

Figure 4.2: Co-reconstitution of $\mathrm{TF}_{\mathrm{o}} \mathrm{F}_{1}$ ATP synthase with Syb into liposomes. Liposomes containing $\mathrm{TF}_{\mathrm{o}} \mathrm{F}_{1}$ ATP synthase and Syb were floated in a Nycodenz step gradient and the flotation fractions analyzed by SDS-PAGE and Coomassie blue staining. Low (left) and high (right) exposure of the Coomassie staining are shown.

As observed for SNAREs, high concentrations of ATP synthase led to instability after fusion as measured again by fluorescence quenching upon anti-A488 addition (Figure 4.3). However, when ATP synthase is reconstituted at a lipid:protein ratio of 8000 , the A488 signal stays stable over time after an initial fast quenching of immediately accessible Ubc6 $\mathrm{A}_{488}$ indicating stable liposomes. When Doa10 is reconstituted with the same lipid:protein ratio (8000), Ubc6 $\mathrm{A}_{\mathrm{A} 88}$ is fully accessible over time to anti-A488. Assuming that the distribution of Doa10 and ATP synthase in liposomes is compara- 
ble at the same lipid:protein ratio, this shows that liposome instability can be avoided without compromising co-reconstitution efficiency. Concluding, a lipid:protein ratio of 8000 is therefore optimal for reconstitution of multipass TM proteins like Doa10.

A

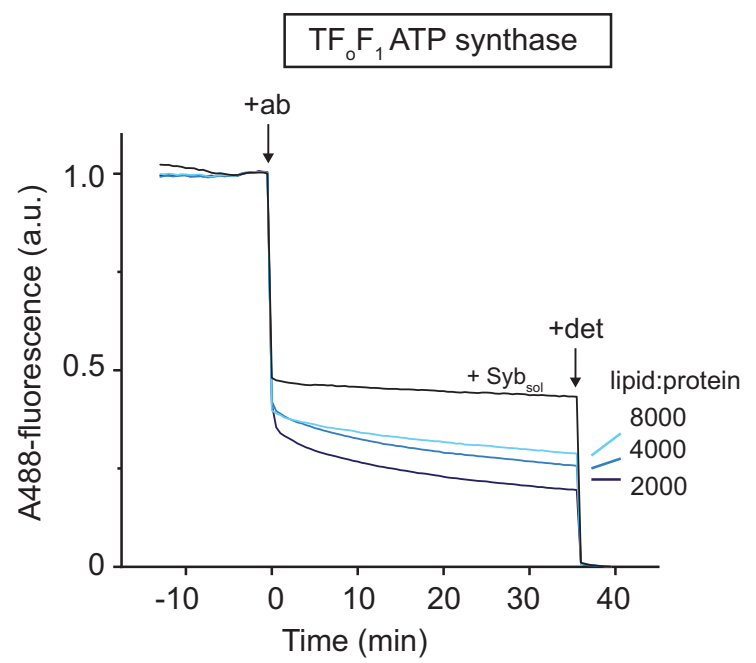

B

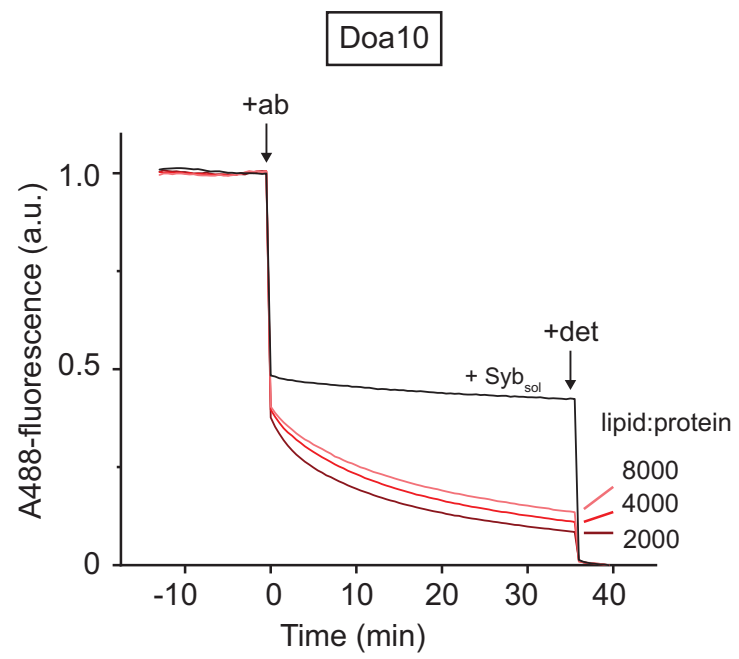

Figure 4.3: Titration of ATP synthase and Doa10 for SNARE-mediated fusion assay. Different lipid:protein ratios (8000, 4000 and 2000) were used for reconstitution of Doa10 or ATP synthase into liposomes to test the influence of protein concentration on liposome stability. ATP synthase (A) or Doa10 (B) were co-reconstituted with Syb and those liposomes fused with liposomes containing Ubc6 ${ }_{\mathrm{A} 488}$ and t-SNARE. The A488 fluorescence upon incubation with anti-A488 antibody $(+\mathrm{ab})$ was measured as a readout for liposome stability and co-reconstitution efficiency. Where indicated, detergent (+det) was added to solubilize the liposomes.)

\subsubsection{Optimization of purification method for Doa10}

I also tested which purification method is most suitable for Doa10 for further reconstitution for the SNARE-mediated fusion assay. I tested two methods to further purify Doa10 after streptavidin affinity chromatography. I compared size-exclusion chromatography (SEC) and sucrose density gradient ultracentrifugation. Whereas the detergent concentration is constant during SEC, density gradients can be prepared with a detergent gradient (with the highest detergent concentration at the top). This has the advantage that excess detergent can be removed during the centrifugation. Doa10 was purified using the detergent GDN and further purified via SEC (150 $\mu$ M GDN) or sucrose density gradient (gradient of 0 to $100 \mu \mathrm{M}$ GDN). The fusion characteristics 
were again assessed by fluorescence quenching upon anti-A488 addition.

When gradient-purified Doa10 was reconstituted, the fluorescence signal decreased slowly over time after the initial fast quenching of wrongly oriented Ubc6 (Figure 4.4). However, when liposomes containing SEC-purified Doa10 were fused with Ubc6 $6_{\text {A488 }}$ liposomes, the extent of initial fluorescence quenching was larger indicating instability of liposomes. This occurred only at a low lipid:Doa10 ratio (2000). At a higher ratio (5000), no difference to gradient-purified Doa10 was observed. To avoid detergent mediated effects, gradient-purified Doa10 was used for functional assays.
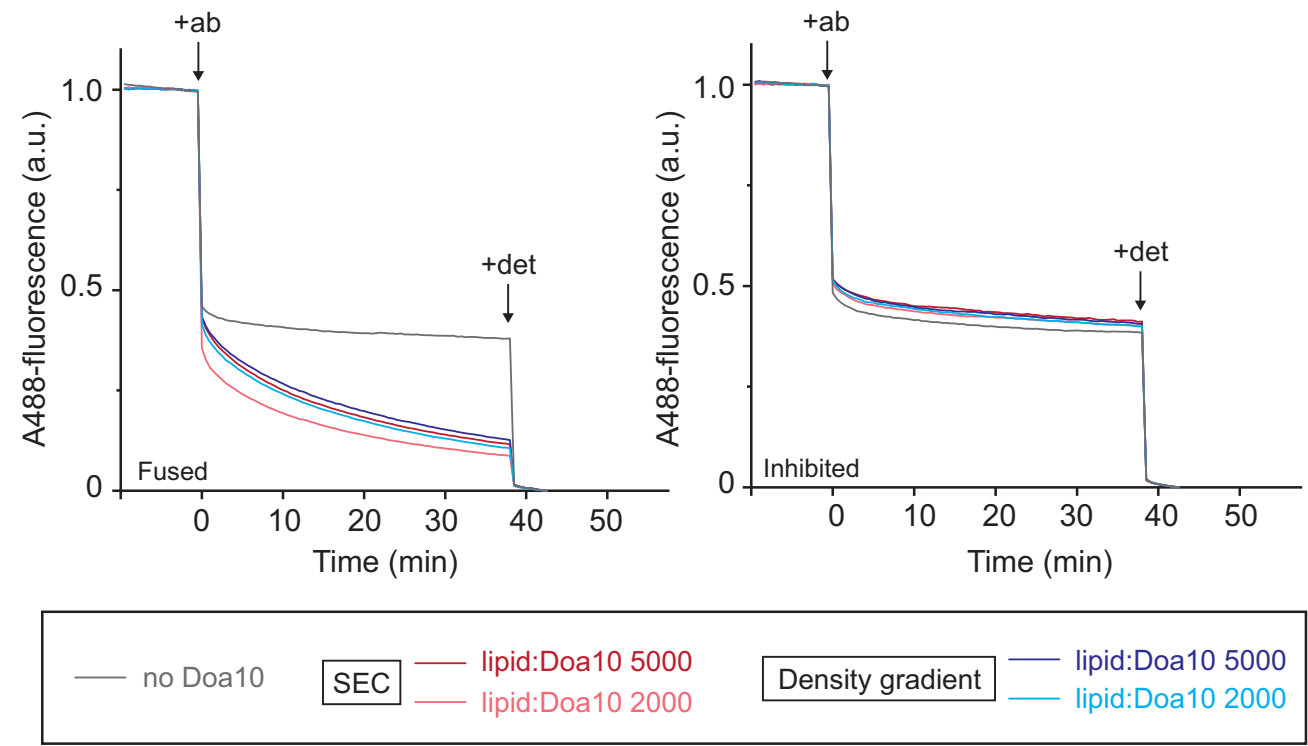

Figure 4.4: Testing SEC and sucrose density gradient centrifugation as purification method for Doa10. Liposomes containing Doa10 purified by SEC (150 $\mu \mathrm{M}$ GDN) or density gradient centrifugation (0 to $100 \mu \mathrm{M}$ GDN gradient) and Syb were fused with liposomes containing Ubc6 $6_{488}$ and t-SNARE and the A488 fluorescence upon incubation with anti-A488 antibody ( $+\mathrm{ab})$ was measured. Where indicated, detergent (+det) was added to solubilize the liposomes. Two different lipid:Doa10 ratios (5000 and 2000) were tested. Fusion was inhibited where indicated with a soluble Syb fragment.

Concluding, these results show that reconstituted membrane proteins affect liposome stability. This effect can be avoided by using high lipid:protein ratios for reconstitution. 


\subsection{Identification of a suitable fluorescent label for Ubc6}

Next, I tested if carboxy-terminal fluorescent labels attached to Ubc6 affect its behaviour. To do so, Ubc6 was carboxy-terminally labeled by sortase-mediated transpeptidation (Popp et al., 2009). Different Ubc6 variants were thereby generated that were labeled with the fluorescent dyes Dylight680 (DL680), DyLight800 (DL800) or AlexaFluor488 (A488). The behaviour of these Ubc6 variants was compared to a Ubc6 construct that does not contain any carboxy-terminal label. It instead is labeled at the cytosolic side at its active-site cysteine with A488 via maleimide chemistry $\left(\mathrm{Ubc}_{\mathrm{C} 87-\mathrm{A} 488}\right)$. I then tested if Doa10 releases these Ubc6 variants.

I co-reconstituted these Ubc6 variants with t-SNARE and fused them to Doa10, Syb liposomes. I then tested for Doa10-mediated release of Ubc6 by incubation with the chaperone Get3, as in Figure 3.4A and 3.4B. After incubation with Get3, liposomes were immobilized via co-reconstituted biotinylated lipids and samples of input and supernatant analyzed by SDS-PAGE. About $45 \%$ of $\mathrm{Ubc}_{\mathrm{C} 87-\mathrm{A} 488}$ were detected in the supernatant (Figure $4.5 \mathrm{C}$ ). A similar fraction of Ubc6 $6_{\mathrm{DL} 680}$ and slightly more Ubc6 $6_{\mathrm{A} 488}$ were released (Figure $4.5 \mathrm{~B}$ and $4.5 \mathrm{D}$ ). In contrast, less Ubc6 $\mathrm{DL}_{800}$ (about $30 \%$ ) was detected in the supernatant (Figure 4.5A). Concluding, a C-terminal fluorescent label on Ubc6 can interfere with the release assay, either by interfering with Doa10-mediated release or with binding of Get3 to the TM anchor of Ubc6. Different fluorescent labels have thereby different effects. Whereas C-terminal labeling of Ubc6 with DL680 does not influence the assay, as its behaviour is comparable to Ubc6 labeled at the cytosolic side, labeling of Ubc6 with A488 increases and labeling with DL800 decreases the amount of Ubc6 detected in the supernatant.

Interestingly, the release efficiency correlates with the molecular weight of the Cterminal label. A488 is the smallest label I tested $(721 \mathrm{~g} / \mathrm{mol})$, whereas DyLight800 is larger $(1075 \mathrm{~g} / \mathrm{mol}$ ) (see Table 4.1 ). This, probably combined with differences in hydrophobicity, may lead to the observed differences in release efficiency. For experiments, I have used Ubc6 labeled with A488 or DL680, unless otherwise indicated.

Table 4.1: Molecular weight of A488, DL680 and DL800 maleimide. Values for molecular weight obtained from Thermo Fisher Scientific.

\begin{tabular}{l|c} 
Dye & Molecular weight $(\mathrm{g} / \mathbf{m o l})$ \\
\hline \hline AlexaFluor488 C5 maleimide & 721 \\
DyLight680 maleimide & 972 \\
DyLight800 maleimide & 1075
\end{tabular}


A
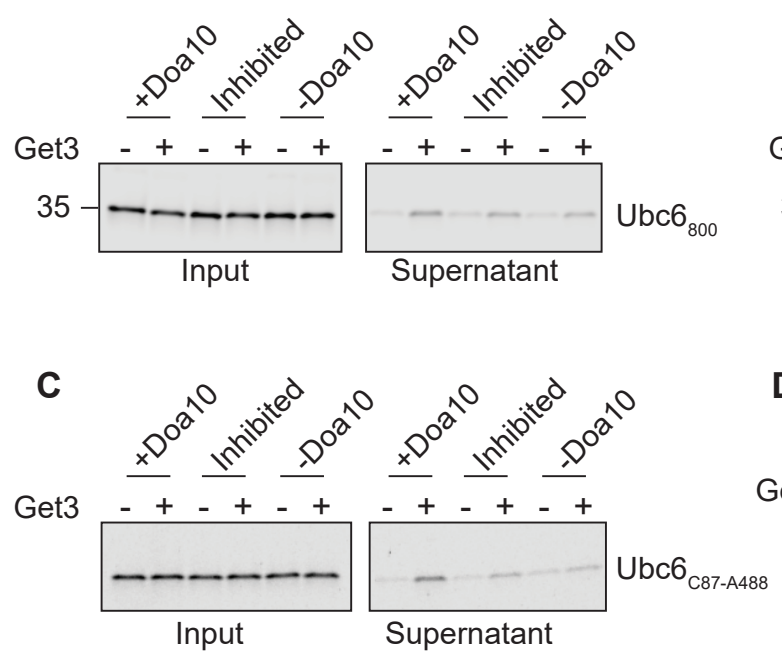

E
B
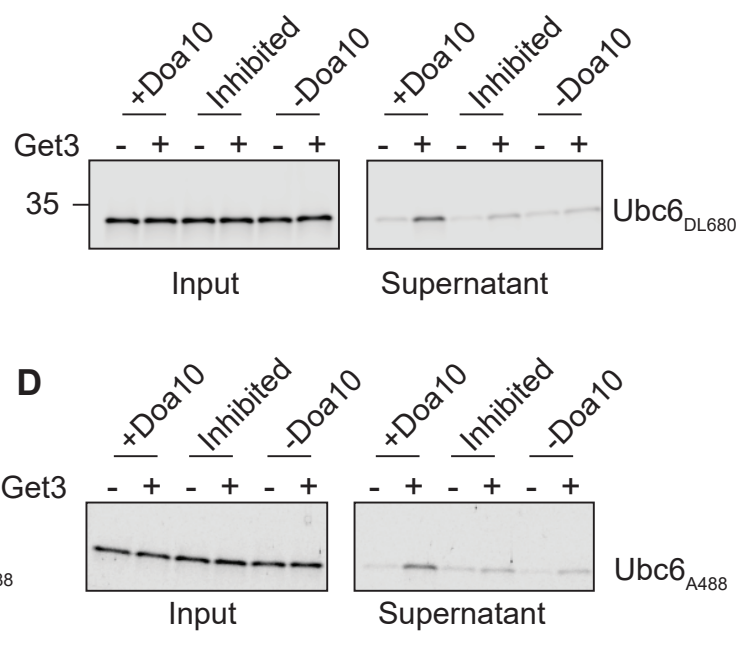

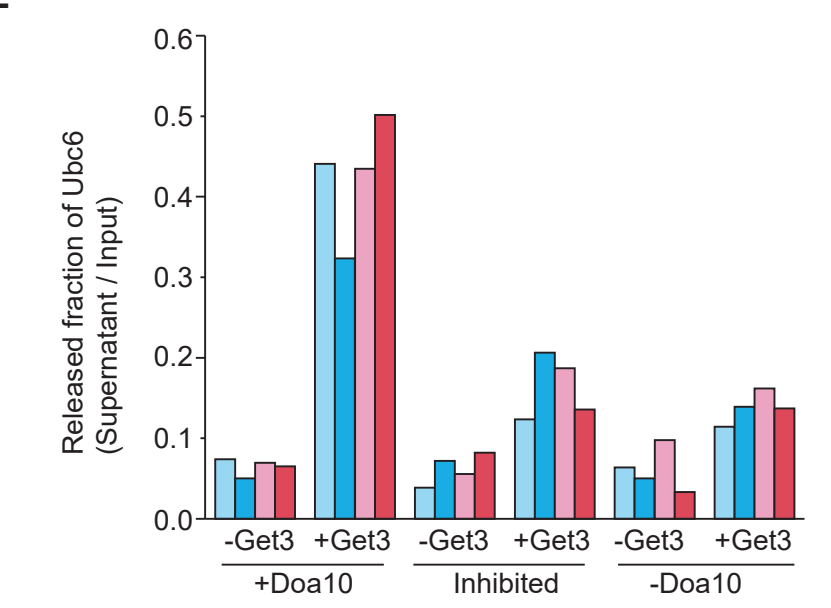

Figure 4.5: Influence of the C-terminal fluorescent label of Ubc6 on substrate behaviour. Different Ubc6 variants $\left(\mathrm{Ubc}_{\mathrm{DL} 680}\right.$, Ubc6 ${ }_{\mathrm{DL} 800}, \mathrm{Ubc}_{\mathrm{A} 488}$, Ubc6 $\left.{ }_{\mathrm{C} 87-\mathrm{A} 488}\right)$ were co-reconstituted with t-SNARE and the liposomes fused with liposomes containing Doa10 and Syb. Liposomes were subsequently incubated with Get3 (f.c. $10 \mu \mathrm{M}$ Get3, $40 \mathrm{nM}$ Doa10, $100 \mathrm{nM}$ Ubc6). Liposomes were immobilized via co-reconstituted biotinylated lipids. Samples of the supernatant and input were analyzed by SDS-PAGE and fluorescence scanning, for (A) Ubc6 DL680, $_{\text {, }}$ (B) $\mathrm{Ubc}_{\mathrm{DL} 800}$, (C) $\mathrm{Ubc}_{\mathrm{C} 87-\mathrm{A} 488}$, (D) Ubc6 ${ }_{\mathrm{A} 488}$. (E) Quantification of (A-D). Fraction of Ubc6 in supernatant relative to input was quantified. 


\section{Identification of structural elements important for Doa10 function}

Doa10 has a TM region comprising 14 TM segments which contains the conserved TD-domain (TM segments 5-7). Studies indicate that this TM region is functionally important. First, mutation of conserved residues in TM segment 5 affects degradation of ERAD substrates (Kreft and Hochstrasser, 2011). Moreover, it has been shown that Doa10 recognizes an intramembrane degron in the substrate Sbh2 (Habeck et al., 2015). The Doa10 TM region might be important for recognition of such a membranelocalized degradation signal. Moreover, it has been hypothesized that the TM domain of Doa10 might have a role in substrate retrotranslocation.

Our established reconstituted system provides some insight into the role of the TM region of Doa10. It recapitulates recognition, ubiquitination and retrotranslocation of Ubc6. In this system, a Doa10 truncation containing only the RING domain and the TM segments 1 and 2 (Doa10 $1-468$ ) is less efficient in Ubc6 ubiquitination compared to full-length Doa10 and is not able to act as a retrotranslocase for Ubc6 (see Figure 3.5). These results indicate that the TM region of Doa10 is important for ubiquitination as well as retrotranslocation of Ubc6. The TD-domain might thereby play a role.

Kreft and Hochstrasser identified Doa10 variants that contain a mutation in the TD-domain and affect degradation of Ubc6 (Kreft and Hochstrasser, 2011). I therefore wanted to test how these Doa10 mutants behave in our reconstituted system. I also sought to identify interaction sites between Ubc6 and Doa10 and therefore optimized a protocol for site-specific photocrosslinking of Ubc6 with Doa10.

\subsection{Characterization of Doa10 variants containing mutations in the TD domain}

Different Doa10 TD mutants have been described that impair degradation kinetics of Ubc6. Doa10 contains a conserved glutamate residue at position 633. Interestingly, mutating this glutamate to glutamine (E633Q) results in enhanced degradation of Ubc6. In contrast, the degradation of Ubc6 is slowed down when the charge is preserved (E633D). Moreover, these mutations of E633 specifically affect degradation of Ubc6, but not of the soluble substrate Deg1-Ura3 or the membrane-bound Deg1-Vma12- 
Ura3 (Kreft and Hochstrasser, 2011).

Impaired degradation kinetics of Ubc6 upon mutation of Doa10 can be the result of a change in Doa10-mediated ubiquitination of Ubc6 in multiple ways. First, interaction of Ubc6 with the ubiquitin ligase can be affected and thus affect substrate recognition as well as ubiquitination. Moreover, during ubiquitination of Ubc6, Doa10 does not only have to bind to substrate, but also coordinate the E2 enzyme Ubc7 with its cofactor Cue1. Altered coordination of the E2 enzyme by the ubiquitin ligase might affect substrate ubiquitination and thus degradation kinetics. In addition, mutation of Doa10 might affect its interaction with other substrates and thus indirectly affect degradation of Ubc6. I directly tested the behaviour of these Doa10 mutants in our established reconstituted system. I first analyzed their behaviour in ubiquitination of Ubc6.

Interestingly, Ubc6 is ubiquitinated by these Doa10 mutants with similar kinetics compared to wildtype Doa10 (Figure 5.1A, B). The ubiquitin chain lengths created only show minor differences. In the presence of Doa10 ${ }_{\mathrm{E} 633 \mathrm{D}}$ or Doa10 $\mathrm{E} 633 \mathrm{Q}$ slightly more monoubiquitinated and concomitantly less polyubiquitinated Ubc6 is generated, compared to Doa10 WT (Figure 5.1C). These small differences might not necessarily be due to the mutation itself, but due to other factors such as small differences in protein concentration, purity or in general variability between different preparations of Doa10. Concluding, I do not observe major differences in Ubc6 ubiquitination in the presence of the Doa10 $\mathrm{E} 633$ mutants. 
A

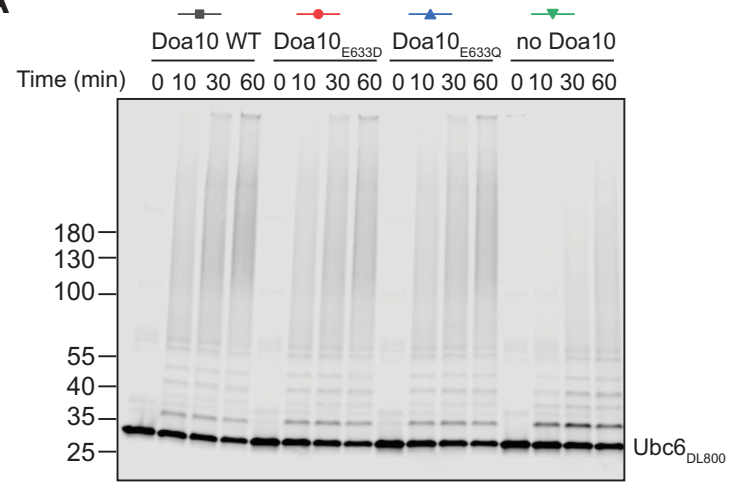

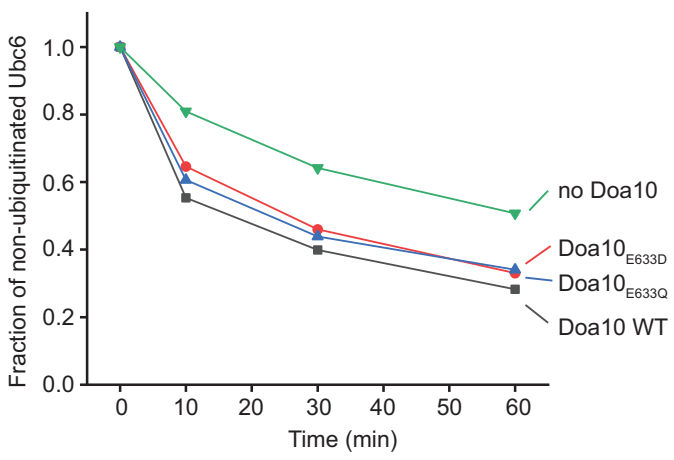

\section{C}

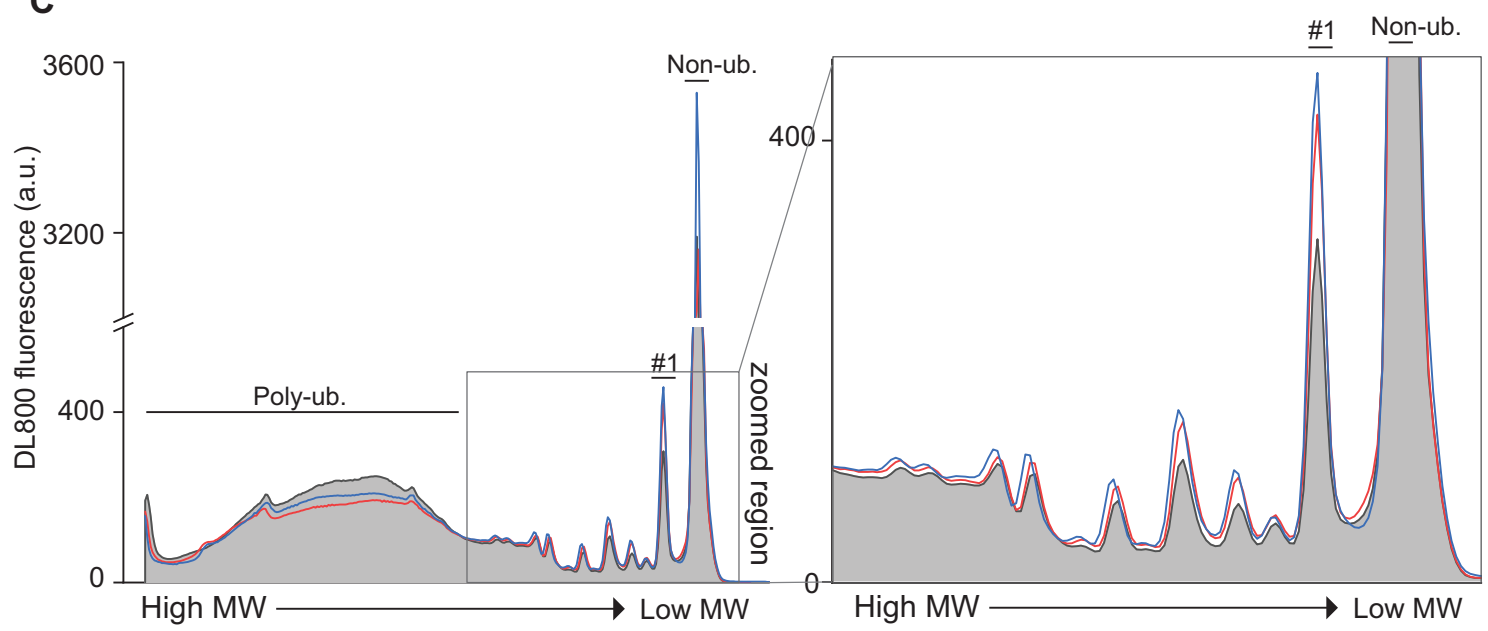

Figure 5.1: Ubiquitination of Ubc6 in the presence of Doa10 $\mathrm{E}_{633 \mathrm{D}}$ and Doa10 6 633Q. Liposomes containing Ubc6 $6_{\mathrm{DL} 800}$ and t-SNARE were fused with liposomes containing Doa10, Cue1 and Syb (lipid:protein ratios of 5000, 2000 and 2000, respectively) and subsequently incubated with ubiquitination machinery (f.c. $0.1 \mu \mathrm{M}$ Uba1, $0.5 \mu \mathrm{M}$ Ubc7, $60 \mu \mathrm{M}$ ubiquitin, $0.2 \mu \mathrm{M}$ Ubc6, $80 \mathrm{nM}$ Doa10, $0.2 \mu \mathrm{M}$ Cue1). (A) Analysis of samples by SDS-PAGE and fluorescence scanning. (B) Quantification of Ubc6 turnover. (C) Analysis of ubiquitin chain length (60 min timepoint).

Different degradation kinetics in vivo could not only be a result of affected ubiquitination, but also different extraction efficiencies. As I observed that Doa10 is a retrotranslocase for Ubc6 (Chapter 3), I wanted to therefore test next if the described Doa10 mutants are capable of retrotranslocating Ubc6 in our reconstituted system. Besides Doa10 $\mathrm{E} 633 \mathrm{D}_{\mathrm{D}}$ and Doa10 $\mathrm{E} 633 \mathrm{Q}$, I also tested two other Doa10 mutants which affect degradation of Ubc6, but also the Doa10 substrate Deg1-Ura3 (Doa10 ${ }_{\mathrm{P} 638 \mathrm{~A}}$ G642A and Doa10 $\mathrm{G636 \textrm {R }}$, (Kreft and Hochstrasser, 2011)). Liposomes con- 
taining these Doa10 mutants or Doa10 WT and Syb were fused with liposomes containing $\mathrm{Ubc}_{\mathrm{A} 488}$ and t-SNARE. Doa10-mediated release was again measured by monitoring the A488-fluorescence upon anti-A488 addition. All tested Doa10 mutants released Ubc6 with similar kinetics compared to wildtype Doa10 (Figure 5.2).

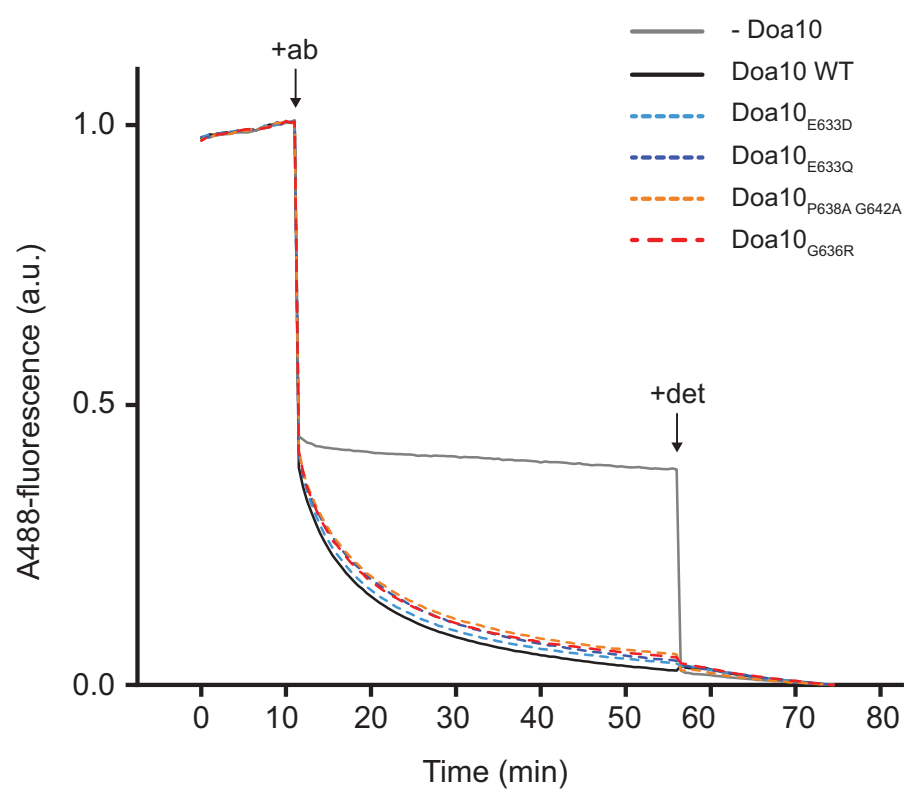

Figure 5.2: Release of Ubc6 by Doa10 TD mutants. Liposomes containing Ubc6 ${ }_{\mathrm{A} 488}$ and t-SNARE were fused with liposomes containing Doa10 (WT, E633D, E633Q, P638A G642A, or G636R) and Syb, and the A488 fluorescence was subsequently measured upon anti-A488 addition (+ab). Where indicated, detergent $(+$ det $)$ was added to solubilize liposomes.

Concluding, I do not observe major differences in ubiquitination or retrotranslocation of Ubc6 in the presence of described Doa10 mutants harboring a mutation in the TD-domain.

\subsection{Identification of interaction sites of Ubc6 with Doa10 by photocrosslinking}

Although mutational analyses of the TD-domain of Doa10 in vivo identified potential interaction sites of Doa10 with Ubc6 (Kreft and Hochstrasser, 2011), the interaction site of Ubc6 and Doa10 is not defined. Moreover, it is unclear if Ubc6 interacts with Doa10 differently in its two states, as E2 enzyme and substrate. Kreft and Hochstrasser (2011) raised the possibility that those interaction sites are distinct. To be able to 
answer these questions I wanted to identify interaction sites between the TM anchor of Ubc6 and Doa10 by site-specific photocrosslinking.

To establish a system that leads to crosslinking of the TM segments of Ubc6 and Doa10, a photocrosslinker was introduced at different positions of the Ubc6 TM anchor by genetic code expansion. I used a crosslinker containing a benzophenone as it is a quite reactive and chemically stable crosslinker that when excited with a wavelength of $360 \mathrm{~nm}$ reacts with $\mathrm{C}-\mathrm{H}$ bonds and thus is protein sequence unspecific (Galardy et al., 1973; Chin et al., 2002).

To incorporate the unnatural amino acid p-Benzoylphenylalanine (BpA) into Ubc6, the amber STOP codon was introduced at different positions of the Ubc6 TM anchor (Figure 5.3A). By co-expression of an orthogonal aminoacyl tRNA synthetase/tRNA pair (Chin et al., 2002) and supplying BpA in the expression medium, BpA was incorporated at sites of Ubc6 containing the amber STOP codon. I first tested the expression of Ubc6 in the presence and absence of BpA and observed that expression of Ubc6 was specific for the presence of BpA (Figure S2A). I confirmed that the band at $50 \mathrm{kDa}$ corresponds to His ${ }_{14}$-SUMO-Ubc6 by adding the SUMO-protease Ulp1 to the lysate to cleave the $\mathrm{His}_{14}$-SUMO tag. I then purified the Ubc6 $6_{\mathrm{BpA}}$ variants by Ni-NTA affinity chromatography (Figure S2B) and directly used the Ulp1-eluted fractions for reconstitution and subsequent crosslinking.

Ubc6 $_{\mathrm{BpA}}$ variants were directly co-reconstituted with Doa10 purified in DMNG. When Ubc6 (WT), Doa10 liposomes are prepared using this direct co-reconstitution protocol, Ubc6 and Doa10 co-float in a Nycodenz step gradient and Ubc6 is efficiently ubiquitinated (Figure S3A and S3B). Liposomes containing different Ubc6 $6_{\mathrm{BPA}}$ variants and Doa10 were exposed to UV-light $(365 \mathrm{~nm})$ and the sample was subsequently enriched for Doa10-containing liposomes by pulldown via the SBP-tag of Doa10 using magnetic streptavidin beads. After elution with sample buffer, samples were analyzed by SDS-PAGE. In the presence of Ubc6 $6_{\mathrm{BpA}}$ variants, two crosslinked bands are visible (Figure 5.3B). Those crosslinks are specific to the presence of Ubc6 and UV-light (data not shown). When wildtype Ubc6 (no BpA incorporated) was co-reconstituted with Doa10 and exposed to UV-light, one crosslinked band appeared as well which had the same migration pattern as the lower crosslinked band of samples containing Ubc6 $6_{\mathrm{BpA}}$. Thus most likely, only the upper crosslinked band is BpA specific.

The upper, BpA-specific band was analyzed by mass spectrometry in collaboration with Iwan Parfentev (Laboratory of Prof. Henning Urlaub, Bioanalytical Mass Spectrometry Group, MPI for Biophysical Chemistry, Göttingen). However, we were not able to identify the crosslinked sites in Doa10 by mass spectrometry. Although the 
protein sequence of Doa10 and Ubc6 was well covered (for Doa10 above $89 \%$ and for Ubc6 above $86 \%$, see table S1), no spectra with high scores were identified. Concluding, whereas the established protocol for photocrosslinking results in detectable crosslinks of Ubc6 with Doa10, we were not able to use this technique for detection of interaction sites by mass spectrometry.

A

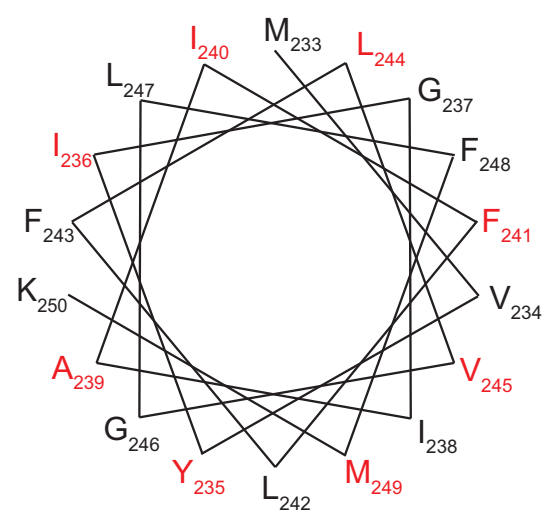

B

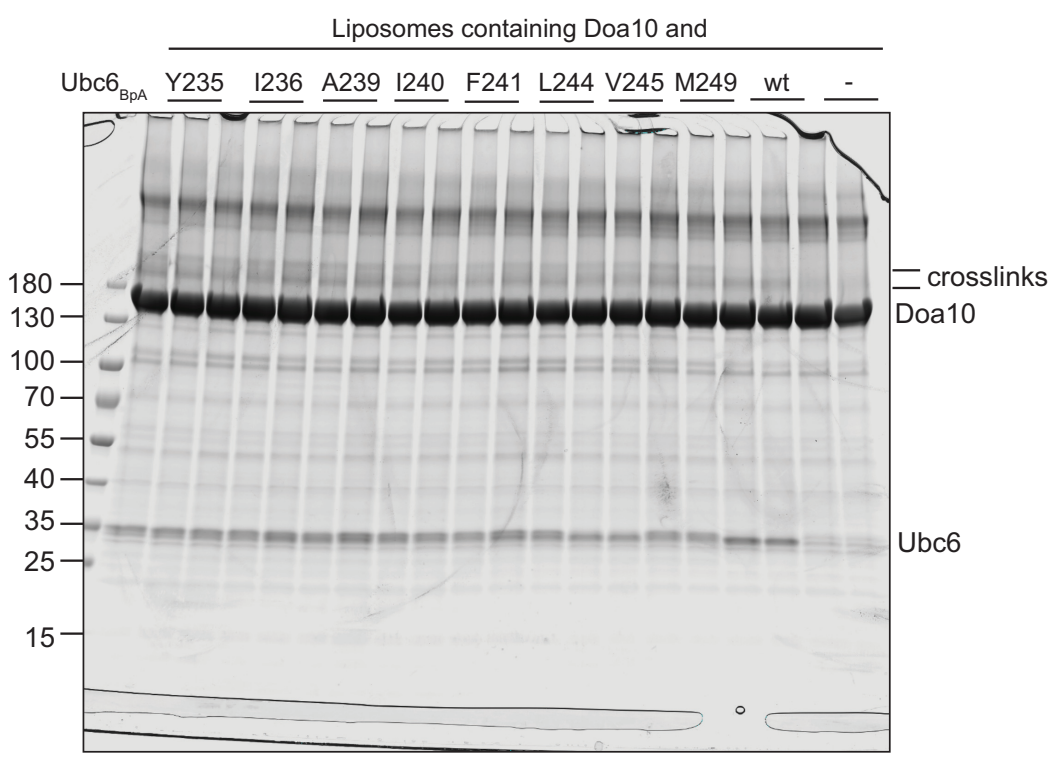

Figure 5.3: Crosslinking of Ubc6 $6_{\mathrm{BpA}}$ variants with Doa10. (A) Peptide wheel presentation of Ubc6 TM (residues 233-250, created with Emboss pepwheel tool). (B) SDS-PAGE analysis of photocrosslinked samples (Coomassie staining). Liposomes containing Ubc6 $6_{\mathrm{BpA}}$ variants and Doa10 were exposed to UV-light (365nm, 4 Joule) and subsequently the sample was enriched for Doa10-containing liposomes by pulldown via the SBP-tag of Doa10. Each sample was loaded in duplicates. The upper crosslinked bands were analyzed by mass spectrometry by Iwan Parfentev (MPI for Biophysical Chemistry, Göttingen). 
I next used this photocrosslinking approach as a readout for the interaction of the TM anchor of Ubc6 with Doa10. Using multiple assays, I have established that Doa10 acts as a retrotranslocase for Ubc6 (Chapter 3). One experiment that led to this conclusion is based on a fluorescence-quenching assay using the antibody antiA488 that binds to the carboxy-terminal A488 label of Ubc6 $\mathrm{A}_{488}$. In the presence of Doa10, the antibody accesses the luminal label of Ubc6 over time indicating that Doa10 retrotranslocates Ubc6 (Figure 3.4A-C). I next tested if the presence of the antibody influences the photocrosslinking of Ubc6 with Doa10. This experiment can give insight into the interaction states of Ubc6 and Doa10 in the photocrosslinking approach, as well as after retrotranslocation of the luminal dye attached to Ubc6.

To test this, I sortase-labeled Ubc6 ${ }_{\mathrm{M} 249 \mathrm{BpA}}$ with A488 at its carboxy-terminal LPETGG tag and co-reconstituted it with Doa10. Liposomes were subsequently treated with TEV-protease to remove the SBP-LPETGG tag from Doa10 which was also labeled as the sortase-labeled Ubc6 was directly used and thus sortase was present during reconstitution (Figure 5.4A). The liposomes were separated from sortase and labeled peptide by flotation in a Nycodenz step gradient. Floated liposomes were preincubated with or without antibody (15 min, RT) and subsequently exposed to UV-light (Figure 5.4B). Samples were analyzed by SDS-PAGE and A488 fluorescence scanning. Again, two crosslinked bands appear after UV-exposure. The band which was shown to be BpA-specific before, disappears when liposomes were preincubated with anti-A488. This indicates that Doa10-mediated retrotranslocation changes the interaction between Ubc6 and Doa10. More specifically, in the presence of Doa10, the anti-A488 antibody shifts the equilibrium towards retrotranslocated Ubc6 which is reflected in a different interaction of Ubc6 with Doa10 as confirmed by photocrosslinking. Moreover, this experiment shows that the photocrosslinking approach (in the absence of the antibody) reports on a state before retrotranslocation by Doa10. 
A

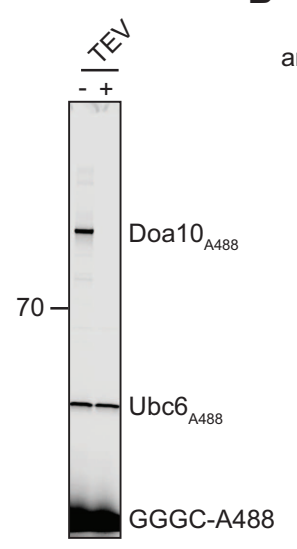

B anti-A488 low exp. high exp.

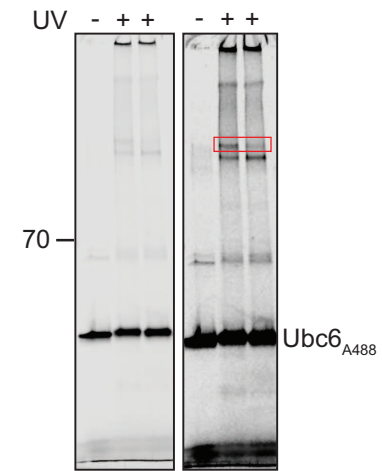

Figure 5.4: Photocrosslinking of liposomes containing Ubc6 $_{\mathrm{M} 249 B \mathrm{BA} A \mathrm{~A} 488}$ and Doa10 after incubation with anti-A488 antibody. (A) Ubc6 ${ }_{\mathrm{M} 249 \mathrm{BpA}}$ was labeled with A488 using Sortase-mediated labeling and the reaction directly used for reconstitution with Doa10 into liposomes. Liposomes were treated subsequently with TEV-protease to cleave the labeled, C-terminal SBP-LPETGG tag from Doa10. Samples before and after TEV-cleavage were analyzed by SDS-PAGE and fluorescence scanning. (B) Liposomes were incubated with or without anti-A488 antibody for $15 \mathrm{~min}$ at RT and then exposed to UV-light (365nm, 4 Joule). As a control for UV-specific bands, a sample was taken before exposure to UV-light (-). Samples were analyzed by SDS-PAGE and fluorescence scanning. Red box indicates BpA-specific, crosslinked band. The same SDS-PAGE gel is shown with two different exposures (exp.).

Summarizing, using Doa10 truncations I show that the TM region of Doa10 is required for ubiquitination as well as spontaneous retrotranslocation by Doa10. Moreover, I have analyzed described Doa10 mutants in Ubc6 ubiquitination and their retrotranslocase activity. However, I have not observed major differences compared to wildtype Doa10 which could be due to technical reasons as well as due to the Doa10 mutants affecting a different process than the ones examined. Moreover, we have optimized a photocrosslinking approach to identify interaction sites between Ubc6 and Doa10. However, due to the requirement of mass-spectrometric analysis of transmembrane proteins this approach is challenging and did not lead to identification of the interaction sites. Thus, alternative approaches that do not rely on mass-spectrometric analysis might be useful in the future. The results of this chapter will be discusssed in detail in Chapter 7. 


\section{Analysis of Doa10-mediated ERAD of Sbh2}

Doa10 recognizes a wide array of potential substrates. Substrates can be soluble proteins in the cytosol or nucleus, as well as membrane proteins. To further characterize Doa10-mediated ERAD and in order to generalize conclusions I obtained by studying Ubc6, I characterized another Doa10 substrate. One well studied Doa10 substrate that is also a tail-anchored membrane protein like Ubc6 is Sbh2. Sbh2 is part of the Ssh1 translocon in S. cerevisiae. S. cerevisiae has two translocons for co-translational translocation, the Sec61 and Ssh1 translocon (Finke et al., 1996). Both translocons are trimeric and contain homologous subunits. The Ssh1 complex consists of Ssh1, Sss1 and Sbh2. Ssh1 and Sbh2 are homologous to the Sec61 translocon components Sec61 and Sbh1, respectively. It has been shown that upon SSH1 deletion or ectopic expression of SBH2, Sbh2 becomes instable and is degraded by Doa10 (Finke et al., 1996; Habeck et al., 2015). This led to the conclusion that Sbh2 is subjected to ERAD when it is not assembled into the Ssh1 complex.

It has been shown that the TM anchor of Sbh2 (aa 57-88) is sufficient for Doa10mediated degradation. This indicated that the Doa10 machinery does not only recognize cytosolic elements of substrates, but can also recognize degrons that are located within a TM segment.

While screens for soluble Doa10 substrates have been carried out (Kopski and Huffaker, 1997; Swanson et al., 2001; Ravid et al., 2006), no screen has been published yet for a membrane-bound substrate of Doa10. The requirements for degradation of a soluble and a membrane protein might be quite different, e.g. for a membrane protein containing an intramembrane degron additional factors might be required for recognition or retrotranslocation. We therefore screened for components necessary for Doa10-mediated degradation of Sbh2, before characterizing it in a reconstituted system.

\subsection{Identification of components necessary for degradation of Sbh2}

Degradation of Sbh2 depends on Doa10, Ubc7, Ubc6, Cdc48 as well as the proteasome (Habeck et al., 2015). In collaboration with Ákos Farkas (Laboratory of Prof. 
Blanche Schwappach-Pignataro, University Medical Center, Göttingen), we designed a tandem fluorescent timer (tFT) screen for components necessary for degradation of Sbh2. This screening method has been established by the Knop lab. A protein of interest is tagged with two fluorescent proteins which have different maturation kinetics (Khmelinskii et al., 2012). Whereas the fast maturing fluorescent protein is detectable shortly after its synthesis and thus gives a readout for the relative abundance of the tagged protein, the slowly maturing fluorescent protein gives a readout for its half-life. The intensity ratio of the slowly and the fast maturing fluorescent protein can therefore be used as a readout for the stability of the protein. As the TM segment as well as the carboxy-terminal residues, but not the cytosolic part of Sbh2 are important for its Doa10-dependent degradation (Habeck et al., 2015), I fused the tFT tag to the amino-terminus of Sbh2 (tFT-Sbh2) to avoid interference of the tag with the degron. For amino-terminal tagging, a tag consisting of the fast maturing superfolder GFP (GFP) and the slowly maturing mCherry protein has been shown to provide information on protein stability (Khmelinskii et al., 2016).

Thus, chromosomal SBH2 was amino-terminally tagged with a tFT tag in a synthetic gene array (SGA) compatible query strain. Sbh2 is only unstable when it is present in excess over its interaction partner Ssh1. Thus, we overexpressed Sbh2 for the screen. To do so, Sbh2 was overexpressed by placing the strong constitutive GPD promotor upstream of SBH2 to drive its expression.

This strain was then crossed with strains from the haploid deletion library (for non-essential genes) (Giaever et al., 2002) and DAmP (decreased abundance by mRNA perturbation) library (for essential genes) (Schuldiner et al., 2005). Strains were analyzed with a high through-put microsocopy setup. tFT-Sbh2 localizes to the ER (Figure 6.1A). Using an automated analysis, cells were identified and the GFP as well as mCherry intensity for each cell was quantified. The values for the GFP and mCherry intensity were used to calculate the GFP/mCherry intensity ratio for each cell. The cell number per well varied between the different strains (57 - 2474 cells/well, Figure $\mathrm{S} 4 \mathrm{~A})$.

We first characterized the distribution of the GFP and mCherry intensities as well as the intensity ratios to determine if the microscopic analysis provides a useful readout on stability of Sbh2 (Figure 6.1B-D). The histograms of the mCherry intensity as well as the intensity ratio show an assymetric distribution with a shift towards higher values indicating stabilized Sbh2. Whereas the GFP signal was well detectable, the signal to noise ratio for the mCherry signal was quite low. Figure $6.1 \mathrm{E}$ shows the images for the GFP and mCherry signal for strains which show either a very high $(\Delta$ doa10) or 
a very low $(\Delta \mathrm{ubp} 8)$ mCherry intensity, to illustrate the low signal to noise ratio for the mCherry intensity. However, the quantification shows that the mCherry signal is sufficient for detection as it is clearly increased in $\Delta$ cue1, $\Delta$ ubc7 and $\Delta$ doa10 (Figure 6.1C). Thus, the results from this screen indicate that information on protein stability can be gained despite the low signal to noise ratio for mCherry. Deletion strains for known ERAD components (Cue1, Ubc6, Doa10, Cdc48) displayed a high GFP as well mCherry intensity indicating that Sbh2 was stabilized in those strains. Moreover, deletion of SSH1 led to a decreased GFP- and mCherry-intensity. This is expected, as in the absence of Ssh1 the whole Sbh2 pool has an exposed degron and thus can be degraded.

We concluded that the screening method gives information on Sbh2 stability and thus decided to repeat the screen with a selected subset of strains to be able to identify hits. From the initially analyzed 5614 strains, 348 strains were selected. Strains which had either a GFP signal higher than 1750, an mCherry signal higher than 75 or a ratio higher than 0.07 were selected. Subsequently a GO-term analysis was used to remove unrelated genes (e.g. mitochondrial or nuclear localization). 
A

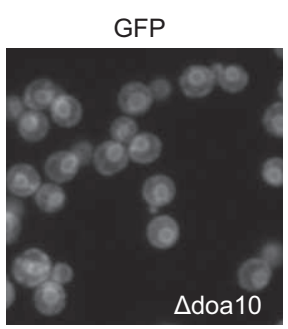

C

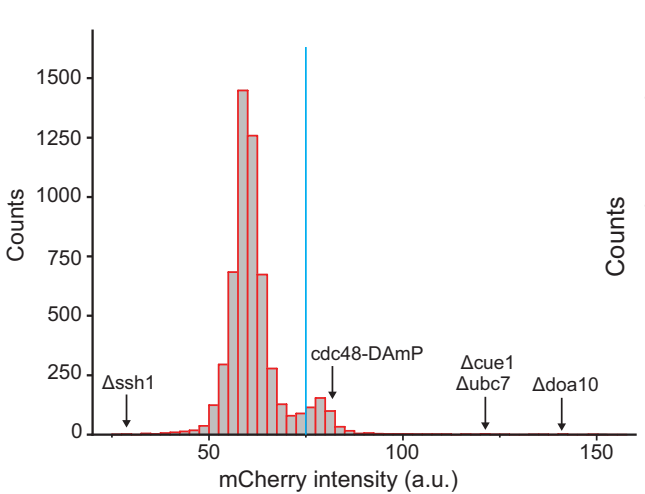

B
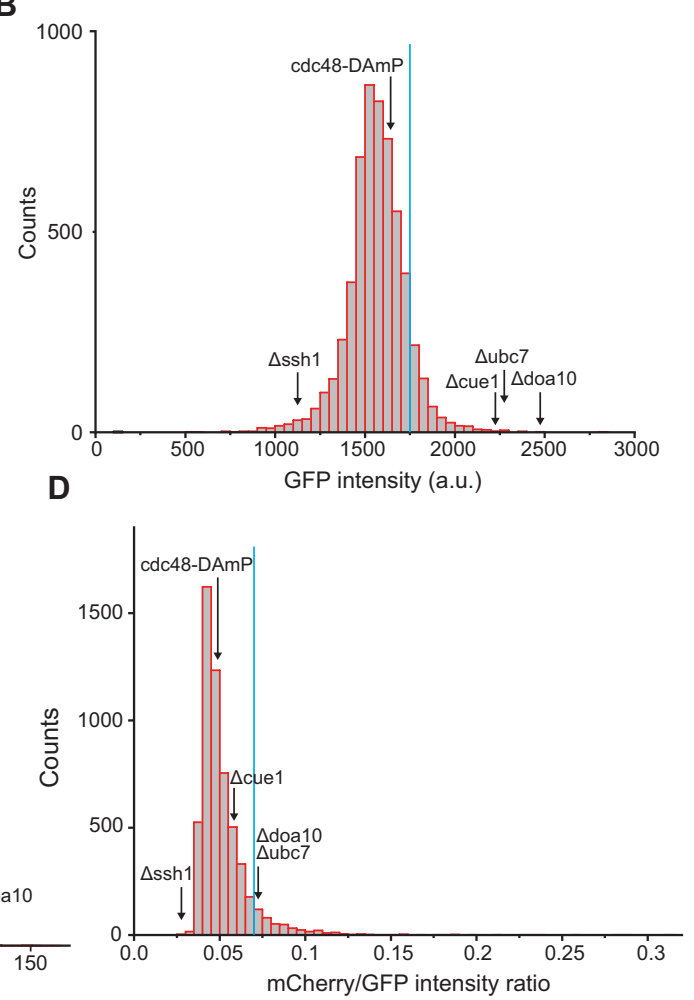

E

GFP

mCherry
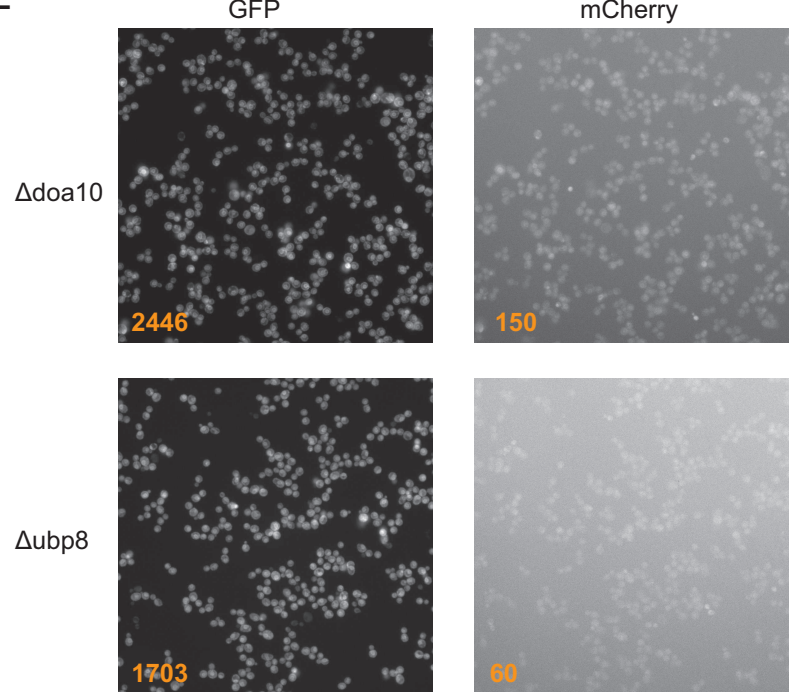

Figure 6.1: Characterization of tFT screen analysis. (A) Localization of tFT-Sbh2 in $\Delta$ doa10. GFP fluorescence for an image acquired for the screen is shown (only part of full image is shown). (B-D) Histograms of GFP (B), mCherry (C) intensity and mCherry/GFP intensity ratio (D). The values for $\Delta$ ssh1, cdc48-DAmP, $\Delta$ ubc7, $\Delta$ cue1 and $\Delta$ doa10 are indicated. The blue line shows the cut-off for selecting hits for the second screen. (E) Images of $\Delta$ doa10 and $\Delta$ ubp8 strains. Images for GFP and mCherry fluorescence are shown and the quantified mean values are shown in orange. Only parts of full image are shown. Microscopy and image analysis was performed by Ákos Farkas (UM, Göttingen). 
We next analyzed the results of both screens for the 348 selected strains. In both experiments, an average of approximately 960 cells was quantified for each strain (Figure S4B and S4C). The values for the GFP and mCherry intensity correlate between the two experiments (Figure 6.2). In contrast, the values for the mCherry/GFP intensity ratio vary between the two experiments. Moreover, we also observed that whereas GFP and mCherry intensity values correlate, they do not correlate for the intensity ratio (data not shown). One explanation for this could be the high signal to noise ratio for the mCherry values. As the intensity ratio is calculated for every cell, this might lead to a high variability. However, as we quantify the GFP and mCherry intensity for every single cell, we do not rely on the ratio for our analysis.

A

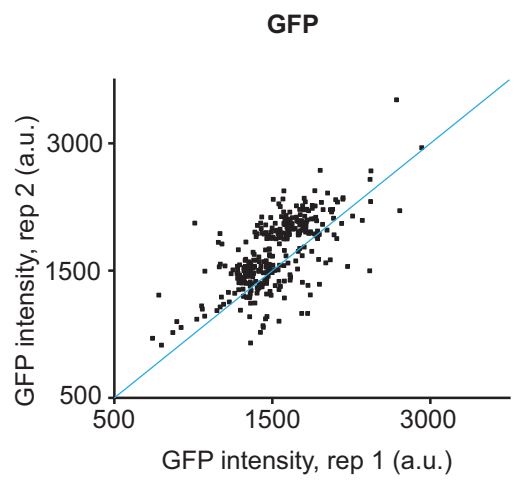

B

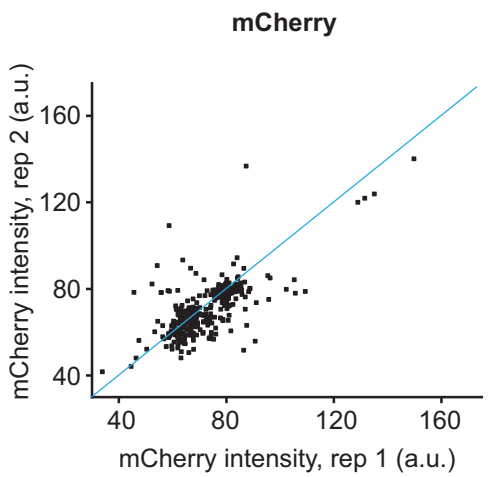

C

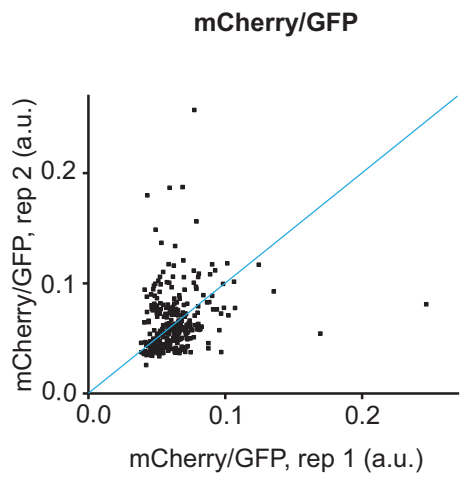

Figure 6.2: Comparison of results for the 348 selected strains of both screens. Scatter plots of values for GFP, mCherry intensity and mCherry/GFP intensity ratio. Results from first repeat (x-axis) are plotted against results from second repeat (y-axis). Each data point represents one analyzed strain. Blue line indicates the $45^{\circ} \mathrm{C}$ line $(\mathrm{y}=\mathrm{x})$.

We therefore selected strains as hits which showed a high GFP (high abundance) as well as a high mCherry (high stability) intensity. Deletion of DOA10, UBC7 as well as CUE1 leads to an increased GFP and mCherry intensity, as expected (Figure 6.3). Our results show that no other deletion affects Sbh2 degradation in a comparably strong manner. A UBC6 deletion was not present in the library and therefore did not show up in the screen. We have identified a few other genes whose deletion affected Sbh2 stability mildly. Deletion of UBP3 which encodes a ubiquitin-specific protease led to stabilization of Sbh2. Moreover, deletion of YGL214W-A, an uncharacterized gene, also stabilized Sbh2. Deletion of those genes did not seem to affect localization of Sbh2 (Figure S5). 

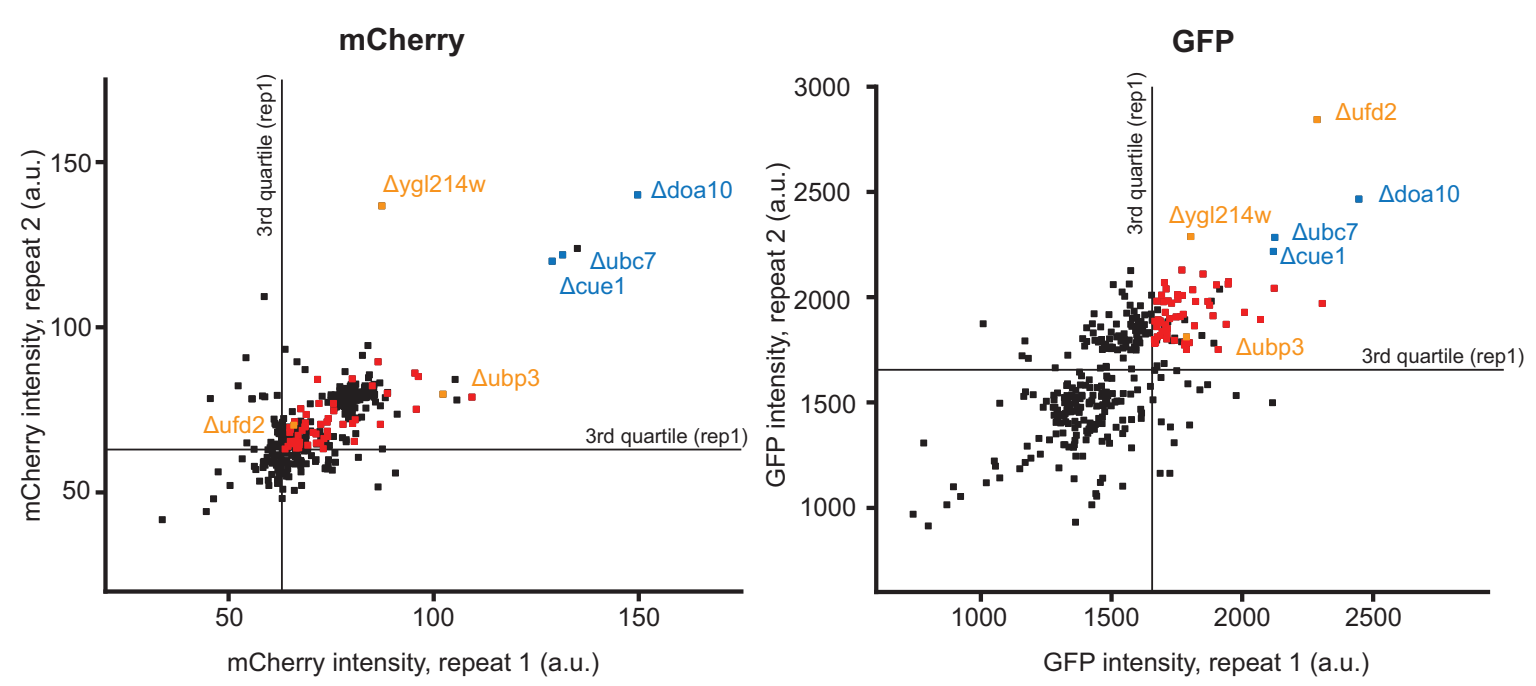

Figure 6.3: Identified hits in the tFT screen. Scatter plots of values for GFP and mCherry intensity of the 348 analyzed strains are shown as in Figure 6.2. Red dots indicate potential hits which were identified as such if both the mCherry as well as the GFP intensity values in both screens were higher than the 3rd quartile value from the first screen (GFP: 1653, mCherry: 63). Known ERAD components are labeled in blue, further hits which might be biologically interesting in yellow. The DAmP strain for CDC48 was only included in the first screen (Figure 6.1) and therefore was not identified in this analysis. The screen was carried out by Ákos Farkas (UM, Göttingen).

UBP3 was an interesting hit in our screen, as deletion of this ubiquitin-specific protease stabilized Sbh2. We hypothesized that it could be involved in substrate deubiquitination necessary for delivery to the proteasome or potentially deubiquitination of Doa10.

Ubp3 is a ubiquitin-specific protease that has been implicated in many cellular processes like ribophagy (Baker et al., 1992; Ossareh-Nazari et al., 2010a) and ERGolgi transport. When UBP3 is deleted, ER to Golgi transport is compromised and ER membranes accumulate. It has been shown that Ubp3 deubiquitinates the COPII subunit Sec23 as well as the COPI subunit Sec27 (Cohen et al., 2003b,a). Moreover, Cdc48 and Npl4 are required for degradation of Sec23 (Ossareh-Nazari et al., 2010b). Deletion of UBP3 suppresses the temperature-sensitive lethality of npl4-1 (Auld et al., 2006).

To verify our screen results, I freshly made a UBP3 deletion strain and measured the mCherry and GFP intensity of ectopically expressed tFT-Sbh2. Measurements were done using a fluorescence plate reader. To ensure that the deletion of UBP3 does 
not affect overall protein stability, we also measured the fluorescence of an ectopically expressed control protein, Sbh1 which is stable in wildtype cells (Habeck et al., 2015). For Sbh1, the mCherry/GFP intensity ratio in $\Delta$ doa10 or $\Delta$ ubp3 is comparable to wildtype indicating that the stability of Sbh1 is not affected by deleting one of these genes (Figure 6.4A). In contrast, Sbh2 is stabilized when DOA10 or UBP3 are deleted. However, the effect of deleting UBP3 is less strong compared to deleting DOA10 (Figure $6.4 \mathrm{~A})$.

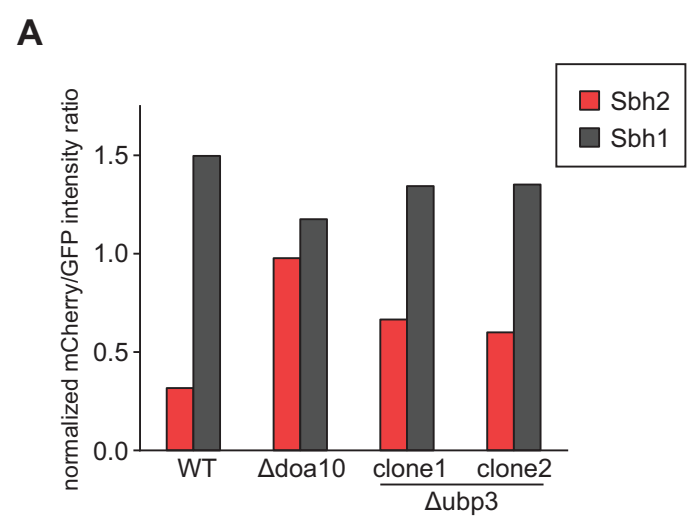

B

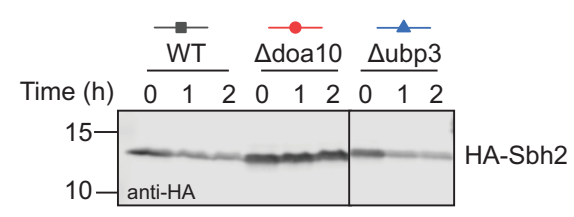

C

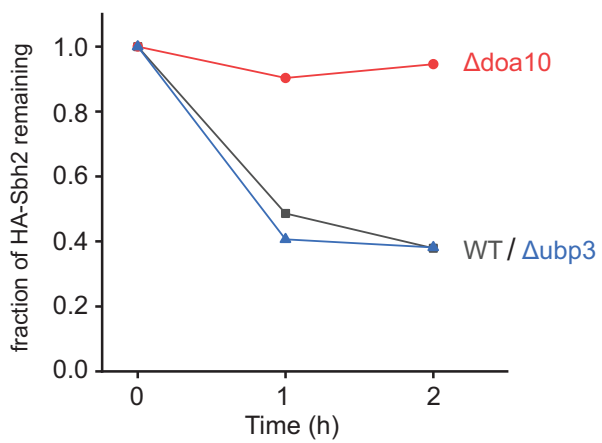

Figure 6.4: Effect of UBP3 deletion on Sbh2 stability. (A) mCherry/GFP intensity ratio of wildtype (WT), $\Delta$ doa10 and $\Delta$ ubp3 (2 clones tested) strains which ectopically expressed tFT-Sbh2 or tFT-Sbh1 from a GPD-promotor. Measurements were performed with a fluorescence plate reader. (B) Degradation of ectopically expressed HA-Sbh2 (from GPD-promotor) in wildtype (WT), $\Delta$ doa10 and $\Delta$ ubp3 cells. After cycloheximide addition, samples were taken at the indicated timepoints. After cell lysis, samples were analyzed by SDS-PAGE and subsequent anti-HA immunoblotting. (C) Quantification of HA-Sbh2 turnover (analyzed from immunoblot in B).

I also measured the degradation kinetics of ectopically expressed, hemagglutinin (HA)-tagged Sbh2 by cycloheximide (CHX) chase. When UBP3 was deleted, cells grew very slowly (data not shown). I observed no difference in the turnover of Sbh2 in a CHX-chase (Figure 6.4B and 6.4C). However, degradation of Sbh2 was quite fast and 
thus the timepoints chosen for the CHX-chase did not resolve the degradation kinetics properly. Concluding, further experiments are necessary to test if Ubp3 has a function in ERAD.

In summary, this screen for components required for degradation of Sbh2 has identified ERAD-components which have been previously shown to be required for degradation of Sbh2 (Habeck et al., 2015). We did not identify any other gene whose deletion had a similarly strong phenotype as deletion of those known ERAD components. This suggested that the machinery for ERAD of Sbh2 might be identified. With this in mind, I set out to reconstitute Sbh2 to directly determine the minimal machinery for Doa10-mediated ERAD of Sbh2.

\subsection{Reconstitution of Doa10-mediated ERAD of Sbh2}

A reconstituted system for Doa10-mediated ERAD of Sbh2 potentially can address many questions. Besides characterization of the minimal machinery for this process, it also allows for investigation of many mechanistic questions regarding ubiquitination and retrotranslocation. Moreover, it can provide a useful tool to be able to compare the behaviour of Ubc6 and Sbh2.

Thus, I next established a reconstituted system for Doa10-mediated ERAD of Sbh2. To do so, I first tested which fluorescent labeling method does not interfere with the substrate behaviour of Sbh2. I engineered Sbh2 variants that contained tags for either sortase-mediated or maleimide-based fluorescent labeling and tested their degradation kinetics by CHX-chase.

\subsubsection{Identification of a suitable Sbh2 construct for reconstitution studies}

I first tested if Sbh2 is suitable for carboxy-terminal Sortase labeling. It has been shown that changing the carboxy-terminal, luminal residues of Sbh2 to the corresponding ones of Sbh1 leads to stabilization of the substrate (Habeck et al., 2015). As it is unclear if this effect is due to a gained interaction with Sec61 or due to masking of the degron, I tested if adding a carboxy-terminal tag to Sbh2 affects its substrate behaviour. Sbh2 was again overexpressed from the GPD promotor. The degradation of HA-Sbh2 containing an 11 aa carboxy-terminal linker followed by an LPETGG tag (HA-Sbh2 ${ }_{\text {linker }}$ ) and HA-Sbh2 WT was assessed by CHX-chase. In contrast to HA-

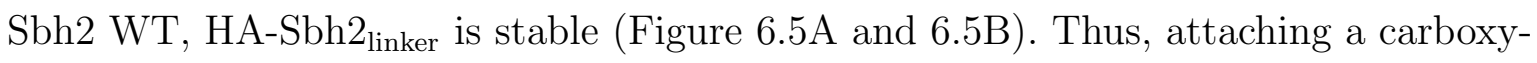
terminal sortase tag to Sbh2 changes its behaviour in Doa10-mediated ERAD and thus a sortase-labeling approach cannot be used for labeling Sbh2. 
A

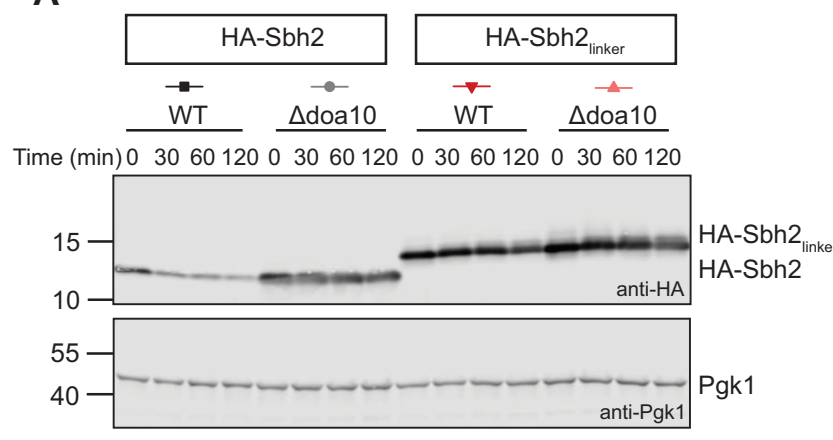

B

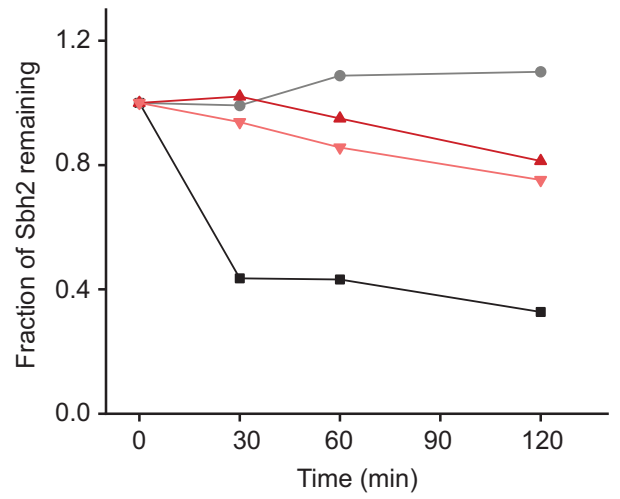

Figure 6.5: Degradation of Sbh2 variants in wildtype and $\Delta$ doa10 cells. (A) HA-tagged Sbh2 as well as HA-tagged Sbh2 variants containing a C-terminal linker (HA-Sbh2 $2_{\text {linker }}$ ) was expressed ectopically from a GPD-promotor. Degradation kinetics were analyzed by CHX-chase, as described in Figure 6.4. Samples were analyzed by SDS-PAGE and subsequent immunoblotting with anti-HA (Sbh2) and anti-Pgk1 (loading control). (B) Quantification of turnover of HASbh2 variants.

I also tested if a carboxy-terminal cysteine $\left(\mathrm{Sbh} 2_{89 \mathrm{C}}\right)$ can be attached to $\mathrm{Sbh} 2$ for labeling using maleimide chemistry. Whereas the degradation kinetics of Sbh2 WT and Sbh2 89 C are comparable, the effect of deleting DOA10 or UBC6 differ slightly between Sbh2 wildtype and Sbh2 $89 \mathrm{C}$ (Figure 6.6), as the degradation of Sbh2 $2_{89 \mathrm{C}}$ is less dependent on Doa10 and Ubc6 compared to Sbh2 WT. Of note, I cannot distinguish with this assay if degradation kinetics are different due to compromised insertion efficiency of Sbh2 into the ER, different interaction of Sbh2 with the Ssh1 translocon or due to different degron recognition of Sbh2 by Doa10. However, to avoid any interference with the intramembrane degron, I decided to use an Sbh2 variant without a carboxyterminal labeling site. As the cytosolic domain of Sbh2 can be exchanged without compromising substrate recognition (Habeck et al., 2015) and wildtype Sbh2 does not contain any cysteine, I introduced a cysteine in the amino-terminal domain of Sbh2 $\left(\mathrm{Sbh} 2_{\mathrm{S} 4 \mathrm{C}}\right)$ for fluorescent labeling. 


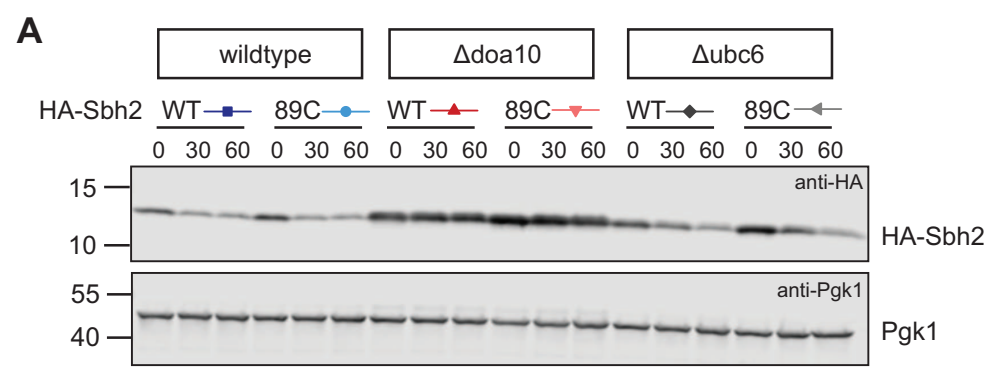

B

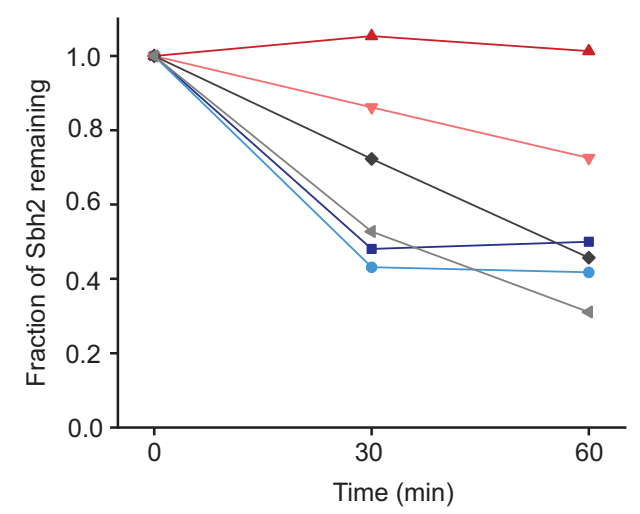

Figure 6.6: Degradation of $\mathrm{Sbh}_{89 \mathrm{C}}$ in wildtype, $\Delta$ doa10 and $\Delta$ ubc6 cells. (A) HA-Sbh2 as well as HA-Sbh2 ${ }_{89 \mathrm{C}}$ were expressed ectopically from a GPD-promotor. Degradation kinetics were analyzed by CHX-chase. Samples were analyzed as described in Figure 6.5. (B) Quantification of turnover of HASbh2 variants.

\subsubsection{Purification and reconstitution of Sbh2}

Sbh2 from S. cerevisiae was expressed in E. coli. Sbh2 $2_{\mathrm{S} 4 \mathrm{C}}$ was purified in DDM by NiNTA affinity chromatography via an amino-terminal His ${ }_{14}$-SUMO tag, subsequently eluted with SUMO-protease Ulp1 and further purified by size exclusion chromatography. Sbh2 was then labeled with DL800 at its cysteine via maleimide chemistry (Figure 6.7A). Although the purification protocol has to be further optimized due to co-purified contaminants, I next tested if Sbh2 can be reconstituted with Doa10 and Ubc6. I directly co-reconstituted Sbh2 with Doa10 (purified in DMNG) with or without Ubc6 into liposomes. All three proteins co-floated in a Nycodenz step gradient indicating that they were reconstituted (Figure 6.7B). 
A

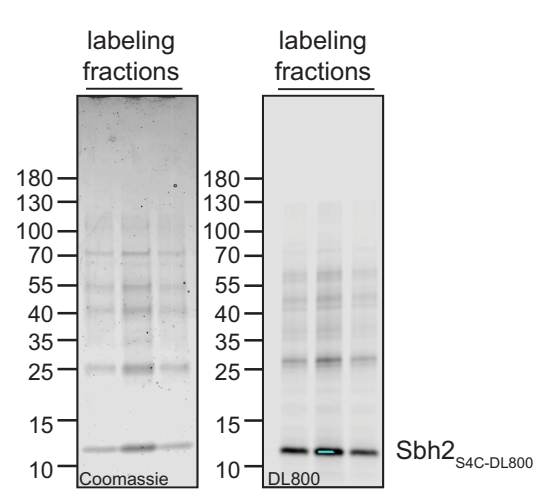

B

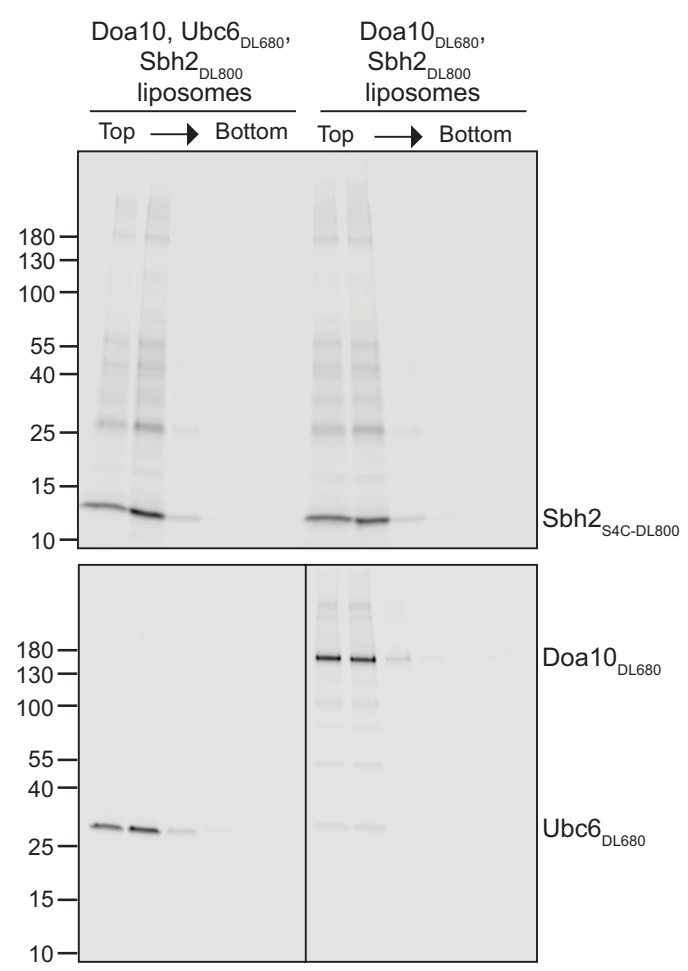

Figure 6.7: Fluorescent labeling and reconstitution of Sbh2. (A) Peak fractions of maleimide labeling reaction of Sbh2 $2_{\mathrm{S} 4 \mathrm{C}}$. Samples of fractions were analyzed by SDS-PAGE and Coomassie staining (left) or fluorescence scanning (right). (B) Liposomes containing Doa10, Ubc6 ${ }_{\text {DL680 }}$, Sbh2 $2_{\mathrm{DL} 800}$ or liposomes

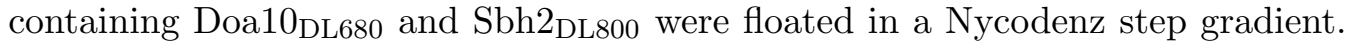
Samples of flotation fractions were analyzed SDS-PAGE and fluorescence scanning (top: DL800, bottom: DL680 fluorescence).

\subsubsection{Characteristics of Doa10-mediated ubiquitination of Sbh2}

I next tested if Doa10 recognizes Sbh2 as a substrate and mediates ubiquitination in the established reconstituted system. I incubated liposomes containing Doa10, Sbh2 and Ubc6 with ubiquitination machinery (Uba1, a soluble Cue1 fragment ( $\left.\mathrm{Cue} 1_{\text {sol }}\right)$, Ubc7, ubiquitin and ATP). To test for the requirement of Ubc6, a second set of liposomes only contained Doa10 and Sbh2. When Sbh2 is co-reconstituted with Ubc6 and Doa10, $50 \%$ of Sbh2 are polyubiquitinated (Figure 6.8). The reaction is mostly complete after $16 \mathrm{~min}$ (Figure 6.8B). Importantly, the decrease of the non-modified band for Sbh2 is dependent on ATP indicating that the decrease of the unmodified band is due to ubiquitination (Figure 6.8C). Interestingly, in the absence of Ubc6, no ubiquitination of Sbh2 occurs. This effect also cannot be rescued by adding a soluble fragment of Ubc6 $\left(\right.$ Ubc6 $\left._{\text {sol }}\right)$. This indicates that Ubc6 is required for priming Sbh2 with ubiquitin, before Ubc7/Cue1-mediated polyubiquitination can occur. In line with this, deletion of 
UBC6 in vivo compromises degradation of Sbh2 (Habeck et al. (2015), also see Figure 6.6). Moreover, the TM anchor of Ubc6 seems to be required for its function as an E2 enzyme.
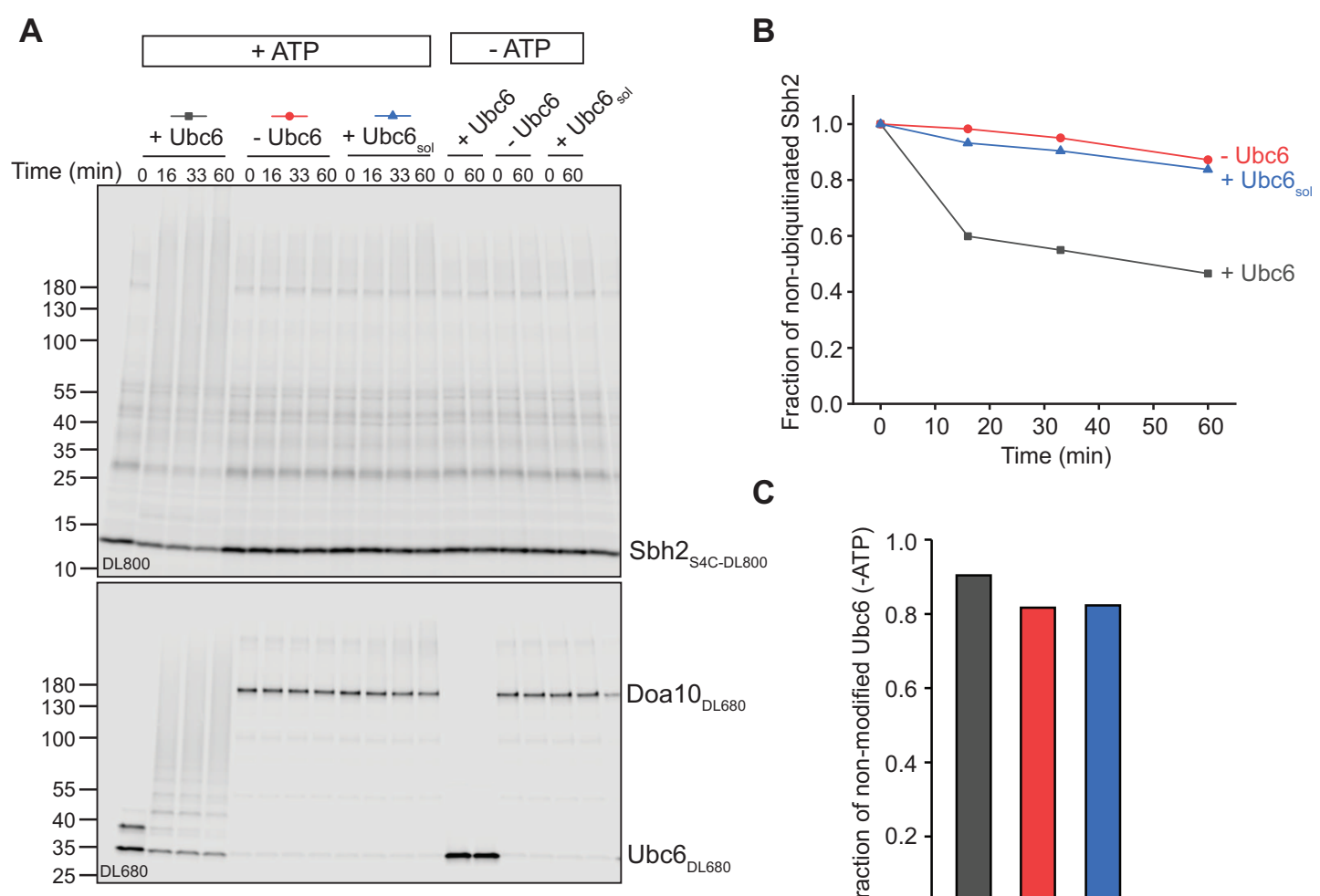

\section{C}

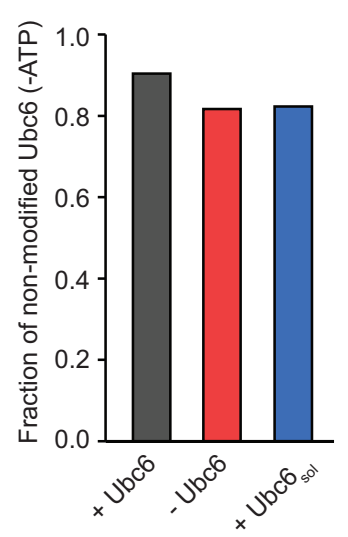

Figure 6.8: Ubiquitination of $\mathrm{Sbh2}$ in a reconstituted system. Liposomes containing Doa10, Ubc6 $6_{\text {DL680 }}$ and Sbh2 ${ }_{\mathrm{S} 4 \mathrm{C}-\mathrm{DL} 800}$ or Doa10 ${ }_{\mathrm{DL} 680}$ and Sbh2 ${ }_{\mathrm{S} 4 \mathrm{C}-\mathrm{DL} 800}$ were incubated with Uba1, Ubc7, a soluble Cue1 fragment $\left(\mathrm{Cue}_{\mathrm{sol}}\right)$, ubiquitin and ATP (f.c. of $0.1 \mu \mathrm{M}$ Uba1, $1 \mu \mathrm{M} \mathrm{Ubc7,} 1 \mu \mathrm{M}$ Cue $1_{\text {sol }}, 120 \mu \mathrm{M}$ ubiquitin, 2.5 mM ATP, $0.1 \mu \mathrm{M}$ Ubc6, $0.1 \mu \mathrm{M}$ Doa10, $0.1 \mu \mathrm{M}$ Sbh2). Where indicated, a soluble

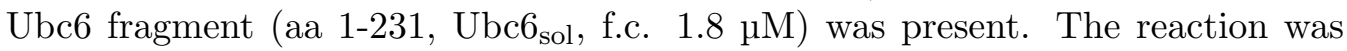
stopped after 0, 16, 33 and 60 min with non-reducing sample buffer. (A) Analysis of samples by SDS-PAGE and fluorescence scanning (top: DL800, bottom: DL680 fluorescence). (B, C) Quantification of (A). (B) Quantification of turnover of Sbh2. Values were normalized to 0 min timepoint. (C) Quantification of turnover of Sbh2 in reactions lacking ATP. Intensity values for $60 \mathrm{~min}$ timepoint were normalized to the respective 0 min timepoint.

Summarizing, we have established a reconstituted system recapitulating ubiquitination of Sbh2. In the presence of Ubc6, Ubc7 and Cue1, Doa10-mediated polyubiquitination occurs. Our results moreover indicate that Doa10 directly recognizes Sbh2. Thus, a minimal machinery for Doa10-mediated ubiquitination of Sbh2 is identified. 


\section{Discussion}

This thesis provides insight into different steps of Doa10-mediated ER-associated protein degradation (ERAD). By establishing a system to co-reconstitute membrane proteins by SNARE-mediated membrane fusion of liposomes, I have recapitulated ubiquitination of Ubc6 as well as extraction of Ubc6 from the membrane. Moreover, I have gained mechanistic insights into the extraction process by showing that Doa10 acts as a retrotranslocase. In an effort to gain further insight into structural elements in Doa10 involved in recognition and processing of Ubc6, I have tested previously described Doa10 mutants and optimized a system for site-specific crosslinking of the transmembrane segment of Ubc6 with Doa10 (in collaboration with Iwan Parfentev, laboratory of Prof. Urlaub, MPI for Biophysical Chemistry, Göttingen). In order to expand our mechanistic understanding to other Doa10 substrates and to be able to draw more general conclusions, I also characterized another Doa10 substrate, the tail-anchored membrane protein Sbh2. To do so, I have first investigated the machinery for Sbh2 degradation in vivo by performing a screen (in collaboration with Ákos Farkas, Laboratory of Prof. Blanche Schwappach-Pignataro, University Medical Center, Göttingen) and also characterized this machinery by recapitulating ubiquitination of Sbh2 in a reconstituted system. In the following section, the results from these different projects are further discussed.

\subsection{Co-reconstitution of membrane proteins by SNARE-me- diated membrane fusion}

Reconstitution of membrane-associated processes often requires the reconstitution of multiple membrane proteins. We have established a system that allows reconstitution of membrane proteins by SNARE-mediated fusion of proteoliposomes. This system has multiple advantages compared to other reconstitution protocols. It enables high co-reconstitution efficiencies and thus makes biochemical studies possible. At the same time, it eliminates artefacts that could arise due to the interaction of membrane proteins in detergent when membrane proteins are directly reconstituted into the same set of liposomes. Moreover, it allows for a higher flexibility when two proteins are coreconstituted whose optimal reconstitution conditions differ such as the detergent used 
for solubilization of the liposomes. However, this is also a limiting factor as SNARE proteins might not be compatible with every detergent and thus reconstitution protocol. Following, I will discuss the characteristics of the established system and compare it to other fusion approaches that have been previously developed.

\subsubsection{SNARE-mediated fusion enables high co-reconstitution efficiencies}

High co-reconstitution efficiencies are an important requirement for a fusion-based coreconstitution approach. The established SNARE-mediated fusion assay allows for high co-reconstitution efficiencies, as Ubc6 is nearly quantitatively co-reconstituted with Doa10. After fusion, pulldown of Doa10 leads to nearly complete pulldown of Ubc6 (Figure A1G). Moreover, efficient polyubiquitination and Doa10-mediated retrotranslocation occur when wildtype Syb and but not when a Syb mutant (Syb $\Delta 84$ ) is reconstituted that allows for docking of liposomes but not full fusion (Figure 3.1, 3.2 and 3.4). These two experiments indicate that the established fusion system enables high co-reconstitution efficiencies and leads to lipid-mixing of the liposome membranes.

Nordlund et al. (2014) have previously used SNARE-mediated fusion to co-reconstitute membrane proteins of the respiratory chain with the ATP synthase (Nordlund et al., 2014). They estimate the co-reconstitution efficiency after fusion by measuring spectrophotometrically the reduction state of encapsulated cytochrome $\mathrm{C}$ that only upon fusion is delivered to cytochrome $\mathrm{C}$ oxidase. With this method, they estimate a fusion efficiency of 70\%. Interestingly, they use the SNAREs SNAP-25A and syntaxin$1 \mathrm{~A}$ which are prone to form a so called 2:1 complex consisting of two syntaxin-1A molecules and one SNAP-25A molecule that is inactive in fusion due to occupying the binding site for synaptobrevin (Syb) (Fasshauer et al., 1997; Margittai et al., 2001; Xiao et al., 2001). Here, we present a strategy which uses the fusogenic $\Delta \mathrm{N}$-complex that consists of SNAP-25A, syntaxin-1A and a soluble Syb fragment (aa 42-96) that inhibits formation of the 2:1 complex and is displaced by full-length Syb during the fusion process (Pobbati, 2006; Hernandez et al., 2012). Our results show that using this $\Delta \mathrm{N}$-complex leads to high co-reconstitution of Ubc6 and Doa10 and is thus useful to co-reconstitute membrane proteins.

\subsubsection{SNARE-mediated fusion allows the use of a lipid composition mim- icking the ER membrane}

Lipid bilayers in a reconstituted system should ideally mimic the lipid composition of the corresponding membrane in vivo. This is a challenge as fusion protocols usually require a certain lipid composition. SNARE-mediated fusion relies on the presence 
of cholesterol (Tong et al., 2009) and $10 \mathrm{~mol} \%$ cholesterol have been used previously for reconstitution of the $\Delta \mathrm{N}$-complex (Pobbati, 2006; Hernandez et al., 2012). In our system, the liposomes used contained $10 \mathrm{~mol} \%$ ergosterol instead of cholesterol, to mimic the ER membrane of $S$. cerevisiae.

An alternative approach has been reported that is based on fusion of liposomes that contain co-reconstituted charged lipids (Biner et al., 2016; Ishmukhametov et al., 2016; Galkin et al., 2018). This approach requires a high concentration of charged lipids. Biner et al. report that up to $30 \mathrm{~mol} \%$ of charged lipids are required for efficient fusion (Biner et al., 2016). Moreover, whereas negatively charged lipids such as phosphatidylserine and phosphatidylinositol occur also in the cell, cationic lipids are not found in the cell (van Meer et al., 2008). This has been a challenge for the reconstitution of ATP synthase as its activity is influenced by the presence of cationic lipids (Ishmukhametov et al., 2016; Galkin et al., 2018).

The presented system for fusion mediated by SNAREs allows the use of a lipid composition roughly mimicking the ER membrane (Tuller et al., 1999; Zinser et al., 1991; van Meer et al., 2008), with the exception that phosphatidylinositol was replaced by phosphatidylcholine. I have not further investigated the effect of the lipid composition on the fusion assay and thus further experiments are required to determine which lipid compositions are compatible with this approach. With this in mind, the lipid composition can be also limiting factor in this system. However, in comparison to a charge-mediated fusion assay it allows the use of naturally occuring lipids.

\subsubsection{Liposome instability is a result of low lipid to protein ratios}

Proteoliposomes represent a suitable system to recapitulate processes at cellular membranes as their lipid bilayer separates two aqueous compartments. For reconstitution of ERAD, the bilayer represents the ER membrane and thus separates the ER lumen and the cytosol. In such a reconstituted system, leakage or instability of the proteoliposomes is unwanted as these compartments have different environments with different protein compositions and a reconstituted system ideally distinguishes these two compartments.

I observed that proteoliposomes are to some extent unstable after fusion (Figure 4.1, 4.3). This instability can be avoided by lowering the amount of membrane proteins reconstituted per liposome without compromising the co-reconstitution efficiency. This effect has been observed before for SNARE-mediated fusion. van den Bogaart et al. (2010) show that proteoliposomes leak after fusion, when the $\Delta \mathrm{N}$-complex is reconstituted at a lipid:protein ratio of 1000. Liposomes were stable when a higher ratio 
$(16,000)$ was used. These results show that the concentration of reconstituted proteins has to be carefully optimized to ensure a functioning and stable system.

The tendency of proteoliposomes to be prone to instability and leakage shows a general disadvantage of reconstitution approaches containing lipid bilayers. Due to the enhanced instability of liposomes when high concentrations of proteins are reconstituted, it is difficult to mimic the protein-rich environment of biological membranes (Quinn et al., 1984; Takamori et al., 2006). One way to overcome this limitation in the future is to combine minimal reconstitution approaches such as proteoliposomes with more complex reconstitution approaches such as vesicles derived from organelles. In a study by Preobraschenski et al. (2014), the neurotransmitter transporter vGLUT has been studied using a combinatorial approach by fusing proteoliposomes with synaptic vesicles. A similar fusion approach using ER-derived vesicles called microsomes and proteoliposomes might be a powerful tool to overcome limitations of proteoliposomes such as lipid bilayer instability in the future.

\subsection{A minimal system for Ubc6 polyubiquitination}

Doa10 functions with the E2 enzymes Ubc6 and Ubc7. Ubc6 itself is unstable and targeted for Doa10-mediated ERAD in a manner dependent on its catalytic activity (Walter et al., 2001; Swanson et al., 2001). I characterized ubiquitination of Ubc6 for two main reasons. First, its behaviour as an E2 enzyme whose autoubiquitination leads to proteasomal degradation is quite interesting and its mechanism as well as its functional implications are not well understood. Moreover, studying Ubc6 reduces the complexity of the reconstituted system, due to its dual function as an E2 enzyme and substrate.

Using the SNARE-mediated fusion assay, I have co-reconstituted Ubc6, Doa10 and Cue1. Ubc6 and Doa10 were thereby in separate liposome sets to ensure they only interact upon fusion and thus in a lipid bilayer context.

\subsubsection{Mono-ubiquitination of Ubc6 depends on its autoubiquitination ac- tivity and is required for subsequent Ubc7/Cue1-mediated polyubiq- uitination}

In the presence of loaded E1 enzyme, Ubc6 ubiquitinates itself on non-cysteine residues. This ubiquitin transfer activity of Ubc6 is stimulated in the presence of Doa10 (Figure 3.1).

Autoubiquitination of E2 enzymes is a common behaviour in vitro (David et al., 
2010). Loaded E2 enzymes are prone to react and in many cases, the RING ubiquitin ligase not only serves as a scaffold to bring together substrate and ubiquitin-loaded E2 enzyme, but stimulates the E2 ubiquitin transfer activity (Yin et al., 2009; Wenzel et al., 2011). Ubc6 thereby transfers multiple mono-ubiquitin moieties onto itself and does not form ubiquitin chains (Figure A1L). This ubiquitin transfer probably occurs intramolecularly, as inactive Ubc6 is stable, even when wildtype Ubc6 is also expressed (Walter et al., 2001).

Mono-ubiquitinated Ubc6 is a substrate for subsequent polyubiquitination by Ubc7, Cue1 and Doa10. In the absence of either of those components, Ubc6 remains monoubiquitinated (Figure 3.2). Ubc7 forms K48-linked ubiquitin chains on Ubc6 (Figure A2D), in agreement with other studies showing that Ubc7 specifically forms K48linked ubiquitin chains (Bazirgan and Hampton, 2008; Bagola et al., 2013). The requirement of mono-ubiquitination can be bypassed by fusing ubiquitin to the aminoterminus of Ubc6 (Figure 3.2). These results are in line with observations in intact cells which show that inactive Ubc6 is degraded when ubiquitin is fused to the aminoterminus of Ubc6 (Weber et al., 2016).

Multiple lines of evidence suggest that the attachment site of ubiquitin during monoubiquitination is not crucial for subsequent polyubiquitination. First, aminoterminal fusion of ubiquitin is sufficient for polyubiquitination. Second, Weber et al. (2016) have identified Serine 196 of Ubc6 as the main ubiquitination site of a soluble Ubc6 fragment in vitro. However, they also show that Ubc6 ${ }_{\mathrm{S} 196 \mathrm{~A}}$ is still degraded in vivo, suggesting that other sites can also be ubiquitinated by Ubc6.

\subsubsection{Proteasomal degradation of Ubc6 might have a regulatory role}

The physiological relevance of proteasomal degradation of Ubc6 is still unclear. Degradation of this highly reactive E2 enzyme could serve a regulatory function. Examples of other E2 enzymes show that autoubiquitination can have a regulatory role for E2 function (reviewed by Stewart et al. (2016)). One such example is Ubc7. When it is not associated with its cofactor Cue1, it assembles a polyubiquitin chain on its active site cysteine which leads to its proteasomal degradation (Ravid and Hochstrasser, 2007). Besides regulation through degradation, it has been also shown that monoubiquitination of E2 enzymes inhibits their ubiquitin transfer activity (Machida et al., 2006; Banka et al., 2015).

Proteasomal degradation of Ubc6 could regulate the activity of the Doa10 complex. In such a model, Ubc6 transfers ubiquitin onto itself when no substrate is bound to the Doa10 complex, leading to Ubc6 polyubiquitination and degradation and thus 
rendering the Doa10 complex less active in substrate ubiquitination. It remains to be tested if such a model holds true. It would be interesting to test if the presence of substrates influences the ubiquitination of Ubc6. Such an experiment could help to understand if other substrates are compared to Ubc6 preferentially ubiquitinated by Doa10.

Concluding, the reconstituted system recapitulates Doa10-mediated polyubiquitination of Ubc6. It identifies the minimal machinery for this process and shows the sequence of steps during polyubiquitination on a mechanistic level. As it recapitulates many observations in intact cells (Walter et al., 2001; Swanson et al., 2001; Weber et al., 2016), it can be concluded that the established reconstituted system is a useful tool to study ERAD of Ubc6.

\subsection{Mechanistic insights into retrotranslocation of membrane proteins}

In ERAD, polyubiquitinated substrates are retrotranslocated back into the cytosol where they are degraded by the proteasome. Our reconstituted system recapitulates this step and provides mechanistic insights into retrotranslocation.

\subsubsection{Cdc48/UN extracts polyubiquitinated Ubc6}

The Cdc48/UN (Ufd1 Npl4) complex extracts polyubiquitinated Ubc6 from the liposome membrane using ATP (Figure 3.3). The minimum ubiquitin chain length on Ubc6 thereby appears to be 5 ubiquitin moieties. This is in agreement with previous studies that have shown that the UN complex binds to ubiquitin chains containing at least 5 ubiquitins in vitro (Bodnar and Rapoport, 2017b). The UN complex is required to recruit Cdc48 to polyubiquitinated substrate (Bodnar and Rapoport, 2017b). In line with this, we observe shorter ubiquitin chains on Ubc6 indicating extraction, only when both Cdc48 and UN are present (Figure 3.3). Thus, the established reconstituted system is sufficient to catalyze not only ubiquitination, but also retrotranslocation of Ubc6.

\subsubsection{Translocation systems contain a driving force and often a protein conduit}

Translocation systems are localized throughout the cell to ensure protein translocation across and insertion into membranes of organelles such as the ER, mitochondria and 
peroxisomes (Agarraberes and Dice, 2001). They share certain characteristics which will be explained further in the following section.

Translocation of proteins requires a driving force. The Sec61/SecY channel provides examples for different driving forces during translocation (reviewed by Rapoport et al. (2017)). During forward co-translational translocation of proteins into the ER, the ribosome "pushes" the polypeptide chain by preventing backsliding. In case of bacterial posttranslational translocation across the plasma membrane, the ATPase SecA pushes substrates. The mechanism is different for posttranslational translocation into the ER where BiP acts as a molecular ratchet during translocation. The presence of an ER-localized sink for the translocating substrate is in this case sufficient for providing directionality for this process (Matlack et al., 1999). Translocation systems not only require a driving force but usually also a protein conduit. The Sec61/SecY channel functions in translocation of soluble proteins as well as insertion of proteins containing transmembrane segments into the ER membrane (Rapoport et al., 2017). Whereas it is established that a protein conduit is usually required for the insertion of membranespanning proteins into a lipid bilayer, it is unclear if it is required for their dislocation as well (also see section 7.3.6).

In ERAD, the pulling force is executed by the Cdc48 ATPase. As Doa10 contains a large TM domain it has been hypothesized that it forms a protein conduit for retrotranslocating substrates (Swanson et al., 2001). This thesis provides evidence for this hypothesis.

\subsubsection{Doa10 facilitates retrotranslocation of Ubc6}

Our results show that Doa10 is a retrotranslocase. Using different assays we show that in the presence of Doa10, a luminal encapsulated dye or protease cleavage site of Ubc6 becomes accessible to the outside over time. Moreover, in the presence of a chaperone, Ubc6 is fully released from the membrane in a Doa10-dependent manner (Figure 3.4).

The Ubc6 TM anchor is sufficient for this release indicating that an intramembrane interaction between Ubc6 and Doa10 is required for this process (Figure 3.6A-D). In line with this, a Doa10 fragment containing TM segments 3-14 and thus lacking the RING domain is capable of retrotranslocating Ubc6 indicating that an interaction between the RING domain and UBC domain is not required for Doa10-mediated retrotranslocation (Figure 3.5A-C).

Interestingly, the identity of the TM segment is important for retrotranslocation as a construct containing the TM anchor of Syb is not released by Doa10. This can have different reasons: higher hydrophobicity of the Syb TM anchor could inhibit the 
spontaneous retrotranslocation by Doa10. Indeed, when comparing the hydropathy profile of Syb and Ubc6, the TM anchor of Ubc6 is less hydrophobic than the one of Syb (Figure 7.1). Alternatively, the Syb TM anchor is not recognized by Doa10 and thus not retrotranslocated.

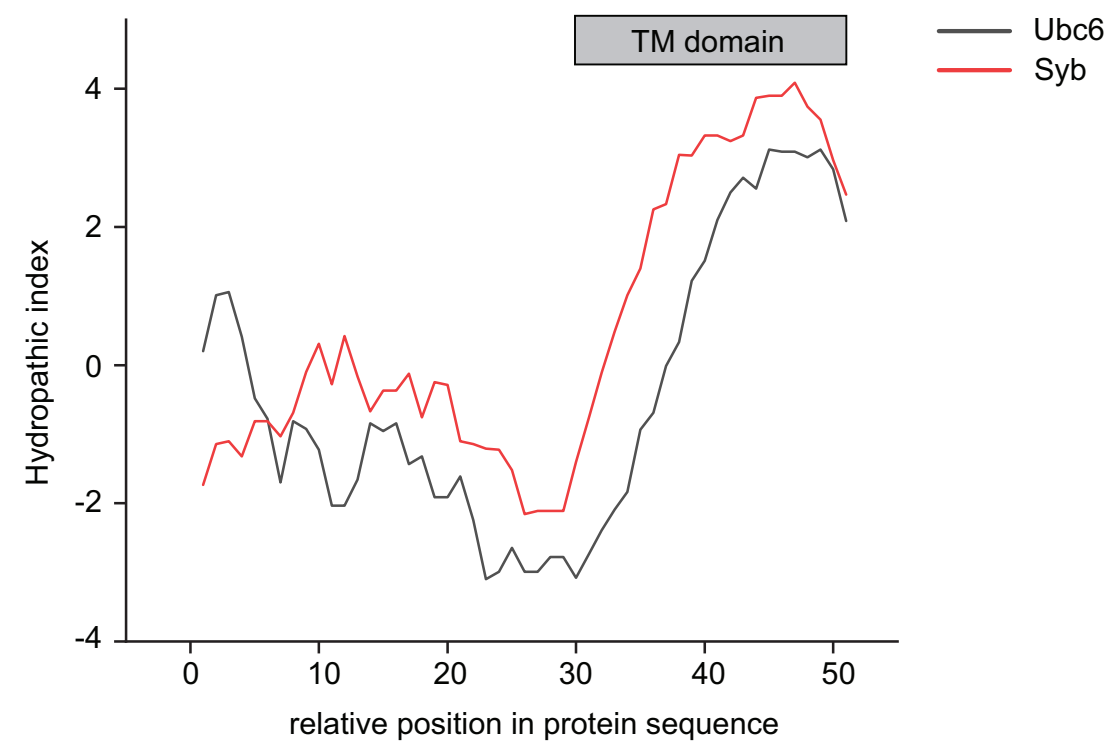

Figure 7.1: Hydropathy profile of Ubc6 and Syb. Hydropathy of the last 50 residues of Ubc6 and Syb (containing the TM anchor) was analyzed according to Kyte and Doolittle (1982) using the ExPasy ProtScale tool (Gasteiger et al., 2005). The position of the TM anchor of Ubc6 and Syb is indicated by a grey box. Hydropathy index as defined by Kyte and Doolittle (1982). The sum of hydropathy values of 9 surrounding residues was calculated for each position (window size $=9$ ).

Ubiquitination experiments indicate that Doa10 indeed recognizes the Ubc6 TM anchor. In contrast to Ubc6 WT, polyubiquitination of a swap mutant containing the Ubc6 cytosolic domain fused to the Syb TM anchor is compromised (Figure 3.6DI). Whereas the kinetics of a single ubiquitin transfer are similar for WT Ubc6 and Ubc6 $6_{\text {SybTM }}$, the kinetics are different when looking at the successive transfer of multiple ubiquitins and the ubiquitin chain length. Thus, the identity of the TM segment is important for polyubiquitination. The ubiquitination experiments thus indicate that Doa10 interacts with the Ubc6 TM anchor, whereas the interaction of Doa10 with the Syb TM anchor is compromised. Interestingly, a Doa10 fragment containing TM segments 3-14 is not only important for retrotranslocation of Ubc6, but is also required for efficient ubiquitination of Ubc6 (Figure 3.5D-H). Concluding, an intramembrane interaction of Doa10 with Ubc6 is required for efficient ubiquitination. This interac- 
tion leads to Doa10-mediated retrotranslocation of the TM anchor of Ubc6. Lacking interaction of Doa10 with the Syb TM anchor compromises ubiquitination as well as Doa10-mediated retrotranslocation. In line with this, experiments in intact cells also indicate that the Ubc6 TM anchor is important for ERAD of Ubc6. The normally stable, soluble E2 enzyme Ubc4 becomes unstable when attached to the TM anchor of Ubc6 (Walter et al., 2001).

\subsubsection{Luminal unfolding occurs concomitant with protein extraction}

Doa10 substrates do not necessarily only contain cytosolic and TM domains, but can also contain luminal domains. I thus tested how a folded luminal domain affects retrotranslocation. To mimick a folded luminal domain, I used a luminal SBP-tag that forms a complex with streptavidin. Doa10-mediated retrotranslocation of Ubc6 is inhibited by a folded luminal domain on Ubc6 (Figure 3.7A-C). Retrotranslocation of this substrate requires ubiquitination and the Cdc48/UN complex (Figure 3.7D-F). During this process, streptavidin stays inside the liposome lumen, whereas the luminal SBP-tag is retrotranslocated to the outside (Figure 3.7G,H). Release of streptavidin from the SBP-tag can be viewed as an unfolding process. This indicates that unfolding of a luminal domain occurs together with substrate extraction. Unfolding at the luminal side is achieved by Cdc48 action on the cytosolic side. Doa10 likely contributes to this process occurring across the membrane by acting as a retrotranslocase.

\subsubsection{Role of the retrotranslocase activity of Doa10}

Our results establish that Doa10 facilitates retrotranslocation of Ubc6 in a reconstituted system. We hypothesize that Doa10 forms a protein conduit for retrotranslocation of other substrates as well. Doa10 is involved in degradation of membrane proteins containing multiple transmembrane segments as well as luminal domains. Examples for such substrates are the multipass transmembrane proteins Ste6* and Pma1 (D378S). Also, an artificial substrate has been described whose luminal domain consists of the $60 \mathrm{kDa}$ protein hemagglutinin neuraminidase and whose degradation partially depends on Doa10 (Vashist and Ng, 2004). The retrotranslocase function of Doa10 might be even more relevant for extraction of such substrates.

It is still unclear how Doa10 forms such a protein conduit. Structural studies are required to get further mechanistic insight. Moreover, we still lack direct evidence that Doa10 and the Cdc48-complex cooperate in extraction. Further experiments are necessary to understand the role of the Doa10 protein conduit during Cdc48-mediated extraction. Besides facilitating retrotranslocation, Doa10 might also be important 
for maintaining the integrity of the ER membrane and for avoiding leakage of small molecules during the extraction process.

\subsubsection{The role of protein conduits for dislocation and insertion of mem- brane proteins into the lipid bilayer}

Dislocation of membrane proteins and the role of protein conduits in this process is investigated not only at the ER membrane. Examples of other dislocation systems can be useful in understanding retrotranslocation in ERAD and thus are introduced and discussed in the following section.

During dislocation of membrane proteins, AAA ATPases do not always cooperate with the ubiquitination machinery, but also can function independent of it. Those AAA ATPases are not soluble like Cdc48, but are anchored to the membrane via one or more TM segments of each of their subunits. Such a dislocation system is often associated with not only an unfolding but also a proteolytic activity of the AAA ATPase (reviewed by Glynn (2017)). Examples are FtsH at the plasma membrane, and its homologs i-AAA as well as m-AAA at the inner mitochondrial membrane (MIM). They all possess proteolytic activity and are involved in the dislocation of membrane proteins into the cytosol (FtsH), intermembrane space (i-AAA) or mitochondrial matrix (m-AAA) (Glynn, 2017).

It has been hypothesized that the TM domain of those AAA ATPases is important for dislocation of membrane proteins. Some studies using mutational analysis of the TM segments indicate a role of the TM domain besides anchoring the AAA ATPase to the membrane (Korbel et al., 2004; Lee et al., 2017). Another example of a membraneembedded AAA ATPase is Msp1. Msp1 is non-proteolytic and is localized at the outer mitochondrial membrane (Nakai et al., 1993). It is involved in proteasomal degradation of tail-anchored proteins (Chen et al., 2014; Okreglak and Walter, 2014) and it has been shown that it is sufficient for dislocation of a tail-anchored protein from the membrane in a reconstituted system using purified components reconstituted into liposomes (Wohlever et al., 2017). Swapping the Msp1 TM domain to the TM domain of Tom70 did neither lead to a phenotypic difference in vivo nor affect dislocation in vitro suggesting that the TM domain of Msp1 was not required for substrate degradation. However, the effect of the TM domain on the kinetics of the dislocation reaction has not been shown yet. Concluding, it has been hypothesized that the TM domain of at least some AAA ATPases is involved in dislocation of membrane proteins, which is supported by some experiments. This suggests that the presence of a protein conduit might be a universal mechanism for the dislocation of membrane proteins. 
Whereas for dislocation of membrane proteins, the presence and role of protein conduits is still unclear, the requirement of protein conduits for the opposite reaction is better understood. The mechanism of insertion for tail-anchored proteins by the GET-pathway provides here a good example, as it is only responsible for insertion of TM segments but is not required to form a protein conduit for luminal segments. The Get1/Get2 heterodimeric complex functions as an insertase that is sufficient to release the tail-anchored protein from Get3 and insert it into the lipid bilayer (Mariappan et al., 2011). It has been shown that the TM domain of Get1/Get2 is not only required to anchor the complex to the membrane, but also provides a binding site for the tailanchored protein and is required for insertion (Wang et al., 2014). This indicates that a protein conduit is required for the delivery of TM segments from the aqueous phase into the lipid environment. Speculatively, this suggests that a protein conduit is also involved in the opposite reaction, such as retrotranslocation of ERAD substrates.

\subsubsection{Role of Ubx2 and Dfm1}

Doa10-mediated retrotranslocation of Ubc6 does not require any other membrane component in a reconstituted system (Figure 3.4). Moreover, the established reconstituted system is sufficient for mediating retrotranslocation of polyubiquitinated Ubc6 (Figure 3.3). Experiments in intact cells indicate that two other components are involved in retrotranslocation, at least for some Doa10 substrates. The membrane protein Ubx2 has been shown to recruit the Cdc48-complex to the Doa10 complex. This recruitment function might be more important in a cellular context than in a reconstituted system. Cdc48 (in complex with different co-factors) carries out many different functions in the cell (Ye et al., 2017). Thus, Ubx2 might be required to ensure that a pool of Cdc48 is localized at the ER. In addition, in our reconstituted system, only $40 \%$ of polyubiquitinated Ubc6 is retrotranslocated. Ubx2 might increase this release efficiency by increasing the local concentration of Cdc48 at the membrane.

The function of the multipass membrane protein Dfm1 is less clear. It has been recently shown that it is required for retrotranslocation of Ste6* (Neal et al., 2018). It probably forms oligomers (Goder et al., 2008), suggesting that it might be involved in the formation of a protein conduit for retrotranslocation of membrane proteins. However, it remains to be tested if it is also involved in retrotranslocation of Doa10 substrates other than Ste6*. Several observations indicate that Dfm1 has multiple roles at the ER membrane.

Dfm1 interacts with components of the Doa10 and Hrd1 complex, which were identified by co-immunoprecipitation of Dfm1 and subsequent mass-spectrometric analy- 
sis (Goder et al., 2008). This study also identified multiple UBX-domain containing proteins. Besides Ubx2, Dfm1 also interacts with Ubx1 and Ubx7. All of these UBXdomain containing proteins interact with Cdc48 (Schuberth et al., 2004). Moreover, binding of Ubx1 and Ubx2 to Cdc48 is mutually exclusive indicating that complexes contain either Ubx1 or Ubx2 (Wilson et al., 2006). Interestingly, both Ubx2 and Dfm1 recruit Cdc48 to the ER membrane. However, only Ubx2 is required for the interaction of Cdc48 with Doa10, suggesting that Dfm1 recruits Cdc48 to the ER membrane when it is not part of the Doa10 complex (Stolz et al., 2010; Schuberth and Buchberger, 2005; Neal et al., 2018). This suggests that Dfm1 might exist in different complexes at the ER. Whereas a complex of Dfm1, Ubx2 and Doa10 (and other ERAD components) is responsible for ERAD of at least some Doa10 substrates, complexes containing Dfm1 and Ubx1 also exist. Interestingly, Ubx1-dependent proteasomal degradation of a substrate has been observed. Asi1 is unstable and its degradation depends on Ubc6, Ubc7, Cue1, Ubx1 and Cdc48 (Pantazopoulou et al., 2016). However, its degradation is independent of the known ER or INM ligases. This indicates that multiple complexes are present at the ER and INM membrane that are involved in proteasomal degradation and that differ not only in their associated ubiquitin ligases but also in accessory components such as Dfm1 and UBX-domain containing proteins.

Using retrotranslocation assays from microsomes it has been shown that increasing the hydrophobicity of TM segments decreases the retrotranslocation efficiency of Doa10 substrates (Guerriero et al., 2017). Thus, retrotranslocation of a multipass TM protein might have different requirements compared to a singlepass TM protein. Speculatively, Dfm1 might cooperate with Doa10 in retrotranslocation of multispanning membrane proteins such as Ste6*. The established reconstituted system can provide a useful tool to study the function of these additional components.

\subsection{Towards structural insights of Doa10}

Multiple conserved regions have been identified in Doa10 such as the TD-domain and the C-terminal element (Swanson et al., 2001; Zattas et al., 2016). Using Doa10 truncations, I have shown that the Doa10 region containing the TM segments 3-14 which contains both the TD-domain as well as the CTE is required for efficient ubiquitination and Doa10-mediated retrotranslocation of Ubc6 (Figure 3.5). 


\subsubsection{Function of the TD-domain}

Mutational analysis of TM5 of Doa10 has identified conserved residues in the TDdomain whose mutation leads to stabilization of Doa10 substrates. Mutation of a motif containing a conserved proline and glycine residue (Doa10 ${ }_{\mathrm{P} 638 \mathrm{~A}} \mathrm{G} 642 \mathrm{~A}$ ) leads to stabilization of a membrane protein (Ubc6) and a soluble substrate (Deg1-Ura3) (Kreft and Hochstrasser, 2011). As the TD-domain comprises mainly membrane-spanning segments, this region might be important for recognition of intramembrane degrons. Kreft and Hochstrasser (2011) have shown that mutation of the residue E633 in the TDdomain affects degradation of Ubc6, but not Vma12-DegAB which contains a cytosolic degron. In our reconstituted system, I have observed no strong differences in Ubc6 polyubiquitination (Figure 5.1).

The discrepancy I observe between my experiments in a reconstituted system compared to studies in intact cells can have multiple reasons. First, I might have performed this experiment under conditions where not Doa10 but another ubiquitination component like Ubc7/Cue1 is limiting which would hide the differences between the Doa10 mutants. Moreover, the difference in vitro might be smaller than the observed difference in vivo due to the absence of competing factors such as deubiquitinases. It has been shown in a reconstituted system that deubiquitination and ubiquitination are competing processes that enhance the discrimination between substrates and nonsubstrates (Zhang et al., 2013).

Third, mutation of conserved residues in the TD-domain affects Doa10 protein levels in vivo (Kreft and Hochstrasser, 2011). Interestingly, Doa10 levels thereby correlate with Ubc6 degradation rates. Compared to WT Doa10, Doa10 6 633Q protein levels are higher and Ubc6 is degraded faster. In contrast, Doa10E633D protein levels are lower and Ubc6 degradation is slowed down. Although this study shows that degradation of a Deg1-containing protein is not compromised to ensure that different Doa10 levels are not the reason for the observed effects, these differences in Doa10 protein levels might have affected degradation of Ubc6.

In addition, mutation of these Doa10 residues might not affect substrate ubiquitination, but rather another step of ERAD. We therefore tested Doa10 mutants described by Kreft and Hochstrasser (2011) for retrotranslocation activity using a fluorescencequenching assay. However, I observed similar retrotranslocation kinetics (Figure 5.2). The methods I have used up to now for measuring Doa10-mediated retrotranslocation are most likely not suitable to measure the kinetics of retrotranslocation and thus monitor differences in retrotranslocation activity. The described assays rely on binding of an antibody, protease cleavage or chaperone binding. Thus, the observed kinetics of 
Ubc6 retrotranslocation depend on the activity of the antibody, protease and chaperone. Concluding, different tools and a more detailed analysis will be necessary to better characterize the behaviour of these Doa10 mutants.

Mutational analysis of the TD-domain of Doa10 has led to a model for the interaction of Doa10 and Ubc6. Kreft and Hochstrasser (2011) showed that when E633 of Doa10 is mutated to glutamine, Ubc6 is degraded faster than in wildtype cells. Interestingly, an inactive Ubc6 version that is stable in wildtype cells, is degraded in cells expressing Doa10E633Q. This degradation is dependent on the presence of Ubc6 WT in the background. The authors hypothesized that Doa10 contains two binding sites for Ubc6, depending on if it acts as E2 enzyme or substrate. Ubc6 transfer from the E2 binding site to the substrate binding site would usually require its intramolecular autoubiquitination. However, in Doa10 E633Q, non-ubiquitinated, catalytically inactive Ubc6 might enter the substrate binding site and a second molecule of WT Ubc6 bound to the E2 binding site might ubiquitinate inactive Ubc6 leading to its Ubc7/Cue1 dependent polyubiquitination. Our results suggest that such a ubiquitination-dependent transfer to a substrate-binding site probably does not occur. This is based on the observation that Doa10 retrotranslocates non-ubiquitinated Ubc6 suggesting that mono-ubiquitination is not required for substrate recognition by Doa10. To further understand the behaviour of Doa10 variants containing a mutation of E633, it would be useful to test if E633 in Doa10 is also important for the degradation of substrates containing an intramembrane degron, or if it is specifically required for Ubc6.

\subsubsection{Identification of interaction sites of the TM anchor of Ubc6 with Doa10 by site-specific crosslinking}

The structural information on the TM region of Doa10 is mostly limited to mutational analysis of the TD-domain (Kreft and Hochstrasser, 2011; Weber et al., 2016). I therefore wanted to identify regions in Doa10 by characterizing the interaction site between Ubc6 and Doa10. Site-specific photocrosslinking is thereby a useful approach. It allows crosslinking of preferentially membrane segments by incorporating the crosslinker in a TM domain. We thus incorporated the photoactivable unnatural amino acid BpA at different positions of the Ubc6 TM anchor. Although we were able to generate crosslinks of Ubc6 $6_{\mathrm{BpA}}$ with Doa10, we did not identify any crosslinked peptides by mass spectrometry (Figure 5.3). This could be due to technical problems associated with the mass-spectrometric analysis of hydrophobic transmembrane segments which is challenging (Calabrese and Radford, 2018). Moreover, a Ubc6 $6_{\mathrm{BpA}}$ variant might 
crosslink to different residues of Doa10 and thus lead to heterogeneity of the sample.

Thus, alternative approaches that do not rely on mass-spectrometry might be useful to identify interaction sites. A Doa10 mutant containing no cysteines (except the RING domain cysteines) would be a useful tool, in case its function is not compromised. Such a Doa10 variant would allow the use of membrane permeable crosslinkers that can be conjugated to cysteines introduced at specific sites of Doa10 via maleimide chemistry. Such an approach has been used to identify interaction sites of the TM domain of Get1/Get2 with the TM anchor of a tail-anchored protein (Wang et al., 2014).

A potentially interesting and not-well studied domain of Doa10 is a putative WWdomain (aa 781-805) (Swanson et al., 2001). WW-domains are 30 aa long domains that often interact with proline-rich ligands and are defined by conserved tryptophane and proline residues (Sudol et al., 1995). 13 WW domains have been identified in S. cerevisiae, such as in the ubiquitin ligases Rsp5 and Doa10 (Hesselberth et al., 2006). Rsp5 has been shown to interact via its WW-domain with a proline-rich motif of an Rsp5 substrate (Shcherbik et al., 2004). Interestingly, Ubc6 and also Doa10 contain a putative WW-domain interaction motif (Swanson et al., 2001; Hesselberth et al., 2006). Indeed, besides interaction with substrates, it has been hypothesized that the WW-domains of Rsp5 and Doa10 also mediate self-interaction, due to the presence of conserved WW-domain binding sites (Hesselberth et al., 2006). However, in contrast to the WW-domain binding site in Doa10, the Doa10 WW-domain itself is not conserved (Hesselberth et al., 2006) and thus might not have a general role for Doa10.

In summary, the identification of structural elements in Doa10 is a challenge especially due to its large TM domain. In vitro approaches are required to complement the results from in vivo mutational analyses. Cross-linking approaches that do not necessarily rely on mass-spectrometric analysis as well as structural studies could give further insight in the future.

\subsection{The machinery for ERAD of Sbh2}

The list of identified Doa10 substrates that are membrane proteins has been growing in the last years. Such membrane-spanning Doa10 substrates have expanded our knowledge of the function of Doa10-mediated ERAD. A defect in biosynthesis per se is hereby not the only signal for degradation. Instead, Doa10-mediated ERAD also occurs in a regulated manner as for squalene monooxygenase which is regulated by intermediates of the sterol synthesis pathway (Foresti et al., 2013). The tail-anchored 
protein Sbh2 is degraded upon deletion of its interaction partner, the translocon subunit Ssh1 (Habeck et al., 2015). For Sbh2 it has been shown that its TM anchor is required for its degradation. The components required for recognition, ubiquitination as well as retrotranslocation of a substrate containing such an intramembrane degron could differ from substrates containing a soluble degron such as Deg1. As screens have been only carried out for such soluble substrates, we performed a screen for components required for degradation of a protein containing a TM domain. We chose the substrate Sbh2 for our screen as it is a well-established substrate of Doa10.

\subsubsection{Tandem fluorescent timer screens are a useful screening tool}

Different screening approaches exist to identify components required for degradation of a protein of interest. These approaches are often based on fusing a reporter protein to a protein of interest. Many reporter proteins are enzymes whose activity is used to measure the stability of the fusion protein. One example for a reporter protein is Ura3, an enzyme involved in uracil biosynthesis. Stabilized substrate allows growth of an auxotrophic strain on medium lacking uracil. Another example is betagalactosidase that cleaves the added chromogenic compound X-Gal. Both of these approaches have been used to characterize the degradation machinery of Deg1-containing fusion proteins (Johnson et al., 1998; Swanson et al., 2001). Recently, a quite sensitive screening method has been developed by the Knop lab termed tandem fluorescent timer screen (Khmelinskii et al., 2012). In this method, two fluorescent proteins with different maturation kinetics are fused to the substrate and thus a readout for protein abundance as well as stability can be provided. Moreover, the fluorescent labeling allows for mea-

suring of not only whole colonies but also single cells. A microscopy-based approach is hereby useful, as the cellular localization of the protein in every sample can be analyzed as well. Moreover, by using the intensity of the fast maturing fluorescent protein, the intensity of the slowly maturing fluorescent protein can be normalized. This eliminates effects on protein abundance during protein synthesis and allows to specifically characterize protein degradation kinetics. Tandem fluorescent timer screens have been used to identify components of the N-end rule pathway as well as components required for degradation of a mislocalized tail-anchored protein (Khmelinskii et al., 2012; Dederer et al., 2019).

\subsubsection{Screening for components required for degradation of Sbh2}

Using this screen, we have identified known components of the ERAD machinery (Figure 6.3). We did not identify any other gene deletion as a similarly strong hit, suggesting 
that all components required for ERAD of Sbh2 might be identified. This is in agreement with a previous study by Carvalho et al. (2006) that has identified components of the Doa10 complex by pulldown of Doa10 and subsequent mass-spectrometric analysis. The results from the screen suggest that all components required for ERAD of a protein containing an intramembrane degron are identified. Moreover, by establishing a reconstituted system, I characterized a minimal machinery for ubiquitination of Sbh2 (see section 7.5.4).

\subsubsection{Potential role of the deubiquitinase Ubp3}

Interestingly, the deubiquitinase Ubp3 appeared as a potential hit in our screen. I verified these results by creating a UBP3 deletion. I was able to confirm the results from the screen using a tFT-construct of Sbh2. However, when I measured the degradation kinetics of Sbh2 using a CHX-chase, I did not observe any strong impairment of Sbh2 turnover (Figure 6.4). This could be a result of tagging, as the tFT-construct contains a GFP-mCherry tag, whereas the CHX-chase construct contains only an HA-tag. The GFP-mCherry tag could create an artefact in $\Delta$ ubp3 cells. However, an alternative explanation is that the CHX-chase is not suitable to report on Sbh2 turnover, as the UBP3 deletion strain grew very slowly and this might have affected the experiment. Whereas strains from an overnight culture were used for measuring the tFT-construct, exponentially growing cells were used in the CHX-chase. Moreover, due to the fast degradation kinetics of Sbh2, the timepoints chosen were not useful to characterize degradation kinetics. Thus, further experiments are required to determine if Ubp3 is involved in ERAD.

What would be a potential role of Ubp3 in ERAD? Deletion of UBP3 stabilizes Sbh2 indicating that Ubp3 promotes ERAD and does not compete with it. It would therefore not be involved in processes such as substrate deubiquitination. This deubiquitinase could either act on Sbh2 downstream of Cdc48 by for example promoting release of substrate from $\mathrm{Cdc} 48$. It has been shown that deubiquitination occurs after Cdc48 action and is required for substrate release from Cdc48 (Stein et al., 2014; Bodnar and Rapoport, 2017b). Whereas the deubiquitinase Otu1 can carry out this function in a reconstituted system, its deletion does not impact ERAD in S. cerevisiae, suggesting the presence of multiple deubiquitinases (Stein et al., 2014). Interestingly, Ubp3 interacts with Cdc48 (Ossareh-Nazari et al., 2010a) and deletion of UBP3 suppresses the temperature-sensitive lethality of the npl4-1 mutant allele (Auld et al., 2006), suggesting that Ubp3 is involved in Cdc48-associated processes.

Alternatively, Ubp3 acts on Doa10 itself. An interesting hypothesis hereby is that 
Ubp3 deubiquitinates autoubiquitinated Doa10 and thus ensures that Doa10 is not subjected to proteasomal degradation. Autoubiquitination of E3-ligases is often used as a readout for E2 activity in vitro. However the relevance of E3 autoubiquitination in vivo is for most E3 ligases not clear. Some examples exist where autoubiquitination leads to either proteasomal degradation or catalytic activation of E3 ligases (reviewed by Deshaies and Joazeiro (2009)). Recent studies indicate that autoubiquitination of Hrd1 has a role in ERAD. Although Hrd1 is stable in wildtype cells, some level of autoubiquitination of Hrd1 can be detected in intact cells (Baldridge and Rapoport, 2016). Upon mutation of certain lysine residues in Hrd1, substrate degradation is compromised, indicating that autoubiquitination is important for Hrd1 function (Baldridge and Rapoport, 2016).

In contrast to Hrd1, it is unclear if autoubiquitination plays a role for Doa10 function. In S. cerevisiae, Doa10 is stable as analyzed by pulse-chase experiments (Zattas et al., 2016). However in the mammalian system, the Doa10 homolog MARCH6 is unstable and its degradation depends on its own activitiy indicating that autoubiquitination of MARCH6 leads to its degradation (Hassink et al., 2005; Zattas et al., 2016). Our reconstituted system shows that Doa10 is autoubiquitinated in the presence of Ubc6 and Ubc7/Cue1 (Figure A2F and A2G). When Doa10 is autoubiquitinated, it gets extracted by the Cdc48-complex to some extent (Figure A3J). Interestingly, the autoubiquitination activity of Doa10 seems to be not required for polyubiquitination or extraction of Ubc6, as $\mathrm{Ub}-\mathrm{Ubc} 6_{\mathrm{C} 87 \mathrm{~A}}$ is ubiquitinated and extracted, in the absence of Doa10 autoubiquitination due to the absence of Ubc6 activity (Figure 3.3F and 3.3G). Moreover, Doa10-mediated retrotranslocation of Ubc6 occurs in the absence of ubiquitinaton machinery and thus also does not require Doa10 autoubiquitination. Concluding, autoubiquitination of Doa10 might be a side-product of its activity. To avoid proteasomal degradation in vivo, deubiquitination of Doa10, and thus Ubp3 might play a role.

As we did not identify any new component strongly required for degradation of Sbh2, I set out to determine the minimal machinery for ERAD of Sbh2. Therefore, I established a reconstituted system using purified components reconstituted into liposomes.

\subsubsection{Minimal machinery for ubiquitination of Sbh2}

In a reconstituted system, Uba1, Ubc6, Ubc7/Cue1 and Doa10 are sufficient to catalyze polyubiquitination of Sbh2 (Figure 6.8). My results further indicate that both Ubc6 and Ubc7 are involved in ubiquitination of Sbh2, as efficient polyubiquitination 
only occurs in the presence of both E2 enzymes. However, these ubiquitination experiments were done with a soluble fragment of Cue1 which reduces the efficiency of polyubiquitination (data not shown), and thus the difference might be less strong when full-length Cue1 is used. Nevertheless, both Ubc6 and Ubc7 seem to have a role in ubiquitination of Sbh2 in vitro, in agreement with stabilization of Sbh2 when UBC6 or UBC7 is deleted in vivo (Habeck et al., 2015).

\subsubsection{Ubc6 and Ubc7 have different functions in Doa10-mediated ERAD}

Multiple lines of evidence indicate that Ubc6 and Ubc7 have different functions in Doa10-mediated ubiquitination. Weber et al. reconstituted autoubiquitination of the Doa10 RING domain by Uba1, Ubc7 and soluble fragments of Ubc6 and Cue1 (Weber et al., 2016). The authors show that Ubc6 initiates a ubiquitin chain by attaching a single ubiquitin and Ubc7 is involved in forming a polyubiquitin chain. Also in our system, I observe that Ubc6 catalyzes monoubiquitination of itself, Doa10 and probably Sbh2 (Figures 3.1, A2F, A2G and 6.8). This monoubiquitination can happen on multiple residues, as I observe no difference of the ubiquitination pattern of Ubc6 when a ubiquitin variant is used in which all lysines are mutated to arginines (Figure A1L). Only in the presence of Ubc7/Cue1, polyubiquitin chains are formed on Ubc6, Doa10 and probably Sbh2. This task division of E2 enzymes is a common feature and probably serves efficient ubiquitination (Deshaies and Joazeiro, 2009). Sequential ubiquitination has also been observed for the E2 enzymes Ubc1 and Ubc4 that function with APC/C. Whereas Ubc4 initiates a ubiquitin chain, Ubc1 elongates this chain (Rodrigo-Brenni and Morgan, 2007). The human E2 enzyme Ubc13 (heterodimer with Msm2) only catalyzes polyubiquitination of its E3 ligase Brca1, when the mono-ubiquitinating E2 Ube2w is present (Christensen et al., 2007). Thus, E2 enzymes can be specific for chain initiation and elongation. Such a task divison also occurs for the Ubc6/Ubc7 pair.

\subsection{Outlook}

This thesis provides mechanistic insights into Doa10-mediated ERAD by recapitulating the ubiquitination and extraction steps using a reconstituted system. Using this system, many questions in Doa10-mediated ERAD can be addressed in the future. We still lack mechanistic information on the function of components such as Ubx2 and Dfm1. Moreover, it will be interesting to expand the substrate repertoire for reconstitution studies to get better insight into processes such as substrate recognition, ubiquitination as well as retrotranslocation. Recent evidence suggests that Doa10 has 
a conserved role in contributing to lipid homeostasis in the cell. Further understanding of Doa10-mediated ERAD of enzymes such as Erg1 and Pgc1 would contribute to deciphering the physiological role of Doa10.

The established reconstituted system is not only a useful tool to further characterize ERAD, but also other processes such as membrane-bound ubiquitination processes (e.g. EGAD at the Golgi), protein translocation across membranes (e.g. peroxisomal import) or dislocation of membrane proteins (e.g. membrane-bound mitochondrial AAA-ATPases).

Our results identify a dislocation activity of a transmembrane protein. This opens the door to many questions. First, it is unclear how Doa10 retrotranslocates Ubc6 and if this retrotranslocation function is relevant for other Doa10 substrates. Structural studies are required to understand this retrotranslocase activity mechanistically. Moreover, further insight into the cooperation of Cdc48 and Doa10 will be useful to understand the universally occuring process of dislocation of membrane proteins. 


\section{Supplement}

A

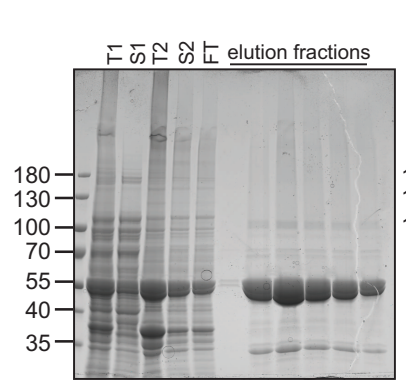

B

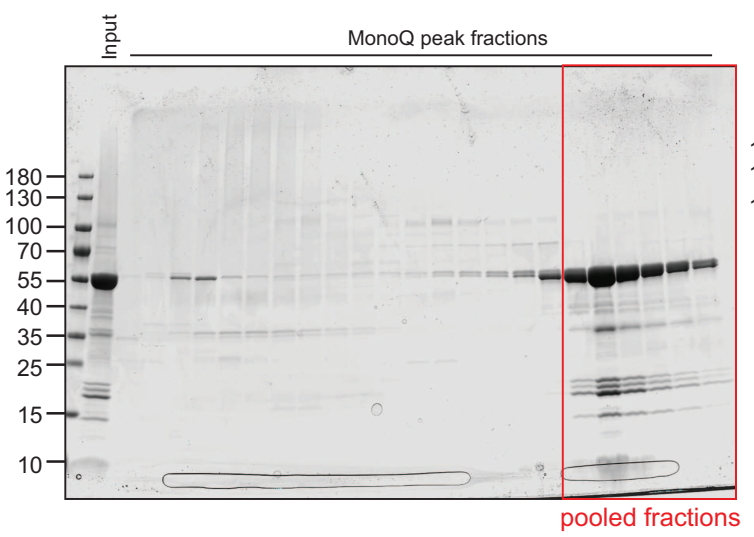

C

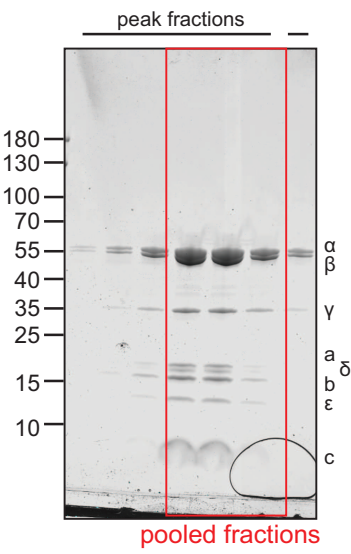

Figure S1: Purification of ATP synthase. ATP synthase was expressed and purified as described previously (Schenck et al., 2009). Samples from the purification procedure were analyzed by SDS-PAGE and Coomassie staining. (A) Samples from solubilization and Ni-NTA affinity chromatography. After lysis, sodium cholate was added (T1). After centrifugation, the supernatant (S1) was discarded and the pellet solubilized with DDM (T2). Unsolubilized material was pelleted by ultracentrifugation and the supernatant (S2) was used for affinity purification. FT indicates unbound material. Imidazole elution fractions are indicated. (B) Samples from MonoQ chromatography. (C) Samples from Superdex200 10/300 chromatography. 


\section{A}

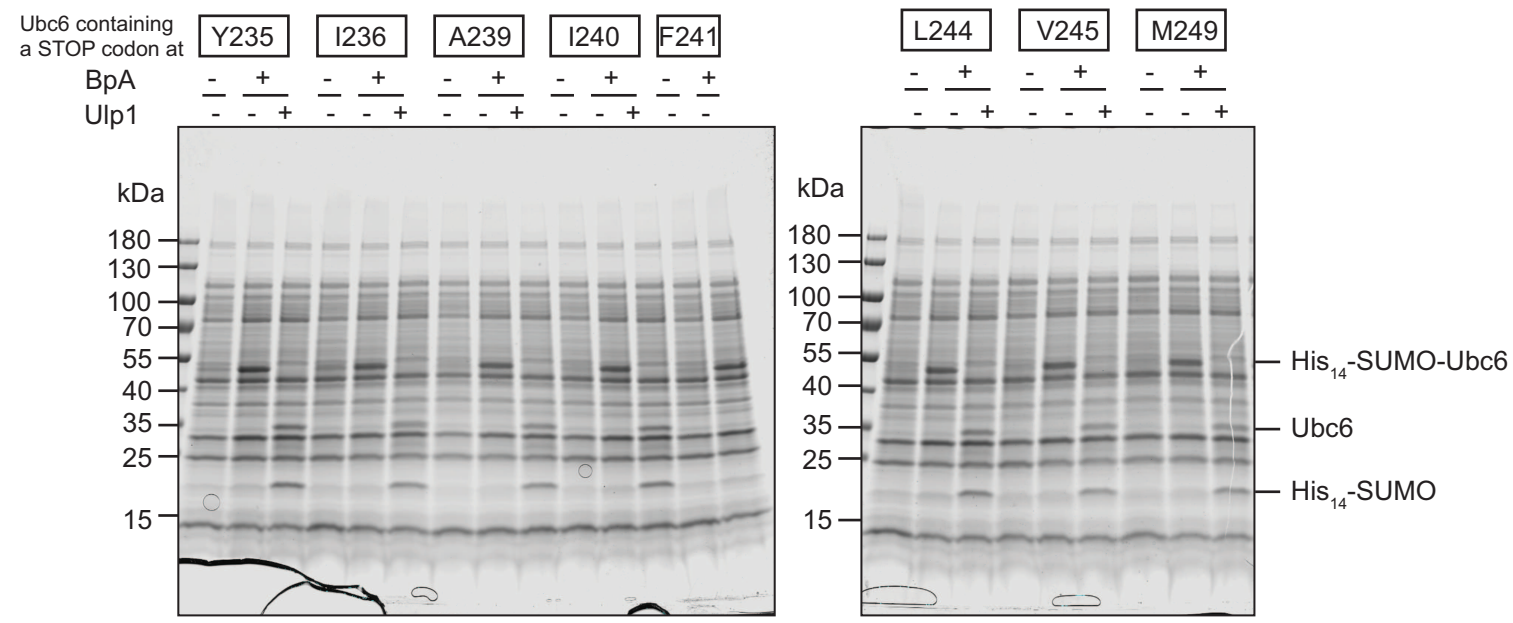

B

$\mathrm{Ubc6}_{\mathrm{BPA}} \frac{\mathrm{Y} 235}{\mathrm{~T} \text { I F } \underline{E(+U)}} \frac{1236}{\mathrm{~T} \text { I F } \underline{E(+U)}} \frac{\mathrm{A} 239}{\mathrm{~T} \text { I F } \underline{E(+U)}} \frac{1240}{T \text { I F } \underline{E(+U)}}$
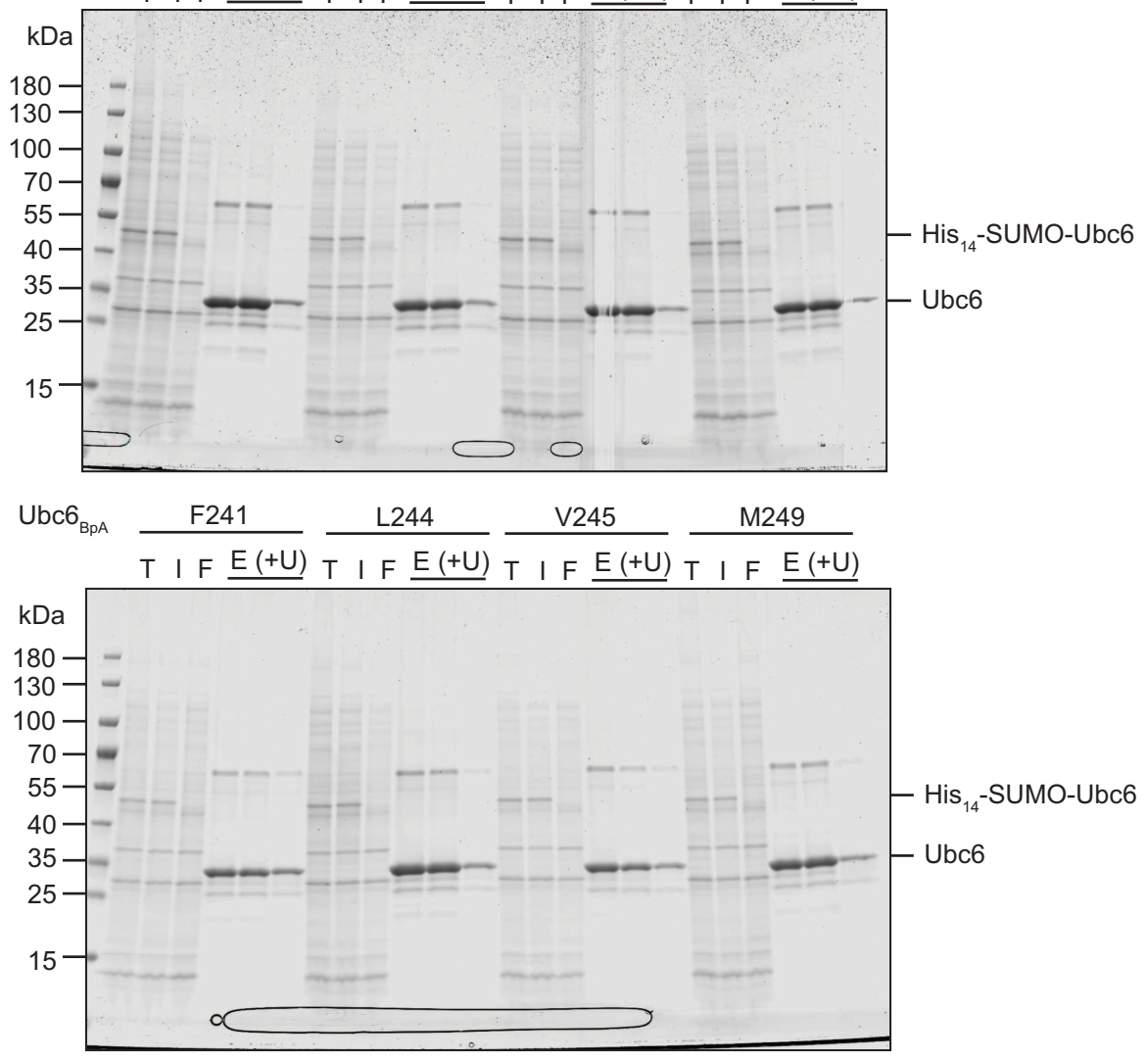

Figure S2: Purification of Ubc6 $\mathbf{B}_{\mathrm{BpA}}$ variants. (A) Test expression of Ubc6 variants containing an amber STOP codon at the indicated position. Expression in the presence $(+)$ or absence $(-)$ of $\mathrm{BpA}$ in the medium. Cells were lysed and membranes subsequently solubilized. (continued) 
Figure S2 (continued): Where indicated, the solubilized fraction was further treated with the SUMO protease Ulp1 to cleave His14-SUMO-Ubc6. Samples were analyzed by SDS-PAGE and Coomassie staining. (B) Purification of Ubc6 $6_{\mathrm{BpA}}$ variants. Samples of the solubilization reaction $(\mathrm{T})$, the supernatant after ultracentrifugation (I), the flowthrough from Ni-NTA affinity chromatography (F) as well as the Ulp1-elution fractions were analyzed by SDS-PAGE and Coomassie staining.

A

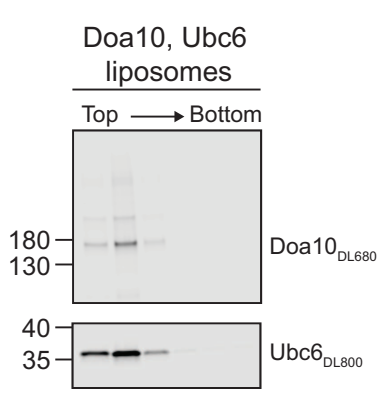

B

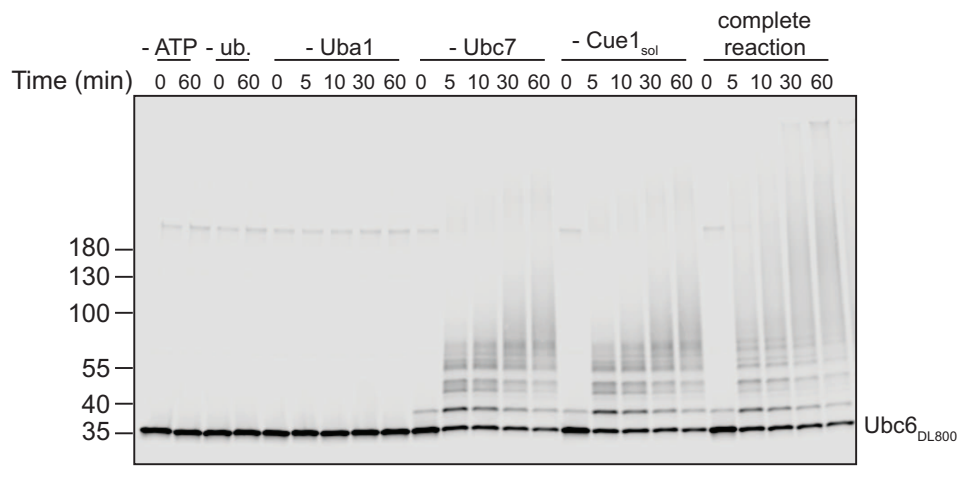

Figure S3: Direct co-reconstitution of Ubc6 and Doa10. Using a direct coreconstitution protocol, Doa10 purified in DMNG and Ubc6 were co-reconstituted. (A) Flotation of Doa10, Ubc6 liposomes in a Nycodenz step gradient. Samples from flotation fractions were analyzed by SDS-PAGE and fluorescence scanning. (B) Ubiquitination of Ubc6. Doa10, Ubc6 liposomes were prepared and incubated with ubiquitination machinery. Where indicated, one component was missing. A soluble Cue1 fragment was used (Cue1 aa 24-203). Final concentrations: $0.1 \mu \mathrm{M}$ Uba1, $1 \mu \mathrm{M}$ Ubc7, $1 \mu \mathrm{M}$ Cue1, $120 \mu \mathrm{M}$ ubiquitin, $2.5 \mathrm{mM}$ ATP, $0.1 \mu \mathrm{M}$ Ubc6, $0.1 \mu \mathrm{M}$ Doa10. 

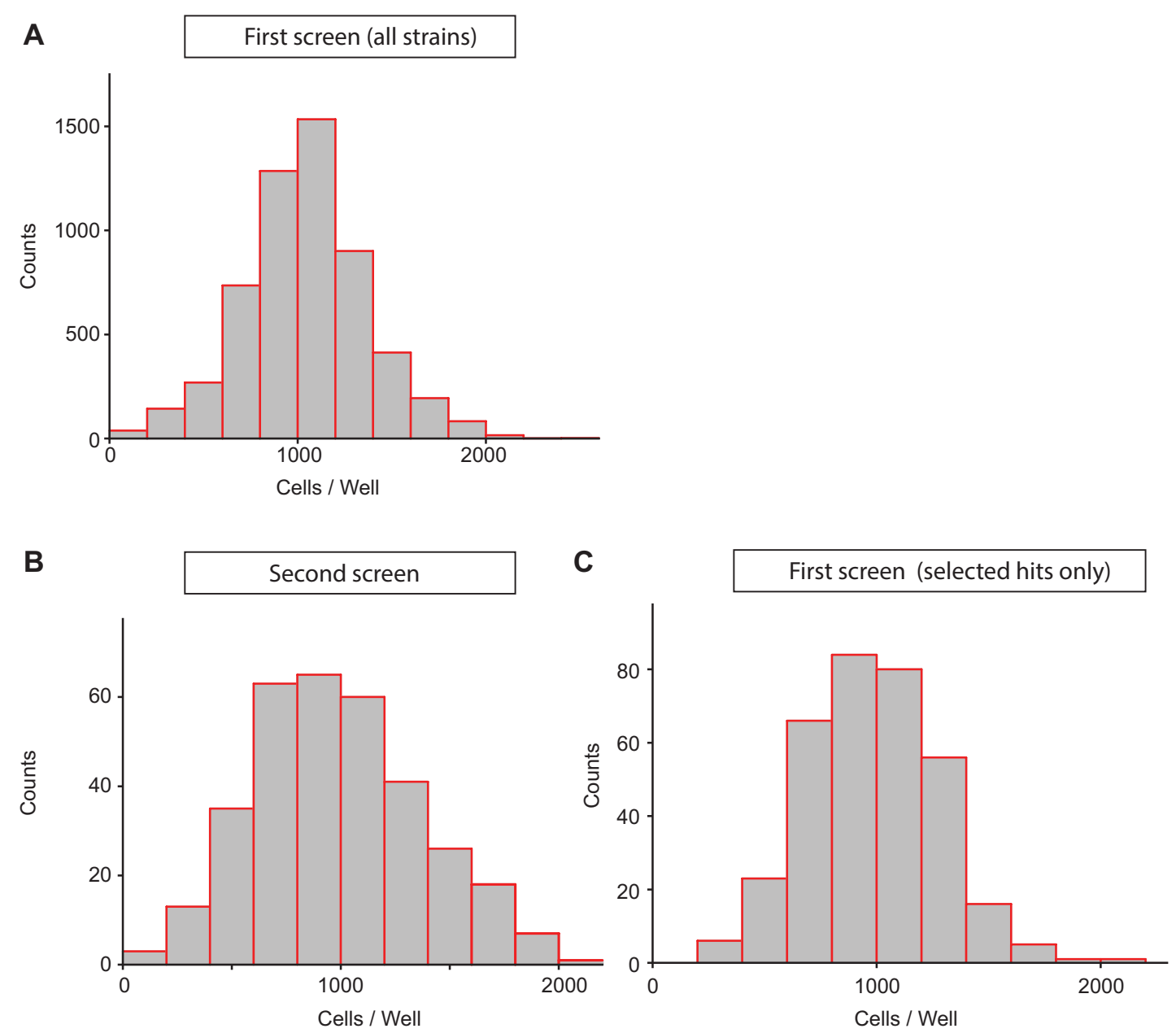

Figure S4: Distribution of cells per well. Histogram of cells per well for (A) first screen (B) second screen (C) first screen (only hits selected for second screen) 
A

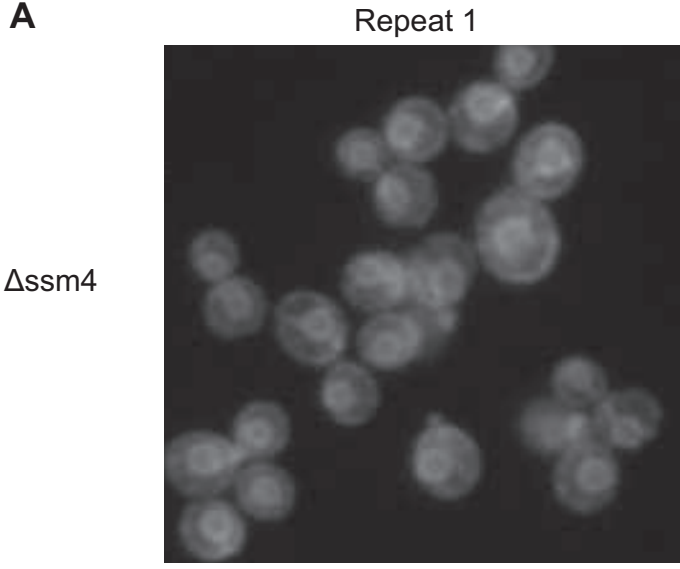

B

ubp3

C

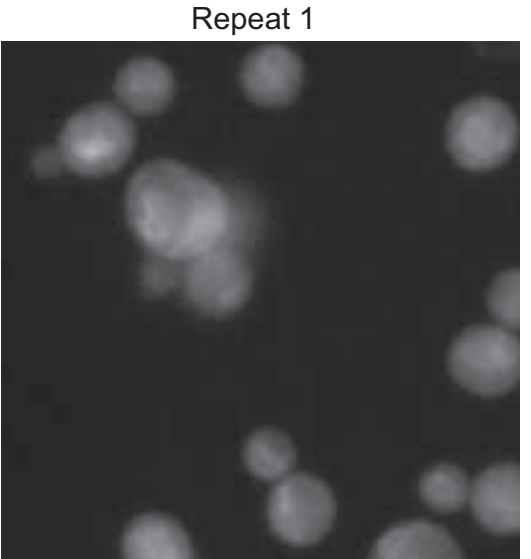

Repeat 1

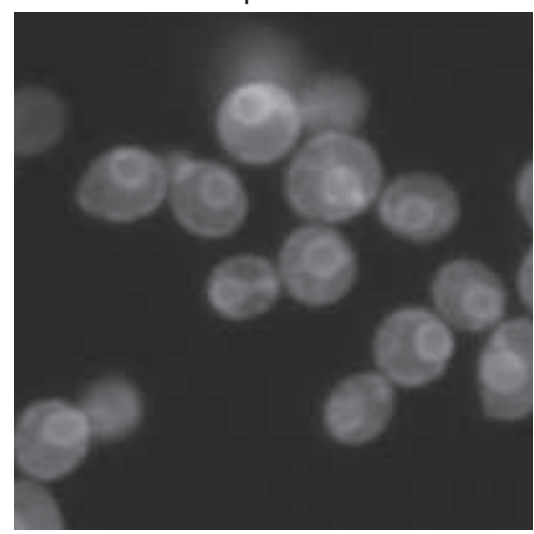

Repeat 2

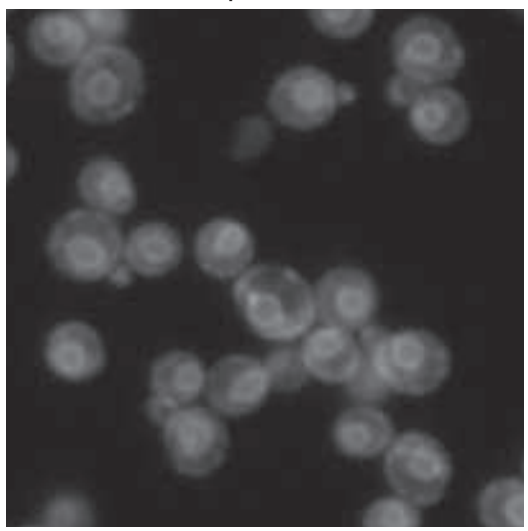

Repeat 2

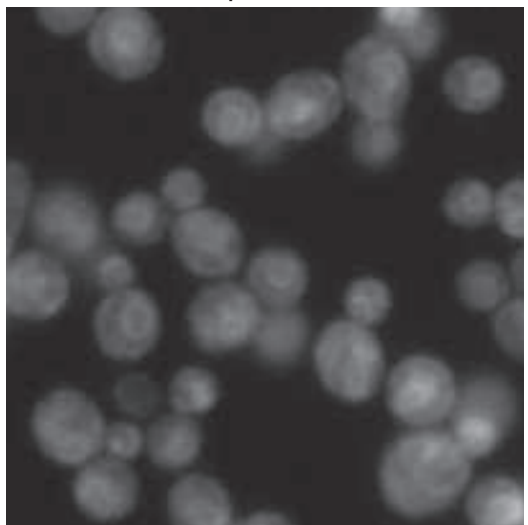

Repeat 2

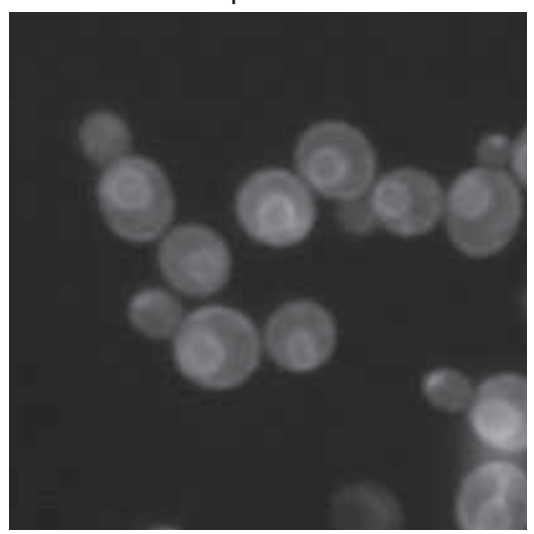

Figure S5: Localization of tFT-Sbh2 in $\Delta \mathrm{ssm} 4, \Delta \mathrm{ubp} 3$ and $\Delta \mathrm{ygl214}$ cells. GFP-fluorescence for a section of the whole image, for (A) $\Delta \operatorname{ssm} 4$, (B) $\Delta \mathrm{ubp} 3$, (C) $\Delta \mathrm{ygl} 214 \mathrm{w}$ strains. 
Table S1: Sequence coverage of Doa10 and Ubc6 after sequential digest with trypsin and chymotrypsin. Compared to digestion with only chymotrypsin (max. coverage for Doa10 83.1, for Ubc6 77.3), the sequential digestion resulted in higher sequence coverage. Mass spectrometric analysis performed by Iwan Parfentev (MPI for Biophysical Chemistry, Göttingen).

\begin{tabular}{l|c|c} 
Liposomes & Doa10 coverage (\%) & Ubc6 coverage (\%) \\
\hline \hline Doa10, Ubc6 $_{\mathrm{Y} 235-\mathrm{BpA}}$ & 91.8 & 87.1 \\
Doa10, Ubc6 $_{\mathrm{I} 236-\mathrm{BpA}}$ & 90.6 & 97.7 \\
Doa10, Ubc6 $_{\mathrm{A} 239-\mathrm{BpA}}$ & 92.3 & 88.7 \\
Doa10, Ubc6 $_{\mathrm{I} 240-\mathrm{BpA}}$ & 91.0 & 86.3 \\
Doa10, Ubc6 $_{\mathrm{F} 241-\mathrm{BpA}}$ & 91.6 & 94.9 \\
Doa10, Ubc6 $_{\mathrm{L} 244-\mathrm{BpA}}$ & 93.1 & 86.3 \\
Doa10, Ubc6 $_{\mathrm{V} 245-\mathrm{BpA}}$ & 91.6 & 87.9 \\
Doa10, Ubc6 $_{\mathrm{M} 249-\mathrm{BpA}}$ & 89.3 & 97.3
\end{tabular}


Table S2: Plasmids used in this thesis.

\section{Plasmid}

Number $\mid$ Source

\begin{tabular}{|c|c|c|}
\hline Doa101-468-SBP in pRS426-pGal1 & 376 & Stein lab \\
\hline Doa10 $_{225-1319-S B P}$ in pRS426-pGal1 & 375 & Stein lab \\
\hline pRS426-pGal1-SBP-SUMO* & 535 & this thesis \\
\hline SBP-SUMO*-Doa10434-1319 in pRS426-pGal1 & 557 & this thesis \\
\hline Ubc6 in K27SUMO & 343 & this thesis \\
\hline $\mathrm{Ubc}_{\mathrm{C} 87 \mathrm{~A}}$ in K27SUMO & 682 & this thesis \\
\hline Ub-Ubc6 ${ }_{\mathrm{C} 87 \mathrm{~A}}$ in K27SUMO & 702 & this thesis \\
\hline 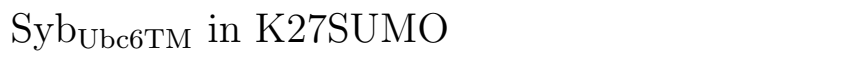 & 509 & this thesis \\
\hline Ubc6 $_{\text {SybTM }}$ in K27SUMO & 536 & this thesis \\
\hline Ub-Ubc6 $6_{\mathrm{C} 87 \mathrm{~A} / \mathrm{SybTM}}$ in K27SUMO & 815 & this thesis \\
\hline Ubc6 (incl. TEV cleavage site) in K27SUMO & 508 & this thesis \\
\hline Ubc6-SBP in pET39b $(+)$ & 633 & this thesis \\
\hline Cue1 in pET39b $(+)$ & 672 & this thesis \\
\hline Get3 in K27SUMO & 504 & this thesis \\
\hline Get3 D57N & 522 & this thesis \\
\hline Get3 I193D & - & $\begin{array}{l}\text { B. Schwappach } \\
\text { Mateja et al. (2009) }\end{array}$ \\
\hline $\begin{array}{l}\text { Syntaxin 1A 183-288, Syb2 49-96 in } \\
\text { pETDuet-1 }\end{array}$ & - & Stein et al. (2007) \\
\hline SNAP25Anocys in pET28a & - & $\begin{array}{l}\text { Fasshauer et al. } \\
\text { (1999) }\end{array}$ \\
\hline Synaptobrevin 2 in pET28a & - & Stein et al. (2007) \\
\hline Doa10 $_{225-1319, E 633 D^{-S B P}}$ in pRS426-pGal1 & 414 & this thesis \\
\hline Doa10 $_{225-1319, E 633 Q^{-S B P}}$ in pRS426-pGal1 & 415 & this thesis \\
\hline Doa10 $225-131$, G636R-SBP in pRS426-pGal1 & 589 & this thesis \\
\hline Doa10 $_{225-1319, P 638 A}$ G642A-SBP in pRS426-pGal1 & 579 & this thesis \\
\hline His $_{14}-\mathrm{SUMO}-\mathrm{Ubc6}$ in pBAD/HisC & 540 & this thesis \\
\hline His $_{14}$-SUMO-Ubc6Y235 TAG & 552 & this thesis \\
\hline His $_{14}$-SUMO-Ubc6 ${ }_{\text {I236 TAG }}$ & 553 & this thesis \\
\hline His $_{14}$-SUMO-Ubc6 ${ }_{\text {A239 TAG }}$ & 554 & this thesis \\
\hline His $_{14}-\mathrm{SUMO}-\mathrm{Ubc} 6_{\mathrm{I} 240} \mathrm{TAG}$ & 555 & this thesis \\
\hline His $_{14}$-SUMO-Ubc6 ${ }_{\mathrm{F} 241 \mathrm{TAG}}$ & 556 & this thesis \\
\hline His $_{14}$-SUMO-Ubc6 ${ }_{\text {L244 TAG }}$ & 561 & this thesis \\
\hline His $_{14}$-SUMO-Ubc6 ${ }_{V 245}$ TAG & 562 & this thesis \\
\hline His $_{14}$-SUMO-Ubc6 $6_{\mathrm{M} 249}$ TAG & 563 & this thesis \\
\hline
\end{tabular}


Table S2 (continued): Plasmids used in this thesis.

\begin{tabular}{l|c|l} 
Plasmid & Number & Source \\
\hline \hline $\begin{array}{l}\text { pGPD-sfGFP-mCherry-HA, pRS415 } \\
\text { (pMAM362) }\end{array}$ & - & $\begin{array}{l}\text { Khmelinskii et al. } \\
(2016)\end{array}$ \\
pGPD-sfGFP-mCherry-HA-Sbh2 in pRS415 & 511 & this thesis \\
pGPD-sfGFP-mCherry-HA-Sbh1 in pRS415 & 515 & this thesis \\
NatMX6-p3nmt1-3xFLAG in pFA6a & - & D. Moazed \\
NatMX6-pGPD-sfGFP-mCherry-HA in pFa6a & 590 & this thesis \\
HA-Sbh2 in pRS415-pGPD & 538 & this thesis \\
HA-Sbh289C in pRS415-pGPD & 699 & this thesis \\
HA-Sbh2 & 565 & this thesis \\
His ${ }_{14}$-SUMO-LPETG in pRS415-pGPD & 695 & this thesis
\end{tabular}

Table S3: S. cerevisiae strains used in this thesis.

\begin{tabular}{|c|c|c|}
\hline Strain & Background & Strain number \\
\hline BY4741 & $\begin{array}{l}\text { MATa his } 3 \Delta 1 \text { leu } 2 \Delta 0 \\
\text { met } 15 \Delta 0 \text { ura } 3 \Delta 0\end{array}$ & \\
\hline$\Delta$ doa10 & BY4741 & yAST099 \\
\hline Doa10-SBP (pRS426-pGal1) & $\Delta$ doa10 & yAST112 \\
\hline Doa10 $1-468_{-}$SBP (pRS426-pGal1) & $\Delta$ doa10 & yAST211 \\
\hline $\begin{array}{l}\text { SBP-SUMO*-Doa10 } \\
\text { (pRS4-1319 } \\
\text { (pR-pGal1) }\end{array}$ & $\Delta$ doa10 & yAST217 \\
\hline Doa10 $_{\text {E633D-SBP (pRS426-pGal1) }}$ & $\Delta$ doa10 & yAST127 \\
\hline Doa10 $_{\mathrm{E} 633 \mathrm{Q}}$-SBP (pRS426-pGal1) & $\Delta$ doa10 & yAST128 \\
\hline Doa10 $_{\mathrm{G} 636 \mathrm{R}} \mathrm{-SBP}$ (pRS426-pGal1) & $\Delta$ doa10 & yAST232 \\
\hline $\begin{array}{l}\text { Doa10 } 1038 \text { A G642A-SBP } \\
\text { (pRS426-pGal1) }\end{array}$ & $\Delta$ doa10 & yAST231 \\
\hline SGA-compatible query strain & $\begin{array}{l}\text { MAT } \alpha \text { his } 3 \Delta 1, \text { leu } 2 \Delta 0, \\
\text { LYS } 2+, \text { met } 15 \Delta 0, \\
\text { ura } 3 \Delta 0, \\
\text { can } 1 \Delta:: S T E 2 \text { prspHIS5, } \\
\text { lyp } 1 \Delta:: \text { STE3pr- LEU2 } \\
\text { (S288C background) }\end{array}$ & $\begin{array}{l}\text { kind gift from } \\
\text { M. Schuldiner }\end{array}$ \\
\hline pGPD-sfGFP-mCherry-Sbh2 & $\begin{array}{l}\text { MAT } \alpha \text { his } 3 \Delta 1, \text { leu } 2 \Delta 0, \\
\text { LYS2 }+, \text { met } 15 \Delta 0, \\
\text { ura3 } \Delta 0, \\
\text { can1 } \Delta:: \text { STE2prspHIS } 5, \\
\text { lyp1 } \Delta:: \text { STE3pr- LEU2 } \\
\text { (S288C background) }\end{array}$ & yAST229 \\
\hline
\end{tabular}


Table S4: Oligonucleotides used for DOA10 cloning. Gibson assembly kit was used for cloning, where indicated site-directed mutagenesis kit (SM) was used.

\begin{tabular}{|c|c|c|}
\hline PCR-product & Sequence & Number \\
\hline $\begin{array}{l}\text { fragments for } \\
\text { transformation } \\
\text { open \#375 } \\
\text { Doa10 Nterm } \\
\text { fragment } \\
\text { (template \#376) }\end{array}$ & $\begin{array}{l}\text { 5'AGAGAGGATGTTTTCAGTAAAATGG }{ }^{3} \text { ' } \\
\text { 5'CATGGTATTGGATCCACTAG } \\
\text { 5' } \\
\text { 5GTAATAAAGTATCAACAAAAATTGTTAATATAC } \\
\text { '' } \\
\text { 5'CCATCATGGGGAATCTTTC }{ }^{3}\end{array}$ & $\begin{array}{l}726 \\
688 \\
727 \\
728\end{array}$ \\
\hline \#535 & $\begin{array}{l}\text { 5' } \text { ACCGGATCGGACTCAGAAG }^{3} \\
\text { 5' } \\
\text { TGAGCTCTCGCTTCCGGA }\end{array}$ & $\begin{array}{l}1154 \\
1155\end{array}$ \\
\hline $\begin{array}{l}\# \mathbf{5 5 7} \\
\text { insert } \\
\text { (vector: } 535, \\
\text { XhoI, SfoI) }\end{array}$ & $\begin{array}{l}\text { 5' acagagaacagattggtggcGATCAAGACGAACAAGAC } \\
\text { 5' } \\
\text { ', acataactaattacatgaccttagcctcctgtttctggtagACT } \\
\text { TTCATCTGGTAAATTTTCTAAAG } \\
{ }^{3}\end{array}$ & $\begin{array}{l}1156 \\
1157\end{array}$ \\
\hline $\begin{array}{l}\# 414 \\
\text { SM, template: } \\
375\end{array}$ & $\begin{array}{l}\text { 5' }{ }^{2} \text { TTCATCGAtTTGGCTGGATTC } \\
\text { ' } \\
\text { 5' }{ }^{\prime} \text { ATAAAGTGAACACCTTGAAAG }\end{array}$ & $\begin{array}{l}807 \\
808\end{array}$ \\
\hline $\begin{array}{l}\# 415 \\
\text { SM, template: } \\
375\end{array}$ & $\begin{array}{l}\text { 5'ATTTTTCATCCAATTGGCTGGATTC }{ }^{3} \\
\text { 5'AAAGTGAACACCTTGAAAGTG }\end{array}$ & $\begin{array}{l}809 \\
810\end{array}$ \\
\hline $\begin{array}{l}\# \mathbf{5 8 9} \\
\text { SM, template: } \\
375\end{array}$ & $\begin{array}{l}\text { 5'CGAATTGGCTAGATTCCCCATTC }{ }^{3} \\
\text { 5'ATGAAAAATAAAGTGAACACCTTG }{ }^{3} \text { ' }\end{array}$ & $\begin{array}{l}1276 \\
1277\end{array}$ \\
\hline $\begin{array}{l}\# \mathbf{5 7 9} \\
\text { SM, template: } \\
375\end{array}$ & $\begin{array}{l}\text { 5' AGCTGCTGTAATGCTAGATTTCTCATTATTTTG }{ }^{3} \\
{ }^{5} \text { AGAATAGCGAATCCAGCCAATTCGATG }^{3}\end{array}$ & $\begin{array}{l}1274 \\
1275\end{array}$ \\
\hline
\end{tabular}


Table S5: Oligonucleotides used for UBC6 cloning. Gibson assembly kit was used for cloning, where indicated site-directed mutagenesis kit (SM) was used.

\begin{tabular}{|c|c|c|}
\hline PCR-product & Sequence & Number \\
\hline $\begin{array}{l}\# \mathbf{3 4 3} \\
\text { insert } \\
\text { (vector: } \\
\text { K27SUMO, SfoI) }\end{array}$ & $\begin{array}{l}\text { 5' }{ }^{\prime} \text { gaacagattggtggcATGGCTACAAAGCAGGCTCA } \\
{ }^{3} \text { ' } \\
\text { agtaccggtaccggcttagcctcctgtttctggtagTTTCATAA } \\
\text { AAAGGCCAACCAAAAAC }^{3}\end{array}$ & $\begin{array}{l}737 \\
780\end{array}$ \\
\hline SM, template:343 & $\begin{array}{l}\text { 5'AGCCCAACACACGATTAGCGCTTTCTATGAGTGATTACCACCCT } \\
\text { 3' } \\
\text { 5'TAATCACTCATAGAAAGCGCTAATCGTGTGTTGGGCTTGA }{ }^{3}\end{array}$ & $\begin{array}{l}1758 \\
1759\end{array}$ \\
\hline $\begin{array}{l}\text { \#702 } \\
\text { insert } \\
\text { open } 682\end{array}$ & 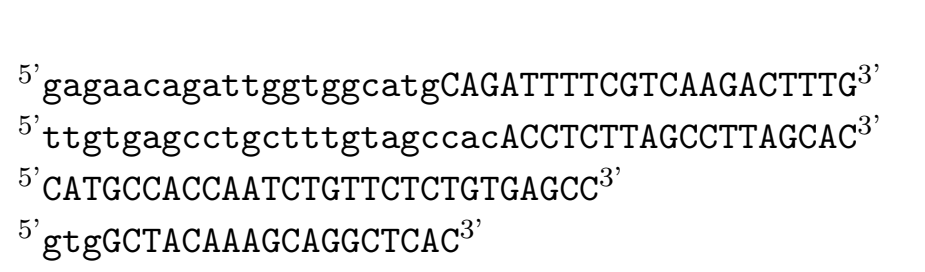 & $\begin{array}{l}1763 \\
1764 \\
1762 \\
1765\end{array}$ \\
\hline $\begin{array}{l}\# \mathbf{5 0 9} \\
\text { insert } \\
\text { open } 343\end{array}$ & $\begin{array}{l}\text { 5' acagagaacagattggtggcATGTCGGCTACCGCTGCCACC }{ }^{3} \text { ' } \\
\text { 5' ataccaatataaaccattgaGGCCCCTGCCTGGAGGGC }{ }^{3} \\
\text { 5' }{ }^{\prime} \text { CAATGGTTTATATTGGTATCG }{ }^{3} \text { ' } \\
\text { 5' GCCACCAATCTGTTCTCTG }{ }^{3}\end{array}$ & $\begin{array}{l}1079 \\
1080 \\
1081 \\
1082\end{array}$ \\
\hline $\begin{array}{l}\# 536 \\
\text { SM }\end{array}$ & $\begin{array}{l}5^{\prime} \text { tggttcaggaggcagtCTACCAGAAACAGGAGGC }{ }^{3} \text { ' } \\
{ }^{\prime} \text { 'gttgcagatccagagccAGTGCTGAAGTAAACGATG }{ }^{3}\end{array}$ & $\begin{array}{l}1152 \\
1153\end{array}$ \\
\hline $\begin{array}{l}\text { \#815 } \\
\text { insert } \\
\text { open } 702\end{array}$ & $\begin{array}{l}5^{\prime} \text { tactaagtttcatgaccagtgatgaagccacgacaggatc }{ }^{3} \\
5^{\prime} \text { ttactagtaccggtaccggcttagcctcctgtttctgg } \\
{ }^{\prime}{ }^{\prime} \\
{ }^{\prime} \text { gccggtaccggtactagtaa' } \\
5^{\prime} \text { actggtcatgaacttagta }\end{array}$ & $\begin{array}{l}2098 \\
2099 \\
2100 \\
2101\end{array}$ \\
\hline $\begin{array}{l}\# \mathbf{5 0 8} \\
\text { insert } \\
\text { SM, template } 343\end{array}$ & $\begin{array}{l}\text { 5' }{ }^{\prime} \text { GTACTTCCAATCGAGTGGCCTACCAGAAACAGGAGGC }{ }^{3} \text { ' } \\
\text { 5'AAGTTTTCAGAGCCACTACCTTTCATAAAAAGGCCAACC }^{3} \text {, }\end{array}$ & $\begin{array}{l}1093 \\
1094\end{array}$ \\
\hline $\begin{array}{l}\# \mathbf{6 3 3} \\
\text { insert } \\
\text { open vector } \\
(\mathrm{pET} 39 \mathrm{~b}(+))\end{array}$ & 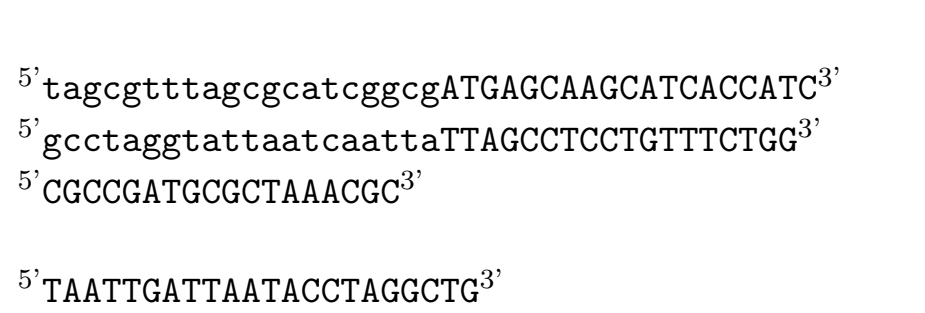 & $\begin{array}{l}1616 \\
1612 \\
1615 \\
1617\end{array}$ \\
\hline
\end{tabular}




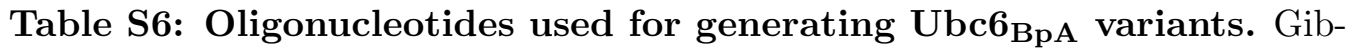
son assembly kit was used for cloning, where indicated site-directed mutagenesis kit (SM) was used.

\begin{tabular}{|c|c|c|}
\hline PCR-product & Sequence & Number \\
\hline $\begin{array}{l}\mathbf{5 4 0} \\
\text { insert } \\
\text { open pBAD- } \\
\text { His14-SUMO }\end{array}$ & $\begin{array}{l}\text { 5' acagagaacagattggtggcATGGCTACAAAGCAGGCTC }{ }^{3} \\
5^{\prime} \text { 'tagcctcctgtttctggtagTTTCATAAAAAGGCCAACCAAAAA } \\
\text { C' }^{3} \\
\text { 5' GCCACCAATCTGTTCTCTG }^{3} \text { ' } \\
{ }^{5}{ }^{\prime} \text { CTACCAGAAACAGGAGGC }{ }^{3}\end{array}$ & $\begin{array}{l}1207 \\
1208 \\
1082 \\
1085\end{array}$ \\
\hline $\begin{array}{l}\mathbf{5 5 2} \\
\text { SM, template } 540\end{array}$ & $\begin{array}{l}\text { 5'TTCAATGGTTTAGATTGGTATCG }{ }^{3} \\
\text { 5' } \text { GAACTATCATTAGGTTCTTTG }^{3}\end{array}$ & $\begin{array}{l}1175 \\
1176\end{array}$ \\
\hline $\begin{array}{l}\mathbf{5 5 3} \\
\text { SM, template } 540\end{array}$ & $\begin{array}{l}\text { 5'AATGGTTTATTAGGGTATCGCTATTTTTTTG }{ }^{3} \text { ' } \\
\text { 5' } \text { GAAGAACTATCATTAGGTTC }^{3}\end{array}$ & $\begin{array}{l}1177 \\
1178\end{array}$ \\
\hline $\begin{array}{l}\mathbf{5 5 4} \\
\text { SM, template } 540\end{array}$ & $\begin{array}{l}\text { 5'TATTGGTATCTAGATTTTTTTGTTTTTGGTTGG }{ }^{3} \\
\text { 5'TAAACCATTGAAGAACTATCATTAG }{ }^{3}\end{array}$ & $\begin{array}{l}1179 \\
1180\end{array}$ \\
\hline $\begin{array}{l}\mathbf{5 5 5} \\
\text { SM, template } 540\end{array}$ & $\begin{array}{l}\text { 5'TGGTATCGCTTAGTTTTTGTTTTTGGTTG }{ }^{3} \\
\text { 5'ATATAAACCATTGAAGAACTATC }{ }^{3}\end{array}$ & $\begin{array}{l}1181 \\
1182\end{array}$ \\
\hline $\begin{array}{l}\mathbf{5 5 6} \\
\text { SM, template } 540\end{array}$ & $\begin{array}{l}\text { 5'TATCGCTATTTAGTTGTTTTTGGTTGG }{ }^{3} \\
\text { 5'CCAATATAAACCATTGAAGAAC }{ }^{3}\end{array}$ & $\begin{array}{l}1183 \\
1184\end{array}$ \\
\hline $\begin{array}{l}\mathbf{5 6 1} \\
\text { SM, template } 540\end{array}$ & $\begin{array}{l}\text { 5' 'TTTTTTGTTTTAGGTTGGCCTTTTTATG } \\
{ }^{3} \\
\text { 5'ATAGCGATACCAATATAAACC }^{3}\end{array}$ & $\begin{array}{l}1185 \\
1186\end{array}$ \\
\hline $\begin{array}{l}\mathbf{5 6 2} \\
\text { SM, template } 540\end{array}$ & $\begin{array}{l}\text { 5'TTTGTTTTTGTAGGGCCTTTTTATGAAACTAC }{ }^{3} \text { ' } \\
\text { 5'AAAATAGCGATACCAATATAAAC }{ }^{3}\end{array}$ & $\begin{array}{l}1187 \\
1188\end{array}$ \\
\hline $\begin{array}{l}\mathbf{5 6 3} \\
\text { SM, template } 540\end{array}$ & $\begin{array}{l}\text { 5' }{ }^{\prime} \text { TGGCCTTTTTTAGAAACTACCAG } \\
\text { 5' } \\
\text { ' } \text { ACCAAAAACAAAAAATAGCG }^{3}\end{array}$ & $\begin{array}{l}1189 \\
1190\end{array}$ \\
\hline
\end{tabular}


Table S7: Oligonucleotides used for cloning CUE1 and GET3. Gibson assembly kit was used for cloning, where indicated site-directed mutagenesis kit (SM) was used.

\begin{tabular}{|c|c|c|}
\hline PCR-product & Sequence & Number \\
\hline $\begin{array}{l}\# 672 \\
\text { insert }\end{array}$ & 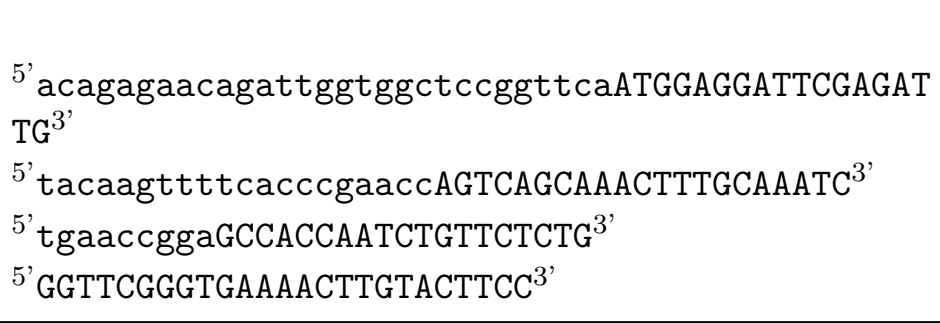 & $\begin{array}{l}1717 \\
1718 \\
1716 \\
1719\end{array}$ \\
\hline $\begin{array}{l}\# \mathbf{5 0 4} \\
\text { insert } \\
\text { (vector: } \\
\text { K27SUMO, SfoI) }\end{array}$ & $\begin{array}{l}\text { 5' acagagaacagattggtggcATGGATTTAACCGTGGAAC }{ }^{3} \text { ' } \\
\text { 5' ttactagtaccggtaccggcCTATTCCTTATCTTCTAACTCATAA } \\
\text { ATG }^{3} \text { ' }\end{array}$ & $\begin{array}{l}1065 \\
1066\end{array}$ \\
\hline $\begin{array}{l}\# \mathbf{5 2 2} \\
\text { SM, template } \\
504\end{array}$ & $\begin{array}{l}\text { 5' GATCTCTACTAATCCTGCCCATAAC }{ }^{3} \\
\text { 5' }{ }^{\prime} \text { AGTAGGAACTGTTTGTTTGG }{ }^{3}\end{array}$ & $\begin{array}{l}1096 \\
1097\end{array}$ \\
\hline
\end{tabular}


Table S8: Oligonucleotides used for SBH2 cloning. (continued) Gibson assembly kit was used for cloning, where indicated site-directed mutagenesis kit (SM) was used.

\begin{tabular}{|c|c|c|}
\hline PCR-product & Sequence & Number \\
\hline $\begin{array}{l}\# 590 \\
\text { pGPD-GFP- } \\
\text { mCherry-HA } \\
\text { open NatMX6- } \\
\text { p3nmt1- } \\
\text { 3xFLAG }\end{array}$ & 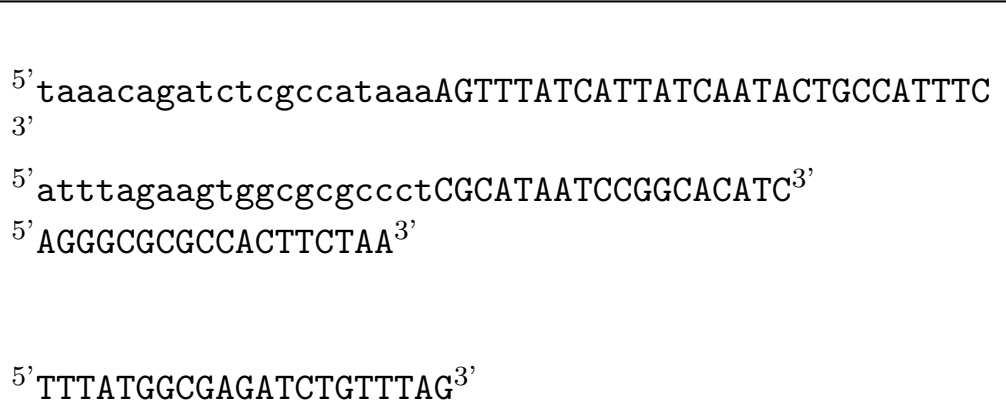 & $\begin{array}{l}1306 \\
1288 \\
1289 \\
1305\end{array}$ \\
\hline $\begin{array}{l}\text { pGPD- } \\
\text { NatMX6-tFT- } \\
\text { Sbh2 } \\
\text { NatMX6- } \\
\text { pGPD-sfGFP- } \\
\text { mCherry-HA } \\
\text { SBH2 5'region } \\
\text { SBH2 3' region }\end{array}$ & 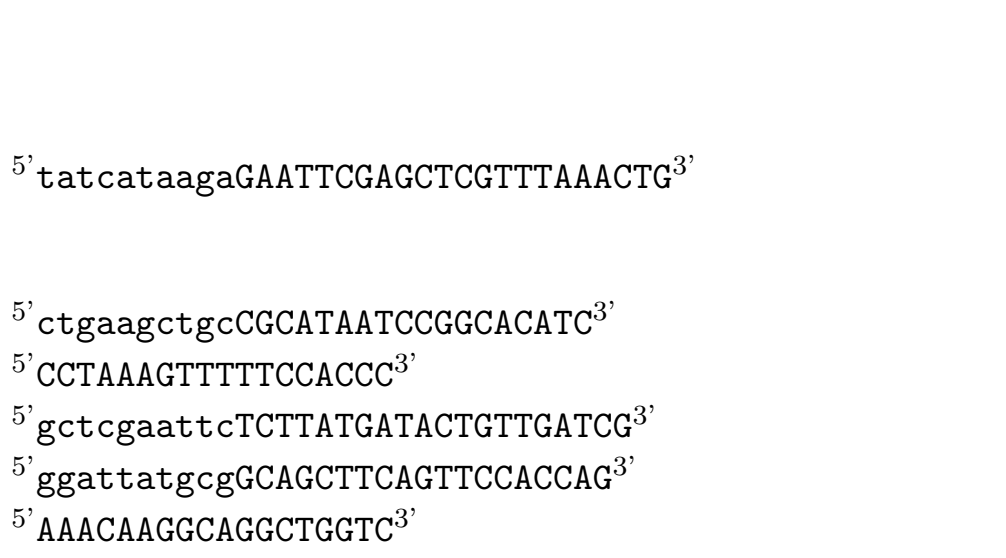 & $\begin{array}{l}1356 \\
1357 \\
1354 \\
1355 \\
1358 \\
1359\end{array}$ \\
\hline open pMAM362 & $\begin{array}{l}\text { 5'taattacatgactcgagttaTATAATGTGTGTAAATTTCGTCAATAGA } \\
\text { TG' } \\
\text { 5' atgagttgtataaactcgactatccgtatgatgtgccggattatgcgA } \\
\text { TGGCAGCTTCAGTTCCAC }{ }^{3} \\
\text { 5' cgcataatccggcacatcatacggataGTCGAGTTTATACAACTCATC } \\
\text { CATC' } \\
\text { 5'TAACTCGAGTCATGTAATTAG }{ }^{3} \text { ' }\end{array}$ & $\begin{array}{l}1073 \\
1074 \\
1067 \\
1068\end{array}$ \\
\hline $\begin{array}{l}\# \mathbf{5 1 5} \\
\text { insert } \\
\text { open pMAM362 }\end{array}$ & $\begin{array}{l}\text { 5' atgagttgtataaactcgactatccgtatgatgtgccggattatgcgA } \\
\text { TGTCAAGCCCAACTCCTC }{ }^{3} \\
5^{\prime} \text { 'taattacatgactcgagttaAAATAACTTACCGGCAACTTTAG } \\
\text { ' } \\
\text { as for generating } \# 511\end{array}$ & $\begin{array}{l}1070 \\
1069\end{array}$ \\
\hline $\begin{array}{l}\text { SM, template } \\
511\end{array}$ & $\begin{array}{l}\text { 5' }{ }^{\prime} \text { ATCCGTATGATGTGCCGG } \\
\text { '' } \\
{ }^{\prime} \text { CATAGCACCGTCGACGGT }^{3}\end{array}$ & $\begin{array}{l}1164 \\
1165\end{array}$ \\
\hline
\end{tabular}


Table S8 (continued): Oligonucleotides used for SBH2 cloning.

\begin{tabular}{|c|c|c|}
\hline PCR-product & Sequence & Number \\
\hline $\begin{array}{l}\# \mathbf{6 9 9} \\
\text { Sbh2 } 2_{89 \mathrm{C}} \\
\text { SM, template } \\
538\end{array}$ & $\begin{array}{l}\text { 5' aatttacacacattatatgtTAACTCGAGTCATGTAATTAG }{ }^{3} \text { ' } \\
\text { 5' }{ }^{\prime} \text { TTACATGACTCGAGTTAacaTATAATGTGTGTAAATTTCGTCAATAG }\end{array}$ & $\begin{array}{l}1787 \\
1788\end{array}$ \\
\hline $\begin{array}{l}\# \mathbf{5 6 5} \\
\text { Sbh2 } \text { linker-LPETGG }_{\text {SM, template }} \\
538\end{array}$ & $\begin{array}{l}\text { 5' } \text { AGGCAGTCTACCAGAAACAGGAGGCTAACTCGAGTCATGTAATTAG }^{3} \text { ' } \\
\text { 5'CCTGAACCAGTTGCAGATCCAGAGCCTATAATGTGTGTAAATTTCGTC }^{3} \text {, }\end{array}$ & $\begin{array}{l}1166 \\
1167\end{array}$ \\
\hline $\begin{array}{l}\# 695 \\
\text { insert } \\
\text { open } 633\end{array}$ & $\begin{array}{l}\text { 5' acagagaacagattggtggcATGGCAGCTTGTGTTCCAC }{ }^{3} \\
\text { 5' } \text { ' }^{\prime} \\
\text { AGAtaggtat }^{3} \\
\text { 5'TAATTGATTAATACCTAGGCTGCTAAAC }{ }^{3} \\
\text { 5'GCCACCAATCTGTTCTCTGTG }\end{array}$ & $\begin{array}{l}1784 \\
1785 \\
1613 \\
878\end{array}$ \\
\hline
\end{tabular}

Table S9: Oligonucleotides used for deleting chromosomal UBP3. Gibson assembly kit was used for cloning, where indicated site-directed mutagenesis kit (SM) was used.

\begin{tabular}{|c|c|c|}
\hline PCR-product & Sequence & Number \\
\hline \multicolumn{3}{|l|}{ UBP3 deletion } \\
\hline \multirow[t]{2}{*}{ KanMX6 cassette } & ${ }^{5}$ 'cattaaaaaaaCGGATCCCCGGGTTAATTAAG ${ }^{3}$ & 1706 \\
\hline & $5^{\prime}$ accccccgtcGAATTCGAGCTCGTTTAAACTG ${ }^{3}{ }^{3}$ & 1707 \\
\hline \multirow[t]{2}{*}{ UBP3 5'region } & $5^{\prime}$ CGACAGGGGCATTGAGCTG $^{3}$ & 1704 \\
\hline & ${ }^{5}$ ' cggggatccgTTTTTTTAATGATGATGGAAAGGAAAGCGG ${ }^{3}$ & 1705 \\
\hline \multirow[t]{2}{*}{ UBP3 3'region } & $5^{5}$ gctcgaattcGACGGGGGGTGGTATTATAG ${ }^{3}$ & 1708 \\
\hline & 5' CATAATATTTTGAAATTCACATTCCTTCTAC ${ }^{3}$ & 1709 \\
\hline \multirow{2}{*}{$\begin{array}{l}\text { whole tagging } \\
\text { cassette }\end{array}$} & 5'CGACAGGGGCATTGAGCTG'3' & 1704 \\
\hline & 5' CATAATATTTTGAAATTCACATTCCTTCTAC ${ }^{3}$ & 1709 \\
\hline \multirow{2}{*}{$\begin{array}{l}\text { control PCR for } \\
\text { deletion }\end{array}$} & 5' CTCACCGGATTCAGTCGTC ${ }^{3}$ & 602 \\
\hline & 5'CATAATATTTTGAAATTCACATTCCTTCTAC ${ }^{3}$ & 1709 \\
\hline
\end{tabular}




\section{References}

Agarraberes, F. A. and Dice, J. (2001). Protein Translocation across Membranes. Biochimica et Biophysica Acta (BBA) - Biomembranes, 1513(1):1-24.

Amm, I., Sommer, T., and Wolf, D. H. (2014). Protein Quality Control and Elimination of Protein Waste: The Role of the Ubiquitin-Proteasome System. Biochimica et Biophysica Acta (BBA) - Molecular Cell Research, 1843(1):182-196.

Auld, K. L., Hitchcock, A. L., Doherty, H. K., Frietze, S., Huang, L. S., and Silver, P. A. (2006). The Conserved ATPase Get3/Arr4 Modulates the Activity of Membraneassociated Proteins in Saccharomyces Cerevisiae. Genetics, 174(1):215-227.

Avci, D. and Lemberg, M. K. (2018). Membrane Protein Dislocation by the Rhomboid Pseudoprotease Dfm1: No Pore Needed? Molecular Cell, 69(2):161-162.

Bagola, K., von Delbrück, M., Dittmar, G., Scheffner, M., Ziv, I., Glickman, M. H., Ciechanover, A., and Sommer, T. (2013). Ubiquitin Binding by a CUE Domain Regulates Ubiquitin Chain Formation by ERAD E3 Ligases. Molecular Cell, 50(4):528-539.

Baker, R. T., Tobias, J., and Varshavsky, A. (1992). Ubiquitin-specific Proteases of Saccharomyces Cerevisiae. Cloning of UBP2 and UBP3, and Functional Analysis of the UBP Gene Family. Journal of Biological Chemistry, 267(32):23364-23375.

Baldridge, R. D. and Rapoport, T. A. (2016). Autoubiquitination of the Hrd1 Ligase Triggers Protein Retrotranslocation in ERAD. Cell, 166(2):394-407.

Banka, P. A., Behera, A. P., Sarkar, S., and Datta, A. B. (2015). RING E3-catalyzed E2 Self-ubiquitination Attenuates the Activity of Ube2e Ubiquitin-conjugating Enzymes. Journal of Molecular Biology, 427(13):2290-2304.

Barlowe, C. K. and Miller, E. A. (2013). Secretory Protein Biogenesis and Traffic in the Early Secretory Pathway. Genetics, 193(2):383-410.

Bays, N. W., Wilhovsky, S. K., Goradia, A., Hodgkiss-Harlow, K., and Hampton, R. Y. (2001). HRD4/NPL4 is required for the proteasomal processing of ubiquitinated ER proteins. Molecular Biology of the Cell, 12(12):4114-4128. 
Bazirgan, O. A. and Hampton, R. Y. (2008). Cue1p Is an Activator of Ubc7p E2 Activity in Vitro and in Vivo. Journal of Biological Chemistry, 283(19):1279712810.

Bhamidipati, A., Denic, V., Quan, E. M., and Weissman, J. S. (2005). Exploration of the Topological Requirements of ERAD Identifies Yos9p As a Lectin Sensor of Misfolded Glycoproteins in the ER Lumen. Molecular Cell, 19(6):741-751.

Biederer, T., Volkwein, C., and Sommer, T. (1997). Role of Cue1p in Ubiquitination and Degradation at the ER Surface. Science, 278(5344):1806-1809.

Biner, O., Schick, T., Müller, Y., and von Ballmoos, C. (2016). Delivery of Membrane Proteins into Small and Giant Unilamellar Vesicles by Charge-mediated Fusion. FEBS Letters, 590(14):2051-2062.

Boban, M., Ljungdahl, P. O., and Foisner, R. (2015). Atypical Ubiquitylation in Yeast Targets Lysine-less Asi2 for Proteasomal Degradation. Journal of Biological Chemistry, 290(4):2489-2495.

Boban, M., Pantazopoulou, M., Schick, A., Ljungdahl, P. O., and Foisner, R. (2014). A Nuclear Ubiquitin-Proteasome Pathway Targets the Inner Nuclear Membrane Protein Asi2 for Degradation. Journal of Cell Science, 127(16):3603-3613.

Boban, M., Zargari, A., Andréasson, C., Heessen, S., Thyberg, J., and Ljungdahl, P. O. (2006). Asi1 Is an Inner Nuclear Membrane Protein That Restricts Promoter Access of Two Latent Transcription Factors. The Journal of Cell Biology, 173(5):695-707.

Bodnar, N. and Rapoport, T. (2017a). Toward an Understanding of the Cdc48/p97 ATPase. F1000Research, 6:1318.

Bodnar, N. O., Kim, K. H., Ji, Z., Wales, T. E., Svetlov, V., Nudler, E., Engen, J. R., Walz, T., and Rapoport, T. A. (2018). Structure of the Cdc48 ATPase with Its Ubiquitin-binding Cofactor Ufd1-Npl4. Nature Structural \&s Molecular Biology, 25(7):616-622.

Bodnar, N. O. and Rapoport, T. A. (2017b). Molecular Mechanism of Substrate Processing by the Cdc48 ATPase Complex. Cell, 169(4):722-735.e9.

Bonifacio, J. S. and Lippincott-Schwartz, J. (1991). Degradation of Proteins within the Endoplasmic Reticulum. Current Opinion in Cell Biology, 3(4):592-600. 
Bordallo, J., Plemper, R. K., Finger, A., and Wolf, D. H. (1998). Der3p/Hrd1p Is Required for Endoplasmic Reticulum-associated Degradation of Misfolded Lumenal and Integral Membrane Proteins. Molecular Biology of the Cell, 9(1):209-222.

Braakman, I. and Hebert, D. N. (2013). Protein Folding in the Endoplasmic Reticulum. Cold Spring Harbor Perspectives in Biology, 5(5):a013201-a013201.

Buchanan, B. W., Lloyd, M. E., Engle, S. M., and Rubenstein, E. M. (2016). Cycloheximide Chase Analysis of Protein Degradation in Saccharomyces Cerevisiae. Journal of Visualized Experiments, (110).

Calabrese, A. N. and Radford, S. E. (2018). Mass Spectrometry-enabled Structural Biology of Membrane Proteins. Methods, 147:187-205.

Carvalho, P., Goder, V., and Rapoport, T. A. (2006). Distinct Ubiquitin-ligase Complexes Define Convergent Pathways for the Degradation of ER Proteins. Cell, 126(2):361-373.

Carvalho, P., Stanley, A. M., and Rapoport, T. A. (2010). Retrotranslocation of a Misfolded Luminal ER Protein by the Ubiquitin-ligase Hrd1p. Cell, 143(4):579591.

Chau, V., Tobias, J., Bachmair, A., Marriott, D., Ecker, D., Gonda, D., and Varshavsky, A. (1989). A Multiubiquitin Chain Is Confined to Specific Lysine in a Targeted Short-lived Protein. Science, 243(4898):1576-1583.

Chen, I., Dorr, B. M., and Liu, D. R. (2011). A General Strategy for the Evolution of Bond-forming Enzymes Using Yeast Display. Proceedings of the National Academy of Sciences, 108(28):11399-11404.

Chen, P., Johnson, P., Sommer, T., Jentsch, S., and Hochstrasser, M. (1993). Multiple Ubiquitin-conjugating Enzymes Participate in the in Vivo Degradation of the Yeast MATalpha2 Repressor. Cell, 74(2):357-369.

Chen, Y.-C., Umanah, G. K. E., Dephoure, N., Andrabi, S. A., Gygi, S. P., Dawson, T. M., Dawson, V. L., and Rutter, J. (2014). Msp1/ ATAD 1 Maintains Mitochondrial Function by Facilitating the Degradation of Mislocalized Tail-anchored Proteins. The EMBO Journal, 33(14):1548-1564.

Chin, J. W., Martin, A. B., King, D. S., Wang, L., and Schultz, P. G. (2002). Addition of a Photocrosslinking Amino Acid to the Genetic Code of Escherichia Coli. Proceedings of the National Academy of Sciences, 99(17):11020-11024. 
Chitwood, P. J., Juszkiewicz, S., Guna, A., Shao, S., and Hegde, R. S. (2018). EMC Is Required to Initiate Accurate Membrane Protein Topogenesis. Cell, 175(6):15071519.e16.

Christensen, D. E., Brzovic, P. S., and Klevit, R. E. (2007). E2-BRCA1 RING Interactions Dictate Synthesis of Mono- or Specific Polyubiquitin Chain Linkages. Nature Structural \&3 Molecular Biology, 14(10):941-948.

Christianson, J. C. and Ye, Y. (2014). Cleaning up in the Endoplasmic Reticulum: Ubiquitin in Charge. Nature Structural \& Molecular Biology, 21(4):325-335.

Civjan, N. R., Bayburt, T. H., Schuler, M. A., and Sligar, S. G. (2003). Direct Solubilization of Heterologously Expressed Membrane Proteins by Incorporation into Nanoscale Lipid Bilayers. BioTechniques, 35(3):556-563.

Cohen, M., Stutz, F., Belgareh, N., Haguenauer-Tsapis, R., and Dargemont, C. (2003a). Ubp3 Requires a Cofactor, Bre5, to Specifically De-ubiquitinate the COPII Protein, Sec23. Nature Cell Biology, 5(7):661-667.

Cohen, M., Stutz, F., and Dargemont, C. (2003b). Deubiquitination, a New Player in Golgi to Endoplasmic Reticulum Retrograde Transport. Journal of Biological Chemistry, 278(52):51989-51992.

David, Y., Ziv, T., Admon, A., and Navon, A. (2010). The E2 Ubiquitin-conjugating Enzymes Direct Polyubiquitination to Preferred Lysines. Journal of Biological Chemistry, 285(12):8595-8604.

Dederer, V., Khmelinskii, A., Huhn, A. G., Okreglak, V., Knop, M., and Lemberg, M. K. (2019). Cooperation of Mitochondrial and ER Factors in Quality Control of Tail-anchored Proteins. eLife, 8.

Deng, M. and Hochstrasser, M. (2006). Spatially Regulated Ubiquitin Ligation by an ER/Nuclear Membrane Ligase. Nature, 443(7113):827-831.

Denic, V., Quan, E. M., and Weissman, J. S. (2006). A Luminal Surveillance Complex That Selects Misfolded Glycoproteins for ER-associated Degradation. Cell, $126(2): 349-359$.

Deshaies, R. J. and Joazeiro, C. A. (2009). RING Domain E3 Ubiquitin Ligases. Annual Review of Biochemistry, 78(1):399-434. 
Doblas, V. G., Amorim-Silva, V., Posé, D., Rosado, A., Esteban, A., Arró, M., Azevedo, H., Bombarely, A., Borsani, O., Valpuesta, V., Ferrer, A., Tavares, R. M., and Botella, M. A. (2013). The SUD1 Gene Encodes a Putative E3 Ubiquitin Ligase and Is a Positive Regulator of 3-hydroxy-3-methylglutaryl Coenzyme a Reductase Activity in Arabidopsis. The Plant Cell, 25(2):728-743.

Dyballa, N. and Metzger, S. (2009). Fast and Sensitive Colloidal Coomassie G-250 Staining for Proteins in Polyacrylamide Gels. Journal of Visualized Experiments, $(30)$.

Enam, C., Geffen, Y., Ravid, T., and Gardner, R. G. (2018). Protein Quality Control Degradation in the Nucleus. Annual Review of Biochemistry, 87(1):725-749.

Espenshade, P. J. and Hughes, A. L. (2007). Regulation of Sterol Synthesis in Eukaryotes. Annual Review of Genetics, 41(1):401-427.

Fasshauer, D., Antonin, W., Margittai, M., Pabst, S., and Jahn, R. (1999). Mixed and Non-cognate SNARE Complexes. Journal of Biological Chemistry, 274(22):1544015446.

Fasshauer, D., Otto, H., Eliason, W. K., Jahn, R., and Brünger, A. T. (1997). Structural Changes Are Associated with Soluble N-Ethylmaleimide-sensitive Fusion Protein Attachment Protein Receptor Complex Formation. Journal of Biological Chemistry, 272(44):28036-28041.

Finger, A., Knop, M., and Wolf, D. H. (1993). Analysis of Two Mutated Vacuolar Proteins Reveals a Degradation Pathway in the Endoplasmic Reticulum or a Related Compartment of Yeast. European Journal of Biochemistry, 218(2):565-574.

Finke, K., Plath, K., Panzner, S., Prehn, S., Rapoport, T. A., Hartmann, E., and Sommer, T. (1996). A Second Trimeric Complex Containing Homologs of the Sec61p Complex Functions in Protein Transport across the ER Membrane of S. Cerevisiae. The EMBO Journal, 15(7):1482-1494.

Finley, D., Ulrich, H. D., Sommer, T., and Kaiser, P. (2012). The Ubiquitin-Proteasome System of Saccharomyces Cerevisiae. Genetics, 192(2):319360.

Flury, I., Garza, R., Shearer, A., Rosen, J., Cronin, S., and Hampton, R. Y. (2005). INSIG: A Broadly Conserved Transmembrane Chaperone for Sterol-sensing Domain Proteins. The EMBO Journal, 24(22):3917-3926. 
Foresti, O., Rodriguez-Vaello, V., Funaya, C., and Carvalho, P. (2014). Quality Control of Inner Nuclear Membrane Proteins by the Asi Complex. Science, 346(6210):751755 .

Foresti, O., Ruggiano, A., Hannibal-Bach, H. K., Ejsing, C. S., and Carvalho, P. (2013). Sterol Homeostasis Requires Regulated Degradation of Squalene Monooxygenase by the Ubiquitin Ligase Doa10/Teb4. eLife, 2.

Forsberg, H., Hammar, M., Andréasson, C., Molinér, A., and Ljungdahl, P. O. (2001). Suppressors of ssy1 and ptr3 Null Mutations Define Novel Amino Acid Sensorindependent Genes in Saccharomyces Cerevisiae. Genetics, 158(3):973-988.

Freemont, P. S., Hanson, I. M., and Trowsdale, J. (1991). A Novel Cysteine-rich Sequence Motif. Cell, 64(3):483-484.

Friedlander, R., Jarosch, E., Urban, J., Volkwein, C., and Sommer, T. (2000). A Regulatory Link between ER-associated Protein Degradation and the Unfoldedprotein Response. Nature Cell Biology, 2(7):379-384.

Furth, N., Gertman, O., Shiber, A., Alfassy, O. S., Cohen, I., Rosenberg, M. M., Doron, N. K., Friedler, A., and Ravid, T. (2011). Exposure of Bipartite Hydrophobic Signal Triggers Nuclear Quality Control of Ndc10 at the Endoplasmic Reticulum/Nuclear Envelope. Molecular Biology of the Cell, 22(24):4726-4739.

Galardy, R. E., Craig, L. C., and Printz, M. P. (1973). Benzophenone Triplet: A New Photochemical Probe of Biological Ligand-Receptor Interactions. Nature New Biology, 242(117):127-128.

Galkin, M. A., Russell, A. N., Vik, S. B., Berry, R. M., and Ishmukhametov, R. R. (2018). Detergent-free Ultrafast Reconstitution of Membrane Proteins into Lipid Bilayers Using Fusogenic Complementary-charged Proteoliposomes. Journal of Visualized Experiments, (134).

Gardner, R., Cronin, S., Leader, B., Rine, J., and Hampton, R. (1998). Sequence Determinants for Regulated Degradation of Yeast 3-Hydroxy-3-MethylglutarylCoA Reductase, an Integral Endoplasmic Reticulum Membrane Protein. Molecular Biology of the Cell, 9(9):2611-2626.

Gardner, R. G., Swarbrick, G. M., Bays, N. W., Cronin, S. R., Wilhovsky, S., Seelig, L., Kim, C., and Hampton, R. Y. (2000). Endoplasmic Reticulum Degradation Requires Lumen to Cytosol Signaling. The Journal of Cell Biology, 151(1):69-82. 
Garza, R. M., Sato, B. K., and Hampton, R. Y. (2009a). In Vitro Analysis of Hrd1p-mediated Retrotranslocation of Its Multispanning Membrane Substrate 3Hydroxy-3-Methylglutaryl (HMG)-CoA Reductase. Journal of Biological Chemistry, 284(22):14710-14722.

Garza, R. M., Tran, P. N., and Hampton, R. Y. (2009b). Geranylgeranyl Pyrophosphate Is a Potent Regulator of HRD-dependent 3-Hydroxy-3-Methylglutaryl-CoA Reductase Degradation in Yeast. Journal of Biological Chemistry, 284(51):3536835380 .

Gasteiger, E., Hoogland, C., Gattiker, A., Wilkins, M. R., Appel, R. D., Bairoch, A., et al. (2005). Protein Identification and Analysis Tools on the Expasy Server. In The Proteomics Protocols Handbook, pages 571-607. Springer.

Gauss, R., Kanehara, K., Carvalho, P., Ng, D. T., and Aebi, M. (2011). A Complex of Pdi1p and the Mannosidase Htm1p Initiates Clearance of Unfolded Glycoproteins from the Endoplasmic Reticulum. Molecular Cell, 42(6):782-793.

Gauss, R., Sommer, T., and Jarosch, E. (2006). The Hrd1p Ligase Complex Forms a Linchpin between ER-lumenal Substrate Selection and Cdc48p Recruitment. The EMBO Journal, 25(9):1827-1835.

Giaever, G., Chu, A. M., Ni, L., Connelly, C., Riles, L., Véronneau, S., Dow, S., Lucau-Danila, A., Anderson, K., André, B., Arkin, A. P., Astromoff, A., Bakkoury, M. E., Bangham, R., Benito, R., Brachat, S., Campanaro, S., Curtiss, M., Davis, K., Deutschbauer, A., Entian, K.-D., Flaherty, P., Foury, F., Garfinkel, D. J., Gerstein, M., Gotte, D., Güldener, U., Hegemann, J. H., Hempel, S., Herman, Z., Jaramillo, D. F., Kelly, D. E., Kelly, S. L., Kötter, P., LaBonte, D., Lamb, D. C., Lan, N., Liang, H., Liao, H., Liu, L., Luo, C., Lussier, M., Mao, R., Menard, P., Ooi, S. L., Revuelta, J. L., Roberts, C. J., Rose, M., Ross-Macdonald, P., Scherens, B., Schimmack, G., Shafer, B., Shoemaker, D. D., Sookhai-Mahadeo, S., Storms, R. K., Strathern, J. N., Valle, G., Voet, M., Volckaert, G., yun Wang, C., Ward, T. R., Wilhelmy, J., Winzeler, E. A., Yang, Y., Yen, G., Youngman, E., Yu, K., Bussey, H., Boeke, J. D., Snyder, M., Philippsen, P., Davis, R. W., and Johnston, M. (2002). Functional Profiling of the Saccharomyces Cerevisiae Genome. Nature, 418(6896):387-391.

Gietz, R. D., Schiestl, R. H., Willems, A. R., and Woods, R. A. (1995). Studies on the transformation of intact yeast cells by the LiAc/SS-DNA/PEG procedure. Yeast, 11(4):355-360. 
Gill, S., Stevenson, J., Kristiana, I., and Brown, A. J. (2011). Cholesterol-dependent Degradation of Squalene Monooxygenase, a Control Point in Cholesterol Synthesis beyond HMG-CoA Reductase. Cell Metabolism, 13(3):260-273.

Gilon, T. (1998). Degradation Signals for Ubiquitin System Proteolysis in Saccharomyces Cerevisiae. The EMBO Journal, 17(10):2759-2766.

Gilon, T., Chomsky, O., and Kulka, R. G. (2000). Degradation Signals Recognized by the Ubc6p-Ubc7p Ubiquitin-conjugating Enzyme Pair. Molecular and Cellular Biology, 20(19):7214-7219.

Glynn, S. E. (2017). Multifunctional Mitochondrial AAA Proteases. Frontiers in Molecular Biosciences, 4.

Goder, V., Carvalho, P., and Rapoport, T. A. (2008). The ER-associated Degradation Component Der1p and Its Homolog Dfm1p Are Contained in Complexes with Distinct Cofactors of the ATPase Cdc48p. FEBS Letters, 582(11):1575-1580.

Grice, G. L. and Nathan, J. A. (2016). The Recognition of Ubiquitinated Proteins by the Proteasome. Cellular and Molecular Life Sciences, 73(18):3497-3506.

Grumati, P., Dikic, I., and Alexandra (2018). ER-phagy at a Glance. Journal of Cell Science, 131(17):jcs217364.

Guerriero, C. J., Reutter, K.-R., Augustine, A. A., Preston, G. M., Weiberth, K. F., Mackie, T. D., Cleveland-Rubeor, H. C., Bethel, N. P., Callenberg, K. M., Nakatsukasa, K., Grabe, M., and Brodsky, J. L. (2017). Transmembrane Helix Hydrophobicity Is an Energetic Barrier during the Retrotranslocation of Integral Membrane ERAD Substrates. Molecular Biology of the Cell, 28(15):2076-2090.

Guna, A., Volkmar, N., Christianson, J. C., and Hegde, R. S. (2017). The ER Membrane Protein Complex Is a Transmembrane Domain Insertase. Science, 359(6374):470-473.

Guo, H., Suzuki, T., and Rubinstein, J. L. (2019). Structure of a Bacterial ATP Synthase. eLife, 8 .

Habeck, G., Ebner, F. A., Shimada-Kreft, H., and Kreft, S. G. (2015). The Yeast ERAD-C Ubiquitin Ligase Doa10 Recognizes an Intramembrane Degron. The Journal of Cell Biology, 209(2):261-273. 
Haenzelmann, P., Buchberger, A., and Schindelin, H. (2011). Hierarchical Binding of Cofactors to the AAA ATPase p97. Structure, 19(6):833-843.

Hampton, R. Y. (1994). Regulated Degradation of HMG-CoA Reductase, an Integral Membrane Protein of the Endoplasmic Reticulum, in Yeast. The Journal of Cell Biology, 125(2):299-312.

Hampton, R. Y., Gardner, R. G., and Rine, J. (1996). Role of 26S Proteasome and HRD Genes in the Degradation of 3-Hydroxy-3-Methylglutaryl-CoA Reductase, an Integral Endoplasmic Reticulum Membrane Protein. Molecular Biology of the Cell, 7(12):2029-2044.

Han, S., Liu, Y., and Chang, A. (2007). Cytoplasmic Hsp70 Promotes Ubiquitination for Endoplasmic Reticulum-associated Degradation of a Misfolded Mutant of the Yeast Plasma Membrane ATPase, PMA1. Journal of Biological Chemistry, 282(36):26140-26149.

Hassink, G., Kikkert, M., van Voorden, S., Lee, S.-J., Spaapen, R., van Laar, T., Coleman, C. S., Bartee, E., Früh, K., Chau, V., and Wiertz, E. (2005). TEB4 Is a C4HC3 RING Finger-containing Ubiquitin Ligase of the Endoplasmic Reticulum. Biochemical Journal, 388(2):647-655.

Hernandez, J. M., Stein, A., Behrmann, E., Riedel, D., Cypionka, A., Farsi, Z., Walla, P. J., Raunser, S., and Jahn, R. (2012). Membrane Fusion Intermediates Via Directional and Full Assembly of the SNARE Complex. Science, 336(6088):15811584 .

Hesselberth, J. R., Miller, J. P., Golob, A., Stajich, J. E., Michaud, G. A., and Fields, S. (2006). Comparative analysis of Saccharomyces cerevisiae WW domains and their interacting proteins. Genome Biology, 7(4):R30.

Hetz, C. and Papa, F. R. (2018). The Unfolded Protein Response and Cell Fate Control. Molecular Cell, 69(2):169-181.

Hetzer, M., Meyer, H. H., Walther, T. C., Bilbao-Cortes, D., Warren, G., and Mattaj, I. W. (2001). Distinct AAA-ATPase p97 Complexes Function in Discrete Steps of Nuclear Assembly. Nature Cell Biology, 3(12):1086-1091.

Hiller, M. M., Finger, A., Schweiger, M., and Wolf, D. H. (1996). ER Degradation of a Misfolded Luminal Protein by the Cytosolic Ubiquitin-Proteasome Pathway. Science, 273(5282):1725-1728. 
Hitt, R. and Wolf, D. H. (2004). Der1p, a Protein Required for Degradation of Malfolded Soluble Proteins of the Endoplasmic Reticulum: Topology and Der1-like Proteins. FEMS Yeast Research, 4(7):721-729.

Hobbs, K. and Tartoff, K. (1987). Improved Media for Growing Plasmid and Cosmid Clones. Focus, 9:9-12.

Horn, S. C., Hanna, J., Hirsch, C., Volkwein, C., Schütz, A., Heinemann, U., Sommer, T., and Jarosch, E. (2009). Usa1 Functions As a Scaffold of the HRD-ubiquitin Ligase. Molecular Cell, 36(5):782-793.

Huyer, G., Piluek, W. F., Fansler, Z., Kreft, S. G., Hochstrasser, M., Brodsky, J. L., and Michaelis, S. (2004). Distinct Machinery Is Required in Saccharomyces Cerevisiae for the Endoplasmic Reticulum-associated Degradation of a Multispanning Membrane Protein and a Soluble Luminal Protein. Journal of Biological Chemistry, 279(37):38369-38378.

Ishida, Y., Yamamoto, A., Kitamura, A., Lamandé, S. R., Yoshimori, T., Bateman, J. F., Kubota, H., and Nagata, K. (2009). Autophagic Elimination of Misfolded Procollagen Aggregates in the Endoplasmic Reticulum as a Means of Cell Protection. Molecular Biology of the Cell, 20(11):2744-2754.

Ishmukhametov, R. R., Russell, A. N., and Berry, R. M. (2016). A Modular Platform for One-step Assembly of Multi-component Membrane Systems by Fusion of Charged Proteoliposomes. Nature Communications, 7(1).

Jahn, R. and Scheller, R. H. (2006). SNAREs - Engines for Membrane Fusion. Nature Reviews Molecular Cell Biology, 7(9):631-643.

Jakob, C. A., Bodmer, D., Spirig, U., Battig, P., Marcil, A., Dignard, D., Bergeron, J. J. M., Thomas, D. Y., and Aebi, M. (2001). Htm1p, a Mannosidase-like Protein, Is Involved in Glycoprotein Degradation in Yeast. EMBO reports, 2(5):423-430.

Jarosch, E., Taxis, C., Volkwein, C., Bordallo, J., Finley, D., Wolf, D. H., and Sommer, T. (2002). Protein Dislocation from the ER Requires Polyubiquitination and the AAA-ATPase Cdc48. Nature Cell Biology, 4(2):134-139.

Johnson, P. R., Swanson, R., Rakhilina, L., and Hochstrasser, M. (1998). Degradation Signal Masking by Heterodimerization of MAT $\alpha 2$ and MATa1 Blocks Their Mutual Destruction by the Ubiquitin-Proteasome Pathway. Cell, 94(2):217-227. 
Jonikas, M. C., Collins, S. R., Denic, V., Oh, E., Quan, E. M., Schmid, V., Weibezahn, J., Schwappach, B., Walter, P., Weissman, J. S., and Schuldiner, M. (2009). Comprehensive Characterization of Genes Required for Protein Folding in the Endoplasmic Reticulum. Science, 323(5922):1693-1697.

Kahya, N., Pécheur, E.-I., de Boeij, W. P., Wiersma, D. A., and Hoekstra, D. (2001). Reconstitution of Membrane Proteins into Giant Unilamellar Vesicles Via Peptideinduced Fusion. Biophysical Journal, 81(3):1464-1474.

Kats, I., Khmelinskii, A., Kschonsak, M., Huber, F., Knieß, R. A., Bartosik, A., and Knop, M. (2018). Mapping Degradation Signals and Pathways in a Eukaryotic N-terminome. Molecular Cell, 70(3):488-501.e5.

Keefe, A. D., Wilson, D. S., Seelig, B., and Szostak, J. W. (2001). One-step Purification of Recombinant Proteins Using a Nanomolar-affinity Streptavidin-binding Peptide, the SBP-tag. Protein Expression and Purification, 23(3):440-446.

Khmelinskii, A., Blaszczak, E., Pantazopoulou, M., Fischer, B., Omnus, D. J., Dez, G. L., Brossard, A., Gunnarsson, A., Barry, J. D., Meurer, M., Kirrmaier, D., Boone, C., Huber, W., Rabut, G., Ljungdahl, P. O., and Knop, M. (2014). Protein Quality Control at the Inner Nuclear Membrane. Nature, 516(7531):410-413.

Khmelinskii, A., Keller, P. J., Bartosik, A., Meurer, M., Barry, J. D., Mardin, B. R., Kaufmann, A., Trautmann, S., Wachsmuth, M., Pereira, G., Huber, W., Schiebel, E., and Knop, M. (2012). Tandem Fluorescent Protein Timers for in Vivo Analysis of Protein Dynamics. Nature Biotechnology, 30(7):708-714.

Khmelinskii, A., Meurer, M., Ho, C.-T., Besenbeck, B., Füller, J., Lemberg, M. K., Bukau, B., Mogk, A., and Knop, M. (2016). Incomplete Proteasomal Degradation of Green Fluorescent Proteins in the Context of Tandem Fluorescent Protein Timers. Molecular Biology of the Cell, 27(2):360-370.

Kim, W., Spear, E. D., and Ng, D. T. (2005). Yos9p Detects and Targets Misfolded Glycoproteins for ER-associated Degradation. Molecular Cell, 19(6):753-764.

Kleiger, G. and Mayor, T. (2014). Perilous Journey: A Tour of the Ubiquitin-Proteasome System. Trends in Cell Biology, 24(6):352-359.

Klug, L. and Daum, G. (2014). Yeast Lipid Metabolism at a Glance. FEMS Yeast Research, 14(3):369-388. 
Knop, M., Finger, A., Braun, T., Hellmuth, K., and Wolf, D. (1996). Der1, a Novel Protein Specifically Required for Endoplasmic Reticulum Degradation in Yeast. The EMBO journal, 15(4):753-763.

Komander, D. and Rape, M. (2012). The Ubiquitin Code. Annual Review of Biochemistry, 81(1):203-229.

Kopski, K. M. and Huffaker, T. C. (1997). Suppressors of the Ndc10-2 Mutation: A Role for the Ubiquitin System in Saccharomyces Cerevisiae Kinetochore Function. Genetics, 147(2):409-420.

Korbel, D., Wurth, S., Käser, M., and Langer, T. (2004). Membrane Protein Turnover by the m-AAA Protease in Mitochondria Depends on the Transmembrane Domains of Its Subunits. EMBO reports, 5(7):698-703.

Kostova, Z., Tsai, Y. C., and Weissman, A. M. (2007). Ubiquitin Ligases, Critical Mediators of Endoplasmic Reticulum-associated Degradation. Seminars in Cell \&6 Developmental Biology, 18(6):770-779.

Kreft, S. G. and Hochstrasser, M. (2011). An Unusual Transmembrane Helix in the Endoplasmic Reticulum Ubiquitin Ligase Doa10 Modulates Degradation of Its Cognate E2 Enzyme. Journal of Biological Chemistry, 286(23):20163-20174.

Kreft, S. G., Wang, L., and Hochstrasser, M. (2006). Membrane Topology of the Yeast Endoplasmic Reticulum-localized Ubiquitin Ligase Doa10 and Comparison with Its Human Ortholog TEB4 (MARCH-VI). Journal of Biological Chemistry, 281(8):4646-4653.

Kruse, K. B., Brodsky, J. L., and McCracken, A. A. (2006). Characterization of an ERAD Gene As VPS30/ATG6 Reveals Two Alternative and Functionally Distinct Protein Quality Control Pathways: One for Soluble Z Variant of Human Alpha-1 Proteinase Inhibitor (A1PiZ) and Another for Aggregates of A1PiZ. Molecular Biology of the Cell, 17(1):203-212.

Kubalová, D., Káňovičová, P., Veselá, P., Awadová, T., Džugasová, V., Daum, G., Malínský, J., and Balážová, M. (2019). The Lipid Droplet Protein Pgc1 Controls the Subcellular Distribution of Phosphatidylglycerol. FEMS Yeast Research, 19(5).

Kyte, J. and Doolittle, R. F. (1982). A Simple Method for Displaying the Hydropathic Character of a Protein. Journal of Molecular Biology, 157(1):105-132. 
Lajoie, M. J., Rovner, A. J., Goodman, D. B., Aerni, H.-R., Haimovich, A. D., Kuznetsov, G., Mercer, J. A., Wang, H. H., Carr, P. A., Mosberg, J. A., Rohland, N., Schultz, P. G., Jacobson, J. M., Rinehart, J., Church, G. M., and Isaacs, F. J. (2013). Genomically Recoded Organisms Expand Biological Functions. Science, 342(6156):357-360.

Lee, S., Lee, H., Yoo, S., and Kim, H. (2017). Molecular Insights into The m-AAA Protease-mediated Dislocation of Transmembrane Helices in the Mitochondrial Inner Membrane. Journal of Biological Chemistry, 292(49):20058-20066.

Li, W., Bengtson, M. H., Ulbrich, A., Matsuda, A., Reddy, V. A., Orth, A., Chanda, S. K., Batalov, S., and Joazeiro, C. A. P. (2008). Genome-wide and Functional Annotation of Human E3 Ubiquitin Ligases Identifies MULAN, a Mitochondrial E3 That Regulates the Organelle's Dynamics and Signaling. PLoS ONE, 3(1):e1487.

Ling, Q., Broad, W., Trösch, R., Töpel, M., Sert, T. D., Lymperopoulos, P., Baldwin, A., and Jarvis, R. P. (2019). Ubiquitin-dependent Chloroplast-associated Protein Degradation in Plants. Science, 363(6429):eaav4467.

Lippincott-Schwartz, J. (1988). Degradation from the Endoplasmic Reticulum: Disposing of Newly Synthesized Proteins. Cell, 54(2):209-220.

Liu, L., Spurrier, J., Butt, T. R., and Strickler, J. E. (2008). Enhanced Protein Expression in the Baculovirus/insect Cell System Using Engineered SUMO Fusions. Protein Expression and Purification, 62(1):21-28.

Liu, Y.-C., Fujimori, D. G., and Weissman, J. S. (2016). Htm1p-Pdi1p Is a Foldingsensitive Mannosidase That Marks N-glycoproteins for ER-associated Protein Degradation. Proceedings of the National Academy of Sciences, 113(28):E4015E4024.

Loayza, D., Tam, A., Schmidt, W. K., and Michaelis, S. (1998). Ste6p Mutants Defective in Exit from the Endoplasmic Reticulum (ER) Reveal Aspects of an ER Quality Control Pathway in Saccharomyces cerevisiae. Molecular Biology of the Cell, 9(10):2767-2784.

Machida, Y. J., Machida, Y., Chen, Y., Gurtan, A. M., Kupfer, G. M., Andrea, A. D., and Dutta, A. (2006). UBE2t Is the E2 in the Fanconi Anemia Pathway and Undergoes Negative Autoregulation. Molecular Cell, 23(4):589-596. 
Mandart, E., Dufour, M. E., and Lacroute, F. (1994). Inactivation of SSM4, a New Saccharomyces Cerevisiae Gene, Suppresses mRNA Instability Due to rna14 Mutations. MGG Molecular \& General Genetics, 245(3):323-333.

Margittai, M., Fasshauer, D., Pabst, S., Jahn, R., and Langen, R. (2001). Homoand Heterooligomeric SNARE Complexes Studied by Site-directed Spin Labeling. Journal of Biological Chemistry, 276(16):13169-13177.

Mariappan, M., Mateja, A., Dobosz, M., Bove, E., Hegde, R. S., and Keenan, R. J. (2011). The Mechanism of Membrane-associated Steps in Tail-anchored Protein Insertion. Nature, 477(7362):61-66.

Mateja, A. and Keenan, R. J. (2018). A Structural Perspective on Tail-anchored Protein Biogenesis by the GET Pathway. Current Opinion in Structural Biology, 51:195-202.

Mateja, A., Paduch, M., Chang, H.-Y., Szydlowska, A., Kossiakoff, A. A., Hegde, R. S., and Keenan, R. J. (2015). Structure of the Get3 Targeting Factor in Complex with Its Membrane Protein Cargo. Science, 347(6226):1152-1155.

Mateja, A., Szlachcic, A., Downing, M. E., Dobosz, M., Mariappan, M., Hegde, R. S., and Keenan, R. J. (2009). The Structural Basis of Tail-anchored Membrane Protein Recognition by Get3. Nature, 461(7262):361-366.

Matlack, K. E., Misselwitz, B., Plath, K., and Rapoport, T. A. (1999). BiP Acts As a Molecular Ratchet during Posttranslational Transport of Prepro- $\alpha$ Factor across the ER Membrane. Cell, 97(5):553-564.

Matsumoto, S., Nakatsukasa, K., Kakuta, C., Tamura, Y., Esaki, M., and Endo, T. (2019). Msp1 Clears Mistargeted Proteins by Facilitating Their Transfer from Mitochondria to the ER. Molecular Cell, 76(1):191-205.

McBratney, S. and Winey, M. (2002). Mutant Membrane Protein of the Budding Yeast Spindle Pole Body Is Targeted to the Endoplasmic Reticulum Degradation Pathway. Genetics, 162(2):567-578.

Mehnert, M., Sommer, T., and Jarosch, E. (2013). Der1 Promotes Movement of Misfolded Proteins through the Endoplasmic Reticulum Membrane. Nature Cell Biology, 16(1):77-86. 
Mehrtash, A. B. and Hochstrasser, M. (2018). Ubiquitin-dependent Protein Degradation at the Endoplasmic Reticulum and Nuclear Envelope. Seminars in Cell 86 Developmental Biology, 93:111-124.

Metzger, M. B., Liang, Y.-H., Das, R., Mariano, J., Li, S., Li, J., Kostova, Z., Byrd, R. A., Ji, X., and Weissman, A. M. (2013). A Structurally Unique E2-binding Domain Activates Ubiquitination by the ERAD E2, Ubc7p, through Multiple Mechanisms. Molecular Cell, 50(4):516-527.

Metzger, M. B., Maurer, M. J., Dancy, B. M., and Michaelis, S. (2008). Degradation of a Cytosolic Protein Requires Endoplasmic Reticulum-associated Degradation Machinery. Journal of Biological Chemistry, 283(47):32302-32316.

Meusser, B., Hirsch, C., Jarosch, E., and Sommer, T. (2005). ERAD: The Long Road to Destruction. Nature Cell Biology, 7(8):766-772.

Mi, H., Muruganujan, A., Ebert, D., Huang, X., and Thomas, P. D. (2018). PANTHER Version 14: More Genomes, a New PANTHER GO-slim and Improvements in Enrichment Analysis Tools. Nucleic Acids Research, 47(D1):D419-D426.

Miller, J. H. (1992). A Short Course in Bacterial Genetics - A Laboratory Manual and Handbook for Escherichia coli and Related Bacteria. Cold Spring Harbor Laboratory Press, 33(4):278-278.

Mumberg, D., Muller, R., and Funk, M. (1994). Regulatable Promoters of Saccharomyces Cerevisiae: Comparison of Transcriptional Activity and Their Use for Heterologous Expression. Nucleic Acids Research, 22(25):5767-5768.

Nakai, M., Endo, T., Hase, T., and Matsubara, H. (1993). Intramitochondrial Protein Sorting. Isolation and Characterization of the Yeast Msp1 Gene Which Belongs to a Novel Family of Putative ATPases. Journal of Biological Chemistry, 268(32):24262-24269.

Nakatsukasa, K., Huyer, G., Michaelis, S., and Brodsky, J. L. (2008). Dissecting the ER-associated Degradation of a Misfolded Polytopic Membrane Protein. Cell, 132(1):101-112.

Nakatsukasa, K. and Kamura, T. (2016). Subcellular Fractionation Analysis of the Extraction of Ubiquitinated Polytopic Membrane Substrate during ER-associated Degradation. PLOS ONE, 11(2):e0148327. 
Nakatsukasa, K., Nishikawa, S., Hosokawa, N., Nagata, K., and Endo, T. (2001). Mnl1p, an Alpha-mannosidase-like Protein in Yeast Saccharomyces Cerevisiae, Is Required for Endoplasmic Reticulum-associated Degradation of Glycoproteins. Journal of Biological Chemistry, 276(12):8635-8638.

Neal, S., Duttke, S. H., and Hampton, R. Y. (2019). Assays for Protein Retrotranslocation in ERAD. In Methods in Enzymology, pages 1-26. Elsevier.

Neal, S., Jaeger, P. A., Duttke, S. H., Benner, C., K.Glass, C., Ideker, T., and Hampton, R. Y. (2018). The Dfm1 Derlin Is Required for ERAD Retrotranslocation of Integral Membrane Proteins. Molecular Cell, 69(2):306-320.e4.

Neuber, O., Jarosch, E., Volkwein, C., Walter, J., and Sommer, T. (2005). Ubx2 Links the Cdc48 Complex to ER-associated Protein Degradation. Nature Cell Biology, $7(10): 993-998$.

Nishikawa, S., Fewell, S. W., Kato, Y., Brodsky, J. L., and Endo, T. (2001). Molecular Chaperones in the Yeast Endoplasmic Reticulum Maintain the Solubility of Proteins for Retrotranslocation and Degradation. The Journal of Cell Biology, 153(5):1061-1070.

Nordlund, G., Brzezinski, P., and von Ballmoos, C. (2014). SNARE-fusion Mediated Insertion of Membrane Proteins into Native and Artificial Membranes. Nature Communications, 5(1):1-8.

Okreglak, V. and Walter, P. (2014). The Conserved AAA-ATPase Msp1 Confers Organelle Specificity to Tail-anchored Proteins. Proceedings of the National Academy of Sciences, 111(22):8019-8024.

Olzmann, J. A. and Carvalho, P. (2018). Dynamics and Functions of Lipid Droplets. Nature Reviews Molecular Cell Biology, 20(3):137-155.

Olzmann, J. A., Kopito, R. R., and Christianson, J. C. (2012). The Mammalian Endoplasmic Reticulum-associated Degradation System. Cold Spring Harbor Perspectives in Biology, 5(9):a013185-a013185.

Omnus, D. J. and Ljungdahl, P. O. (2014). Latency of Transcription Factor Stp1 Depends on a Modular Regulatory Motif That Functions As Cytoplasmic Retention Determinant and Nuclear Degron. Molecular Biology of the Cell, 25(23):38233833 . 
Ossareh-Nazari, B., Bonizec, M., Cohen, M., Dokudovskaya, S., Delalande, F., Schaeffer, C., Dorsselaer, A. V., and Dargemont, C. (2010a). Cdc48 and Ufd3, New Partners of the Ubiquitin Protease Ubp3, Are Required for Ribophagy. EMBO reports, 11(7):548-554.

Ossareh-Nazari, B., Cohen, M., and Dargemont, C. (2010b). The Rsp5 Ubiquitin Ligase and the AAA-ATPase Cdc48 Control the Ubiquitin-mediated Degradation of the COPII Component Sec23. Experimental Cell Research, 316(20):3351-3357.

Pantazopoulou, M., Boban, M., Foisner, R., and Ljungdahl, P. O. (2016). Cdc48 and Ubx1 Participate in a Pathway Associated with the Inner Nuclear Membrane That Governs Asi1 Degradation. Journal of Cell Science, 129(20):3770-3780.

Pfeiffer, A., Stephanowitz, H., Krause, E., Volkwein, C., Hirsch, C., Jarosch, E., and Sommer, T. (2016). A Complex of Htm1 and the Oxidoreductase Pdi1 Accelerates Degradation of Misfolded Glycoproteins. Journal of Biological Chemistry, 291(23):12195-12207.

Plemper, R. K., Egner, R., Kuchler, K., and Wolf, D. H. (1998). Endoplasmic Reticulum Degradation of a Mutated ATP-binding Cassette Transporter Pdr5 Proceeds in a Concerted Action of Sec61 and the Proteasome. Journal of Biological Chemistry, 273(49):32848-32856.

Pobbati, A. V. (2006). N- to C-terminal SNARE Complex Assembly Promotes Rapid Membrane Fusion. Science, 313(5787):673-676.

Popp, M. W.-L., Antos, J. M., and Ploegh, H. L. (2009). Site-specific Protein Labeling Via Sortase-mediated Transpeptidation. Current protocols in protein science, $56(1): 15-3$.

Popp, M. W.-L. and Ploegh, H. L. (2011). Making and Breaking Peptide Bonds: Protein Engineering Using Sortase. Angewandte Chemie International Edition, 50(22):5024-5032.

Preobraschenski, J., Zander, J.-F., Suzuki, T., Ahnert-Hilger, G., and Jahn, R. (2014). Vesicular Glutamate Transporters Use Flexible Anion and Cation Binding Sites for Efficient Accumulation of Neurotransmitter. Neuron, 84(6):1287-1301.

Printen, J. A. and Sprague, G. F. (1994). Protein-protein Interactions in the Yeast Pheromone Response Pathway: Ste5p Interacts with All Members of the MAP Kinase Cascade. Genetics, 138(3):609-619. 
Quinn, P., Griffiths, G., and Warren, G. (1984). Density of Newly Synthesized Plasma Membrane Proteins in Intracellular Membranes II. Biochemical Studies. The Journal of Cell Biology, 98(6):2142-2147.

Rabinovich, E., Kerem, A., Frohlich, K.-U., Diamant, N., and Bar-Nun, S. (2002). AAA-ATPase p97/Cdc48p, a Cytosolic Chaperone Required for Endoplasmic Reticulum-associated Protein Degradation. Molecular and Cellular Biology, $22(2): 626-634$.

Rape, M. and Kirschner, M. W. (2004). Autonomous Regulation of the Anaphasepromoting Complex Couples Mitosis to S-phase Entry. Nature, 432(7017):588595.

Rapoport, T. A., Li, L., and Park, E. (2017). Structural and Mechanistic Insights into Protein Translocation. Annual Review of Cell and Developmental Biology, 33(1):369-390.

Ravid, T. and Hochstrasser, M. (2007). Autoregulation of an E2 Enzyme by Ubiquitinchain Assembly on Its Catalytic Residue. Nature Cell Biology, 9(4):422-427.

Ravid, T. and Hochstrasser, M. (2008). Diversity of Degradation Signals in the Ubiquitin-Proteasome System. Nature Reviews Molecular Cell Biology, 9(9):679-689.

Ravid, T., Kreft, S. G., and Hochstrasser, M. (2006). Membrane and Soluble Substrates of the Doa10 Ubiquitin Ligase Are Degraded by Distinct Pathways. The EMBO Journal, 25(3):533-543.

Rigaud, J.-L. and Lévy, D. (2003). Reconstitution of Membrane Proteins into Liposomes. In Methods in Enzymology, pages 65-86. Elsevier.

Rodrigo-Brenni, M. C. and Morgan, D. O. (2007). Sequential E2s Drive Polyubiquitin Chain Assembly on APC Targets. Cell, 130(1):127-139.

Ruggiano, A., Foresti, O., and Carvalho, P. (2014). ER-associated Degradation: Protein Quality Control and Beyond. The Journal of Cell Biology, 204(6):869-879.

Ruggiano, A., Mora, G., Buxó, L., and Carvalho, P. (2016). Spatial Control of Lipid Droplet Proteins by the ERAD Ubiquitin Ligase Doa10. The EMBO Journal, 35(15):1644-1655. 
Samant, R. S., Livingston, C. M., Sontag, E. M., and Frydman, J. (2018). Distinct Proteostasis Circuits Cooperate in Nuclear and Cytoplasmic Protein Quality Control. Nature, 563(7731):407-411.

Sato, B. K. and Hampton, R. Y. (2006). Yeast Derlin Dfm1 Interacts with Cdc48 and Functions in ER Homeostasis. Yeast, 23(14-15):1053-1064.

Sato, B. K., Schulz, D., Do, P. H., and Hampton, R. Y. (2009). Misfolded Membrane Proteins Are Specifically Recognized by the Transmembrane Domain of the Hrd1p Ubiquitin Ligase. Molecular Cell, 34(2):212-222.

Schenck, S., Wojcik, S. M., Brose, N., and Takamori, S. (2009). A Chloride Conductance in VGLUT1 Underlies Maximal Glutamate Loading into Synaptic Vesicles. Nature Neuroscience, 12(2):156-162.

Schindelin, J., Arganda-Carreras, I., Frise, E., Kaynig, V., Longair, M., Pietzsch, T., Preibisch, S., Rueden, C., Saalfeld, S., Schmid, B., Tinevez, J.-Y., White, D. J., Hartenstein, V., Eliceiri, K., Tomancak, P., and Cardona, A. (2012). Fiji: An Open-source Platform for Biological-image Analysis. Nature Methods, 9(7):676682.

Schmidt, O., Weyer, Y., Baumann, V., Widerin, M. A., Eising, S., Angelova, M., Schleiffer, A., Kremser, L., Lindner, H., Peter, M., Fröhlich, F., and Teis, D. (2019). Endosome and Golgi-associated Degradation (EGAD) of Membrane Proteins Regulates Sphingolipid Metabolism. The EMBO Journal, 38(15).

Schneider, C. A., Rasband, W. S., and Eliceiri, K. W. (2012). NIH Image to ImageJ: 25 years of image analysis. Nature methods, 9(7):671.

Schoebel, S., Mi, W., Stein, A., Ovchinnikov, S., Pavlovicz, R., DiMaio, F., Baker, D., Chambers, M. G., Su, H., Li, D., Rapoport, T. A., and Liao, M. (2017). Cryo-EM Structure of the Protein-conducting ERAD Channel Hrd1 in Complex with Hrd3. Nature, 548(7667):352-355.

Schuberth, C. and Buchberger, A. (2005). Membrane-bound Ubx2 Recruits Cdc48 to Ubiquitin Ligases and Their Substrates to Ensure Efficient ER-associated Protein Degradation. Nature Cell Biology, 7(10):999-1006.

Schuberth, C. and Buchberger, A. (2008). UBX domain proteins: Major regulators of the AAA ATPase Cdc48/p97. Cellular and Molecular Life Sciences, 65(15):23602371. 
Schuberth, C., Richly, H., Rumpf, S., and Buchberger, A. (2004). Shp1 and Ubx2 Are Adaptors of Cdc48 Involved in Ubiquitin-dependent Protein Degradation. EMBO reports, 5(8):818-824.

Schuldiner, M., Collins, S. R., Thompson, N. J., Denic, V., Bhamidipati, A., Punna, T., Ihmels, J., Andrews, B., Boone, C., Greenblatt, J. F., Weissman, J. S., and Krogan, N. J. (2005). Exploration of the Function and Organization of the Yeast Early Secretory Pathway through an Epistatic Miniarray Profile. Cell, 123(3):507519.

Schuldiner, M., Metz, J., Schmid, V., Denic, V., Rakwalska, M., Schmitt, H. D., Schwappach, B., and Weissman, J. S. (2008). The GET Complex Mediates Insertion of Tail-anchored Proteins into the ER Membrane. Cell, 134(4):634-645.

Schultz, M. L., Krus, K. L., Kaushik, S., Dang, D., Chopra, R., Qi, L., Shakkottai, V. G., Cuervo, A. M., and Lieberman, A. P. (2018). Coordinate regulation of mutant NPC1 degradation by selective ER autophagy and MARCH6-dependent ERAD. Nature Communications, 9(1).

Schägger, H. (2006). Tricine-SDS-PAGE. Nature Protocols, 1(1):16-22.

Schägger, H. and von Jagow, G. (1987). Tricine-Sodium Dodecyl Sulfatepolyacrylamide Gel Electrophoresis for the Separation of Proteins in the Range from 1 to $100 \mathrm{kDa}$. Analytical Biochemistry, 166(2):368-379.

Scott, D., Oldham, N. J., Strachan, J., Searle, M. S., and Layfield, R. (2014). Ubiquitinbinding Domains: Mechanisms of Ubiquitin Recognition and Use As Tools to Investigate Ubiquitin-modified Proteomes. Proteomics, 15(5-6):844-861.

Shcherbik, N., Kee, Y., Lyon, N., Huibregtse, J. M., and Haines, D. S. (2004). A Single PXY Motif Located within the Carboxyl Terminus of Spt23p and Mga2p Mediates a Physical and Functional Interaction with Ubiquitin Ligase Rsp5p. Journal of Biological Chemistry, 279(51):53892-53898.

Shen, H.-H., Lithgow, T., and Martin, L. (2013). Reconstitution of Membrane Proteins into Model Membranes: Seeking Better Ways to Retain Protein Activities. International Journal of Molecular Sciences, 14(1):1589-1607.

Shevchenko, A., Wilm, M., Vorm, O., and Mann, M. (1996). Mass Spectrometric Sequencing of Proteins from Silver-stained Polyacrylamide Gels. Analytical Chemistry, 68(5):850-858. 
Shiber, A., Breuer, W., Brandeis, M., and Ravid, T. (2013). Ubiquitin Conjugation Triggers Misfolded Protein Sequestration into Quality Control Foci When Hsp70 Chaperone Levels Are Limiting. Molecular Biology of the Cell, 24(13):2076-2087.

Shyu, P., Ng, B. S. H., Ho, N., Chaw, R., Seah, Y. L., Marvalim, C., and Thibault, G. (2019). Membrane Phospholipid Alteration Causes Chronic ER Stress through Early Degradation of Homeostatic ER-resident Proteins. Scientific Reports, $9(1): 1-15$.

Simocková, M., Holic, R., Tahotná, D., Patton-Vogt, J., and Griac, P. (2008). Yeast Pgc1p (YPL206c) Controls the Amount of Phosphatidylglycerol Via a Phospholipase C-type Degradation Mechanism. Journal of Biological Chemistry, 283(25):17107-17115.

Smoyer, C. J., Smith, S. E., Gardner, J. M., McCroskey, S., Unruh, J. R., and Jaspersen, S. L. (2019). Distribution of Proteins at the Inner Nuclear Membrane Is Regulated by the Asi1 E3 Ligase in Saccharomyces Cerevisiae. Genetics, 211(4):1269-1282.

Sommer, T. and Jentsch, S. (1993). A Protein Translocation Defect Linked to Ubiquitin Conjugation at the Endoplasmic Reticulum. Nature, 365(6442):176-179.

Stefanovic, S. and Hegde, R. S. (2007). Identification of a Targeting Factor for Posttranslational Membrane Protein Insertion into the ER. Cell, 128(6):1147-1159.

Stein, A., Radhakrishnan, A., Riedel, D., Fasshauer, D., and Jahn, R. (2007). Synaptotagmin Activates Membrane Fusion through a $\mathrm{Ca}^{2+}$-dependent Trans Interaction with Phospholipids. Nature Structural \& Molecular Biology, 14(10):904-911.

Stein, A., Ruggiano, A., Carvalho, P., and Rapoport, T. A. (2014). Key Steps in ERAD of Luminal ER Proteins Reconstituted with Purified Components. Cell, 158(6):1375-1388.

Stewart, M. D., Ritterhoff, T., Klevit, R. E., and Brzovic, P. S. (2016). E2 Enzymes: More Than Just Middle Men. Cell Research, 26(4):423-440.

Stolz, A., Schweizer, R. S., Schäfer, A., and Wolf, D. H. (2010). Dfm1 Forms Distinct Complexes with Cdc48 and the ER Ubiquitin Ligases and Is Required for ERAD. Traffic, 11(10):1363-1369. 
Studier, F. W. (2005). Protein Production by Auto-induction in High-density Shaking Cultures. Protein Expression and Purification, 41(1):207-234.

Stuerner, E., Kuraku, S., Hochstrasser, M., and Kreft, S. G. (2012). Split-Doa10: A Naturally Split Polytopic Eukaryotic Membrane Protein Generated by Fission of a Nuclear Gene. PLoS ONE, 7(10):e45194.

Sudol, M., Chen, H. I., Bougeret, C., Einbond, A., and Bork, P. (1995). Characterization of a Novel Protein-binding Module - the WW Domain. FEBS Letters, 369(1):67-71.

Suzuki, T., Ueno, H., Mitome, N., Suzuki, J., and Yoshida, M. (2002). F0 of ATP Synthase Is a Rotary Proton Channel. Journal of Biological Chemistry, 277(15):1328113285.

Swanson, R., Locher, M., and Hochstrasser, M. (2001). A Conserved Ubiquitin Ligase of the Nuclear Envelope/Endoplasmic Reticulum That Functions in Both ER-associated and Mata2 Repressor Degradation. Genes 8 Development, 15(20):2660-2674.

Szathmary, R., Bielmann, R., Nita-Lazar, M., Burda, P., and Jakob, C. A. (2005). Yos9 Protein Is Essential for Degradation of Misfolded Glycoproteins and May Function As Lectin in ERAD. Molecular Cell, 19(6):765-775.

Takamori, S., Holt, M., Stenius, K., Lemke, E. A., Grønborg, M., Riedel, D., Urlaub, H., Schenck, S., Brügger, B., Ringler, P., .., and Jahn, R. (2006). Molecular Anatomy of a Trafficking Organelle. Cell, 127(4):831-846.

Tan, J. M., Cook, E. C., van den Berg, M., Scheij, S., Zelcer, N., and Loregger, A. (2019). Differential Use of E2 Ubiquitin Conjugating Enzymes for Regulated Degradation of the Rate-limiting Enzymes HMGCR and SQLE in Cholesterol Biosynthesis. Atherosclerosis, 281:137-142.

Taxis, C., Hitt, R., Park, S.-H., Deak, P. M., Kostova, Z., and Wolf, D. H. (2003). Use of Modular Substrates Demonstrates Mechanistic Diversity and Reveals Differences in Chaperone Requirement of ERAD. Journal of Biological Chemistry, 278(38):35903-35913.

Theesfeld, C. L. and Hampton, R. Y. (2013). Insulin-induced Gene Protein (INSIG)dependent Sterol Regulation of Hmg2 Endoplasmic Reticulum-associated Degradation (ERAD) in Yeast. Journal of Biological Chemistry, 288(12):8519-8530. 
Tong, A. H. Y. and Boone, C. (2006). Synthetic Genetic Array Analysis in Saccharomyces Cerevisiae. In Yeast Protocols, pages 171-192. Humana Press.

Tong, A. H. Y. and Boone, C. (2007). 16 High-throughput Strain Construction and Systematic Synthetic Lethal Screening in Saccharomyces cerevisiae. In Methods in Microbiology, pages 369-707. Elsevier.

Tong, J., Borbat, P. P., Freed, J. H., and Shin, Y.-K. (2009). A Scissors Mechanism for Stimulation of SNARE-mediated Lipid Mixing by Cholesterol. Proceedings of the National Academy of Sciences, 106(13):5141-5146.

Travers, K. J., Patil, C. K., Wodicka, L., Lockhart, D. J., Weissman, J. S., and Walter, P. (2000). Functional and Genomic Analyses Reveal an Essential Coordination between the Unfolded Protein Response and ER-associated Degradation. Cell, 101(3):249-258.

Tsuchiya, H., Ohtake, F., Arai, N., Kaiho, A., Yasuda, S., Tanaka, K., and Saeki, Y. (2017). In vivo Ubiquitin Linkage-type Analysis Reveals That the Cdc48Rad23/Dsk2 Axis Contributes to K48-linked Chain Specificity of the Proteasome. Molecular Cell, 66(4):488-502.e7.

Tuller, G., Nemec, T., Hrastnik, C., and Daum, G. (1999). Lipid Composition of Subcellular Membranes of an FY1679-derived Haploid Yeast Wild-type Strain Grown on Different Carbon Sources. Yeast, 15(14):1555-1564.

Twomey, E. C., Ji, Z., Wales, T. E., Bodnar, N. O., Ficarro, S. B., Marto, J. A., Engen, J. R., and Rapoport, T. A. (2019). Substrate Processing by the Cdc48 ATPase Complex Is Initiated by Ubiquitin Unfolding. Science, 365(6452):eaax1033.

van den Bogaart, G., Holt, M. G., Bunt, G., Riedel, D., Wouters, F. S., and Jahn, R. (2010). One SNARE Complex Is Sufficient for Membrane Fusion. Nature Structural \& Molecular Biology, 17(3):358-364.

van Meer, G., Voelker, D. R., and Feigenson, G. W. (2008). Membrane Lipids: Where They Are and How They Behave. Nature Reviews Molecular Cell Biology, $9(2): 112-124$.

Varshavsky, A. (1991). Naming a Targeting Signal. Cell, 64(1):13-15.

Vashist, S. and Ng, D. T. (2004). Misfolded Proteins Are Sorted by a Sequential Checkpoint Mechanism of ER Quality Control. The Journal of Cell Biology, 165(1):4152. 
Vasic, V., Denkert, N., Schmidt, C. C., Riedel, D., Stein, A., and Meinecke, M. (2020). Hrd1 forms the retrotranslocation pore regulated by auto-ubiquitination and binding of misfolded proteins. Nature Cell Biology, 22(3):274-281.

von Delbrück, M., Kniss, A., Rogov, V. V., Pluska, L., Bagola, K., Löhr, F., Güntert, P., Sommer, T., and Dötsch, V. (2016). The CUE Domain of Cue1 Aligns Growing Ubiquitin Chains with Ubc7 for Rapid Elongation. Molecular Cell, 62(6):918-928.

Voth, W., Schick, M., Gates, S., Li, S., Vilardi, F., Gostimskaya, I., Southworth, D. R., Schwappach, B., and Jakob, U. (2014). The Protein Targeting Factor Get3 Functions As ATP-independent Chaperone under Oxidative Stress Conditions. Molecular Cell, 56(1):116-127.

Walter, J., Urban, J., Volkwein, C., and Sommer, T. (2001). Sec61p-independent Degradation of the Tail-anchored ER Membrane Protein Ubc6p. The EMBO Journal, 20(12):3124-3131.

Wang, F., Chan, C., Weir, N. R., and Denic, V. (2014). The Get1/2 Transmembrane Complex Is an Endoplasmic-reticulum Membrane Protein Insertase. Nature, 512(7515):441-444.

Wang, Q. and Chang, A. (1999). Eps1, a Novel PDI-related Protein Involved in ER Quality Control in Yeast. The EMBO Journal, 18(21):5972-5982.

Wang, Q. and Chang, A. (2003). Substrate Recognition in ER-associated Degradation Mediated by Eps1, a Member of the Protein Disulfide Isomerase Family. The EMBO Journal, 22(15):3792-3802.

Wangeline, M. A. and Hampton, R. Y. (2018). "Mallostery" - ligand-dependent Protein Misfolding Enables Physiological Regulation by ERAD. Journal of Biological Chemistry, 293(38):14937-14950.

Weber, A., Cohen, I., Popp, O., Dittmar, G., Reiss, Y., Sommer, T., Ravid, T., and Jarosch, E. (2016). Sequential Poly-ubiquitylation by Specialized Conjugating Enzymes Expands the Versatility of a Quality Control Ubiquitin Ligase. Molecular Cell, 63(5):827-839.

Weber, T., Zemelman, B. V., McNew, J. A., Westermann, B., Gmachl, M., Parlati, F., Söllner, T. H., and Rothman, J. E. (1998). SNAREpins: Minimal machinery for membrane fusion. Cell, 92(6):759-772. 
Wenzel, D. M., Lissounov, A., Brzovic, P. S., and Klevit, R. E. (2011). UBCH7 Reactivity Profile Reveals Parkin and HHARI to Be RING/HECT Hybrids. Nature, 474(7349):105-108.

Wilson, J. D., Liu, Y., Bentivoglio, C. M., and Barlowe, C. (2006). Sel1p/Ubx2p Participates in a Distinct Cdc48p-dependent Endoplasmic Reticulum-associated Degradation Pathway. Traffic, 7(9):1213-1223.

Wohlever, M. L., Mateja, A., McGilvray, P. T., Day, K. J., and Keenan, R. J. (2017). Msp1 Is a Membrane Protein Dislocase for Tail-anchored Proteins. Molecular Cell, 67(2):194-202.e6.

Xiao, W., Poirier, M. A., Bennett, M. K., and Shin, Y.-K. (2001). The Neuronal t-SNARE Complex Is a Parallel Four-helix Bundle. Nature Structural Biology, $8(4): 308-311$.

Xu, P., Duong, D. M., Seyfried, N. T., Cheng, D., Xie, Y., Robert, J., Rush, J., Hochstrasser, M., Finley, D., and Peng, J. (2009). Quantitative Proteomics Reveals the Function of Unconventional Ubiquitin Chains in Proteasomal Degradation. Cell, 137(1):133-145.

Yang, B., Wu, Y.-J., Zhu, M., Fan, S.-B., Lin, J., Zhang, K., Li, S., Chi, H., Li, Y.-X., Chen, H.-F., Luo, S.-K., Ding, Y.-H., Wang, L.-H., Hao, Z., Xiu, L.-Y., Chen, S., Ye, K., He, S.-M., and Dong, M.-Q. (2012). Identification of Cross-linked Peptides from Complex Samples. Nature Methods, 9(9):904-906.

Ye, Y., Meyer, H. H., and Rapoport, T. A. (2001). The AAA ATPase Cdc48/p97 and Its Partners Transport Proteins from the ER into the Cytosol. Nature, 414(6864):652656.

Ye, Y., Tang, W. K., Zhang, T., and Xia, D. (2017). A Mighty "Protein Extractor" of the Cell: Structure and Function of the p97/Cdc48 ATPase. Frontiers in Molecular Biosciences, 4:39.

Yin, Q., Lin, S.-C., Lamothe, B., Lu, M., Lo, Y.-C., Hura, G., Zheng, L., Rich, R. L., Campos, A. D., Myszka, D. G., Lenardo, M. J., Darnay, B. G., and Wu, H. (2009). E2 Interaction and Dimerization in the Crystal Structure of TRAF6. Nature Structural \& Molecular Biology, 16(6):658-666.

Zargari, A., Boban, M., Heessen, S., Andréasson, C., Thyberg, J., and Ljungdahl, P. O. (2007). Inner Nuclear Membrane Proteins Asi1, Asi2, and Asi3 Function 
in Concert to Maintain the Latent Properties of Transcription Factors Stp1 and Stp2. Journal of Biological Chemistry, 282(1):594-605.

Zattas, D., Berk, J. M., Kreft, S. G., and Hochstrasser, M. (2016). A Conserved Cterminal Element in the Yeast Doa10 and Human MARCH6 Ubiquitin Ligases Required for Selective Substrate Degradation. Journal of Biological Chemistry, 291(23):12105-12118.

Zattas, D. and Hochstrasser, M. (2014). Ubiquitin-dependent Protein Degradation at the Yeast Endoplasmic Reticulum and Nuclear Envelope. Critical Reviews in Biochemistry and Molecular Biology, 50(1):1-17.

Zelcer, N., Sharpe, L. J., Loregger, A., Kristiana, I., Cook, E. C. L., Phan, L., Stevenson, J., and Brown, A. J. (2014). The E3 Ubiquitin Ligase MARCH6 Degrades Squalene Monooxygenase and Affects 3-Hydroxy-3-Methylglutaryl Coenzyme A Reductase and the Cholesterol Synthesis Pathway. Molecular and Cellular Biology, 34(7):1262-1270.

Zhang, Z.-R., Bonifacino, J. S., and Hegde, R. S. (2013). Deubiquitinases Sharpen Substrate Discrimination during Membrane Protein Degradation from the ER. Cell, 154(3):609-622.

Zinser, E., Sperka-Gottlieb, C., Fasch, E.-V., Kohlwein, S. D., Paltauf, F., and Daum, G. (1991). Phospholipid Synthesis and Lipid Composition of Subcellular Membranes in the Unicellular Eukaryote Saccharomyces Cerevisiae. Journal of Bacteriology, 173(6):2026-2034. 


\section{Acknowledgements}

I would like to thank Dr. Alexander Stein who has been an extremely supportive supervisor. His passion for science was a great source of motivation and I really enjoyed working on this exciting $\mathrm{PhD}$ project.

I would also like to thank the members of my thesis advisory committee, Prof. Blanche Schwappach-Pignataro and Prof. Holger Stark, for all the advice and helpful discussions on my project. Moreover, Blanche Schwappach-Pignataro has been a wonderful mentor whose advice helped me a lot. I am also grateful to Prof. Reinhard Jahn and Dr. Alex Faesen for their scientific input in countless lab seminars. Moreover, I would like to thank Ákos Farkas and Iwan Parfentev for their strong efforts during our collaborations.

A really big thank you goes to all my colleagues. They have created a wonderful and supportive lab environment. I would like to thank Iris, for her technical assistance and for being such an enthusiastic person keeping our group together. Nupur is an amazing colleague whom I am grateful for all the technical assistance in protein purification. I would also like to thank Aikaterini for being an amazing lab mate and Niels Denkert for always calming me down and finding a logical solution. I am really grateful for Anuruti, for being an amazing lab mate who always has such a positive spirit and motivating words. I would also like to thank my bench mate Vedran who made these last four years an amazing experience. I am very grateful for our friendship and our endless scientific discussions.

Moreover, I would like to thank all the members of the Neurobiology Department, for their scientific input during lab seminars as well as for creating an amazing working atmosphere.

I am very grateful to the IMPRS Molecular Biology Program, and would like to especially thank Dr. Steffen Burkhardt and Kerstin Grüniger for all their help and dedication during my whole time in Göttingen.

I would like to thank my friends who made the last years really special. A big thank you to Shama for being such an amazing and supportive friend. Thank you to Vedran and Benji for being so enthusiastic about science communication, and life in general. I would like to thank my room mate and friend Tahere for being an amazing friend with unlimited support in all areas of life. Thank you to Madhobi, Swati, Isaac, Oleh, 
Judith, Matteo and Tomas! I would also like to thank Marieta who brought music back into my life.

Ich kann mich nicht genug bei meinen Eltern bedanken, die mit ihrer unendlichen Liebe immer hinter mir stehen. Danke, dass ihr immer für mich da seid! A really big thank you goes to my sister Christina whom I can always count on and who always provides me with another view on life with her amazing humor. I especially want thank Thomas whose support has been endless, who always understands and finds the right words and with whom work and life in general is simply so much better. 


\section{Doa10 is a membrane protein retrotranslocase in ER-associated protein degradation}

Claudia C Schmidt, Vedran Vasic, Alexander Stein*

Research Group Membrane Protein Biochemistry, Max Planck Institute for Biophysical Chemistry, Göttingen, Germany

*For correspondence: alexander.stein@mpibpc.mpg.de

Competing interests: The authors declare that no competing interests exist.

Funding: See page 27

Received: 15 March 2020 Accepted: 28 May 2020 Published: 26 June 2020

Reviewing editor: Ramanujan S Hegde, MRC Laboratory of Molecular Biology, United Kingdom

(c) Copyright Schmidt et al. This article is distributed under the terms of the Creative Commons Attribution License, which permits unrestricted use and redistribution provided that the original author and source are credited.

\begin{abstract}
In endoplasmic reticulum-associated protein degradation (ERAD), membrane proteins are ubiquitinated, extracted from the membrane, and degraded by the proteasome. The cytosolic ATPase Cdc48 drives extraction by pulling on polyubiquitinated substrates. How hydrophobic transmembrane (TM) segments are moved from the phospholipid bilayer into cytosol, often together with hydrophilic and folded ER luminal protein parts, is not known. Using a reconstituted system with purified proteins from Saccharomyces cerevisiae, we show that the ubiquitin ligase Doa10 (Teb-4/MARCH6 in animals) is a retrotranslocase that facilitates membrane protein extraction. A substrate's TM segment interacts with the membrane-embedded domain of Doa10 and then passively moves into the aqueous phase. Luminal substrate segments cross the membrane in an unfolded state. Their unfolding occurs on the luminal side of the membrane by cytoplasmic Cdc48 action. Our results reveal how a membrane-bound retrotranslocase cooperates with the Cdc48 ATPase in membrane protein extraction.
\end{abstract}

\section{Introduction}

The endoplasmic reticulum (ER) is a major site for protein folding and maturation in the endomembrane system of the eukaryotic cell. A conserved quality control pathway called ER-associated protein degradation (ERAD) removes misfolded, unassembled and mistargeted proteins from the ER into the cytosol where they are degraded by the proteasome (Christianson and Ye, 2014; Mehrtash and Hochstrasser, 2019; Ruggiano et al., 2014). ERAD thus contributes to protein homeostasis. Its malfunction results in ER stress (Hwang and Qi, 2018), and it has been linked to several human diseases (Guerriero and Brodsky, 2012; Qi et al., 2017). ERAD is part of the ubiquitin proteasome system. Studies in the yeast Saccharomyces cerevisiae identified two universally conserved membrane-embedded ubiquitin ligases that ubiquitinate ERAD substrates, Hrd1 (SYVN1 in human) (Bordallo et al., 1998; Hampton et al., 1996; Kikkert et al., 2004; Nadav et al., 2003) and Doa10 (TEB-4/MARCH6 in animals, SUD-1 in Arabidopsis thaliana) (Doblas et al., 2013; Hassink et al., 2005; Swanson et al., 2001). In higher eukaryotes, a larger variety of ubiquitin ligases plays a role in ERAD (Olzmann et al., 2013).

Substrates of ERAD can be soluble luminal proteins, or membrane proteins that either need to be moved across or extracted from the ER membrane. This process, termed retrotranslocation or dislocation, requires the AAA protein Cdc48 (VCP or p97 in animals) (Bays et al., 2001; Garza et al., 2009; Jarosch et al., 2002; Nakatsukasa et al., 2008; Rabinovich et al., 2002; Ye et al., 2001). Cdc48 is recruited to substrates by its cofactors Ufd1 and Npl4 which interact with polyubiquitin chains with lysine 48 linkage (Meyer et al., 2002; Ye et al., 2003). The Cdc48 complex is thought to generate a pulling force that drives extraction of polyubiquitinated proteins from the membrane. This notion is based on biochemical experiments with soluble proteins (Bodnar and Rapoport, 2017; Olszewski et al., 2019) and recent cryo-EM structures (Bodnar et al., 2018; Cooney et al., 
eLife digest The inside of a cell contains many different compartments called organelles, which are separated by membranes. Each organelle is composed of a unique set of proteins and performs specific roles in the cell. The endoplasmic reticulum, or ER for short, is an organelle where many proteins are produced. Most of these proteins are then released from the cell or sorted to other organelles. The ER has a strict quality control system that ensures any faulty proteins are quickly marked for the cell to destroy. However, the destruction process itself does not happen in the ER, so faulty proteins first need to leave this organelle. This is achieved by a group of proteins known as endoplasmic reticulum-associated protein degradation machinery (or ERAD for short).

To extract a faulty protein from the ER, proteins of the ER and outside the ER cooperate. First, an ERAD protein called Doa10 attaches a small protein tag called ubiquitin to the faulty proteins to mark them for destruction. Then, outside of the ER, a protein called Cdc48 'grabs' the ubiquitin tag and pulls. But that is only part of the story. Many of the proteins made by the ER have tethers that anchor them firmly to the membrane, making them much harder to remove.

To get a better idea of how the extraction works, Schmidt et al. rebuilt the ERAD machinery in a test tube. This involved purifying proteins from yeast and inserting them into artificial membranes, allowing closer study of each part of the process. This revealed that attaching ubiquitin tags to faulty proteins is only one part of Doa10's role; it also participates in the extraction itself. Part of Doa10 resides within the membrane, and this 'membrane-spanning domain' can interact with faulty proteins, loosening their membrane anchors. At the same time, Cdc48 pulls from the outside. This pulling force causes the faulty proteins to unfold, allowing them to pass through the membrane.

Given these findings, the next step is to find out exactly how Doa10 works by looking at its threedimensional structure. This could have implications not only for the study of ERAD, but of similar quality control processes in other organelles too. A build-up of faulty proteins can cause diseases like neurodegeneration, so understanding how cells remove faulty proteins could help future medical research.

2019; Twomey et al., 2019), which showed that processive threading of a substrate through the central pore of the Cdc48 hexamer under ATP consumption leads to unfolding.

Apart from the Cdc48 ATPase, membrane proteins of the ERAD machinery are thought to contribute to retrotranslocation. For soluble substrates, Hrd1 forms part of a retrotranslocon pore from the ER lumen to the cytosol (Baldridge and Rapoport, 2016; Carvalho et al., 2010; Stein et al., 2014; Vasic et al., 2020), but other components such as the Derlin Der1 are also involved, as shown by biochemical data and a recent cryo-EM structure of the Hrd1 complex (Mehnert et al., 2014; Wu et al., 2020). Less is known about retrotranslocation of membrane proteins. The machinery that mediates this process needs to be quite versatile, because substrates can exhibit different topologies. They may contain one or multiple transmembrane (TM) segments, stretches of hydrophilic amino acids in luminal loops and tightly folded domains. How these structurally and physicochemically diverse elements move across a phospholipid bilayer during the extraction process is not known. Multipass transmembrane proteins such as members of the Derlin family (Der1 and Dfm1 in yeast; Derlin-1,-2 in animals), Hrd1 and Doa10 have been suggested to act as retrotranslocases for membrane proteins (Carvalho et al., 2010; Hampton and Sommer, 2012; Lilley and Ploegh, 2004; Neal et al., 2018; Swanson et al., 2001; Ye et al., 2004).

Another unresolved but linked question regards the folding state of luminal domains during the retrotranslocation process. It is unclear whether luminal domains are moved across the membrane in a folded state, if unfolding occurs prior to retrotranslocation, potentially by a separate ER luminal machinery, or if unfolding is directly coupled to retrotranslocation (Brodsky, 2012; Shi et al., 2019).

To address these questions, we investigated ERAD mediated by the ubiquitin ligase Doa10 from S. cerevisiae. Doa10 is a $150 \mathrm{kDa}$ protein with $14 \mathrm{TM}$ segments (Kreft et al., 2006). Its substrates include single- and multi-spanning membrane proteins of the ER and inner nuclear membrane, but also soluble proteins of the cyto- and nucleoplasm (Ravid et al., 2006). No completely soluble, luminal substrates of Doa10 have been described. The degrons of Doa10 substrates can be cytoplasmic (Furth et al., 2011; Swanson et al., 2001), or within the TM region (Habeck et al., 2015). 
Furthermore, Doa10 regulates sterol metabolism in plants, fungi and animals by degrading squalene monooxygenase (Doblas et al., 2013; Foresti et al., 2013). Through degradation of mislocalized membrane proteins, Doa10 has a role in maintaining organelle identity (Dederer et al., 2019; Matsumoto et al., 2019; Ruggiano et al., 2016).

Doa10 works in concert with two ubiquitin conjugating enzymes (E2), the tail-anchored membrane protein Ubc6 and the soluble cytoplasmic protein Ubc7 (Swanson et al., 2001). Ubc7 is anchored to the ER membrane by Cue1 (Biederer et al., 1997). Experiments with soluble cytoplasmic protein fragments showed that Ubc6 and Ubc7 have different roles in the build-up of polyubiquitin chains. Ubc6 initiates ubiquitin chains by transferring the first ubiquitin moiety, whereas Ubc7 extends ubiquitin chains with mainly lysine 48 linkage (Weber et al., 2016). Importantly, Ubc6 is itself an unstable protein and degraded in a Doa10-dependent manner (Swanson et al., 2001; Walter et al., 2001). Similarly, Ubc6 homologues in plants and mammals have been shown to be unstable and are degraded by the proteasome (Lam et al., 2014). Interestingly, in case of the Arabidopsis thaliana Ubc6 homologue Ubc32, and the mammalian Ube2J1, Hrd1 was identified as the ubiquitin ligase involved (Burr et al., 2011; Chen et al., 2016).

Here, we developed a reconstituted system with purified proteins to investigate the role of Doa10 in membrane protein retrotranslocation. This system allowed us to mechanistically investigate membrane protein extraction, without relying on indirect read-outs of downstream reactions such as proteasomal degradation. We show that Doa10 is a membrane protein retrotranslocase. Furthermore, we show how Doa10 cooperates with the Cdc48 ATPase in the extraction of proteins with folded luminal domains.

\section{Results}

We reconstituted purified and fluorescently labeled Doa10 and its substrate Ubc6 into separate liposome populations, together with complementary SNARE proteins (Figure 1A and Figure 1-figure supplement 1, A to F). The tail-anchored (TA) membrane protein Ubc6 was chosen as a model substrate, to limit the number of membrane proteins in our system and thus its complexity. To achieve efficient liposome fusion, we employed previously well-characterized engineered versions of rat SNAREs involved in synaptic vesicle exocytosis (Cypionka et al., 2009; Hernandez et al., 2012). Indeed, mixing of the two liposome sets led to SNARE-mediated co-reconstitution of Ubc6 and Doa10 (Figure 1-figure supplement 1G). This approach ensures that Doa10 and Ubc6 only interact in the phospholipid bilayer, avoiding non-native interactions that can occur when membrane proteins are mixed in the presence of detergents for co-reconstitution. Protease protection experiments showed that Doa10 was reconstituted mostly in the correct orientation (Figure 1-figure supplement $1 \mathrm{H}$ ). For Ubc6, $45 \%$ was correctly oriented, another $45 \%$ wrong-side out oriented, and a minor fraction not properly membrane inserted (Figure 1-figure supplement 1, I to K).

We postulated that retrotranslocase activity of Doa10 facilitates release of a substrate into the aqueous solution, but that such an event should be energetically disfavored unless the membranereleased state was stabilized by chaperones and re-insertion prevented (Figure 1B). To test this hypothesis, we incubated Ubc6 liposomes with Get3, a chaperone for TA proteins (Mateja et al., 2009) that also interacts with Ubc6 (Figure 1-figure supplement 2A). Liposomes were then immobilized to separate soluble and membrane-bound proteins (Figure 1-figure supplement 2B). In the presence of Get3, $43 \pm 4 \%$ Ubc6 was released from liposomes with co-reconstituted Doa10 (Figure $1 C, D$ and Figure 1-figure supplement 2C). When co-reconstitution of Doa10 with Ubc6 was prevented by inhibiting SNARE-mediated fusion or when Ubc6 liposomes were fused with liposomes lacking Doa10, only 7-9\% of Ubc6 were found in the soluble fraction, representing the fraction of Ubc6 sticking to the outside of the liposome surface. In the absence of Get3, or when we used a Get3 mutant defective in TA protein binding (Get3 I193D) (Mateja et al., 2009), we observed no, or drastically reduced release, respectively. Nucleotide hydrolysis was not required for Ubc6 release and an ATPase deficient mutant (Get3 D57N) behaved indistinguishably from wild-type (WT) Get3. This suggests that Doa10 allows for passive movement of its substrate Ubc6 out of the membrane.

Release of Ubc6 from the liposome membrane involves movement of the TM anchor and the luminally encapsulated C-terminus across the lipid bilayer. To directly measure exposure of the C-terminus upon retrotranslocation we used a Ubc6 variant labeled with an AlexaFluor488 (A488) 
A<smiles>CC1CCCCC1C</smiles>
t-SNARE

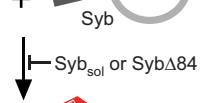

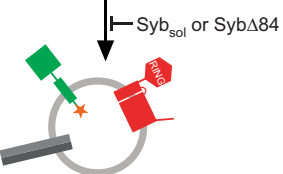

C
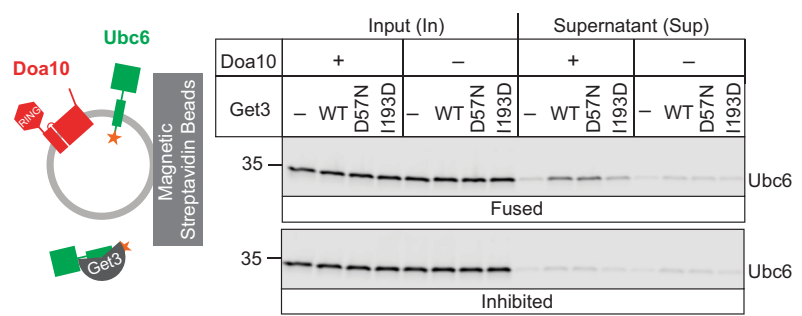

B

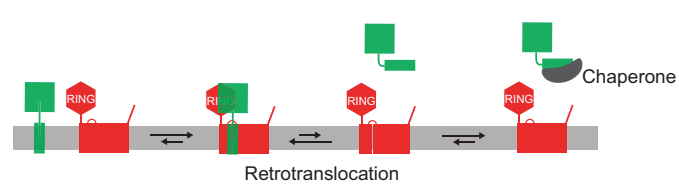

Retrotranslocation
E

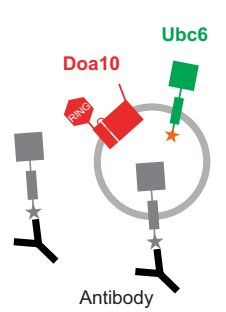

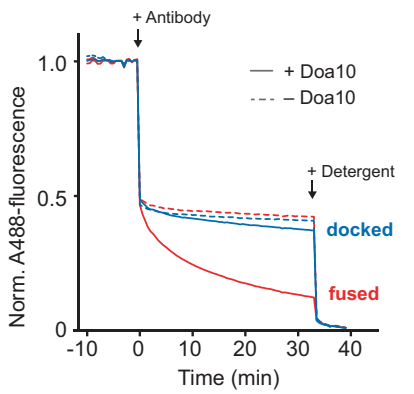

$\mathbf{F}$

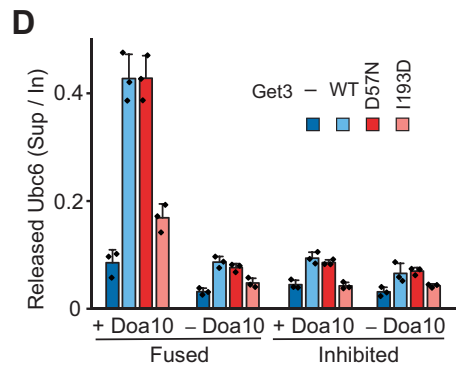

G
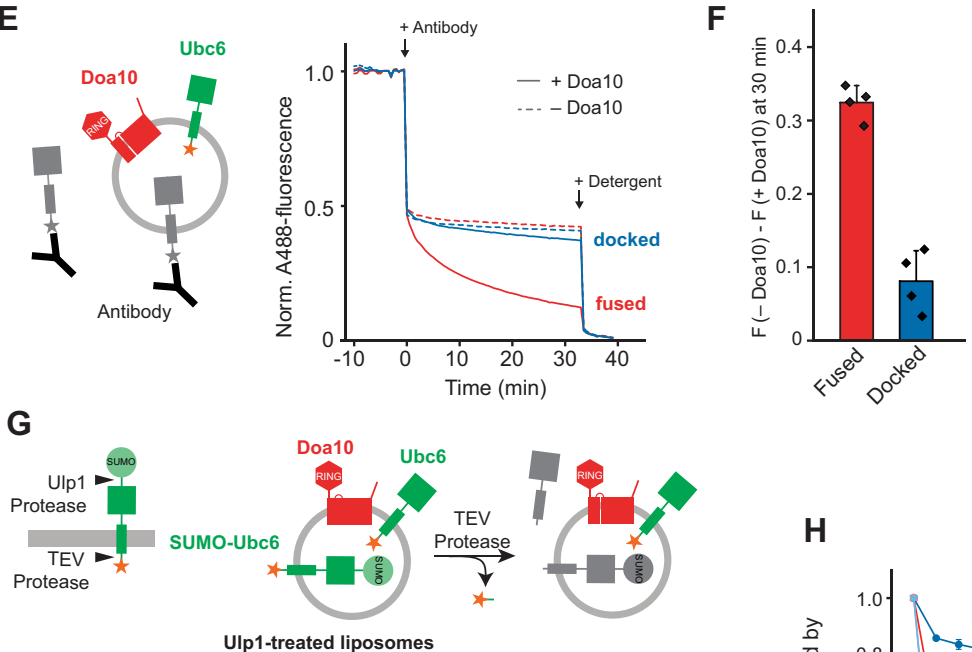

Ulp1-treated liposome
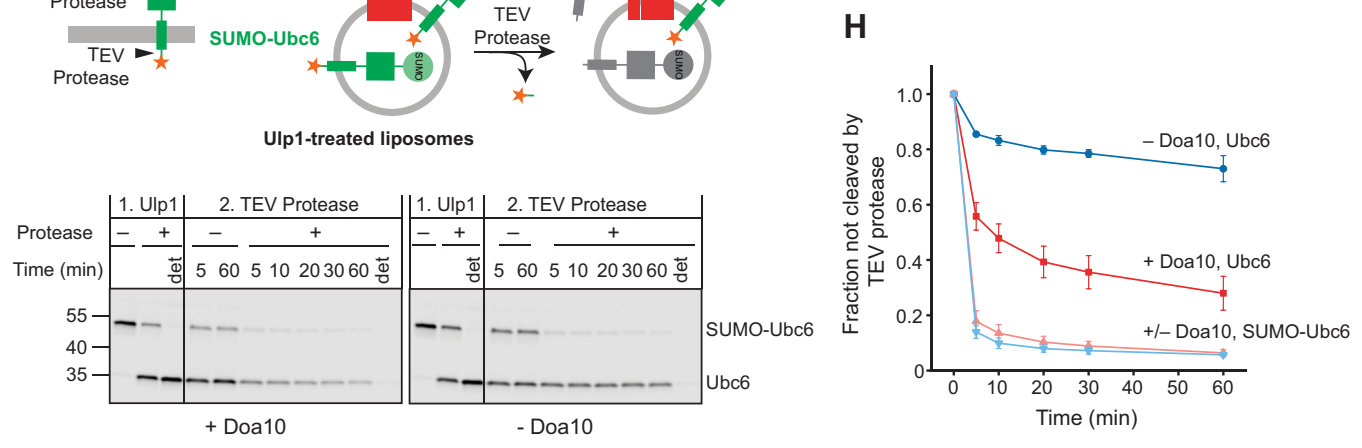

Figure 1. Retrotranslocation of Ubc6 by Doa10. (A) SNARE-mediated co-reconstitution of Ubc6 and Doa10. Engineered versions of SNAREs involved in

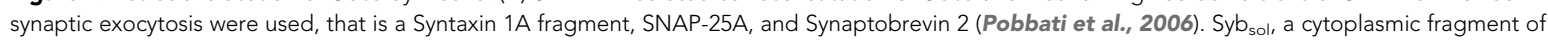
Synaptobrevin (Syb). Syb $\Delta 84$, Syb mutant that results in a docked state (Hernandez et al., 2012). See Figure 1-figure supplement 1, E to K for characterization of liposomes. (B) Working hypothesis for retrotranslocation by Doa10. (C) Membrane release of Ubc6 in the presence of Get3. Figure 1 continued on next page 
Figure 1 continued

Fluorescently labeled Ubc6 was co-reconstituted with Doa10 by SNARE-mediated fusion (+ Doa10), as shown in (A). Where indicated, Ubc6 liposomes were fused to liposomes lacking Doa10 (- Doa10), or fusion was inhibited with Syb sol (Inhibited). After incubation with the indicated Get3 variants or buffer, liposomes were immobilized (Figure 1-figure supplement 2B). Input and supernatant samples were analyzed by SDS-PAGE and fluorescence scanning. Final concentrations (f.c.): $0.1 \mu \mathrm{M}$ Ubc6, $40 \mathrm{nM}$ Doa10, $10 \mu \mathrm{M}$ Get3. (D) Quantification (mean $\pm \mathrm{SD}$ ) of three independent experiments as in (C). (E) Retrotranslocation of Ubc6, measured as quenching of a C-terminal AlexaFluor488 (A488) label by an antibody. Liposomes were generated as shown in (A). Where indicated, liposomes lacked Doa10 (- Doa10), or co-reconstitution was inhibited by using Syb $\Delta 84$ (docked). Arrows indicate addition of the quenching antibody or of solubilizing amounts of detergent (Triton X-100). F.c.: $0.2 \mu \mathrm{M}$ Ubc6, 80 nM Doa10. (F) Quantification $($ mean \pm SD) of four experiments as in (E). The fraction of accessible dye after 30 min was compared between conditions with and without Doa10. F, normalized fluorescence. (G) Retrotranslocation of Ubc6, measured by a protease protection assay. Ubc6 with an N-terminal SUMO tag (SUMO-Ubc6) and a TEV protease cleavage site between the C-terminus and the fluorescent dye was used. Arrow heads indicate cleavage sites for Ulp1 and TEV protease. SUMO-Ubc6 liposomes with or without Doa10 were incubated with Ulp1. Ulp1-treated liposomes were then incubated with buffer or TEV protease. Indicated reactions contained detergent to solubilize liposomes (det). Aliquots were taken at the indicated times and analyzed by SDS-PAGE and fluorescence scanning. F.c. during incubation with TEV protease: $0.1 \mu \mathrm{M}$ Ubc6, $40 \mathrm{nM}$ Doa10, $10 \mu \mathrm{M}$ TEV protease. (H) Quantification (mean \pm SD) of the fraction of Ubc6 and SUMO-Ubc6 inaccessible to TEV protease, from three experiments as in (G). Band intensities from samples treated with TEV protease were normalized to the corresponding band intensities of samples without TEV protease.

The online version of this article includes the following source data and figure supplement(s) for figure 1:

Source data 1. This file contains the quantification of fluorescently labeled Ubc6 (Figure 1D) as well as of Rhodamine-labeled lipids (Figure 1-figure supplement 2B).

Source data 2. This file contains the quantification of the quenched fraction of Ubc6 in samples containing Doa10 compared to samples lacking Doa10, as shown in Figure 1F.

Source data 3. This file contains the quantification of the TEV-protected fraction of SUMO-Ubc6 and Ubc6 shown in Figure $1 \mathrm{H}$.

Figure supplement 1. Quality control of liposomes.

Figure supplement 1-source data 1. This file contains the quantification of the TEV-and Ulp1 cleaved fraction of SUMO-Ubc6, as well as of Ulp1cleaved Ubc6 that is accessible to TEV protease, as shown in Figure 1-figure supplement $1 \mathrm{~K}$.

Figure supplement 2. Retrotranslocation in the presence of Get3.

Figure supplement 3. Co-reconstitution with ATP synthase.

dye at the C-terminus. An anti-A488 antibody quenches A488 fluorescence and reports on accessibility of the C-terminus (Figure 1E). In the absence of Doa10, we observed a sudden decrease in fluorescence by $50 \%$ upon antibody addition, corresponding to the fraction of wrong-side out protein that exposes its C-terminus on the outside of liposomes. Upon solubilization of liposomes, the antibody quenches the fluorescence of all A488 epitopes. However, in the presence of Doa10, the sudden decrease in fluorescence was followed by a slower decrease to about $10 \%$ of the original fluorescence signal within $30 \mathrm{~min}$. Thus, in the presence of Doa10, the luminally-encapsulated part of Ubc6 becomes accessible to the antibody over time. Ubc6 and Doa10 need to reside in the same membrane as we observed only minor quenching above background when we used a mutant SNARE that only supports liposome docking (Figure 1E,F).

As an alternative read-out for retrotranslocation, we used a protease protection assay. To identify correctly oriented Ubc6 we used an N-terminal SUMO fusion (SUMO-Ubc6) and Ulp1 protease. To monitor retrotranslocation, we introduced a TEV protease cleavage site between the C-terminus of Ubc6 and the fluorescent dye. This cleavage site resides in the liposome lumen and would only become accessible upon retrotranslocation (Figure 1G). Ulp1 incubation resulted in a shift of correctly oriented protein in SDS-PAGE. We then added TEV protease and followed cleavage over time (Figure 1G,H). Wrong-side out SUMO-Ubc6 was completely accessible to TEV protease and was cleaved independently of the presence of Doa10 within $5 \mathrm{~min}$. Strikingly, only in the presence of Doa10, Ulp1-cleaved, and thus right-side out Ubc6 was also accessible to TEV cleavage over longer incubation times, indicating retrotranslocation. In liposomes lacking Doa10, only a small fraction of Ulp1-cleaved Ubc6 was accessible to TEV protease, corresponding to the not properly reconstituted Ubc6. Together, Get3 capture, antibody accessibility, and protease protection assays show that Doa10 facilitates movement of the Ubc6 TM across the membrane into the aqueous phase. Comparison of the fraction of correctly oriented protein and the released fraction shows that retrotranslocation is very efficient in all three assays. Thus, Doa10 is a retrotranslocase.

Importantly, we also tested if another unrelated multipass membrane protein leads to Ubc6 retrotranslocation by destabilizing the lipid bilayer. To this end, we purified the TF $F_{0}$ ATP synthase from Bacillus PS3, which contains 20 TM segments (Guo et al., 2019). Using the same reconstitution 
A
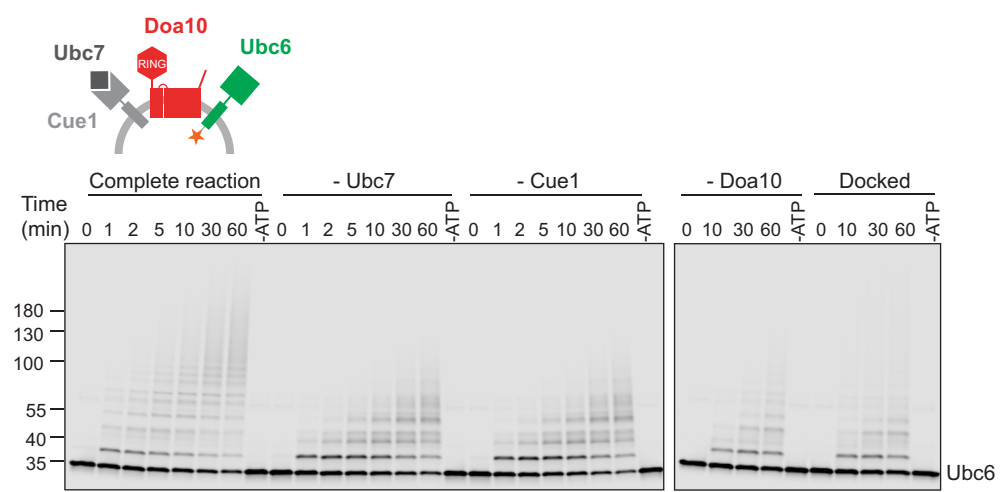

B

\section{C}
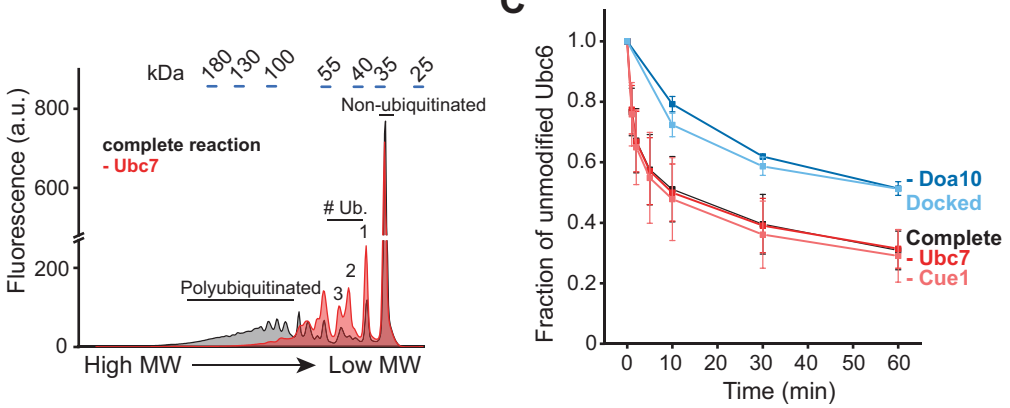

D
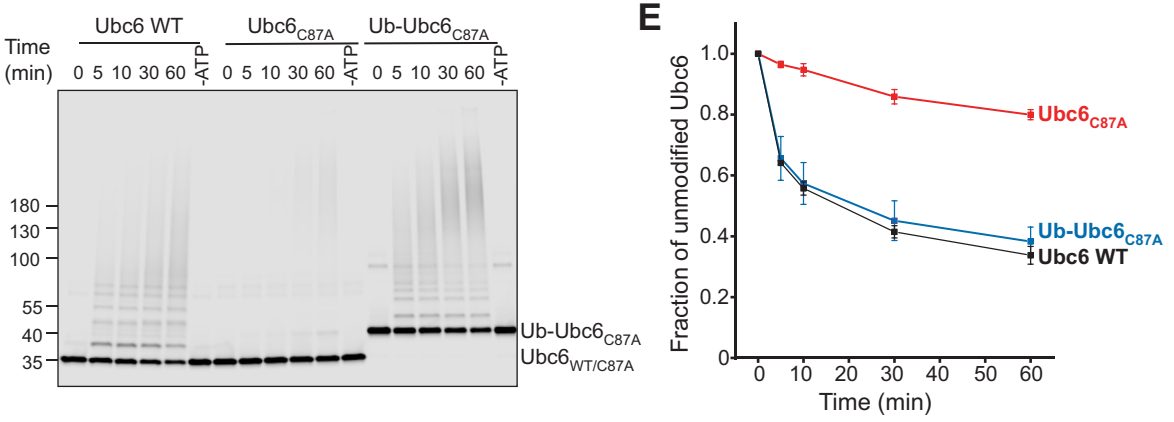

$\mathbf{F}$
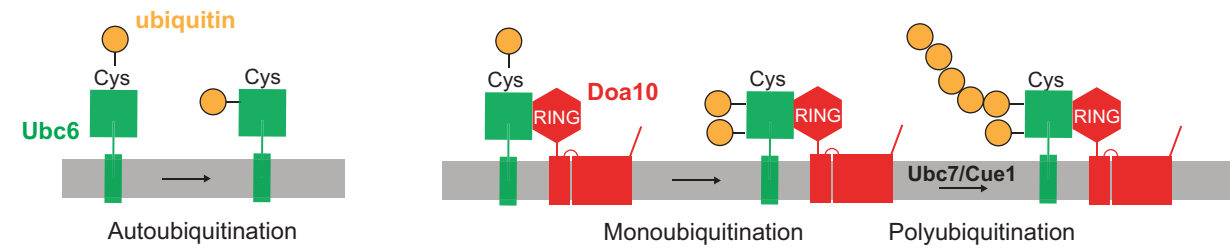

Figure 2. Ubiquitination of Ubc6. (A) Time course of ubiquitination of Ubc6. Final concentrations in the complete reaction: $40 \mathrm{nM}$ Doa10, $10 \mathrm{nM}$ Cue 1

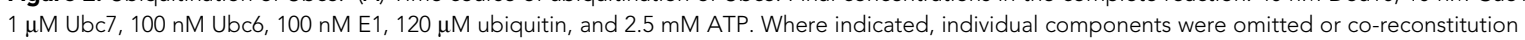
was inhibited by using Syb $\Delta 84$ (Docked). For each reaction, a 60 min sample in the absence of ATP is shown. Samples were analyzed by SDS-PAGE and fluorescence scanning. (B) Analysis of ubiquitin-chain length on Ubc6 from an experiment as in (A). Line-scans were performed on fluorescence images Figure 2 continued on next page 
Figure 2 continued

for the complete reaction and in the absence of $\mathrm{Ubc7}$ at $\mathrm{t}=30 \mathrm{~min}$. Approximate molecular weights are indicated on top. \# $u b$. denotes number of ubiquitin moieties attached. (C) Quantification (mean \pm SD) of the fraction of unmodified Ubc6 from three experiments as in (A). (D) Time course of ubiquitination of Ub-Ubc6 ${ }_{\mathrm{C} 87 \mathrm{~A}}$ compared to $\mathrm{Ubc6}$ WT and $\mathrm{Ubcb}_{\mathrm{c} 87 \mathrm{~A}}$ in the presence of Doa10, Cue1, and Ubc7. Concentrations and analysis as in (A). (E) Quantification (mean \pm SD) of the fraction of unmodified Ubc6 variants from three experiments as in (D). (F) Model for ubiquitination of Ubc6. Ubc6 autoubiquitination activity results in transfer of ubiquitin from its active site cysteine to a non-cysteine residue (Weber et al., 2016). In the presence of Doa10, this activity is enhanced and Ubc6 is multi-monoubiquitinated. Ubc7/Cue1 are then required to form polyubiquitin chains on monoubiquitinated Ubc6.

The online version of this article includes the following source data and figure supplement(s) for figure 2:

Source data 1. This file contains the quantification of the fraction of unmodified Ubc6 from three experiments as in Figure 2A, as shown in in Figure $2 C$. Source data 2. This file contains the quantification of the fraction of unmodified Ubc6 from three experiments as in Figure 2D, as shown in Figure 2E. Source data 3. This file contains the quantification of the fraction of unmodified Ubc6 from three experiments as in Figure 2-figure supplement 1C, as shown in Figure 2-figure supplement 1D.

Figure supplement 1. E3-independent and -dependent ubiquitination of Ubc6.

protocol as for Doa10, we co-reconstituted ATP synthase with Ubc6 (Figure 1-figure supplement $3 A, B)$ and then tested for retrotranslocation of Ubc6 using the antibody accessibility assay. Under these conditions, only a minor fraction of Ubc6 becomes accessible to the antibody (Figure 1-figure supplement $3 C, D$ ). We conclude that retrotranslocation of Ubc6 is not due to some non-specific perturbation of the membrane caused by any multipass TM protein.

The observation that traps such as Get3 or the antibody are sufficient to drive retrotranslocation to completion suggests that Doa10 allows membrane-inserted and retrotranslocated soluble states of Ubc6 to exist in an equilibrium (Figure 1B). In the absence of Get3 or the anti-A488 antibody, the membrane-embedded state of the substrate is energetically favored. Get3 or the anti-A488 antibody shift the equilibrium towards the soluble state by binding to retrotranslocated Ubc6. Thus, traps bind retrotranslocated Ubc6 and prevent reinsertion. In the cell, retrotranslocation requires Cdc48 activity (Garza et al., 2009; Nakatsukasa et al., 2008; Ye et al., 2001), suggesting that the pulling force generated by $\mathrm{Cdc} 48$ provides the directionality.

To test this directly, we next investigated membrane extraction of Ubc6 by Cdc48. As Cdc48 acts on polyubiquitin chains, we first reconstituted Ubc6 polyubiquitination. Degradation of Ubc6 requires its own E2 activity, the E2 Ubc7 and its adapter, the membrane-anchored Cue1 (Biederer et al., 1997; Swanson et al., 2001; Walter et al., 2001). Using the fusion system, we coreconstituted Doa10 and Cue1 with Ubc6. When we added ubiquitin activating enzyme (E1), ubiquitin, ATP, and Ubc7, we observed robust polyubiquitination of Ubc6 (complete reaction, Figure 2A). In the absence of Ubc7 or Cue1, polyubiquitination was abolished, and we observed ubiquitin adducts of lower molecular weight (Figure 2A,B). These represent multiple monoubiquitinations, as the ubiquitination pattern was very similar when we used a ubiquitin mutant in which all lysines are mutated to arginine (Ubiquitin $\mathrm{KO}$ ) and that thus cannot form ubiquitin chains (Figure 2-figure supplement 1A). Monoubiquitination also occurred in the absence of Doa10 (Figure 2-figure supplement $1 B$ ), but was enhanced in its presence (Figure 2-figure supplement $1 C, D$ ). Kinetics of transfer of the first ubiquitin onto Ubc6 were independent of the presence of Ubc7/Cue1 (Figure 2C), suggesting that monoubiquitination is a prerequisite for Ubc7-dependent polyubiquitination. This is indeed the case, as a catalytically inactive Ubc6 mutant (Ubc6 $c 87 A$ ) was not ubiquitinated, but an $\mathrm{N}$-terminal fusion of ubiquitin with inactive Ubc6 (Ub-Ubc6 $6_{C 87 A}$ ) was a substrate for Ubc7-dependent polyubiquitination (Figure 2D,E and Figure 2-figure supplement 1E). Together, these results establish that after active site loading of Ubc6 with ubiquitin, Doa10 catalyzes Ubc6monoubiquitination, followed by Ubc7/Cue1-dependent polyubiquitination (Figure 2F). These results agree with observations made in intact cells and with recombinant soluble fragments of Doa10 and Ubc6 (Walter et al., 2001; Weber et al., 2016). Furthermore, they indicate that our reconstituted system faithfully recapitulates the in vivo ubiquitination pathway for Ubc6.

Next, we tested for membrane extraction of polyubiquitinated Ubc6 by the Cdc48 ATPase. To this end, we immobilized Doa10/Ubc6 liposomes after the ubiquitination reaction, then incubated with Cdc48 and its co-factors Ufd1 and Npl4 (UN), and analyzed soluble and membrane-bound fractions (Figure 3A and Figure 3-figure supplement 1,A to D). We observed Cdc48- and ubiquitination-dependent extraction of Ubc6 (Figure $3 A, B$ and Figure 3-figure supplement 1E,F). 


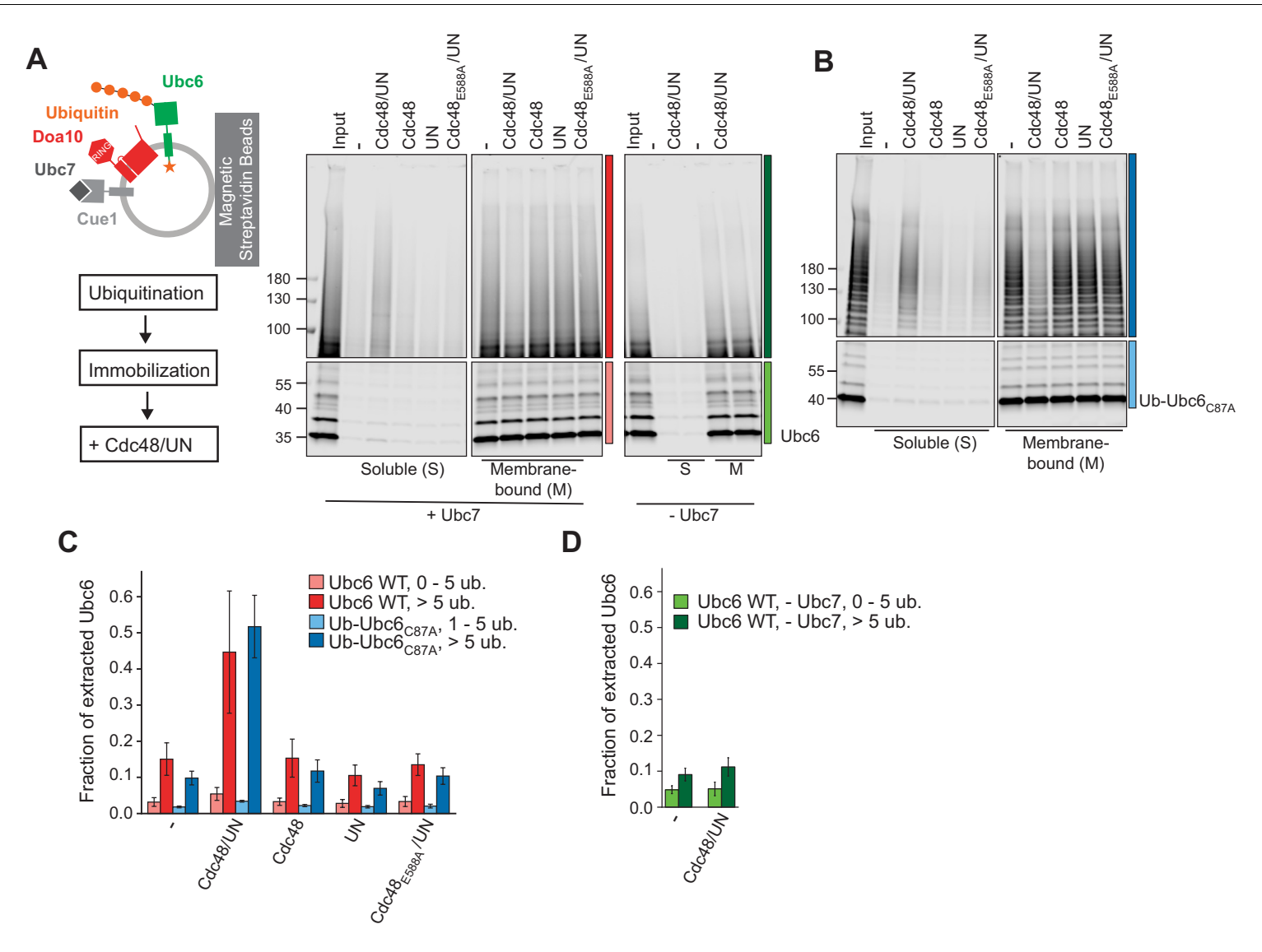

Figure 3. Cdc48-mediated Membrane Extraction of Ubc6. (A) Extraction of Ubc6 by Cdc48 and Ufd1/Npl4 (UN). After ubiquitination, liposomes were immobilized (Figure 3-figure supplement 1,A to D). One bead equivalent was removed, and bound protein was eluted with SDS sample buffer (Input). Beads were then incubated with the indicated components. Soluble (S) and membrane-bound (M) material were analyzed by SDS-PAGE and fluorescence scanning. Colored bars indicate categorization of ubiquitin chain length as used for quantification in (C) and (D). For better visibility, bottom and top gel parts are scaled differently. See Figure 3-figure supplement 1E for uncut image. Final concentrations: 50 nM Ubc6, 20 nM Doa10, $0.1 \mu \mathrm{M}$ Cdc48 hexamer, $0.1 \mu \mathrm{M}$ Ufd1 and Npl4. (B) As in (A), but with Ub-Ubc6 $c 87 \mathrm{~A}$ instead of Ubc6. See Figure 3-figure supplement 1F for uncut image. (C) Quantification (mean \pm SD) of three experiments as in (A) and (B). Ubiquitinated species were categorized according to ubiquitin chain length, as indicated in (A) and (B). The signal in the soluble fraction was normalized to that in the input. (D) Quantification (mean \pm SD) of three experiments as in (A), when ubiquitination was performed in the absence of Ubc7.

The online version of this article includes the following source data and figure supplement(s) for figure 3 :

Source data 1. This file contains the quantification of the fraction of extracted Ubc6 from three experiments as in Figure $3 \mathbf{A}, \mathbf{B}$, as shown Figure $3 \mathbf{C}, \mathbf{D}$. Figure supplement 1. Cdc48-mediated extraction.

Extraction efficiency was dependent on the length of ubiquitin chains, with five ubiquitin moieties being minimally required (Figure 3-figure supplement 1G). In the presence of Cdc48/UN, $45 \pm$ $17 \%$ of Ubc6 molecules with more than five attached ubiquitin moieties were extracted, compared to $15 \pm 4 \%$ in the absence of the $\mathrm{Cdc} 48$ complex (Figure $3 \mathrm{~A}, \mathrm{C}$ ). No extraction above this background was observed when either Cdc48 or Ufd1/Npl4 were omitted. Furthermore, ATP hydrolysis by the $\mathrm{Cdc} 48$ complex was necessary, as $\mathrm{Cdc} 48_{\mathrm{E} 588 \mathrm{~A}}$ was inactive. Polyubiquitin chains were required because we found no extraction above background when ubiquitination was performed in the absence of Ubc7 or less than five ubiquitins were attached (Figure $3 A, C, D)$. Similar observations 


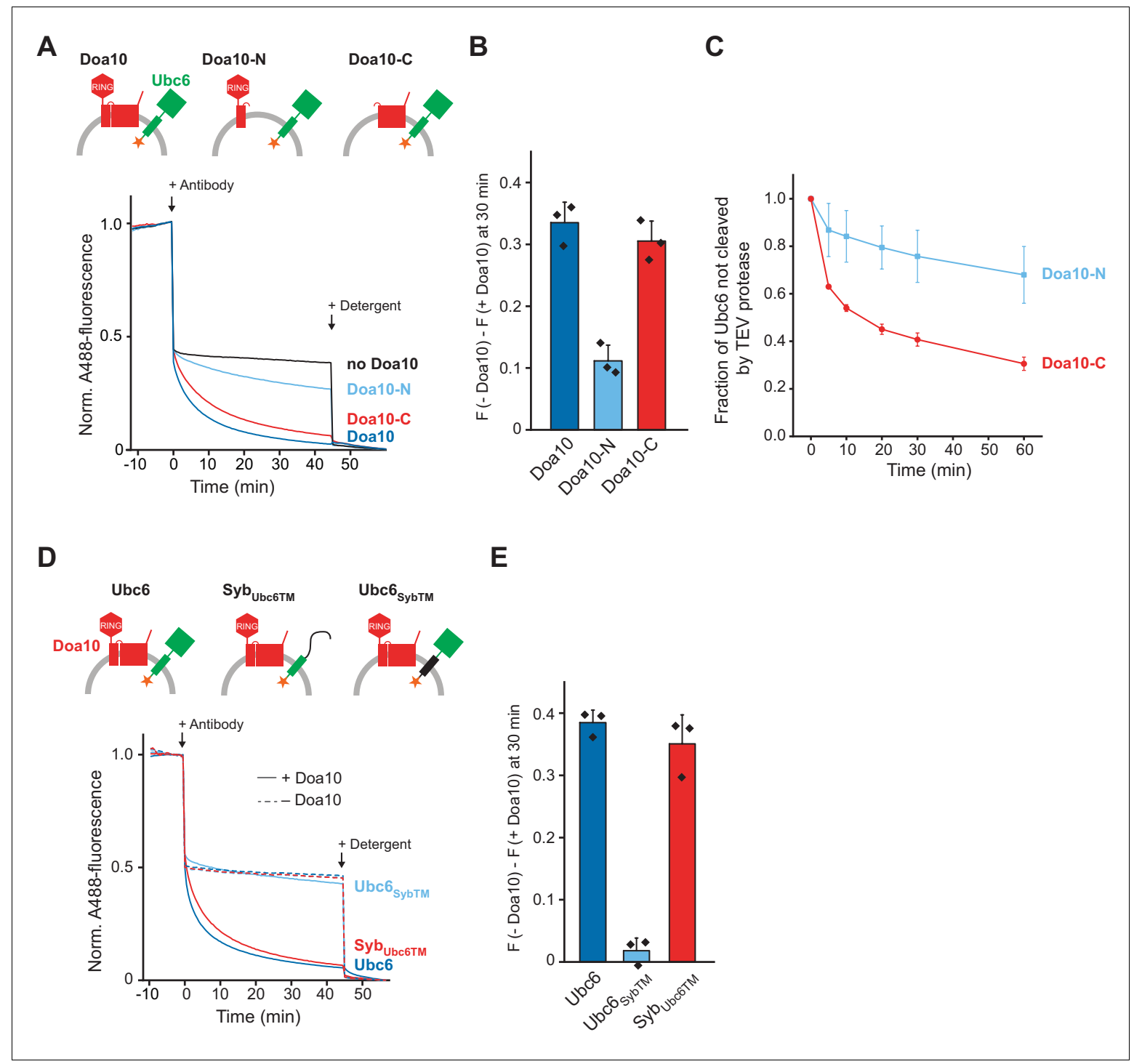

Figure 4. Structural Determinants for Retrotranslocation. (A) Retrotranslocation of Ubc6 by Doa10 variants, as measured by accessibility of a fluorescence quenching antibody to a C-terminal A488 dye on Ubc6, as described in Figure 1E. Ubc6 liposomes containing the indicated Doa10 variants were used. Doa10-N, residues 1-468; Doa10-C, residues 434-1319. Arrows indicate addition of antibody or detergent. Final concentrations (f. c.): $0.2 \mu \mathrm{M}$ Ubc6, $80 \mathrm{nM}$ Doa10 variants. (B) Quantification (mean \pm SD) of three experiments as in (A). The fraction of accessible dye after 30 min was compared between conditions with the indicated Doa10 variant and without Doa10. F, normalized fluorescence. (C) Retrotranslocation of Ubc6 by Doa10 variants, as measured by accessibility of TEV protease to the C-terminus of Ubc6, as described in Figure 1G. SUMO-Ubc6 liposomes with either Doa10-N or Doa10-C were treated with Ulp1 to identify right-side out oriented Ubc6. TEV protease was added and samples at different time points were analyzed by SDS-PAGE and fluorescence scanning. Quantification as in Figure 1H, but only for Ulp1-cleaved Ubc6. F.c. during incubation with TEV protease: $0.1 \mu \mathrm{M}$ Ubc6, $40 \mathrm{nM}$ Doa10 variants, $10 \mu \mathrm{M}$ TEV protease. (D) Retrotranslocation of Ubc6 variants measured as in (A). A488-labeled Ubc6, Ubc6 SуьтM, or Sybubc6TM were directly co-reconstituted with Doa10 because Sybubc6TM was incompatible with SNARE-mediated co-reconstitution. Liposomes containing Doa10 were affinity-purified for this experiment (Figure 4-figure supplement 1A,B). (E) Quantification (mean \pm SD) of three experiments as in (D). The fraction of accessible dye after 30 min was compared between conditions with and without Doa10.

Figure 4 continued on next page 
Figure 4 continued

The online version of this article includes the following source data and figure supplement(s) for figure 4:

Source data 1. This file contains the quantification of the quenched fraction of Ubc6 in samples containing Doa10 or its variants compared to samples lacking Doa10 from three experiments as in Figure 4A, as shown in Figure 4B.

Source data 2. This file contains the quantification of the TEV-protected fraction of Ubc6 from three experiments, as shown in Figure $4 C$.

Source data 3. This file contains the quantification of the quenched fraction of Ubc6 or its variants in samples containing Doa10 compared to samples lacking Doa10 from three experiments as in Figure 4D, as shown in Figure 4E.

Figure supplement 1. Antibody accessibility assay for Ubc6/Syb chimera.

Figure supplement 1-source data 1. This file contains numerical values for data shown in Figure 4-figure supplement 1E.

were made when we used Ub-Ubc6 ${ }_{\mathrm{C} 87 \mathrm{~A}}$ instead of WT Ubc6 to increase the efficiency of polyubiquitination (Figure 3B,C). Together, these observations show that the Cdc48 complex provides the driving force for the extraction of a polyubiquitinated membrane protein. We currently do not understand what limits the efficiency of $\mathrm{Cdc} 48$ mediated extraction in our assay. It is possible that a stabilizing chaperone or an accessory factor such as the Cdc48 co-factor Ubx2 would contribute to complete extraction (Neuber et al., 2005; Schuberth and Buchberger, 2005).

To define structural elements in Doa10 important for its retrotranslocase activity, we generated two truncated versions of Doa10 that encompassed either only the N-terminal RING domain and the first two TM segments (Doa10-N), or the C-terminal part containing TM segments 3-14 (Doa10-C) (Figure 1-figure supplement $1, F$ to $H$ ). The sites of truncation were chosen based on the finding that in the yeast Kluyveromyces lactis, Doa10 is expressed as two separate polypeptides with similar boundaries (Stuerner et al., 2012). We then tested if those Doa10 variants retrotranslocate Ubc6 using the antibody accessibility assay. Doa10-C behaved similarly to full-length Doa10, whereas Doa10-N resulted in only minor quenching above background (Figure 4A,B). Corresponding observations were made when we tested for retrotranslocation using the protease protection assay (Figure 4C). These results show that TM segments 3-14 in Doa10 are sufficient to mediate retrotranslocation of Ubc6.

To test for structural elements in Ubc6 relevant for retrotranslocation, we generated mutants in which either its TM anchor or its cytoplasmic part were replaced with the corresponding segments of the TA protein synaptobrevin (Ubc6 SybTM and Sybubc6TM, respectively). We then tested for retrotranslocation of these mutants using the antibody accessibility assay (Figure 4-figure supplement 1A,B). We only observed retrotranslocation of the Ubc6 TM, but not of the Syb TM (Figure 4D,E). A similar experimental setup also allowed us to exclude leakage or liposome rupture as the cause for antibody accessibility (Figure 4-figure supplement 1,C to E). Thus, the identity of the substrate's TM segment is important for retrotranslocation.

Next, we tested how these mutations in the TM domains of Doa10 and Ubc6 affect ubiquitination of Ubc6. To specifically test for effects on polyubiquitination, we again used Ub-Ubc6 $6_{C 87 A}$, for which the initial ubiquitination steps (ubiquitin loading and monoubiquitination) are bypassed. Replacement of the Ubc6 TM with the TM of Syb mildly affected polyubiquitination as seen by the emergence of shorter ubiquitin chains in the case of the Syb TM (Figure 5A,B; Figure 5-figure supplement $1 A, B$ ). To test for effects of TM replacement on monoubiquitination of Ubc6, we compared Ubc6 and Ubc6 SybTM. Doa10-dependent monoubiquitination of Ubc6 SybTM was impaired (Figure 5C,D; Figure 5-figure supplement 1C,D), while E3-independent autoubiquitination of this mutant was unaffected (Figure 5-figure supplement 1E,F). Thus, the Ubc6 TM anchor contributes to the efficient Doa10-dependent ubiquitination of Ubc6, indicating a more efficient recruitment to Doa10.

Moreover, efficient ubiquitination of Ubc6 requires the TM domain of Doa10. Ubc6 polyubiquitination by Doa10-N was less efficient compared to full-length Doa10 (Figure 5E,F). This was not due to impaired E3 activity, because co-reconstitution of Doa10-N and Doa10-C together restored ubiquitination to WT levels. Monoubiquitination in the absence of Ubc7 was similarly affected (Figure 5figure supplement $2, \mathrm{~A}$ to $\mathrm{C}$ ). We conclude that the Doa10 region that includes TMs 3-14 plays a role in ubiquitination of Ubc6. Both ubiquitination and retrotranslocation of Ubc6 are sensitive to changes in the membrane-embedded regions of Ubc6 and Doa10, indicating a specific interaction of Doa10 with the TM domain of Ubc6. Previous observations suggested that the identity of the TM 
A
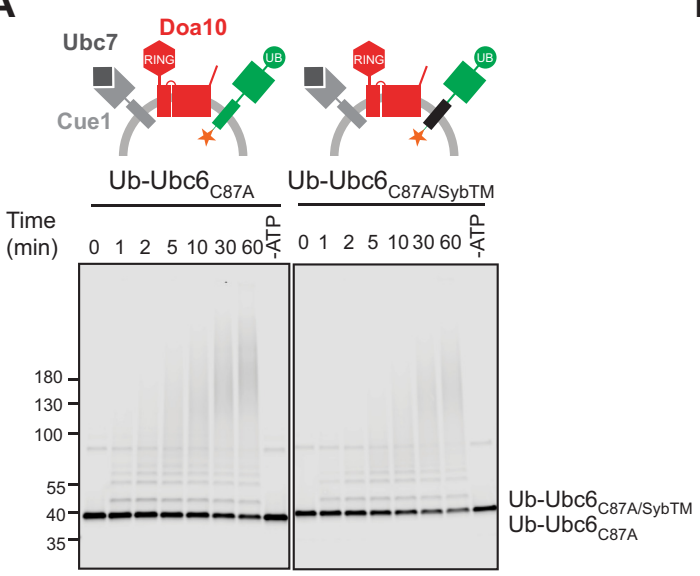

C

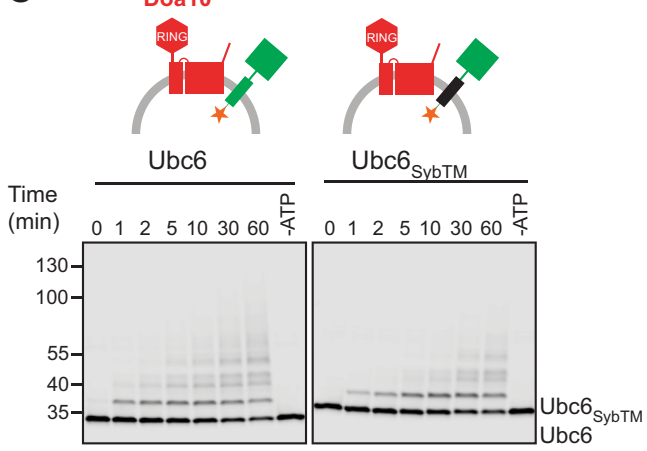

E

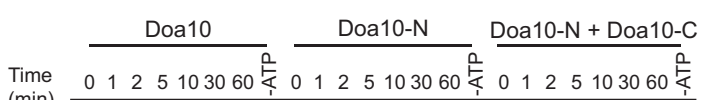

(min) $0125103060 \varangle 0125103060 \ll 0125103060 \varangle$
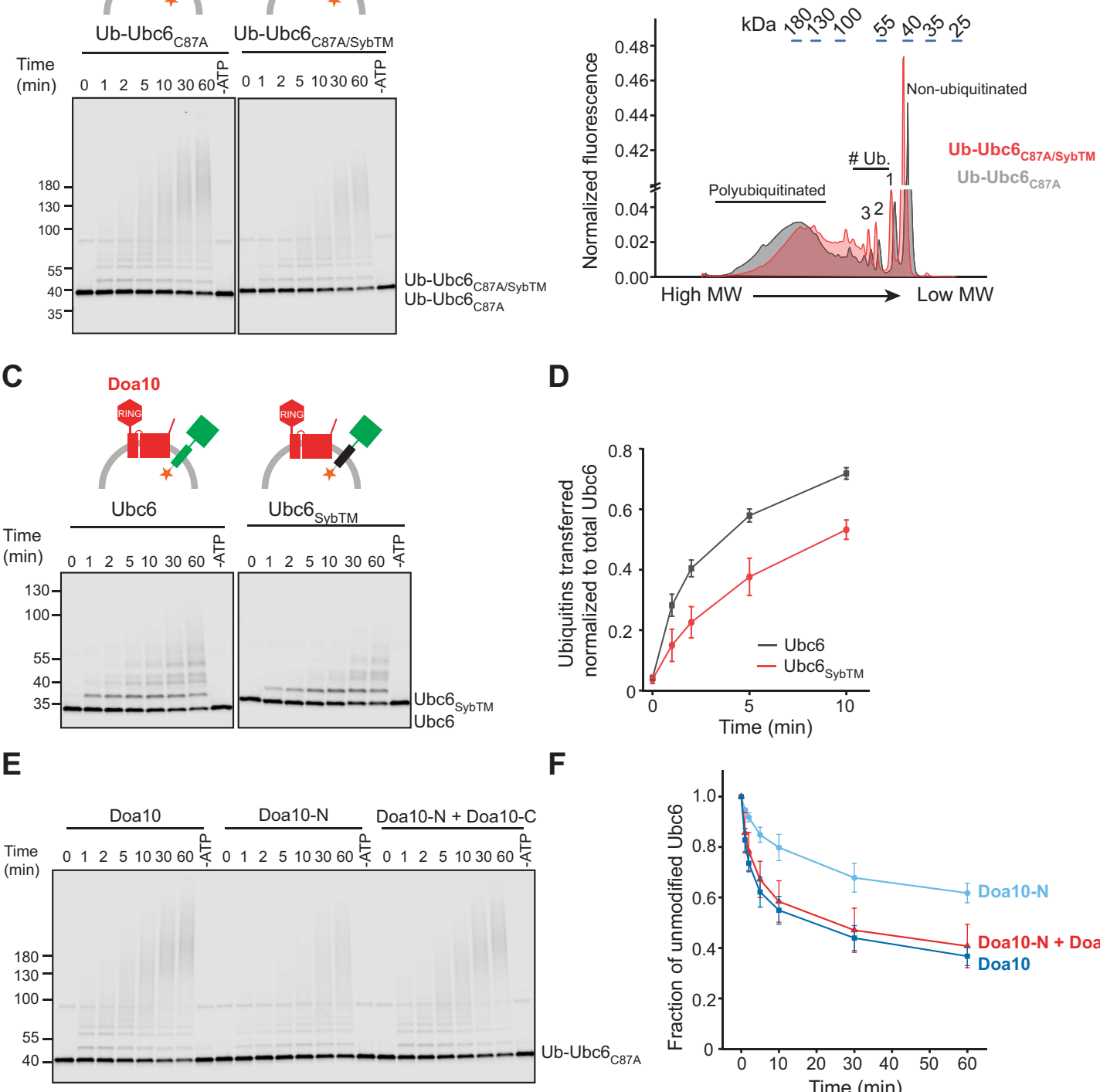

D

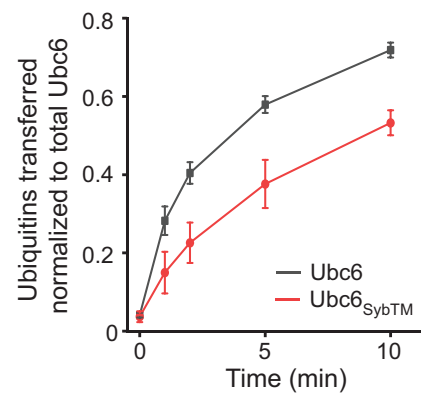

$\mathbf{F}$

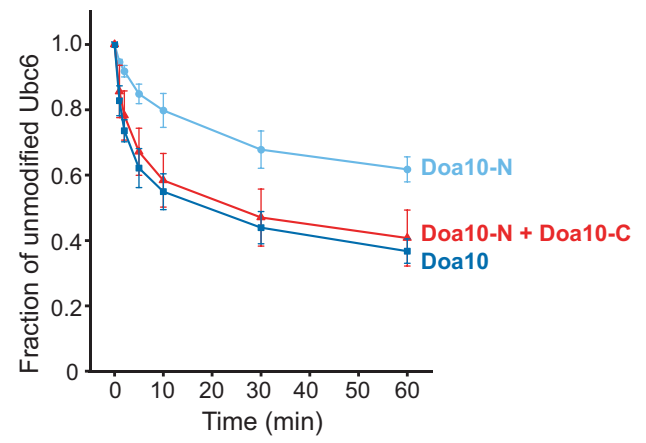

Figure 5. Structural Determinants for Ubiquitination. (A) Time course of ubiquitination of Ub-Ubc6c87A or Ub-Ubc6 C87A/SybTM by Doa10 in the presence of Cue1/Ubc7. For each reaction, a 60 min sample in the absence of ATP is shown. Samples were analyzed by SDS-PAGE and fluorescence scanning. Final concentrations: $40 \mathrm{nM}$ Doa10, $10 \mathrm{nM}$ Cue1, $1 \mu \mathrm{M} \mathrm{Ubc7,} 100 \mathrm{nM}$ Ubc6 variants, $100 \mathrm{nM}$ E1, $120 \mu \mathrm{M}$ ubiquitin, and $2.5 \mathrm{mM}$ ATP. See Figure 5-

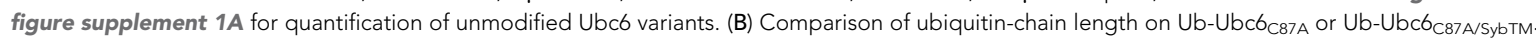
Line-scans were performed on fluorescence images of two representative gel samples (30 min timepoint) as in (A). Approximate molecular weights are Figure 5 continued on next page 
Figure 5 continued

indicated on top. \# Ub., number of ubiquitin moieties attached. (C) Time-course of Ubc6 WT or Ubc6 sybTM ubiquitination in the absence of Ubc7/Cue1. Analysis and concentrations as in (A). See Figure 5-figure supplement $1 \mathrm{C}$ for quantification of unmodified Ubc6 variants. (D) Quantification (mean \pm SD) of total ubiquitin-transfer to Ubcb or UbcbsybTM from three experiments as in (C). Intensities of Ubcb variants with one to four ubiquitin moieties attached were determined as described in Figure 5-figure supplement 1D, summed up for each time point and normalized to total Ubc6 in the reaction. (E) Time course of ubiquitination of Ub-Ubc6 $6_{C 87 A}$ by Doa10 variants in the presence of Cue1/Ubc7. Liposomes contained Ub-Ubc6 $\mathrm{C87A}$ and either full-length Doa10, only Doa10-N, or both Doa10-N and -C. Analysis and concentrations as in (A). (F) Quantification (mean \pm SD) of unmodified Ub-Ubcb ${ }_{\text {C87A }}$ from three experiments as in (E).

The online version of this article includes the following source data and figure supplement(s) for figure 5:

Source data 1. This file contains the quantification of the number of ubiquitins ( $\mathrm{n}$ ) transferred per Ubc6 or Ubc6 SybTM (Figure 5-figure supplement 1D) as well as the quantification of the number of total ubiquitin transferred from three experiments as in Figure 5C, as shown in Figure 5D.

Source data 2. This file contains the quantification of the fraction of unmodified Ubc6 from three experiments as in Figure $\mathbf{5 E}$, as shown in Figure $\mathbf{5 F}$. Figure supplement 1. Ubiquitination of Ubcb/Syb chimera.

Figure supplement 1-source data 1. This file contains the quantification of the fraction of unmodified Ubc6 as shown in Figure 5-figure supplement $1 \mathrm{~A}, \mathrm{C}$ and $\mathrm{F}$.

Figure supplement 2. Ubc6 ubiquitination by Doa10 variants.

Figure supplement 2-source data 1. This file contains the quantification of the fraction of unmodified Ubc6 as shown in Figure 5-figure supplement 2B.

Figure supplement 2-source data 2. This file contains the quantification of the number of total ubiquitin transferred in presence of different Doa10 variants from three experiments, as shown in Figure 5-figure supplement $2 \mathrm{C}$.

segment played a role in substrate degradation (Habeck et al., 2015; Ruggiano et al., 2016; Walter et al., 2001). Our results show that the TM domain of Doa10 recognizes substrates and thereby contributes to the specificity of substrate selection. The observation that the mutant version of Ubc6 (Ubc6 SybTM $_{\text {) }}$ is still ubiquitinated to some extent suggests that other factors might contribute to substrate discrimination. Deubiquitinating enzymes have previously been shown to sharpen substrate selectivity in ERAD and might also play such a role in the context of Doa10-mediated ERAD (Zhang et al., 2013).

Substrates of Doa10 exhibit a wide range of topologies. They may contain multiple TM segments, such as the misfolded variants of the multi-spanning membrane proteins Pma1 and Ste6, called Pma* and Ste6*, respectively (Huyer et al., 2004; Wang and Chang, 2003), or luminal folded domains (Vashist and Ng, 2004). We next asked the question how the presence of an additional luminal polypeptide segment or an interaction with another luminal protein affects retrotranslocation. We appended a streptavidin binding peptide (SBP) to the C-terminus of Ubc6 (Ubc6-SBP), and formed a complex with streptavidin (Figure 6-figure supplement 1A,B). When we co-reconstituted this complex with Doa10, we observed no quenching over time upon antibody addition (Figure 6A, B). When we added biotin, which breaks the high affinity SBP-streptavidin interaction (Keefe et al., 2001), we observed Doa10-dependent quenching over time. This was only the case when biotin was used, but much reduced when we used a biotinylated protein, which is still capable of dissociating streptavidin from Ubc6-SBP on the outside of liposomes (Figure 6-figure supplement 1, C to E), but cannot pass the membrane. Together, this shows that a protein-protein interaction on the luminal side of the membrane, mimicking the presence of a folded domain, acts as an anchor and prevents retrotranslocation of Ubc6.

Finally, we tested if this anchoring can be overcome by the Cdc48 complex. Liposomes containing Doa10 and Ubc6-SBP in complex with streptavidin were incubated with ubiquitination mix followed by the addition of Cdc48 complex and anti-A488 antibody. Retrotranslocation occurred depending on polyubiquitination and Cdc48 activity (Figure $6 C, D$ and Figure 6-figure supplement 1F). In the absence of ubiquitin, when Ubc7 was omitted, or when we used the catalytically inactive Cdc48 ${ }_{\mathrm{E} 588 \mathrm{~A}}$, no fluorescence quenching above background was observed. Importantly, streptavidin remained encapsulated in liposomes in reactions where Ubc6-SBP was extracted (Figure 6E,F). Thus, Cdc48 action on the cytosolic side of the membrane leads to dissociation of streptavidin from the SBP-tag in the liposome lumen. As this reaction entails the breaking of bonds that are comparable to the intramolecular interactions that keep a protein folded, we interpret the dissociation of the SBP tag from streptavidin as unfolding. Our results thus show that Doa10 retrotranslocates a luminal protein segment in an unfolded state. Cdc48, acting on cytoplasmic polyubiquitin chains, generates a 
A

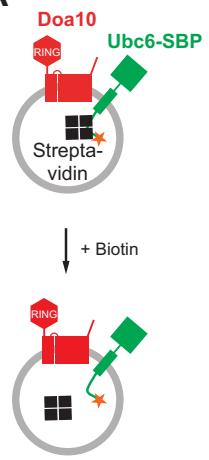

C

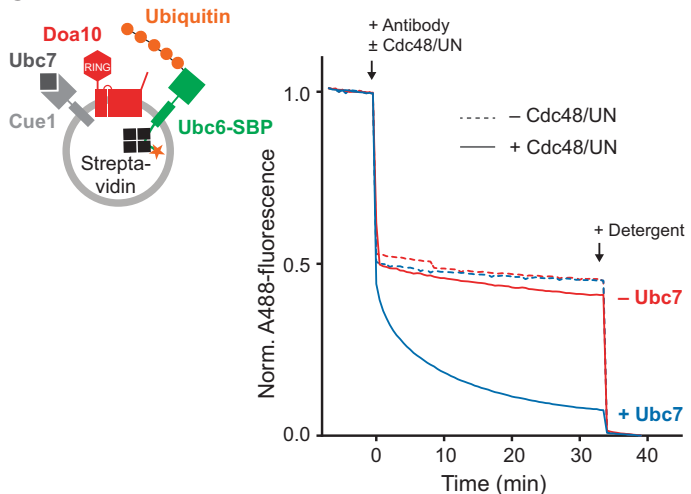

E

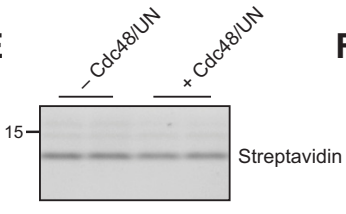

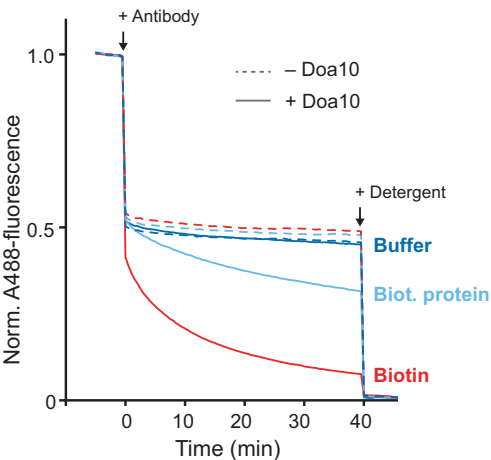

B

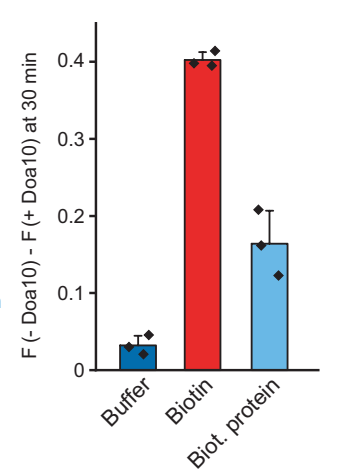

D

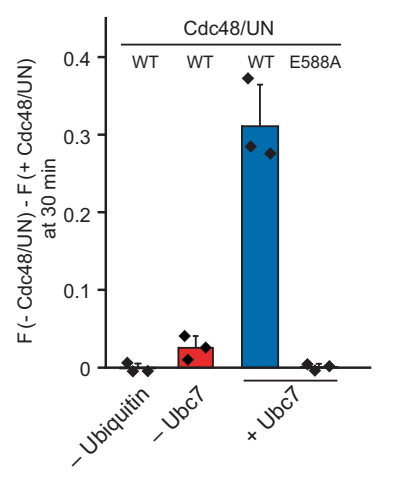

$\mathbf{F}$

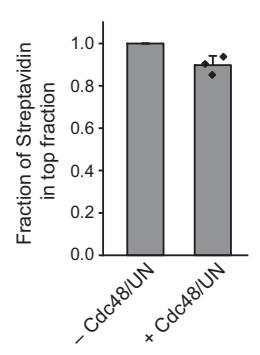

Figure 6. Effect of a Luminal Domain on Retrotranslocation. (A) Retrotranslocation of Ubc6 with a C-terminal streptavidin binding peptide (SBP), reconstituted in complex with streptavidin, was measured by accessibility of a fluorescence quenching antibody to a C-terminal A488 dye on Ubc6, as described Figure 1E. Liposomes were incubated with buffer, biotin or a biotinylated protein prior to addition of the antibody. Final concentrations: 0.2 $\mu \mathrm{M}$ Ubc6, $80 \mathrm{nM}$ Doa10, $0.25 \mu \mathrm{M}$ streptavidin, $1.5 \mu \mathrm{M}$ biotin or biotinylated protein. (B) Quantification (mean $\pm \mathrm{SD}$ ) of three experiments as in (A). The fraction of accessible dye after 30 min was compared between conditions with and without Doa10. F, normalized fluorescence. (C) Effect of Cdc48 and Ufd1/Npl4 (UN) on retrotranslocation of Ubc6-SBP in complex with streptavidin, measured using the antibody accessibility assay as in (A). Prior to the fluorescence measurement, liposomes were incubated with ubiquitination mix with or without Ubc7. Arrows indicate when antibody, with or without Cdc48/UN, or detergent were added. Final concentrations: $0.17 \mu \mathrm{M}$ Ubc6-SBP, 68 nM Doa10, $0.17 \mu \mathrm{M}$ hexameric Cdc48, Ufd1, and Npl4. See Figure 6-figure supplement $1 F$ for gel samples of ubiquitination reaction. (D) Quantification (mean \pm SD) of three experiments as in (C). The fraction of accessible dye after 30 min was compared between conditions with and without Cdc48/UN. In addition, experiments lacking ubiquitin or with the Cdc48 mutant E588A were quantified. (E) Determination of liposome-encapsulated streptavidin after extraction. Samples from experiments as in (D) Figure 6 continued on next page 
Figure 6 continued

were taken at $\mathrm{t}=30 \mathrm{~min}$. Biotin was added, and liposomes floated in a Nycodenz gradient. Co-floating streptavidin was detected in SDS-PAGE using stain-free technology. Two replicates are shown for each condition. (F) Quantification (mean \pm SD) of the relative amount of streptavidin co-floating from three experiments as in (E). Each data point represents the mean of two replicates as shown in (E).

The online version of this article includes the following source data and figure supplement(s) for figure 6:

Source data 1. This file contains the quantification of the quenched fraction of Ubc6 in samples containing Doa10 compared to samples lacking Doa10 from three experiments as in Figure 6A, as shown in Figure 6B.

Source data 2. This file contains the quantification of the quenched fraction of Ubc6 in samples containing Cdc48/UN compared to samples lacking Cdc48/UN from three experiments as in Figure 6C, as shown in Figure 6D

Source data 3. This file contains the quantification of streptavidin in the top flotation fraction from three experiments as in Figure $6 E$, as shown in Figure 6F.

Figure supplement 1. Liposomes with Ubc6-SBP and streptavidin.

mechanical force that results in luminal unfolding und drives retrotranslocation. The integrity of the membrane is maintained in this process.

\section{Discussion}

During extraction of a protein from the membrane, an energetic barrier must be overcome that depends on the hydrophobicity of its TM domain (Botelho et al., 2013; Guerriero et al., 2017). Our results provide evidence that Doa10 contributes to overcoming the energetic barrier for membrane protein extraction. This is demonstrated by passive movement of a tail-anchored membrane protein into the aqueous phase in the presence of Doa10. In the absence of a folded luminal domain, factors such as Get3 or an antibody that trap the retrotranslocated state are sufficient to drive the reaction, bypassing the requirement for ubiquitination and $\mathrm{Cdc} 48$.

ERAD also entails retrotranslocation of less hydrophobic sequences, such as those in luminal loops or domains, across the hydrophobic core of the membrane. We show that, once dissociated from streptavidin, a 57 amino acid long luminal protein segment that includes the SBP-tag is retrotranslocated by Doa10 without markedly affecting retrotranslocation kinetics. Thus, Doa10 can also accommodate these less hydrophobic sequences in the retrotranslocation process.

A major unresolved question concerns the fate of luminal domains during retrotranslocation. Some studies suggested that luminal domains cross the ER membrane in a folded state, based either on the observation that substrates containing tightly folded domains were retrotranslocated in the first place or that they were detected in a folded state after retrotranslocation into the cytosol (Fiebiger et al., 2002; Petris et al., 2014; Shi et al., 2019; Tirosh et al., 2003). Others suggested that retrotranslocation requires unfolding of luminal protein segments, including reduction of disulfide bonds, prior to retrotranslocation (reviewed in Ellgaard et al., 2018). Some of these differences might be explained by the fact that different ubiquitin ligase complexes were involved that might have different requirements for retrotranslocation. Our data show that Doa10 does not accommodate a folded domain during retrotranslocation. Instead, unfolding of polypeptide segments on the luminal side of the membrane is a direct consequence of $\mathrm{Cdc} 48$ acting on cytoplasmically attached polyubiquitin chains.

It is unclear if chaperone-driven retrotranslocation of Ubc6 in the absence of ubiquitination or Cdc48 action also occurs in the cell. Genetic and biochemical experiments in yeast showed that degradation of TM domain containing Doa10 substrates, including Ubc6, strictly depends on Cdc48, Ufd1 and Npl4 (Foresti et al., 2013; Habeck et al., 2015; Huyer et al., 2004; Neuber et al., 2005; Ravid et al., 2006; Ruggiano et al., 2016; Wang and Chang, 2003). In the case of the strongly hydrophobic, multi-spanning Doa10 substrate Ste6*, fractionation experiments also showed that the Cdc48 complex is required for the retrotranslocation step (Nakatsukasa et alo, 2008; Nakatsukasa and Kamura, 2016; Neal et al., 2018). However, these experiments do not exclude the possibility that a relatively mildly hydrophobic protein such as Ubc6 retrotranslocates into the cytosol in a Doa10-dependent, but ubiquitination- and Cdc48-independent manner. Speculatively, such a chaperone-stabilized cytoplasmic pool would not be a substrate for proteasomal degradation but rather for chaperone-assisted reinsertion into the ER membrane, and might therefore be difficult to detect. Furthermore, as chaperones would only be able to capture a substrate that has emerged 
from the membrane, we expect such chaperone-driven retrotranslocation to be sensitive to the overall hydrophobicity of the TM segment. Interestingly, several studies showed that chaperones play an important role in ERAD of membrane proteins at different stages of the ERAD process. In yeast, Hsp70 and Hsp40 chaperones promote ubiquitination of Ste6* and a misfolded variant of Pma1 (Han et al., 2007; Nakatsukasa et al., 2008). In mammals, the Bag6-Ubl4a-Trc35 chaperone complex facilitates ERAD of membrane proteins by stabilizing a soluble cytoplasmic state (Claessen and Ploegh, 2011; Claessen et al., 2014; Ernst et al., 2011; Wang et al., 2011; Xu et al., 2012). If such chaperones also act as a sink in a putative Cdc48-independent retrotranslocation process, remains to be determined.

Using a reconstituted system, we disentangled different ERAD subreactions, that is substrate recruitment, ubiquitination, retrotranslocation, and membrane extraction. This allowed us to identify two activities of Doa10: retrotranslocase and ubiquitin ligase. The interplay of Doa10 and the Cdc48 complex during the extraction process remains to be further explored. Studies showed that Cdc48 is recruited to the Doa10-complex via Ubx2 (Neuber et al., 2005; Schuberth and Buchberger, 2005). This recruitment is dependent on the ubiquitination activity of Doa10, suggesting that Cdc48-recruitment requires either autoubiquitination of Doa10 or substrate ubiquitination. Moreover, the Derlin Dfm1, which also interacts with Cdc48 through its carboxy-terminal SHP-box, has been shown to be required for degradation of Ste6* (Neal et al., 2018). Our experimental system is expandable and can be used to explore how Dfm1, Ubx2 or other factors affect retrotranslocation by Doa10 and Cdc48.

How exactly Doa10 facilitates release of proteins from the membrane is unclear. Structural information on Doa10 is necessary to further elucidate its mechanism of action. We speculate that TM segments access a binding site in Doa10 through a lateral gate. This might promote delipidation of TM segments and breaking of helix-helix interactions in multi-spanning membrane proteins. Quality control pathways for membrane proteins that require their extraction from the membrane exist not only in the ER, but also in other organelles. In the Golgi, mitochondria and chloroplasts, membrane proteins are removed from the organelle for proteasomal degradation in the cytosol. These processes are dependent on ubiquitination and Cdc48 (Heo et al., 2010; Ling et al., 2019; Schmidt et al., 2019; Tanaka et al., 2010). Moreover, extraction of membrane proteins also occurs by membrane-bound AAA ATPases that often have not only unfolding, but also proteolytic activity. In mitochondria, Msp1 and the FtsH-related AAA metalloproteases m-AAA and i-AAA are examples for membrane-bound AAA ATPases (Glynn, 2017). For m-AAA mediated extraction into the mitochondrial matrix, a contribution of the TM domain was shown, suggesting a retrotranslocase activity (Korbel et al., 2004; Lee et al., 2017). We propose that membrane-bound retrotranslocases generally contribute to AAA protein-driven extraction of membrane proteins.

\section{Materials and methods}

Key resources table

\begin{tabular}{|c|c|c|c|}
\hline $\begin{array}{l}\text { Reagent type } \\
\text { (species) or resource }\end{array}$ & $\begin{array}{l}\text { Source or } \\
\text { reference }\end{array}$ & Identifiers & $\begin{array}{l}\text { Additional } \\
\text { information }\end{array}$ \\
\hline Gene (S. cerevisiae) & DOA10 & YIL030C & Amplified from BY4741 \\
\hline Gene (S. cerevisiae) & UBC6 & YER100W & Amplified from BY4741 \\
\hline Gene (S. cerevisiae) & UBA1 & YKL210W & Amplified from BY4741 \\
\hline Gene (S. cerevisiae) & UBC7 & YMR022W & Amplified from BY4741 \\
\hline Gene (S. cerevisiae) & CUE1 & YMR264W & Amplified from BY4741 \\
\hline Gene (S. cerevisiae) & CDC48 & YDL126C & Amplified from BY4741 \\
\hline Gene (S. cerevisiae) & UFD1 & YGR048W & Amplified from BY4741 \\
\hline Gene (S. cerevisiae) & NPL4 & YBR170C & Amplified from BY4741 \\
\hline Gene (S. cerevisiae) & GET3 & YDL100C & Amplified from BY4741 \\
\hline Gene & Synaptobrevin 2 & NP_036795 & \\
\hline
\end{tabular}

(Rattus norvegicus)

Continued on next page 


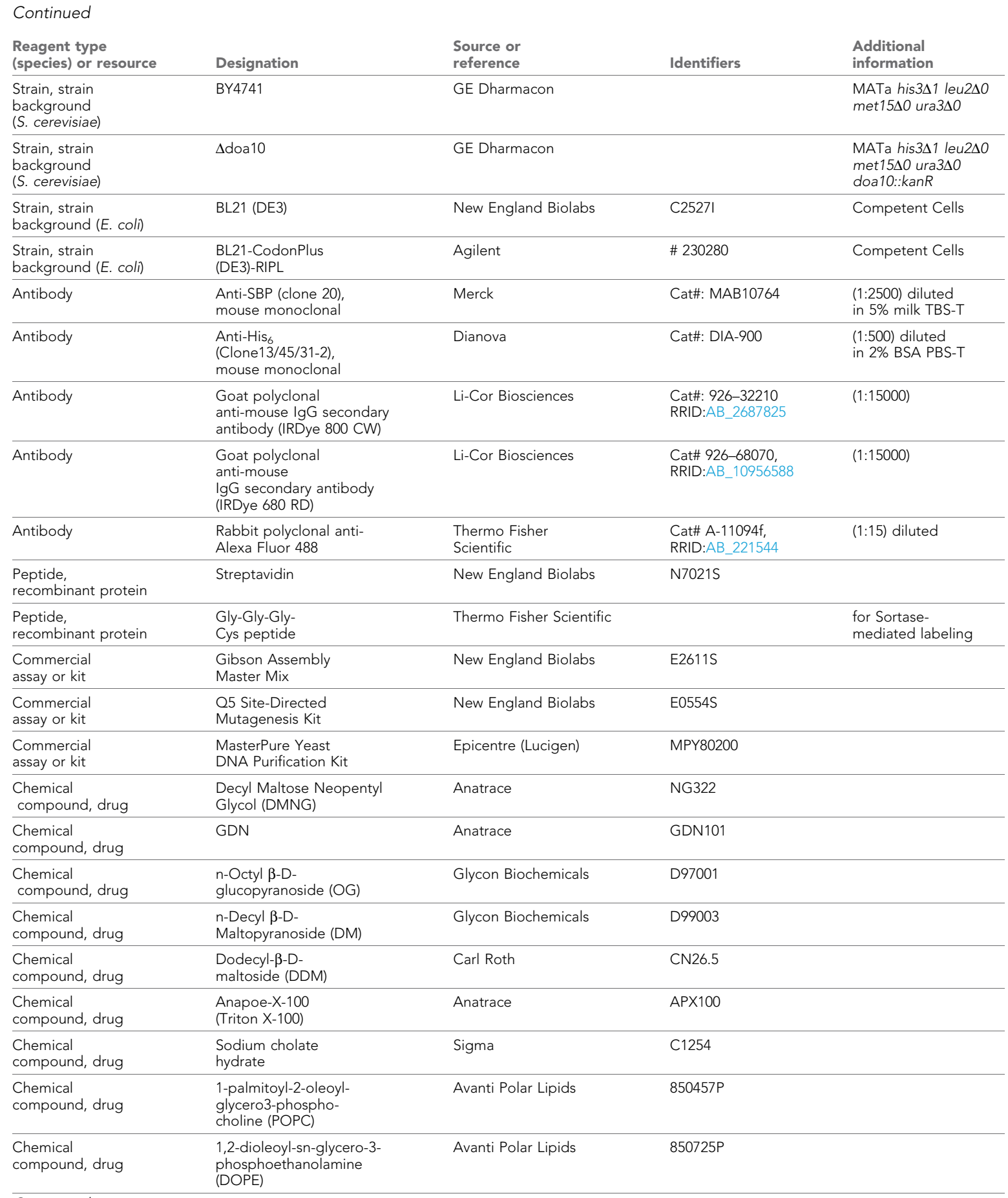

Continued on next page 


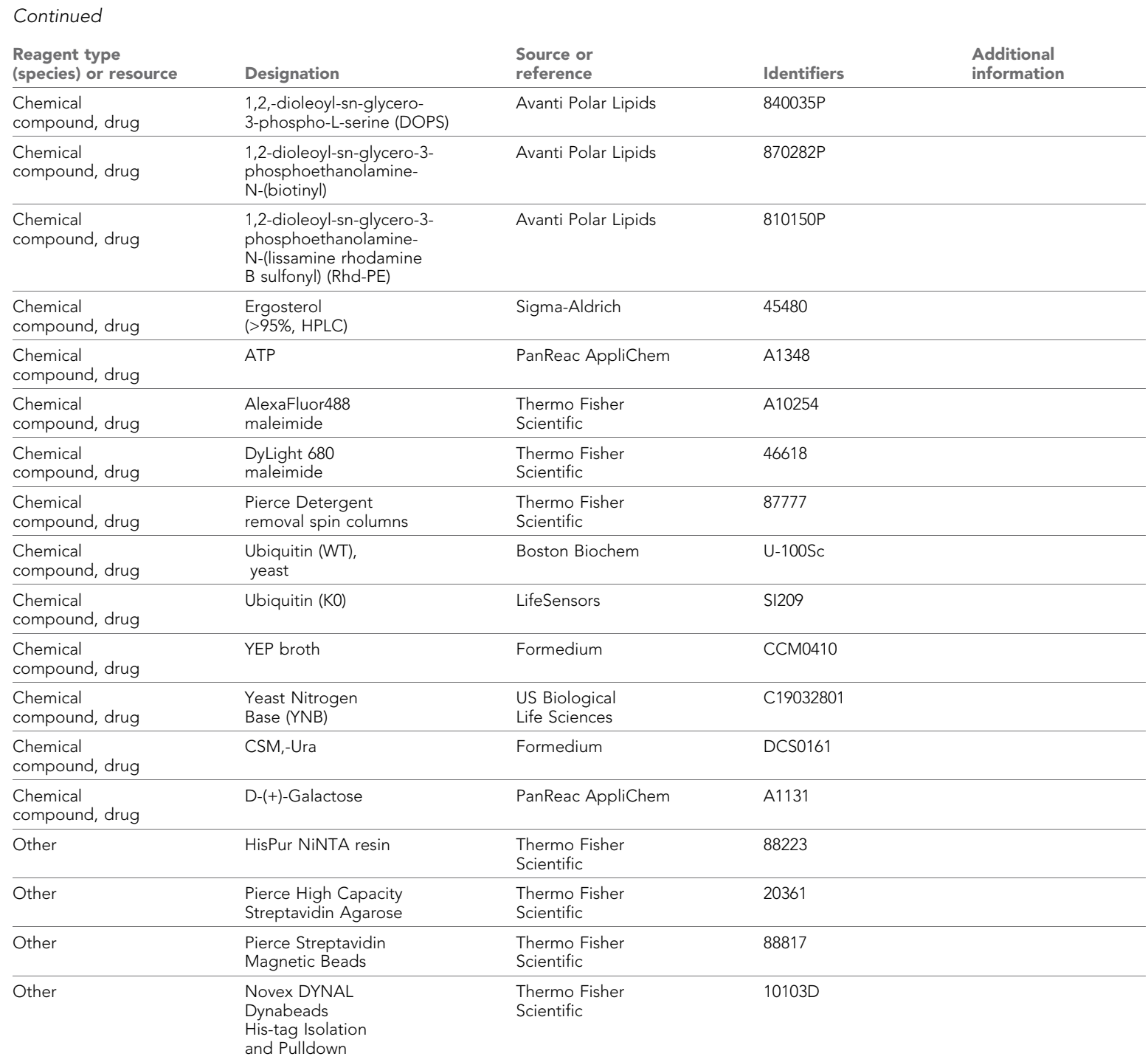

\section{Strains used for protein expression}

For protein expression in E. coli, BL21-CodonPlus (DE3)-RIPL competent cells (Agilent) were used. Where indicated, BL21 (DE3) competent cells (NEB) were used instead. To express Doa10 in S. cerevisiae, we used a doa10 deletion strain derived from BY4741.

\section{Constructs}

All sequences were from S. cerevisiae, except for SNARE proteins which were from rattus norvegicus.

\section{Doa10}

As full-length DOA10 is toxic for E. coli (Mandart et al., 1994), the DOA10 sequence was split in two parts and cloned into two separate plasmids, similarly to as described before (Swanson et alo, 
2001). Sequences coding for Doa10 (amino acids (aa) 1-468, Doa10-N) and Doa10 (aa 225-1319), were both cloned into a pRS426-pGal1 plasmid (Mumberg et al., 1994) using Xhol/Spel restriction sites (plasmids \#376 and \#375, respectively). At the carboxy terminus, both constructs were appended with a tobacco etch virus (TEV) protease cleavage site followed by a streptavidin-binding peptide (SBP) tag (Keefe et al., 2001), in which the single lysine was mutated to arginine, and a short sequence for sortase-mediated labeling with fluorescent dyes (Popp et al., 2009). The full C-terminal tag for Doa10 had the sequence GSGENLYFOSGGGMDERTTGWRGGHVVEGLAGELE QLRARLEHHPQGQREPLPETGG. A plasmid containing full-length Doa10 was subsequently generated in S. cerevisiae (DOA10 deletion strain) by homologous recombination. To do so, plasmid \#375 was linearized (starting from Doa10 residue 225) and an N-terminal fragment was generated from plasmid \#376 (Doa10 residues 1-257) by PCR. The N-terminal fragment contained 80 to $100 \mathrm{nt}$ overlaps with the linearized plasmid. Both PCR-products were co-transformed into S. cerevisiae. Correct homologous recombination was confirmed by sequencing of the PCR-amplified insert after preparation of total DNA of the generated strain (yAST112). The construct for expression of Doa10-C (aa 434-1319) contained an N-terminal SBP-SUMO* tag (Liu et al., 2008) and a C-terminal sortase (LPETGG) tag.

\section{Ubc6}

UBC6 from S. cerevisiae and its variants were cloned into the K27SUMO vector using the Sfol restriction site (Stein et al., 2014). This vector encodes an N-terminal His ${ }_{14}$-SUMO-tag. Ubc6 and its variants were appended with a C-terminal LPETGG tag for sortase-mediated labeling. Expression constructs for Ubc6 and its variants were generated by Gibson assembly (NEB) and site-directed mutagenesis (NEB):

Ubc6sybTM contained the cytosolic Ubc6 domain (aa 1-231) fused to the sequence of the transmembrane (TM) domain of rat synaptobrevin 2 (Syb, aa 96-116). Vice versa, Sybubc6TM contained the cytosolic part of Syb (aa 1-95) fused to the Ubc6 TM domain (aa 232-250). Constructs containing a Syb TM domain contained a linker between the TM domain and the LPETGG tag for sortase labeling with the sequence GSGSATGSGGS.

To generate catalytically inactive Ubc6, the active-site Cys (C87) was mutated to Ala (Ubc6c87A).

Ub-Ubc6 $6_{C 87 A}$ and $U b-U b c 6_{C 87 A / S y b T M}$ contained ubiquitin ${ }_{V 76}$ (aa 1-76) which was inserted between the sequence encoding the His ${ }_{14}$-SUMO tag and the respective Ubc6 variant. For efficient Ulp1-cleavage, a linker sequence (coding for GSG) was inserted between the His ${ }_{14}-\mathrm{SUMO}$ tag and ubiquitin.

SUMO-Ubc6 contained a C-terminal TEV-cleavage site flanked by linker sequences which was introduced between the Ubc6 TM domain and the LPETGG tag resulting in GSGS-ENLYFQSSGLPETGG.

Ubc6-SBP contained a C-terminal TEV-cleavage site separated from Ubc6 by a linker (GSGENLYFOSGGG) followed by an SBP-tag and residues LPETGG for sortase-mediated labeling. The coding sequence for His ${ }_{14}$-SUMO-Ubc6-SBP was inserted into a pET39b(+) vector (Novagen) right after the DsbA signal sequence.

\section{Cue1}

Cue1 was engineered with an $\mathrm{N}$-terminal His ${ }_{14}$-SUMO-tag and a C-terminal TEV-cleavage site followed by an SBP-tag separated from Cue1 by a linker (resulting in the same C-terminal tag as the one for Ubc6-SBP). A short linker (coding for SGS) was introduced between the His ${ }_{14}$-SUMO tag and Cue1. The coding sequence for this construct was inserted into the pET39b(+) vector (Novagen) right after the DsbA signal sequence. After TEV-cleavage during purification, the sequence for the C-terminal end of Cue 1 is GSGENLYFO.

\section{Get3}

The coding sequence for Get3 (and Get $3_{D 57 \mathrm{~N}}$ ) was inserted into the K27SUMO vector using the Sfol restriction site. The expression construct contained an $\mathrm{N}$-terminal His ${ }_{14}-\mathrm{SUMO}$ tag.

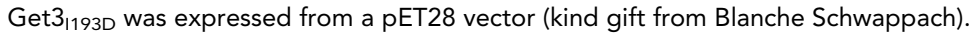


SNARES

All constructs for expression of SNAREs have been previously described (Hernandez et al., 2012; Stein et al., 2007).

\begin{tabular}{ll} 
Construct & Plasmid Number \\
\hline Doa10 (aa 1-468, Doa10-N) in pRS426-pGal1 & 376 \\
\hline Doa10 (aa 225-1319) in pRS426-pGal1 & 375 \\
\hline Doa10 (aa 434-1319, Doa10-C) in pRS426-pGal1 & 557 \\
\hline Ubc6 in K27SUMO & 343 \\
\hline Ubc6c87A in K27SUMO & 682 \\
\hline Ub-Ubc6 c87A in K27SUMO & 702 \\
\hline Sybubc6TM in K27SUMO & 509 \\
\hline Ubc6sybTM in K27SUMO & 536 \\
\hline Ub-Ubc6c87A/SybTM in K27SUMO & 815 \\
\hline Ubc6 (incl. C-terminal TEV cleavage site) in K27SUMO & 508 \\
\hline Ubc6-SBP in pET39b(+) & 633 \\
\hline Cue1 in pET39b(+) & 672 \\
\hline Get3 in K27SUMO & 504 \\
\hline Get3 D57N in K27SUMO & 522 \\
\hline Get3 I193D & Mateja et al., 2009 \\
\hline Syntaxin 1A 183-288, Syb2 49-96 in pETDuet-1 & Stein et al., 2007 \\
\hline SNAP25A nocys in pET28a & Fasshauer et al., 1999 \\
\hline Synaptobrevin 2 in pET28a & Stein et al., 2007 \\
\hline
\end{tabular}

\section{Expression and purification of proteins}

For expression of Doa10, yeast cells were grown in synthetic complete medium containing $2 \%(\mathrm{w} / \mathrm{v})$ Glucose and amino acid drop-out supplements at $30^{\circ} \mathrm{C}$ (Formedium). An overnight culture (grown for $24 \mathrm{hr}$ ) was diluted 1:50 into fresh medium. After $24 \mathrm{hr}$ of growth, expression was induced by addition of yeast extract, peptone and galactose to final concentrations of $1 \%, 2 \%$, and $2 \%(\mathrm{w} / \mathrm{v})$, respectively. After 17-19 hr, the cells were harvested at $3000 \times$ g, washed once with $\mathrm{ddH}_{2} \mathrm{O}$, resuspended in a minimal amount of $\mathrm{H}_{2} \mathrm{O}$ and stored at $-80^{\circ} \mathrm{C}$.

To prepare cell lysates, $150 \mathrm{~g}$ of cells were resuspended in $900 \mathrm{~mL}$ of cold $\mathrm{H}_{2} \mathrm{O}$ and incubated with $2 \mathrm{mM} \mathrm{DTT}$ for $15 \mathrm{~min}$ on ice. All subsequent steps were done at $4^{\circ} \mathrm{C}$. The cells were pelleted at $3000 \times \mathrm{g}$ and resuspended in lysis buffer $(20 \mathrm{mM} \mathrm{HEPES} / \mathrm{KOH} \mathrm{pH} 7.5,5 \mathrm{mM}$ potassium acetate, 600 $\mathrm{mM}$ mannitol, $0.5 \mathrm{mM}$ EDTA). PMSF $(1 \mathrm{mM})$ and Pepstatin $\mathrm{A}(2 \mu \mathrm{M})$ were added freshly. The cell suspension was then added to a bead beating chamber (total volume $300 \mathrm{~mL}$ ) filled up to $1 / 3$ with zirkonia beads. Cells were lysed in a Bead Beater (Biospec Products) with cycles of $20 \mathrm{~s}$ on and $2 \mathrm{~min}$ breaks in between for $50 \mathrm{~min}$. Beads were filtered off, and the lysate was centrifuged at $1500 \times \mathrm{g}$ for $10 \mathrm{~min}$. The supernatant was subsequently centrifuged at $40,000 \mathrm{xg}$ for $45 \mathrm{~min}$ in a Ti45 rotor. The pelleted crude membrane fraction was resuspended in $200 \mathrm{~mL}$ lysis buffer by douncing and again pelleted at $180,000 \times \mathrm{g}$ for $30 \mathrm{~min}$. The pellet was resuspended in $40 \mathrm{~mL}$ buffer, frozen in liquid nitrogen, and stored at $-80^{\circ} \mathrm{C}$. The total protein concentration of the membrane fraction was determined using the Pierce660 nm Protein Assay (Thermo Scientific).

To purify SBP-tagged Doa10, the membrane fraction was solubilized with $1.3 \%(\mathrm{w} / \mathrm{v})$ GDN (Anatrace) at a protein concentration of 3-4 mg/mL in $20 \mathrm{mM} \mathrm{HEPES} / \mathrm{KOH}$ pH7.4, $300 \mathrm{mM}$ potassium chloride, $0.5 \mathrm{mM}$ TCEP, $5 \mathrm{mM}$ magnesium acetate, supplemented with $1 \mathrm{mM}$ PMSF and 1 Pierce cOmplete EDTA-free protease inhibitor cocktail (Roche) per $100 \mathrm{~mL}$ solubilization volume. After $1 \mathrm{hr}$ solubilization, insoluble material was pelleted at $40,000 \mathrm{rpm}$ for $30 \mathrm{~min}$ in a Ti45 rotor. The supernatant was added to $4 \mathrm{~mL}$ Pierce High Capacity Streptavidin Agarose slurry (Thermo Fisher Scientific) and incubated for $3 \mathrm{hr}$. The beads were filtered off and washed with $4 \times 25 \mathrm{~mL}$ of wash buffer (20 
$\mathrm{mM}$ HEPES/KOH pH 7.4, $150 \mathrm{mM}$ potassium chloride, $5 \mathrm{mM}$ magnesium acetate, $0.5 \mathrm{mM}$ TCEP, 150 $\mu \mathrm{M}$ GDN). Doa10 was eluted with wash buffer supplemented with $2 \mathrm{mM}$ biotin. Doa10 was further purified by sucrose density gradient ultracentrifugation. Gradients were prepared with two solutions where the less dense solution contained GDN (solution A: $20 \mathrm{mM} \mathrm{HEPES/KOH} \mathrm{pH} \mathrm{7.4,} 150 \mathrm{mM}$ potassium chloride, $2 \mathrm{mM}$ magnesium acetate, $10 \%$ (w/v) sucrose, $0.5 \mathrm{mM}$ TCEP, $100 \mu \mathrm{M}$ GDN, solution B: $20 \mathrm{mM} \mathrm{HEPES} / \mathrm{KOH}$ pH7.4, $150 \mathrm{mM}$ potassium chloride, $2 \mathrm{mM}$ magnesium acetate, 25\% (w/ v) sucrose, $0.5 \mathrm{mM}$ TCEP). Gradients were prepared using a gradient mixer (Gradient Master, Biocomp Instruments) at RT and kept at $4^{\circ} \mathrm{C}$. $500 \mu \mathrm{L}$ sample was loaded on top of the gradient. After centrifugation at 40,000 rpm for $19 \mathrm{hr}$ in a SW41Ti rotor, the gradient was harvested in $500 \mu \mathrm{L}$ fractions. Doa10-containing fractions were concentrated with Amicon Ultra Centrifugal Filters (Merck) using a $100 \mathrm{kDa}$ cut-off. The same protocol was used for purification of SBP-tagged Doa10-N and Doa10-C.

For bacterial expression, an overnight culture was diluted 1:50 into Terrific Broth and grown at $37^{\circ} \mathrm{C}$. At an $\mathrm{OD}_{600}$ of 0.5 , the cells were shifted to $18^{\circ} \mathrm{C}$ and expression was induced with $0.5 \mathrm{mM}$ IPTG. After approximately $20 \mathrm{hr}$ of induction, cells were harvested at $4000 \mathrm{rpm}$, resuspended in buffer Im30 (50 mM Tris/ $/ \mathrm{HCl}$ pH8.0 (at $\left.4^{\circ} \mathrm{C}\right), 500 \mathrm{mM} \mathrm{NaCl}, 30 \mathrm{mM}$ Imidazole) and stored at $-20^{\circ} \mathrm{C}$. Cells were lysed using a microfluidizer (17,000 psi, two passages). Immediately afterwards, $1 \mathrm{mM}$ PMSF was added. Cell debris and unbroken cells were pelleted (1500 x g, $10 \mathrm{~min})$. A membrane fraction was prepared by ultracentrifugation of the supernatant $(40,000 \mathrm{rpm}, 45 \mathrm{~min}$, Ti45 rotor). The pellet was resuspended in buffer $\operatorname{Im} 30$ by douncing, frozen in liquid nitrogen, and stored at $-80^{\circ} \mathrm{C}$.

Ubc6 and its variants were purified as described (Vasic et al., 2020).

To purify Ubc6-SBP, an additional purification step was included to ensure that only full-length Ubc6-SBP was purified. After size-exclusion chromatography, the protein was diluted to $0.5 \mathrm{mg} / \mathrm{mL}$ and bound to Pierce High Capacity Streptavidin Agarose (Thermo Scientific). After washing the beads with buffer containing $20 \mathrm{mM}$ HEPES/ $\mathrm{KOH}$ pH 7.4, $250 \mathrm{mM}$ sodium chloride, $0.2 \mathrm{mM}$ TCEP, $0.03 \%(w / v)$ n-dodecyl- $\beta$-D-maltopyranoside (DDM, Carl Roth), Ubc6-SBP was eluted with buffer supplemented with $2 \mathrm{mM}$ biotin.

To purify SUMO-Ubc6 (containing a C-terminal TEV cleavage site), protein was eluted from the Ni-NTA resin with buffer containing $50 \mathrm{mM}$ Tris/ $\mathrm{HCl} \mathrm{pH} 8.0$ (at $4^{\circ} \mathrm{C}$ ), $500 \mathrm{mM}$ sodium chloride, $0.03 \%$ $(\mathrm{w} / \mathrm{v}) \mathrm{DDM}$ and $500 \mathrm{mM}$ imidazole, and then further purified by size-exclusion chromatography (Superdex 200), as for Ubc6.

To purify Get3, bacterial lysate was cleared by ultracentrifugation $\left(40,000 \mathrm{rpm}, 45 \mathrm{~min}, 4^{\circ} \mathrm{C}\right.$, Ti45 rotor) and the supernatant incubated with Ni-NTA slurry $(6 \mathrm{ml}$ slurry for $6 \mathrm{~L}$ of culture) for $2 \mathrm{hr}$. Beads were filtered off and washed with $4 \times 50 \mathrm{~mL}$ buffer $\operatorname{Im} 30$ and $50 \mathrm{~mL}$ of buffer $\operatorname{Im} 10(20 \mathrm{mM}$ Tris/HCl pH8.0 (at $4^{\circ} \mathrm{C}$ ), $200 \mathrm{mM} \mathrm{NaCl}, 10 \mathrm{mM}$ Imidazole). Get3 was eluted from beads by cleavage with Ulp1 as described above. The elution fractions were supplemented with $1 \mathrm{mM}$ DTT and further purified by size-exclusion chromatography using a Superdex 200 HiLoad16/60 column (GE Healthcare) equilibrated with $20 \mathrm{mM}$ HEPES/KOH pH 7.4, $200 \mathrm{mM} \mathrm{NaCl}, 1 \mathrm{mM}$ DTT.

Uba1, Ubc7, Cdc48 and Ufd1/Npl4 were purified as described (Stein et alo, 2014). Cue1 was purified as described (Vasic et al., 2020).

To express the t-SNARE complex, plasmids encoding syntaxin-1a (aa 183-288), synaptobrevin-2 (aa 49-96) (pETDuet-1 vector) and SNAP-25A (pET28a vector) were co-transformed into BL21 (DE3) E. coli cells (NEB) and expressed as described previously (Stein et alo, 2007). Briefly, at an $\mathrm{OD}_{600}$ of 0.5 , the cells were shifted to $18^{\circ} \mathrm{C}$ and induced with $0.5 \mathrm{mM} \mathrm{IPTG}$. After approximately $20 \mathrm{hr}$ of induction, the cells were harvested at $4000 \mathrm{rpm}$, resuspended in buffer $1 \mathrm{~m} 8(50 \mathrm{mM}$ Tris/ $\mathrm{HCl}$ pH8.0 at $4^{\circ} \mathrm{C}, 500 \mathrm{mM} \mathrm{NaCl}, 8 \mathrm{mM}$ Imidazole) and stored at $-20^{\circ} \mathrm{C}$. After cell lysis using a microfluidizer in the presence of $1 \mathrm{mM}$ PMSF and Complete protease inhibitor, the lysate was cleared by ultracentrifugation (40,000 rpm, $30 \mathrm{~min}$, Ti45 rotor). The pellet was solubilized in buffer Im8 supplemented with $5 \%(\mathrm{w} / \mathrm{v})$ sodium cholate (Sigma), $2 \mathrm{M}$ urea, $200 \mathrm{mM}$ sucrose and $1 \mathrm{mM}$ PMSF (30 min, RT). Insoluble material was pelleted by ultracentrifugation $\left(40,000 \mathrm{rpm}, 30 \mathrm{~min}, 4^{\circ} \mathrm{C}\right.$, Ti45 rotor). HisPur Ni-NTA resin $\left(6 \mathrm{~mL}\right.$ for $6 \mathrm{~L}$ of culture) was added to the supernatant and incubated for $3 \mathrm{hr}$ at $4^{\circ} \mathrm{C}$ while rotating. Beads were filtered off and washed with $4 \times 50 \mathrm{~mL}$ wash buffer $(20 \mathrm{mM}$ Tris/ $\mathrm{HCl} \mathrm{pH}$ 8.0 (at $4^{\circ} \mathrm{C}$ ), $500 \mathrm{mM} \mathrm{NaCl}, 8 \mathrm{mM}$ imidazole, $200 \mathrm{mM}$ sucrose, $2 \%$ (w/v) octyl glucoside (OG, Glycon Biochemicals)). Protein was eluted with wash buffer supplemented with $400 \mathrm{mM}$ Imidazole. $1 \mathrm{mM}$ DTT and $0.05 \mathrm{mg} / \mathrm{mL}$ of thrombin $(100 \mathrm{x}$ stock prepared in $50 \%(\mathrm{w} / \mathrm{v})$ glycerol) were added to the elution fractions and incubated at $4{ }^{\circ} \mathrm{C}$ overnight. The solution was then diluted to a conductivity of 
$15 \mathrm{mS} / \mathrm{cm}$ with buffer A (20 mM Tris/HCl pH7.4 (RT), $1 \mathrm{mM} \mathrm{DTT,} 200 \mathrm{mM}$ sucrose, $2 \%$ (w/v) OG). The protein was further purified by ion exchange chromatography on a Mono $\mathrm{Q}$ column (GE healthcare) equilibrated with $20 \mathrm{mM}$ Tris/ $\mathrm{HCl}$ pH7.4 (RT), $150 \mathrm{mM} \mathrm{NaCl}, 1 \mathrm{mM}$ DTT, $200 \mathrm{mM}$ sucrose, $2 \%$ $(\mathrm{w} / \mathrm{v}) \mathrm{OG}$ and eluted in a gradient until $450 \mathrm{mM} \mathrm{NaCl}$ (elution at $\sim 25 \mathrm{mS} / \mathrm{cm}$ ).

ATP synthase (from Bacillus PS3) was expressed and purified as described previously (Schenck et al., 2009; Suzuki et al., 2002). Shortly, ATP synthase was purified in the detergent DDM via a His $10^{-}$tag attached to the $\beta$ subunit. After Ni-NTA affinity chromatography, the dialysis step was omitted and the sample was directly further purified via ion exchange chromatography (MonoQ). Detergent was exchanged to GDN subsequently by size exclusion chromatography (Superose 6 16/60, equilibrated with $10 \mathrm{mM} \mathrm{HEPES}, 100 \mathrm{mM} \mathrm{KCl}, 5 \mathrm{mM} \mathrm{MgCl} 2$ and $50 \mu \mathrm{M} \mathrm{GDN}, \mathrm{pH}$ 7.4). The protein was stored at $4^{\circ} \mathrm{C}$ for up to two weeks.

To express Syb, a plasmid encoding His ${ }_{6}$-thrombin-Syb was transformed into BL21 (DE3) E. coli cells (NEB). Expression and preparation of a membrane fraction were done as described above, in buffer Im15 (50 mM Tris/HCl pH 8.0, $500 \mathrm{mM} \mathrm{NaCl}, 15 \mathrm{mM}$ imidazole). The membrane fraction was solubilized in buffer $\operatorname{Im} 15$ supplemented with $2.5 \%(\mathrm{w} / \mathrm{v})$ sodium cholate for $30 \mathrm{~min}$. After ultracentrifugation, solubilized material was incubated with Ni-NTA slurry $(6 \mathrm{~mL}$ for $6 \mathrm{~L}$ culture) for $3 \mathrm{hr}$. Beads were filtered off and washed with $2 \times 50 \mathrm{~mL}$ wash buffer $\operatorname{lm} 15$ supplemented with $1.5 \%$ (w/v) sodium cholate and subsequently with $4 \times 50 \mathrm{~mL}$ wash buffer $\operatorname{Im} 15$ supplemented with $5 \mathrm{mM}$ decylmaltoside (DM, Glycon Biochemicals). Protein was eluted with buffer Im15 containing $400 \mathrm{mM}$ imidazole and $5 \mathrm{mM}$ DM. The solution was dialyzed overnight against $10 \mathrm{mM}$ MOPS, $50 \mathrm{mM} \mathrm{NaCl}, 1 \mathrm{mM}$ DTT, $1 \mathrm{mM}$ EDTA pH 7.0 (10 kDa MWCO), in the presence of $0.05 \mathrm{mg} / \mathrm{mL}$ thrombin. The protein was further purified by ion exchange chromatography on a MonoS column (GE healthcare) equilibrated with $10 \mathrm{mM}$ MOPS, $50 \mathrm{mM} \mathrm{NaCl}, 1 \mathrm{mM}$ EDTA and $1 \mathrm{mM} \mathrm{DTT} \mathrm{pH} 7.0$ and eluted in a salt gradient to $500 \mathrm{mM} \mathrm{NaCl}$.

\section{Sortase-mediated labeling}

Proteins were labeled at their C-terminal LPETGG tag with the previously described technique sortase-mediated transpeptidation (Popp et al., 2009). A peptide with the sequence GGGC was labeled at its cysteine residue with a maleimide dye. Peptide dissolved in $100 \mathrm{mM} \mathrm{HEPES/KOH} \mathrm{pH}$ 7.4 was added to dye (DyLight680 maleimide or DyLight800 maleimide, Thermo Scientific) in 1.5fold molar excess. After labeling at RT for $2 \mathrm{hr}$, the reaction was stopped with $10 \mathrm{mM}$ DTT. To label peptide with AlexaFluor 488 C5 Maleimide (Thermo Scientific), peptide and dye were both dissolved in $100 \mathrm{mM} \mathrm{HEPES} / \mathrm{KOH} \mathrm{pH} 7.4$ and then mixed in a 1:1 molar ratio. A pentamutant P94R/D160N/ D165A/K190E/K196T of SrtA from S. aureus was purified from E. coli (Chen et alo, 2011).To label proteins, 3-fold molar excess of labeled peptide, $10 \mathrm{mM} \mathrm{CaCl}_{2}$ and SortA were added to the protein. SortA was added to $1 / 7$ of the total concentration of reactants (peptide and protein). After labeling for $16-20 \mathrm{hr}$ at $4^{\circ} \mathrm{C}$, the reaction was separated by size-exclusion chromatography.

\section{Reconstitution into proteoliposomes}

Preparation of protein-free liposomes

The following lipids were purchased from Avanti Polar Lipids: 16:0-18:1 PC (POPC, 1-palmitoyl-2oleoyl-glycero3-phosphocholine), 18:1 ( $\triangle 9$-Cis) PE (DOPE, 1,2-dioleoyl-sn-glycero-3-phosphoethanolamine), 18:1 PS (DOPS, 1,2,-dioleoyl-sn-glycero-3-phospho-L-serine), 18:1 Biotinyl PE (Biotinyl-PE, 1,2-dioleoyl-sn-glycero-3-phosphoethanolamine-N-(biotinyl)), 18:1 Liss Rhod PE (Rhd-PE, 1,2-dioleoyl-sn-glycero-3-phosphoethanolamine-N-(lissamine rhodamine B sulfonyl)). Ergosterol ( $\geq 95 \%$, HPLC) was purchased from Sigma-Aldrich.

Large unilamellar liposomes were prepared by reverse-phase evaporation as described (Hernandez et al., 2012). Briefly, lipids were dissolved in chloroform and mixed at a molar ratio of 60:20:10:10 (POPC: DOPE: DOPS: Ergosterol). Chloroform was subsequently removed using a rotary evaporator by lowering the pressure step-wise to $20 \mathrm{mbar}$. The lipid film was then dissolved in $1 \mathrm{~mL}$ diethyl ether (when preparing $1 \mathrm{~mL}$ of liposomes with a final concentration of $20 \mathrm{mM}$ lipid) and 300 $\mu \mathrm{L}$ of buffer $\mathrm{L}$ (20 mM HEPES/KOH pH7.4, $150 \mathrm{mM}$ potassium chloride, $5 \mathrm{mM}$ magnesium acetate) was added. The sample was sonicated for $1 \mathrm{~min}$ on ice (Branson Sonifier $450,100 \%$ duty cycle, microtip limit 1). Afterwards the ether was removed at 500 mbar. After $10 \mathrm{~min}, 700 \mu \mathrm{L}$ of buffer $\mathrm{L}$ was added and the pressure was gradually decreased to $100 \mathrm{mbar}$ until diethyl ether was completely 
removed. The volume was adjusted to $1 \mathrm{~mL}$ with $\mathrm{ddH}_{2} \mathrm{O}$. The resulting lipid suspension was extruded through a polycarbonate filter ( $11 \mathrm{x}$ through a $0.4 \mu \mathrm{M}$ filter, $21 \mathrm{x}$ through a $0.1 \mu \mathrm{M}$ filter) using the Mini extruder kit (Avanti Polar Lipids). Protein-free liposomes were used for up to 2 weeks after preparation. For pulldown experiments via co-reconstituted biotinylated lipids, lipids were used in a molar ratio of 57.5:20:10:10:0.5:2 (POPC: DOPE: DOPS: Ergosterol: Rhd-PE: Biotinyl-PE).

\section{Reconstitution of proteins into liposomes}

To reconstitute proteins into liposomes, protein-free liposomes were mixed with detergent and proteins and subsequently incubated for $1 \mathrm{hr}$ at RT prior to detergent removal. The detergent concentration used for solubilization can be described by the R-value (Rigaud and Lévy, 2003). The R-value is defined as the ratio of the total detergent concentration above the critical micellar concentration and the total lipid concentration $\left(R=\left[D_{\text {total }}-D_{C M C}\right] /[\right.$ lipid $]$ ).

To co-reconstitute Ubc6 and t-SNARE, protein-free liposomes (4 mM final lipid concentration)

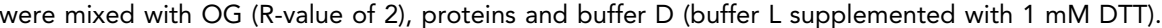
t-SNARE and Ubc6 were reconstituted at a molar lipid: protein ratio of 1000 and 2000, respectively. After incubation for $1 \mathrm{hr}$ at RT, the detergent was removed by dialysis against a $1000 \mathrm{x}$ volume of buffer D at RT in two steps using dialysis cassettes (16 hr with $2000 \mathrm{kDa}$ cut-off, $2 \mathrm{hr}$ with 10,000 kDa cut-off; Slide-a-Lyzer from Thermo Scientific). Biobeads (SM-2 resin, Bio-Rad) were added to the

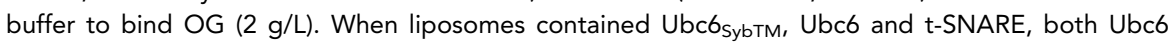
variants were reconstituted at a molar lipid: protein ratio of 2000.

To reconstitute Doa10 and Syb, protein-free liposomes (4 $\mathrm{mM}$ final lipid concentration) were mixed with DM (R-value of 0.55 ), proteins and buffer $T$ (buffer $L$ supplemented with $0.1 \mathrm{mM}$ TCEP). Doa10 and Syb were reconstituted at a molar lipid: protein ratio of 5000 and 2000 , respectively. For ATP synthase, also a molar lipid: protein ratio of 5000 was used. For ubiquitination experiments, Cue1 was co-reconstituted with Doa10 and Syb at a molar lipid: protein ratio of 20,000. After incubation for $1 \mathrm{hr}$ at RT, the detergent was removed by incubation with resin from Pierce detergent removal spin columns (Thermo Scientific) in three subsequent steps (45 mg washed resin to $130 \mu \mathrm{L}$ reconstitution mix in each step). Resin incubation was performed while rotating the sample, at RT for 20 min each. Doa10 truncations were reconstituted at the same lipid: protein ratio, also when both Doa10 truncations were co-reconstituted for the rescue experiment in Figure $5 \mathrm{E}, \mathrm{F}$ and Figure 5figure supplement 2 .

After reconstitution into separate liposomes, Ubc6 and Doa10 were subsequently co-reconstituted by SNARE-mediated fusion. Both liposomes sets were therefore mixed by diluting them 1:10 into buffer $\mathrm{T}$ (unless otherwise indicated) and incubated for $1 \mathrm{hr}$ at $30^{\circ} \mathrm{C}$. To inhibit fusion, t-SNARE liposomes were preincubated with 7-fold excess of a soluble Syb fragment (aa 1-95, Syb sol $_{\text {) }}$ for 5 min at RT prior to addition of Syb liposomes.

For the experiment in Figure 4, D and E, Doa10 was co-reconstituted with either Ubc6sybTM, SybUbc6TM, or Ubc6 directly in a 1-step protocol. For this protocol, Doa10 purified in DMNG (f.c. 0.5 $\mathrm{mM}$ ) was used. Protein-free liposomes (10 $\mathrm{mM}$ final lipid concentration) were mixed with DMNG (Rvalue of 1.5), proteins and buffer T. Ubc6 and Doa10 were both reconstituted at a molar lipid: protein ratio of 10,000. After incubation for $1 \mathrm{hr}$ at RT, the detergent was removed by incubation with Pierce detergent removal spin columns that were pre-washed with buffer $T$ in three subsequent steps (one spin column for $100 \mu \mathrm{L}$ reconstitution mix in each step). Incubation was performed at RT for 10,20 and $30 \mathrm{~min}$ and the sample eluted by centrifugation at 3,500 rpm for $2 \mathrm{~min}$ in a table top centrifuge. To reconstitute Ubc6 or its variants alone (without Doa10), protein-free liposomes (10 $\mathrm{mM}$ final lipid concentration) were mixed with OG (R-value of 2.0), proteins and buffer T. Ubc6 was reconstituted at a molar lipid: protein ratio of 10,000. After incubation for $1 \mathrm{hr}$ at RT, the detergent was removed by adding resin from Pierce detergent removal spin columns in three subsequent steps $(40,60,60 \mathrm{mg}$ resin to $160 \mu \mathrm{L}$ reconstitution mix in step 1,2 and 3, respectively). Resin incubation was performed while rotating the sample, at RT for $20 \mathrm{~min}$ each and the sample eluted by centrifugation at 3,500 rpm for $2 \mathrm{~min}$ in a table top centrifuge.

\section{Flotation of liposomes}

To test for reconstitution of proteins, liposomes were floated in a Nycodenz step gradient. Nycodenz stocks were prepared in buffer L. $50 \mu \mathrm{L}$ of liposomes were mixed with $50 \mu \mathrm{L}$ of $80 \%(\mathrm{w} / \mathrm{v})$ 
Nycodenz and overlaid with $40 \mu \mathrm{L}$ of $30 \%$ and $15 \%(\mathrm{w} / \mathrm{v})$ Nycodenz and $40 \mu \mathrm{L}$ of buffer $\mathrm{L}$. The gradients were ultracentrifuged at 50,000 rpm for $1 \mathrm{hr}$ at $4^{\circ} \mathrm{C}$ (S55-S rotor). The gradient was disassembled in six fractions, starting from the top of the gradient. Fractions were analyzed by SDSPAGE.

Protease protection

To check the orientation of Ubc6 reconstituted into liposomes, trypsin protease was used. Liposomes were diluted (1:10 in buffer D) and incubated with $6.6 \mu \mathrm{g} / \mathrm{mL}$ trypsin (Roche) at RT. The detergent control contained in addition 1\% Triton-X100 (TX100, Anatrace, Anapoe-X-100). The reaction was stopped with $4 \mathrm{mM} \mathrm{PMSF}$ and samples were analyzed by SDS-PAGE.

The orientation of Doa10 TEV-SBP or Doa10-N $\mathrm{N}_{\text {TEV-SBP }}$ in liposomes was determined by assessing the accessibility of the C-terminal TEV-cleavage site to TEV-protease. Liposomes were diluted 1:10 into buffer $\mathrm{D}$ and incubated with $10 \mu \mathrm{M}$ TEV-protease at RT. The detergent control contained in addition $1 \%$ Triton-X100. The reaction was stopped by addition of SDS-sample buffer and samples were analyzed by SDS-PAGE and subsequent Western blotting against the SBP-tag. To determine the orientation of SBP-SUMO*-Doa10-C, the accessibility of the N-terminal SBP-SUMO* to Ulp1* protease was assessed (protocol as described above for TEV-protease).

The reconstitution quality of Ubc6 was assessed with a Ubc6 construct containing an N-terminal SUMO tag and a C-terminal TEV-cleavage site followed by a fluorescent dye (SUMO-Ubc6). Liposomes containing SUMO-Ubc6 and t-SNARE were diluted into buffer T 1:20 (f.c. $0.1 \mu \mathrm{M}$ Ubc6). Ulp1 and/or TEV-protease were added to a f.c. of $10 \mu \mathrm{M}$ each.

\section{Pulldowns}

For pulldown experiments via the SBP-tag of Doa10, $20 \mu \mathrm{L}$ of the fusion reaction (supplemented with $0.25 \mathrm{mg} / \mathrm{mL}$ bovine serum albumin (BSA)) were incubated with $20 \mu \mathrm{L}$ of Pierce Streptavidin Magnetic Beads (Thermo Scientific) prewashed with buffer B (buffer T supplemented with $0.25 \mathrm{mg} /$ $\mathrm{mL}$ BSA). After binding for $1 \mathrm{hr}$ (rotating, RT), the supernatant of the binding reaction was taken off, the beads washed three times with $100 \mu \mathrm{L}$ of buffer $\mathrm{B}$ and bound proteins eluted with $20 \mu \mathrm{L}$ of buffer B supplemented with $2 \mathrm{mM}$ biotin. Samples from input, supernatant and elution fractions were analyzed by SDS-PAGE.

For pulldowns via the His-tag of ATP synthase, the fusion reaction was supplemented with 200 $\mathrm{mM}$ imidazole and $0.4 \mathrm{mg} / \mathrm{mL}$ BSA, and incubated with $20 \mu \mathrm{L}$ magnetic Dynabeads (His-tag Isolation and Pulldown, ThermoFisher Scientific) (f.c. of $0.2 \mu \mathrm{M}$ Ubc6, $80 \mathrm{nM}$ ATP synthase). After binding for 30 min (rotating, RT), the supernatant was removed. Samples of input and supernatant were analyzed by SDS-PAGE and immunoblotting for His-tagged $\beta$-subunit of ATP Synthase.

\section{Assays for release by Doa10}

Get3 capture assay

Liposomes were prepared with protein-free liposomes containing 2 mol\% biotinyl-PE and 0.5 mol\% Rhd-PE and fused as described above. The fusion reaction was diluted 1:2 into buffer T (f.c. Ubc6 $=0.1 \mu \mathrm{M}$ ) and incubated with an excess of Get3 (f.c. $10 \mu \mathrm{M}$ ). After incubation at RT for $16 \mathrm{hr}$, the reaction was diluted to a f.c. of lipid of $0.2 \mathrm{mM}(1: 2$ dilution) and $0.25 \mathrm{mg} / \mathrm{mL}$ BSA was added. The diluted mix was then added to an equal volume of Pierce Streptavidin Magnetic beads (Thermo Scientific, prewashed with buffer B). After binding for $1 \mathrm{hr}$, the supernatant was removed. Input and supernatant samples were analyzed by SDS-PAGE, and the Rhodamine fluorescence measured as described below.

For the turbidity assay, Ubc6 (in $0.03 \%$ (w/v) DDM) was diluted 1:25 into buffer L (f.c. of $1.8 \mu \mathrm{M}$ Ubc6) in the presence or absence of Get3 (f.c. $1.8 \mu \mathrm{M}$ or $3.6 \mu \mathrm{M}$ ). The optical density at $360 \mathrm{~nm}$ was measured using a UV-2401PC spectrophotometer (Shimadzu Corporation).

Protease protection assay

For this experiment, both sets of liposomes (SUMO-Ubc6, t-SNARE and Syb liposomes containing no, full-length or truncated Doa10 versions) were diluted 1:5 for the fusion reaction. First, Ulp1 cleavage was performed (f.c. $2 \mu \mathrm{M}$ Ulp1, $0.2 \mu \mathrm{M}$ Ubc6, $0.08 \mu \mathrm{M}$ Doa10). For the subsequent TEV-cleavage, the Ulp1-cleaved sample was diluted 1:2 and incubated with $10 \mu \mathrm{M}$ TEV-protease. During TEV- 
cleavage, $0.5 \mathrm{mM}$ DTT was present. Detergent controls contained 1\% TX100. Reactions were stopped by adding SDS sample buffer.

Antibody accessibility assay

AlexaFluor 488 fluorescence was measured in a Tecan Genios Pro microplate reader using 495/10 $\mathrm{nm}$ and $535 / 25 \mathrm{~nm}$ for excitation and emission, respectively. The fluorescence of $30 \mu \mathrm{L}$ of the fusion reaction was measured in a 96-well plate (Corning, REF 3686) with a f.c. of Ubc6 (labeled with A488) of $0.2 \mu \mathrm{M}$. After the signal was stable, the measurement was stopped, anti-Alexa Fluor 488 polyclonal antibody (Invitrogen, \#A-11094) was added (diluted 1:15) and the measurement started again. After approx. $40 \mathrm{~min}, 1 \mu \mathrm{L}$ TX100 (f.c. 1\%) was added to solubilize the liposomes. To analyze the fluorescence traces, the three measurements (equilibration, antibody and detergent addition) were merged. The background (stabilized A488 signal after detergent addition) was subtracted from all measurements. The fluorescence traces were subsequently normalized to the average signal of the last 10 timepoints before antibody addition. To quantify the fraction of released Ubc6, the difference between the normalized values of the samples with and without Doa10 $30 \mathrm{~min}$ after antibody addition was calculated.

To test for the release of the Ubc6/Syb chimera, liposomes containing Doa10 directly co-reconstituted with A488-labeled Ubc6, Ubc6 Гуртм or Syb Чвсьтм were first subjected to a pulldown via the SBP-tag of Doa10. Liposomes were diluted 1:8 (f.c. Ubc6 $0.125 \mu \mathrm{M}, 0.25 \mathrm{mg} / \mathrm{mL}$ BSA) and $50 \mu \mathrm{L}$ of diluted liposomes were added to $50 \mu \mathrm{L}$ of Pierce Streptavidin Magnetic Beads (Thermo Scientific) prewashed with buffer B. For the controls, Ubc6-only liposomes were also diluted 1:8 (in the presence or absence of 1:8 diluted Doa10-liposomes) and incubated with beads. After binding for $1 \mathrm{hr}$ (rotating, RT), the supernatant of the binding reaction was taken off, the beads washed three times with $200 \mu \mathrm{L}$ of buffer B and bound Doa10-liposomes eluted with $40 \mu \mathrm{L}$ of buffer B supplemented with $2 \mathrm{mM}$ biotin. Samples from input, supernatant and elution fractions were analyzed by SDSPAGE. $30 \mu \mathrm{L}$ of eluted fractions (Doa10-containing liposomes) were then added into a 96-well plate. Liposomes lacking Doa10 (containing Ubc6, Ubc6 Sуьтм or SybUbс6тM) were diluted 1:15 in buffer T. The antibody quenching assay was then carried out as described above.

\section{Ubiquitination assays}

All ubiquitination reactions were performed at $30^{\circ} \mathrm{C}$ in a thermocycler. The fusion reaction was diluted 1:2 (f.c. of $0.1 \mu \mathrm{M}$ Ubc6, $0.01 \mu \mathrm{M}$ Cue1 and $0.04 \mu \mathrm{M}$ Doa10). The following components were used at the indicated concentrations unless stated otherwise: $0.1 \mu \mathrm{M}$ Uba1 (E1), $1 \mu \mathrm{M}$ Ubc7, $120 \mu \mathrm{M}$ ubiquitin (from S. cerevisiae, R and D Systems) and $2.5 \mathrm{mM}$ ATP. All reactions contained 0.1 $\mathrm{mg} / \mathrm{mL}$ BSA. The ubiquitin mutant K0 (Lifesensors) is derived from human ubiquitin. Reactions were stopped by adding reducing SDS-sample buffer and samples were analyzed by SDS-PAGE.

\section{Analysis of ubiquitination reactions}

To analyze the fraction of non-modified protein, the non-ubiquitinated band was quantified and normalized to the $0 \mathrm{~min}$ timepoint. To analyze the ubiquitin chain profile, the fluorescence intensity was quantified along a vertical axis starting from the top of the gel using the line scan function in ImageJ (Figure $2 \mathrm{~B}$ and Figure $5 \mathrm{~B}$ ). When different Ubc6-variants were compared (Ubc6 vs Ubc6 sybTM $_{\text {), the }}$ line scan values were normalized to the integral of the whole scan (Figure $5 \mathrm{~B}$ ), to account for different sortase labeling efficiencies. To quantify the kinetics for generation of mono-, di-, tri- and tetraubiquitinated species, each band corresponding to one, two, three and four ubiquitins was quantified for every time point and normalized to the non-ubiquitinated band at the $0 \mathrm{~min}$ timepoint (Figure 5-figure supplement 1D). To calculate the total number of ubiquitins transferred relative to total Ubc6, the values for species modified with 1 to 4 ubiquitins obtained as above were summed up for each timepoint (Figure 5D and Figure 5-figure supplement 2C).

\section{Measuring extraction by the Cdc48-complex}

Proteoliposomes were prepared with protein-free liposomes containing 2 mol\% biotinyl-PE and 0.5 mol\% Rhd-PE. Fusion and ubiquitination was carried out as described above. To immobilize liposomes after ubiquitination, the ubiquitination reaction was diluted 1:2 to a final lipid concentration of $0.2 \mathrm{mM}$ total lipid (f.c. of $0.05 \mu \mathrm{M}$ Ubc6) and BSA was added to a f.c. of $0.25 \mathrm{mg} / \mathrm{ml}$. The diluted 
mix was then added to an equal volume of Pierce Streptavidin Magnetic Beads (Thermo Scientific, prewashed with buffer B). After incubation for $1 \mathrm{hr}$ at RT (rotating), the unbound fraction was removed and the beads were subsequently washed $3 \times$ with buffer $B$. The beads were then resuspended in the same volume of buffer B and $30 \mu \mathrm{L}$ of the suspension aliquoted in a PCR-strip. The buffer was removed and the beads resuspended in $1 \times$ extraction mixes or $1 \times$ SDS sample buffer. 1 $x$ extraction mixes contained $0.25 \mathrm{mg} / \mathrm{mL}$ BSA and where indicated $0.1 \mu \mathrm{M} \mathrm{Cdc48} \mathrm{(hexamer)} \mathrm{and} 0.1$ $\mu \mathrm{M} U \mathrm{fd} 1 / \mathrm{Np} / 4$. Beads were incubated for $30 \mathrm{~min}$ at $30^{\circ} \mathrm{C}$. The supernatant was removed (containing extracted and soluble proteins). After washing the beads $3 \times$ with buffer $B$, the bound proteins were eluted by adding $30 \mu \mathrm{L}$ of $1 \times$ SDS sample buffer. Samples of the supernatant and the elution fractions were analyzed by SDS-PAGE. To quantify the liposome immobilization efficiency, the Rhodamine fluorescence was measured in a Tecan Genios Pro microplate reader using 550/10 nm and $590 / 20 \mathrm{~nm}$ for excitation and emission, respectively. To quantify the protein immobilization efficiency, the DyLight680 (Ubc6/Ub-Ubc6 $c 87 \mathrm{~A})$ fluorescence was measured using the Odyssey scanner (384-well plate, transparent bottom).

Analysis of extraction reactions

To quantify the extraction efficiency of Ub-Ubc6 ${ }_{C 87 \mathrm{~A}}$ relative to its ubiquitination status (Figure 3figure supplement 1G), bands corresponding to Ub-Ubc6 $6_{C 87 A}$ modified with 1 to 10 ubiquitins were quantified separately and normalized to the corresponding band of the input sample (beads treated with sample buffer).

To quantify the fraction of Ubc6 in the supernatant (Figure 3C,D), the fluorescence intensity for Ubc6 modified with 0-5 ubiquitins and for Ubc6 modified with more than five ubiquitins was quantified by drawing a single rectangular box around the respective area using Image Studio and subsequently normalized to the Input (beads treated with $1 \times$ SDS sample buffer). For Ub-Ubc6 $6_{c 87 A}$, the unmodified band was counted as monoubiquitinated.

\section{Experiments with Ubc6-SBP}

To reconstitute Ubc6-SBP into proteoliposomes, Ubc6-SBP was preincubated with a 1.25-fold molar excess of tetrameric streptavidin (NEB) for $15 \mathrm{~min}$ at RT in the presence of $0.03 \%$ DDM to allow for complex formation. The reconstitution conditions were otherwise the same as for the co-reconstitution of Ubc6 and t-SNARE (with $2 \mu \mathrm{M}$ Ubc6-SBP and $2.5 \mu \mathrm{M}$ Streptavidin).

To assess the orientation of Ubc6-SBP in liposomes (Figure 6-figure supplement 1B), a TEV protease protection assay was carried out as described above, except that liposomes were diluted 1:5.

A biotinylated nanobody was used for the biotinylated protein control. To test if the biotinylated nanobody (anti-GFP, construct for expression kindly provided by Dirk Görlich; purified and biotinylated essentially as described in Pleiner et alo, 2018 is completely biotinylated (Figure 6-figure supplement 1C), biotinylated nanobody was supplemented with $0.25 \mathrm{mg} / \mathrm{mL}$ BSA and incubated with magnetic streptavidin beads that were prewashed with buffer B or prewashed with buffer $B$ supplemented with $10 \mathrm{mM}$ biotin. After binding for $45 \mathrm{~min}$ at RT, samples of input and supernatant fractions were analyzed by SDS-PAGE and stain-free scanning using a GelDoc EZ Imager.

For the antibody accessibility assay, the fusion reaction was first incubated with $1.5 \mu \mathrm{M}$ biotin or biotinylated protein for $10 \mathrm{~min}$ at RT, before the fluorescence measurements were started as described above. A 6-fold molar excess of biotin/biotinylated protein over streptavidin was used. Fluorescence traces were processed and analyzed as described above.

To check if biotinylated protein is capable of releasing Streptavidin from Ubc6-SBP, a flotation assay was used. Liposomes containing Ubc6-SBP and t-SNARE were incubated with Streptavidin for $5 \mathrm{~min}$ at RT (f.c. $0.9 \mu \mathrm{M}$ Streptavidin, $1.4 \mu \mathrm{M}$ Ubc6-SBP). A 10-fold excess of biotin or biotinylated nanobody was then added and after incubation for another $5 \mathrm{~min}$, a sucrose density gradient $(40 \%$ $(\mathrm{w} / \mathrm{v}), 30 \%(\mathrm{w} / \mathrm{v}), 15 \%(\mathrm{w} / \mathrm{v})$ and buffer T layer) was assembled. Flotation was carried out as described above. Samples were analyzed by SDS-PAGE and stain-free scanning using a GelDoc EZ Imager. Streptavidin and Ubc6-SBP levels were quantified using ImageJ. Intensity values for Streptavidin were normalized to Ubc6-SBP levels and subsequently to the buffer control.

To measure extraction of Ubc6-SBP by the Cdc48-complex using the antibody accessibility assay, fusion and ubiquitination reactions were carried out as described above, except that liposomes were 
diluted 1:5 instead of 1:10 for the fusion reaction. After $30 \mathrm{~min}$ of ubiquitination (total volume of 30 $\mu \mathrm{l}$ with f.c. $0.2 \mu \mathrm{M}$ Ubc6-SBP, $0.08 \mu \mathrm{M}$ Doa10, $0.02 \mu \mathrm{M}$ Cue1, $0.1 \mu \mathrm{M}$ Uba1, $1 \mu \mathrm{M}$ Ubc7, $120 \mu \mathrm{M}$ ubiquitin and $2.5 \mathrm{mM} \mathrm{ATP)}$, additional $2.5 \mathrm{mM}$ ATP were added and the fluorescence measurement was started (plate reader preheated to $30^{\circ} \mathrm{C}$ ). After $10 \mathrm{~min}$, anti-A488 antibody (1:15 diluted) and 3 $\mu \mathrm{L}$ of $10 \times \mathrm{Cdc} 48 / \mathrm{UN}$ mix were added. $10 \times \mathrm{Cdc} 48 / \mathrm{UN}$ mix contained $2 \mu \mathrm{M}$ Cdc48 (hexamer), $2 \mu \mathrm{M}$ $\mathrm{Ufd} 1 / \mathrm{Npl} 4$ as well as $1 \mathrm{mM}$ ATP. To quantify the fraction of extracted Ubc6-SBP, fluorescence traces were processed as described above and the difference between the normalized values of samples with and without Cdc48/UN 30 min after antibody addition was calculated.

To test, if streptavidin stays in the liposome lumen during extraction (Figure $6 E, F$ ), samples were floated after the extraction assay in a Nycodenz step gradient as described above. The samples were prepared with the following modifications: To ensure sufficient detection levels, the liposomes were diluted 1:4 for the fusion reaction. The ubiquitination reaction (f.c. $0.3 \mu \mathrm{M}$ Ubc6, $0.12 \mu \mathrm{M}$ Doa10, $0.03 \mu \mathrm{M}$ Cue1, $0.1 \mu \mathrm{M}$ Uba1, $1 \mu \mathrm{M}$ Ubc7, $120 \mu \mathrm{M}$ ubiquitin and $2.5 \mathrm{mM}$ ATP) was carried out as described above. After $30 \mathrm{~min}$ of ubiquitination, the extraction assay was performed in the

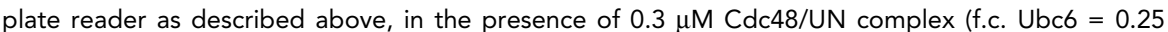
$\mu \mathrm{M})$. After $30 \mathrm{~min}, 2 \mathrm{mM}$ biotin was added to $50 \mu \mathrm{L}$ sample, a Nycodenz step gradient was assembled and the flotation carried out as described above. Samples were analyzed by SDS-PAGE and stain-free scanning using a GelDoc EZ Imager. Streptavidin levels were quantified using ImageJ. Intensity values were normalized to the sample without Cdc48/UN.

\section{Analysis}

Samples were mixed with SDS sample buffer (stock used as $3 \times$ contained $12 \%(w / v)$ SDS, 30\% (w/v) glycerol, $0.05 \%$ Coomassie blue G-250, $150 \mathrm{mM}$ Tris/ $\mathrm{HCl} \mathrm{pH} \mathrm{7.0 \%} \mathrm{and} 6 \%(\mathrm{v} / \mathrm{v})$ B-mercaptoethanol for reducing sample buffer (Schägger, 2006). Samples were heated at $70^{\circ} \mathrm{C}$ (Streptavidin-containing samples were boiled) and analyzed by SDS-PAGE using CRITERION TGX stain-free precast gels (BioRad).

Fluorescent proteins were detected using an Odyssey scanner (Li-Cor) for DyLight680 and DyLight800-labeled proteins, and an FLA-700 fluorescence scanner (Fujifilm) for AlexaFluor488labeled proteins. To detect streptavidin, samples were run on CRITERION TGX stain-free precast gels (Bio-Rad) and scanned with a GelDoc EZ Imager (Bio-Rad). Colloidal Coomassie staining was used (Dyballa and Metzger, 2009). SBP-tagged proteins were analyzed by western blotting where indicated. After transfer on a nitrocellulose membrane using the Trans-Blot Turbo Transfer System (Bio-Rad), the membrane was blocked with 5\% skim milk powder (dissolved in TBS-T) for $1 \mathrm{hr}$ at RT. A 1:2500 dilution of anti-SBP antibody (clone 20, mouse monoclonal, MAB10764, Millipore), and a 1:15,000 dilution of secondary antibody (goat anti-mouse, IRDye $800 \mathrm{CW}$ or IRDye 680RD) were used for detection. For the analysis of His-tagged ATP synthase, the nitrocellulose membrane was blocked with $2 \%(\mathrm{w} / \mathrm{v})$ BSA (dissolved in PBS containing $0.1 \%(\mathrm{v} / \mathrm{v})$ Tween and $0.1 \%(\mathrm{v} / \mathrm{v})$ Triton-X100). A 1:500 dilution of anti-His 6 tag antibody (Dia-900, Clone13/45/31-2, mouse monoclonal, Dianova), and a 1:15,000 dilution of secondary antibody (goat anti-mouse, IRDye $800 \mathrm{CW}$ ) were used for detection. Antibodies were diluted in the respective blocking buffer. Gels were quantified using ImageStudio Lite (Li-Cor). Fiji (ImageJ) was used for quantification of ubiquitin chain profiles (plot profile function) as well as streptavidin (gel analyzer function) (Schindelin et al., 2012).

\section{Acknowledgements}

We thank Blanche Schwappach (UM Göttingen, Germany) for providing Get3 constructs, Iris Bickmeyer and Nupur Nupur for technical assistance, and Marina Rodnina, Alex Faesen, Tom Rapoport and Blanche Schwappach for comments on the manuscript. This work was supported by the European Research Council (ERC) under the Horizon2020 research and innovation program (grant \# 677770), by the Deutsche Forschungsgemeinschaft SFB1190, TP15 (both to AS), and the Boehringer Ingelheim Fonds (to VV). 


\section{Additional information}

Funding

\begin{tabular}{lll} 
Funder & Grant reference number & Author \\
\hline $\begin{array}{l}\text { H2020 European Research } \\
\text { Council }\end{array}$ & 677770 & Alexander Stein \\
\hline $\begin{array}{l}\text { Deutsche Forschungsge- } \\
\text { meinschaft }\end{array}$ & SFB1190, TP15 & Alexander Stein \\
\hline
\end{tabular}

The funders had no role in study design, data collection and interpretation, or the decision to submit the work for publication.

Author contributions

Claudia C Schmidt, Conceptualization, Data curation, Formal analysis, Validation, Investigation, Visualization, Methodology, Writing - original draft, Writing - review and editing; Vedran Vasic, Methodology; Alexander Stein, Conceptualization, Supervision, Funding acquisition, Methodology, Writing original draft, Project administration, Writing - review and editing

Author ORCIDs

Claudia C Schmidt (iD) https://orcid.org/0000-0002-7642-7081

Vedran Vasic (iD https://orcid.org/0000-0003-2575-1006

Alexander Stein (iD https://orcid.org/0000-0002-2696-0611

Decision letter and Author response

Decision letter https://doi.org/10.7554/eLife.56945.sa1

Author response https://doi.org/10.7554/eLife.56945.sa2

\section{Additional files}

Supplementary files

- Transparent reporting form

Data availability

All data generated or analysed during this study are included in the manuscript and supporting files.

\section{References}

Baldridge RD, Rapoport TA. 2016. Autoubiquitination of the Hrd1 ligase triggers protein retrotranslocation in ERAD. Cell 166:394-407. DOI: https://doi.org/10.1016/j.cell.2016.05.048, PMID: 27321670

Bays NW, Wilhovsky SK, Goradia A, Hodgkiss-Harlow K, Hampton RY. 2001. HRD4/NPL4 is required for the proteasomal processing of ubiquitinated ER proteins. Molecular Biology of the Cell 12:4114-4128. DOl: https://doi.org/10.1091/mbc.12.12.4114, PMID: 11739805

Biederer T, Volkwein C, Sommer T. 1997. Role of Cue1p in Ubiquitination and degradation at the ER surface. Science 278:1806-1809. DOI: https://doi.org/10.1126/science.278.5344.1806, PMID: 9388185

Bodnar NO, Kim KH, Ji Z, Wales TE, Svetlov V, Nudler E, Engen JR, Walz T, Rapoport TA. 2018. Structure of the Cdc48 ATPase with its ubiquitin-binding cofactor Ufd1-Npl4. Nature Structural \& Molecular Biology 25:616622. DOl: https://doi.org/10.1038/s41594-018-0085-x, PMID: 29967539

Bodnar NO, Rapoport TA. 2017. Molecular mechanism of substrate processing by the Cdc48 ATPase complex. Cell 169::722-735. DOI: https://doi.org/10.1016/j.cell.2017.04.020

Bordallo J, Plemper RK, Finger A, Wolf DH. 1998. Der3p/Hrd1p is required for endoplasmic reticulum-associated degradation of misfolded lumenal and integral membrane proteins. Molecular Biology of the Cell 9:209-222. DOI: https://doi.org/10.1091/mbc.9.1.209, PMID: 9437001

Botelho SC, Tatsuta T, von Heijne G, Kim H. 2013. Dislocation by the m-AAA protease increases the threshold hydrophobicity for retention of transmembrane helices in the inner membrane of yeast mitochondria. Journal of Biological Chemistry 288:4792-4798. DOI: https://doi.org/10.1074/jbc.M112.430892, PMID: 23283966

Brodsky JL. 2012. Cleaning Up: ER-Associated Degradation to the Rescue. Cell 151:1163-1167. DOI: https:// doi.org/10.1016/j.cell.2012.11.012 
Burr ML, Cano F, Svobodova S, Boyle LH, Boname JM, Lehner PJ. 2011. HRD1 and UBE2J1 target misfolded MHC class I heavy chains for endoplasmic reticulum-associated degradation. PNAS 108:2034-2039. DOI: https://doi.org/10.1073/pnas.1016229108, PMID: 21245296

Carvalho P, Stanley AM, Rapoport TA. 2010. Retrotranslocation of a misfolded luminal ER protein by the ubiquitin-ligase Hrd1p. Cell 143:579-591. DOI: https://doi.org/10.1016/j.cell.2010.10.028, PMID: 21074049 Chen I, Dorr BM, Liu DR. 2011. A general strategy for the evolution of bond-forming enzymes using yeast display. PNAS 108:11399-11404. DOI: https://doi.org/10.1073/pnas.1101046108, PMID: 21697512

Chen Q, Zhong Y, Wu Y, Liu L, Wang P, Liu R, Cui F, Li Q, Yang X, Fang S, Xie Q. 2016. HRD1-mediated ERAD tuning of ER-bound E2 is conserved between plants and mammals. Nature Plants 2:16094. DOI: https://doi. org/10.1038/nplants.2016.94, PMID: 27322605

Christianson JC, Ye Y. 2014. Cleaning up in the endoplasmic reticulum: ubiquitin in charge. Nature Structural \& Molecular Biology 21:325-335. DOI: https://doi.org/10.1038/nsmb.2793, PMID: 24699081

Claessen JH, Sanyal S, Ploegh HL. 2014. The chaperone BAG6 captures dislocated glycoproteins in the cytosol. PLOS ONE 9:e90204. DOI: https://doi.org/10.1371/journal.pone.0090204, PMID: 24594942

Claessen JH, Ploegh HL. 2011. BAT3 guides misfolded glycoproteins out of the endoplasmic reticulum. PLOS ONE 6:e28542. DOI: https://doi.org/10.1371/journal.pone.0028542, PMID: 22174835

Cooney I, Han H, Stewart MG, Carson RH, Hansen DT, Iwasa JH, Price JC, Hill CP, Shen PS. 2019. Structure of the Cdc48 segregase in the act of unfolding an authentic substrate. Science 365:502-505. DOI: https://doi.org/ 10.1126/science.aax0486, PMID: 31249134

Cypionka A, Stein A, Hernandez JM, Hippchen H, Jahn R, Walla PJ. 2009. Discrimination between docking and fusion of liposomes reconstituted with neuronal SNARE-proteins using FCS. PNAS 106:18575-18580. DOI: https://doi.org/10.1073/pnas.0906677106, PMID: 19843696

Dederer V, Khmelinskii A, Huhn AG, Okreglak V, Knop M, Lemberg MK. 2019. Cooperation of mitochondrial and ER factors in quality control of tail-anchored proteins. eLife 8:e45506. DOl: https://doi.org/10.7554/eLife. 45506, PMID: 31172943

Doblas VG, Amorim-Silva V, Posé D, Rosado A, Esteban A, Arró M, Azevedo H, Bombarely A, Borsani O, Valpuesta V, Ferrer A, Tavares RM, Botella MA. 2013. The SUD1 gene encodes a putative E3 ubiquitin ligase and is a positive regulator of 3-hydroxy-3-methylglutaryl coenzyme a reductase activity in arabidopsis. The Plant Cell 25:728-743. DOI: https://doi.org/10.1105/tpc.112.108696, PMID: 23404890

Dyballa N, Metzger S. 2009. Fast and sensitive colloidal coomassie G-250 staining for proteins in polyacrylamide gels. Journal of Visualized Experiments 3:1431. DOI: https://doi.org/10.3791/1431

Ellgaard L, Sevier CS, Bulleid NJ. 2018. How Are Proteins Reduced in the Endoplasmic Reticulum? Trends in Biochemical Sciences 43:32-43. DOI: https://doi.org/10.1016/j.tibs.2017.10.006

Ernst R, Claessen JH, Mueller B, Sanyal S, Spooner E, van der Veen AG, Kirak O, Schlieker CD, Weihofen WA Ploegh HL. 2011. Enzymatic blockade of the ubiquitin-proteasome pathway. PLOS Biology 8:e1000605. DOI: https://doi.org/10.1371/journal.pbio.1000605, PMID: 21468303

Fasshauer D, Antonin W, Margittai M, Pabst S, Jahn R. 1999. Mixed and non-cognate SNARE complexes characterization of assembly and biophysical properties. The Journal of Biological Chemistry 274:15440-15446. DOI: https://doi.org/10.1074/jbc.274.22.15440, PMID: 10336434

Fiebiger E, Story C, Ploegh HL, Tortorella D. 2002. Visualization of the ER-to-cytosol dislocation reaction of a type I membrane protein. The EMBO Journal 21:1041-1053. DOl: https://doi.org/10.1093/emboj/21.5.1041, PMID: 11867532

Foresti O, Ruggiano A, Hannibal-Bach HK, Ejsing CS, Carvalho P. 2013. Sterol homeostasis requires regulated degradation of squalene monooxygenase by the ubiquitin ligase Doa10/Teb4. eLife 2:e00953. DOI: https://doi. org/10.7554/eLife.00953, PMID: 23898401

Furth N, Gertman O, Shiber A, Alfassy OS, Cohen I, Rosenberg MM, Doron NK, Friedler A, Ravid T. 2011. Exposure of bipartite hydrophobic signal triggers nuclear quality control of $\mathrm{Ndc} 10$ at the endoplasmic reticulum/nuclear envelope. Molecular Biology of the Cell 22:4726-4739. DOI: https://doi.org/10.1091/mbc e11-05-0463

Garza RM, Sato BK, Hampton RY. 2009. In vitro analysis of Hrd1p-mediated retrotranslocation of its multispanning membrane substrate 3-hydroxy-3-methylglutaryl (HMG)-CoA reductase. Journal of Biological Chemistry 284:14710-14722. DOI: https://doi.org/10.1074/jbc.M809607200, PMID: 19324879

Glynn SE. 2017. Multifunctional Mitochondrial AAA Proteases. Frontiers in Molecular Biosciences 4:34. DOl: https://doi.org/10.3389/fmolb.2017.00034

Guerriero CJ, Reutter KR, Augustine AA, Preston GM, Weiberth KF, Mackie TD, Cleveland-Rubeor HC, Bethel NP, Callenberg KM, Nakatsukasa K, Grabe M, Brodsky JL. 2017. Transmembrane helix hydrophobicity is an energetic barrier during the retrotranslocation of integral membrane ERAD substrates. Molecular Biology of the Cell 28:2076-2090. DOl: https://doi. org/10.1091/mbc.e17-03-0184, PMID: 28539401

Guerriero CJ, Brodsky JL. 2012. The delicate balance between secreted protein folding and endoplasmic reticulum-associated degradation in human physiology. Physiological Reviews 92:537-576. DOI: https://doi. org/10.1152/physrev.00027.2011, PMID: 22535891

Guo H, Suzuki T, Rubinstein JL. 2019. Structure of a bacterial ATP synthase. eLife 8:e43128. DOI: https://doi.org/ 10.7554/eLife.43128, PMID: 30724163

Habeck G, Ebner FA, Shimada-Kreft H, Kreft SG. 2015. The yeast ERAD-C ubiquitin ligase Doa10 recognizes an intramembrane degron. Journal of Cell Biology 209:261-273. DOI: https://doi.org/10.1083/jcb.201408088, PMID: 25918226 
Hampton RY, Gardner RG, Rine J. 1996. Role of $26 \mathrm{~S}$ proteasome and HRD genes in the degradation of 3hydroxy-3-methylglutaryl-CoA reductase, an integral endoplasmic reticulum membrane protein. Molecular Biology of the Cell 7:2029-2044. DOI: https://doi.org/10.1091/mbc.7.12.2029, PMID: 8970163

Hampton RY, Sommer T. 2012. Finding the will and the way of ERAD substrate retrotranslocation. Current Opinion in Cell Biology 24:460-466. DOI: https://doi.org/10.1016/j.ceb.2012.05.010, PMID: 22854296

Han S, Liu Y, Chang A. 2007. Cytoplasmic Hsp70 promotes ubiquitination for endoplasmic reticulum-associated degradation of a misfolded mutant of the yeast plasma membrane ATPase, PMA1. Journal of Biological Chemistry 282:26140-26149. DOI: https://doi.org/10.1074/jbc.M701969200, PMID: 17631501

Hassink G, Kikkert M, van Voorden S, Lee SJ, Spaapen R, van Laar T, Coleman CS, Bartee E, Früh K, Chau V, Wiertz E. 2005. TEB4 is a C4HC3 RING finger-containing ubiquitin ligase of the endoplasmic reticulum. Biochemical Journal 388:647-655. DOI: https://doi.org/10.1042/BJ20041241, PMID: 15673284

Heo JM, Livnat-Levanon N, Taylor EB, Jones KT, Dephoure N, Ring J, Xie J, Brodsky JL, Madeo F, Gygi SP, Ashrafi K, Glickman MH, Rutter J. 2010. A stress-responsive system for mitochondrial protein degradation. Molecular Cell 40:465-480. DOI: https://doi.org/10.1016/j.molcel.2010.10.021, PMID: 21070972

Hernandez JM, Stein A, Behrmann E, Riedel D, Cypionka A, Farsi Z, Walla PJ, Raunser S, Jahn R. 2012. Membrane fusion intermediates via directional and full assembly of the SNARE complex. Science 336:15811584. DOI: https://doi.org/10.1126/science.1221976, PMID: 22653732

Huyer G, Piluek WF, Fansler Z, Kreft SG, Hochstrasser M, Brodsky JL, Michaelis S. 2004. Distinct machinery is required in Saccharomyces cerevisiae for the endoplasmic reticulum-associated degradation of a multispanning membrane protein and a soluble luminal protein. Journal of Biological Chemistry 279:38369-38378. DOI: https://doi.org/10.1074/jbc.M402468200, PMID: 15252059

Hwang J, Qi L. 2018. Quality control in the endoplasmic reticulum: crosstalk between ERAD and UPR pathways. Trends in Biochemical Sciences 43:593-605. DOI: https://doi.org/10.1016/j.tibs.2018.06.005, PMID: 30056836 Jarosch E, Taxis C, Volkwein C, Bordallo J, Finley D, Wolf DH, Sommer T. 2002. Protein dislocation from the ER requires polyubiquitination and the AAA-ATPase Cdc48. Nature Cell Biology 4:134-139. DOI: https://doi.org/ 10.1038/ncb746, PMID: 11813000

Keefe AD, Wilson DS, Seelig B, Szostak JW. 2001. One-step purification of recombinant proteins using a nanomolar-affinity streptavidin-binding peptide, the SBP-Tag. Protein Expression and Purification 23:440-446. DOI: https://doi.org/10.1006/prep.2001.1515, PMID: 11722181

Kikkert M, Doolman R, Dai M, Avner R, Hassink G, van Voorden S, Thanedar S, Roitelman J, Chau V, Wiertz E. 2004. Human HRD1 is an E3 ubiquitin ligase involved in degradation of proteins from the endoplasmic reticulum. Journal of Biological Chemistry 279:3525-3534. DOI: https://doi.org/10.1074/jbc.M307453200, PMID: 14593114

Korbel D, Wurth S, Käser M, Langer T. 2004. Membrane protein turnover by the m-AAA protease in mitochondria depends on the transmembrane domains of its subunits. EMBO Reports 5:698-703. DOI: https:// doi.org/10.1038/sj.embor.7400186, PMID: 15205678

Kreft SG, Wang L, Hochstrasser M. 2006. Membrane topology of the yeast endoplasmic reticulum-localized ubiquitin ligase Doa10 and comparison with its human ortholog TEB4 (MARCH-VI). Journal of Biological Chemistry 281:4646-4653. DOI: https://doi.org/10.1074/jbc.M512215200, PMID: 16373356

Lam SY, Murphy C, Foley LA, Ross SA, Wang TC, Fleming JV. 2014. The human ubiquitin conjugating enzyme UBE2J2 (Ubc6) is a substrate for proteasomal degradation. Biochemical and Biophysical Research Communications 451:361-366. DOl: https://doi.org/10.1016/j.bbrc.2014.07.099, PMID: 25083800

Lee S, Lee H, Yoo S, Kim H. 2017. Molecular insights into the m-AAA protease-mediated dislocation of transmembrane helices in the mitochondrial inner membrane. Journal of Biological Chemistry 292:2005820066. DOI: https://doi.org/10.1074/jbc.M117.796763, PMID: 29030426

Lilley BN, Ploegh HL. 2004. A membrane protein required for dislocation of misfolded proteins from the ER. Nature 429:834-840. DOI: https://doi.org/10.1038/nature02592, PMID: 15215855

Ling Q, Broad W, Trösch R, Töpel M, Demiral Sert T, Lymperopoulos P, Baldwin A, Jarvis RP. 2019. Ubiquitindependent chloroplast-associated protein degradation in plants. Science 363:eaav4467. DOI: https://doi.org/ 10.1126/science. aav4467

Liu L, Spurrier J, Butt TR, Strickler JE. 2008. Enhanced protein expression in the baculovirus/insect cell system using engineered SUMO fusions. Protein Expression and Purification 62:21-28. DOI: https://doi.org/10.1016/j. pep.2008.07.010, PMID: 18713650

Mandart E, Dufour ME, Lacroute F. 1994. Inactivation of SSM4, a new Saccharomyces cerevisiae gene, suppresses mRNA instability due to rna14 mutations. Molecular and General Genetics MGG 245:323-333. DOI: https://doi.org/10.1007/BF00290112, PMID: 7816042

Mateja A, Szlachcic A, Downing ME, Dobosz M, Mariappan M, Hegde RS, Keenan RJ. 2009. The structural basis of tail-anchored membrane protein recognition by Get3. Nature 461:361-366. DOI: https://doi.org/10.1038/ nature08319, PMID: 19675567

Matsumoto S, Nakatsukasa K, Kakuta C, Tamura Y, Esaki M, Endo T. 2019. Msp1 clears mistargeted proteins by facilitating their transfer from mitochondria to the ER. Molecular Cell 76::191-205. DOI: https://doi.org/10. 1016/j.molcel.2019.07.006

Mehnert M, Sommer T, Jarosch E. 2014. Der1 promotes movement of misfolded proteins through the endoplasmic reticulum membrane. Nature Cell Biology 16:77-86. DOI: https://doi.org/10.1038/ncb2882, PMID: 24292014 
Mehrtash AB, Hochstrasser M. 2019. Ubiquitin-dependent protein degradation at the endoplasmic reticulum and nuclear envelope. Seminars in Cell \& Developmental Biology 93:111-124. DOI: https://doi.org/10.1016/j. semcdb.2018.09.013, PMID: 30278225

Meyer HH, Wang Y, Warren G. 2002. Direct binding of ubiquitin conjugates by the mammalian p97 adaptor complexes, p47 and Ufd1-Npl4. The EMBO Journal 21:5645-5652. DOl: https://doi.org/10.1093/emboj/ cdf579, PMID: 12411482

Mumberg D, Müller R, Funk M. 1994. Regulatable promoters of Saccharomyces cerevisiae: comparison of transcriptional activity and their use for heterologous expression. Nucleic Acids Research 22:5767-5768. DOI: https://doi.org/10.1093/nar/22.25.5767, PMID: 7838736

Nadav E, Shmueli A, Barr H, Gonen H, Ciechanover A, Reiss Y. 2003. A novel mammalian endoplasmic reticulum ubiquitin ligase homologous to the yeast Hrd1. Biochemical and Biophysical Research Communications 303:9197. DOI: https://doi.org/10.1016/S0006-291X(03)00279-1, PMID: 12646171

Nakatsukasa K, Huyer G, Michaelis S, Brodsky JL. 2008. Dissecting the ER-associated degradation of a misfolded polytopic membrane protein. Cell 132:101-112. DOI: https://doi.org/10.1016/j.cell.2007.11.023, PMID: 18191224

Nakatsukasa K, Kamura T. 2016. Subcellular fractionation analysis of the extraction of ubiquitinated polytopic membrane substrate during ER-Associated degradation. PLOS ONE 11:e0148327. DOI: https://doi.org/10. 1371/journal.pone.0148327, PMID: 26849222

Neal S, Jaeger PA, Duttke SH, Benner C, K.Glass C, Ideker T, Hampton RY. 2018. The Dfm1 derlin is required for ERAD retrotranslocation of integral membrane proteins. Molecular Cell 69::306-320. DOI: https://doi.org/10. 1016/j.molcel.2017.12.012

Neuber O, Jarosch E, Volkwein C, Walter J, Sommer T. 2005. Ubx2 links the Cdc48 complex to ER-associated protein degradation. Nature Cell Biology 7:993-998. DOI: https://doi.org/10.1038/ncb1298

Olszewski MM, Williams C, Dong KC, Martin A. 2019. The Cdc48 unfoldase prepares well-folded protein substrates for degradation by the $26 \mathrm{~S}$ proteasome. Communications Biology 2:29. DOI: https://doi.org/10. 1038/s42003-019-0283-z, PMID: 30675527

Olzmann JA, Kopito RR, Christianson JC. 2013. The mammalian endoplasmic reticulum-associated degradation system. Cold Spring Harbor Perspectives in Biology 5:a013185. DOI: https://doi.org/10.1101/cshperspect. a013185, PMID: 23232094

Petris G, Casini A, Sasset L, Cesaratto F, Bestagno M, Cereseto A, Burrone OR. 2014. CD4 and BST-2/tetherin proteins retro-translocate from endoplasmic reticulum to cytosol as partially folded and multimeric molecules. Journal of Biological Chemistry 289:1-12. DOI: https://doi.org/10.1074/jbc.M113.512368, PMID: 24257748

Pleiner T, Bates M, Görlich D. 2018. A toolbox of anti-mouse and anti-rabbit lgG secondary nanobodies. Journal of Cell Biology 217:1143-1154. DOI: https://doi.org/10.1083/jcb.201709115, PMID: 29263082

Pobbati AV, Stein A, Fasshauer D. 2006. N- to C-terminal SNARE complex assembly promotes rapid membrane fusion. Science 313:673-676. DOI: https://doi.org/10.1126/science.1129486, PMID: 16888141

Popp MW, Antos JM, Ploegh HL. 2009. Site-specific protein labeling via sortase-mediated transpeptidation. Current Protocols in Protein Science 15:3. DOI: https://doi.org/10.1002/0471140864.ps1503s56, PMID: 193657 88

Qi L, Tsai B, Arvan P. 2017. New insights into the physiological role of endoplasmic Reticulum-Associated degradation. Trends in Cell Biology 27:430-440. DOI: https://doi.org/10.1016/j.tcb.2016.12.002, PMID: 2 8131647

Rabinovich E, Kerem A, Fröhlich KU, Diamant N, Bar-Nun S. 2002. AAA-ATPase p97/Cdc48p, a cytosolic chaperone required for endoplasmic reticulum-associated protein degradation. Molecular and Cellular Biology 22:626-634. DOI: https://doi.org/10.1128/MCB.22.2.626-634.2002, PMID: 11756557

Ravid T, Kreft SG, Hochstrasser M. 2006. Membrane and soluble substrates of the Doa10 ubiquitin ligase are degraded by distinct pathways. The EMBO Journal 25:533-543. DOI: https://doi.org/10.1038/sj.emboj. 7600946

Rigaud JL, Lévy D. 2003. Reconstitution of membrane proteins into liposomes. Methods in Enzymology 372:6586. DOl: https://doi.org/10.1016/S0076-6879(03)72004-7. PMID: 14610807

Ruggiano A, Foresti O, Carvalho P. 2014. Quality control: er-associated degradation: protein quality control and beyond. The Journal of Cell Biology 204:869-879. DOI: https://doi.org/10.1083/jcb.201312042, PMID: 24637321

Ruggiano A, Mora G, Buxó L, Carvalho P. 2016. Spatial control of lipid droplet proteins by the ERAD ubiquitin ligase Doa10. The EMBO Journal 35:1644-1655. DOI: https://doi.org/10.15252/embj.201593106, PMID: 27357570

Schägger H. 2006. Tricine-SDS-PAGE. Nature Protocols 1:16-22. DOI: https://doi.org/10.1038/nprot.2006.4, PMID: 17406207

Schenck S, Wojcik SM, Brose N, Takamori S. 2009. A chloride conductance in VGLUT1 underlies maximal glutamate loading into synaptic vesicles. Nature Neuroscience 12:156-162. DOI: https://doi.org/10.1038/nn. 2248, PMID: 19169251

Schindelin J, Arganda-Carreras I, Frise E, Kaynig V, Longair M, Pietzsch T, Preibisch S, Rueden C, Saalfeld S, Schmid B, Tinevez JY, White DJ, Hartenstein V, Eliceiri K, Tomancak P, Cardona A. 2012. Fiji: an open-source platform for biological-image analysis. Nature Methods 9:676-682. DOl: https://doi.org/10.1038/nmeth.2019, PMID: 22743772

Schmidt O, Weyer Y, Baumann V, Widerin MA, Eising S, Angelova M, Schleiffer A, Kremser L, Lindner H, Peter M Fröhlich F, Teis D. 2019. Endosome and Golgi-associated degradation (EGAD) of membrane proteins regulates 
sphingolipid metabolism. The EMBO Journal 38:e101433. DOI: https://doi.org/10.15252/embj.2018101433, PMID: 31368600

Schuberth C, Buchberger A. 2005. Membrane-bound Ubx2 recruits Cdc48 to ubiquitin ligases and their substrates to ensure efficient ER-associated protein degradation. Nature Cell Biology 7:999-1006. DOI: https:// doi.org/10.1038/ncb1299, PMID: 16179952

Shi J, Hu X, Guo Y, Wang L, Ji J, Li J, Zhang ZR. 2019. A technique for delineating the unfolding requirements for substrate entry into retrotranslocons during endoplasmic reticulum-associated degradation. Journal of Biological Chemistry 294:20084-20096. DOI: https://doi.org/10.1074/jbc.RA119.010019, PMID: 31748412

Stein A, Radhakrishnan A, Riedel D, Fasshauer D, Jahn R. 2007. Synaptotagmin activates membrane fusion through a Ca2+-dependent trans interaction with phospholipids. Nature Structural \& Molecular Biology 14 904-911. DOI: https://doi.org/10.1038/nsmb1305, PMID: 17891149

Stein A, Ruggiano A, Carvalho P, Rapoport TA. 2014. Key Steps in ERAD of Luminal ER Proteins Reconstituted with Purified Components. Cell 158:1375-1388. DOI: https://doi.org/10.1016/j.cell.2014.07.050

Stuerner E, Kuraku S, Hochstrasser M, Kreft SG. 2012. Split-Doa10: a naturally split polytopic eukaryotic membrane protein generated by fission of a nuclear gene. PLOS ONE 7:e45194. DOI: https://doi.org/10.1371/ journal.pone.0045194, PMID: 23071509

Suzuki T, Ueno H, Mitome N, Suzuki J, Yoshida M. 2002. F(0) of ATP synthase is a rotary proton channel obligatory coupling of proton translocation with rotation of c-subunit ring. The Journal of Biological Chemistry 277:13281-13285. DOI: https://doi.org/10.1074/jbc.M111210200, PMID: 11815616

Swanson R, Locher M, Hochstrasser M. 2001. A conserved ubiquitin ligase of the nuclear envelope/endoplasmic reticulum that functions in both ER-associated and Matalpha2 repressor degradation. Genes \& Development 15:2660-2674. DOI: https://doi.org/10.1101/gad.933301, PMID: 11641273

Tanaka A, Cleland MM, Xu S, Narendra DP, Suen DF, Karbowski M, Youle RJ. 2010. Proteasome and p97 mediate mitophagy and degradation of mitofusins induced by parkin. The Journal of Cell Biology 191:13671380. DOI: https://doi.org/10.1083/jcb.201007013, PMID: 21173115

Tirosh B, Furman MH, Tortorella D, Ploegh HL. 2003. Protein unfolding is not a prerequisite for endoplasmic reticulum-to-cytosol dislocation. Journal of Biological Chemistry 278:6664-6672. DOI: https://doi.org/10.1074/ jbc.M210158200, PMID: 12482753

Twomey EC, Ji Z, Wales TE, Bodnar NO, Ficarro SB, Marto JA, Engen JR, Rapoport TA. 2019. Substrate processing by the Cdc48 ATPase complex is initiated by ubiquitin unfolding. Science 365:eaax1033. DOI: https://doi.org/10.1126/science.aax1033, PMID: 31249135

Vashist S, Ng DT. 2004. Misfolded proteins are sorted by a sequential checkpoint mechanism of ER quality control. Journal of Cell Biology 165:41-52. DOI: https://doi.org/10.1083/jcb.200309132, PMID: 15078901

Vasic V, Denkert N, Schmidt CC, Riedel D, Stein A, Meinecke M. 2020. Hrd1 forms the retrotranslocation pore regulated by auto-ubiquitination and binding of misfolded proteins. Nature Cell Biology 22:274-281. DOI: https://doi.org/10.1038/s41556-020-0473-4, PMID: 32094691

Walter J, Urban J, Volkwein C, Sommer T. 2001. Sec61p-independent degradation of the tail-anchored ER membrane protein Ubc6p. The EMBO Journal 20:3124-3131. DOI: https://doi.org/10.1093/emboj/20.12.3124, PMID: 11406589

Wang Q, Chang A. 2003. Substrate recognition in ER-associated degradation mediated by Eps1, a member of the protein disulfide isomerase family. The EMBO Journal 22:3792-3802. DOI: https://doi.org/10.1093/emboj/ cdg378, PMID: 12881414

Wang Q, Liu Y, Soetandyo N, Baek K, Hegde R, Ye Y. 2011. A ubiquitin ligase-associated chaperone holdase maintains polypeptides in soluble states for proteasome degradation. Molecular Cell 42:758-770. DOI: https:// doi.org/10.1016/j.molcel.2011.05.010, PMID: 21636303

Weber A, Cohen I, Popp O, Dittmar G, Reiss Y, Sommer T, Ravid T, Jarosch E. 2016. Sequential Polyubiquitylation by specialized conjugating enzymes expands the versatility of a quality control ubiquitin ligase. Molecular Cell 63:827-839. DOI: https://doi.org/10.1016/j.molcel.2016.07.020, PMID: 27570077

Wu X, Siggel M, Ovchinnikov S, Mi W, Svetlov V, Nudler E, Liao M, Hummer G, Rapoport TA. 2020. Structural basis of ER-associated protein degradation mediated by the Hrd1 ubiquitin ligase complex. Science $\mathbf{3 6 8}$ eaaz2449. DOI: https://doi.org/10.1126/science.aaz2449

Xu Y, Cai M, Yang Y, Huang L, Ye Y. 2012. SGTA recognizes a noncanonical ubiquitin-like domain in the Bag6Ubl4A-Trc35 complex to promote endoplasmic reticulum-associated degradation. Cell Reports 2:1633-1644. DOI: https://doi.org/10.1016/j.celrep.2012.11.010, PMID: 23246001

Ye Y, Meyer HH, Rapoport TA. 2001. The AAA ATPase Cdc48/p97 and its partners transport proteins from the ER into the cytosol. Nature 414:652-656. DOI: https://doi.org/10.1038/414652a, PMID: 11740563

Ye Y, Meyer HH, Rapoport TA. 2003. Function of the p97-Ufd1-Npl4 complex in retrotranslocation from the ER to the cytosol: dual recognition of nonubiquitinated polypeptide segments and polyubiquitin chains. The Journal of Cell Biology 162:71-84. DOI: https://doi.org/10.1083/jcb.200302169, PMID: 12847084

Ye Y, Shibata Y, Yun C, Ron D, Rapoport TA. 2004. A membrane protein complex mediates retro-translocation from the ER lumen into the cytosol. Nature 429:841-847. DOI: https://doi.org/10.1038/nature02656

Zhang ZR, Bonifacino JS, Hegde RS. 2013. Deubiquitinases sharpen substrate discrimination during membrane protein degradation from the ER. Cell 154:609-622. DOI: https://doi.org/10.1016/j.cell.2013.06.038, PMID: 23 890821 


\section{elife}

\section{Figures and figure supplements}

Doa10 is a membrane protein retrotranslocase in ER-associated protein degradation

\section{Claudia C Schmidt et al}


A<smiles>CC1CCCC(C)CC1</smiles>

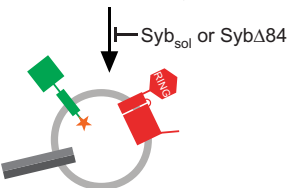

C
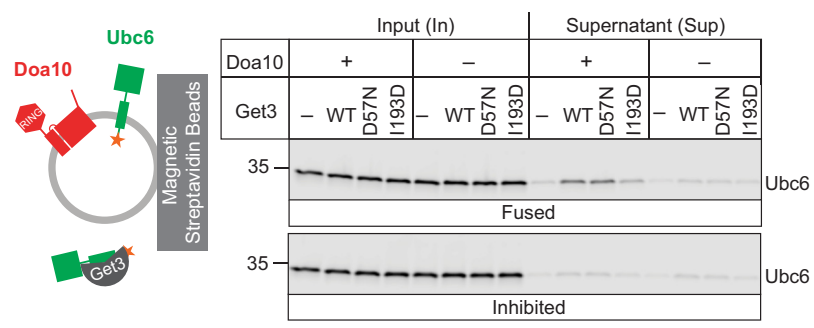

B

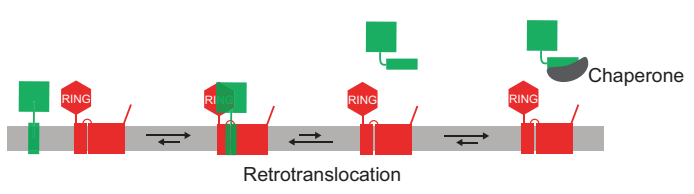

D

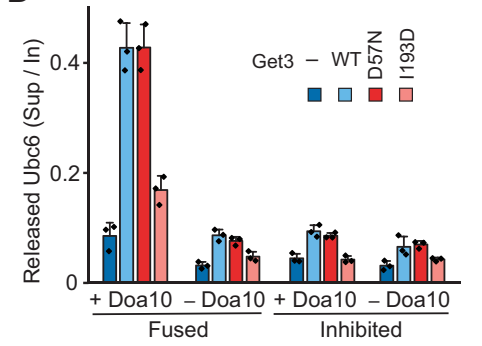

E

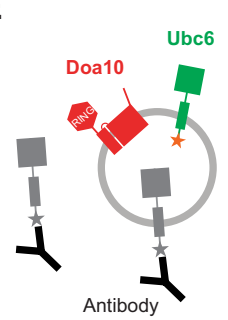

G
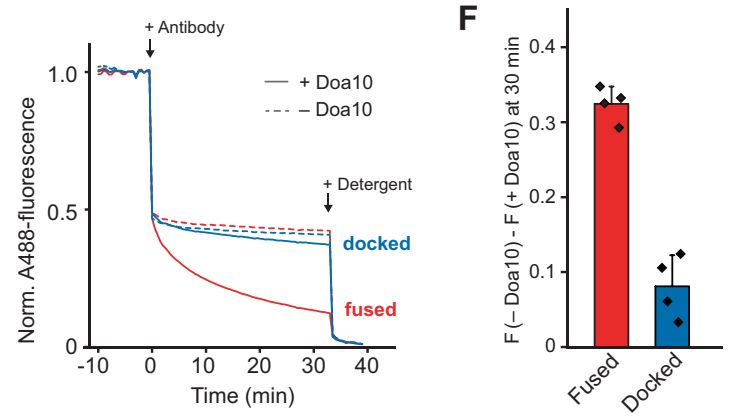

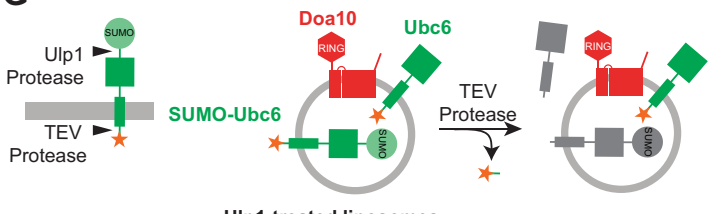

Ulp1-treated liposomes

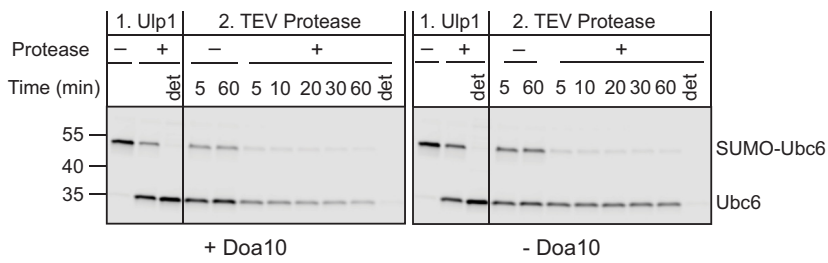

H

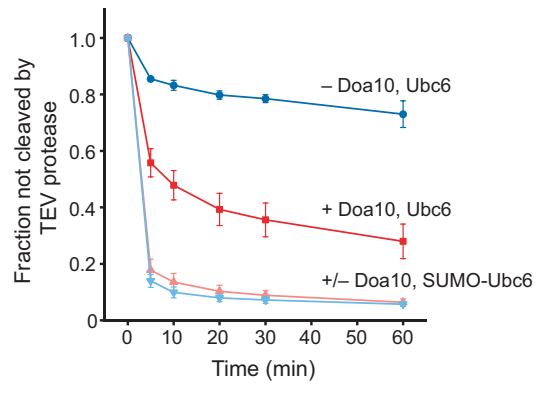

Figure 1. Retrotranslocation of Ubc6 by Doa10. (A) SNARE-mediated co-reconstitution of Ubc6 and Doa10. Engineered versions of SNAREs involved in

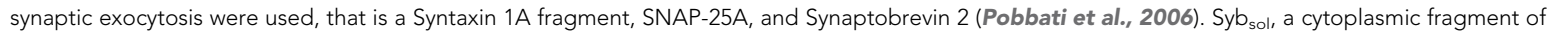
Figure 1 continued on next page 
eLife

Biochemistry and Chemical Biology | Cell Biology

Figure 1 continued

Synaptobrevin (Syb). Syb $\Delta 84$, Syb mutant that results in a docked state (Hernandez et al., 2012). See Figure 1-figure supplement 1, E to K for characterization of liposomes. (B) Working hypothesis for retrotranslocation by Doa10. (C) Membrane release of Ubc6 in the presence of Get3. Fluorescently labeled Ubc6 was co-reconstituted with Doa10 by SNARE-mediated fusion (+ Doa10), as shown in (A). Where indicated, Ubc6 liposomes were fused to liposomes lacking Doa10 (- Doa10), or fusion was inhibited with Sybsol (Inhibited). After incubation with the indicated Get3 variants or buffer, liposomes were immobilized (Figure 1-figure supplement 2B). Input and supernatant samples were analyzed by SDS-PAGE and fluorescence scanning. Final concentrations (f.c.): $0.1 \mu \mathrm{M}$ Ubc6, $40 \mathrm{nM}$ Doa10, $10 \mu \mathrm{M}$ Get3. (D) Quantification (mean \pm SD) of three independent experiments as in (C). (E) Retrotranslocation of Ubc6, measured as quenching of a C-terminal AlexaFluor488 (A488) label by an antibody. Liposomes were generated as shown in (A). Where indicated, liposomes lacked Doa10 (- Doa10), or co-reconstitution was inhibited by using Syb $\Delta 84$ (docked). Arrows indicate addition of the quenching antibody or of solubilizing amounts of detergent (Triton X-100). F.c.: $0.2 \mu \mathrm{M}$ Ubc6, $80 \mathrm{nM}$ Doa10. (F) Quantification $($ mean \pm SD) of four experiments as in (E). The fraction of accessible dye after $30 \mathrm{~min}$ was compared between conditions with and without Doa10. $\mathrm{F}$, normalized fluorescence. (G) Retrotranslocation of Ubc6, measured by a protease protection assay. Ubc6 with an N-terminal SUMO tag (SUMO-Ubc6) and a TEV protease cleavage site between the C-terminus and the fluorescent dye was used. Arrow heads indicate cleavage sites for Ulp1 and TEV protease. SUMO-Ubc6 liposomes with or without Doa10 were incubated with Ulp1. Ulp1-treated liposomes were then incubated with buffer or TEV protease. Indicated reactions contained detergent to solubilize liposomes (det). Aliquots were taken at the indicated times and analyzed by SDS-PAGE and fluorescence scanning. F.c. during incubation with TEV protease: $0.1 \mu \mathrm{M}$ Ubc6, $40 \mathrm{nM}$ Doa10, $10 \mu \mathrm{M}$ TEV protease. (H) Quantification (mean \pm SD) of the fraction of Ubc6 and SUMO-Ubc6 inaccessible to TEV protease, from three experiments as in (G). Band intensities from samples treated with TEV protease were normalized to the corresponding band intensities of samples without TEV protease. 


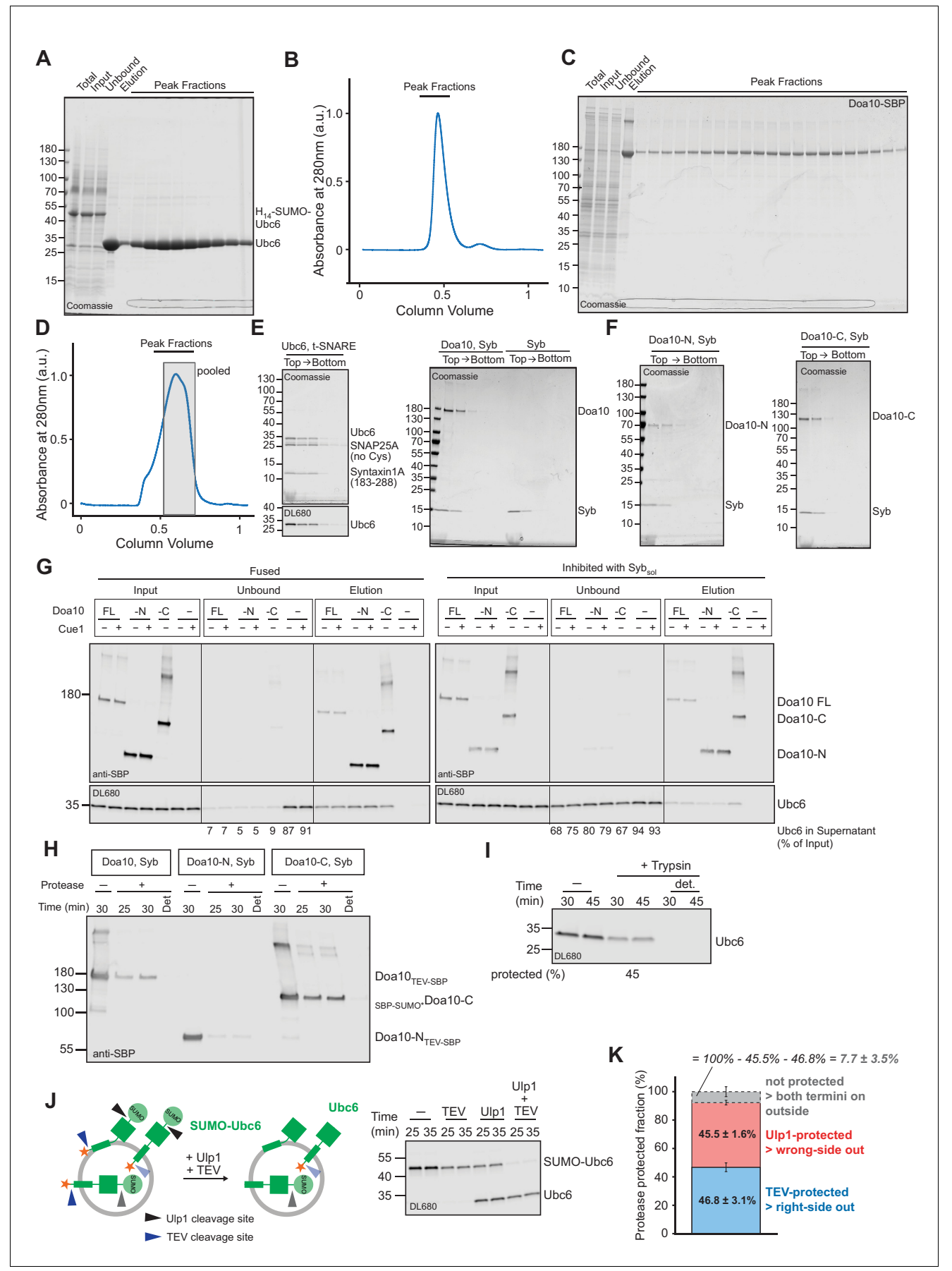

Figure 1-figure supplement 1. Quality control of liposomes. (A) Coomassie Blue stained SDS-PAGE showing purification of Ubc6 from an E. coli membrane fraction by immobilized nickel ion chromatography and gel filtration. The protein was expressed with an $\mathrm{N}$-terminal $\mathrm{H}_{14}$-SUMO tag that was Figure 1-figure supplement 1 continued on next page 
Figure 1-figure supplement 1 continued

cleaved with Ulp1 on beads. Samples of the membrane fraction before and after solubilization are denoted as Total and Input, respectively. (B) Chromatogram for gel filtration of Ubc6 on a Superdex 200 column. Peak fractions were analyzed by SDS PAGE, as shown in (A). (C) Coomassie Blue stained SDS-PAGE showing purification of SBP-tagged Doa10 from S. cerevisiae by streptavidin-affinity chromatography and gel filtration (Superose six column). SBP, streptavidin binding peptide. (D) Chromatogram for gel filtration of Doa10 purification shown in (C). Shaded area indicates fractions that were pooled. (E) Liposomes with co-reconstituted t-SNARE and Ubc6, fluorescently labeled with DyLight680 (DL680, left), and liposomes with coreconstituted Doa10 and synaptobrevin (Syb, right) were subjected to a Nycodenz step gradient. After ultracentrifugation, the gradient was fractionated and analyzed by SDS-PAGE and Coomassie Blue staining (top) and fluorescence scanning (bottom). (F) Liposomes containing Syb and truncated versions of Doa10 used in Figure 4, Figure $\mathbf{5}$ and Figure 5-figure supplement 2 were analyzed as in (E). (G) Analysis of SNARE-dependent interaction of liposomes. Liposomes containing Syb and different SBP-tagged Doa10 versions were mixed with Ubcb (fluorescently labeled)/t-SNARE

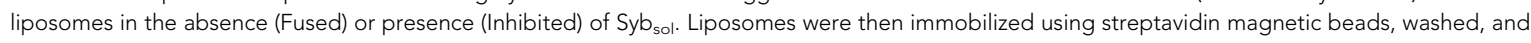
eluted with biotin. Input, unbound, and elution fractions were analyzed by SDS-PAGE followed by Western blotting using an anti-SBP antibody to detect Doa10 variants (top), or fluorescence scanning to detect Ubc6 (bottom). Numbers below unbound fractions indicate the depletion of Ubc6 relative to input fractions. Doa10-N, and -C, refer to variants of Doa10 used in Figure 4, Figure 5 and Figure 5-figure supplement 2. Doa10-FL, full length Doa10. (H) Liposomes containing different Doa10 versions were subjected to either TEV protease (Doa10 TEV-SBP and Doa10-N TEV-SBP) or a mutant version of Ulp1 (sBP-SUMO*Doa10-C Liu et al., 2008) for the indicated times in the absence or presence of Triton-X100 (det), and analyzed by SDS-PAGE and Western blotting using an anti-SBP antibody. Protease cleavage sites are only accessible in correctly oriented Doa10. (I) Liposomes containing fluorescently labeled Ubcb and t-SNARE were subjected to a tryptic digest for the indicated times in the absence or presence of Triton-X100 (det). Samples were analyzed by SDS-PAGE and fluorescence scanning. The percentage of protected protein is indicated at the bottom. (J) Evaluation of Ubc6 reconstitution by protease protection. Liposomes containing Ubc6 with an N-terminal SUMO-moiety, and a TEV cleavage site between the TM anchor and the fluorescent dye (SUMO-Ubc6), were incubated with either TEV protease, Ulp1, or both proteases for the indicated times. Samples were analyzed by SDS-PAGE and fluorescence scanning. Ulp1 cleavage results in a size-shift in SDS-PAGE, TEV cleavage removes the dye, indicating correctly and wrongly oriented protein, respectively. Accessibility to both proteases indicates improper reconstitution. (K) Quantification (mean \pm SD) of the fraction of non-cleaved Ubc6 from three experiments as in $(\mathrm{J}) .46 .8 \pm 3.1 \%$ of SUMO-Ubc6 was protected from TEV protease and is thus right-side out oriented. $45.5 \pm 1.6 \%$ of SUMO-Ubc6 was protected from Ulp1 protease and is thus wrong-side out oriented. This indicates that a fraction of SUMO-Ubc6 ( $7.7 \pm 3.5 \%)$ was not protected from either protease. In line with this, $26.9 \pm 2.5 \%$ Ulp1-cleaved Ubc6 was also cleaved by TEV protease, corresponding to $14.7 \pm 1.4 \%$ of total Ubc6. Thus, we estimate that about $10 \%$ of Ubc6 is not properly reconstituted. 


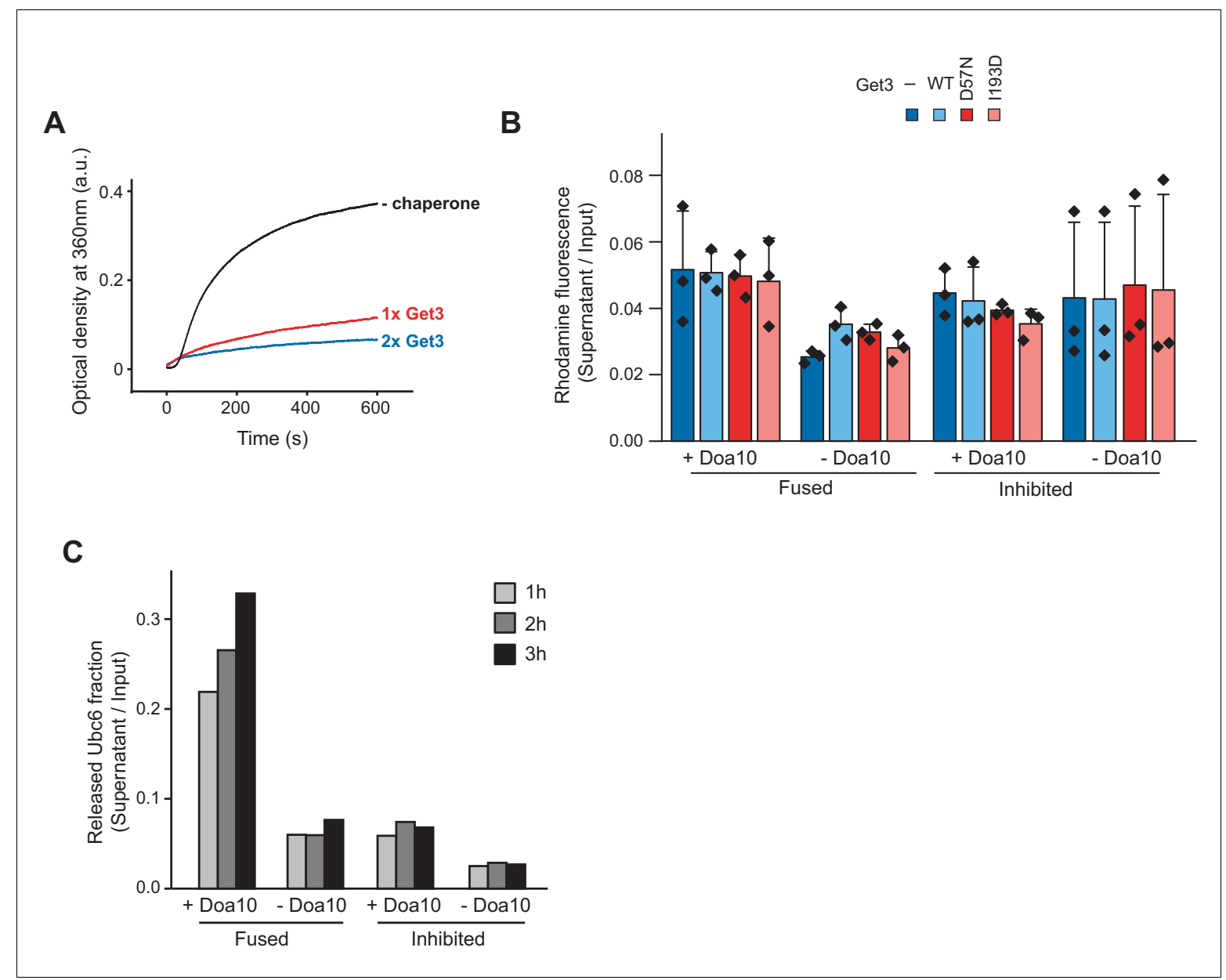

Figure 1-figure supplement 2. Retrotranslocation in the presence of Get3. (A) Aggregation of Ubc6 in detergent-free buffer. Ubc6 in $0.03 \%$ ( $w / v$ ) dodecyl maltoside was diluted 25-fold (f.c. $1.8 \mu \mathrm{M}$ ) into buffer containing no, $1.8 \mu \mathrm{M}$ or $3.6 \mu \mathrm{M}$ Get3. Optical density was measured at $360 \mathrm{~nm}$. (B) Immobilization efficiency of liposomes used in Figure $1 C, D$ was quantified (mean \pm SD) via co-reconstituted fluorescent Rhodamine-phosphatidyl ethanolamine (PE). $n=3$ independent experiments. (C) Release of Ubc6 in the presence of Get3, as in Figure 1C, $D$, but for shorter incubation times. 
A

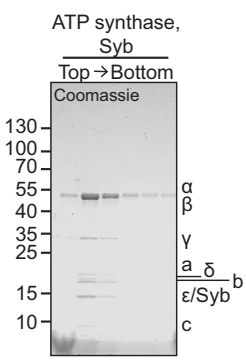

C

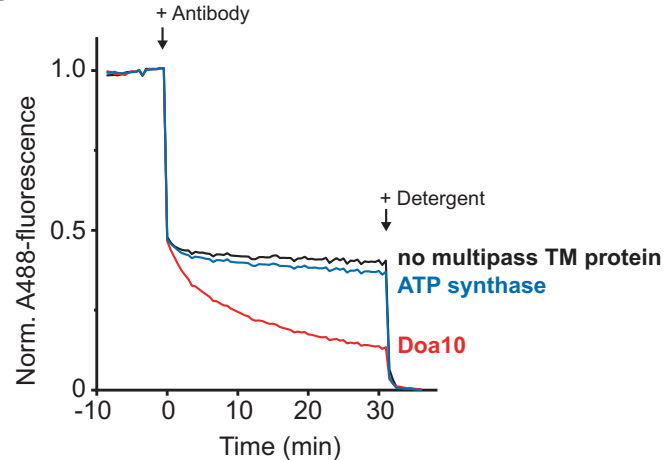

B

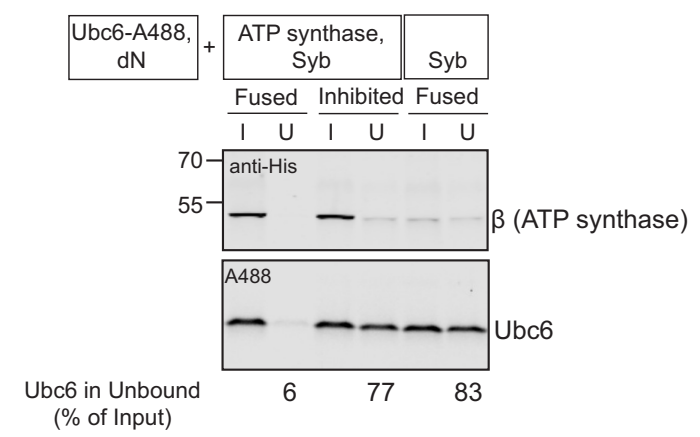

D

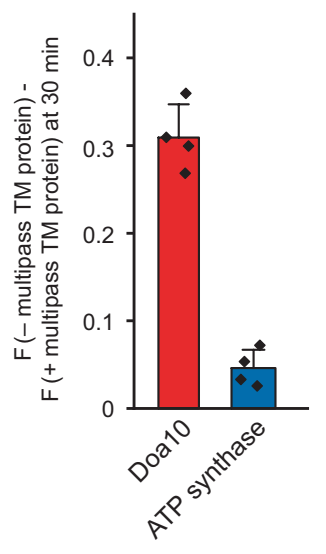

Figure 1-figure supplement 3. Co-reconstitution with ATP synthase. (A) Liposomes with co-reconstituted ATP synthase and synaptobrevin (Syb) were subjected to a Nycodenz step gradient. After ultracentrifugation, the gradient was fractionated and analyzed by SDS-PAGE and Coomassie Blue staining. Bacillus PS3 ATP synthase is a multiprotein complex with a subunit composition of $\alpha_{3} \beta_{3} \gamma \delta \varepsilon \mathrm{ab}_{2} \mathrm{c}_{20}$ (Guo et alo, 2019). (B) Analysis of coreconstitution efficiency of ATP synthase and Ubc6 upon SNARE-mediated fusion. Liposomes containing Syb and ATP synthase (tagged with His 10 -tag at $\beta$-subunit) were mixed with Ubc6 (fluorescently labeled)/t-SNARE liposomes in the absence (Fused) or presence (Inhibited) of Syb $b_{\text {sol. }}$ Liposomes were then immobilized via the His-tag of ATP synthase with magnetic beads. Input (I) and unbound (U) fractions were analyzed by SDS-PAGE followed by Western blotting using an anti-His antibody to detect ATP synthase (top), or fluorescence scanning to detect Ubcb (bottom). Numbers below unbound fractions indicate the depletion of Ubc6 relative to input fractions. (C) Retrotranslocation of Ubcb in the presence of Doa10 or ATP synthase, measured as quenching of a C-terminal AlexaFluor488 (A488) label by an antibody. Where indicated, liposomes lacked Doa10 or ATP synthase (no multipass TM protein). Arrows indicate addition of the quenching antibody or of solubilizing amounts of detergent (Triton X-100). F.c.: 0.2 $\mu \mathrm{M}$ Ubc6, $80 \mathrm{nM}$ Doa10/80 nM ATP synthase. (D) Quantification (mean \pm SD) of four experiments as in (C). The fraction of accessible dye after 30 min was compared between conditions with and without a multipass TM protein. F, normalized fluorescence. 
A
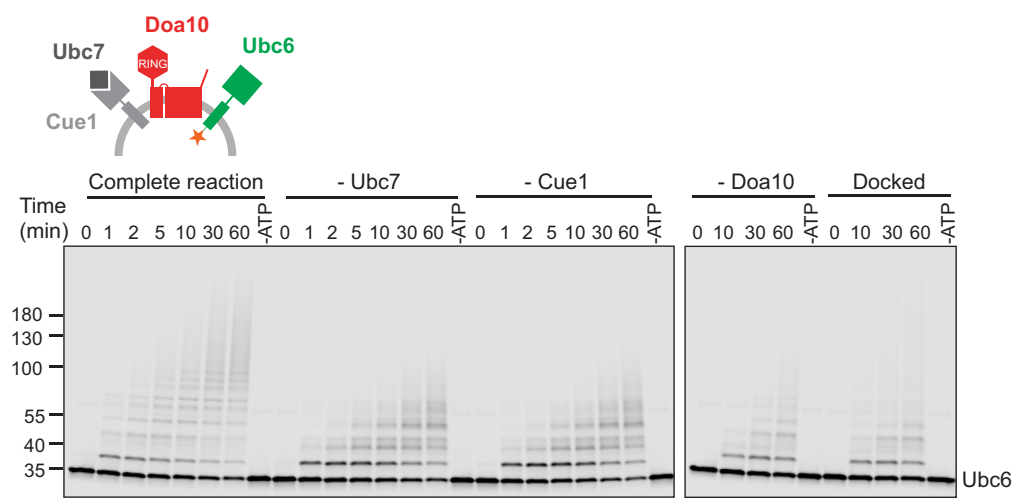

B

\section{C}
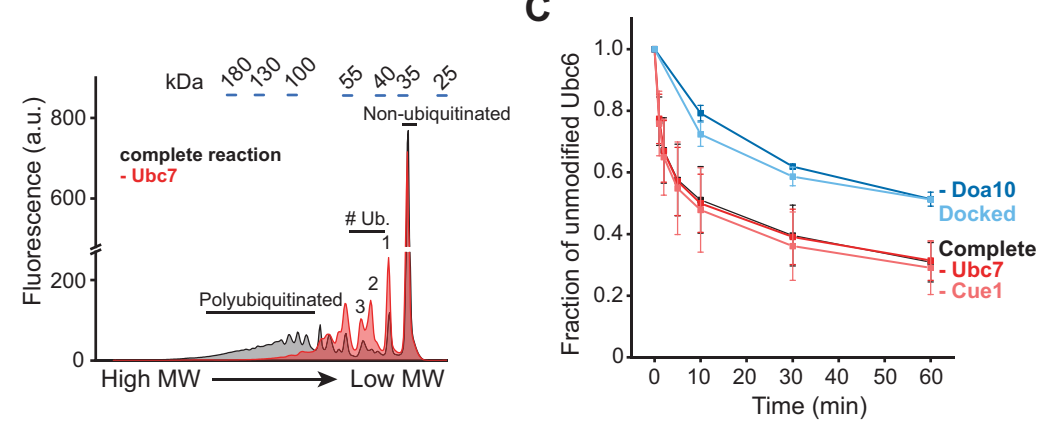

D
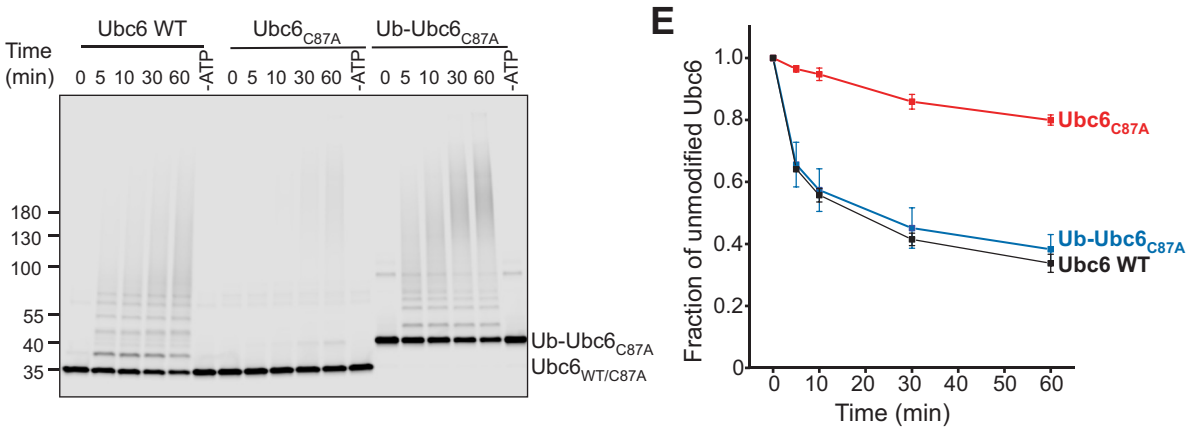

$\mathbf{F}$

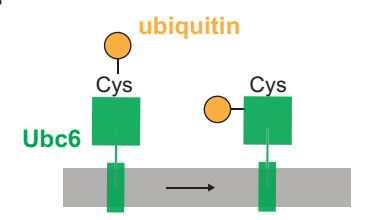

Autoubiquitination

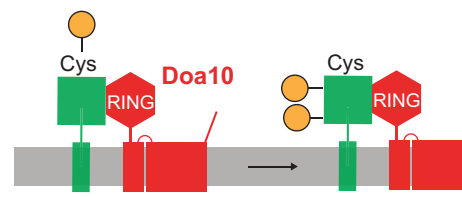

Monoubiquitination

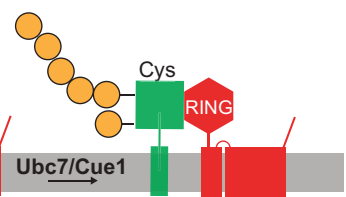

Polyubiquitination

Figure 2. Ubiquitination of Ubc6. (A) Time course of ubiquitination of Ubc6. Final concentrations in the complete reaction: $40 \mathrm{nM}$ Doa10, $10 \mathrm{nM}$ Cue1, $1 \mu \mathrm{M}$ Ubc7, $100 \mathrm{nM}$ Ubc6, $100 \mathrm{nM} \mathrm{E1}, 120 \mu \mathrm{M}$ ubiquitin, and $2.5 \mathrm{mM}$ ATP. Where indicated, individual components were omitted or co-reconstitution Figure 2 continued on next page 
eLife

Biochemistry and Chemical Biology | Cell Biology

Figure 2 continued

was inhibited by using Syb $\Delta 84$ (Docked). For each reaction, a $60 \mathrm{~min}$ sample in the absence of ATP is shown. Samples were analyzed by SDS-PAGE and fluorescence scanning. (B) Analysis of ubiquitin-chain length on Ubc6 from an experiment as in (A). Line-scans were performed on fluorescence images for the complete reaction and in the absence of $\mathrm{Ubc7}$ at $\mathrm{t}=30 \mathrm{~min}$. Approximate molecular weights are indicated on top. \# ub. denotes number of ubiquitin moieties attached. (C) Quantification (mean \pm SD) of the fraction of unmodified Ubc6 from three experiments as in (A). (D) Time course of ubiquitination of Ub-Ubc6 ${ }_{\mathrm{C} 87 \mathrm{~A}}$ compared to $\mathrm{Ubc6}$ WT and $\mathrm{Ubcb}_{\mathrm{C} 87 \mathrm{~A}}$ in the presence of Doa10, Cue1, and Ubc7. Concentrations and analysis as in (A). (E) Quantification (mean \pm SD) of the fraction of unmodified Ubcb variants from three experiments as in (D). (F) Model for ubiquitination of Ubc6. Ubc6 autoubiquitination activity results in transfer of ubiquitin from its active site cysteine to a non-cysteine residue (Weber et al., 2016). In the presence of Doa10, this activity is enhanced and Ubc6 is multi-monoubiquitinated. Ubc7/Cue1 are then required to form polyubiquitin chains on monoubiquitinated Ubc6. 
A

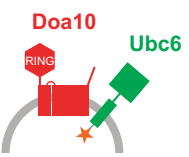

Ubiquitin WT Ubiquitin K0

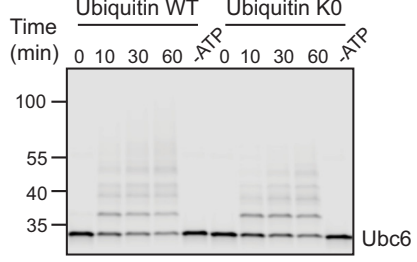

C

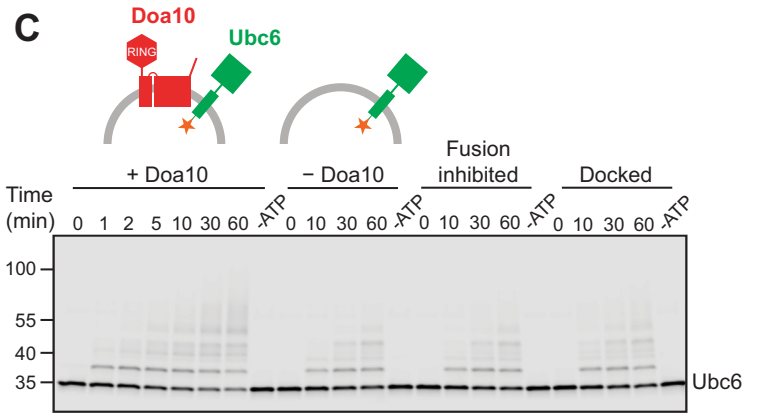

B

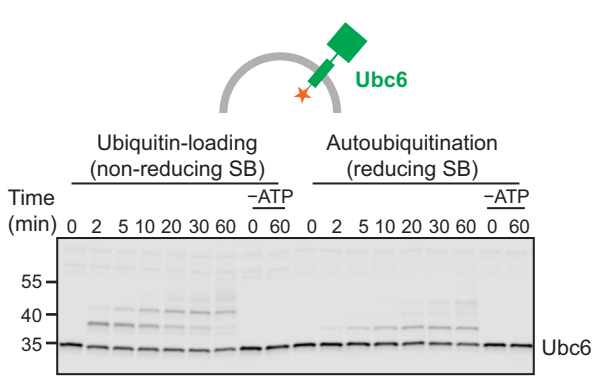

D

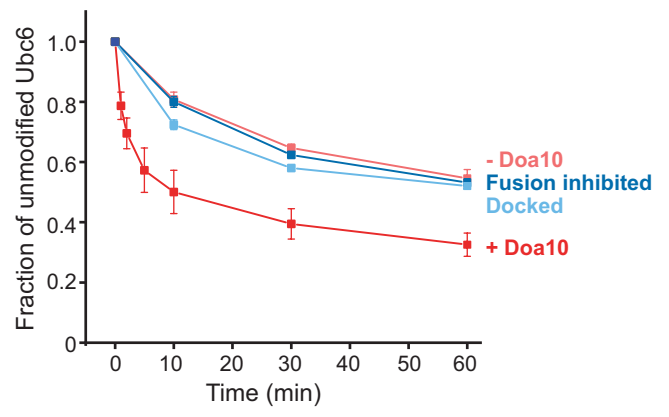

E Ub-Ubc6 ${ }_{C 77 A}$,

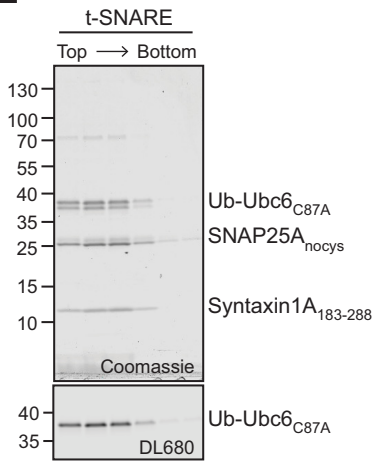

Figure 2-figure supplement 1. E3-independent and -dependent ubiquitination of Ubc6. (A) Time course of ubiquitination of Ubc6 in the absence of Ubc7/Cue1 using either WT ubiquitin or a ubiquitin mutant with all Lys mutated to Arg (K0). $40 \mathrm{nM}$ Doa10 and $0.1 \mu \mathrm{M}$ Ubc6 in liposomes were incubated with $0.1 \mu \mathrm{M} \mathrm{E1}, 120 \mu \mathrm{M}$ ubiquitin, and $2.5 \mathrm{mM}$ ATP. A 60 min sample in the absence of ATP is shown for each reaction. Samples were analyzed by SDS-PAGE and fluorescence scanning. (B) Doa10-independent autoubiquitination of Ubc6. Liposomes containing fluorescently labeled Ubc6 were incubated with E1, ubiquitin, and ATP. Samples at indicated time points were analyzed by SDS-PAGE under non-reducing (left) and reducing conditions (right) and fluorescence scanning. Reactions lacking ATP are denoted as -ATP. Final concentrations: $0.1 \mu \mathrm{M}$ Ubc6, $0.1 \mu \mathrm{M}$ Uba1, $120 \mu \mathrm{M}$ ubiquitin, $2.5 \mathrm{mM}$ ATP. SB, SDS sample buffer. (C) Time course of ubiquitination of Ubcb in the absence of Ubc7/Cue1. Ubc6 liposomes containing or lacking Doa10 were used. Where indicated, co-reconstitution was inhibited using either a soluble Syb fragment (Syb $b_{\text {sol }}$ ) or a Syb mutant (Syb $\Delta 84)$. Concentrations and analysis as in (A). (D) Quantification (mean \pm SD) of the fraction of unmodified Ubc6 from three experiments as in (C). (E) Ub-Ubc6 $6_{C 87 A} / t-S N A R E$ liposomes were subjected to a Nycodenz step gradient. After ultracentrifugation, the gradient was fractionated and analyzed by SDS-PAGE and Coomassie Blue staining (top) and fluorescence scanning (DL680, bottom). 

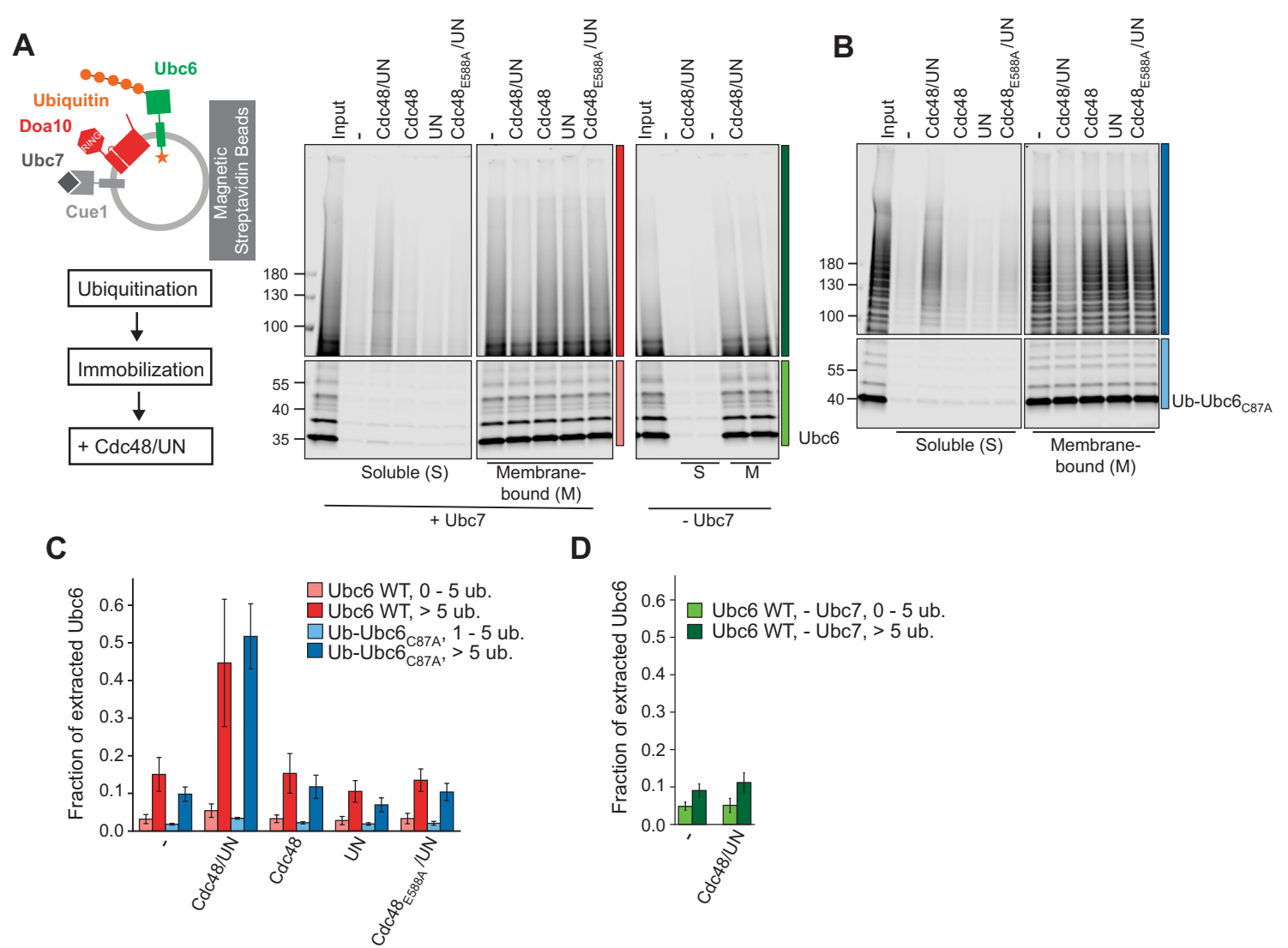

D

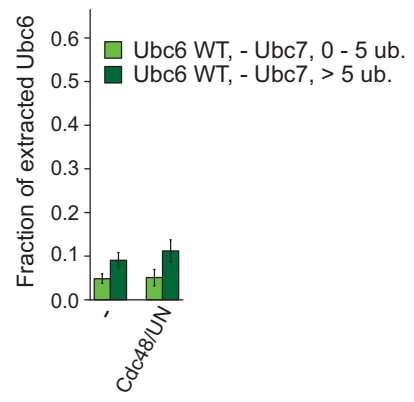

Figure 3. Cdc48-mediated Membrane Extraction of Ubc6. (A) Extraction of Ubc6 by Cdc48 and Ufd1/Npl4 (UN). After ubiquitination, liposomes were immobilized (Figure 3-figure supplement 1,A to D). One bead equivalent was removed, and bound protein was eluted with SDS sample buffer (Input). Beads were then incubated with the indicated components. Soluble (S) and membrane-bound (M) material were analyzed by SDS-PAGE and fluorescence scanning. Colored bars indicate categorization of ubiquitin chain length as used for quantification in (C) and (D). For better visibility, bottom and top gel parts are scaled differently. See Figure 3-figure supplement 1E for uncut image. Final concentrations: $50 \mathrm{nM}$ Ubc6, $20 \mathrm{nM}$ Doa10, $0.1 \mu \mathrm{M}$ Cdc48 hexamer, $0.1 \mu \mathrm{M}$ Ufd1 and Npl4. (B) As in (A), but with Ub-Ubc6c87A instead of Ubc6. See Figure 3-figure supplement 1F for uncut image. (C) Quantification (mean \pm SD) of three experiments as in (A) and (B). Ubiquitinated species were categorized according to ubiquitin chain length, as indicated in (A) and (B). The signal in the soluble fraction was normalized to that in the input. (D) Quantification (mean \pm SD) of three experiments as in (A), when ubiquitination was performed in the absence of Ubc7. 
A

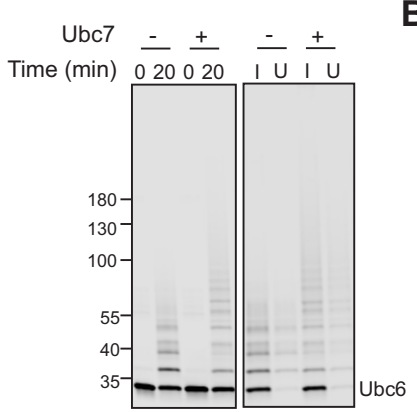

D

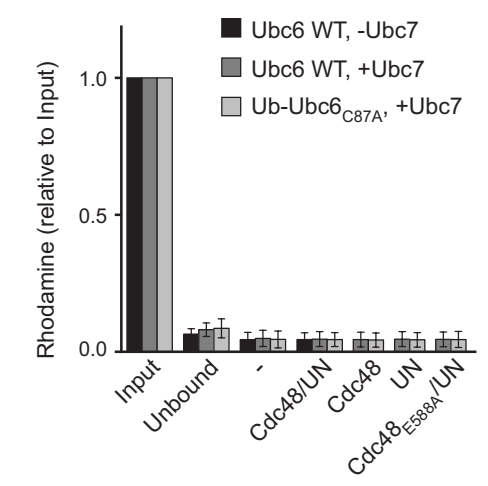

$\mathbf{F}$

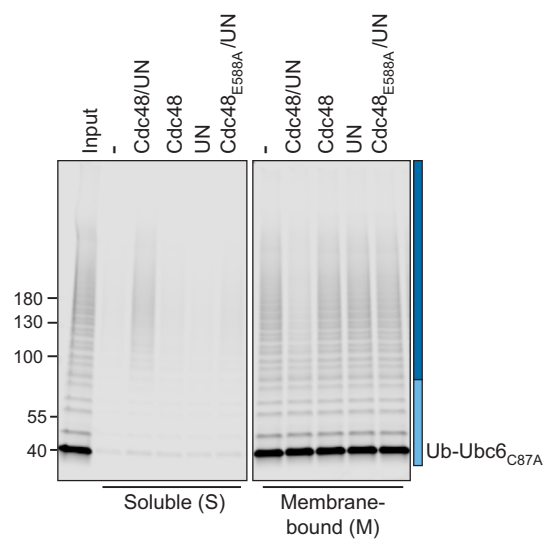

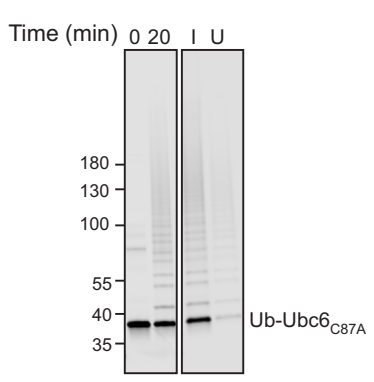

C
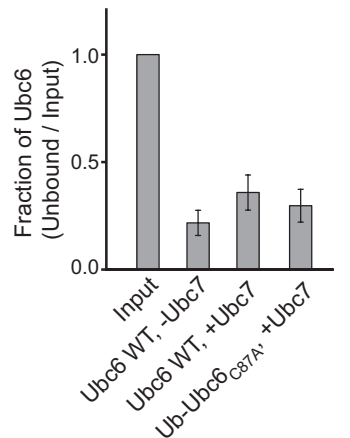

E

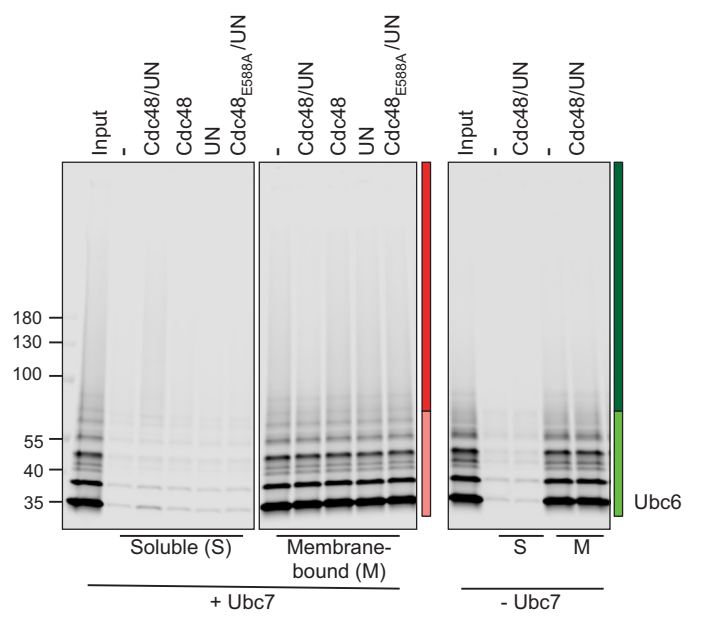

G

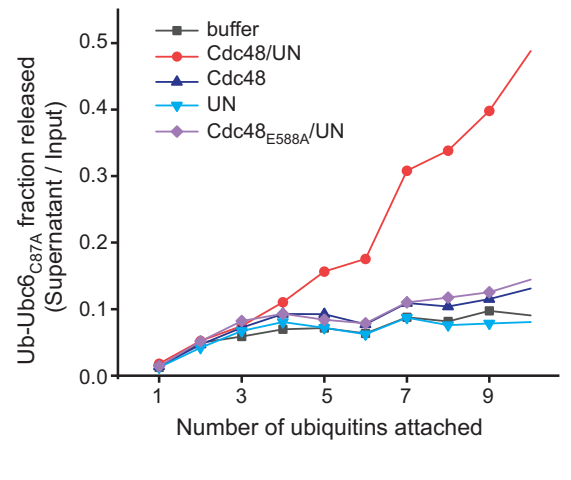

Figure 3-figure supplement 1. Cdc48-mediated extraction. (A) Samples from ubiquitination and immobilization under conditions described in Figure 3A. Liposomes containing Ubc6, Doa10 and Cue1 were incubated with E1, ubiquitin, and ATP, with or without Ubc7. After 20 min, the Figure 3-figure supplement 1 continued on next page 
eLife

Biochemistry and Chemical Biology | Cell Biology

Figure 3-figure supplement 1 continued

ubiquitination reaction was stopped by adding EDTA. Liposomes were then immobilized to streptavidin magnetic beads. Input (I) and unbound (U) fractions were analyzed by SDS-PAGE and fluorescence scanning. (B) Samples from ubiquitination and immobilization under conditions described in Figure 3B. As in (A), but with Ub-Ubcb $c 87 \mathrm{~A}$ instead of $\mathrm{Ubc6}$ and ubiquitination in presence of Ubc7. (C) Quantification (mean \pm SD) of protein immobilization efficiency from three experiments as in (A) and (B). (D) Quantification (mean \pm SD) of the efficiency of liposome immobilization in experiments described in Figure 3A,B, measuring the fluorescence of Rhodamine-PE that was co-reconstituted into liposomes. Rhodamine content of Input and Unbound fraction from immobilization reaction (as in (A) and (B)) as well as of the soluble fraction after extraction (as in Figure $3 \mathbf{A}, \mathbf{B}$ ) was determined. $n=3$ independent experiments. (E) Uncut image with uniform scaling of Figure 3A. (F) Uncut image with uniform scaling of Figure 3B. (G) Comparison of extraction efficiency of $\mathrm{Ub}-\mathrm{Ubcb}_{\mathrm{C} 87 \mathrm{~A}}$ modified with ubiquitin chains of different length as shown in Figure $3 B$. 


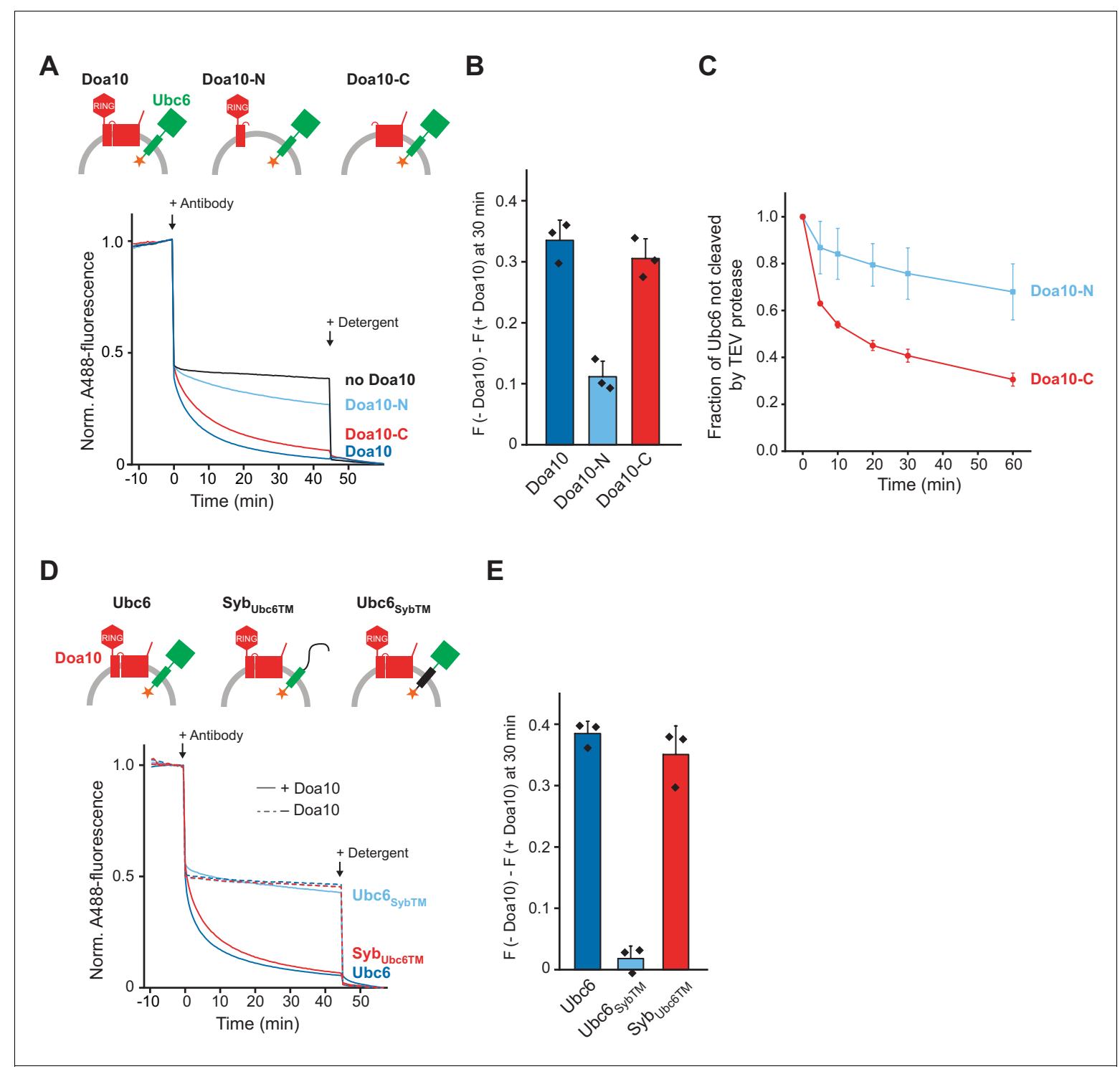

Figure 4. Structural Determinants for Retrotranslocation. (A) Retrotranslocation of Ubc6 by Doa10 variants, as measured by accessibility of a fluorescence quenching antibody to a C-terminal A488 dye on Ubc6, as described in Figure 1E. Ubc6 liposomes containing the indicated Doa10 variants were used. Doa10-N, residues 1-468; Doa10-C, residues 434-1319. Arrows indicate addition of antibody or detergent. Final concentrations (f. c.): $0.2 \mu \mathrm{M} \mathrm{Ubc6,} 80 \mathrm{nM}$ Doa10 variants. (B) Quantification (mean $\pm \mathrm{SD}$ ) of three experiments as in (A). The fraction of accessible dye after 30 min was compared between conditions with the indicated Doa10 variant and without Doa10. F, normalized fluorescence. (C) Retrotranslocation of Ubc6 by Doa10 variants, as measured by accessibility of TEV protease to the C-terminus of Ubc6, as described in Figure 1G. SUMO-Ubc6 liposomes with either Doa10-N or Doa10-C were treated with Ulp1 to identify right-side out oriented Ubc6. TEV protease was added and samples at different time points were analyzed by SDS-PAGE and fluorescence scanning. Quantification as in Figure 1H, but only for Ulp1-cleaved Ubc6. F.c. during incubation with TEV protease: $0.1 \mu \mathrm{M}$ Ubc6, $40 \mathrm{nM}$ Doa10 variants, $10 \mu \mathrm{M}$ TEV protease. (D) Retrotranslocation of Ubc6 variants measured as in (A). A488-labeled Ubc6, Ubc6 SуьтM, or SybUbс6тM were directly co-reconstituted with Doa10 because Sybubc6TM was incompatible with SNARE-mediated co-reconstitution. Liposomes containing Doa10 were affinity-purified for this experiment (Figure 4-figure supplement 1A,B). (E) Quantification (mean \pm SD) of three experiments as in (D). The fraction of accessible dye after 30 min was compared between conditions with and without Doa10. 
A

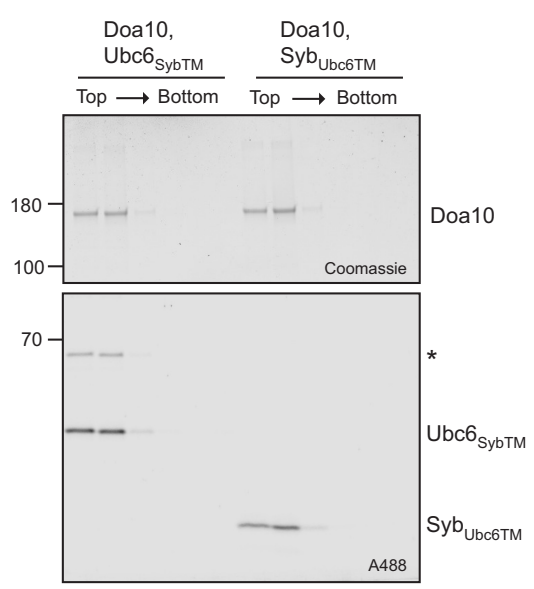

C

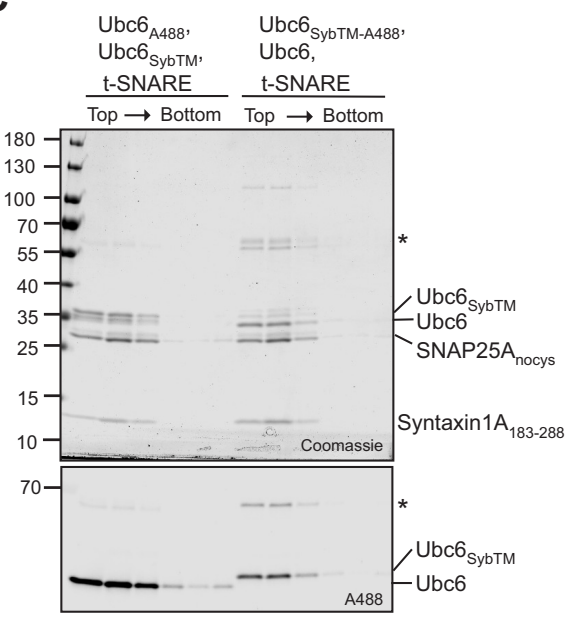

B

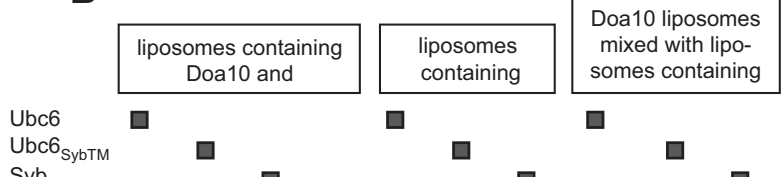

$\mathrm{Syb}_{\text {Ubc6TM }}$

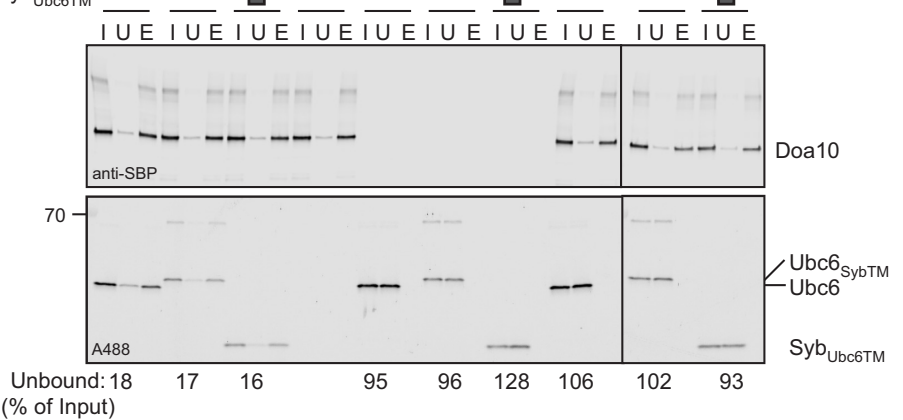

D

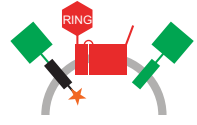

E

Ubc6 $_{\text {Sy }}$

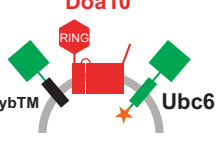

A488 attached to:

$\square$ Ubc6

_+ Doa10

$\square$ Ubc6 $_{\text {SybTM }}$

... - Doa10
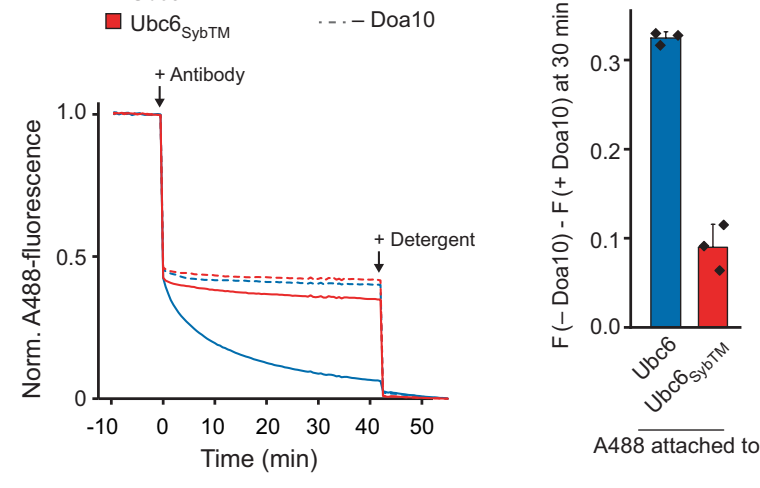

Figure 4-figure supplement 1. Antibody accessibility assay for Ubcb/Syb chimera. (A) Doa10 liposomes directly co-reconstituted with fluorescently labeled Ubc6 SуbтM $_{\text {or Syb Ubс6тM }}$ were subjected to a Nycodenz step gradient. After ultracentrifugation, the gradient was fractionated and analyzed by

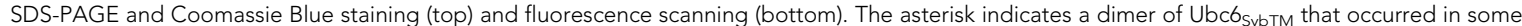
sortase mediated labeling reactions of this construct. (B) Affinity-purification of Doa10-SBP liposomes for experiment in Figure 4D using streptavidin beads. Inputs (I), unbounds (U) and biotin-elutions (E) were analyzed by SDS-PAGE and by either Western Blot using an anti-SBP antibody (Doa10-SBP), or fluorescence scanning (Ubcb/Syb chimera). As an indication of the specificity of the pull-down, we also tested for binding of liposomes lacking Doa10, and for co-purification of Ubc6 liposomes, when Doa10-SBP and Ubc6 were reconstituted in separate liposome sets. Numbers at the bottom indicate the percentage of $\mathrm{Ubcb}$ in the unbound fraction. Note that different intensities are due to different labeling efficiencies. (C) Liposomes containing t-SNARE, Ubc6 SybTM and Ubc6, either fluorescently labeled on Ubc6 SybTM or Ubc6, were subjected to a Nycodenz step gradient. After ultracentrifugation, the gradient was fractionated and analyzed by SDS-PAGE and Coomassie Blue staining (top) and fluorescence scanning (bottom). The asterisk indicates a dimer of Ubc6 Syb $_{\text {Ty }}$ that occurred in some sortase mediated labeling reactions of this construct. (D) Antibody accessibility assay with liposomes containing both, Ubc6 and Ubc6 SybTM, and with or without Doa10. In separate liposomes populations, A488 was either attached to Ubc6 Figure 4-figure supplement 1 continued on next page 
eLife Research article

Biochemistry and Chemical Biology | Cell Biology

Figure 4-figure supplement 1 continued

or Ubc6 SybTM. Arrows indicate addition of antibody or solubilizing detergent. Final concentrations: $0.2 \mu \mathrm{M}$ for Ubc6 and Ubc6 SybTM, $80 \mathrm{nM}$ Doa10. (E) Quantification (mean \pm SD) of three experiments as in (D). The fraction of accessible dye after 30 min was compared between conditions with and without Doa10. F, normalized fluorescence. 
A

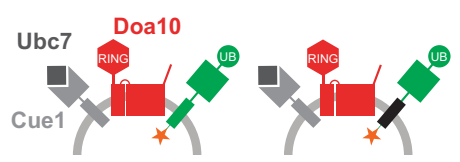

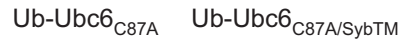

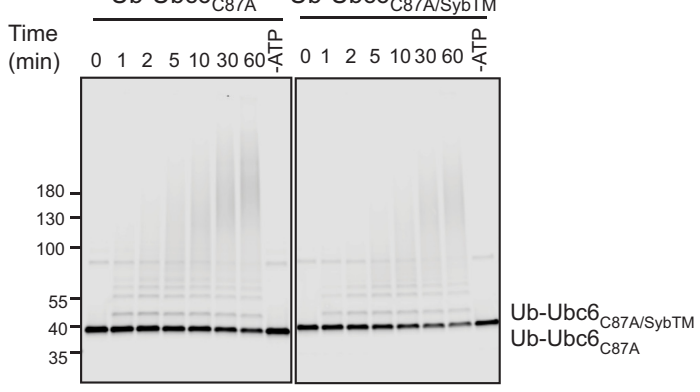

C

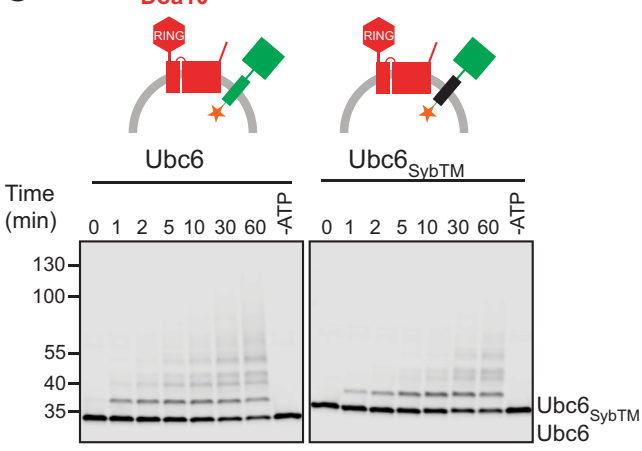

E

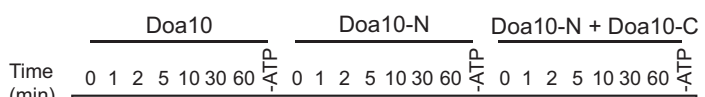

(min) $0125103060<0125103060 \varangle 0125103060<$
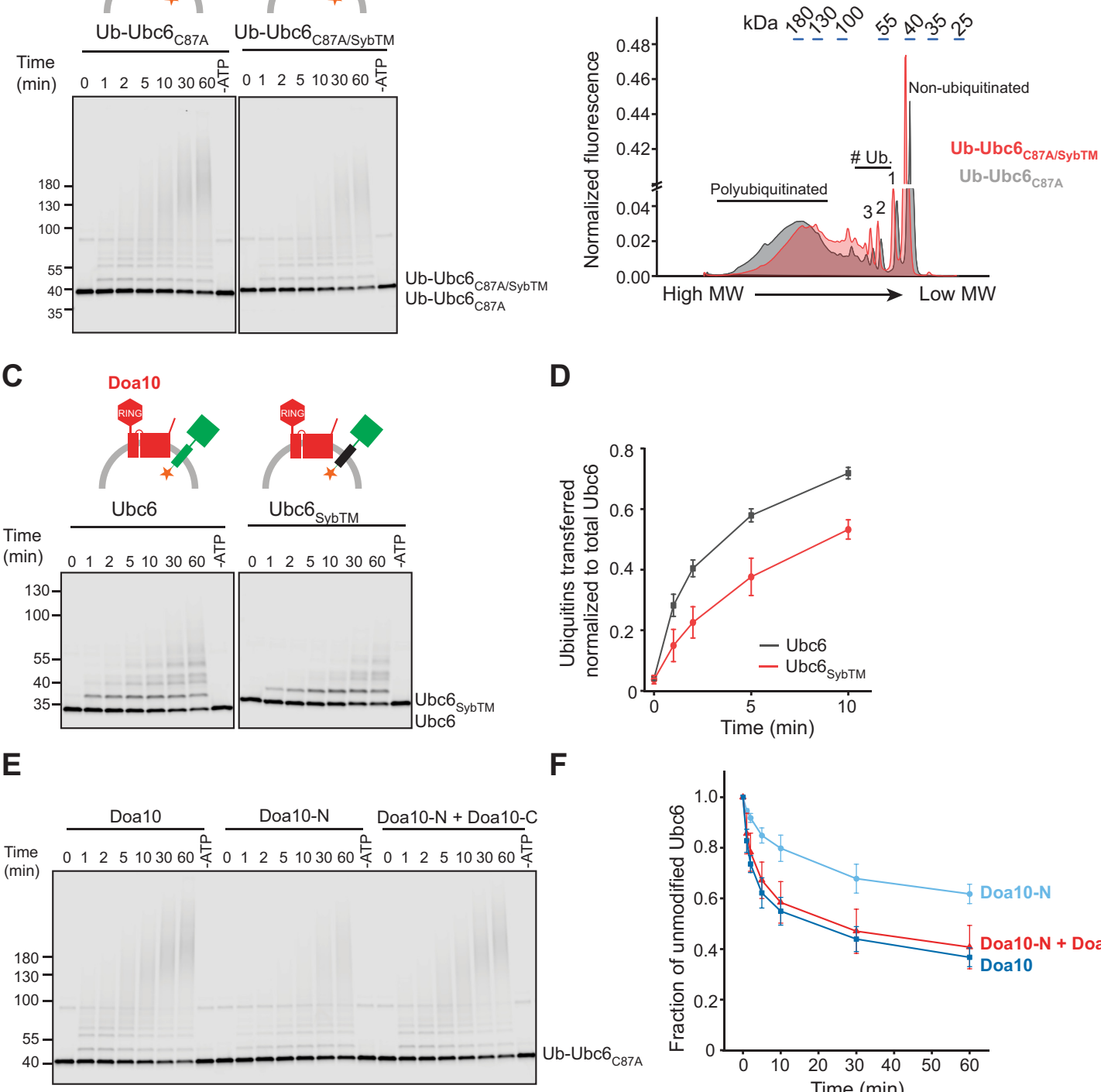

D

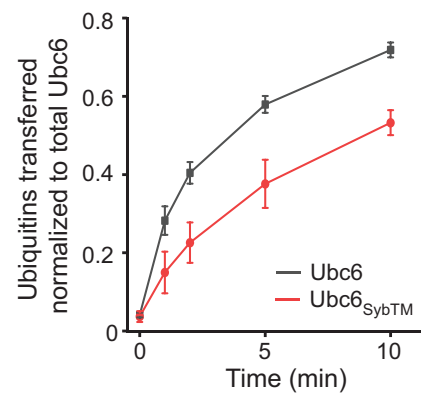

$\mathbf{F}$

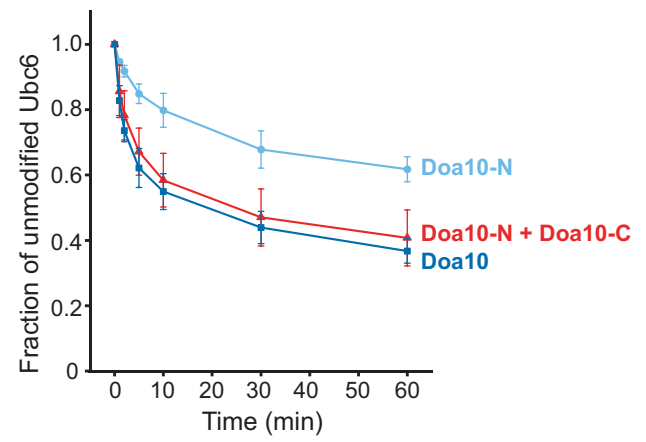

Figure 5. Structural Determinants for Ubiquitination. (A) Time course of ubiquitination of Ub-Ubc6 $\mathrm{C} 87 \mathrm{~A}$ or Ub-Ubc6 $\mathrm{C87A} / \mathrm{Syb}_{\mathrm{B} M}$ by Doa10 in the presence of Cue1/Ubc7. For each reaction, a 60 min sample in the absence of ATP is shown. Samples were analyzed by SDS-PAGE and fluorescence scanning. Final concentrations: 40 nM Doa10, 10 nM Cue1, $1 \mu$ M Ubc7, 100 nM Ubc6 variants, 100 nM E1, $120 \mu$ M ubiquitin, and 2.5 mM ATP. See Figure 5figure supplement $1 \mathrm{~A}$ for quantification of unmodified Ubc6 variants. (B) Comparison of ubiquitin-chain length on Ub-Ubc6 $c 87 \mathrm{~A}$ or Ub-Ubc6 $\mathrm{C} 87 \mathrm{~A} / \mathrm{SybTM}_{\mathrm{B}}$. Figure 5 continued on next page 
eLife

Biochemistry and Chemical Biology | Cell Biology

Figure 5 continued

Line-scans were performed on fluorescence images of two representative gel samples (30 min timepoint) as in (A). Approximate molecular weights are indicated on top. \# Ub., number of ubiquitin moieties attached. (C) Time-course of Ubc6 WT or Ubc6sybTM ubiquitination in the absence of Ubc7/Cue1. Analysis and concentrations as in (A). See Figure 5-figure supplement 1C for quantification of unmodified Ubc6 variants. (D) Quantification

(mean $\pm \mathrm{SD}$ ) of total ubiquitin-transfer to Ubc6 or Ubc6 SybTM from three experiments as in (C). Intensities of Ubcb variants with one to four ubiquitin moieties attached were determined as described in Figure 5-figure supplement 1D, summed up for each time point and normalized to total Ubc6 in the reaction. (E) Time course of ubiquitination of Ub-Ubc6 $\mathrm{C}_{87 \mathrm{~A}}$ by Doa10 variants in the presence of Cue1/Ubc7. Liposomes contained Ub-Ubcb $\mathrm{C87A}$ and either full-length Doa10, only Doa10-N, or both Doa10-N and -C. Analysis and concentrations as in (A). (F) Quantification (mean \pm SD) of unmodified Ub-Ubc6 ${ }_{\text {C87A }}$ from three experiments as in (E). 
A

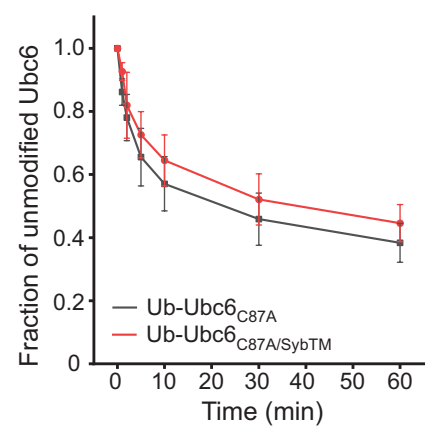

C

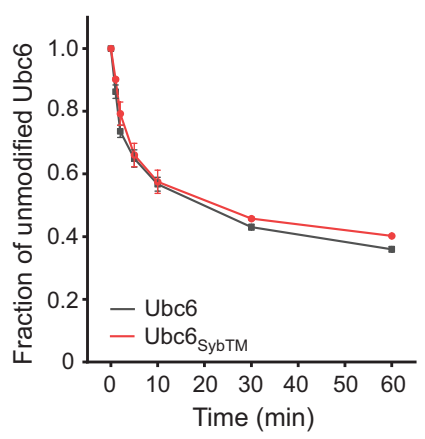

E

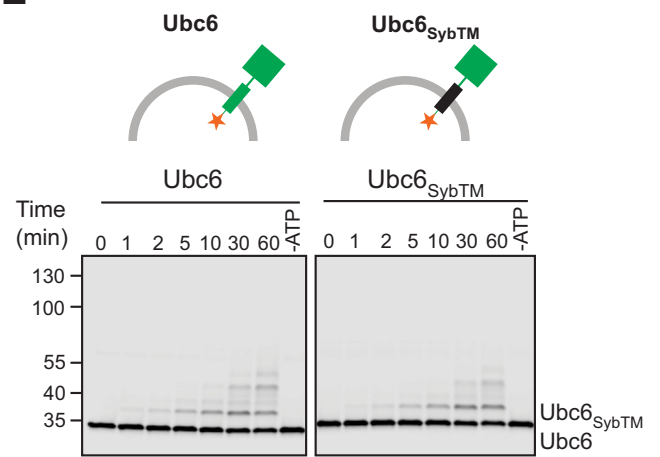

B Ubc6 SурTM,,$\quad$ Ub-Ubc6 $6_{\text {C87ASYbTM, }}$

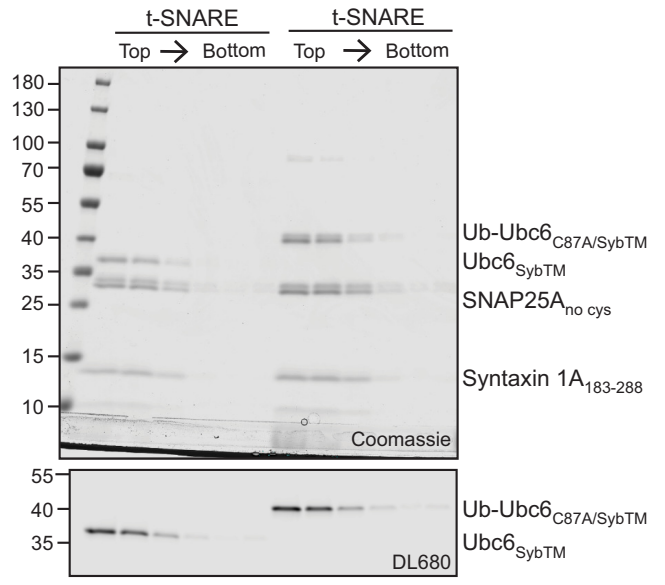

D

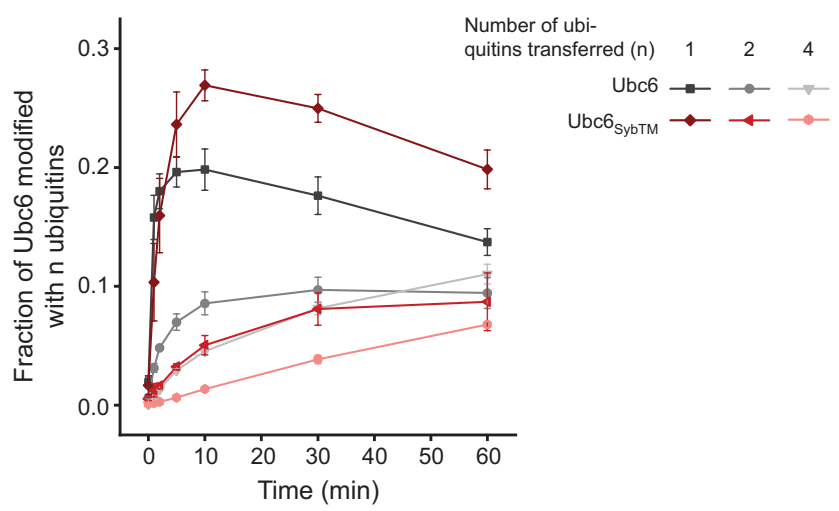

$\mathbf{F}$

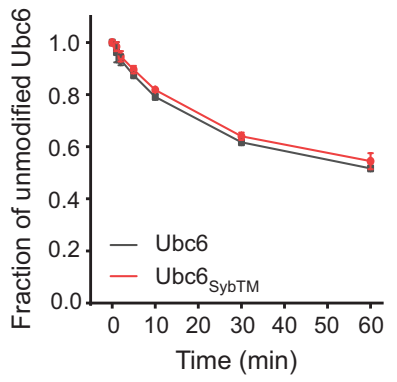

Figure 5-figure supplement 1. Ubiquitination of Ubcb/Syb chimera. (A) Quantification (mean \pm SD) of the fraction of unmodified Ub-Ubc6 $c 87 A$ or Ub-

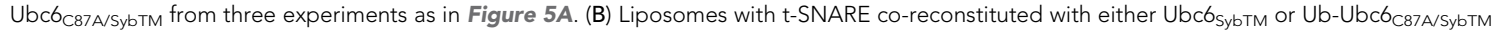
(fluorescently labeled) were subjected to a Nycodenz step gradient. After ultracentrifugation, the gradient was fractionated and analyzed by SDS-PAGE Figure 5-figure supplement 1 continued on next page 
eLife Research article

Biochemistry and Chemical Biology | Cell Biology

Figure 5-figure supplement 1 continued

and Coomassie Blue staining (top) and fluorescence scanning (bottom). (C) Quantification (mean \pm SD) of the fraction of unmodified Ubcb or UbcbsybTM from three experiments as in Figure 5C. (D) Quantification (mean \pm SD) of mono-, di-, and tetra-ubiquitinated Ubcb species relative to total Ubcb from three experiments as in Figure 5C. (E) Time course of E3-independent autoubiquitination of Ubcb and Ubc6sybTM. Liposomes containing the indicated Ubc6 variants (100 nM) were incubated with $100 \mathrm{nM} \mathrm{E1}, 120 \mu \mathrm{M}$ ubiquitin, and $2.5 \mathrm{mM}$ ATP. A 60 min sample in the absence of ATP is shown for each reaction. Samples were analyzed by SDS-PAGE and fluorescence scanning. (F) Quantification (mean \pm SD) of the fraction of unmodified Ubcb or UbcbsybTM from three experiments as in (E). 


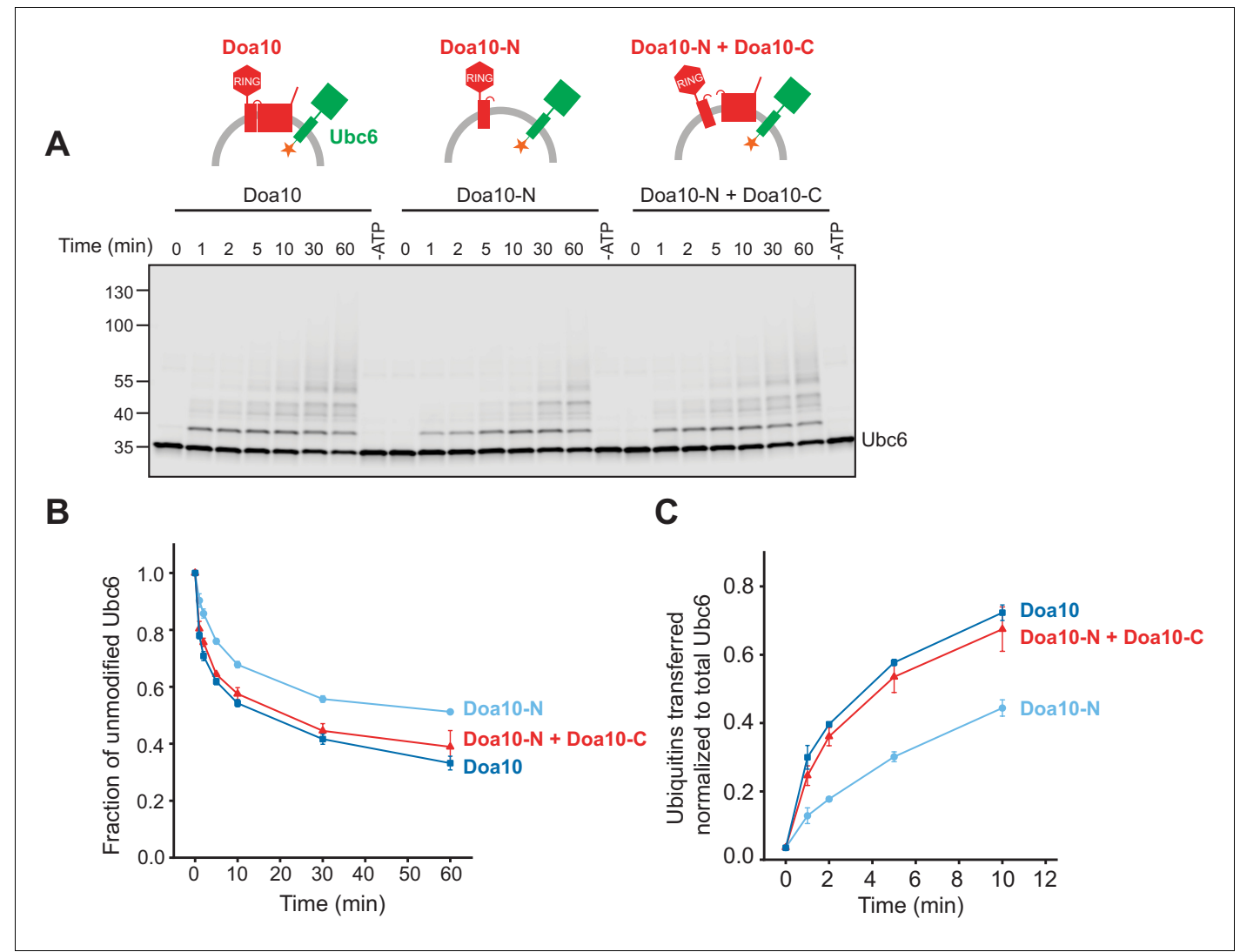

Figure 5-figure supplement 2. Ubc6 ubiquitination by Doa10 variants. (A) Time course of Ubc6 ubiquitination in the presence of different Doa10 variants in the absence of Ubc7/Cue1. Indicated liposomes were incubated with $100 \mathrm{nM} \mathrm{E1,} 120 \mu \mathrm{M}$ ubiquitin, and $2.5 \mathrm{mM}$ ATP (f.c. 100 nM Ubc6, 10 nM Cue1, and 40 nM for Doa10-variants). A 60 min sample in the absence of ATP is shown for each reaction. Samples were analyzed by SDS-PAGE and fluorescence scanning. (B) Quantification (mean \pm SD) of the fraction of unmodified Ubc6 from three experiments as in (A). (C) Quantification $($ mean \pm SD) of total ubiquitin-transfer relative to Ubc6 from three experiments as in (A). Intensities of Ubc6 with one to four ubiquitin moieties attached were summed up for each time point and normalized to total Ubc6 in the reaction, as described in Figure 5D and Figure 5-figure supplement $1 D$. 
A

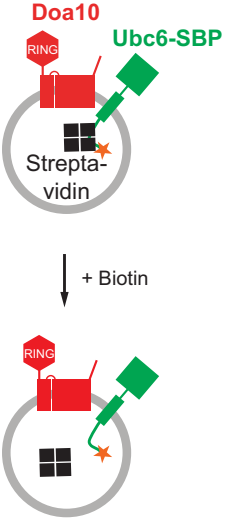

C

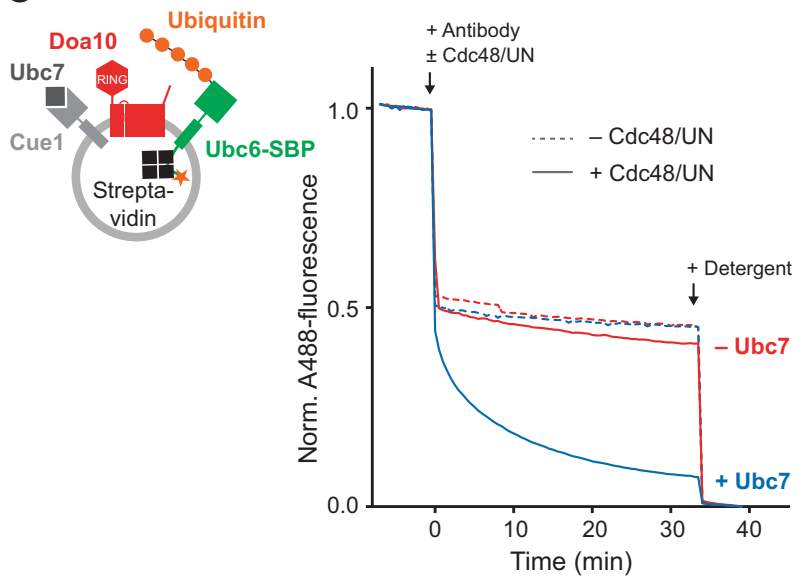

B

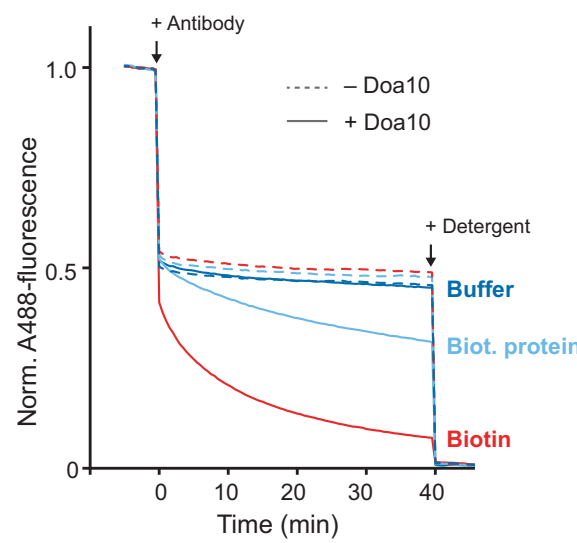

D
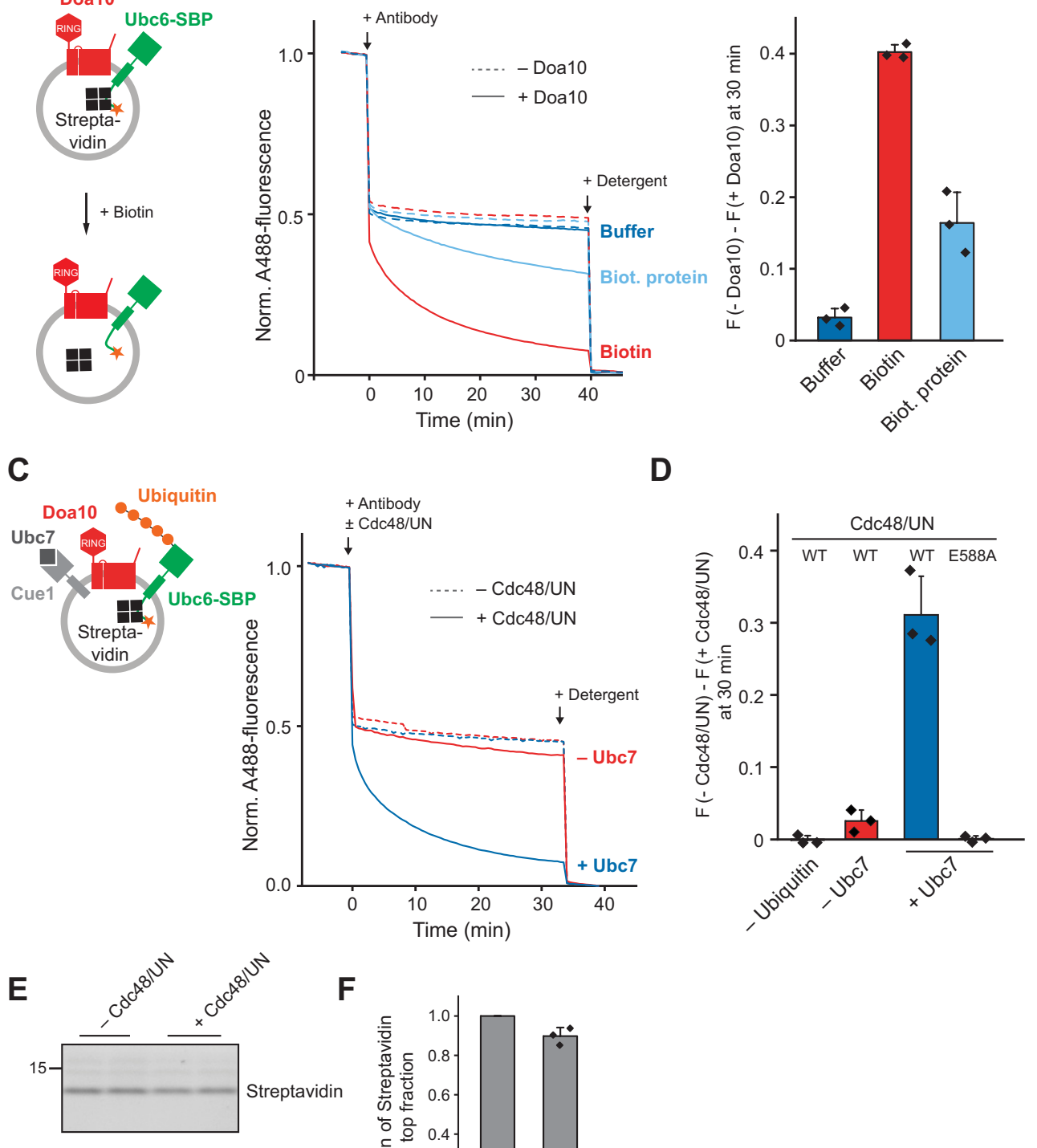

$\mathbf{F}$

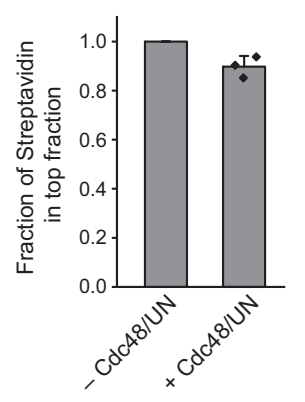

Figure 6. Effect of a Luminal Domain on Retrotranslocation. (A) Retrotranslocation of Ubc6 with a C-terminal streptavidin binding peptide (SBP), reconstituted in complex with streptavidin, was measured by accessibility of a fluorescence quenching antibody to a C-terminal A488 dye on Ubc6, as described Figure 1E. Liposomes were incubated with buffer, biotin or a biotinylated protein prior to addition of the antibody. Final concentrations: 0.2 Figure 6 continued on next page 
eLife

Biochemistry and Chemical Biology | Cell Biology

Figure 6 continued

$\mu \mathrm{M}$ Ubc6, $80 \mathrm{nM}$ Doa10, $0.25 \mu \mathrm{M}$ streptavidin, $1.5 \mu \mathrm{M}$ biotin or biotinylated protein. (B) Quantification (mean \pm SD) of three experiments as in (A). The fraction of accessible dye after 30 min was compared between conditions with and without Doa10. F, normalized fluorescence. (C) Effect of Cdc48 and Ufd1/Npl4 (UN) on retrotranslocation of Ubc6-SBP in complex with streptavidin, measured using the antibody accessibility assay as in (A). Prior to the fluorescence measurement, liposomes were incubated with ubiquitination mix with or without Ubc7. Arrows indicate when antibody, with or without Cdc48/UN, or detergent were added. Final concentrations: $0.17 \mu \mathrm{M}$ Ubc6-SBP, $68 \mathrm{nM}$ Doa10, $0.17 \mu \mathrm{M}$ hexameric Cdc48, Ufd1, and Npl4. See Figure 6-figure supplement $1 \mathrm{~F}$ for gel samples of ubiquitination reaction. (D) Quantification (mean \pm SD) of three experiments as in (C). The fraction of accessible dye after 30 min was compared between conditions with and without Cdc48/UN. In addition, experiments lacking ubiquitin or with the Cdc48 mutant E588A were quantified. (E) Determination of liposome-encapsulated streptavidin after extraction. Samples from experiments as in (D) were taken at $\mathrm{t}=30 \mathrm{~min}$. Biotin was added, and liposomes floated in a Nycodenz gradient. Co-floating streptavidin was detected in SDS-PAGE using stain-free technology. Two replicates are shown for each condition. (F) Quantification (mean \pm SD) of the relative amount of streptavidin co-floating from three experiments as in (E). Each data point represents the mean of two replicates as shown in (E). 
A

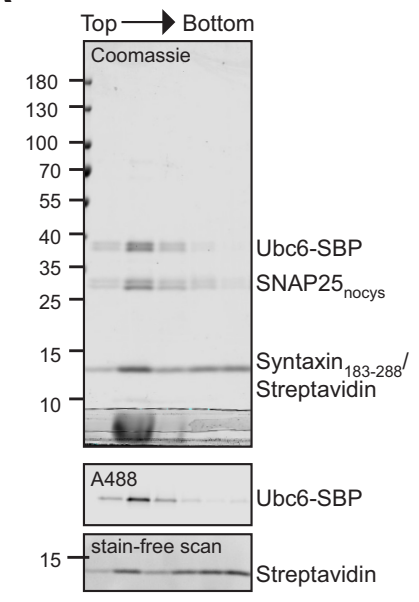

B

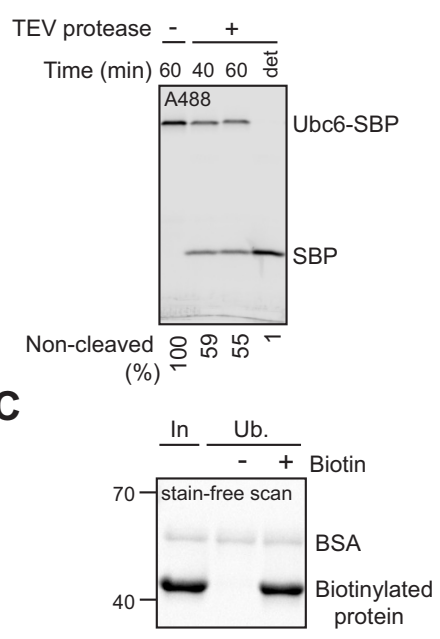

D

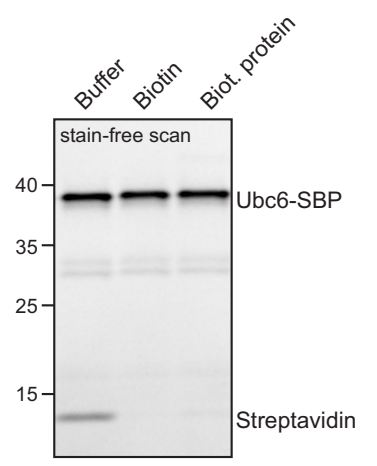

E

\section{F}

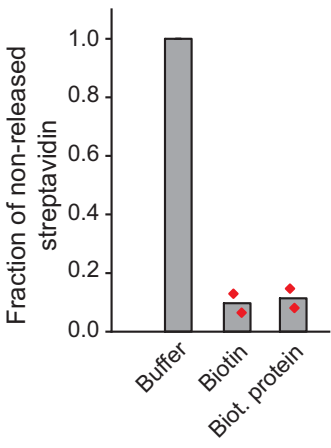

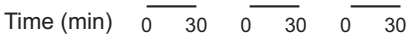

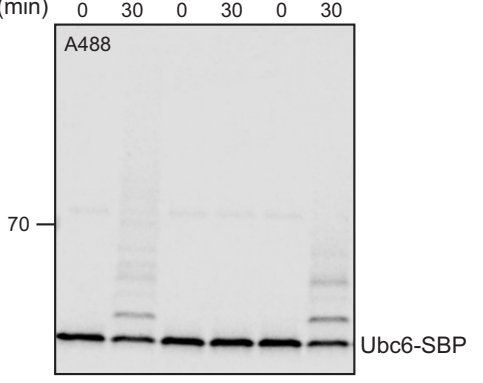

Figure 6-figure supplement 1. Liposomes with Ubc6-SBP and streptavidin. (A) Liposomes containing t-SNARE and fluorescently labeled Ubc6-SBP in complex with streptavidin were subjected to a Nycodenz step gradient. After ultracentrifugation, the gradient was fractionated and analyzed by SDSPAGE and Coomassie Blue staining (top), fluorescence scanning (middle), and using stain-free dye technology (bottom) to distinguish between streptavidin and Syntaxin, which is not visible with this technique as it lacks tryptophan residues. (B) The orientation of Ubc6-SBP in liposomes was assessed by testing for accessibility of TEV-protease to the TEV-cleavage site between the Ubc6 TM anchor and the SBP-tag. Liposomes were incubated with buffer or TEV protease with or without TX-100 (det) for the indicated times. Samples were analyzed by SDS-PAGE and fluorescence scanning. Numbers at the bottom indicate the fraction of protein right-side out oriented. (C) Streptavidin affinity pulldown of biotinylated protein in the presence and absence of biotin showing complete biotinylation of the protein. Samples of Input (In) and unbound (Ub) fractions were analyzed by SDSPAGE and scanning of the gel using stain-free dye technology. (D) Streptavidin was added to liposomes containing fluorescently labeled Ubc6-SBP. After addition of buffer, biotin, or biotinylated protein, liposomes were floated in a sucrose step gradient. The top fraction of the gradient was analyzed by SDS-PAGE and the gel scanned using stain-free dye technology to visualize streptavidin. Ubc6 gives a strong signal here, because the fluorescent dye is also detected with the scanner used. (E) Quantification of co-floating streptavidin from two experiments as in (D). Streptavidin signal was first normalized to Ubc6-SBP signal and then normalized to the signal in buffer only control. (F) Ubiquitination of fluorescently labeled Ubc6-SBP. Samples of the ubiquitination reaction from experiments as in Figure $6 C$ were analyzed by SDS-PAGE and fluorescence scanning. 